\title{
1995 \\ Laboratory-Directed Research and Development Annual Report
}

Compiled by

D. P. Cauffman, Chief Scientist

D. L. Shoaf, Manager, Academic and Professional Affairs

D. A. Hill, Senior Administrative Specialist

A. B. Denison, Senior Consulting Scientist 


\section{Abstract}

This report summarizes progress of the Lockheed Idaho Technologies Company (Lockheed Idaho) Laboratory-Directed Research and Development (LDRD) program during the fiscal year 1995 at the Idaho National Engineering Laboratory (INEL). The report includes progress reports from one hundred eighty-two individual $R \& D$ projects in five categories. The LDRD program is under the auspices of DOE Order 5000.4A.

\section{Report}

This report is issued by the Idaho National Engineering Laboratory (INEL). The INEL is a multiprogram engineering and science laboratory operated by Lockheed Idaho Technologies Company for the Department of Energy.

Inquiries about the INEL LDRD Program may be directed to the LDRD Office:

Debonny L. Shoaf

Lockheed Idaho Technologies Company

P. O. Box 1625- 2214

Idaho Falls, Idaho 83415

Telephone 208-526-0430

e-mail dls@inel.gov.

This report was prepared as an account of work sponsored by the United States Government. Neither the United States nor the United States Department of Energy, nor their employees, subcontractors, or their employees, (1) make any warranty, express or implied, or assumes any legal liability of any information, apparatus, product, or process disclosed, or represents that its use would not infringe privately owned rights; not (2) endorses or approves, either expressly or by implication any apparatus, product, or process or the use thereof covered by this report. 


\section{DISCLAMMER}

Portions of this document may be illegible in electronic image products. Images are produced from the best available original document. 


\section{Contents}

The Scientific Method . . . . . . . . . . . . . 1

Laboratory-Directed Research and

Development Program Overview .......

National Integrated

Environmental Management

Assessment Methodology for Enhanced

Bioremediation of Hydrocarbons in

the Petroleum Industry . ...............

Catalyst-Assisted Sonochemical Treatment

of Hazardous Organic Wastes . . . . . . . . . . .

Synthesis, Characterization, and the Modes

of Interaction in Volatile Organic Compound

Sensor Materials .....................

Integration of Sample Introduction Techniques,

Torch Design, and Optics for the Determination

of Trace Metals in Biological Samples with

ICPAES .........................

Removal of Chlorinated Solvents from Air

Streams Using Anaerobic Biofiltration ........

Using Sawdust for Microbial

Abatement of Acid Mine Drainage ..........

High-Energy Computational Dosimetry for

Neutron Radiotherapy ...............

Photocatalytic Treatment of Anionic

Cyanides

Catalytic Destruction of Halogenated

Organics at Supercritical Fluid Conditions ......

Destruction of PCBs in Oil Using Gamma

Irradiation and Ozone...$\ldots \ldots \ldots \ldots \ldots$

Bioprocess Development for the Remediation

of $\mathrm{Cr}(\mathrm{VI})$ - and $\mathrm{U}(\mathrm{VI})$-Contaminated Soils and

Groundwater ......................

Ultraviolet Volatile Organic Compound

Destruction Technology, Liner Lifetime

and By-product Testing with Hazardous

Waste Disposal Considerations ............

Portable Photon Analysis Spectrometer

for TRU and Gamma Assay . . . . . . . . . . . . .

Conceptual Design of a 55-Gallon Waste

Container Refurbishment System
Photodissociation of Mercuric Chloride

Treatability Study $\ldots \ldots \ldots \ldots \ldots \ldots \ldots$.

INEL-University of Idaho Laboratory for

Liquid Extraction and Ion Exchange Research ...

Fracture Mechanics-Based Design Methodologies for Metal Matrix Composite Material .........

Develop, Build, and Test Assay System

to Measure Transuranic Waste and Fission

Product Contamination in Subsurface Soil ..... .

Biodecontamination of Massive Concrete

Structures .......................

Catalyst Assisted In Situ Bioremediation . . . . . 32

Sustainable Water Resources Management .... 33

High-Sensitivity Nondestructive Transuranic

Waste Examination for Characterization ..... 35

Flexible Fish Conduit Passive System . . . . . . . 36

Development of a Methodology for

Assessment of Power Plant Emissions

on Air Quality-related Values ............ 38

Passive Containment of Volatile Organic

Soil Vapors . . . . . . . . . . . . . . . . . . . . . . 39

Development of Vadose Zone Monitoring

Instruments ................... 40

Intelligent Sensor Data Acquisition Analysis . . . 41

Modeling Underground Structures ......... 42

Neural Analysis Techniques for Field-

Deployable Environment Sensors ...........

Grouping and Modeling Ecosystem

Components for Ecological Risk Assessment ... . 45

Water Shutoff in Producing Oil Wells

Using Bacteria .................. 46

Estimating Sorption Properties of

Clay Materials .....................

Identification of a Surfactant/Alcohol/Polymer System for Safe Mobilization of TCE . . . . . . . . 49

Aquatic Vascular Plant Control . . . . . . . . . 51

Bioprocessing of Indigo Textile Dye

Waste Effluent ................... 
Removal of Methanol from Forest Products

Industry Off-gases by Biofiltration .........

Using Nutrient-Diffusing Substrata to

Conduct Rational Bioremediation ...........

Intermediate/Field-Scale Model for

Predicting Microbial Transport ............

Prompt Gamma Neutron Activation Analysis

Methods Development for Waste Assay

Applications . . . . . . . . . . . . . . . .

Real-Time Asbestos Analysis by Raman

Spectroscopy..$\ldots \ldots \ldots \ldots \ldots \ldots \ldots$

Rapid Agrochemical Sensor

In Situ Biodegradation of High-Energy

Materials ............................

Rapid Laser Assay of Surface Contamination

during Decontamination and

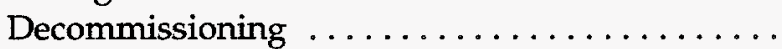

Measuring the Component Ratio in Oil/

Water/Gas Mixtures and Determining

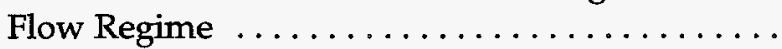

Polyethylene Sulfur Cement: Feasibility as

a Waste Encapsulation Material for Low-Level

and Mixed Wastes ......................

In-Field Assay for Parts-per-Million Levels

of Chromium in Environmental Media ........

Low-Temperature Reductive Catalytic

Destruction of Halogenated Organics

Periphyton Communities for Environmental

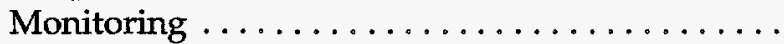

Tolo Lake Mammoth Excavation ............

Alternate Material Stainless Steel Lined Concrete . .

Oxidative Processes for the Remediation of

Contaminated Soils .....................

Solid Phase Microextraction ............ 72

Synthesis and Characterization of $\mathrm{Pt} / \mathrm{Pd}$

Compounds for a Fiber Optic Based

Sensor for Volatile Organic Compounds ...... . 73

ICPP Testbed Framework and Field Tests .... 74

Electrocatalytic Vapor Sensor . . . . . . . . . . 75

Characterization of Fuel Processing/Waste Form

Chemistry by High-Temperature Mass

Spectrometry
Telepresence for Mobile Robotics in Nuclear

Environments .....................

Sodium-Bearing Waste Treatment Technology

for Separating the Nitrate Anion and Alkali

Salts Using Ion-Exchange . . . . . . . . . . . .

The Effect of Thermal Treatment of INEL Soil

Prior to Cementitious Solidification .........

Automatic Pressurized Filtering of Environmental

Samples for Remote Applications ...........

Position Tracking for Remote Subsurface

Characterization Systems ..............

Evaluation of Delphi Oxidation for Activated

Carbon ........................

Evaluation of EET Proprietary Decontamination . .

Nuclear Operations and Nuclear Materials

Dispositioning ..................

ATR Radioisotope Production and

Purifications Study .................

Neutron Delivery System for Fast-Neutron

Radiotherapy ....................

Real-time Measurement Dosimetry for

Boron Neutron Capture Therapy . . . . . . . . .

Mixed Oxide Fuels Testing in the Advanced

Test Reactor ...................... 92

General Purpose Time-Dependent Particle

Transport with Isotope Generation and Depletion . .

Development of a Simplified Version of

SCDAP/RELAP5 for Simulator Applications .... .

Nonfertile Fuel Fabrication Development and

Irradiation Performance Assessment . . . . . . . . 96

Photoneutron Studies for Radiotherapy

Feasibility Study of Using INEL-Developed

Transport Tools to Calculate Photodynamic

Dosimetry .........................

Kinetic Modeling of the ITER Dissipative

Diverter .........................

Automated Neutron Probe Monitoring . . . . . . . 101

Tritium Production by Irradiation of $3 \mathrm{He}$. . . . . 103

Technetium Separation System . . . . . . . . . 105

On-Site F-18 Production . . . . . . . . . . . . 105

WERC University Design Competition

107 
National Infrastructure Technology

Test/Evaluation . . . . . . . . . . . . . . . . 109

Microstructural Influences on Corrosion

Advanced Combined Environments Test

Station Scoping Studies . . . . . . . . . . . 112

Characterization of Aged Materials with NDE

and Internal Friction Measurements

National Derived Use

Technology Transfer Center

Nondestructive Examination of Bottom-

Crimped Seam on 55-gallon Drums

High-Transition Temperature SQUID

(Superconducting Quantum Interference

Devices)-Sensor Nondestructive Evaluation .....

Robust Nondestructive Evaluation Using

Photorefractive Nonlinear Optics

Polymers-Water Separations, Ozone/Oxygen

Separations, Platform, and Laser-Assisted

Polymerization

Iron Aluminide Filters for Improved

Efficiency Fossil Energy Conversion

Wear Coatings for Aluminum Automobile

Engine Components

Factors That Determine the Microstructure

of Spray Deposited Materials ............ 126

Microbiology of Sugar Plant Flume Systems . . . 128

Alkylation Catalysts for Operation at

Supercritical Fluid Conditions

Mixed Microbial Encapsulated Fertilizer

Amendments

Sensors for the Control of Thermal Spray

Processes ... . . . . . . . . . . . . . . . . . . . . 131

Heat Transfer Through Solid Cryogen ....... 133

Concentration Technologies ............. 133

Site-Specific Technologies for Agriculture:

Pilot Project . . . . . . . . . . . . . . . . 134

Development of Nondestructive Fatigue

Monitor ....................... 136

Advanced Barcode (Holotag) Reader . . . . . . . 137

Transportation Network Core Software ...... 138
Optical Sensor Development for High-Voltage

Metering and Protection Applications

Development of Chemically Selective

Surfaces for Adsorption and Detection

of Gas-phase Contaminants . . . . . . . . . . . 140

Alloys by Design . . . . . . . . . . . . 142

Improved Process Control Thermocouples . . . . 143

National Infrastructure Renewal . . . . . . . . . 144

Optical Measurement Technology . . . . . . . . . 146

Velocity Sensor for Control of Thermal Spray ... 148

Hybrid Electric Vehicle Evaluation ......... 149

Demand Side Management Control

System for Electric Vehicles ............... 150

Development of Intelligent Cooperative

Miniature Robot Systems . . . . . . . . . . . . . . . 151

Natural Gas Locomotives and

Transportable Power . . . . . . . . . . . . . . . . . . 152

Loom Feedback Controls . . . . . . . . . . . . . . 153

Highly Dynamic Materials Process

Modeling Using Particle Methods . . . . . . . . . 155

Advanced Plasma Processes for Black

Liquor Gasification ................. 156

Joining and Testing of Small-Scale

Automotive Components ... . . . . . . . . . . 159

Process Control Development for

Ultracapacitor Electrodes by Liquid

Injection Plasma Deposition . . . . . . . . . . . 161

Miniature Alternative Power Supply,

Solid Oxide Fuel Cell . . . . . . . . . . . . . . 162

Miniature Alternative Power Supply

Thermophotovoltaic ................ 163

Optimization-Based SIMPLEV . . . . . . . . . 164

Hybrid Vehicle Component Hardware-

in-the-Loop Feasibility Study . . . . . . . . . . . . 165

Software Metrics Program . . . . . . . . . . . . . . 167

Manufacturing Research ............. 168

Building Materials . . . . . . . . . . . . . 170

Smart HVAC Systems Design $\ldots \ldots \ldots \ldots \ldots 171$

High-Temperature Porous Cement Filters for

the Paper and Pulp Industry . . . . . . . . . . 173 
Silent Discharge Plasma for Treatment of

Automobile Exhaust . . . . . . . . . . . . . . . . . . . 175

Evaluation of Liquid Metal Technologies

Applicable to Radioactively Contaminated

Scrap Metal Recycling . . . . . . . . . . . . . 176

INEL Metal Recycle: Induction Melting . . . . . 177

Process Industries Industrial Improvement

Using Integrated Process Evaluation

Depleted Uranium Flywheels for

Stationary Applications . . . . . . . . . . . . 180

Simulation-based Prototyping for

Manufacturing-based Engineering $\ldots \ldots \ldots \ldots 180$

Readiness ..................... 182

Butterfat Management $\ldots \ldots \ldots \ldots \ldots \ldots \ldots$

Advanced Process Diagnostics System ........ 185

Miniature Tag and Covert Communicator . . . . . 186

INEL Multimedia Object Database $\ldots \ldots \ldots \ldots 188$

General User Interface Development

Environment ....................... 189

Intelligent Data Access and Retrieval System .... 190

C++ SAGE

Fractional Frame Augmentation

Development of Prompt Gamma Assay

Method for Mining of Phosphate Ore ....... . 192

Develop Intelligent Farm Management

System Database Prototype . . . . . . . . . . . . 194

Measurement of Potato Water Content . . . . . . 194

Numerical Investigation of Dynamic Pressure

Seals in Geologically Active Basins . . . . . . . . 196

Evaluation of Potential Antimicrobial

Properties of Malted Barley and Its

Agricultural Applications

Biological System for Organic Acid Removal

with Minimal Effluent . . . . . . . . . . . . . . . . . . . 198

Isolation of Gossypol Degrading Bacteria . . . . . 199

System for Growing Conifer Embryos . . . . . . 201

Improved Transformation and Regeneration

Techniques for Cotton . . . . . . . . . . . . . 202

Compressed Gas Safety Valve ........... 203
Internal-Reforming Solid Oxide Fuel Cell

Thermo-Chemical Modeling . . . . . . . . . . . . 204

Advanced Methods for Adaptive Control

for Automated Traffic Management ........ 206

System Dynamics Model for Intermodalism . . . 207

Alternate Joining Techniques for Textiles . . . . . 208

Real-Time, On-Loom Knit and Hand

Characterization Using Diffractive Optical

Methods .......................... 209

Micromechanical Analysis Capability

for Composite Materials . . . . . . . . . . . . 210

Metal Matrix Composites from Novel

Nitride Preforms . . . . . . . . . . . . . . . . . . . 212

Cooperative Industrial Research with

General Electric Lighting Products

Lifetime Extension and Increased

Reliability for Refinery Structures . . . . . . . 213

Solar Bimodal Technologies Demonstration . . . . 214

Weight in Motion (WIM) Sensor and

Signal Analysis Technology . . . . . . . . . . 216

Communications Spectrum Needs for

Short-Range Intelligent Vehicle

Highway Systems Communications

Developing a Nonlinear Electromagnetic

Modeling Capability Using the Finite-

Difference Time Domain Method

Electrochemical-based Processing

Technologies

Nonaqueous Processing in the

Textile Industry $\ldots \ldots \ldots \ldots \ldots \ldots \ldots \ldots . \ldots 221$

Offline Manufacturing Simulation . . . . . . . 222

Spray Forming Titanium Alloys into

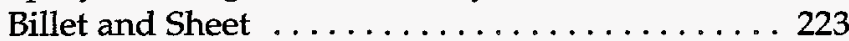

Industrially Compatible Laser Ultrasonics . . . . 225

Optimization of Automobile Component

Shapes ...................... 227

Mediator ..................... 228

Gas-Driven Photovoltaic Generators . . . . . . . . 229

Measurement of Crude Oil Corrosivity . . . . 230

Installation of Precession $x$-Ray Camera

for Single Crystal Analysis . . . . . . . . . . . 232 
Direct Production of Low-Cost High-Quality Titanium Powder by Mechanical Alloying 232

Petroleum Tanks Inspection Delivery System . . 233

Long-Term Research Initiatives . . . . . . 235

Molecular Biology 237

Nuclear Physics 239

Condensed Matter 242

Theoretical Chemistry 247

Computational Mechanics 249

Nonconventional Bioprocessing 252

Radiation Measurements
Nuclear Fuels and Materials 256

Experimental Thermal Science 259

Appendix A-Author Index A-1

Appendix B-Project Number and Title Index $\ldots \ldots \ldots \ldots \ldots \ldots \ldots \ldots \ldots \ldots$ B-1

Appendix C-Project Performance

Measures

Appendix D-Relevance to Major National Programs .................. D-1

Appendix E-1995 Refereed Publications ... E-1 Appendix F-Acronyms .......... F-1 
What hopes and fears does the scientific method imply for mankind? I do not think that this is the right way to put that question. Whatever this tool in the hand of man will produce depends entirely on the nature of the goals alive in this mankind. Once these goals exist, the scientific method furnishes means to realize them. Yet it cannot furnish the very goals. The scientific method itself would not have led anywhere, it would not even have been born without a passionate striving for clear understanding.

-Albert Einstein

Out of My Later Years 
Idaho National Engineering Laboratory LDRD FY 1995 Annual Report 


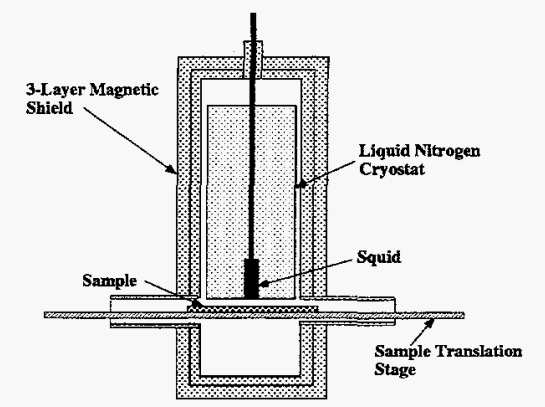

High tc SQUID-Sensor NDE (see page 119).

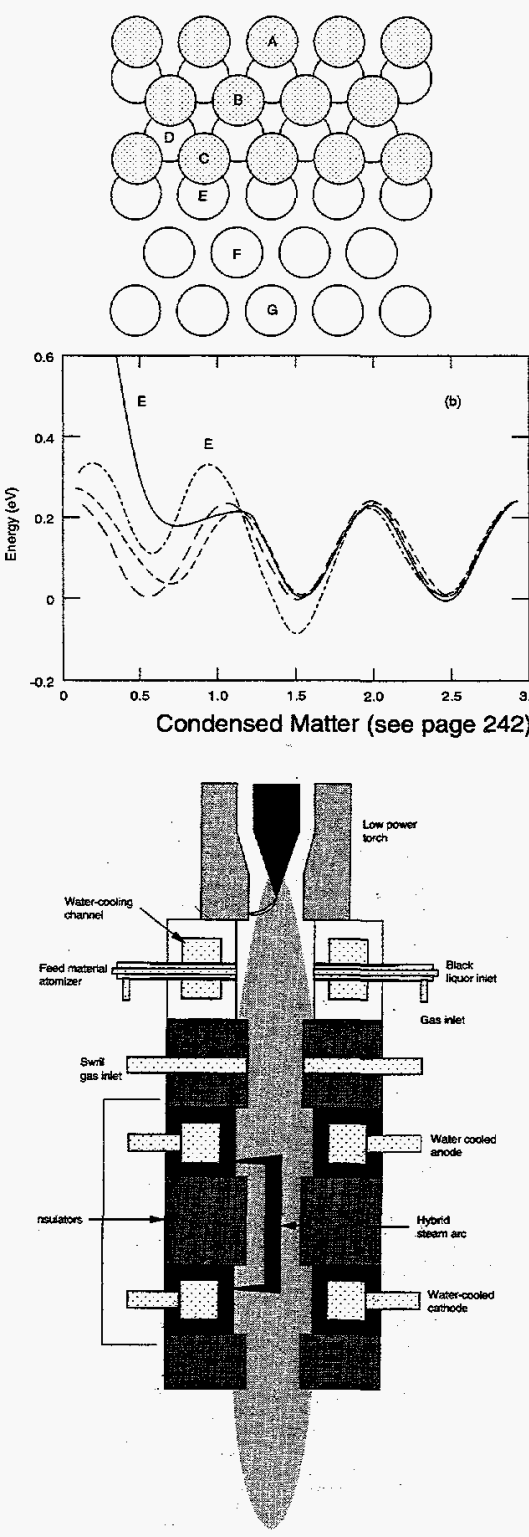

Plasma Processes for Black Liquor Gasification (see page 156)

\section{Laboratory-Directed Research and Development Program Overview}

\section{The Laboratory-Directed} Research and Development Program (LDRD) is a key component of the discretionary research conducted by Lockheed Idaho Technologies Company (Lockheed Idaho) at the Idaho National Engineering Laboratory (INEL). The threefold purpose and goal of the LDRD program is to maintain the scientific and technical vitality of the INEL, respond to and support new technical opportunities, and enhance the agility and flexibility of the national laboratory and Lockheed Idaho to address the current and future missions of the Department of Energy.

The LDRD Program is a key link between technology users and technology developers in formulating a strategically aligned research and development portfolio that addresses both current and future technology needs of the INEL, the Department of Energy, and the U.S. national needs. The LDRD Program promotes synergistic partnerships with universities to engage the diverse research resources that complement those of the INEL. Collaboration with other national laboratories maximizes the value of the LDRD investment. Based on an understanding of the technology needs of industrial sectors, the Laboratory can leverage its existing resources to bridge the gap between embryonic or emerging technologies and their industrial applications. LDRD projects are the seed corn research and development that sustains the vitality and value of the national laboratories. Technologies developed through LDRD projects generate worldrenowned scientific expertise and licensable intellectual property that strengthen the economic capabilities of the United States.

Lockheed Idaho Technologies Company became the management and operating contractor at the Idaho National Engineering Laboratory at the outset of Fiscal Year 1995. The LDRD portfolio was reoriented to more closely align research to support new missions and business objectives. The INEL's relatively new status as the lead Environmental Restoration and Waste Management (EM) Laboratory in the DOE complex is reflected in the distribution of research projects in the portfolio.

The LDRD portfolio comprises five major categories, or thrusts. The National Integrated Environmental Management (NIEM) Thrust encompasses a wide variety of operational and innovative research and development programs directed at waste management, environmental restoration, pollution prevention, and integrated environmental management. The products of this thrust would be faster and cheaper cleanup, commercialization of environmentally derived technologies, and the ability to influence environmental policy and programmatic decisionmaking within and outside the DOE complex.

The Nuclear Operations and Nuclear Materials Dispositioning (NO\&NMD) Thrust comprises the wide variety of nuclear research and development programs at the INEL. Research extends over both industrial and medical uses of radiation science and technology, with emphasis on significant commercial potential and on building 


\section{FY-95 LDRD Program Statistics}

Total projects

Smallest project

Largest project

Average project

Average project (FTE)
182

$\$ 9 \mathrm{~K}$

$\$ 406 \mathrm{~K}$

$\$ 84 \mathrm{~K}$

0.75 major technical contributions to nuclear materials production, fissile materials dispositioning, and nuclear nonproliferation.

The projects in the National Infrastructure Technology Test/Evaluation Center (NITTEC) Thrust focus on capabilities and products that would minimize risks and costs in sustaining the U.S. infrastructure. Beginning with aging nuclear plants, this research enhances the safety and economic security of the nation by minimizing risks and costs for extending structural life, maintenance and surveillance of existing structures, rehabilitation and retrofit of structures affected by natural disasters, and design and construction of new structures

The National Derived Use and Technology Transfer Center (NDUTTC) Thrust facilitates dual use of investment of public resources (made for public benefit) for both DOE mission support and for derived, industrial use in the areas of energy, environment, and national security, aided by innovative policies, procedures, contracts, and industry- government partnerships. This thrust is expected to lead to large-scale and engineering prototype demonstration projects at the INEL.

Long-Term Research Initiatives (LTRI) sustain core competencies or develop such competencies in areas deemed critical for future missions. The research activities tend to be fundamental in nature, focusing on the creation of new knowledge rather than on near-term application. The remaining eighty-one percent of the LDRD portfolio comprises more highly focused projects that span the technology development spectrum from fundamental to applied.

Research and development is of value because it enhances our ability to address the technological needs of our existing customers, and because it enables us to address the needs of potential customers. The projects in the FY-95 portfolio were selected based on their potential to realize a return on investment. This annual report

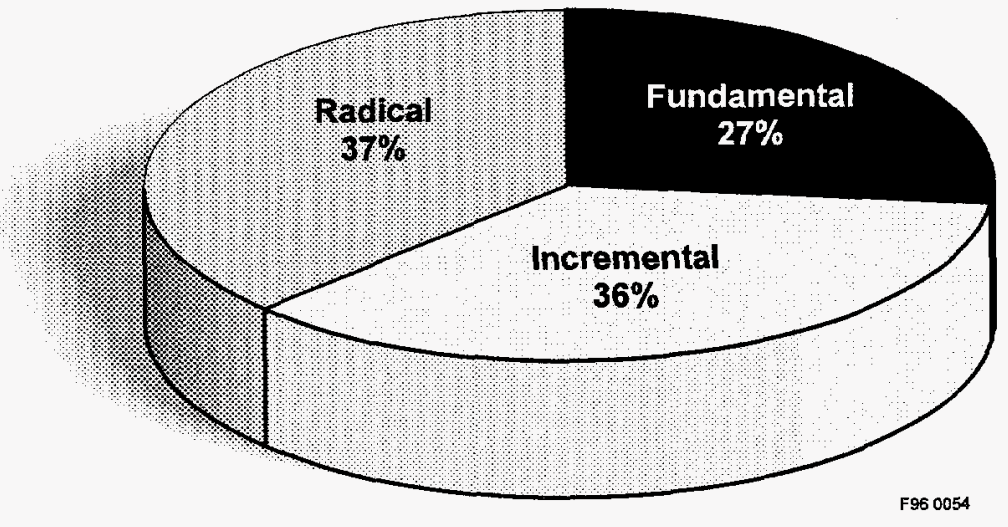

Classification categories taken from Third Generation R\&D, Roussel, Saad, Erickson (1991)

catalogues and organizes information about these projects in a variety of ways that illustrate the balance in the portfolio.

The following sections of the report present summaries of each of the 182 projects funded in Fiscal Year 1995 grouped by the five strategic thrust areas.

The figure below displays the FY-95 LDRD portfolio categorized by potential return on investment. The three R\&D-type categories are from Third Generation RED, Roussel, Saad, and Erickson (1991). Approximately $36 \%$ of the FY-95 LDRD portfolio pursued incremental research, focusing on novel exploitation of existing scientific and engineering knowledge (generally, low risk, moderate reward). Approximately $37 \%$ of the portfolio pursued radical research, focusing on creation of new knowledge for specific business objectives (generally higher risk, high reward). And approximately $27 \%$ of the portfolio pursued fundamental research, focusing on the creation of new knowledge to broaden and deepen understanding of a scientific or engineering area (generally, high risk, uncertain applicability to identified business needs). The portfolio was generally balanced in terms of anticipated risk and expected return on investment.

The figure at the top of the following page displays the FY-95 LDRD portfolio categorized according to the maturity of the technologies pursued. Again, the categories were taken from Third Generation RED. Because the role of the LDRD Program is to be the company's seed corn, an understanding of technology maturity is extremely important. From it is gained insight useful in developing $R \& D$ strategies, marketing decisions, estimating probability 


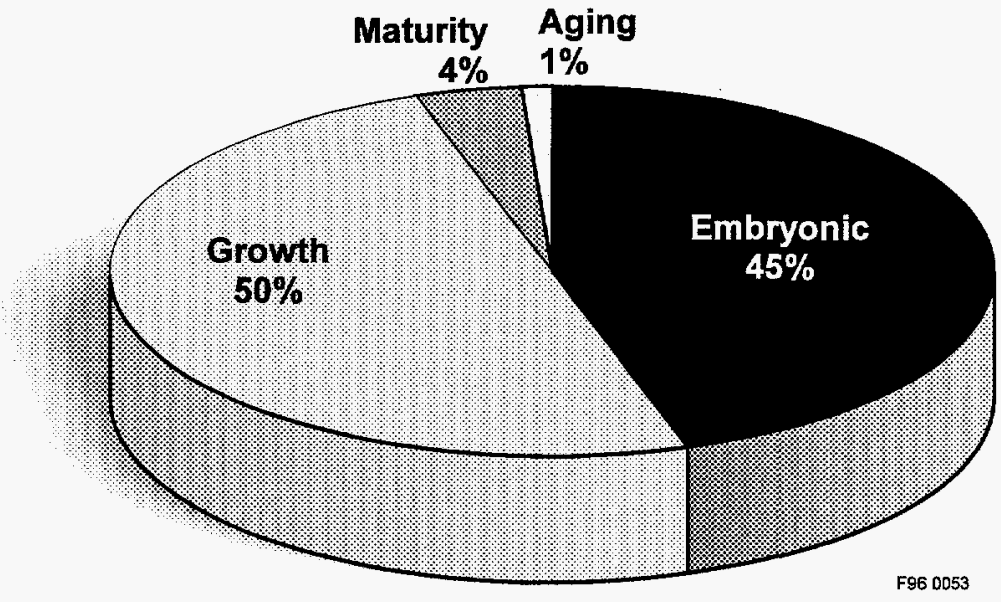

$R \& D$ technology maturity taken from Third Generation R\&D, Roussel, Saad, Erickson (1991)

of success, etc. Approximately $45 \%$ of the LDRD portfolio pursued embryonic technologies. Embryonic research is characterized by application within 7 to 15 years and high durability of technical advantage. Approximately $50 \%$ of the LDRD portfolio pursued growth technologies. Growth research is characterized by application within 2 to 7 years and moderate durability of technical advantage. Only $4 \%$ of the portfolio focuses on mature technologies, (within 1 to 4 years and fair dura- bility of technical advantage). Less than $1 \%$ of the portfolio focuses on aging technologies (within 1 to 4 years with very short durability of technical advantage).

Appended to this report are indexes to the LDRD project summaries organized by author (Appendix A) and by title and project number (Appendix B). Specific performance measures for each LDRD project are presented in Appendix $\mathrm{C}$ and summarized in the table below. A matrix presents the FY-95 LDRD projects

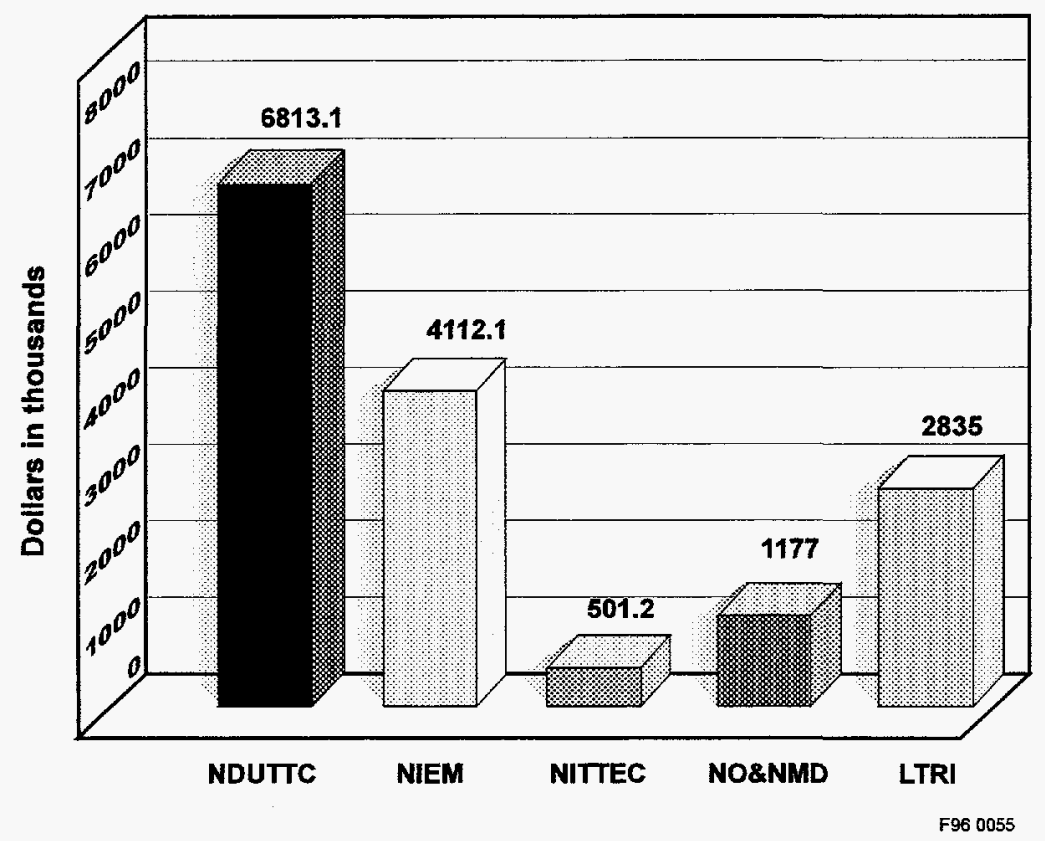

FY-95 Performance Measures

Refereed publications

Symposia organized or chaired

Invited presentations

Patent disclosures*

Patent applications*

Patents awarded*

Licensing agreements*

Copyrights*

Cooperative research and development agreements Work for Others agreements Students supported

*These data represent a snapshot of multiyear LDRD Program intellectual property pursuits as of end of fiscal year 1995.

showing application to major national programs (Appendix D). Also included is a list of refereed publications generated through LDRD work (Appendix E), and a list of acronyms used in this publication is attached (Appendix F).

Questions concerning specific LDRD projects or the Lockheed Idaho LDRD Program can be directed to Debonny Shoaf, LDRD Office, Lockheed Idaho Technologies Company, either at P. O. Box 1625, MS 2214, Idaho Falls, Idaho 83415; or by e-mail, DLS@inel.gov; or by telephone, 208-526-0430. 


\title{
National Integrated Environmental Management
}

\author{
Strategic Thrust
}

Treat ideas emerge from the common cauldron of
privalectual activity, and are rarely cooked up in
-James R. Newman original recipes.
Scientific American 


\section{Assessment Methodology for Enhanced Bioremediation of Hydrocarbons in the Petroleum Industry LDRD 102}

\section{J. H. Wolfram}

Many sites in the world are contaminated with toxic and hazardous organic chemicals, including petroleum products. The contaminating substances often have migrated through the vadose soil zone and are entering the ground water in the aquifers. Bioremediation using intrinsic microorganisms is a potential way of achieving clean-up. However, there have not been adequate ways to assess its potential, methods to optimize its rates of removal of the contaminants, or techniques to monitor its progress in the field. The results and methods developed are now being used to expedite clean-up and assessment for the progress of bioremediation at other sites in the State of Montana. A contract with Montana's Leaking Underground Storage Tank program is adopting some of the knowledge we have developed over the period of this project. There have been many spinoffs of this work, both at the academic and commercial levels. Some of this work is still ongoing at Montana State University, supported by the petroleum industry.

\section{Objectives}

- Monitor the bioremediation status at the Michigan siteState East Bay.

- Monitor the bioremediation status in the vadose zone at the Montana site.

- Develop respirometry techniques for determining the parameters critical for optimizing the rate of clean-up in the vadose zone.

- Perform mesoscale tests using a glass aquifer system to determine the dissolved oxygen demand in an aquifer contami- nated with BTEX (benzene, toluene, ethyl benzene, xylene) compounds.

\section{Accomplishments}

The site at State East Bay near Traverse City, Michigan, has been monitored for dissolved oxygen, $\mathrm{pH}, \mathrm{BTEX}$ levels, and cell enumeration since December 1991. Water samples were collected from each of the monitoring wells as well as the purge wells and tested for the above parameters. This year we have seen a dramatic reduction in one of the aquifers. The contours for the site are shown in the figure. A core sample was also gathered in an area where cleanup has occurred.
The Montana site became contaminated as a result of a pipeline rupture in 1988-1989, and about 185,000 gallons of unleaded gasoline leaked out. The vadose zone became saturated with hydrocarbons and over the years began weeping hydrocarbons into the aquifer below the bed rock level. In 1994, a trench was dug across the contaminated area down to bed rock at 28 feet. The soil was unsaturated but hydrocarbons were easily detected. Several prefabricated sensing and transfer pipes were installed, and the soil was returned to its natural setting.

The vadose zone below the 9-foot interval was devoid of oxygen, and at the 25-foot interval not only was oxygen depleted but high levels of

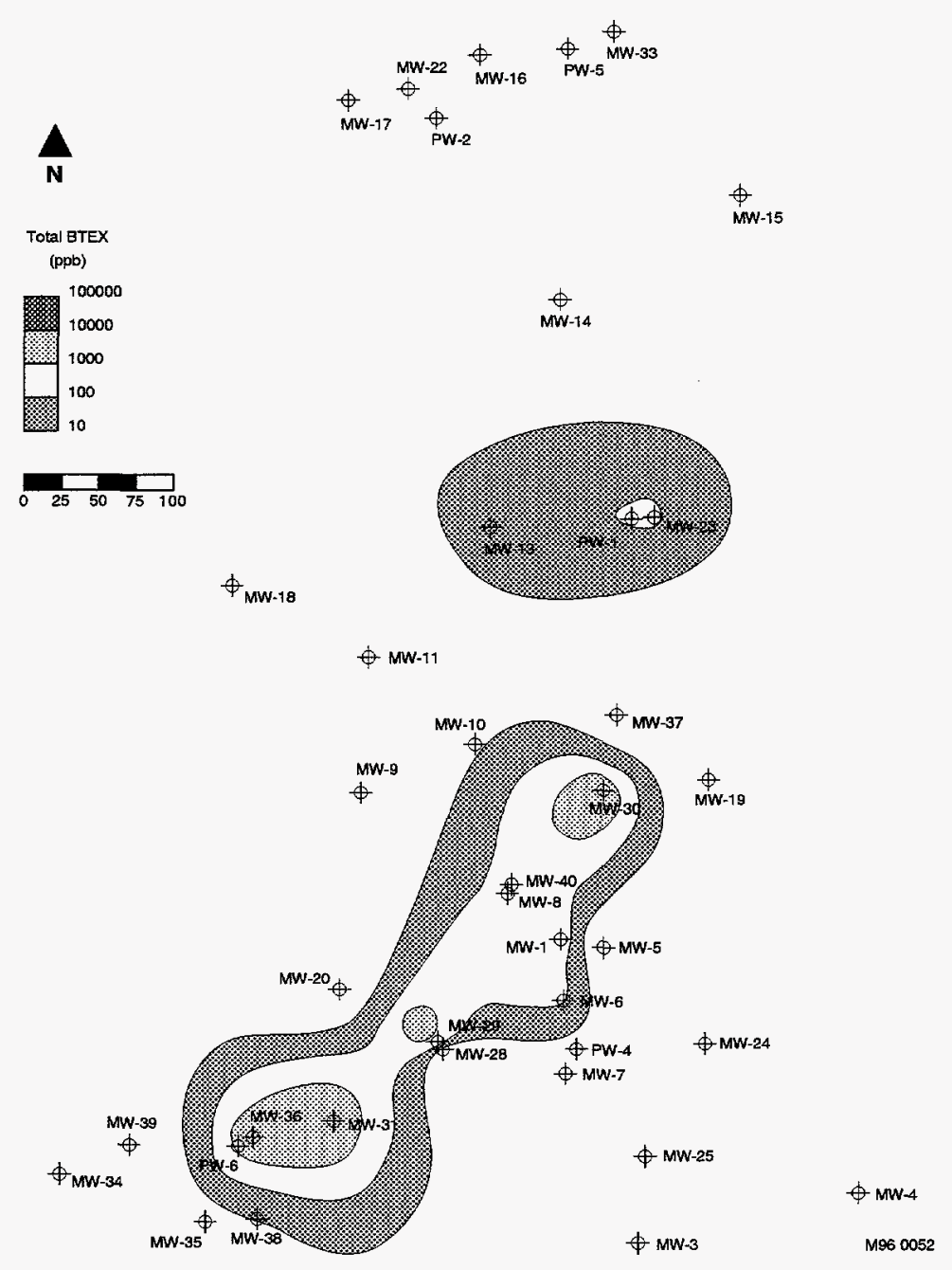


carbon dioxide and methane were found. These results were very surprising, different than what the experts would have predicted.

A respirometry technique was developed that showed the importance of the soil moisture content to the rate of bioremediation in these unsaturated soils. The soil moisture content was related to the bioavailability of selected aromatic hydrocarbons. A nondimensional bioavailability factor was generated, expressed either by a ratio of soil biodegradation rates to liquid biodegradation rates or as a function of degradation and sorption parameters. These results are important new information for optimizing the clean-up rate in arid areas.

The mesoscale glass aquifer has been designed and fabricated. Small Clark electrodes have been inserted into the apparatus for monitoring the oxygen levels in the saturated porous media. The aquifer has been filled with porous media taken from the coring at the Michigan site. This material has the intrinsic microbial flora present and will approximate what is taking place in the saturated aquifer that is contaminated with BTEX compounds. The work is still ongoing at Montana State University, where a graduate student is being mentored. The support is coming from Conoco.

\section{New or Increased Technical Capabilities}

Experience has been gained in assessing, testing, and implementing bioremediation treatments for in situ cleanups, and the knowledge is now assisting Montana State's Leaking Underground Storage Tank program. This group has signed a contract with MSU to assist them in developing faster and less costly ways of remediating the 1000 sites that exist in the state. Two students are being trained in this new technology, who will become the next generation of remediation engineers.

\section{Business Development Opportunities}

A number of states and petroleum companies could use our services in their daily remediation needs. All states have many leaking underground storage tanks, and many of the tanks sites have had migration of the petroleum hydrocarbons. Most small consulting engineering firms do not know how to implement or assess this new technology. They are afraid of bidding it in their proposals and are reluctant to try it on sites in progress of clean-up. Either a consulting service could be developed around this expertise, or some kind of on-the-job-training service that describes the fundamentals of implementation of bioremediation in the field and how to monitor its progress, and how to make modifications if needed during the cleanup process.

\section{Catalyst-Assisted Sonochemical Treatment of Hazardous Organic Wastes} LDRD 242

$$
\text { R. B. Wright }
$$

This project investigated the catalyst assisted sonochemical treatment of hazardous organic wastes. Sonochemistry is the use of acoustic cavitation to induce chemical reactions. Cavitation is the formation of gas bubbles (or cavities) in a liquid and occurs when the pressure within the liquid drops sufficiently lower than the vapor pressure of the liquid. Cavitation can occur from a variety of causes: turbulent flow, laser heating, electrical discharge, boiling, radiolysis, or acoustic irradiation. The project dealt only with acoustic cavitation which resulted from irradiation of liquids at frequencies of $20 \mathrm{kHz}$. Given that high temperatures and pressures can be induced in liquids during their sonication, we examined this phenomenon as it related to the destruction of hazardous organic liquids, specifically liquid halocarbons (VOCs) found in numerous waste sites [for example, carbon tetrachloride $\left(\mathrm{CCl}_{4}\right)$, methylene chloride $\left(\mathrm{CH}_{2} \mathrm{Cl}_{2}\right)$, and trichloroethylene ( $\mathrm{TCE}, \mathrm{CHCl}=\mathrm{CCl}_{3}$ )].

\section{Objectives}

The specific objective of this project was the determination of the effectiveness of sonochemical processing for the destruction of hazardous halogenated organics (VOCs) to less hazardous products. The project explored the chemical products resulting from sonochemical treatment of carbon tetrachloride, methylene chloride, and trichloroethylene and the affects of acoustic frequency, acoustic intensity, and bulk temperature on the products formed and the conversion efficiency of the process as outlined by the following test sequence:

- Sonication of neat liquid halocarbons.

- Sonication of halocarbon liquids mixed with water, methanol isopropanol, hexane or acetone (even if the halocarbon is not soluble in the solvent the sonication process causes emulsification of the two liquids).

- Sonication of neat liquid halocarbons with the addition of soluble (for example chloroplatinic acid, $\mathrm{H}_{2} \mathrm{PtCl}_{6}$, or palladium chloride, $\mathrm{PdCl}_{2}$ ) or supported (on gamma-alumina powder for example) $\mathrm{Pt}$ and Pd catalysts (note: the insoluble supported catalysts will become dispersed in the liquid during sonication).

- Sonication of halocarbon liq- 
uids mixed with water, methanol, isopropanol, hexane or acetone with the addition of soluble or supported Pt and Pd catalysts.

Prior to conducting the above experiments, a suitable sonication reactor was assembled from parts procured from a commercial source (see the figure). A gas chromatograph (GC) and a coupled gas chromatograph-mass spectrometer (GC-MS) were used for the qualitative and quantitative analysis of the reaction products formed during the sonochemical treatments.

\section{Accomplishments}

When sound passes through a liquid, it consists of expansion (negative pressure) waves and compression (positive pressure) waves. These cause preexisting bubbles to grow and recompress. This acoustic cavitation can lead to an implosive collapse of such cavities which can cause high-energy chemical processes to occur. Esti- mates of the high temperatures and pressures created during the collapse of the cavities range from $5,000 \mathrm{~K}$ to $10,000 \mathrm{~K}$ and $1,000 \mathrm{~atm}$ to $10,000 \mathrm{~atm}$. Sonochemistry is strongly influenced by a variety of external parameters, including acoustic frequency, acoustic intensity, bulk liquid temperature, static pressure, choice of ambient gas, and choice of solvent.

The efficiency and selectivity of the sonochemical treatment of the VOCs was studied by varying those parameters (given above and discussed below) which directly influence the sonochemical process and also by introducing a catalyst into the reactor during sonication. By varying these parameters and by the use of a catalyst it was possible to convert the VOCs to less harmful products. (It may also be possible to control the selectivity of the process to form specific nonhazardous value-added products useful in other industrial processes.) This project demonstrated the efficacy of the sonochemical process as

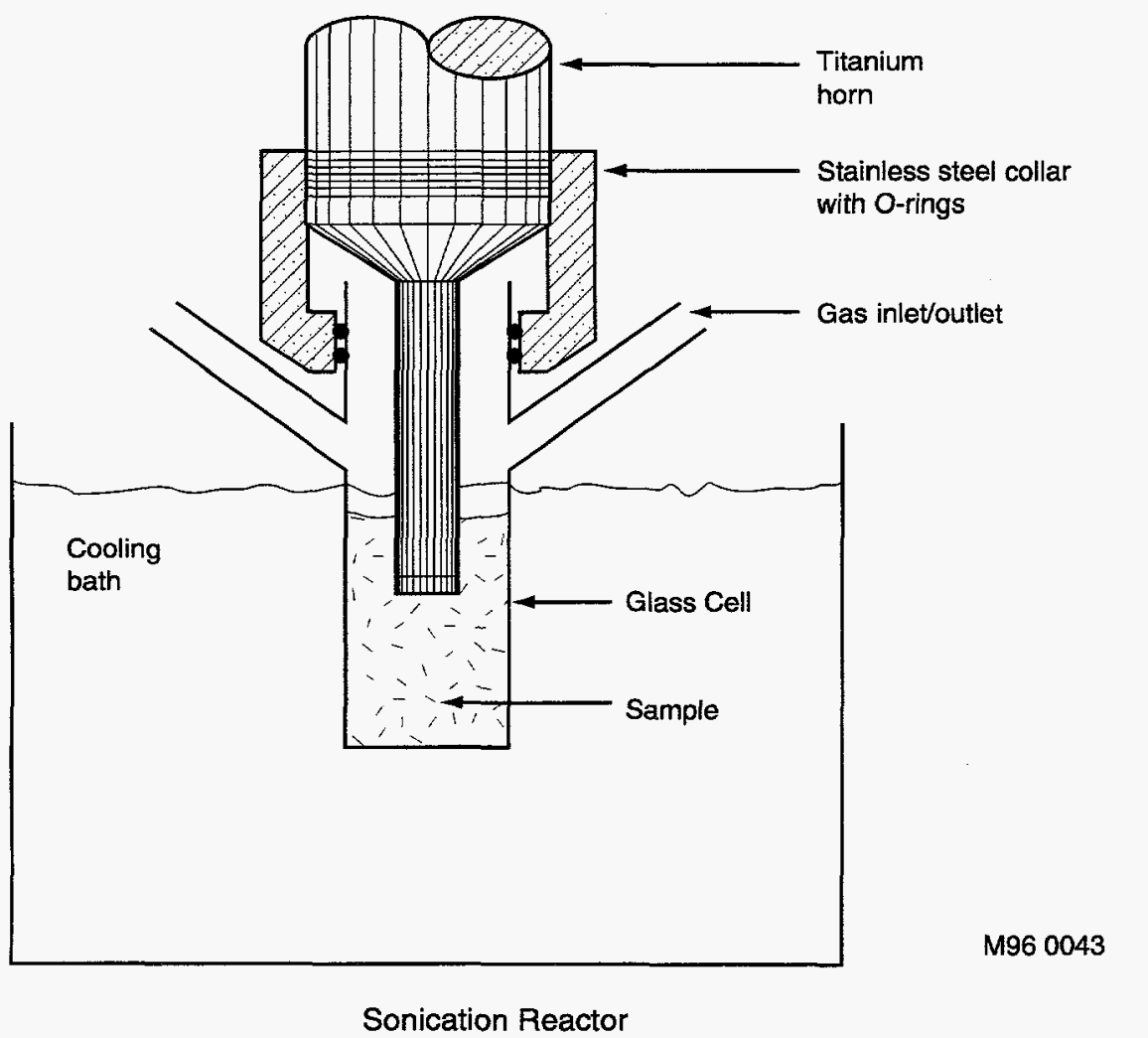

a new method for the remediation of hazardous VOCs. The results indicate that liquid wastes containing up to 10 volume $\%$ of selected halocarbons can be converted with up to $100 \%$ efficiency to $\mathrm{CO}, \mathrm{CO}_{2}$ and $\mathrm{HCl}$ at room temperature and atmospheric pressure after sonication at $20 \mathrm{kHz}$ for 1 hour.

The project examined the conversion of carbon tetrachloride $\left(\mathrm{CCl}_{4}\right)$, methylene chloride $\left(\mathrm{CH}_{2} \mathrm{Cl}_{2}\right)$, and trichloroethylene $\left(\mathrm{CCl}_{2}=\mathrm{CCl}_{2}\right)$ when mixed (10 volume \% concentration) with methanol $(\mathrm{CH} 30 \mathrm{H})$, isopropanol [ $\left.\left(\mathrm{CH}_{3}\right)_{2} \mathrm{CHOH}\right]$, hexane $\left(\mathrm{C}_{6} \mathrm{H}_{14}\right)$, acetone $\left(\mathrm{CH}_{3} \mathrm{COCH}_{3}\right)$, and water. The halocarbons were also mixed with combinations of these solvents. A marked dependence of reaction was observed on the choice of resolvent used. The addition of $100 \mathrm{mg}$ of a 1 weight $\% \mathrm{Pt}$ on gamma-alumina catalyst to the mixture during sonication further promoted the sonochemical assisted decomposition to approximately $100 \%$ for all of the compounds. The $\mathrm{pH}$ of the solution decreased to approximately 1 to 2 in all cases where there was a decrease in the amount of halocarbon after sonication. This is a new and very exciting observation! That is, the addition of heterogeneous catalysts to the mixture during sonochemical processing can indeed further increase the rate and conversion efficiency at which the halocarbons decompose. Our results (a Patent Idea Record is being submitted), and results previously reported in the literature, would indicate that sonochemical treatment of aqueous and nonaqueous liquid wastes is a new, easily implemented and potentially very economical method of remediating liquids that contain hazardous organic compounds.

\section{New or Increased Technical Capabilities}

This project addressed the development of a low temperature 
and pressure process (i.e., the reactor itself is operating under these conditions, but the effect is induced by the high temperatures and pressures generated on the microscopic level), which can be scaled to large volume processing, and showed the secondary waste streams which are generated (for example, $\mathrm{CO}, \mathrm{CO}_{2}$, $\mathrm{HCl}$ and $\mathrm{HF}$ ) are easily treatable.

Sonochemistry offers the potential, as yet not demonstrated on a commercial scale, for the remediation of VOCs; if not the selective conversion to valueadded products, then at least to the more easily handled gaseous products. This approach may also be applicable to the destruction of other hazardous chemicals such as PCBs, dioxins, herbicides, germicides, insecticides, freons and chemical warfare agents. The technology is attractive as it is a low temperature and low pressure process, and large-scale sonochemical equipment and process reactors have, and are being used for other industrial applications. It is not currently known if batch or continuous processing of the waste is the optimal method of treatment.

The further development of this technology will permit the INEL to become a major force in the implementation of sonochemical processing as a means for remediating hazardous compound containing liquid waste streams.

\section{Business Development Opportunities}

This LDRD permitted the establishment of a working relationship with a company that has developing industrial capabilities in the area of sonochemistry: Montec Associated, Inc. of Billings, MT. With continued DOE support is should be possible to combine the INEL's experience in this area and the new catalyst assisted findings with the promising large-scale, low-frequency sonochemical processing technology owned by Montec Associated, Inc. (trade-marked as
ResonantSonics) to establish a joint project that would lead to the commercialization of this remediation methodology and the establishment of licensable patent coverage of the findings.

\section{Synthesis, Characterization, and the Modes of Interaction in Volatile Organic Compound Sensor Materials LDRD 247}

\section{G. A. Moore, J. R. Kennedy}

Chemical sensors play an ever increasing role in industrial process control, emission monitoring, waste site characterization, and remediation activities. This effort was directed towards the discovery and characterization of novel volatile organic compound (VOC) sensing materials that could be used in high temperature environments such as petroleum refinery cracking towers, incinerators, or flue stacks. The approach taken here was to synthesize crystalline inorganic compounds which have molecular level pore structures and chemical stability up to about $500^{\circ} \mathrm{C}$. Characterization of the synthesized materials include powder $x$-ray diffraction to establish the crystal structure and Differential Scanning Calorimetry (DSC) to determine the chemical stability of the materials and their reactivity with guest molecules. To this end, attempts to prepare pure quantities of the compound $\mathrm{TaPS}_{6}$ were carried out. The compound was chosen since it is stable up to about $600^{\circ} \mathrm{C}$, its crystal structure is already known (contains channels with free diameter of about $4.65 \AA$ ) is structurally related to a number of other potentially useful phases, and since many of its physical properties are still unknown (as well as apparently an optimized synthetic procedure), their determination, measurement, and application to sensing devices could form the basis for a patent.

\section{Objectives}

- $\quad$ Prepare $\mathrm{TaPS}_{6}$ in sufficient quantity to measure physical properties. Likely to entail optimizing synthetic procedure and equipment/apparatus setup and adjustment at INEL laboratories.

- Measure assorted physical properties of target compound if enough compound can be prepared. These may include $x$-ray data, thermal behavior (differential scanning calorimetry), and, if possible, electrical conductivity and optical data.

- Expose/react target compound with volatile organic compounds at various temperatures and determine any change in properties.

- Prepare and submit patent application on findings and/or prepare, submit, and publish experimental findings in refereed journal if warranted.

- Present experimental findings as part of lecture at the spring 1996 national meeting of the American Chemical Society.

\section{Accomplishments}

The title compound $\mathrm{TaPS}_{6}$ was prepared by heating a stoichiometric mixture of the elements at $600^{\circ} \mathrm{C}$ for several days in an evacuated quartz ampoule. The structure is illustrated in the Figure and shows channels running in three dimensions with a free diameter of about $4.65 \AA$. Thus, small molecules can be anticipated to enter the channels, the presence of which should alter physical properties of the host material and provide a means for the materials to be used in sensor devices. By preparing the material at higher temperatures, it was hoped that it would be stable over a temperature range 


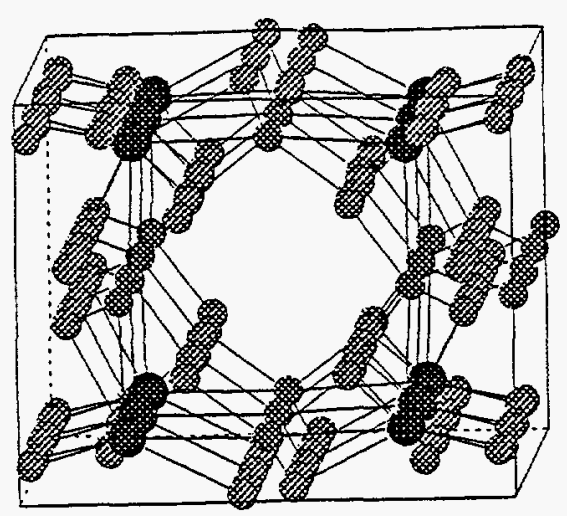

Structure of $\mathrm{TaPS}_{6}$, Showing Channel Running in One Dimension of Lattice

up to about $500^{\circ} \mathrm{C}$. In addition, it was hoped that the guest/host interactions would also occur at elevated temperatures for application in higher temperature processes.

$\mathrm{TaPS}_{6}$ could not be prepared in pure form, being contaminated with an as yet unidentified second phase. Since there are a number of compounds with stoichiometries close to $\mathrm{TaPS}_{6}$, the contamination is probably one of these structurally related phases. Thermal testing was performed to identify the stability and reactivity of the mixed material. Differential scanning calorimetry (DSC) revealed that the phases are stable over the temperature range 22 to $500^{\circ} \mathrm{C}$. Investigations on the reactivity of the host material mixture with methanol using the DSC technique show that reaction/interaction does indeed take place. Endothermic transitions occur at about 110,140, 161, and $169^{\circ} \mathrm{C}$. Since no clear transition is observed at ca. $65^{\circ} \mathrm{C}$ (the boiling point of methanol at 1 atmosphere pressure), it can be proposed that the transition at $110^{\circ} \mathrm{C}$ is the higher pressure liquidgas transition for methanol (experiment performed in sealed vessel).

The two transitions at $140^{\circ} \mathrm{C}$ and $161^{\circ} \mathrm{C}$ are intriguing since they must represent some type of interaction between the host material mixture and the gaseous methanol. It is not clear what the nature of these interactions is. Since the pure phase has not yet been secured, further measurements on the physical properties have not been performed.

Preliminary experiments have shown that $\mathrm{TaPS}_{6}$ and/or a related material will interact with gaseous methanol at elevated temperatures. The intimate mechanism(s) of the interactions are not yet known, nor are the effects on the physical properties of the material(s). The results are, however, extremely encouraging since it appears that these and related materials are feasible candidates for applications in chemical sensing devices operating in environments at temperatures far above $100^{\circ} \mathrm{C}$.

\section{New or Increased Technical Capabilities}

These preliminary results have given the INEL new expertise and experience in the area of high temperature chemical sensing materials. Specifically, $\mathrm{TaPS}_{6}$ and or a related phase have been shown to interact with gaseous methanol at temperatures between $100^{\circ} \mathrm{C}$ and $200^{\circ} \mathrm{C}$. These results are encouraging to pursue further studies to achieve sensing capabilities at temperatures above $200^{\circ} \mathrm{C}$. Continued work in this area would be to further and more fully investigate the $\mathrm{TaPS}_{6}$ material as well as the entire $\mathrm{Ta} / \mathrm{P} / \mathrm{S}$ phase region around this stoichiometry. Expanded efforts should be directed at designing new materials whose structures would accommodate guest compounds as well as investigating known materials for their propensity to interact in this manner.

\section{Integration of Sample Introduction Techniques, Torch Design, and Optics for Determining Trace Metals in Biological Samples with Inductively Coupled Plasma Atomic Emission Spectrometer}

LDRD 262

W. F. Bauer

There is a need for multielement analytical techniques to determine trace metal concentrations in biological samples. These analytical techniques must be suitable for adaptation to and routine use in clinical or toxicological laboratory environment. While there are many elemental spectroscopic techniques available for trace metal determinations in widely varied sample matrices, current commercial instrumentation is not necessarily easy to use and, therefore, is not readily adaptable to many clinical environments. The goal of this work was to develop analytical techniques and instrumentation for trace metal analysis in biological samples that would be specifically geared toward clinical and toxicological laboratories. Flow injection analysis, direct injection inductively coupled plasma torches, and direct analysis of slurried microliter samples of whole blood were to be investigated. The ultimate product was expected to be first-to-the-market, user friendly turn-key analytical systems for trace metal analysis in biological fluids. Interest was expressed in instrumentation with adequate sensitivity, dynamic range and the ability to provide short turnaround times that is also suitable for operation by technicians in a clinical or mobile laboratory setting.

\section{Objectives}

- Acquire a direct injection nebulizer (DIN)/plasma torch for the ARL 3520 inductively coupled plasma atomic emission spectrometer (ICPAES) and assess the ability of this torch 
to handle slurries of whole blood when operated in a flow injection analysis mode.

- Determine figures of merit for several elements using conventionally viewed ICP with DIN/plasma torch and FIA.

- Evaluate significant spectral and ionic interferences likely to be encountered in the analysis of whole blood using an axially viewed ICPAES instrument. Multivariate analysis tools were to be employed to compensate for these interferences.

Accomplishments

A series of 20 standards were prepared to evaluate the spectral and ionic interferences expected in the trace element analysis of whole blood by ICPAES and the ability of the analysis tool known as partial least squares (PLS) to compensate for these effects. These standards contained both the major $(n=7)$ and trace elements $(n=9)$ of blood in appropriate concentration ranges for each element. The concentrations were varied randomly with respect to each other so that there was little possibility that any two elements changed concentration in the same way. This would allow the PLS algorithm to determine what effect each element would have on every other element and define factors for each interaction. Net intensities were acquired at a total of 25 wavelengths using a conventionally viewed ICPAES instrument with a minitorch. Wavelengths were selected to represent the selected elements well when considering the concentration ranges expected, i.e., most sensitive lines for trace elements and less sensitive lines for macro elements. Initial results using the PLS algorithm were quite promising. For the macro elements and the most sensitive trace elements, correlation coefficients of $\sim 0.99$ were readily obtained. The less sensitive trace elements gave correlations of 0.96-0.98. A larger training set (i.e., more standards) and/or full peak profiles at each wavelength instead of a single intensity are expected to significantly enhance the correlations and lower the standard error. The net result should be significantly lower detection limits. This set of standards was also to be run on an axially viewed ICPAES with an array detector, so that both the sensitivity and the amount of information could be significantly enhanced. Unfortunately, a state-of-the-art, axially viewed ICPAES instrument is not available at the INEL, and the identified industrial collaborator with such an instrument was forced to change jobs, and contact was temporarily lost. This part of the project was not pursued farther.

A DIN/plasma torch was also purchased for in-house use, however, there were significant delays in getting all of the necessary parts to operate this torch. The net result was that other commitments for direct work were made and the project was not resumed due to time constraints. Less than $1 / 3$ of the total allocated funds were spent.

\section{New or Increased Technical Capabilities}

Even though there were circumstances that prevented this project from being completed, the work that was done did serve to increase capabilities at the INEL. The acquisition of the DIN/plasma torch will be useful in allowing the analysis of very small sample volumes with no waste. This will be useful for both conventional elemental determinations and speciation using hyphenated techniques such as HPLC-ICPAES. This tool will be useful for many missions at the INEL, including environmental, waste characterization and research support. The successful application of PLS to solve interfer- ence problems in ICPAES may also find application in the analysis of samples with very complex matrices and, possibly, development of lower-cost lower-resolution instruments for specific problems, e.g., effluent, waste stream, or process monitoring.

\section{Business Development Opportunities}

No new business opportunities were achieved during this early development stage.

\section{Removal of Chlorinated Solvents from Air Streams Using Anaerobic Biofiltration LDRD 303}
B. D. Lee

Subsurface soils and sediments contaminated with halocarbons, such as carbon tetrachloride or perchloroethylene (PCE), are often remediated using soil vapor extraction or air stripping of groundwater, producing a contaminant laden off-gas stream. This research seeks to optimize the operating parameters for a biofilter as part of the development of a commercially available vapor treatment system for the complete removal of chlorinated solvents such as PCE or carbon tetrachloride from off-gas streams. Our objectives are significant for the continued development of vapor-phase treatment systems for chlorinated solvents, and successful completion of them will ensure the INEL continued leadership in the development of gasphase bioreactors industry wide. Currently, there are soil vapor extraction operations removing carbon tetrachloride from subsurface soils at Hanford and at the INEL. Activated carbon has been used as the method for remediating off-gas streams created from these processes. The biofiltration system being developed has the potential to regenerate these carbon beds, or it could be used to treat the off-gas stream directly from the soil vapor extraction system. Continued development of these types of biofil- 
ters at the INEL will increase economic competitiveness of industries in the United States with industries in Europe, who are using biological treatment systems for their off-gases in many situations.

\section{Objectives}

- Determine optimum nutrient conditions for the removal of chlorinated solvents from air streams.

- Determine the inhibitory effects of mixtures of dechlorination products.

- Determine the inhibitory effect of oxygen on the removal of carbon tetrachloride.

- Determine a method for separating chlorinated solvents from oxygen in off-gas stream.

Accomplishments

Removal of carbon tetrachloride was determined using continuous-flow, laboratory-scale biofilters having a volume of $1.4 \mathrm{~L}$. The biofilter bed medium was compostbased and amended with lava rock to facilitate flow, and with calcite to control $\mathrm{pH}$. The biofilters were operated at an empty bed residence time of 28 minutes. Bed medium microorganisms were supplied with methanol as the primary source of carbon. Tests were run using inlet methanol concentrations of $500,1,000,1,500$, and 2,000 ppmv in an attempt to determine the effect of carbon source concentration on removal of carbon tetrachloride. Initial testing was done using nitric oxide (NO) as an added electron acceptor. In later testing, time-released fertilizer was added as a potential source of nitrogen in the absence of NO. Biofilters were operated for approximately 1 month without carbon tetrachloride in the feed stream. After establishing an anaerobic consortia on methanol, we fed carbon tetrachloride to the biofilters at a target inlet concentration of 50 ppmv. Maximum removal efficiencies in the range of $40 \%$ were achieved at methanol concentrations of greater than 1,000 ppmv in biofilters that had been amended with some form of nitrogen oxide compound, either in the form of fertilizer or as NO. The volumetric productivity of the biofilter under operating conditions was $0.3 \mathrm{~g}$ of carbon tetrachloride ( $\mathrm{m}^{3}$ of bed $)^{-1} \mathrm{hr}^{-1}$. Dechlorination products, such as chloroform, never appeared in the outlet of the biofilter, indicating that the carbon tetrachloride was completely degraded by the bed medium microorganisms. Since breakdown products were not observed, no inhibition studies were performed. Formation of acid from the release of chlorine from the solvent may cause decreased $\mathrm{pH}$ in the bed, leading to a loss in activity. The bed medium pH remained above 8 during the test period, indicating the calcite added to the bed was an adequate method of controlling decreasing $\mathrm{pH}$. Tests to determine the effect of oxygen on carbon tetrachloride removal are in progress. An oxygen concentration of $5 \%$ has been added to the biofilter inlet. Results are not yet adequate for discussion, but initial results indicate that oxygen has not decreased carbon tetrachloride removal. We need more time to adequately prove this hypothesis.

We chose an activated carbonbased oxygen/chlorinated solvent separation system owing to the natural sorption/desorption characteristics of the activated carbon. This system will allow for a steady, continuous flow of contaminant to the biofilter. This is advantageous because the biofilter could also be used to regenerate carbon canisters on site, whereas they are currently being regenerated away from the remediation site at increased cost to the operation.
New or Increased Technical Capability

Developing the anaerobic biofilter for removing chlorinated solvents will bring unique capabilities to the INEL. Continuous-flow anaerobic biofiltration has not been attempted at other national laboratories, in industry, or by academia. The research performed will also lead to increased expertise in the area of biofiltration. The Biotechnology Department at the INEL has been involved in the development of biofilters for numerous compounds, including petroleum hydrocarbons, chlorinated solvents, alcohols, terpenes, and nitrogen oxide compounds.

\section{Business Development Opportunities}

Successful completion of this research project will lead to the design and fabrication of a commercializable treatment unit for vapor-phase chlorinated solvents. The treatment system could be used by environmental remediation firms. Envirogen and Radian have already shown interest through ongoing dialog, which could lead to a licensing agreement. Consulting firms, such as Metcalf and Eddy have demonstrated interest in codeveloping a biofilter for the removal of chlorinated solvents from off-gas streams produced from sparging of contaminated groundwater.

\section{Using Sawdust for Microbial Abatement of Acid Mine Drainage LDRD 0306}

$$
\text { K. S. Noah }
$$

This research is determining whether sawdust can be used to support a bioprocess for the remediation of acid mine drainage (AMD). The proposed process is based on the 
metabolic activity of sulfate-reducing bacteria (SRB). SRB anaerobically reduce sulfates to sulfides, which in turn complex with metal ions to form metal sulfide precipitates; SRB also participate in nitrate reduction. As $S R B$ consume $\mathrm{H}+$ during the reduction of sulfates and nitrates, bicarbonate alkalinity is generated, resulting in neutralization of the solution as well. In an AMD environment, microbial activity is limited by the availability of organic substrates. Sawdust, a readiIy available, high-volume, low-cost forest industry waste product, could provide the necessary nutrients to support the SRB, which in turn, remediate the AMD. The mining industry is in need of passive systems that will provide long-term, low-cost, low-maintenance, self-sustaining technologies for AMD treatment, compared to conventional technologies. Sawdust reactors could provide a passive in situ immobilizing environment for the $S R B$ to precipitate metals, reduce sulfates and nitrates, and neutralize acidic water, hence meeting these criteria. Thus, an in situ biological process using sawdust may offer a long-term, cost-effective, alternative to conventional AMD treatments.

\section{Objectives}

- Perform batch studies to determine microbial toxicity of Kennecott leachate used in previous flow-through test.

- Revive bacteria in current reactors.

- Optimize the process with Kennecott's metal-laden leachate by examining the effects of flowrate, amount of sawdust, etc., on sulfate reduction, metal removal, and neutralization of the leachate.

- Determine preliminary design for scale-up of this process.

- Pursue funding for small-scale field trials, including selection of suitable field locations.

\section{Accomplishments}

Batch studies. After approximately 3 months of treatment of Kennecott leachate in FY-94, the leachate $\mathrm{pH}$ was unchanged, and no significant sulfate or metal reduction had occurred. A minimal inhibitory study was then conducted by adding various concentrations of the leachate to vials containing SRB, in which nutrients were not limited. We observed $90 \%$ sulfate reduction at full strength, suggesting nutrient limitation.

Revive bacteria. The bacteria in the reactor system were revived by passing a synthetic AMD solution containing lactate through the reactor. Sulfate reduction occurred, the $\mathrm{pH}$ increased, and the bacteria once again became viable.

Optimization. In order to determine the optimal process variables using Kennecott leachate, several factors were investigated, including substrate. For this study, flow of leachate through the revived reactor cells was started again at full strength; once again, the $\mathrm{pH}$ remained low, and no metal or sulfate reduction occurred after 2 months of continuous flow. Optimization of the process with this leachate was never achieved owing to process failure. Therefore, investigation of process variables that may have caused the failure, i.e., microbial toxicity or nutrient limitations, were further investigated. These studies revealed that the SRB were unable to revive themselves with lactate addition in the presence of leachate, suggesting leachate toxicity to the organisms, or N or P limitation. Nitrate and phosphate fertilizers were then added to the leachate and to the reactors. After 6 weeks with N/P-supplemented leachate, the $\mathrm{pH}$ has not changed, and no metal or sulfate reduction has occurred. Thus, either leachate toxicity to non-SRB microorganisms or carbon limitation at a preSRB step in the process is the likely cause of the process failure.

Scale-up and funding. We investigated this process for treatment of AMD for three agencies, including Cyprus Climax Metals Company, California State University at Fresno (through Dr. Karl Longley of the California Regional Water Quality Control Board), and the Idaho Division of Environmental Quality (for a mine in Northern Idaho). Packed columns using downflow were used for each treatment. Based on the results of the Idaho Division of Environmental Quality laboratory trial, a pilotscale system was designed for use in a field trial. We are currently negotiating with officials from this mine to begin a field trial on site in early FY-96. We have also identified several other potential sponsors and locations for field trials, which are discussed below.

New or Increased Technical Capability

Treatment of AMD was successful in the laboratory-scale bioreactor system. Biocatalytic remediation of AMD had not previously been attempted at the INEL; thus, this represents a new technical capability at the INEL and an increased capability in biological mining technologies in general. The next step for this system is field trials, which will allow us to study the process on site and also provide working examples to obtain further funding for additional development and design. We believe that our current understanding of the process, along with results obtained from potential field trials, will allow us to engineer full-scale systems that can successfully remediate AMD streams from both operational and abandoned mine sites more cheaply than conventional liming. 


\section{Business Development Opportunities}

Dr. Karl Longley is awaiting our results to see whether this technology will remediate his AMD; if so, this will provide an additional opportunity for a field trial. Thompson Creek Mines was willing to provide a site but could not provide funding. Other possible funding agencies are the Department of Transportation offices in several states, e.g., Idaho, Montana, etc., whose interest would arise from acid drainage from pyritic rock exposed (and later oxidized) during highway construction in mountainous areas. A one-page proposal for a field trial has been submitted to Cyprus Climax Metals Company.

Although the process failed with Kennecott leachate, some results obtained during this work allowed us to write a one-page proposal to study their heap pads. We negotiated the technical work scope under a new invited sponsored WFO contract during the last week of FY-95. Finally, we are currently working with Lockheed Idaho ES\&H, Lockheed Idaho Technology Transfer, a product manager from the Lockheed Idaho Mining and Minerals/Alternative Energy Products office, Idaho Division of Environmental Quality, and the owners of the Northern Idaho mine site to finalize the proposal for the field trial at their mine.

\section{High-Energy Computational Dosimetry for Neutron Radiotherapy} LDRD 1172

\section{W. Nigg, F. J. Wheeler}

This project is developing advanced computational methods and software for neutron transport and radiation dosimetry suitable for clinical application to fast-neutron therapy for cancer with neutron capture augmentation. The workscope is in support of Lockheed Idaho commitments to the lonix/Lockheed Idaho/Washington State University Cooperative Research and Development Agreement (CRADA) established in May, 1995.

\section{Objectives}

An extremely important aspect of all forms of radiotherapy for cancer is the need for pretreatment computation and optimization of the radiation dose distribution in the target volume. This so-called treatment planning process achieves the optimal therapeutic ratio (tumor dose relative to critical normal tissue dose) for every individual patient by a systematic procedure for specifying the appropriate irradiation parameters to be employed for a given treatment. These parameters include angle of therapy beam incidence, beam aperture and shape, and beam intensity as a function of position across the beam front. This project focuses on the extension of current INEL-developed advanced computational methods for Neutron Capture Therapy (NCT) to permit application to computational dosimetry and treatment planning of fast neutron radiotherapy with or without NCT augmentation. This will extend the range of clinical applicability of the INEL methodology to all known forms of neutron therapy, offering access to a much broader sector of the radiotherapy planning software market.

The current version of the INEL NCT dosimetry software package (referred to in the copyright documentation as BNCT_rtpe) has been licensed for beta testing to a number of NCT research centers worldwide, including Brookhaven National Laboratory, where it is currently used for human therapy planning. INEL is recognized worldwide as the leading resource for this partic- ular technology. The INEL dosimetry software is, however, limited in application to therapy modalities involving neutrons having energies of $17 \mathrm{MeV}$ or less. This is more than adequate for current practice in neutron capture therapy, but not for fast neutron therapy, where neutron energies up to about $66 \mathrm{MeV}$ are typically encountered. The objective of this work is to extend the range of applicability of the INEL software to neutrons having energies of $66 \mathrm{MeV}$.

Upon completion of the workscope, we will have completed software validated against experiment and independent computational methods. All of the existing geometric reconstruction algorithms, radiation transport algorithms, and radiation dose calculational algorithms that have been developed for neutron capture therapy are still applicable, though the radiation transport methodology will need to be augmented with an explicit recoil-proton calculational option in order to properly handle the direct fast neutron component of the total absorbed dose from high-energy neutrons. This will be based on a standard continuous slowing down model based on proton range-energy relationships that are well known. In addition, it will be necessary to include the effects of certain highenergy threshold interactions in the dose calculations. The complete package will be benchmarked against standard models from the literature as well as against appropriate measurements in tissueequivalent phantoms.

\section{Accomplishments}

Although the project was initiated in late FY-95, some initial tasks have been completed. We have identified and obtained an appropriate high-energy cross section data base for the elements that 
must be represented in biophysical models of radiation transport in tissue. In addition, we have initiated the incorporation of this data base into a new, experimental version of the currently-existing INEL neutron capture therapy dosimetry software.

\section{New or Increased Technical Capability}

One purpose of the CRADA is to integrate INEL expertise in neutron capture therapy with that of our CRADA partner in fast neutron therapy to create a synergistic product. Our niche will be in the field of computational dosimetry for all forms of neutron therapy, where we hope to hold a position of international technical and commercial leadership.

\section{Business Development Opportunities}

The INEL has been engaged for a number of years in the developing computational dosimetry and treatment planning methods and software for low-energy neutron capture therapy applications. We hold a significant intellectual property position in this technology. One anticipated outcome of this work will be a licensable, revenue-generating software package for computational dosimetry and treatment planning applicable to all forms of neutron therapy, both low- and high-energy. The software will then be licensed on a nonexclusive basis to the Ionix Corporation for clinical implementation. This will satisfy our commitments under the current CRADA scope of work. We will retain rights to license the software package to other interested clinical parties as well. Even in the absence of the planned CRADA, an enhanced version of the INEL dosimetry software that allows computations appropriate to fast neutron therapy would be expected to have the potential for considerable commercial value.

\section{Photocatalytic Treatment of Anionic Cyanides}

LDRD 1201

\section{K. L. Gering, J. J. Rosentreter}

This project assessed the performance of photocatalysis for the oxidation (destruction) of both simple and complexed anionic cyanides in water, where our focus was to reduce levels of cyanide in a given mock wastewater while producing nontoxic reaction products. Specifically, we investigated the use of ultraviolet (UV) and simulated solar energy to promote the oxidation of aqueous cyanide. Dr. Jeffrey Rosentreter and two graduate students from ISU performed the laboratory experiments that led to several clear and significant findings. Using an assortment of UV and solar-simulating lamps, the ISU researchers determined that the mechanisms involved with cyanide oxidation involve a twostep process: photoaquation (dissociation) and photo-oxidation. It was found that the photo-oxidation step occurred appreciably in solar-type wavelengths only when a photocatalyst was used. Upon performing a screening study of candidate catalyst materials, it was determined that $\mathrm{TiO}_{2}$ was the most practical photo-catalyst for our study. It was clearly shown that anionic cyanide was effectively reduced in water containing cyanidebearing compounds when subjected to the combination of simulated sunlight and $\mathrm{TiO}_{2}$. Another significant aspect of the ISU work was the development of a novel piezoelectric device that provides real-time measurements of cyanide in solution; this device alone has excellent potential as a commercial analytical instrument.

Dr. Kevin L. Gering from Lockheed Idaho was Project Manager for this work, and performed a preliminary Engineering Feasibility Study for the use of solar energy for aqueous cyanide oxidation, based on the ISU data. This feasibility study will serve as the basis for a treatment prototype envisioned for a follow-on project (if funded).

\section{Objectives}

- Construct flow cell photolysis reactors.

- Identify photo-active cyanide species.

- Identify and test candidate photocatalysts.

- Test treatment system using simulated sunlight.

- Write and present a paper for the ACS National Meeting (August 1995).

- Write a summary report for ISU laboratory results.

- Perform an engineering feasibility study based on the ISU data.

\section{Technical Accomplishments}

All of the technical objectives above were accomplished by Lockheed Idaho and ISU. The laboratory investigation of solar treatment of cyanides, carried out by ISU project, produced several clear and significant findings. Three laboratoryscale photo-reaction cells were designed and fabricated. These cells accommodated three irradiation sources: the far UV (working wavelength $\approx 254 \mathrm{~nm}$ ), the near UV (working wavelength $\approx 365 \mathrm{~nm}$ ), and a simulated solar spectrum (wavelengths between 320 and $800 \mathrm{~nm}$ ). Aqueous solutions containing simple and complex anionic cyanides (from sources such as $\mathrm{KCN}, \mathrm{K}_{3} \mathrm{Fe}(\mathrm{CN})_{6}, \mathrm{~K}_{3} \mathrm{Co}(\mathrm{CN})_{6}$, $\mathrm{K}_{3} \mathrm{Cr}(\mathrm{CN})_{6}, \mathrm{~K}_{2} \mathrm{Zn}(\mathrm{CN}) 4$, etc.) were interrogated using all three irradiation sources. Two homogeneous (no catalysis) photochemical reactions were identified: (1) the photoaquation reaction is most prevalent at the near-UV and solar wavelengths, wherein the cyanide anions are dissociated from metal- 
cyanide complexes, and (2) photooxidation reactions occur most readily in the far-UV region. Since our focus was to evaluate the use of solar energy to accomplish photo-oxidation of cyanide, photocatalysts that would enhance photo-oxidation at solar wavelengths were investigated. Candidate photocatalysts were evaluated through a theoretical and experimental screening study. A solid semiconductor material composed of pure titanium dioxide $\left(\mathrm{TiO}_{2}\right)$ was determined to be the most useful photocatalyst for our work. This material is beneficial in that it is inexpensive, easy to handle, stable, nontoxic, and dramatically effective in stimulating photo-oxidation using near-UV and/or simulated solar irradiation. The use of $\mathrm{TiO}_{2}$ was found to be essential for the solar treatment of anionic cyanides, wherein a heterogeneous photochemical reaction mechanism would occur, converting the cyanide $\left(\mathrm{CN}^{-}\right)$to cyanate $\left(\mathrm{CNO}^{-}\right)$, a nontoxic, nonregulated, stable reaction product. The oxidation of the cyanide occurs by way of a reaction step that involves production of hydroxy radicals $(\bullet \mathrm{OH})$ from water molecules available at the surface of the photocatalyst particles; the radicals then proceed to oxidize the $\mathrm{CN}^{-}$to $\mathrm{CNO}^{-}$. Our studies have clearly shown that cyanide levels drop more rapidly when $\mathrm{TiO}_{2}$ is present, with a subsequent rise of nontoxic cyanate. Many of the results for this project were presented (and well received) at the 210th National ACS Meeting held this year in Chicago.

The photochemical studies placed a heavy demand on timely, accurate cyanide detection. This demand was met by the creation of a novel analytical device sensitive to anionic cyanide that responds in a real-time mode. This device, based on piezoelectric principles, was developed in tandem with the treatment technology and should reside as an integral part of any future prototype treatment system. In addition, the accuracy and speed of the cyanide detection device make it an excellent candidate to be a marketable analytical instrument.

Finally, we performed a preliminary engineering feasibility study for the conceptual design of a portable prototype treatment system based on the use of solar energy (see the figure below). This study, based on the laboratory data from ISU, considers the practical engineering aspects of using actual sunlight as an energy source for the oxidation of aqueous cyanide found in natural and anthropogenic sources. The feasibility study will serve as an excellent foundation for the prototype development and fabrication proposed for the second year (FY-96) of this work. One application of the prototype will be the treatment of wastewaters at remote geographic locations (e.g., abandoned mines and leach ponds). The prototype should be attractive as a marketable technology.

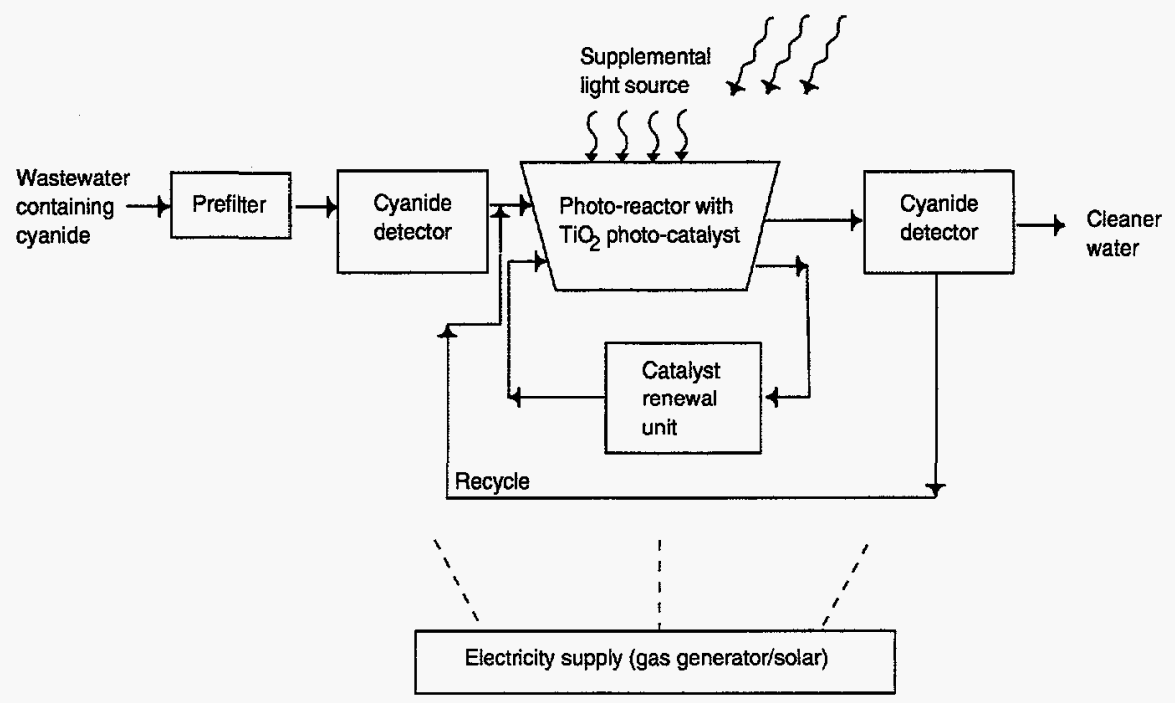

Conceptual block-flow diagram of the prototype treatment system for the photocatalytic destruction of cyanide in wastewater 


\section{Catalytic Destruction of Halogenated Organics at Supercritical Fluid Conditions}

LDRD 1202

\section{M. Ginsoar, S. K. Janikowski}

This project is developing a lowtemperature reductive catalytic process for the destruction of halogenated organics for the treatment of hazardous and low-level mixed wastes. The treatment process is a viable reductive alternative to the oxidative destruction of halogenated organics.

At the INEL, and throughout the $D O E$ and $D O D$ systems, there are significant quantities of hazardous halogenated organics mixed with hazardous and mixed hazardous/radioactive wastes. In addition, U.S. industry produces thousands of tons of halogenated wastes per year. The preferred method of destruction of these mixed and hazardous wastes is hightemperature incineration. This method for the processing of halogenated organics, is known to be incomplete, producing significantly more toxic compounds than the original wastes, such as PCBs, dioxins, and dibenzofurans. These products of combustion are uncontrollably released into the atmosphere.

Development of the reductive lowtemperature catalytic dehalogenation process would provide an economically viable alternative to high-temperature oxidative destruction of halogenated organics. Halogens are effectively substituted with hydrogen, which is donated by the processing solvent. All testing performed to date has demonstrated high levels of compound destruction and reaction products always less hazardous than the feed materials. The reaction products are collected in a liquid and can be analyzed prior to final disposal. Thus, this process has a high potential to be an effective, economically efficient, novel treatment technology for detoxification of chemically hazardous mixed waste streams.

\section{Objectives}

- Develop a bench-scale, continuous flow, experimental reactor unit capable of studying the catalytic reaction at supercritical temperatures and pressures.

- Demonstrate the ability to dehalogenate halocarbons at supercritical fluid (SCF) conditions.

- Reduce hydrogen donating solvent usage in the catalytic dehalogenation reaction.

- Control catalyst activity maintenance at supercritical fluid conditions.

\section{Accomplishments}

In FY-95, a bench-scale, continuous flow experimental reactor unit was built. This unit enabled us to study the reductive catalytic destruction of halogenated organics at supercritical fluid conditions. The experimental unit can study reaction temperatures up to $350^{\circ} \mathrm{C}$ and pressures from ambient to 6000 psig.

Five chlorinated hydrocarbons were tested and all found to dehalogenate at the conditions tested. The compounds were $1,1,1$ trichloroethane, trichloroethylene, tetrachloroethylene, methylene chloride, and chlorobenzene. The reactions occurred over an industrial catalyst using solvents that donated hydrogen to the halogenated molecules. Concentrations of the chlorinated organics in the processing solvent were typically 2,000 ppm; however, 1,1,1 trichloroethane was studied at concentrations up to $100,000 \mathrm{ppm}$. Reaction rates were observed to be an order of magnitude higher near the solvent critical pressure than at ambient pressures for a set temperature. Reaction rates were observed to moderately increase with temperature at a given SCF pressure in the range of 1.03 to 1.12 times the solvent critical temperature. Levels of reaction rates were highest for the chloro-alkane and decreased in the following order: chloro-alkanes $>$ chloro-alkenes > chloro-aromatics.

Catalyst activity maintenance was studied using 1,1,1 trichloroethane over the industrial catalyst in a solvent at ambient and SCF pressures. Catalyst activity for the dehalogenation reaction was found to be stable at both SCF and ambient pressure conditions for six hours time-on-line. However, at the lowest amount of catalyst studied, the reaction rate was observed to decline with time. This loss of activity is attributed to the loss in hydrogen production rate, which, with small quantities of catalyst, limits the overall dehalogenation rate.

All of the solvents tested were found to produce hydrogen at reaction conditions. The hydrogen production reaction in most cases proceeded through the reforming reaction, producing a side product of carbon monoxide. In most cases, the hydrogen production rate increased with pressure up to the solvent critical point and then decreased with increasing pressure. This behavior is typical of a cross-over from kinetically controlled reaction rates to equilibrium limited reaction. Gas generation rates generally increased with catalyst loading, up to the equilibrium limit. These observations suggest that pressures significantly in excess of the solvent critical pressure may limit the solvent-to-halocarbon ratio.

New or Increased Technical Capability

New or increased technical capabilities at the INEL resulting from this project are as follows:

- A reductive catalytic process to destroy halogenated compounds in hazardous and mixed waste is being developed. 
- An experimental unit capable of studying continuous flow catalytic reactions in a fixed bed reactor in either gas, liquid, or supercritical phases at temperatures up to $350^{\circ} \mathrm{C}$ and pressures up to 6,000 psig is now available at the INEL.

- Expertise studying catalytic reactions in supercritical fluids has increased.

\section{Business Development Opportunities}

The industrial applications of the process are highly significant. We anticipate that at the end of FY-96 the process will be sufficiently mature to transfer the technology to industry.

In conjunction with LDRD 8615, eight companies contacted have expressed interest in assisting in development and licensing the technology once it is developed. All are potential CRADA partners for the scale-up and technology transfer tasks. The interested companies are ETAS Corporation, ENSR Operations, Northeast Hazardous Substance Research Center, Geotextiles Environmental Supply and Installation, Burlington Environmental, Inc., Laidlow Environmental Services, Inc., Rollins Environmental Services, Inc., and The 3M Company. ETAS Corporation has signed a letter of intent to CRADA with Lockheed Idaho on the development of this technology.

\section{Destruction of PCBs in Oil Using Gamma Irradiation and Ozone LDRD 1203}

\section{K. J. Liekhus, B. J. Mincher}

This project investigated whether the addition of ozone to a simulated waste oil containing polychlorobiphenyl (PCB) would accelerate the rate of $P C B$ destruction. More rapid destruction of PCBs in mixed waste oil would be beneficial to the Idaho National Engineering Laboratory and other sites.

\section{Objectives}

Identify the relative rate of PCB destruction in samples of simulated waste oil exposed to gamma irradiation with and without simultaneous exposure to ozone. The two cases investigated were

- PCB 155 (hexachlorobiphenyl) in iso-octane.

- Aroclor 1260 in hydraulic oil.

\section{Accomplishments}

The change in the concentration of PCB 155 as a function of the absorbed radiation dose in ozonated and nonozonated iso-octane is shown in the figure. The rate of decomposition is initially similar in both solutions up to a radiation dose rate of $1 \mathrm{MRad}$; however, the ozonated solution experienced no further significant loss in PCB concentration. In contrast, the PCB concentration in nonozonated isooctane decreased steadily out to an absorbed dose rate of $4 \mathrm{MRad}$, where PCB 155 was no longer detected. A linear regression was performed on the data for the nonozonated iso-octane, and a slope of $0.66 \mathrm{MRad}-1$ was obtained with a correlation coefficient of 0.8 . This slope is pseudo-first-order and agrees with previously measured values at similar dose rates and concentrations indicating pseudo-first-order kinetics.

The change in the concentration of Aroclor 1260 as a function of the absorbed radiation dose in ozonated and nonozonated hydraulic oil was also measured. Aroclor 1260 consists of a mixture of PCB congeners with various degrees of chlorine substitution. A linear regression of the data from the nonozonated hydraulic oil indicates a slope of $0.05 \mathrm{MRad}-1$. The limited data do not indicate a significant difference in the rate of PCB destruction with the addition of ozone.

The free radical yields for isooctane and hydraulic oils are similar and iso-octane is a reasonable surrogate for hydraulic oil irradiations. However, the rate of PCB 155 destruction was shown to be negatively affected by the presence of ozone, while no difference in the destruction rate was observed for Aroclor 1260 in the presence of

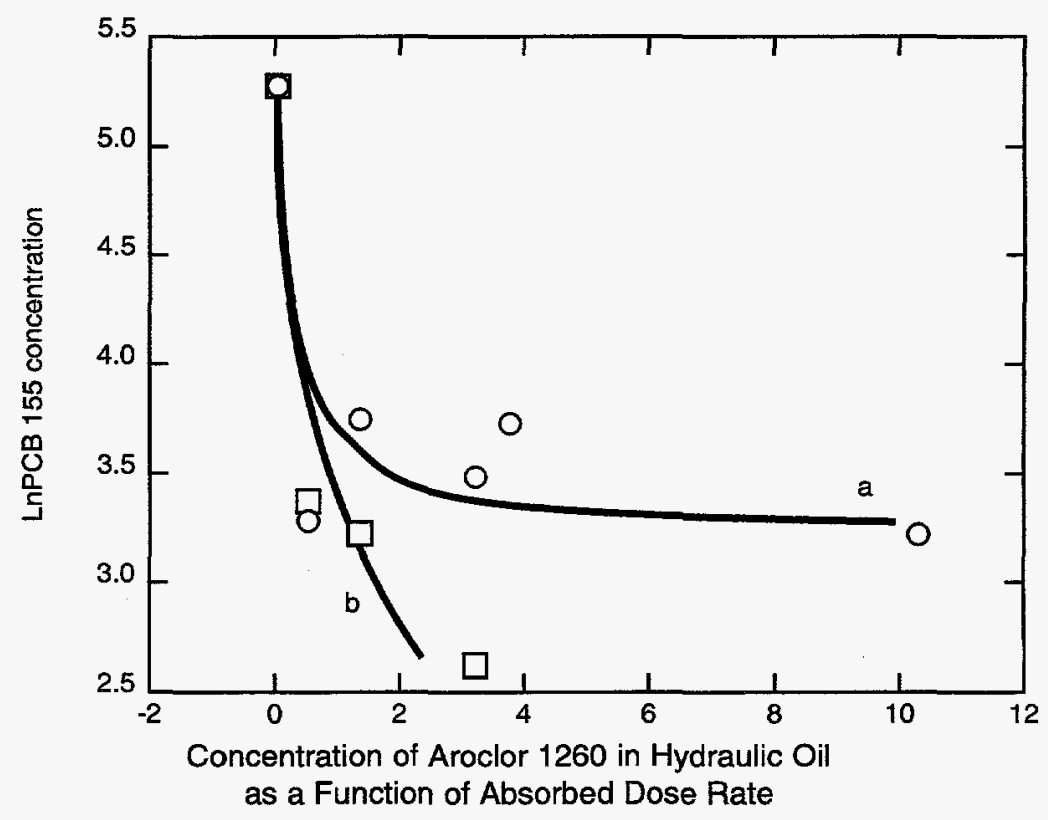

Idaho National Engineering Laboratory LDRD FY 1995 Annual Report 
ozone. We attribute this observation to the difference in the relative PCB destruction rates. Since the rate constant is an order of magnitude less than for PCB 155, the concentrations of Aroclor 1260 in the hydraulic oil with and without ozone will probably not diverge until at significantly greater dose rates.

\section{New or Increased Technical Capability}

The test results did not support the original hypothesis that the addition of ozone would accelerate the rate of $\mathrm{PCB}$ destruction in waste oils. Ozone competes for the free electrons that are required in decomposition of $\mathrm{PCB}$ compounds by free-electron capture. This information will be submitted as a technical note to the Journal of Applied Radiation and Isotopes.

\section{Business Development Opportunities}

No business relationships were developed or maintained during this research. A potential area of business development involves the polymer used to make the boxes that held the oil samples. The polymer steadily darkened as a result of exposure to gamma radiation. Potential business development involves using the polymer to create a dosimeter that is inexpensive to measure total adsorbed radiation dose and is simple to use.

\section{Bioprocess Development for the Remediation of $\mathrm{Cr}(\mathrm{VI})$ - and $\mathrm{U}(\mathrm{VI})$ - Contaminated Soils and Groundwater}

LDRD 1233

$$
\text { C. E. Turick }
$$

Hexavalent chromium [Cr(VI)] is a high priority environmental restoration (ER) problem at many DOE sites and at other government and private sites.
This research successfully addressed the development of a bioreactor capable of maintaining very high densities of bacteria capable of biochemically reducing $\operatorname{Cr}(V I)$ to the less toxic and insoluble $\mathrm{Cr}(\mathrm{III})$, leading to the subsequent removal of chromium from contaminated effluents through precipitation. The targeted problems include $\mathrm{Cr}(\mathrm{VI})$-contaminated aqueous streams from pump and treat operations and from liquid effluents discharged from soil wash processes. The Cr(III) produced by the bacterial reduction of $\mathrm{Cr}(\mathrm{VI})$ can be precipitated out of solution through physical-chemical means without the need to drastically adjust $\mathrm{pH}$, which is a common problem in conventional technologies. Additional applications for this technology include treatment and recycling of $\mathrm{Cr}(\mathrm{VI})$-containing waste streams, and in-situ $\mathrm{Cr}(\mathrm{VI})$ reduction. This is the only acceptable in-situ remediation technology for $\mathrm{Cr}(\mathrm{VI})$-contaminated soils.

The operation of the bioreactor relies on previous biotechnology findings at INEL. These findings constitute the key intellectual property incorporated in a patent for this technology. Bioreactor experiments have demonstrated this technology to be at least an order of magnitude more efficient than current technologies. The benefit to INEL from this project is an economical means of removing $\mathrm{Cr}(\mathrm{VI})$ and related metals from contaminated soils, sediments, and contained wastes and mixed wastes, such as those found throughout most DOE sites, and transfer of INEL technology to industry for commercialization.

\section{Objectives}

- Evaluate market for technology and most likely customers or partners. Understand present technology and competition.

- Evaluate potential process inhibitors associated with various effluents.

- Develop and operate a high throughput bioreactor.

\section{Accomplishments}

The technology and market evaluation took the form of a market analysis. Technological areas that fit this technology include:

- $\mathrm{Cr}(\mathrm{VI})$ reduction of soil wash effluents from ex situ remediation activities

- $\mathrm{Cr}(\mathrm{VI})$ reduction of aqueous streams from pump and treat remedial activities

- In situ bioreduction of $\mathrm{Cr}(\mathrm{VI})$ followed by $\mathrm{Cr}$ (III) complexation to the soil matrix

- Treatment of $\mathrm{Cr}(\mathrm{VI})$-containing waste streams from industries such as electroplaters and metal finishers

- Treatment of $\mathrm{Cr}(\mathrm{VI})$-contaminated mixed wastes and hazardous wastes stored at government facilities.

The economic potential in these areas is in excess of $\$ 5$ billion. The market analysis was used by the intellectual property committee to decide to pursue a patent for this technology. A copy of the market analysis can be obtained from the principal investigator.

We tested the incorporation of a method for encouraging $\mathrm{Cr}(\mathrm{VI})$ bioreduction in a bioreactor. The method we used is based on previous work at INEL and is the major component of the intellectual property used for a patent application for this bioprocess. We successfully demonstrated $\mathrm{Cr}$ (VI) bioreduction in a bioreactor containing a mixed culture of soil bacteria. The bioreactor contained an environment that favored the growth of bacteria capable of growing on the toxic and soluble $\mathrm{Cr}(\mathrm{VI})$ and converting it to benign and less soluble Cr(III). The dominant population in the bioreactor consisted of 
$\mathrm{Cr}(\mathrm{VI})$-reducing bacteria. As the bioprocess continued, the environment in the bioreactor selected for the best strains of $\mathrm{Cr}$ (VI)-reducing bacteria. A high throughput bioreactor was then designed and operated, demonstrating the potential for the use of this technology for industrial purposes.

Because the bioprocess is designed to select for $\mathrm{Cr}(\mathrm{VI})$-reducing bacteria from a mixed culture, the input does not have to be sterile. Any contaminating organisms not capable of $\mathrm{Cr}(\mathrm{VI})$ reduction will be outcompeted. Bacterial contaminants capable of $\mathrm{Cr}(\mathrm{VI})$ reduction will be incorporated into the bioreactor population if they can grow as fast or faster than those already present. This strategy allows for improved reactor kinetics with time.

Potential inhibitors that might be encountered in a pump and treat operation for $\mathrm{Cr}(\mathrm{VI})$ remediation from groundwater were examined with this technology. We found that the strategy of high selection pressure on the microbial population prevented observable inhibition from occurring. This technology is more robust than anticipated, and may in fact be virtually fool proof.

There is evidence that certain features of the bioreactor design could be used to concentrate the $\mathrm{Cr}$ (III) in the reactor until a high concentration is obtained. The $\mathrm{Cr}$ (III) could then be desorbed in concentrations high enough to be recycled rather than being disposed of as a waste.

New Or Increased Technical Capability

- Bioreactor for reduction of $\mathrm{Cr}(\mathrm{VI})$ and related metals

- In situ bioremediation of $\mathrm{Cr}(\mathrm{VI})$ and related metals

- Bioreactor for recovery of $\mathrm{Cr}$ (III) for waste minimization.

Business Development Opportunities

With this project, Lockheed Idaho has an opportunity to generate revenue in the following areas:

- Reduction of Cr(VI) in soil wash effluents from ex-situ remediation activities

- Reduction of $\mathrm{Cr}(\mathrm{VI})$ in aqueous streams from pump and treat remedial activities

- In situ bioreduction of $\mathrm{Cr}$ (VI) followed by $\mathrm{Cr}(\mathrm{III})$ complexation to the soil matrix

Treatment of $\mathrm{Cr}(\mathrm{VI})$-containing waste streams from industries such as electroplaters and metal finishers

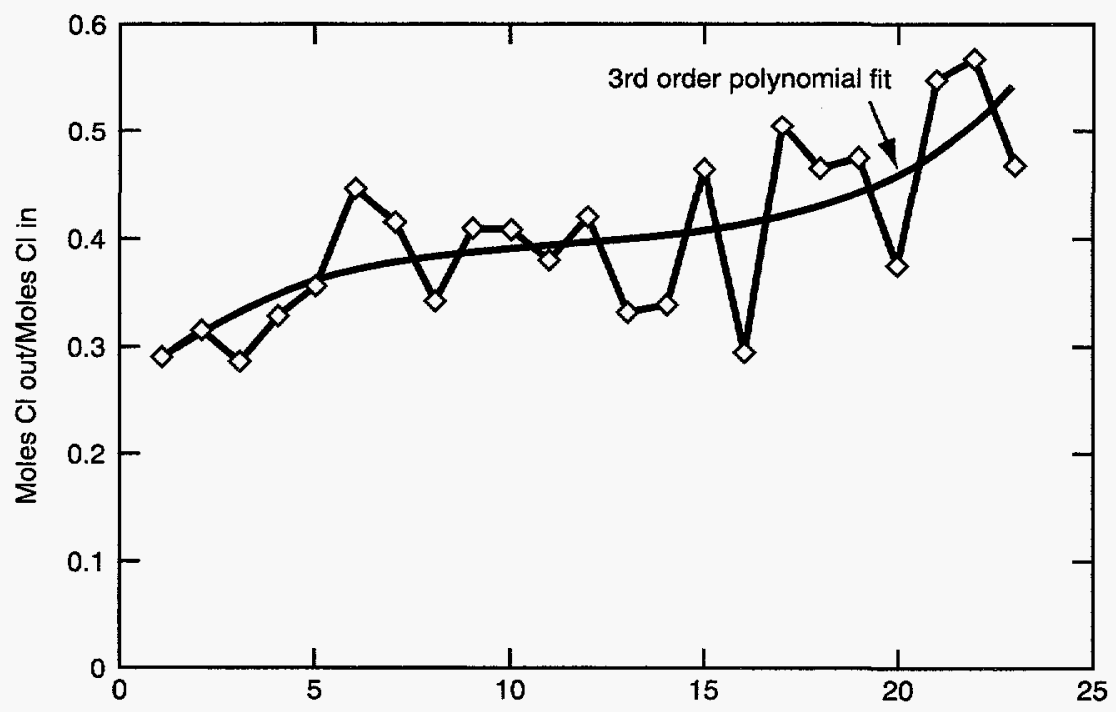

- $\mathrm{Cr}(\mathrm{VI})$ treatment of mixed wastes and hazardous wastes stored at government facilities.

The economic potential in these areas is in excess of $\$ 5$ billion.

\section{Ultraviolet VOC Destruction Technology, Liner Lifetime and By-product Testing with Hazardous Waste Disposal Considerations LDRD 1306}

\section{R. S. Lickson}

The purpose of this project was to evaluate the effectiveness of a new volatile organic compound (VOC) treatment technology being developed by Process Technologies, Inc. (PTI) of Boise, Idaho. This technology uses low pressure ultraviolet (UV) lamps located within a reaction chamber to break apart the chemical bonds making up a VOC molecule. A dry, porous reagent material (liner) is attached to the inner surface of the reaction chamber and chemically reacts with the gaseous by-products of the VOC destruction. The chemical reaction forms a stable, solid reaction product (inorganic salt) within the reagent liner, and has the potential to completely eliminate the generation of any hazardous secondary waste. This LDRD has performed bench scale testing of the PTI treatment technology with specific concentrations of carbon tetrachloride (CCL4). CCL4 was selected because it is the major subsurface contaminate at the Idaho National Engineering Laboratory (INEL) and at the Hanford Nuclear Reservation. Future research and application of this technology at the INEL may greatly assist in the cleanup of VOC contaminated air, soil and groundwater at the Radioactive Waste Management Complex (RWMC). The proposed research may also be useful eliminating $V O C$ emissions from industrial operations such as semiconductor manufacturing, pharmaceutical, foundries and other critical industries where compliance under the new 
Clean Air Act Amendments may soon be required.

\section{Objectives}

- Perform bench scale testing of a new volatile organic compound (VOC) treatment technology.

- Develop liner lifetime data for the destruction of carbon tetrachloride (CCL4).

- Analyze system by-products which may compromise system operation and liner disposal.

- Perform Toxicity Characteristic Leaching Procedure (TCLP) testing and validation on liner material to ensure disposal as nonhazardous waste.

- Perform Secondary Electron Microscope (SEM) analysis of liner cross-sections to determine the degree of liner saturation.

- Evaluate test results to optimize process, document system operating and lifecycle costs verses other VOC destruction technologies.

- Document results and propose additional areas of research and application of this technology at the INEL.

\section{Accomplishments}

Bench scale testing of this technology was accomplished in two phases. In Phase 1, two reactor chambers were connected in series. Each reaction chamber consisted of a 4-inch-diameter aluminum tube, 58 inches long, with a 0.5 -inchthick reagent liner material rotary molded to the inside wall of the tube. Three low-pressure UV lamps placed longitudinally within each tube provided photon flux at the desired wavelength. CCL4 at a concentration of approximately $5,000 \mathrm{ppm}$ was introduced into the tubes at a flow rate of 2.2 liters per minute. A CCL4 destruction efficiency of $99.7 \%$ was observed. The effective liner life was determined by measuring the amount of chloride in the effluent gas. The amount of chloride exiting the reactor, expressed as a percentage of chloride introduced into the system on a molar basis, rose significantly after approximately 23 days (412 continuous hours) of operation.

In Phase 2, two 5-inch-diameter tubes with 1-inch-thick liner material were connected in series. This configuration allowed the same effective flow area and light spacing within the reaction chamber, and maintained the same CCL4 destruction efficiency, inlet concentration, and flowrate as Phase 1. The Phase 2 test ran for approximately 70 days ( 1482.4 continuous hours), and was terminated a little earlier than desired due to project completion deadlines. The results for Phase 2, for moles of chloride in the discharge gas expressed as a percentage of moles of chloride in the inlet, again showed a significant rise in chloride output signaling the end of effective liner operation.

The SEM examination of the spent liner material indicated that reagent utilization was actually greatest along the outside radius of the liner. It had always been assumed that the reagent utilization was greatest along the inside surface of the liner, where the liner has the greatest exposure to the gas flow and the UV lamps. The SEM analysis, as measured by the chlorine to calcium ratio, showed that the liner exposed to the UV had a chloride to calcium ratio of less than 0.5 to 1 . The chloride to calcium ratio increased to a value approaching 2:1 at the outer radius of the liner at the junction with the aluminum tube. This discovery was the premise for increasing the liner thickness to 1 inch for the Phase 2 test.
The increased liner thickness in Phase 2 equated to an increase in reagent volume of 2.3 times, and an increase in reagent mass of 2.05 times. However, the run-time for the Phase 2 test was 3.6 times longer than the Phase 1 test. This would indicate a better utilization of the additional liner material added for the Phase 2 test. By adding to the reagent liner thickness, additional liner lifetime and better liner utilization has been achieved.

TCLP tests were performed on spent reagent liner material from both Phase 1 and Phase 2 tests. All samples passed TCLP testing, indicating liner disposal as nonhazardous waste or liner recycling by a cement manufacturer is feasible.

PTI had estimated the cost to destroy CCL4 to be approximately $\$ 7.50 / \mathrm{lb}$. CCL4, with the replacement of the spent liner material accounting for $40 \%$ of this cost, or $\$ 3.00 / \mathrm{lb}$. With the improvements made by this LDRD, spent liner replacement cost has been reduced by more than $70 \%$, to less than $\$ 1.00 / 1 \mathrm{~b}$. This will reduced the overall cost of CCL4 destruction to less than $\$ 5.50 / \mathrm{lb}$, with the potential of further cost reductions with additional process improvements. Information from Hanford vapor vacuum extraction (VVE) operations have indicated carbon bed regeneration costs of $\$ 5.68$ per pound of VOC have been experienced.

\section{New or Increased Technical Capability}

Knowledge of the reaction chemistry between the CCL4 gas and reagent liner has been developed, as well as effective liner lifetime and system cost. Application of this technology to waste cleanup at the INEL and other DOE sites is now better defined. 


\section{Business Development Opportunities}

PTI is working as a subcontractor to the INEL to help develop their technology for the destruction of CCL4. Discussion for the development of a CRADA are ongoing.

\section{Portable Photon Analysis Spectrometer for TRU and $\gamma$ Assay} LDRD 2104

\section{R. J. Gehrke}

This project is developing a commercially available, battery-powered, portable multichannel analyzer, incorporating on a PC platform the dualenergy puiser technology developed at the INEL and the VAXGAP spectral analysis programs used by the Radiation Measurements Laboratory at the Test Reactor Area of the INEL. With these capabilities, laboratory quality analyses can be conducted in the field on $L x$-ray emitting TRU and $\gamma$-ray emitting radionuclides.

\section{Objectives}

- Design and build a low-power dual-energy pulser for use in battery-powered portable multichannel analyzers.

- Engineer the necessary software to analyze the $x$-ray and $\gamma$-ray portions of a spectrum to operate on a PC computer. Pursue commercial development of x-ray, $\gamma$-ray, and pulser software to operate the PPAS with a PC with Canberra Industries. Canberra holds a nonexclusive license for the dual-energy pulser and has shown strong interest in this project.

- Develop a background reduction technique.

- Make operational a prototype
Portable Photon Analysis Spectrometer.

\section{Accomplishments}

A dual-energy precision pulser provides a $50-\mathrm{Hz}$ low and highpulse to the test input of the preamplifier that, when digitized, fall in the bottom tenth and top tenth regions of a 8192-channel analyzer. These pulser peaks are then calibrated against a calibrated energy scale and assigned a $\gamma$-ray equivalent energy. The pulser peaks, which follow all energy scale drifts, can be used to measure the gain and zero intercept of any subsequent spectrum. The pulser measures pile up by measuring the number of pulses stored compared to the number injected into the preamplifier. It also detects any noise or temperature change causing drift in the energy scale through increases in the width of its peaks. Use of the pulser information verifies that the spectrometer electronics were operating correctly during the spectrum accumulation.

We needed a dual-energy, pulser-equipped battery-powered multichannel analyzer system that would consume as little power as possible. Consequently, we designed a dual-energy low-power, precision pulser. Its power requirements are 3 watts for 5 seconds at power-on time, followed by 1.2 watts after the warm up. The commercial analyzer We chose the Canberra Inspector analyzer because it is very light, is totally programmable, and Canberra has a nonexclusive license for the dual-energy pulser. Since the Inspector incorporates programmable gate arrays, it was necessary for Canberra to incorporate the pulser $A D C$ interface circuitry into their chip. They agreed to do this at no charge.

In order to redesign the VAXGAP family of spectral analysis programs to operate on a PC, a number of modifications were required. To reduce the complexity of this task, we chose an $\alpha$-class Digital Equipment Corporation computer and the Windows NT operating system from Microsoft. Some of the computer algorithms previously written in FORTRAN had to be rewritten in the $C$ programming language, the language of choice for the Windows environment. A high-quality real-time spectral data display program, written by L. East was incorporated on the PC. At this time, all calculational algorithms operate on the PC platform.

Algorithms to communicate between the Inspector and the PC were developed by D. Femec. This is the last piece required for a complete, operable spectrometer system. Completion of job-specific tasks as encountered with the application of $\mathrm{x}$ - and $\gamma$-ray analyses will be completed in FY-96; much work also remains in debugging various programmed algorithms so that they work smoothly together. System performance testing will occur in FY-96.

Weight will be a severe limitation to a portable Ge spectrometer. Specific design of thin passive shields to reduce the spectral continuum less than $\sim 200 \mathrm{keV}$ will be pursued as the application requires.

\section{New or Increased Technical Capability}

A dual-energy-equipped, portable, battery-powered Ge or $\mathrm{Si}(\mathrm{Li})$ spectrometer with advanced and tested spectral analysis algorithms is a major advance for in-field measurements. This is confirmed by the fact that Canberra Industries intends to incorporate the pulser in their next generation of Inspector.

\section{Business Development Opportunities}

Since the pulser is licensed by Canberra Industries, its use in Canberra products will result in royal- 
ties to Lockheed Idaho Technologies Company and to the inventors.

\section{Conceptual Design of a 55-Gallon Waste Container Refurbishment system} LDRD 3203

\section{J. R. Wolf, D. J. Varacalle}

The objective of this project was to develop and demonstrate an INEL capability to use thermal spray coatings to solve corrosion problems in hazardous waste storage containers. The research was directed towards identifying and solving specific containment problems for DOE waste storage facilities. The use of thermal spray coatings to refurbish 55-gallon waste containers enhances structural integrity, protects the containers from corrosion, and extends container lifetime. By eliminating the use of overpack containers, thermal spray coatings can effect a significant reduction in Transuranic (TRU) waste generation, with a corresponding reduction in storage volume and significant cost savings in the future (i.e., the cost of transportation for 83-gallon overpacks). Collaborative efforts with Hobart-Tafa Technologies (Bow, NH) and Walker Sandblasting (ldaho Falls, ID) were established relative to this LDRD; these efforts involved the use of equipment and donation of manpower and materials to accomplish the research.

\section{Objectives}

- Optimize surface preparation techniques for undegraded surfaces (i.e., new or slightly corroded substrates)

- Optimize coating process parameters and attributes (i.e., thickness, adhesion, corrosion resistance) for aluminum and zinc coatings.

\section{Accomplishments}

We conducted several series of surface modification and coating application experiments that included a variety of parameteric studies. The coatings and substrates were characterized and evaluated by a number of techniques. These included Rockwell and Vickers hardness tests, optical metallography, x-ray diffraction, image analysis, surface profilometry, and deposition efficiency.

We investigated surface modification (grit blasting) of the waste containers to ensure the bond strength of the as-sprayed coatings. Two grit blast devices were studied using a Box full-factorial design parametric study. For each device, operating parameters were varied around the blast media, the air pressure, the work distance, and the number of passes on the substrate. Roughness of the substrates was substantially increased by using this process. The two devices used for the abrasive blasting experiments were an Econoline RA 36-1 Blast Cabinet, and a Clemco $100 \mathrm{lb}$. capacity pressurized-pot blaster. Three types of abrasive blast media were used for the experiments. These were 35 mesh copper slag, 35 mesh silica sand, and 60 mesh alumina grit. Five major studies were conducted, using different combinations of abrasive blasting devices, blast media, and working parameters.

The effects analysis indicates the Clemco equipment roughened the substrates more than the Econoline equipment. Copper slag grit resulted in more surface modification than the other grits for the Econoline machine, while there was little difference between the silica and copper grits used in the Clemco machine. The surfaces produced by grit blasting with the Econoline system appear to be relatively free from embedded grit or surface scale particles, while for- eign particles were frequently seen embedded in the surfaces produced with the Clemco system.

We then conducted a series of experimental coating experiments to determine if aluminum and zinc coatings applied with a TWEA spray system could perform as anti-corrosion coatings for waste container applications. Box fullfactorial experiments were used to determine the optimum combination of process parameters. The process parameters varied in the experiments were orifice diameter, flow pattern, and primary pressure. The coating attributes evaluated included porosity and deposition efficiency for all of the samples. Selected samples were also tested for hardness, roughness, and corrosion resistance. Porosity was determined using image analysis.

By examining the optimum levels of the process parameters, we identified a design coating for this particular waste application for the TWEA experiments. After the effects analysis was completed, we conducted ANOVA (analysis of variance) calculations for each experiment. The optimum coating for this application would have low porosity and high deposition efficiency with low roughness and high hardness. Included in these considerations is the ability of the coating to galvanically protect the substrate, which was determined with corrosion testing for selected coatings.

As of this writing, one paper has been published on this work; it was presented at the National Thermal Spray Conference.

New or Increased Technical Capability

- Fabrication of new coating systems using a new thermal spray process at the INEL.

- Technology transfer to industry (Tafa, Walker).

- University collaboration with 
the Basic Industry Research Laboratory (BIRL) at Northwestern (Tom Bernecki).

- Industrial collaboration with Tubalcain (Walt Riggs), Walker Sandblasting (John Walker), Tafa, Inc. (Gary Irons).

Business Development Opportunities

Tafa and BIRL personnel involved INEL personnel (Varacalle) in proposals to the government (DOD, EPA) and EPRI. Major proposals to the federal and state governments will be pursued with the team constructed on this LDRD for infrastructure applications in FY-96.

\section{Photodissociation of Mercuric Chloride Treatability Study LDRD 3710}

\section{R. E. McAtee}

Photodissociation is a process in which light energy is used to dissociate a molecule into molecular fragments and/or atoms. This technology was selected to be studied as a potential treatability process for the mercury waste at the Idaho National Engineering Laboratory (INEL). We selected mercuric chloride for the study as a representative mercury waste, using gas phase mercury chloride in order to reduce the presence of intermolecular forces which would complicate the study of the photodissociation process. Since mercuric chloride is a solid at room temperature, temperatures and pressures needed to sublimate the solid into gas were determined. Solid-vapor and liquid-vapor coexistence curves in a pressure versus temperature diagram were prepared for mercury chloride. This showed that the sublimation temperature was reasonably achievable. However, because of the need to maintain a constant pressure in the dissociation process, and because the mercury chloride speciation is unknown, keeping the pressure constant would be difficult. Since the method of removing the mercury has not been investigated, the photodissociation process may not be a useful technique to yield metallic mercury in a waste treatment process.

Another approach to using light energy for the photodissociation of mercury chloride is the sunlight photoreduction, titanium-dioxide-catalyzed conversion of mercuric chloride to metallic mercury. This process is currently being studied by an Associated Western Universities (AWU) scholarship student at Idaho State University.

\section{Objectives}

- Review the literature for ultraviolet light photodissociation of mercury chloride.

- Prepare mercury chloride solid-vapor/liquid-vapor coexistence curves in a pressure versus temperature phase diagram.

- Purchase materials and equipment and fabricate the photocell/test reactor.

- Perform the experiments according to the experimental plan and report the results.

\section{Accomplishments}

- The photocell/test reactor was fabricated (see the figure).
- The photodissociation process using the test cell was determined impractical as a technique to yield metallic mercury in a waste treatment process, so the experimental studies were not performed.

Currently, the photodissociation of mercury chloride using a process of sunlight photoreduction, titanium-dioxide-catalyzed conversion of mercuric chloride to metallic mercury is being studied. No data are available at this time.

New or Increased Technical Capability

A successful conclusion to this research study will furnish the capability to treat mercury waste at the INEL.

Business Development Opportunities

No industrial contacts have been made at this time.

\section{INEL-University of Idaho Laboratory for Liquid Extraction and Ion Exchange Research LDRD 4078}

\section{A. L. Olson}

This project addresses research concerning removal of hazardous

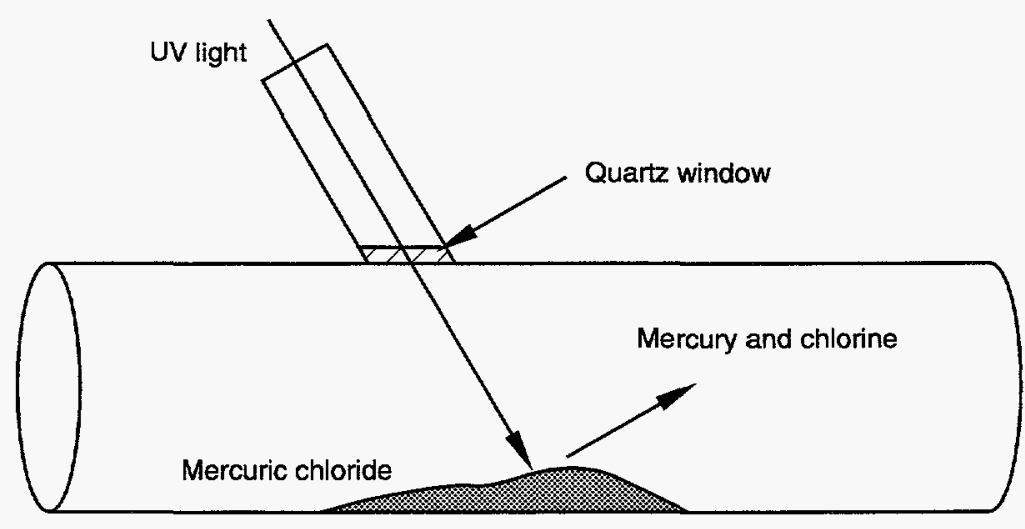

UV Test Cell 
materials from radioactive, acidic liquid wastes. The research has been conducted over a three year period. The project involves the fabrication of a liquid extraction and ion exchange pilot scale laboratory at the University of Idaho (UI) for joint UI-INEL research. Separation of hazardous materials from Idaho Chemical Processing Plant (ICPP) radioactive liquid wastes is currently being considered as a treatment before ultimate disposal of the waste. The research is being conducted to (a) provide a technical basis for potentially decreasing the volume of mixed, low-level INEL waste that must be successfully immobilized; (b) provide data concerning the ability to separate hazardous material from liquid wastes; and (c) to provide an experienced personnel pool for activities pertinent to the INEL mission. Project activities include (a) identification of liquid extraction and ion exchange agents for removal of hazardous materials, specifically from acidic, aqueous waste solutions; (b) laboratory scale evaluation of potential liquid extraction and ion exchange agents; (c) fabrication of a pilot scale equipment laboratory: (d) evaluation of candidate separating agents in the laboratory: and (e) correlation of experimental data with current computer models. While the data generated by this project will be applicable mostly to DOE mixed liquid wastes, we expect that portions of the data can be extrapolated to some types of industrial wastes. Typical industries for which the data may be applicable include metal finishing wastes, mining wastes, pigments from paint preparation, and plastics and organic chemicals manufacture.

\section{Objectives}

- Complete fabrication of the pilot-scale equipment laboratory and verify nominal pulse column flooding parameters.

- Complete selection of laboratoryscale evaluation of liquid extraction agents.
- Complete laboratory evaluation of ion exchange agents for lead.

- Complete ion exchange pilot scale equipment tests.

- Submit report summarizing work completed in FY-95.

\section{Accomplishments}

We completed the fabrication of the solvent extraction and ion exchange pilot scale laboratory equipment during FY-95. The 24-ft-high, two-in.-diameter pulsed liquid extraction column with auxiliary pumps, pulser, tanks, and piping was constructed, and flooding tests were performed to allow comparison with equipment at the Idaho Chemical Processing Plant (ICPP). Testing was conducted at a range of pulser-induced amplitude frequency products, aqueous to organic phase ratios (A/O ratios), and pulse amplitudes to validate the equipment setup and provide data for comparison. Flooding velocity data were obtained for the UI column, the INEL column (in Building 637 at ICPP), and a similar column at Hanford. The UI flooding test results compare favorably to previous results, giving a degree of confidence to future generated data.

We also fabricated a 5-ft-high, 2-in.-diameter ion exchange column and tested it for operability. We also identified agents for separating lead from ICPP tank farm liquid waste simulant. Several candidate ion exchange agents were evaluated. Sybron Ionac SR-5 and polyantimonic acid were found to have favorable distribution coefficients for lead in the unneutralized and partially neutralized $(\mathrm{pH}$ around 1.5) simulant. The resin was able to decrease lead concentrations in the tank farm simulant from about $280 \mathrm{ppm}$ down to less than $10 \mathrm{ppm}$. This translates to distribution coefficients $(\mathrm{Kd})$ of between 55 and $1143 \mathrm{ml}$ of sample per gram of resin. Polyantimonic acid was prepared in the laboratory, and investigations were initiated for immobilization on a silica substrate for evaluation in the ion exchange column. Other ion exchange agents were investigated, including Sybron Ionac AFP 329, Sybron Ionac SR-4, Zeotech Chabazite, Zeotech Zeobrite M, Zeotech Zeocarb $\mathrm{M}$, ammonium molybdophosphate, crystalline silico titanate, and tungsten oxide, but no significant lead removal was observed. One candidate liquid extraction agent was found, ammonium pyrillidine dithiocarbamate, which could remove about $80 \%$ of the lead in one contact. Other agents that were investigated included methyl isobutyl ketone, tributyl phosphate, dibutyl phosphate, 18 crown 6 ether, bis(2ethylhexyl) hydrogen phosphate, and tris(2ethylhexyl) phosphate. These agents did not show any appreciable distribution of lead into the extracting agent in laboratory experiments.

Analytical capabilities for laboratory investigations were enhanced with the acquisition of a PerkinElmer atomic absorption spectrophotometer. It is on loan from the INEL. The laboratory now consists of a small scale vortex mixer, small ion exchange column with pumps, $\mathrm{pH}$ meter, and atomic absorption spectrophotometer for evaluation of selected agents for lead and other heavy metals removal. Work is currently continuing on evaluation of other liquid extraction agents and on preparation of bulk quantities of polyantimonic acid for immobilization and evaluation in the lab scale and pilot scale ion exchange columns. A copy of ASPEN software was also obtained for correlating liquid extraction and ion exchange results. 
New or Increased Technical Capability

There have been several benefits to the INEL from this project. A laboratory for joint INEL-UI use has been put in place, tested, and is now available for research on a variety of liquid separation techniques. This is a very valuable resource. Expertise and experience has been gained by three UI personnel concerning problems and solutions at the INEL directly related to it mission of environmental management. This experience base will hopefully increase and provide a personnel pool for INEL in the future. Three candidate agents have been identified which have the potential for separating lead from low $\mathrm{pH}$ or acidic type wastes; just as important, several other separating agents have been identified as not being acceptable for lead separations from acidic solutions. This work has provided valuable technical information to the data base for lead separations from liquid, low $\mathrm{pH}$ environments.

\section{Business Development Opportunities}

Contact has been made with Zeotech Corporation concerning development of an engineered form of polyantimonic acid for evaluation in laboratory and pilot scale environments. This has been an informal arrangement to date, with only exchanges of technical information. Data from near term evaluations of polyantimonic acid immobilized upon a zeolite substrate will be used to further this relationship, perhaps developing into a CRADA, and leading to a commercial application for acid wastes.

\section{Fracture Mechanics-based Design Methodologies for Metal Matrix \\ Composite Material \\ LDRD 4090}

W. G. Reuter

Proven design methodologies, based on fracture mechanics concepts, do not exist for metal-matrix composites (MMCs), but are required to efficiently design these materials for use in industrial applications. Fracture mechanics concepts are presently used, for homogeneous metallic structures, to predict (a) crack growth resulting from fatigue, stress corrosion cracking, hydrogen assisted cracking, etc., and (b) the critical crack size that will initiate crack growth or failure.

These capabilities provide the necessary tools needed to predict (a) fitness-for-service when a defect is discovered that exceeds the allowable values, provided in the Codes and Standards, in a structure, (b) a minimum operating lifetime, or (c) an allowable lifetime extension for structural components. The last two items relate only to structural components where fracture mechanics concepts play a significant role in the lifetime.

The Idaho National Engineering Laboratory (INEL) has been developing tools for predicting the fracture process in structural components fabricated from monolithic ceramics and homogeneous metallic materials and recently initiated research into developing similar tools for nonhomogeneous metallic (weldments) structures and for composites. The initial desire was to develop predictive tools for composites where the strengthening agent was either a particulate, a whisker, or a continuous fiber. The desired approach is to use planestrain fracture toughness (KIc) or Jlc (including CTOD) measured from small, standard fracture toughness specimens to predict the fracture process in structural components.
Objectives

- Developing an understanding of the fracture process.

- Develop methods for using fracture toughness data to predict failure of simulated structural components.

- Identify limits of applicability for methods developed.

\section{Accomplishments}

We tested DWA-supplied composites and obtained preliminary test fracture toughness results. Those specimens containing machined notches experienced either a negligible shift ( $D$ specimens) or a slight shift in fracture toughness as a function of notch root radius (B specimens). The preliminary fracture toughness results show a negligible influence of the ratio of crack depth (a) to specimen width (W). This suggests that the material has negligible sensitivity to constraint. If true, this should improve the accuracy of predicting failure for structural components. A review of the fracture surfaces showed substantial tunneling of the precrack and of the slow crack growth. Therefore, it is necessary to side groove all future tests of this material.

Completeness of the machining and testing of materials supplied by Duralcan-USA is scheduled after the DWA results are completed.

The fracture surfaces show considerable tunneling of both the precrack and the crack growth that occurs during the test. These results showed the need to machine side grooves for all subsequent tests.

Preliminary fracture toughness results provided no valid KIc values. These results show an apparent lack of sensitivity of $\mathrm{KQ}$ to constraint. 
The identification of some of the limits of applicability for methods developed in Item 2 must await completion of the tests of the Duralcan-USA specimens since the ductility of these materials differs from that of the DWA supplied material.

This study shows that standard fracture toughness tests and technology are capable of being used to predict some of the fracture process for structural components fabricated from MMCs.

\section{Business Development Opportunities}

As this work presently stands, it is premature to offer definitive answers to industry concerning crack propagation in metal matrix materials. Nevertheless, the prelininar results have stimulated interest in further studies from General Motors and the Office of Energy Efficiency and Renewable Energy (DOE). A white paper in response to the interest at EE-DOE (Sid Diamond.

\section{Develop, Build, and Test an Assay System to Measure Transuranic Waste and Fission Product Contamination in Subsurface Soil LDRD 4110}

\section{V. Mclssac}

The purpose of this project is to design, build, and experimentally evaluate field-deployable radiological assay instruments capable of measuring directly low-level concentrations of actinide and ${ }^{90} \mathrm{Sr}$ contamination in subsurface soil. Because the actinides and ${ }^{90} \mathrm{Sr}$ are important isotopes included in the calculation of radiological risk, their concentrations in the subsurface soil at radioactive waste repository sites will have to be characterized and monitored. Since traditional, radiochemical methods used to measure ${ }^{90} \mathrm{Sr}$ and alpha-emitting actinides such as ${ }^{230} \mathrm{Th}, 235 \mathrm{U}$, ${ }^{239} \mathrm{Pu}$, and ${ }^{241} \mathrm{Am}$ in soil are complicated, time consuming, and very expensive, this project could have a major impact by lowering the cost of remediating and closing buried radioactive waste remediation sites. It could also have a major impact on the cost to clean up potentially contaminated Operable Units at DOE facilities.

Three different assay instruments were designed during FY-95. Two of the three instruments were built and subjected to preliminary tests; the third remains conceptual at this time. A specially designed xenon proportional counter $x$-ray detector that detects actinide contamination in soil by measuring $L$-shell $x$-rays was built by TGM Detectors, Inc. and delivered to the INEL in August. A triple proportional counter beta detector that measures ${ }^{90} \mathrm{Sr}$ contamination in soil by measuring high-energy beta particles emitted by ${ }^{90} Y$ was fabricated at the INEL. Testing of these two instruments was initiated in August. A planar model of the triple proportional counter $90 \mathrm{Sr}$ detector was loaned to Idaho State University (ISU) and used by a graduate student for his Masters thesis work and by ISU faculty members to evaluate the effectiveness of commercially available decontamination reagents to remove ${ }^{90} \mathrm{Sr}$ from surfaces of lead bricks, shot, and chips. ISU faculty members are now writing two journal articles describing their work. A conceptual design of a downhole gridded ionization chamber alpha spectrometer was developed, and an invention disclosure record was submitted for the device. Ordela, Inc., Oak Ridge, Tennessee, a manufacturer of custom gridded ionization chambers, signed a nondisclosure agreement for the down-hole ionization chamber and expressed an interest in building a prototype.

\section{Objectives}

- Survey technologies that could be used to measure actinides and ${ }^{90} \mathrm{Sr}$ in subsurface soil.

- Build a triple proportional counter ${ }^{90} \mathrm{Sr}$ detector and procure a Xe-filled $x$-ray proportional counter.

- Perform limited experimental evaluations of the two detectors.

\section{Accomplishments}

A cylindrical triple proportional counter ${ }^{90} \mathrm{Sr}$ detector was fabricated and tested using ${ }^{137} \mathrm{Cs}$ and ${ }^{90} \mathrm{Sr}$ point sources. The device consists of three concentrically arranged proportional counters. The outer two counters are operated in coincidence to detect the high energy beta particles emitted during the decay of ${ }^{90} \mathrm{Y}$. The center counter is operated in anticoincidence with the outer two counters, and is thus operated as a guard detector to minimize the background count rate from cosmic rays and natural terrestrial radiation. Several design problems were discovered and resolved during testing. Overall, the instrument operated as expected and should have a detection sensitivity of less than $10 \mathrm{pCi} / \mathrm{g}$ for ${ }^{90} \mathrm{Sr}$ with a 10 minute count time.

A custom designed xenon proportional counter $x$-ray detector was received from TGM Detectors, Inc. in August. It was selected for evaluation to take advantage of an $x$-ray spectral analysis method already developed at the INEL for germanium photon spectrometers. A used MicroVAX 3300 was purchased in FY-95 to control the operation of the instrument and analyze the collected $x$-ray spectra using available software programs. The body of the counter, which is cylindrical in shape, has an outside diameter of two inches and is 14-inches long. To allow efficient 
penetration of the $x$-rays into the counting gas, two 0.010 -inch-thick beryllium windows are installed in the wall of the counter. The energy resolution of the detector at $6 \mathrm{keV}$ was measured to be $17 \%$, which is sufficiently good to resolve the actinide $x$-rays of interest. Experimental results for a $1 \mathrm{nCi} / \mathrm{g} 239 \mathrm{Pu}$ soil standard indicate the detection sensitivity of the instrument will be less than $100 \mathrm{nCi} / \mathrm{g}$ with a $10-$ minute count time.

A conceptual design for a small-diameter, flow-through Frisch grid ionization chamber alpha spectrometer was developed based in part on our experience with the large-area gridded ionization chambers evaluated for the Rapid Transuranic Monitoring Laboratory. Unlike the long-range alpha detector developed at LANL and the electret distributed by RADELEC, INC., the proposed device will identify specific actinide isotopes by measuring the pulse-height spectrum of the alpha activity in the soil. The other two devices are clever gross alpha counters. The ionization chamber will identify specific alpha-emitting isotopes such as ${ }^{230} \mathrm{Th},{ }^{234} \mathrm{U}$, ${ }^{238} \mathrm{Pu},{ }^{239} \mathrm{Pu}$, and ${ }^{241} \mathrm{Am}$. Ordela, Inc., agreed to collaborate on the development of a down-hole gridded ionization chamber alpha spectrometer.

\section{New or Increased Technical Capabilities}

Douglas Greenwell, who is manager of the Soils Restoration Department at the INEL, expressed a strong interest in the instrument for use in characterizing surficial ${ }^{90} \mathrm{Sr}$ contamination at a number of sites at the INEL. Professor Brey at ISU has requested that the planar model of the detector be returned to ISU as soon as practical so that they may continue using it to measure the effectiveness of decontamination reagents to remove ${ }^{90} \mathrm{Sr}$ from lead bricks. The limited experimental results for the xenon x-ray detector obtained to date indicate the instrument will be capable of quantifying $100 \mathrm{nCi} / \mathrm{g}$ ${ }^{239} \mathrm{Pu}$ in soil with a 10 -minute count time.

\section{Business Development Opportunities}

Lockheed Idaho is filing a patent application for the triple proportional counter ${ }^{90} \mathrm{Sr}$ detector. Canberra Nuclear and one other vendor have agreed to sign nondisclosure agreements for the detector. The prognosis is very good that a licensing agreement will be signed in FY-96.

\section{Biodecontamination of Massive Concrete Structures} LDRD 4171

\section{R. D. Rogers, M. A. Hamilton}

This $L D R D$ responds to the Needs Statement $D D-7$ of ID/IP Decontamination and Decommissioning of Facilities Integrated Demonstration Program. The task identifies an innovative technology to decontaminate concrete. It is estimated that a developed biological process could reduce the costs associated with existing D\&D technologies, which rely on physical removal and disposal of contaminated portions of buildings and structures. The proposed technology is an in situ process that should be applicable for use on concrete floors, walls, unlined reactor pools, and, possibly, rubble. The process has the potential to reduce the waste volume and contamination levels of secondary contaminated waste generated during $D \& D$ activities by fifty percent and, therefore, save the Department of Energy considerable valuable resources as well as reduce costs.

The project involves the use of a naturally occurring, nonpathogenic, microbiological process. The microorganisms used for the process are known to be responsible for the microbially influenced degradation (MID) of concrete. The process will pose no health risks, and the spread of MID organisms will be controlled through the manipulation of key nutrients. The concrete surface, loosened by the bacterial activity, should be removed with little physical effort.

\section{Objectives}

- Develop a biodecontamination process. An accelerated testing technology, already developed at INEL for cement solidified waste forms, will be adapted to concrete surfaces. This will allow rapid evaluation of conditions (physical, chemical, and biological) that affect MID of these concrete surfaces. Information from this work will be used in the design of a laboratory-scale biodecontamination test chamber, which will be built and tested.

- Conduct an evaluation of the process of MID at manmade structures. Evaluate data obtained from the literature and select massive concrete structures currently hosting the natural occurrence of MID. The purpose of this effort is to begin to develop a data base for understanding the key environmental conditions that initiate and sustain the MID process.

- Develop an ongoing effort to determine facilities at DOE sites that could benefit from the use of a passive decontamination process. Select a facility at which a field test demonstration could be conducted.

\section{Accomplishments}

The technical focus has been on developing an understanding of the MID process and how it can be adapted for the decontamination of concrete surfaces. We decided that of the three types of MID microorganisms that exist in nature, the 
thiobacilli species would be most suitable for continued work. Based on past experience, data from the literature, and a visit to the laboratory of the world's authority on MID, a design was developed for an MID test cell. The cell was designed to be attached to a concrete wall so that real-time studies can be conducted on the conditions required to promote and enhance the development of the MID process. Conditions in the design criteria included temperature, humidity, sulfur source and concentration, processing time, and application of the inoculum source. Two $20 \times 20 \times 6$-cm rectangular prototype test cells have been manufactured for laboratory studies. They have been attached to blocks of concrete and we have initiated test development. Based on experience of designing, fabricating, installing, and initial operation of the prototype laboratory cells, four $1 \times 1-\mathrm{m}$ cells are currently being manufactured. These cells will be used in the field evaluation of the MID decontamination process.

In conjunction with the above work, an accelerated MID test program was started to determine the effect of thiobacilli sp on epoxy coatings used to seal contaminated concrete surfaces. This study differs from those using test cells in that the coated specimen is being sprayed at predetermined intervals with a liquid culture of thiobacilli. Past experience with this method has shown it to be a way to accelerate the affects of MID. After six weeks of testing, the exposed coating appears to be lifting from the concrete surface. Successful penetration of the coating will add significantly to the importance of the MID biodecontamination process.

Many of the numerous, contaminated facilities at the INEL have been evaluated as possible sites for both the field evaluation study and as locations for field demonstration of the MID process. A site has been selected for conducting the field evaluation of the process. It is fitting that this initial work will be conducted at EBR-I, which is the facility where nuclear energy was first used to produce electricity. Selection of the facility for field demonstration will occur after the successful completion of the field evaluation study.

New or Increased Technical Capabilities

This project is improving the INEL capability in applied biotechnology. The INEL is becoming a recognized world leader in research in the area of biological effects on concrete. It is expected that the INEL will be a resource in developing methods to control MID activity.

\section{Business Development Opportunities}

A successfully demonstrated decontamination technology will be readily sought after by those involved in D\&D operations. As an outgrowth of the work funded by this LDRD, British Nuclear Fuel ltd (BNFL) signed a Co-operative Research and Development Agreement (CRADA) on August 22, 1995. The intent of the CRADA is the further development of the MID decontamination process to the point that BNFL will be able to use it as a commercial process. The reported worth of the CRADA is $\$ 3 \mathrm{M}$ over a three-year period.

\section{Catalyst Assisted In Situ Bioremediation LDRD 4172}

\section{B. D. Lee, W. A. Apel}

At least 60 waste sites at 18 Department of Energy (DOE) facilities and numerous industrial facilities have the need for in situ treatment technologies to remediate mixtures of chlorinated solvents as the primary contaminant in groundwater and unsaturated subsurface soils. Current methods used for remediation of these subsurface contaminants include pump and treat, followed by air sparging, hydraulic containment, or soil vapor extraction. Catalyst-assisted in situ bioremediation represents a technology that can replace or be used to complement current physical methods for total remediation of a contaminated site. The primary advantages forwarded by coupling chemical catalysis with biologically mediated electron transfer are increased applicability (site to site) and increased dechlorination rates. Microorganisms that establish a reducing environment in the subsurface might not have the ability to carry out reductive dechlorination. By adding a catalyst and coupling it to the microbial system, dehalogenation can occur regardless of the ability of the indigenous microorganisms to carry out reductive dechlorination.

\section{Objectives}

- Determine the ability of the microbial population to dechlorinate subsurface soils and groundwater.

- Assay transition metal catalyst to determine reaction rates as well as substrate reactivity.

\section{Accomplishments}

We performed microbial testing using batch experiments to enrich for sulfate reducing bacteria (SRB) from subsurface soil from Dover Air Force Base (AFB). The experiments were set up using lactate as the carbon source. Testing was done at 15 and $30^{\circ} \mathrm{C}$ to determine differences in acclimation as effected by temperature. Groundwater temperature in most aquifers is in the range of 10 to $15^{\circ} \mathrm{C}$.

Experiments were set up for acclimation with and without tetrachloroethylene (PCE). Studies were made on lactate removal by the cultures during SRB enrichment, and upon subsequent 
respikes with lactate. Visual examination of the test vials showed the formation of ferrous sulfide, an indication that sulfate reduction was occurring. As the concentration of PCE in the reaction vial increased, the removal of lactate decreased. The removal rate of lactate at $30^{\circ} \mathrm{C}$ was greater than at $15^{\circ} \mathrm{C}$. PCE removal results indicated that dechlorination of PCE did not occur over the test period. These experiments are continuing to determine the amount of time required for acclimation to the solvent. Experiments are in progress to determine whether the addition of vitamin B12 decreases the amount of time required for dechlorination to occur.

We are using differential soil bioreactors to determine the dechlorination of PCE under accelerated and catalyst-assisted conditions. The differential soil bioreactors were fabricated out of stainless steel for continuous flow testing. The system has been leak checked and calibrated for operation. The reactors have been loaded with soil from Dover AFB and will be operated at a temperature of $15^{\circ} \mathrm{C}$.

Catalysts were synthesized for the project using vitamin B-12 as the standard. Vitamin B-12 is a cobalt-containing macrocycle that has demonstrated dechlorination activity with a reducing agent and coupled to electron transfer from microorganisms. Six catalysts were synthesized and tested for their reactivity towards dechlorination.

Table 1

Loss of methylene chloride after 24 hours of reaction with various catalysts at room temperature.

\begin{tabular}{lcccc}
\multicolumn{5}{c}{$\begin{array}{c}\text { Table 1 } \\
\text { Loss of methylene chloride after 24 hours of reaction } \\
\text { with various catalysts at room temperature. }\end{array}$} \\
Catalyst & $\begin{array}{l}\text { \% Area /Mole } \\
\text { Difference }\end{array}$ & $\begin{array}{l}\text { Relative } \\
\text { Rank/Mole }\end{array}$ & $\begin{array}{l}\text { \% Area/Weight } \\
\text { Difference }\end{array}$ & $\begin{array}{l}\text { Relative } \\
\text { RankWeight }\end{array}$ \\
\hline CMC & 28.2 & & & \\
CPC & 12.9 & 0.82 & 31.3 & 0.39 \\
CSC & 18.4 & 0.38 & 30.1 & 0.38 \\
CPPC & 11.6 & 0.54 & 29.2 & 0.37 \\
NPC & 34.3 & 0.34 & 26.8 & 0.34 \\
IPC & 13.9 & 1.00 & 80 & 1.00 \\
Vitamin B-12 & 18.5 & 0.40 & 32.5 & 0.41 \\
& & 0.54 & 21.5 & 0.27 \\
\hline
\end{tabular}

Three of the catalysts were synthesized using cobalt as the central metal, and different macrocyclic ligands were attached to the metal. A second set of catalysts varied the central metal using the same porphyrin. Dechlorination activity of the catalysts for methylene chloride was tested using borohydride as the chemical reducing agent. Because of borohydride activity with the methylene chloride, controls were run to determine the remaining methylene chloride in the reaction vials. Loss of methylene chloride can be seen in Table 1. The catalyst designated NPC gave significantly better destruction of methylene chloride than the other catalyst synthesized, including vitamin B-12, on either a weight or mole basis. Based on molar concentration, a close second was achieved by catalyst $\mathrm{CMC}$, followed by vitamin B-12. Screening tests will be done to couple the synthesized catalysts to the electron transfer from the sulfate reducing bacteria isolated during the first task during FY-96.

\section{New or Increased Technical Capability}

INEL is gaining research expertise related to in situ bioremediation of chlorinated solvents through this research project. A more detailed database comparing intrinsic, accelerated, and catalystassisted in situ bioremediation will be developed through this research. Continued evaluation of the differential soil bioreactor as an engineering tool will be achieved through this project. Acceleration of the in situ process can greatly decrease the amount of time for the government and industry to clean up subsurface soils and sediments.

Business Development Opportunities

Internal communication has occurred between work organizations because of the type of expertise required on this project. Collaboration with DuPont is occurring on the project as well. The INEL is also involved in the Remediation Technologies Development Forum, a consortium of private industry companies and the government, studying different methods of remediation to clean up contaminated sites. There is collaboration rather than competition to solve similar environmental problems.

\section{Sustainable Water Resources Management LDRD 4204}

$$
\text { J. I. Mills }
$$

Sustainable management of water resources is a complex and volatile challenge facing the countries of the world today. There are many areas where water demands are beginning to exceed supply, or where surface and ground waters are being polluted and aquatic plants and animals are threatened. Finding solutions to these and similar issues is becoming increasingly difficult as more diverse groups of stakeholders are involving themselves in the management of water resources.

The INEL is pioneering the application of systems analysis for identifying and optimizing long-term water management strategies and facilitating resolution of conflict and misunderstanding among stakeholders. We developed a water resource management and decision making software package for the Snake River Basin 
using input from a wide range of water resource stakeholders. This PCbased tool allows diverse stakeholders to rapidly assess the effects of proposed solutions to complex water management issues. An important feature is its ability to simulate the basin hydrology, including surface and ground water, agriculture water use and economic impacts, and hydroelectric generation and sales.

\section{Objectives}

- Identify the Snake River Basin water resource stakeholders and the issues that are of most interest to them.

- Define a systems model for the Basin that will assess key stakeholder issues.

- Develop a PC-based computer simulation of the Basin's water resources, including a userfriendly interface.

- Hold a stakeholder workshop to introduce the model and obtain feedback on its effectiveness and usefulness to the stakeholders.

\section{Accomplishments}

A team composed of personnel from INEL, industry (Rocky Mountain Institute), a university (Washington State University), and two consultants with water expertise was formed to perform this LDRD project. Stakeholders that were pursuing water issues in the Snake River Basin were identified, first by interest and then by name. Important areas of interest included agriculture and aquiculture, electric power generation, environ- mental concerns (including preservation of endangered species), water rights (including claims by Indian tribes), and water management by state and federal agencies. Contacts were made with numerous individuals in each of these areas to identify specific issues that were of interest or concern.

Results were shared among team members using e-mail to create a virtual office environment.

Using the stakeholder-established priority issues, we developed a PC-based systems simulation of the Snake River Basin. The simulation is similar to Management Flight Simulators that have proven successful in other fields, such as electric power or insurance. Use of the program can help stakeholders examine issues and options and approach consensus by conducting

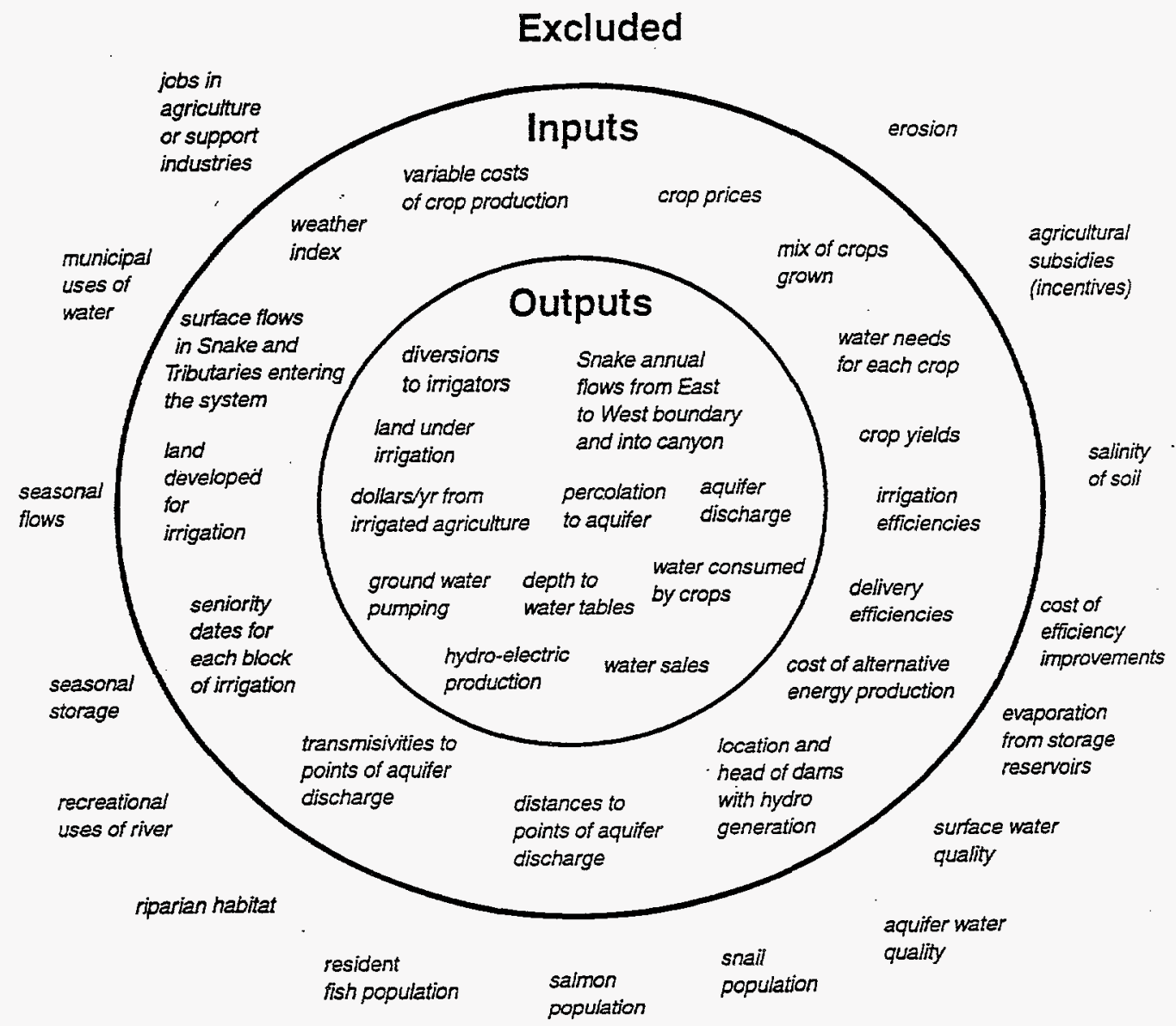

System Boundary for the Snake River Explorer 
what if explorations. The figure

below presents a bulls eye diagram showing what is included and excluded from the simulation.

A hydrological model that couples the surface and ground water is the primary engine of the simulation. Storage in individual reservoirs is not included. Annual precipitation can be specified as either average, dry, wet, or a random combination of these three. The aquifer is segmented into four regions, and river recharge is concentrated in two areas along the river. Aquifer level in each of the segments is calculated. Farming areas are modeled in five broadly defined irrigation districts. Three districts use surface water exclusively, and two use pumping exclusively. Each irrigation district has four farms represented, where different crops can be grown (each requiring different amounts of water) and economic benefits can be derived. The simulator includes the capability to examine how water use efficiency and land following affects river flows. The program includes a simple model of water rights that determines the amount of land under irrigation in each of the irrigation districts.

Electric power generation in the Basin is calculated based on the flow and distribution of water along the river. The amount of energy that could be generated by dams downstream of the Basin, using water flowing from the basin, is also calculated. The economic value of the electric power is combined with the economic value of crops grown to provide an economic indicator called the Gross Snake Product. The simulator includes the capability to plot cumulative measures of water and economic performance over a 100-year simulation period.
A workshop with eighteen stakeholders representing a wide range of interests was held in Boise. Feedback from the stakeholders indicated that they expected the integrated systems approach taken with the simulation to be important in the resolution of future water resource management issues. Generally, participants in the workshop agreed that the existing model needs the addition of reservoir storage and improved information for water rights to make it more realistic.

\section{New or Increased Technical Capability}

The INEL now has a demonstrated capability in development and use of water resource decisionmaking tools. Using this tool as a lever, the INEL should work with local, national, and international entities to enhance the simulation and provide water management consulting and educational services. In addition, there should be collaboration with universities, government agencies, and the private sector to examine other important natural resource issues, such as land use management, soil erosion, water quality, and preservation of endangered species.

\section{Business Development Opportunities}

There has been close cooperation with the Alternate Energy Products directorate and the Environmental Life Sciences Products directorate. Contacts for possible development of CRADAs are currently being planned with the Bureau of Reclamation, the Idaho Department of Water Resources, and the World Bank.

\section{High-Sensitivity \\ Nondestructive Analysis for \\ Transuranic Waste Characterization \\ LDRD 4275}

\section{Y. D. Harker, G. K. Becker}

The purpose of this project was to evaluate two methods for producing a high sensitivity/high penetration nondestructive assay method for determining the total activity in a 208-liter (55-gallon) drum containing waste contaminated with transuranic and uranium compounds. The major need for this technology is in determining the activity concentrations down to 1 $n C i / g$ so that segregation of waste into TRU, low level, or shallow land burial categories can be determined based on the criteria established by the Environmental Protection Agency. Waste forms that are of particular concern are the sludges stored at the Radioactive Waste Management Complex. For these waste forms, existing assay techniques cannot penetrate the center of the waste, and thus fail to meet EPA accuracy requirements. Considering that more than $50 \%$ of the waste stored at RWMC is in this waste form, it is imperative that we develop a new method for assaying such wastes. We judged two methods of active interrogation to have the penetration and sensitivity to meet this challenge. The first is fast neutron interrogation and the second is photon interrogation. This LDRD project was funded to look at these two approaches and determine their potential with respect to meeting the criteria of assaying waste like the sludge waste forms.

\section{Objectives}

- Test the feasibility of using He4 fast neutron detectors in conjunction with a fast neutron $(800 \mathrm{keV})$ interrogation neutron source to stimulate fissions in plutonium. 
- Test the feasibility of using $\mathrm{He}-3$ thermal neutron detectors inside a moderator assembly in conjunction with an interrogation source of high-energy Bremsstrahlung photons (6.5 $7.5 \mathrm{MeV}$ ) to stimulate photo fissions in plutonium.

\section{Accomplishments}

The basic premise of the concept being studied is to use an interrogation neutron source whose energy is below the lower energy limit of sensitivity for the $\mathrm{He}-4$ neutron detectors. Using this concept, one could interrogate with fast neutrons and at the same time detect the higher-energy neutrons created by fissions without detecting the interrogating neutrons. This would enhance the sensitivity of the method over conventional neutron interrogation concepts, which use pulsed interrogation neutron sources and count fission neutrons following the interrogation. During FY-94 we tested the new concept using $230 \mathrm{keV}$ interrogation neutrons and reported the results in the annual report for FY-94 LDRD 7905. From the results of the FY-94 project, we concluded that the concept should be tested further using higher energy interrogation neutrons ( 800 $\mathrm{keV}$ ) to improve the penetrability into plutonium chunks. This became one of the objectives for FY-95.

For the FY-95 tests, we used the same detector configuration fastened to a 208-liter (55-gallon) drum as was used in FY-94; only the energy of the interrogation neutrons was changed. As in FY94, the concept using a higher neutron energy demonstrated the desired sensitivity, although there was some increase in background readings due to a small sensitivity in the He-4 detectors to the $800 \mathrm{keV}$ interrogation neutrons. These tests showed that the higher energy neutron interrogation provides a marked improvement in assaying plutonium in the form of dense chunks. However, even at the higher interrogation energy, there is a significant number of neutrons being thermalized in the matrix. This will be a source of uncertainty in all neutron based interrogation systems.

With respect to the second objective for FY-95, we performed a small-scale test using a small moderated He- 3 detector assembly that had been designed for use with photon interrogation. Originally the plan called for a sensitivity test using a large moderated $\mathrm{He}-3$ detector assembly and a 208-liter (55-gallon) drum. The large He-3 detector assembly was not designed for this use, so we modified the test plan to use a smaller neutron detector designed specifically for photo neutron detection. The main objective of the smaller scale tests was to determine the penetrability of the interrogating photons. It was clear that photon interrogation has the desired penetration capability. What remains to be done is to design and fabricate a larger detector assembly with the capability of accommodating the photon flash. Current knowledge indicates that this can be accomplished by extending the design used by the smaller detector to a larger scale. Therefore, the conclusion of this project is that photon interrogation using the right mix of interrogation source and detector design is a viable approach to meet the sensitivity and penetration requirements for waste assay at RWMC.

\section{New or Increased Technical Capabilities}

As stated, this project was created to address a need for nondestructive assay at the INEL RWMC. We found that both fast neutron interrogation and photon interrogation have the potential to meet this need. Fast neutron interrogation has the sensitivity and has improved penetrability over conventional thermal neutron interrogation. Photon interrogation has the desired penetrability. While sensitivity was demonstrated in this project, the results provided have shown that better sensitivity can be achieved with the right design of detector circuitry.

\section{Business Development Opportunities}

This project was directed. toward a specific need at INEL; however, this development could benefit other sites. It will require more large scale testing before commercialization can be considered.

\section{Flexible Fish Conduit Passage System LDRD 4374}

\section{B. N. Rinehart}

This report summarizes the action taken during FY-95 on the conceptual design of an in-reservoir submerged conduit for transporting juvenile salmon and steelhead (known as smolts) downriver to increase the fish runs, particularly wild salmon, in the Columbia River Basin. Efforts to control the waters of the Columbia River Basin for positive gains have not always yielded completely positive results. A less-than-desired consequence is the impact that reservoirs and dams have had, and continue to have, on the Basin's salmon populations. A concept to minimize this impact is the development and operation of an in-reservoir conduit saimon passage system. The system would minimize smolt mortality rates and provide a speedier and less stressful downstream passage to the ocean.

\section{Objectives}

The Idaho National Engineering Laboratory (INEL) has proposed an in-reservoir submerged 
conduit for smolt transportation as an alternative to an open concretelined channel. The method of fish collection is not addressed in this report, since it is a separate problem that must be addressed for all conduit configurations. Several fish collection techniques have been proposed by biologists, and the United States Army Corps of Engineers (Corps) is presently addressing this issue. The fish would be taken through or past the dams by a fish passage designed by the Corps. The details of the dam modifications are being addressed by the Corps and, therefore, are not addressed here. The conduit will provide for the safe downstream passage of juvenile salmon and steelhead within the Columbia River Basin. Transporting mature fish back to their respective spawning areas is not within the scope of this fish transportation system.

\section{Accomplishments}

Several conduit configurations are being evaluated. All are flexible, in-reservoir configurations. As presently envisioned, all configurations would be sized for $5000 \mathrm{~m} / \mathrm{h}$ $(3.1+$ miles per hour) flow and would consist of a flexible, thin membrane conduit with appropriate means of maintaining flow velocity within the required limits. The conduit would be submerged under shipping traffic routes and shoreline boating access lanes. Resting areas would be provided by either conduit rest areas, or by in-reservoir net pens.

This fish transportation concept is designed to satisfy four basic biological requirements. In addition, the concept is also designed to meet the basic engineering and legal requirements. The four basic biological requirements are as follows:

- Rapid transit of smolts. The transmit system should pro- vide a time for transit between the point of hatching and the ocean that is roughly equivalent to the historical river speed, which is approximately 3 to 5 feet per second.

- Maintenance of river sense trail. A transport system must supply continuous input to the development of a river sense trail if mature fish are expected to return to the proper spawning area. The migratory habits of fish indicate that fish accumulate knowledge or a sense of the river path to the ocean as they travel the path downstream, and this sense trail is replayed in reverse when they return to spawn.

- Biological and environmental compatibility with fish. Any transportation system must provide a proper environment for the fish during their passage. This environment includes proper temperature, oxygen, rest areas, feeding opportunities, protection from predators, etc. The materials and the transportation process themselves must also be harmless to the fish.

- Reservoir use compatibility. Many reservoir uses must be considered in the design and placement of a fish transportation system. These uses include short-based activities that cannot be blocked in the access to the reservoir, reservoir navigation (both through and across the reservoir), and variations in reservoir heights resulting from electrical production and irrigation demands.

The advantages and unique options of the proposed conduit system are as follows:

- Usable in any reservoir. The conduit system can be used as a submerged system and, if required, as a surface system.

- Accommodates reservoir use, surface, and shore. The conduit system has the capability of being placed submerged or on the surface. This makes it usable in any reservoir, including those that have barge traffic, boats, skiers, and fisherman.

- Oxygenation. The system allows for the addition of oxygen if needed. If so, the pressurized water supply to the water jet ring can be supersaturated with oxygen and exhausted into the conduit to supply oxygen to the fish. Medication or anything else dissolvable in water could also be supplied

- Underwater storage. Since the conduit will not be needed year round, it can be sunk and stored on the bottom for most of the year, owing to its flexibility and water-tolerant design. Recharging the floatation devices would bring it to its operational grade when needed.

- Variable location. The lightweight and flexible nature of the conduit system allows it to be moved if future demands on the reservoir so dictate. Modifications in its linear makeup (substitution of underwater sections for surface sections) would also be possible.

- Simple, mass-produced components. There are only two basic components to the system: the conduit sections and the water-jets sections (the underwater section is a fully closed surface section). The water-jet sections are used to connect the conduit sections together. By consisting of only two basic components, the sys- 
tem allows for full use of massproduction techniques.

- Easily installed on site. The fabrication process for the conduit consists of unrolling the conduit sections from the back of a construction boat, attaching the floats, and connecting the sections with the pumping sections.

- Simple operation from one location. The pressurized water for the pumping section is supplied from one or both ends of the conduit. Control of the pressured water constitutes control of the conduit.

\section{New or Increased Technical Capabilities}

The INEL has the capability to provide a broad spectrum of support services to the conduit team to ensure timely and efficient design. The team comprises the Department of Energy (DOE), Bonneville Power Administration (BPA), and the U.S. Army Corps of Engineers (Corps). Support will be derived from INEL personnel with subcontractors used for special expertise. The various forms of support include, but are not limited to, cost and schedule engineering support, technical analysis, and design engineering support for the flexible inreservoir conduit.

\section{Business Development Opportunities}

Salmon passage is a critical issue to the Pacific Northwest, where over $50 \%$ of the area's electricity demand is met by hydropower generation. The flexible conduit is a viable solution to help solve the salmon passage problem and still maintain a valuable source of electricity. The INEL is one of the primary proponents of the flexible conduit system. If decisionmakers select this option, INEL will be in a position to provide the needed technical support.

\section{Methodology for Assessment of Power Plant Emissions on Air Quality-related Values \\ LDRD 4550}

$$
\text { G. J. White }
$$

Section 160 of the Clean Air Act (CAA) requires Federal land managers to adopt measures "to preserve, protect, and enhance the air quality in national parks, national wildemess areas, national monuments, national seashores, and other areas of special national or regional natural, recreation, scenic, or historic value." The CAA also contains "prevention of significant deterioration" ( $P S D$ ), provisions aimed at protecting and enhancing the air quality in wilderness areas and other locations of special scenic, recreational, historical, or natural value. The focus of this $\angle D R D$ is on the evaluation of ecological indicators of impacts to air-quality-related values from pollutants emitted by coal-fired electric utilities. We have developed a method for assessing the ability of various ecological indicators to assess these impacts.

\section{Objectives}

- Develop and implement a conceptual framework to establish and prioritize criteria for evaluating potential ecological indicators of changes in air-quality-related values (AQRVs).

- Identify specific methods for applying indicators to assess changes in the status of AQRVs for use by the electric utility companies, responsible federal land managers, and other interested parties.

- Develop a prototype methodology for implementing an $A Q R V$ monitoring program in Class I areas.

- Finalize and maintain a data base containing information pertinent to the AQRV issue, including legislation and standards, sampling and monitoring techniques, available data, etc.

\section{Accomplishments}

The overall objective of this work was to produce a standardized method by which indicators of air pollutant impacts to air-qualityrelated values (AQRVs) in Class I areas may be identified and evaluated. To accomplish this, a conceptual framework for establishing and ranking criteria to be used in evaluating various potential ecological indicators of changes to AQRVs was developed. The criteria thus identified were then evaluated using this conceptual framework. Some criteria were identified as being critical to the success of a monitoring effort (must criteria), whereas others were determined to be desirable but not necessarily essential (want criteria). Indicators that do not meet all the established must criteria are eliminated from consideration. A ranking system was then put into place by which the relative importance of each want criteria may be established.

The ranking system was evaluated during a workshop conducted under separate funding during which members of the environmental subcommittee of Western Utilities Supply and Transmission (WEST), an organization of energy producers in the western U.S., were introduced to the process. Specific methods for applying indicators to assess changes in the status of AQRVs were evaluated using this process during the workshop, and feedback from the attendees resulted in minor alterations in the evaluation procedure for indicator criteria.

A draft prototype methodology for implementing AQRV monitoring programs in Class I areas was developed before the WEST workshop. This methodology 
included descriptions of the legislative authority regarding protection of Class I wilderness areas, the overall process by which land managers determine Class I areas and AQRVs at risk, and the process by which ecological indicators should be evaluated in terms of their ability to assess AQRV status. The methodology was revised following the workshop.

A database of information pertinent to the AQRV issue was expanded and continues to be maintained. This database includes pertinent legislation and standards, techniques for sampling and monitoring using ecological indicators, and data available to date.

New or Increased Technical Capabilities

The process described above has been designed to provide a useable, cost-effective method for developing AQRV monitoring programs for Class I areas, a method that is acceptable to the electric utility industry, regulatory and non-regulatory government agencies, and other interested parties. As such, it supports the needs of western coal-fired utilities. Completion of this LDRD has allowed INEL to remain on the forefront of issues related to monitoring and assessment of air-pollution-related impacts to ecological systems in wilderness and other remote areas. This will allow us to act as an unbiased expert to help industry comply with federal monitoring and assessment requirements in Class I areas.

\section{Business Development Opportunities}

This project has been conducted as a collaborative effort with Western Energy Supply and Transmission (WEST). WEST has funded a series of workshops designed to evaluate and improve the procedure developed as part of this LDRD. The first workshop was held in Sun Valley in June, and was attended by members of the WEST environmental subcommittee. A revised version of the process will be presented and discussed at a second workshop scheduled for December in Denver, to be attended by personnel from the various Federal agencies involved with $A Q R V$ issues. This second workshop will enable us to incorporate input from the Federal agencies to the process. A third workshop designed to evaluate the decision-making process will be held in Pocatello in early 1996. The results of the three workshops will be reported in March to the WEST environmental subcommittee during their annual meeting in Phoenix. At the conclusion of the Phoenix meeting, the WEST Management Committee will decide on follow-up funding to field test the procedure. Furthermore, although developed for the electric utility industry, the process has direct application and is being marketed to other industries, including the petroleum and mining industries.

\section{Passive Containment of Volatile Organic Soil Vapors \\ LDRD 4579}

\section{W. C. Downs}

The migration of contaminant vapor plumes in the vadose (unsaturated) zone at many DOE and industrial sites is a topic of major concern. Locally, evidence indicates that unmitigated migration of the contaminant plume at the Radioactive Waste Management Complex (RWMC) will eventually contaminate the ground water and move beyond the RWMC boundaries. The objective of this effort was to demonstrate, in the field, the feasibility of passive air injection to create a pressure buffer beneath the contaminant plume, preventing its further ver- tical migration downward. Previous work has documented the existence of pressure gradients between the atmosphere and subsurface soil gas. This project makes use of these pressure gradients to inject fresh air into the subsurface beneath the contaminant plume at the RWMC. We designed and constructed a one-way valve (check valve) and used it to prevent the exhaustion of the injected air, thus maintaining the increased subsurface pressure. Injected air flows of up to 2 cubic feet per minute were observed, and tracer studies showed a radius of influence of at least 20 feet.

\section{Objectives}

- Design and fabricate a oneway injection valve.

- Instrument the test well.

- Collect flow and pressure data.

- Perform tracer test for radius of influence.

- Submit project report.

\section{Accomplishments}

We designed a one-way valve (check valve) that admits fresh air into a well whenever atmospheric pressures exceed pressures in the subsurface. The valve was engineered for simplicity of maintenance and minimal expense. It was made of polyvinyl chloride (PVC) parts (purchased from a local hardware store) and a thin Lexan sheet. Some machine work was done on the PVC. In operation, the thin Lexan sheet, or diaphragm, lifts to open at a pressure differential of only 0.06 millibars, admitting air into the well.

In this application, we mounted the valve directly to the end of the 4-inch pipe stem protruding from the borehole. In the borehole, the pipe stem connects on its lower end to a straddle-packer, which functions essentially like a well screen, allowing access to a 3-meter isolated interval of the borehole. 
The well was instrumented with an anemometer and a pressure transducer to record flow and pressure in the pipe.

A multi-level sampling well located 20 feet from the injection well provided pressure measurements in the subsurface. The well was configured with eight sample ports at various depths in the subsurface. Pressure transducers were connected to the sample tubes, and one to the atmosphere. Fifteenminute data were fed to a data acquisition system.

We performed a tracer test by injecting a pulse of sulfur hexafluoride (SF6) through a thin tube lowered to the center of the straddlepacker. Tracer concentrations were monitored in the multi-level sampling well at a port horizontal to the straddle-packed interval.

Pressure data showed that the subsurface interval adjacent to the straddle-packer was pressurized by high atmospheric pressures at land surface. We observed that when the atmospheric pressure dropped, the pressure in Port 5 (adjacent to the straddle-packer) remained higher than pressures above or below.

Tracer data show rapid movement of $\mathrm{SF}_{6}$ from the straddlepacker to the sample port 20 feet away. With all other modeling parameters held constant, a diffusion coefficient $\left(1.0 \mathrm{~m}^{2} /\right.$ day) twice as large as those used successfully in other INEL modeling studies $\left(0.5 \mathrm{~m}^{2} /\right.$ day) was necessary to match the observed data. This result indicates tracer movement, not just tracer diffusion.

Pressure increases observed at the depth of air injection indicate that passive injection does establish a buffer zone in the subsurface which may prevent further downward migration of soil vapors, effectively isolating the underlying aquifer from contamination. Tracer studies indicate that at the loca- tion studied in this report, a radius of influence of at least 20 feet has been established. This is supported by modeling studies of arrivaltime and breakthrough shape, which indicate that diffusion alone does not account for the rapid arrival of the tracer.

\section{New or Increased Technical Capabilities}

These results are the first published data showing the use of air injection as a vadose-zone plume containment technology. It was performed using inexpensive equipment, relying on natural atmospheric pressure fluctuations as a driving force.

\section{Business Development Opportunities}

During the course of this research, we made contact with the UnoCal Oil Company of California and reached a tentative agreement to investigate the potential of passive injection at one of their sites. The interest is centered around the concept of supplying air to microorganisms in the subsurface to stimulate their biodegradation of contaminant fuel hydrocarbons. This technology, known as bioventing, is presently accomplished with electrical motors and blowers. The passively powered technology demonstrated at the INEL is of interest because of its low cost, low maintenance properties. Business development funds have been requested to pursue this opportunity.

\section{Development of Vadose Zone Monitoring Instruments LDRD 4581 \\ J. M. Hubbell}

This LDRD focused on developing vadose zone monitoring instruments to rapidly and effectively char- acterize, monitor and sample the unsaturated zone. This LDRD built, tested, and refined the design for four vadose zone instruments invented at the INEL including the portable tensiometer, deep tensiometer, suction bailer, and a field matric potential sensor (FMPS). The portable and deep tensiometer and FMPS measures matric potential (soil tension) for calculation of flux rates and direction of flow in the unsaturated zone. The suction bailer collects water from unsaturated materials or thin saturated layers for analysis of chemical or radiochemical constituents. Vadose zone instruments are used to detect contaminant or water movement before the contaminant reaches the water table. These instruments can be utilized at numerous sites throughout the world including waste disposal sites such as the RWMC, infiltration ponds (ICPP, TAN, etc.), inactive tailing ponds or other sites were contaminant movement is a concern. Patents applications have been prepared on the first two of these inventions and inventions disclosures have been filed on the remaining two inventions. These vadose zone instruments will reduce costs and decrease uncertainty in: site characterization, monitoring the facility while in operation, and post-closure monitoring for subsurface contaminants and water movement. They will provide data previously unattainable for determining if the site has the potential for contaminant or water movement to the water table.

\section{Objectives}

The overall objective of this LDRD is to refine and develop four vadose zone monitoring and sampling instruments. Specific objectives are:

- Build, test, and evaluate a new deep tensiometer design. Perform proof of principle for this innovative design.

- Refine and evaluate the design of the portable tensiometer. Test the reliability of the measurements to standard instru- 
ments in laboratory and field experiments. Test the range of geologic materials that can be monitored.

- Build, test and evaluate the suction bailer. Refine the design for both unsaturated and saturated conditions.

- Fabricate a prototype field matric potential sensor and laboratory test the conceptual design.

Test results will be documented on the deep tensiometer, portable tensiometer, suction bailer and field matric potential sensor. Patent applications will be submitted for the deep and portable tensiometers.

\section{Accomplishments}

All of the FY95 objectives were met for this LDRD. The deep tensiometer design was built, tested, and resultant data evaluated. The second design of the deep tensiometer that allows an unlimited depth of installation was proven in field tests and tested in the field for over five months. Development of the deep tensiometer spawned two additional designs for specialized applications and reduced the overall costs of the downhole portion of the instrument by a factor of 10. A patent application for the deep tensiometer was submitted to the Patents and Trademark Office (PTO).

The design of the portable tensiometer was evaluated and refined. The reliability of the measurements to standard tensiometers was tested in laboratory and field experiments. The range of geologic materials that can be monitored was tested and a solution successfully tested to allow use in course grained materials. A design was built and tested to allow continuous logging in small-diameter wells (1.5 inches). A patent appli- cation was submitted to the PTO on the portable tensiometer.

Demonstration suction bailers were built, tested and evaluated in the laboratory. The design was refined for use in both unsaturated and saturated conditions. Laboratory tests were preformed on the porous stainless steel and ceramics to use in the suction bailer.

A prototype field matric potential sensor was fabricated and laboratory tested indicating that this concept is viable. The design was refined to reduce the time to collect matric potential data, increase the reliability and make the instrument easier to maintain and use.

\section{New or Increased Technical Capabilities}

All of these instruments have been invented at the INEL and so there is no work being performed anywhere else in the world on these devices. The INEL is becoming the world leader on tensiometer design. These instruments will allow accurate measurement of matric potential at depths previously not possible. They have application for basic research to test the underlying theories of water transport in the vadose zone and can be used for field measurement of moisture/contaminant transport at waste disposal and agricultural facilities. These technologies need to be used in field tests and the results published.

\section{Business Development Opportunities}

Business development opportunities varied by the individual instruments. We have worked with several companies but at the same time have tried to limit the relationship to protect the Lockheed Idaho intellectual rights. The deep tensiometer is the most developed instrument and subsequently has had the most interest/interactions with outside organizations. Three companies have shown interest in this device with one of them offering to sell the device, once manufactured. This device and the portable tensiometer will probably be added to the ASTM standards on tensiometry, thereby increasing its visibility and making it a standard instrument. A presentation was given at ER-95 in August 95 on the portable tensiometer. This needs to be followed by publication of the results to obtain a peer-review and to advertise the capabilities of this tensiometer. These devices present new concepts that will be initially unfamiliar to the scientific community but following the peer-review the instruments should become the work horses for vadose zone monitoring. The suction bailer and field matric potential sensor have only been viewed by few in the scientific community, but we have received favorable responses on these devices. All of these instruments have a large potential to be built and marketed for use at environmental and agricultural sites.

\section{Intelligent Sensor Data Acquisition Analysis LDRD 4863 \\ R. E. Polk}

This study was conducted to determine the extent of the current state of the art for the use of fuzzy logic and neural networking processes (intelligent/expert data processing) as a means of interfacing chemical sensor systems and analytical instrumentation to a central data collection system. The data collection system would then use expert data processing to perform real time data validations that fulfill CERCLA/RCRA requirements. We reviewed the literature; held discussions with vendors of computers, computer software, and analytical instruments; and conducted interviews with researchers develop- 
ing data systems for chemical sensors and analytical instruments to determine the technology gaps in real-time data validation processes. The study revealed that a broad interpretation of the definition of the term data validation existed. The opinion of most people was that real-time data validation meeting CERCLA/RCRA requirements was available. However, our investigation showed that a complete program did not exist. Of the programs investigated in the study, some may have application as part of a real-time data validation process. The results of this investigation show the technology gaps and give direction for preparing a proposal to gather and coordinate the research teams needed to develop a comprehensive real-time data validation process that meets CERCLA/RCRA requirements.

\section{Objectives}

- Determine if a method is available to perform real-time data validation that fulfill CERCLA/RCRA requirements, and if no method exists, determine whether there are research organizations developing a method.

- Review the current analytical data reduction methods, instrumentation validation processes, research studies for computer analytical instrumentation and computer-sensor interfaces, and bar coding methods for maintaining sample history, integrity, and quality.

- Develop a test plan for realtime data validation and prepare a LDRD proposal for gathering a qualified team to develop a real-time data validation process meeting the requirements for CERCLA/ RCRA.

\section{Accomplishments}

We conducted a comprehensive literature search for real-time data validation processes that would address all issues and requirements for CERCLA and RCRA. We also searched for computerized controls and data reduction systems for sensors and analytical instruments.

We conducted interviews with researchers developing data systems for chemical sensors and analytical instruments and with vendors for computers, computer software, and analytical instruments.

We developed a test plan from our evaluation that we used to prepare an LDRD proposal for FY-96.

New or Increased Technical Capabilities

The information gathered in this program shows the need for a real-time data validation System that will meet the CERCLA/RCRA requirements. This system would greatly reduce the time and cost of the current method of manually performing data validation. Realtime data validation will find widespread application at DOE, DoD, BLM, etc., state agencies, and private industry.

\section{Business Development Opportunities}

Numerous business opportunities will be pursued in the followon work scheduled for FY-96. We will approach several interested parties for possible participation in CRADA, business spinoff, and other business activities. We will approach the analytical instrumentation industry early, both because they have a potential for direct benefit derived from competitive advantage and would therefore have a vested interest, and because they may speed up and enhance the process of system development. Second, the regulatory community will be contacted, largely because this system of data management has been an unfulfilled regulatory objective for decades and would provide a clear and present advantage to their oversight and control capacity.

\section{Modeling Underground Structures \\ LDRD 4864}

\section{A. J. Edwards, L. E. Freeman}

The immediate objective of this research was to produce models that allow the prediction of expected gravity and gravity gradient signatures for two underground structures on the INEL site. A broader objective is to establish the INEL as a test bed for testing technologies for detecting underground facilities. The detection and characterization of underground facilities was identified as the number two national priority in Deputy Secretary of Defense John Deutch's Report on Nonproliferation and Counterproliferation Activities and Programs presented to Congress in May 1994. DOE-HQ/NN-20 has established an interlaboratory program to develop and test underground detection technologies. This project is an integral part of the INEL strategic plan to develop a product line in underground detection technology conceptualization, design, fabrication, demonstration, and testing.

We generated computer code to calculate the gravity anomaly and the gravity gradient anomalies associated with underground structures. The code was validated and then used to generate results for the BORAX reactor site and for the Initial Engine Test (IET) facility. These sites are being proposed as test sites for underground facility detection technologies. The code generates signatures by forward modeling from three-dimensional mass anomaly information. Results can be displayed as an undulating surface or a contour map representation. The code is more flexible in this respect than existing commercially available 2-1/2-D or 2-3/4-D gravity modeling codes.

\section{Objectives}

- Generate software to model the gravity anomaly produced by density anomalies. 
- Generate software to model the elements of the gravity gradient tensor produced by density anomalies.

- Apply these models to the BORAX and IET underground structures.

\section{Accomplishments}

We created and validated models allowing prediction of gravity anomalies and gravity gradient anomalies. When given information representing the mass anomalies in the subsurface, they correctly calculate the gravity anomaly and the gravity gradient anomalies that would be observed at the surface. The mass anomaly information is input in finite element form, allowing 3-D representation of the structure.

We applied the models to the BORAX reactor and the IET site. The figure shows a typical mass anomaly horizontal cross section through the BORAX reactor and the gradient in the $X$ direction of the $X$ component of gravity $(X X$ gravity gradient) that would be observed at the surface. For the BORAX reactor, the floor is at grade level. The reactor pits and utilities trenches are seen in black. At the north end, the high density concrete walls of the BORAX-V reactor pit are seen in white. The XX gravity gradient is only one of the 9 elements of the gravity gradient tensor. The ripples across the plot are a result of the irregular shape of the basalt bedrock which lies about 5 meters below the surface. The noisy area in the center is a representation of the reactor itself. Similar data were obtained for the IET site.

Note that although commercially available code exists that will calculate the gravity anomaly from $2-1 / 2$-D or $2-3 / 4-D$ density anom- alies, the commercial package only computes the $\mathrm{ZZ}$ gravity gradient.

An invention disclosure was filed with the Technology Transfer Office regarding the software developed on this project. A detailed technical report with a more complete description of this project is available.

\section{New or Increased Technical Capabilities}

The models developed through this project have established the INEL as a leader in gravity and gravity gradient modeling, a new capability for the INEL. As a result, the likelihood that the INEL will be selected as an underground facility detection test bed has been increased.

\section{Business Development Opportunities}

Informal contacts have been made with two companies that are active in gravity and gravity gradient measurement:

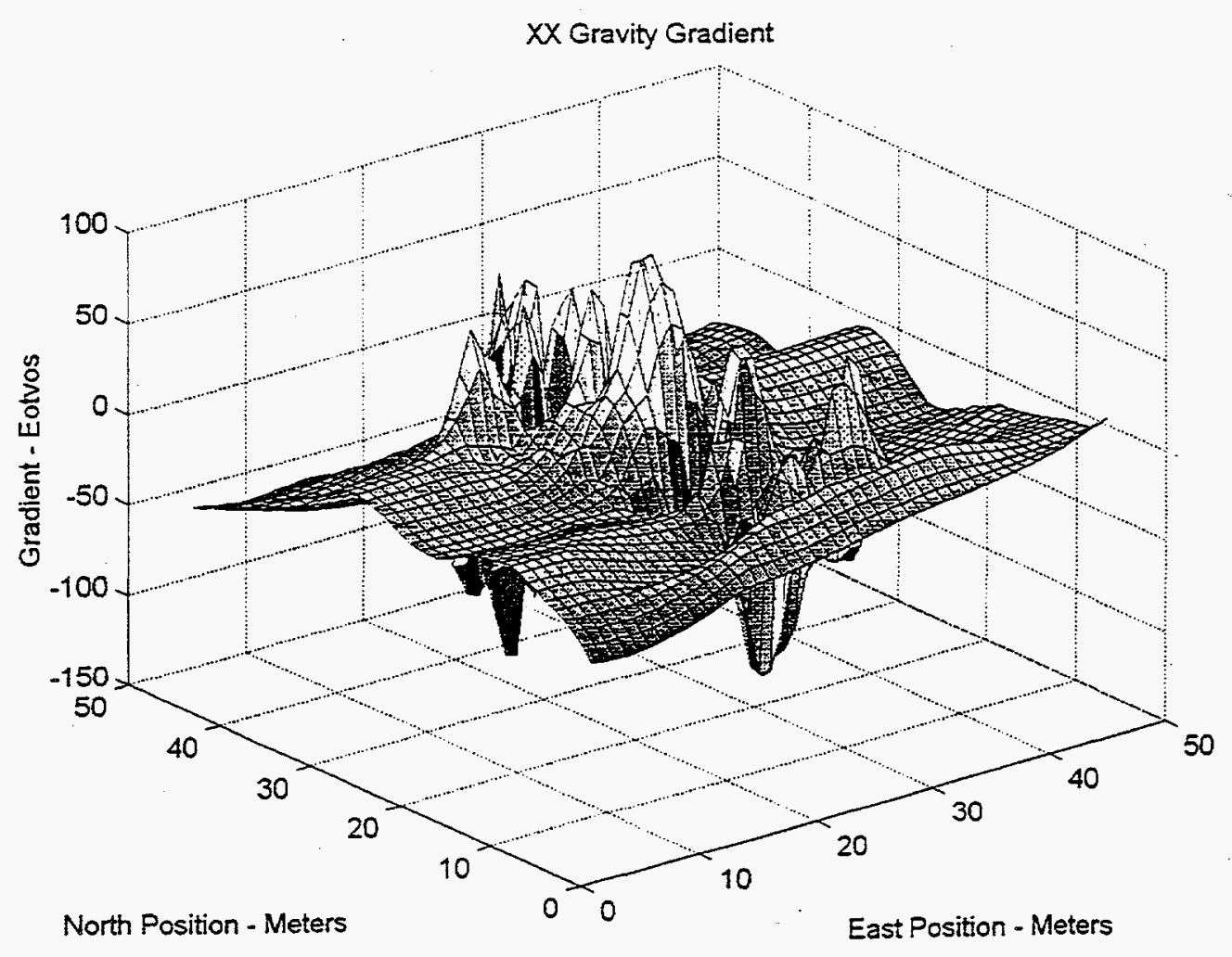

XX Gravity Gradient 
- Bell Aerospace has a full-tensor gravity gradiometer survey system that might be demonstrated for underground facility detection at the INEL.

- GWR Instruments is developing a portable superconducting gravity meter and has recently been awarded a Small Business Innovation Research grant to pursue a portable vertical component gravity gradiometer based on the same technology.

Contacts with both of these companies have the potential to develop into CRADAs or some other similar working arrangement.

\section{Neural Analysis Techniques for Field-Deployable Environmental Sensors LDRD 4865}

$$
\text { D. K. Kotter }
$$

We developed neural analysis techniques to add artificial intelligence to field-deployable environmental sensors. We performed proof-of-concept tests using the electrocatalytic vapor sensor (EVS). We demonstrated that the EVS sensor responded to various gases by creating a unique electromagnetic signature. The signature was fully characterized through the use of neural networks. We demonstrated that advanced pattern recognition algorithms can be developed to reduce false and nuisance alarms and reduce manpower requirements for environmental monitoring scenarios. We propose that neural signal processing can be used to enhance several other INEL sensor technologies.

\section{Objectives}

- Initiate collaboration with Lockheed Martin Research and Development Center (RDD).

- Evaluate neural network software tools.
- Design and implement a neural net training test bed.

- Collect test data with the electrocatalytic vapor sensor.

- Generate a special neural network algorithm.

- Develop a portable PC-based control and data acquisition system.

- Perform system-level bench tests.

\section{Accomplishments}

\section{RDD collaboration}

We gave a presentation on Lockheed Idaho sensor capabilities to key corporate personnel. An intercompany work transfer was put in place to support adapting key corporate neural network techniques to Lockheed Idaho sensors. G. Cordes supported this technology transfer through a one-month outplant assignment.

Neural network software evaluations

We evaluated several neural network packages, including Mathworks MathLab, NASA NETS-3, and Ward Systems NeuralShell to determine the optimum methods for processing sensor data. We determined that the proprietary Lockheed GRNN algorithm quickly converged to a correct solution with only $1 \%$ of the training data required of commercial back-propagation methods.

Training test bed

We configured a test bed to emulate sensor field conditions, with critical parameters such as gas concentration, flow rate, and temperature carefully controlled. This provided an environment for training the neural network with known data/conditions and for validating the system with noisy data.

\section{EVS data collection.}

We set up a test matrix for collecting the sensor data sets necessary for development and training of the neural network. Multiple data sets of $\mathrm{NO}_{\mathrm{x}}$ and $\mathrm{SO}_{\mathrm{x}}$ at concentrations of 100, 50,10 and 5 ppm were collected. Preprocessing methods, such as two-point averaging and smoothing of the data, were used to compensate for fluctuations from electronic noise. Data collected over long periods of time exhibited excessive drift that greatly affected the accuracy of the sensor. The experimental setup was modified in an attempt to improve stability, with marginal benefit. We recommend that future enhancements include improved sensor temperature regulation, printed circuit board mounting of the sensor, and expanding the neural network to compensate for drift trends.

Neural network algorithm

We generated a neural network algorithm for use with the EVS sensor. We were able to improve spectral identification by scanning the raw sensor data and doing feature extraction. This greatly reduce the complexity of analyzing the sensor's output and provided near real-time analysis. The neural net was highly accurate on estimating the contamination levels of a single test gas. Results obtained with the proprietary Lockheed neural network algorithms (GRNN) were benchmarked and validated using a commercial neural network toolbox, with GRNN being an order of magnitude more sensitive. Experiments were limited to identification of a two-gas mixture. Future development will center on the identification of several VOC components in a mixture.

Control and data acquisition

The neural analysis software and control hardware was configured into a lap-top computer. A software-based graphical user interface was developed. A UNIX operating system was installed on the system to allow execution of the 
RDD neural network source code. This provided a subset of the development system used at RDD and allowed benchtop experimentation with live sensor data. The prototype system was configured for operation of the EVS.

Sensor system bench testing

Experts at the Lockheed Martin Research Center performed extensive training and validation of the neural network using sensor data collected at Lockheed Idaho. Laboratory experiments were performed to see how the sensor data acquisition/analysis routine worked with noisy, overlapping, highly nonlinear, and noncontinuous data. Preliminary results show that the neural network classifiers often outperform classical techniques.

\section{Summary}

Bench tests have verified that neural analysis techniques can be used to make intelligent sensor systems that can be optimized for the environment where they are deployed. A sensor can be retrained to monitor specific waste streams without hardware modification. Many sensor systems must operate in environments of extreme noise and variants. Nonlinear conditions can easily be introduced into the sensor system and cause deviation from calibration parameters.

Neural networks easily compensate for these varying conditions through their extremely flexible and fuzzy operation.

New or Increased Technical Capabilities

A major initiative was to aggressively expand the technology base of the Sensor Products Directorate by transferring neural network technology from the Lockheed Idaho Research Center. Intercompany integrated product/proposal teams were established. The core capability now exists to adapt specialized Corporate RDD signal processing techniques to existing Lockheed Idaho technologies. Neural nets can improve the per- formance of Lockheed Idaho sensors by proving the ability to analyze the spectra from multi-sensor, multi-component mixtures, and accurately determine sample composition even when signal to noise limits are less than one. This would allow field analysis in harsh environments without complex sampling/separation methods and without strictly controlled environment conditions. The end product would be the development of lowcost, field-portable systems with analysis capabilities equivalent to rival laboratory-based instruments of much greater cost and complexity.

\section{Business Development Opportunities}

An intercompany work transfer was established with Lockheed Martin Corporate. This provided extensive visibility of the Lockheed Idaho sensor technologies, with the goal of collaborating on future developments and proposals. The application of the electrocatalytic vapor sensor in support of bioremediation tasks was presented to representatives of the petroleum industry.

\section{Grouping and Modeling Ecosystem Components for Ecological Risk Assessment} LDRD 5203

\section{N. L. Hampton}

The primary purpose of this research was to evaluate and refine a newly developed methodology for conducting screening-level ecological risk assessments (SLERAs). High economic potential exists for developing transferrable risk assessment technology to more quickly and cost effectively address regulatory requirements at the INEL as well as other government and industrial hazardous waste sites. An approach to SLERA that incorporates functional grouping has been developed at the INEL for the assessment of terrestrial ecosystems. The methodology is based on using a combination of ecological and toxicological data to model exposure for a hypothetical species representing all members of a functional group. This approach was compared with the more traditional method of modeling exposures for individual endpoint or indicator species through a series of sensitivity analyses to identify model input parameters critical to SLERA exposure calculations using both functional groups and individual species. The most sensitive model parameters, regardless of whether groups or individuals are modeled, were shown to include toxicity reference values (TRVs) and uptake and bioaccumulation factors for plants and animals (PUFs and BAFs). Exposures calculated for individual receptors were shown to be less conservative when extrapolating potential exposure to similar receptors. These results will be used to refine the functional grouping methodolagy for ecological risk assessment at terrestrial sites having multiple contaminants and to focus limited resources on collecting the most important ecological or toxicological input data. An extension of this project scope (year-end funding) allowed a preliminary evaluation to determine the feasibility of developing broadly applicable plant uptake adjustment factors for radioactive contaminants (N. Hampton, R. VanHorn, G. Harris). Late year funding was also approved to support preliminary investigation of wetland areas associated with the Big Lost River. Aquatic samples and field observations were collected for two major areas on the INEL including the Big Lost River sinks and playas and RWMC spreading area $A$ (R. Rope).

\section{Objectives}

- Refine methodology for grouping receptors for ecological risk assessment.

- Use existing data to define ranges and medians for the ecologically-based screening level (EBSL) model parameters, and perform sensitivity analy- 
ses to identify critical parameters in the EBSL model.

- Compare EBSLs calculated using the functional group method with the individual species methodology and determine the usefulness or functional grouping for building or reducing conservatism in calculated EBSLs.

- Document project results (draft informal report).

- Present the results of this research at the annual meeting of the Society of Environmental Toxicology and Chemistry (SETAC).

- Conduct a preliminary assessment of soil properties and plant characteristics affecting plant radionuclide uptake and summarize available plant uptake/bioavailability data for radionuclides over a range of soil $\mathrm{pH}$ (extended project scope).

- Evaluate wetland indicators (vegetation, hydrology, and soil) for areas of the INEL which received water from upstream releases to the Big Lost River (primarily the Big Lost River Sinks and playas). 1995 is the first year since 1984 in which significant stream flows have reached and remained on the INEL [extended project scope (R. Rope)].

\section{Accomplishments}

This research supports the development of systematic, repeatable and more cost effective methodology for addressing CERCLA related remediation activities for hazardous waste sites, specifically, ecological risk assessment. Ranges and median values for individual model parameters were developed from existing data or estimated. These values were then used to perform a suite of sensitivi- ty studies to identify which model parameters most significantly affect the calculated contaminant exposure level for receptors. The model was tested using both grouped (functional grouping) and individual receptor calculations.

EBSLs for individual species were shown to be less conservative exposure indicators for similar receptors. A draft informal report titled An Evaluation of Ecologically-based Screening Levels for Single and Grouped Receptors, INEL-95/0286, was prepared to document the original scope of this proposal. An abstract summarizing the results of this project was submitted and accepted for presentation at the SETAC meeting in November, 1995. A preliminary assessment of soil properties and plant characteristics affecting plant radionuclide uptake in varying soils was conducted. Aquatic samples and field data were collected at three wetland sites on the INEL.

\section{New or Increased Technical Capabilities}

This research builds upon existing INEL risk assessment capabilities and expertise at both the individual and group level. Significant contributions to ecological risk assessment methodology have been developed at the INEL and have received positive review by government agencies and industry at the local and regional level. This study was intended to enhance the application of these new methodologies and was used to focus research on the next step in development and application of ecological risk assessment methodology for terrestrial sites having complex contaminant issues. Efforts will now be concentrated on addressing techniques for decreasing the uncertainty associated with the most sensitive exposure model parameters and developing methods to quantify and reduce the uncertainties associated with exposure assessment in general.
Two proposals have been developed and submitted as a continuation of this research. The first addresses the potential for reduction of model uncertainty associated with TRVs and the second, a tool for calculating more realistic PUF values for site specific soil conditions.

\section{Business Development Opportunities}

Scientists and risk assessors from the INEL and Dames and Moore collaborated to develop the EBSL model for use in INEL screening-level ecological risk assessments. The results of this research has potential for benefiting risk assessments conducted across the country by Dames and Moore as well as other contractors.

\section{Water Shutoff in Producing Oil Wells Using Bacteria LDRD 5205}

\section{E. P. Robertson, C. P. Thomas, G. A. Bala}

The purpose of this project was to determine the ability of bacterial agents to reduce the influx of water into producing oil wells. Many oil fields produce large amounts of water along with the oil because of encroaching brine. Reducing the produced water volume would result in more favorable field economics by decreasing fluid lifting costs and water disposal costs, as well as increasing oil production.

Current industry techniques for addressing this problem involve the use of polymers cross-linked with a metal ion (usually chromium III or aluminum) to plug porous rock. Bacterial plugging technology is expected to be more economical and environmentally compatible compared to polymers cross-linked with metal ions.

Six different bacterial strains (in a variety of media) were selected for plugging experiments based on their reported ability to produce polymer. 
Initial tests were done in one-dimensional flow experiments using sandpacks of crushed sandstone. The best plugging culture was applied to a two-dimensional physical model of an oil reservoir filled with crushed sandstone and saturated with both oil and water. Results from the reservoir physical model show that treating the production well with the selected bacteria preferentially plugs the water zones and significantly reduces the produced water-oil ratio.

The use of this technology could have a large economic impact on the regional and national oil and gas industry. Potential benefits to the INEL include licensing contacts with oil industry service companies.

\section{Objectives}

- Construct one-dimensional sandpacks.

- Construct a bench scale twodimensional sandpack (reservoir physical model).

- Select bacterial strain to be used in reservoir physical model based on performance in one-dimensional sandpacks.

- Run control experiments in reservoir physical model.

- Test optimized bacterial system in reservoir physical model for capability of reducing water production and effect on oil production.

- Evaluate economic efficiency of bacterial system for water shutoff in producing wells.

\section{Accomplishments}

One-dimensional flow sandpacks were constructed and used to evaluate different bacterial strains for their ability to plug porous media. Three pore volumes of bacteria were then injected in the outlet end of each column. The inlet lines and sandface were then flushed with deionized water to displace bacteria that may cause facial plugging during subsequent permeability tests.

We selected six different bacterial strains for experimentation from the open literature based on their ability to produce polymer. All six strains were tested for their ability to plug porous media using the one-dimensional sandpacks. Sandpack permeability was measured as a function of the amount of liquid injected through the column. Bacterial inoculation and incubation can affect the permeability of the column drops significantly. We selected B. circulans to be applied in the two-dimensional reservoir physical model based on these results.

A bench-scale physical model of a producing oil reservoir was constructed, filled with crushed Berea sandstone, and then saturated with water. We then introduced rude oil into the model and allowed time for the system to come to equilibrium and steadystate flowing conditions. Control experiments on the physical model established baseline production data that we used to compare data obtained after bacterial application.

We studied the effect of applying bacteria to the two-dimensional reservoir physical model. We measured water cut and the percent of the production liquid that is water as a function of the number of pore volumes produced from the model. Before bacterial inoculation, the water cut reached $95 \%$ after 0.3 pore volumes were produced. After bacterial inoculation, the produced water cut dropped to $50 \%$; a $47 \%$ reduction from the pre-treatment level.

An economic program was written to evaluate the efficiency of the bacterial water shutoff system. The oilfield economic and production variables needed for the model were provided by Phillips Petroleum Company and are indicative of the Smackover oil field in southern
Arkansas where water production is high. The process appears to be economically attractive. Based on results from this project, the bacterial process would pay out in 40 days for a typical Smackover well. The main cost driver for microbial water shutoff is the nutrient cost. No effort has been made to economically optimize the nutrient recipe, but lowering nutrient cost should be easily accomplished for field application.

\section{New or Increased Technical Capabilities}

The INEL has benefitted from this project by expanding the use of biotechnology into this important area in the oil and gas industry. The process has been proposed by other researchers, but to the best of our knowledge this is the first time reducing produced water in oil reservoirs using bacteria has been shown to work.

\section{Business Development Opportunities}

This process has potential for business opportunities. However, more work (both in the laboratory and in the field) is necessary before commercially marketing the process. An oil field service company, TIORCO Inc., Denver, Colorado, expressed interest in the results of the project. We have worked with this company in the past, but though they are interested they have no experience nor expertise in using microbes in oil field applications, so CRADA opportunities with them appear small. We have also discussed the reservoirs using bacteria has been shown to work.

\section{Business Development Opportunities}

This process has potential for business opportunities. However, more work (both in the laboratory and in the field) is necessary before commercially marketing the process. An oil field service company, TIORCO Inc., Denver, 
Colorado, expressed interest in the results of the project. We have worked with this company in the past, but though they are interested they have no experience nor expertise in using microbes in oil field applications, so CRADA opportunities with them appear small. We have also discussed the work with Phillips Petroleum Company, Bartlesville, Oklahoma. They also are interested in the outcome. No other serious discussions with external businesses have been made to date.

\section{Estimating Sorption Properties of Clay Materials} LDRD 5301

\section{R. W. Smith, C. A. Dicke}

Radionuclide mobility is a primary concern in risk and performance assessments and in the design of remedial actions for contaminated sites. Transport models are used in Risk and Performance Assessments to predict contaminant concentration distributions. These concentration distributions are then used to evaluate dose to the public by groundwater consumption. Typically, transport models utilize empirical data to describe contaminant interactions with the porous media. The empirical data describe bulk geochemical interactions, often in the form of a distribution coefficient, and offer no insight into the mechanisms controlling contaminant retention. In addition, site-specific distribution coefficients are not available for transport calculations. This can lead to over or underestimation of contaminant migration. Decisions based on these predictions can result in additional expense in siting new disposal facilities and remediation of old disposal facilities because the factors controlling the contaminant migration are not known. Methods developed in this research will provide a technology to predict adsorption behavior based on mechanisms related to site mineralogy and water chem- istry. Therefore, changes in contaminant sorption behavior resulting from variations in solution chemistry (e.g., $\mathrm{pH}$ dependence or chemical composition) can be modeled. This project enhances the understanding of contaminant behavior in sediments and provides an improved basis for prediction of contaminant mobility.

\section{Objectives}

- Convert the FITEQL nonlinear optimization program to the FORTRAN computer language from BASIC to provide a more efficient implementation of the code.

- Determine acidity constants and surface complexation constants on $\mathrm{Fe}, \mathrm{Al}, \mathrm{Si}$, and $\mathrm{Mn}$ oxides.

- Develop predictive algorithms to estimate SCM constants based on effective charge, ionic radius and hydrolysis behavior of the aqueous ions involved (c.f., Smith and Jenne 1991).

- Derive surface acidity constants for clay minerals such as kaolinite and smectites. (Task will run into FY-96).

- Derive SCM constants for adsorption onto clay minerals such as kaolinite and other clay minerals (Task will run into FY-96).

\section{Accomplishments}

The ability to predict the mobility of hazardous metals, metalloids, and radionuclides in the subsurface on a mechanistic basis has a wide range of applications at the INEL, other DOE facilities, and in the private sector. Mobility of contaminants is a primary concern in designing disposal facilities and remedial activities for active and inactive hazardous and mixed waste sites. Many current risk assessment and hydrologic solute transport computer codes repre- sent contaminant retardation with sorption isotherms (e.g., linear, langmuir, and freundlich).

Isotherms are strongly dependent on experimental conditions and are not readily extrapolated to other conditions. In addition, risk assessment is commonly conducted prior to site characterization resulting in the selection of adsorption parameters with a bias toward availability rather than appropriateness (i.e., other conditions). To compensate for the lack of sitespecific sorption parameters, what are thought to be conservative values for retardation parameters are often used. These conservative retardation parameters may result in overestimating the spatial extent of contamination and underestimating the concentration of contaminants in soils and sediment near the sources of contamination. Also, the use of nonsite-specific adsorption parameters are not necessarily conservative and may result in predictions that underestimate contaminant migration.

Finally, sorption isotherms do not explicitly reflect the physical processes that control retardation (e.g., surface adsorption, surface precipitation, surface absorption, or surface induced redox reactions), limiting their usefulness for modeling sites where system parameters (e.g., pH, Eh TDS, etc.) may change significantly through time.

The mechanistic diffuse doublelayer model (DLM) as implemented by Dzombak and Morel (1990) is being used to estimate sorption constants. In order to develop a consistent set of DLM parameters, a bibliography containing over 300 references dealing with adsorption of various metals and radionuclides onto silica, aluminum, iron, and manganese oxides and clay minerals has been established. The data from these studies are currently being extracted to determine oxide surface properties from titra- 
tion data and DLM complexation parameters using the nonlinear optimization program FITEQL. The work of Dzombak and Morel (1990) has been used as a reference point to begin parameter estimations. Surface acidity constants have been determined for the simple oxides and surface complexation constants for uranium, neptunium, and plutonium have been determined based on adsorption experiments for hydrous ferric oxide and goethite.

An algorithm to predict DLM constants using the methods of Brown and Sylva (1985) based on effective charge, ion size, and hydrolysis behavior of the aqueous ions involved for sorption on oxides has been developed. Predictions based on these relationships expand the number of hazardous metals and radionuclides that can be addressed using the DLM to describe adsorption (see the figure below). This algorithm can be used to estimate adsorption constants for metals and radionuclides that have no available data for iron oxides. An abstract on the surface complexation of actinides on iron oxides has been submitted for peer-reviewed publication in Scientific Basis for Nuclear Waste Management and a manuscript is in preparation for submission to Radiochimica Acta documenting the predictive algorithm for iron oxide adsorption.

\section{New or Increased Technical Capabilities}

The combination of consistent adsorption constants and identification of the solid phase controlling adsorption of metals and radionuclides in INEL sediments will allow prediction of adsorption parameters for transport models based on soil mineralogy and water chemistry. The impact of varying water chemistry and solid phases can then be addressed using models with confirmatory experiments rather than extensive laboratory studies.

Enhanced capability to simulate contaminant migration in the subsurfaces is beneficial because long-lived radionuclide decay is slow relative to the effective lifetimes of engineered hydrologic barriers thereby exposing these

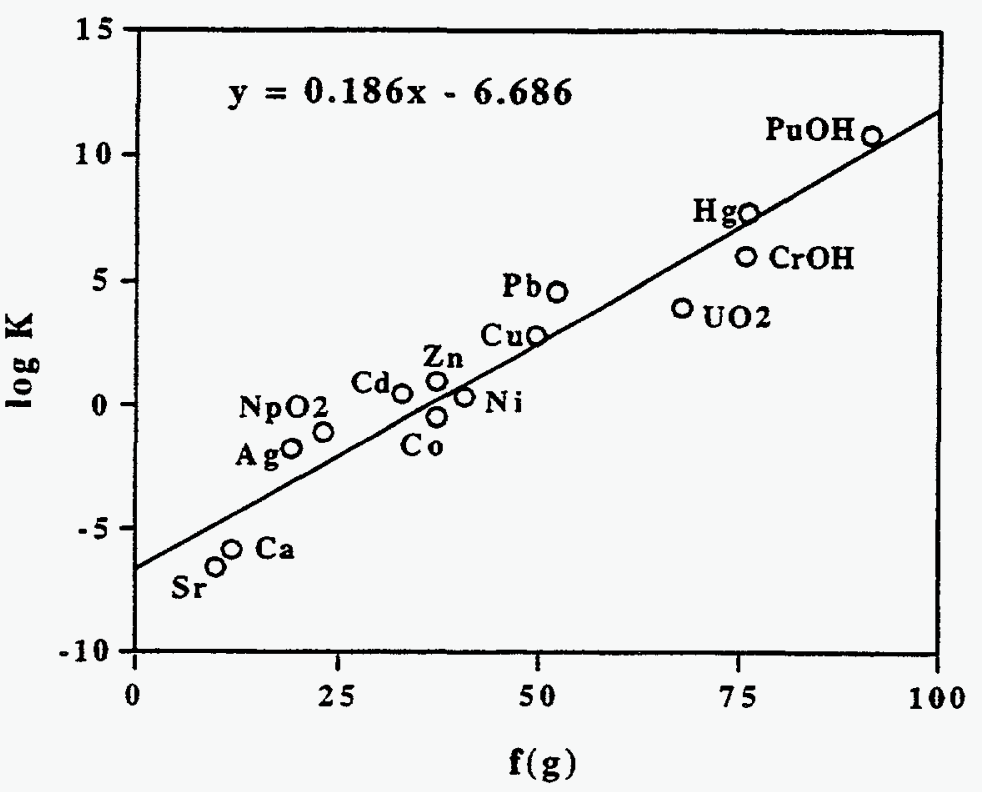

Relationship Between Charge, lonic Radius, and Hydrolysis Behavior Function

[f(g)] and the Adsorption Constant for Metals and Radionuclides of Varying Oxidation States (Plus 1 to 4 Oxidation States) contaminants to leaching and subsequent migration for long time periods. Currently, most migration simulations are based on hydrologic solute transport codes that represent retardation using linear sorption isotherms derived from studies of bulk geochemical interactions within a limited range of experimental conditions which limits predictive capability. However, predictions of actinide an metal ion behavior based on mechanistic understanding can be extrapolated to diverse conditions including new investigation sites. In addition, adsorption simulations based on a mechanistic understanding enhances the ability to make cost effective decisions on remediation and disposal options.

\section{Business Development Opportunities}

This work will aid in transport modeling for Performance and Risk Assessments and remedial activities involving metals and radionuclides at facilities across the INEL. Other DOE, DOD, and commercial facilities will also benefit from this technology because it will provide a means to determine contaminant interactions with porous media quickly and cost effectively.

\section{Identification of a Surfactant/Alcohol/Polymer System for Safe Mobilization of TCE LDRD 5303 \\ G. M. Shook}

We have focused our efforts on identifying a chemical mixture (surfactant, alcohol, and polymer [SAP]) in order to solubilize a significant amount of TCE in a micro-emulsion. A microemulsion is any stable mixture of surfactant, NAPL, water, and alcohol. The initial design criteria used in this study was to minimize the density of the resulting microemulsion, however, 
theoretical and numerical studies have shown that vertical migration of a DNAPL is controlled by two dimensionless scaling groups: a gravity number and the effective aspect ratio. Embedded in these two scaling groups are four design parameters: injection rate, micro-emulsion phase density and viscosity, and well spacing. Numerical simulations show that the extent of vertical migration can be predicted on the basis of the two scaling groups. Through manipulation of the design parameters, we can design a cleanup in which vertical migration is predicted and wells screened sufficiently deeply for full capture. This has been tested at the laboratory scale. Excellent agreement was obtained between the predicted and observed migration. This demonstrates that hydraulic control over the contaminant can be maintained, and that we can aggressively address DNAPL remediation. A schematic of the process is given in the figure.

\section{Objectives}

- Develop theoretical arguments using inspectional analysis to show vertical migration of DNAPLs is controlled by two scaling groups.

- Perform sensitivity studies to illustrate the relationship between migration and the scaling groups.

- In laboratory experiments, identify surfactant and alcohol pairs for solubilization of TCE.

- Perform corefloods using the chemical pairs identified in Task 3 to evaluate solubilization potential of TCE. Simulate all of the corefloods.

- Perform two-dimensional laboratory experiments to demonstrate vertical migration is predictable and controllable. Use theoretical and numerical arguments from tasks 1 and 2 to predict vertical migration. Compare against experimentallyobserved migration.

\section{Accomplishments}

Our primary objectives in this project were: to determine the factors that control the vertical migration of a dense, organic contaminants in the subsurface, to derive expressions that describe those controlling factors, and to demonstrate through laboratory experiments the validity of the approach. Through the use of inspection analysis we have written the partial differential equations describing flow in a two-dimensional cross section in dimensionless terms. The advantage of using dimensionless equations is that the solutions apply to all problems that are geometrically similar; thus, solutions are applicable to both lab and field scale. In doing so, we have shown that the vertical migration of a more dense phase (either a DNAPL or a microemulsion that contains a significant amount of a DNAPL) is a function of only two dimensionless groups: a gravity number, $\mathrm{Ng}$, and an effective aspect ratio.

Also within these dimensionless groups are four design parameters: microemulsion phase density and viscosity, respectively, injection rate, and well spacing. These are design parameters, since, by selecting their value, we are able to partially control the value of the two scaling groups. It is important to realize that the specific value of any given parameter within the scaling group is unimportant; it is the value of the group that describes the vertical migration. Therefore, we are able to manipulate the amount of migration of the contaminant.

Numerical simulations of DNAPL migration as a function of these scaling groups has been conducted. The vertical migration of the contaminant is a function of the two scaling groups, and is predictable. We have performed two experiments to validate this theory. In both cases, the predicted migration agreed within $5 \%$ of the observed migration in the experiment. We have therefore shown that the migration of DNAPLs in the subsurface is a predictable quantity, and that we are able to design a remediation strategy that captures the full plume.

New or Increased Technical Capabilities

This is the only approach that we are aware of in which DNAPL migration is predicted on the basis of design parameters and aquifer

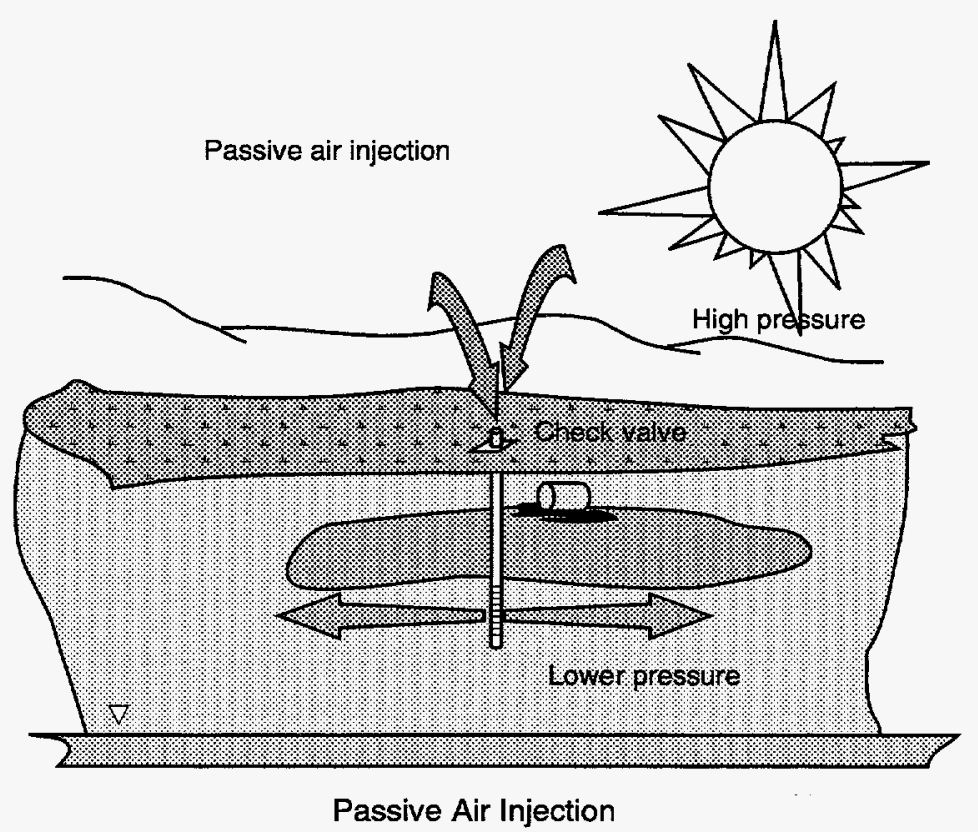

Idaho National Engineering Laboratory LDRD FY 1995 Annual Report 
properties, and full capture of the plume can be ensured. We believe a pilot scale field test will demonstrate this new technology to the environmental field, and we will be able to use this new technology in support of many DOE and DOD field sites.

\section{Business Development Opportunities}

We have submitted a white paper (preproposal) on this technology to various $\mathrm{DOE}$ and $\mathrm{DOE}$ program managers, including managers at LLNL, various U.S. Air Force bases, the Air Force Center for Environmental Excellence, and the DOE Plume Focus Group. This white paper describes the technology, and solicits partial funding for a field test. We have further formed a tentative working group that includes INEL, University of Texas, and private (Intera Company) researchers, all of whom have extensive experience with this type of technology. We believe that a successful field demonstration will enable the INEL to take the lead in treating DNAPL-contaminated sites throughout the world.

\section{Aquatic Vascular Plant Control LDRD 5601}

\section{P. A. Pryfogle, B. N. Rinehart}

The purpose of the program was to assess the extent of nuisance algal and aquatic plant growth in hydropower waterways in the Northwest United States. One objective of the program was to identify a specific aquatic plant growth problem and become more involved with the processes developing and implementing a control strategy. This effort was to help establish a more realistic understanding of aquatic plant growth problems and become aware of the constraints and requirements associated with control/eradication techniques.

\section{Objectives}

- Evaluate the extent of nuisance aquatic plant and algae growth in the Pacific Northwest.

- Establish a data-base on the problems identified and the control strategies that have been used to alleviate the problems.

- Examine regulatory (local, state, and federal) concerns associated with treatment methods.

- Select a location with problem growth and become involved it the abatement processes.

- Write and submit summary papers to refereed journals.

\section{Accomplishments}

The extent of the problem with nuisance aquatic plants and algae is quite large costing the public millions of dollars not only for treatment and control but also for damages directly and indirectly to the environment and use of aquatic ecosystems. Agriculture and hydro-power generation facilities probably represent the largest industrial sector directly impacted by aquatic plants and algae.

Whereas human uses associated with recreation and aesthetics are other areas impacted by excessive aquatic plant and algae growth.

A data base on the problems throughout the Northwest was not assembled since it was determined that there are several resources currently available for similar information. The U.S. Department of Interior and the U.S. EPA maintain records for the licensing and use of pesticides for the control of aquatic weeds. Both agencies have databases available providing information on use and areas of application. Similarly, several chemical companies that produce aquatic plant control products, publish pamphlets and guides that aid in the identification of aquatic plants, offer suggested herbicides for their control, and briefly state regulatory concerns for their use.

Information on algae growth problems was similar to aquatic plants but it was somewhat sketchy. Algal problems tend to be a little harder to track. Often algal growth problems are treated and then forgotten with little or no record of decisions made. The use of copper sulfate or copper-based products are commonly used, however the general movement is to remove this option through tighter regulations on the use of copper in aquatic environments. The main concern here was the potential build up of heavy metals (copper) in the environment over repeated and extended periods of application.

The majority of the regulations are associated with state and federal agencies; however, other users in watersheds may also dictate regulations guarding their interests as well. These interests are associated with recreational uses, public health criteria, and other public uses. This may create a need for a case by case study to determine the general and specific regulations associated with each problem growth site.

A specific problem related to excessive algae growth in hydrogeneration canals was identified. A collaborative effort was established between Lockheed Idaho Technologies Company (Lockheed Idaho) and Pacific Gas \& Electric Company (PG\&E) to assist in developing a resolution to their algae problems. Their particular problem was caused by filamentous algal growth in their transfer canals resulting in an inability to maintain adequate flow for optimum power generation. Over the course of this year's effort we established baseline water quality information, identified the algae 
associated with the problem, and initiated a couple studies aimed at remediating the nuisance algae growth. In addition a cooperative research and development agreement (CRADA) is under development between Lockheed Idaho and PG\&E for continuing work on this particular problem.

\section{New or Increased Technical Capabilities}

This effort provided an opportunity to expand on the existing skills at the INEL to develop environmental measurement processes. Our involvement in the selection of water quality criteria and sampling strategy was requested by $P G \& E$ for establishing base-line information on the hydro-generation canals. Plus our involvement with this problem has provided an opportunity for us to receive recognition in working with aquatic plant and algae problems.

\section{Business Development Opportunities}

There are several areas where business potentials have been developed. We are underway with the creation of a CRADA agreement between Lockheed Idaho and $P G \& E$. One particular aspect of this agreement offers us the opportunity to test facilities that would be hard to find or construct otherwise. A second opportunity lies in the development of biological materials for the control of nuisance algae growth. The production of an environmentally friendly product may capture a percentage of the lucrative aquatic herbicide industry and offer a substantial return-on-investment for Lockheed Idaho. Similarly, a technique is under study for sampling, making observations, and taking measurements in high flow open canal systems. This technique also carries certain product value.

\section{Bioprocessing of Indigo Textile Dye Waste Effluent}

LDRD 5608

J. K. Polman

The project was designed to discover solutions for treating indigocontaining waste effluents from textile dyeing plants. This is important to the denim textile industry, which is a large business in this country. The proposed solution was to use a biocatalyst to decolorize the indigo dye found in effluents, making them more suitable for discharge to public waters. The biocatalyst was to be discovered and patented by INEL researchers, providing revenue for the INEL and an expandable business opportunity for the local and regional community. $A$ biocatalyst that decolorized indigo was discovered and characterized, and an invention disclosure has been filed. An additional business opportunity was discovered in the use of the biocatalyst for fading denim fabric. This is the subject of an LDRD proposed for fiscal year 1996.

\section{Objectives}

- Discover and characterize biocatalysts that decolorize indigo dye.

- Use these biocatalysts to develop bioprocesses for decolorizing indigo dye in waste effluents and denim products.

\section{New or Increased Technical Capabilities}

To date, we have discovered six indigo carmine decolorizing microorganisms out of 390 strains tested. One of these organisms is outstanding in its ability to decolorize, and we focussed all of our subsequent work on that microorganism. This particular creature is one that we predicted would degrade indigo based on a specific physiological property (which we cannot disclose due its enabling nature). These are the things we have found out about this unique biocatalyst:

- It is extracellular, and thus is easily purified from cultures. This is a very beneficial property with regards to preparing the biocatalyst for use in an industrial formulation. No cell rupture is required; cell rupture, as the term implies, consumes a lot of energy.

- Oxygen enhances the decolorization reaction and may be a co-reactant. If oxygen is required to obtain good decolorization, the cost of decolorization increases significantly.

- It decolorizes insoluble indigo. This is the most relevant form of the indigo dyes we studied.

- It has a molecular size less than 3,000 daltons. This is unusual for a biocatalyst. Because of its small size, it may not be an enzyme.

- It is stable at room temperature for long periods (days). This is also an indication it is not an enzyme. The observation is a good one from a product standpoint, because the product will be stable during storage and shipping.

- It is sensitive to extreme heat. This suggests that the catalyst is organic rather than inorganic.

- It tolerates very high concentrations of surfactant. Since surfactants are often present in textile dye waste streams, this property will be beneficial to its use in harsh waste mixtures.

So far we have been unable to use the biocatalyst to fade indigodyed denim. This is probably due to either the inaccessibility of the indigo to the biocatalyst or to the complexation or fixation of the indigo to the fabric. An LDRD 
proposed for FY-96 to addresses this problem.

We envision bioprocess schemes in which (a) effluents stored in a holding tank are treated with a commercial preparation of our biocatalyst, and (b) denim products are washed in a biocatalyst detergent which fades them.

\section{New or Increased Technical Capabilities}

If the decolorization process is patented, the INEL will own a process for treating indigo-dyed products in an industry which prefades ninety-eight percent of the 600 million indigo-dyed garments produced in the United States each year. This translates into a lot of money for the INEL. The effort requires that we continue the research with denim-fading so that a method for using the biocatalyst to access fabric-entrapped indigo can be developed. The accomplishment of discovering a new indigo-decolorizing microorganism adds to INEL Biotechnology's capability of finding the right bug for the right job.

\section{Business Development Opportunities}

The problem of decolorizing indigo was first posed to us by Ned Lindsey, the Vice President of Technical Services at Avondale Mills in Sylacauga, Alabama.

Based on his interest, we proposed to use LDRD funding in Fy-95 for finding an indigo-decolorizing biocatalyst and implementing it in the treatment of waste or the fading of denim. We have communicated our progress (without enablement) to Mr. Lindsey during the entire project. We also visited the Avondale plant to collect samples, obtain denim, and get an idea of the plant layout.

We have also communicated our research progress to Art Toompas (Corporate Environmental Manager) and Steve Sheffey (Director of Product Development) of Cone Mills in Greensboro, North
Carolina. They have both expressed interest in our work.

A marketing assessment was performed by LITCO's Business Development Department this summer. The final results of that study have not been communicated to us yet, but we were informed by the researcher Brad Goodson that Levi Strauss had been approached with the idea of fading denim with our biocatalyst. They were interested in our results.

\section{Removal of Methanol from Forest Products Industry Off-gases by Biofiltration} LDRD 5614

$$
\text { W. A. Apel }
$$

Methanol vapors are a major air contaminant resulting from composite board manufacturing processes. In addition, methanol, regardless of source, represents the largest point source air pollutant in the world, with total emissions being in the range of 143 million pounds per year. With the advent of Title III, Clean Air Act Amendments of 1990, methanol is regulated as a hazardous air pollutant. Conventional methods for control of methanol vapors include technologies such as regenerative thermal oxidation and carbon sorption. While these technologies are reasonably effective, they are expensive. Biofiltration is an emerging technology that has the potential to effectively and economically control methanol vapor emissions, with the primary end products being water and carbon dioxide. The purpose of this project was to evaluate the potential of developing a biofiltration technology for this purpose, with special emphasis on controlling methanol emissions from forest product industry sources. Laboratory-scale, continuous-flow biofilters were constructed and subjected to air streams containing various concentrations of methanol vapors. Microbes indigenous to the wood waste bed medium used in the biofilters actively degraded the methanol vapors over an inlet concentration range varying between 30 and 2,000 ppm(v). In much of testing, with an empty bed residence time of 2 minutes (equating to approximately a 1 minute packed bed residence time), removal was in excess of $90 \%$. This equates to an absolute removal rate of $25 \mathrm{~g}$ methanol per $\mathrm{m}^{3}$ of bed per hour at an $850 \mathrm{ppm}(\mathrm{v})$ inlet concentration.

\section{Objectives}

- Evaluate rates of methanol physical sorption to biofilter bed media components.

- Evaluate bed ripening times.

- Determine methanol removal rates over a variety of methanol vapor concentrations.

- Evaluate effects of temperature on methanol biodegradation.

- Perform a preliminary economical evaluation of methanol biofiltration in comparison with competitive conventional technologies.

\section{Accomplishments}

Continuous flow biofilters were constructed from glass process pipe. The biofilters were packed with a bed medium consisting of waste wood chips.

Methanol vapors were delivered to the biofilters in a continuous air stream at an empty bed retention time of two minutes. This equates to a packed bed residence time of approximately one minute.

Methanol concentrations in the inlet and outlet streams were monitored via gas chromatography.

Adsorption experiments show that the bed medium's propensity for methanol adsorption depended on the moisture content of the bed. Methanol adsorption was higher when the bed was moist and lower when the bed was dry. Under nor- 
mal operating conditions, the beds were saturated with methanol less than one hour after start up, so that methanol removal observed hours or days after startup was attributable to microbial degradation, not physical sorption.

Initial experiments with the wood chip bed show that inoculation with nonindigenous microbes was unnecessary. After relatively short exposures to methanol streams, the organisms indigenous to wood chip bed medium removed methanol at high rates. This result was not especially surprising, since in the literature a variety of microbes have been shown to grow on methanol as a sole energy and carbon source.

Methanol removal approached $100 \%$ at inlet concentrations up to approximately $7.5 \times 10^{5} \mu \mathrm{g}$ per $\mathrm{m}^{3}$ of air (see the figure below). The probable operating range in the real world is 2.5 to $5.0 \times 10^{5} \mu \mathrm{g}$ methanol per $\mathrm{m}^{3}$ of air. At concentrations above $7.5 \times 10^{5} \mu \mathrm{g}$ methanol per $\mathbf{~ m}^{3}$ air, removal efficiencies gradually fell.

The volumetric productivity of methanol removal by the beds was linear over the entire range tested.
At a methanol concentration of $5 \times 10^{5} \mu \mathrm{g}$ methanol per $\mathrm{m}^{3}$ air, the methanol removal rate was $12 \mathrm{~g}$ methanol per $\mathrm{m}^{3}$ of bed per hour. Extrapolation of these data show that methanol removal is viable at full scale with a reasonable sized biofilter.

Final experiments evaluating the effects of temperature on methanol removal are in progress and will be completed by the end of the fiscal year. At this point it appears that methanol removal is feasible over the entire mesophilic range. Thermophilic removal is currently being investigated.

Economic estimates comparing biofiltration with regenerative thermal oxidation for methanol removal in composite board plants indicate that a plant of typical size can save approximately $\$ 2$ million in capital costs and about $\$ 0.5$ million per year in operating costs with biofiltration.

\section{New or Increased Technical Capabilities}

The INEL has expanded its biofiltration expertise into the biofiltration of methanol as a result of this project. This fills an important niche in our experience base,

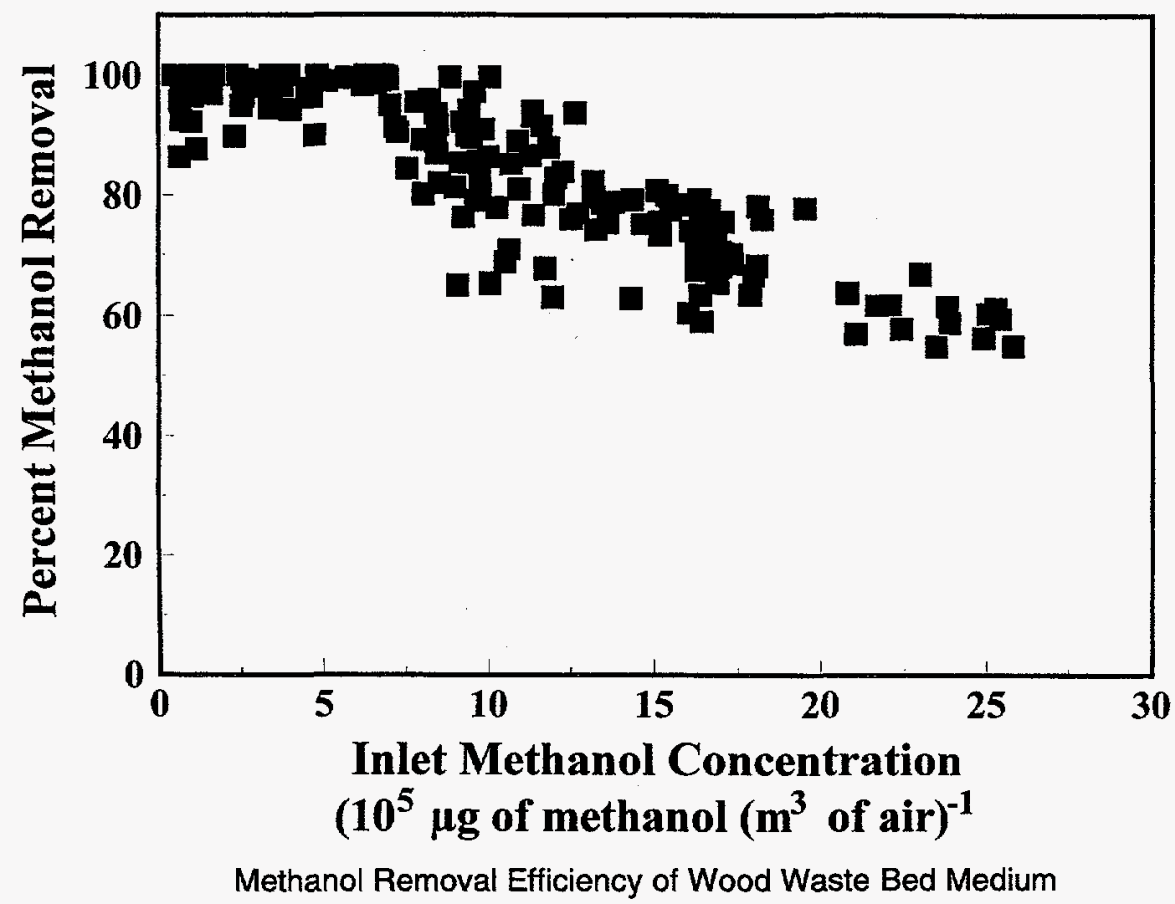

Idaho National Engineering Laboratory LDRD FY 1995 Annual Report 
as in situ bioleaching or microbial enhanced oil recovery, stands to benefit from the technology. U.S. economic competitiveness may be bolstered by using this tool to diagnose microbial nutrient deficiencies in groundwaters or soils. Bioremediation of hazardous waste is forecast to be a $\$ 1.5$ billion industry by the year 2000 (see Bioprocessing Technology, May 1991, Vol. 5), an estimate based on the potential for bioremediation to address waste issues for which current technologies pose exorbitant costs, daunting technical challenges, or significant health risks. To effectively perform in situ bioremediation, it is necessary to sustain the essential introduced or indigenous microorganisms with the appropriate nutrients.

\section{Objectives}

- Determine NDS effectiveness for predicting nutrient limitations in Hanford bioremediation with B. Peyton (Pacific Northwest Laboratory) and R. Gillis (Montana State University) in comparative studies with conventional devices for biofilm attachment

- Determine the nutrient limitations of microbes that leach copper from chalcopyrite in collaboration with the U.S. Bureau of Mines and Cyprus Minerals, Inc.

- Complete commercial assessment of the NDS device with INEL Technology Transfer Group.

\section{Accomplishments}

In FY-95, an internal market analysis was conducted that determined that approximately 100 NDS units per year could be used at approximately 200 sites per year within the United States. J. Billings of Billings and Associates, Inc. provides a higher estimate of 1000 sites. It was also estimated that application of the device needed to be combined with other technologies that would be needed for the complete characterization of a waste site before in situ bioremediation is implemented. The estimates were based only upon bioremediation sites, and did not include sites that used other types of in situ bioprocessing. It was determined that both a need and a suitable position existed that allowed pursuit of a patent. In May 1995, we filed a patent application on the NDS apparatus and all versions of a device that might allow the diagnostic characterization of nutrient limitations in groundwaters. In July 1995, data from the NDS studies were presented in a forum of DOE investigators from the Subsurface Science Program. This represented an opportunity for future studies to include consideration of use of the NDS system for determining nutrient deficiencies in subsurface microbial communities.

The other goals, namely (a) determination of nutrient limitation in subsurface microbial communities from Hanford and recommendation regarding nutrient supplements to enable bioremediation, (b) determination of mining industry needs for in situ biomining diagnostic tests, and (c) determination of nutrient limitation in subsurface microbes at the Mineral Park Site, were not realized. This failure occurred because of an unpredictable loss of funding late in FY95 connected with projects at both the Hanford and Mineral Park sites, which led to lost chances for in situ incubation of NDS devices at contaminated or mine sites. In both cases, the assumptions and prerequisites (access to samples and boreholes) that had been identified in the original work plan as being potential limiting factors were at fault. The loss of these opportunities had nothing to do with the technology described in this report, but rather with the volatility of support of collaborators and potential collaborators.
Once it was determined that these tasks were not going to be realized, a portion of the LDRD research funds were returned to the LDRD office.

\section{New or Increased Technical Capabilities}

This research is important because it represents the ability to define conditions under which aquifer microorganisms become non-limited through nutrient supplement, thus enabling such organisms to be used for in situ remediation activities. Ultimately, the NDS device can be used to determine whether biological remediation is even feasible or whether other technologies are more reasonable. The INEL, other DOE sites, and industrial sites have organic and inorganic groundwater contaminants that can be biologically degraded or biologically immobilized. The advantages of using microorganisms for in situ contaminant degradation or immobilization are that

- Microorganisms can be highly specific for targeted wastes.

- Complete degradation of organic contaminants is possible, yielding nontoxic by-products of metabolism.

- Bioprocesses are less expensive than conventional technologies.

- Direct contact of the microorganisms with the waste is possible in remote locations of the subsurface.

Further, it is known that microorganisms in many aquifers are under starvation conditions, yet the limiting nutrients are rarely understood. This is an important technology for defining the ecological constraints on subsurface microbial populations. In addition, this technology can be used in more basic research scenarios such as projects in the DOE Subsurface Science Program. We envision 
applications in biohydrometallurgical, agricultural, and enhanced oil recovery settings. The research contributes to a strength that the INEL exhibits in characterizing the biological, chemical, and physical features of terrestrial subsurface environments.

\section{Business Development Opportunities}

Several important business development opportunities exist as a result of this funding:

- A patent application is under consideration for this technology, which may serve to benefit the INEL.

- Collaborations have been established with B. Peyton (Pacific Northwest Laboratory) and one continues with R. Gillis (Montana State University). The three parties seek to test this device alongside other biological coupons for determining microbial biofilms in subsurface settings. A proposal, which will be co-authored by the three parties, is planned for submission to the new DOE Office of Health and Environmental Research In Situ Bioremediation Program.

- Application of the device has been discussed with J. Billings (Billings and Associates, Inc.), who showed interest in possible collaborations with the INEL in this and other remediation technologies. Billings is also an important contact with regard to the licensing of new in situ remediation technologies.

- Data acquired through the use of the device was presented to F. Wobber (DOE-HQ, Office of Energy Research), and opportunities for continued funding and development may exist through that route, especially with regard to possible remediation alternatives at the Test Area North injection well.
- Technical contacts have been made with the Idaho Groundwater Protection Section, G. Johnson (hydrologist, University of Idaho) and M. Odom from DuPont Inc., with which the INEL has a memorandum of agreement.

\section{Intermediate/Field-Scale Model for Predicting Microbial Transport LDRD 6701}

\section{A. L. Schafer-Perini}

Laboratory studies have shown that microorganisms can be effectively used in porous media to transform and entrain contaminants. However, when applied at the field scale, the use of microorganisms for remediation has not been entirely successful. This is due in part to (a) our inability to predict known processes in the subsurface at an appropriate scale, and (b) an incomplete understanding of the physical processes controlling biological transport and transformation at a field scale. Both issues are related through space and time scales and their definition in the predictive modeling context. The primary objectives of this project were to develop a methodology for incorporating micrometer scale laboratory observations into the kilometer scale models necessary for predicting field-scale transport and transformation.

To accomplish the model development and validation, an interdisciplinary study integrating laboratory observations of microbial movement through heterogeneous geochemical environments with a new predictive modeling approach was proposed. Observations of laboratory microscale behavior in porous media columns were to be incorporated into a predictive model using particle-grid techniques that will allow movement of appropriate scale representative elementary volumes (REVs). The predictive model would use a decision-tree based logic in combination with Eulerian-Lagrangian techniques to simulate the microscale microbial-geochemical system as it is transported through the kilometer-scale hydrodynamic system. The predictive model was to be refined through hypotheses testing and prediction against known and observed microbial migration.

\section{Objectives}

Overall project objectives were to continue the development of a model to accurately simulate existing, ongoing, and future biological transport and transformation experiments conducted by the Center for Biofilm Engineering (CBE), and funded through the NSF and CBE's Industrial Partners. INEL's contribution would be in the form of basic research for model development (FY-94), providing training in use of the model, and updating the model as additional laboratory results became available. Primary objectives for the FY-94 work included modifying an existing three-dimensional particle-grid code to incorporate filtration, multicomponent reactions, mass dependent permeability and porosity, and the effects of geochemical heterogeneity for application to existing laboratory results. FY-94 modifications included addition of relative permeability-biomass curves, effective porosity-biomass relationships, heterogeneous reactivity for the inclusion of geochemical reactions, inclusion of filtration, and the inclusion of multi-component reaction equations.

FY-95 objectives included calibration of the model to laboratory data, and further modification of the code as necessary to match observed behavior. The specific tasks were defined as follows:

- Define laboratory experiments that can be used to test and quantify the relative permeabilityporosity-filtration parameters for a specific contaminantgeochemical-hydrological 
system. This system will consists of $\mathrm{JP} 4$ in a clean sand column.

- Calibrate the model against the laboratory data.

- Make modifications to the modeling approach to bring the model in line with the observed laboratory results.

\section{Accomplishments}

FY-95 Technical accomplishments included successful modification of the FY-94 conservative transport code to incorporate a random walk representation of the advective dispersion equation. In addition, a literature search was conducted to select a filtration model for simulating the filtration of colloidal sized particles in packedbed systems. This was implemented but not tested. The transport code was then loosely coupled to a flow code to allow the interchange of data. This allows incorporation of relative permeability-biomass and porosity-biomass relationships to allow modification of hydraulic conductivity as a function of increasing biofilm thickness. Laboratory experiments were then defined for testing/quantifying relk-phi-filtration parameters for JP4/TCE systems in conjunction with CBE (Task 1). The second and third tasks assumed that CBE would be able to run the experiments in FY-95. However, CBE's funding and scheduling did not allow the completion of those experiments. Therefore, Tasks 2 and 3 will be postponed until the experiments can be rescheduled.

New or Increased Technical Capabilities

At this point, a model has been developed that will accurately simulate biological transport and transformation experiments and is ready for calibration to predict these interactions in the subsurface.
Business Development Opportunities

Commercialization of this type of code typically results in royalties, after validation and benchmarking, this code could be sold outright. An additional and probably more lucrative long-term financial benefit could be realied through code application. Applications could for example include work on the TAN remedial action at the INEL, and in conjunction with the remedial action under design at the March AFB (an INEL ongoing activity).

\section{Prompt Gamma Neutron Activation Analysis Methods Development for Waste Assay Applications LDRD 7907}

\section{R. J. Gehrke}

The purpose of this LDRD was to develop on-line methods for the assay of hazardous and mixed low-level waste by prompt gamma neutron activation analysis (PGNAA). The focus during $F Y-95$ was the preincineration assay of combustible low-level radioactive waste for chlorine, where the chlorine emissions at the incinerator are limited by EPA regulation to $4 \mathrm{lb} / \mathrm{h}$. Preincineration assay virtually eliminates the possibility of unintentional chlorine release above permit levels, by redirecting excessive amounts to other treatment and disposal options.

\section{Objectives}

- Optimize the experimental configuration to provide high and relatively uniform sensitivity for the detection of chlorine regardless of its position in the combustible waste container.

- Demonstrate that assay can be performed on-line within an assay time compatible with an incineration rate of 6 boxes per hour and a sensitivity of $2 / 3 \mathrm{lb}$ per box.

- Present the results of this study at the Annual Meeting of the American Nuclear Society held June 1995 in Philadelphia.

- Pursue a patent for the use of PGNAA for preincineration assay of combustible waste (Invention EGG-PI-768).

\section{Accomplishments}

To provide assurance that chlorine will not be released in quantities exceeding permit levels $(4 \mathrm{lb} / \mathrm{h})$, we developed a PGNAA method for the on-stream preincineration assay of low-level radioactive combustible waste for its chlorine content. The assay system we developed consists of three $\sim 5-\mu g^{252}$ californium neutron sources located in a plane at strategic locations on each of three sides of the $60 \times$ $60 \times 60-\mathrm{cm}$ cardboard waste box to be assayed and incinerated. Located on the fourth side is either a germanium or a scintillation $\gamma$-ray spectrometer to detect the activated prompt $\gamma$-rays from chlorine. To provide a complete assay of a waste box, counts are recorded at three positions along the length of the box, each with equal count times. Each of the three spectral counts can be analyzed separately or simply combined into one averaged spectrum. By taking three counts at equidistant positions along the length of the box, a reasonable averaged estimate of the chlorine content is obtained.

To determine the sensitivity and its uniformity over the assay volume, we took measurements of a surrogate combustible waste box containing rolls of paper towels arranged in a $5 \times 5$ cross-sectional matrix. Approximately 55 rolls of 
paper towels were used to fill the entire box (it was necessary to cut some of the rolls). This matrix arrangement approximates the material and density of real waste and simplified positioning of a sample polyvinyl chloride (PVC) plastic rod. We placed a PVC rod, $12.7 \mathrm{~cm}$ in diameter, $20 \mathrm{~cm}$ in length, and with a mass of $3800 \mathrm{~g}$, at the same depth in each of the 25 matrix positions by removing a roll of paper towel and replacing it with the plastic rod. With this arrangement, we acquired prompt capture $\gamma$-ray spectra for $1000 \mathrm{~s}$ using a germanium spectrometer. The results from these measurement for the $1165 \mathrm{keV}$ chlorine capture $\gamma$-ray indicated that a relative sensitivity within a factor of three could be achieved irrespective of the location of the PVC rod in the box. The absolute sensitivity was sufficient to meet emission limits of $4 \mathrm{lb}$ chlorine per hour in an incinerator burning four boxes per hour. It should be noted that the relative sensitivity can be made more uniform at some loss of sensitivity by moving the germanium detector back a few $\mathrm{cm}$. Under these conditions, the sensitivity may not vary more than a factor of two over the entire assay volume of the box. The lower limit-of-detection is estimated to be $33 \mathrm{~g}$ of PVC.

The use of 252 califormium source with higher activities is being considered in order to reduce the count time for assay of the entire box, so that 6 boxes can be assayed per hour.

We submitted a patent idea record to patent the use of PGNAA for preincineration assay of combustible waste (Invention EGG-PI768). The company declined to pursue a patent at this time. The results of this investigation were presented at the Annual Meeting of the American Nuclear Society; the paper was published in the trans- actions of the meeting (R. J. Gehrke, R. J. Pawelko, R. C. Greenwood, 1995, Application of PGNAA to Preincineration Assay of Combustible Waste for Chlorine, ANS Transactions 72,117 ).

New or Increased Technical Capabilities

As a result of this study, it is clear that on-stream assay of waste by PGNAA can be performed in an efficient and accurate manner.

Further, by strategic placement of the neutron sources and the detectors, it is possible to achieve relatively uniform sensitivity over the assay volume. This experience will be of great help in a new Mixed Waste Focus Area Technical Task Plan for the assay of containerized waste.

\section{Business Development Opportunities}

External individuals and companies following the developments of this LDRD include Don Steinman of Martin Marietta Specialties Components, and David Weigel of SEG (Scientific Ecology Group).

\section{Real-Time Asbestos Analysis by Raman Spectroscopy} LDRD 8104

$$
\text { J. C. Ingram }
$$

Contamination of asbestos represents a significant decontamination and decommissioning (D\&D) problem for most DOE sites, including the INEL. Currently, determination of asbestos in asbestos-containing material relies on collecting samples of the material in question, sending the samples to an off-site laboratory, and analysis of the material using polarized light microscopy. This type of approach is time consuming and expensive. The objective of this program was to investigate the use of Raman spectroscopy for detection of asbestos in real world samples. The proposed approach was to use a fiber-optic-based Raman spectrometer for on-site asbestos detection. Two investigative approaches were taken in these proof-of-principle studies: (a) optimization of the instrumentation for asbestos detection using a laboratorybased spectrometer with fiber optic attachments, and (b) application of difference spectroscopy to minimize background interferences. The results of this work indicate that asbestos detection by Raman spectroscopy is severely hampered by the combination of weak Raman signal from the asbestos and strong interferences from background materials (building and construction materials). Based on these results, we concluded that onsite asbestos detection by Raman spectroscopy is not feasible at the present time. However, with the increasing developments in chemometrics, we suggest that the use of Raman spectroscopy be revisited in the near future in combination with multicomponent analysis and/or neural networks in order to overcome the difficulties identified by the present work.

\section{Objectives}

- Analyze Raman asbestos by itself using a fiber-optic-based Raman spectrometer.

- Analyze Raman interferant materials.

- Analyze Raman model mixed samples, asbestos and interferant materials.

- Analyze Raman real world samples.

- Analyze scoping asbestos and interferants using a fiberoptic-based infrared spectrometer. (This task was not performed, because the instrument that was to be used was not available.)

\section{Accomplishments}

Two approaches were taken in order to determine the feasibility of Raman spectroscopy for on-site asbestos detection. The first 
approach involved the optimization of instrumentation and was performed at the INEL using two different Raman spectrometers. The second approach involved the development of difference spectroscopy and was performed at the University of Arizona. These approaches are discussed below.

Approach 1. Initial work was performed using a commercially available fiber-optic-based Raman spectrometer (RAMAN ONE, Chromex). This instrument is very rugged, as it contains an air-cooled detector and a robust laser. It also has fairly low sensitivity, which translates to the need for strong Raman signal in order to achieve detection with this instrument. Consequently, Raman analyses of the standard asbestos samples with this instrument were unsuccessful due to their weak Raman signal.

In the next set of experiments, we equipped an existing research Raman spectrometer with a highly sensitive, liquid-nitrogen-chilled detector and superior optics with a fiber optic sampling assembly. The premise for these experiments was to construct a fiber-optic-based Raman instrument with a premium detector and optics with which to detect asbestos. Upon establishing detection parameters, a more rugged design would be proposed. We found, however, that asbestos detection using the premium fiberoptic-based Raman instrument was difficult in the case of analyses of standard materials, and that asbestos was undetectable for mixed samples.

Approach 2. The objective of using difference spectroscopy was to overcome the problem of interference from the building and construction materials typically associated with asbestos-containing materials. We hoped that by taking a difference spectrum between the Raman spectra of the interfer- ant material and the asbestos/ interferant mixed materials, detection of the asbestos could be achieved. However, we found that the asbestos signal was concealed in the interference signal and could not be successfully extracted at asbestos contents less than $20 \%$. Even at asbestos contents in excess of $20 \%$, detection of asbestos was very difficult.

The combination of the results from the two different approaches led us to the conclusion that the use of Raman spectroscopy for onsite asbestos detection is not feasible at the present time. We suggest that as chemometric methods for data processing develop, the use of Raman spectroscopy in combination with some type of multicomponent analysis be examined for asbestos detection.

\section{New or Increased Technical Capabilities}

New technical capability at the INEL as a result of this project was the acquisition of the fiber optic sampling assembly. Previous to this project, the laboratory Raman system was limited to sampling through a microscope. The fiber optic sampling assembly offers the capability of performing a number of analyses, including gas-phase, in-situ reactions, and heat sensitive materials. Additionally, the analysis of asbestos and other related silicate materials by Raman spectroscopy broadened the technical expertise of the INEL staff in both handling these types of materials and in the spectral interpretation.

\section{Business Development Opportunities}

Initial contacts were made with Spectral Instruments concerning the production of an instrument; however, no further discussions were warranted.

\author{
Rapid Agrochemical \\ Sensor \\ LDRD 8106 \\ J. C. Ingram
}

Challenges in development of new methods for pesticide analysis include complex sample matrices, the presence of multiple pesticides, low volatilities and/or highly polar molecules. In many cases, the critical step in these analysis methods is extraction of the analyte of interest and/or sample cleanup before analyses, which is particularly problematic for solid samples. Direct surface analysis of the sample offers a means for streamlining pesticide analysis by minimizing the sample preparation step (including extraction of the analyte). Secondary mass ion spectrometry (SIMS) instrumentation developed at the INEL is demonstrating tremendous potential in this area through its ability to detect and identify chemicals of various types on environmental samples, including vegetation, soil and rocks, stainless steel, glass, etc. We performed a scoping study to evaluate the applicability of SIMS to detection of agrochemicals on various sample types. The study consisted of a matrix of 20 agrochemicals on four substrates, analyzed using a SIMS instrument with single-stage mass spectrometry capability. This instrument detected 16 of the 20 pesticides on all four substrates.

\section{Objectives}

- Select agrochemicals and sample types for SIMS detectability study.

- Conduct SIMS detectability study of agrochemicals on various samples.

- Evaluate SIMS detectability study.

- Define instrumentation requirements needed to develop 
a field-portable instrument of reasonable cost.

- Establish collaborations with members of the agrochemical community.

\section{Accomplishments}

A summary of the results of the static SIMS analyses of the pesticides on the four samples are presented in Table 1. The table lists the 20 pesticides and the four sample materials tested. The results of this scoping study indicate that 16 of 20 of the pesticides tested were positively detected in varying degrees on at least three of the four sample types by direct analysis of the sample surface using static SIMS. Of the 80 different pesticide/sample combinations ( 20 pesticides $x 4$ sample types), $46 \%$ of the combinations gave strong detection. These results are attributed to the fact that many of the pesticides have one or more of the following properties: (a) polar, (b) nonvolatile, and (c) easily ionized. Of the four pesticides that were considered difficult to detect by static SIMS, only one of them, trifluralin, was not detected on any of the samples. This result was not surprising, as previous analyses of nitro-organic compounds (like tri-

Table 1

Summary of Pesticide Detection Results

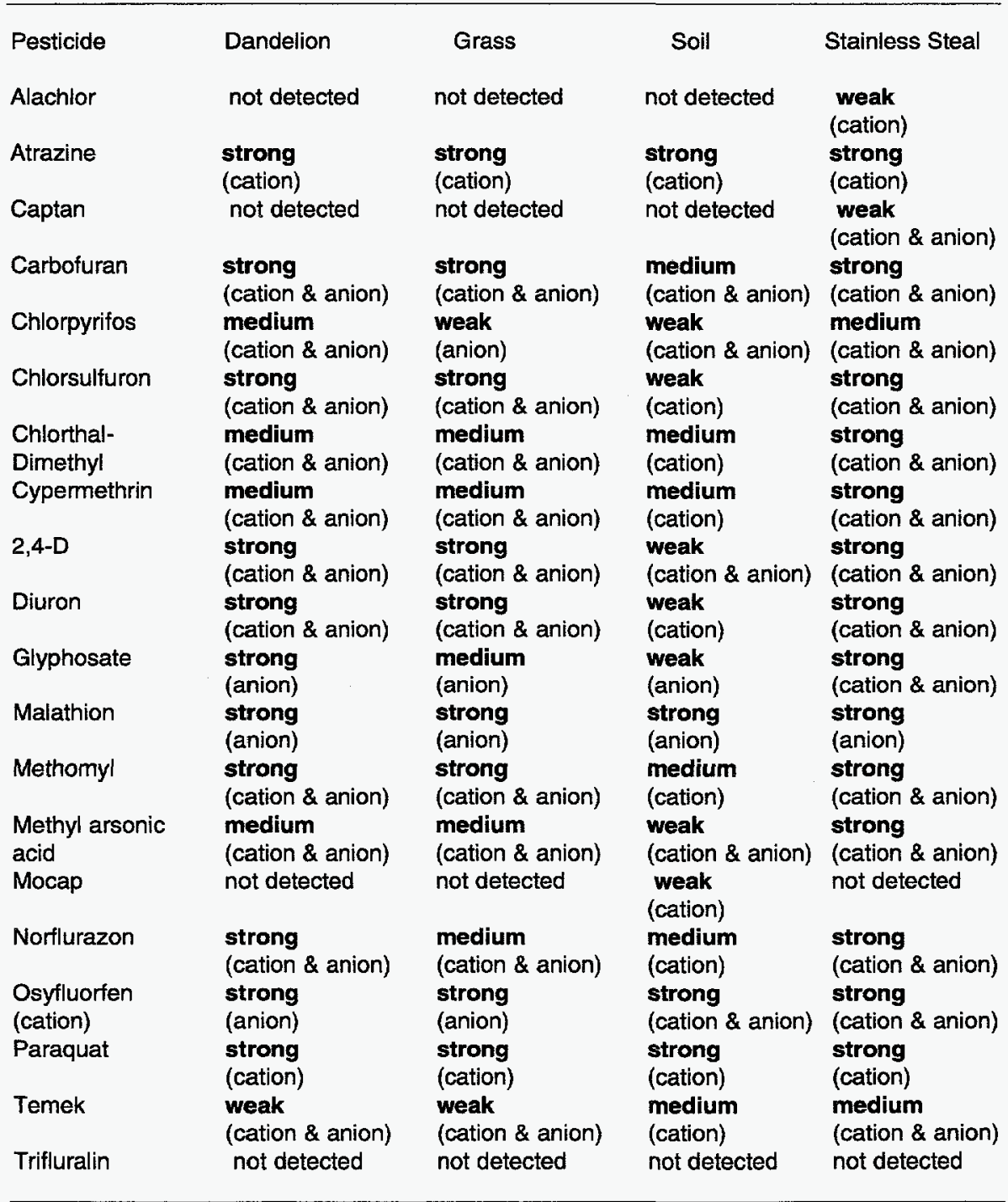

fluralin) in this laboratory indicate that this class of compounds is difficult to detect by static SIMS.

Also listed in table is the type of ion (cation/anion) used for detection of the pesticides. In $60 \%$ of the pesticide/sample combinations detected by static SIMS, both cations and anions were used for identification. Detection in the rest of the combinations was divided such that in $25 \%$ of the cases only cations used for identification and in $15 \%$ of the cases only anions were used for identification. The ability to collect both the cation and anion spectra in a nearly simultaneous manner, as was performed in this study, is advantageous in providing sufficient information for detection.

Although not specifically investigated, the results of this study also demonstrate the power of SIMS for probing the surface chemistry of the pesticides on the sample. In comparing SIMS results from the four sample types, some trends were observed. SIMS detection of pesticides adsorbed on the stainless steel is, in most cases, the most facile. We believe that this is due in part to the lower surface area of the stainless steel compared to the other samples, and the fact that the absorption of the pesticides into the stainless steel, which constitutes a loss, is minimal compared to the other sample types. A second trend observed is the similarity in SIMS spectra acquired from the dandelion leaves, the grass, and the stainless steel. In the case of the data collected from the soil, only 2 of the 20 pesticides used anions only for detection; for 10 of the 20 pesticides adsorbed at soil, cations only were used for detection. These results are in contrast to the other three sample types, in which only 2 of the 20 pesticides required cations only for detection. This phenomenon is explained in part by the enhanced 
ability of the soil to protonate the pesticides as compared to the other sample types studied.

One of the project tasks was to define instrumentation requirements to develop a field portable unit. Mass spectrometry instrumentation is advancing at a high rate, particularly in the field of ion trap mass spectrometers, which is the type of mass spectrometer best suited for this work. Benchtop systems currently cost approximately $\$ 80,000$, and are available from several commercial vendors. We are working with one vendor (Teledyne) to ensure that SIMS capabilities can be easily added as an option to their commercial instruments. Teledyne is also developing a new system that is designed to be field portable; thus, we envision that SIMS will be a viable option for performing field measurements.

\section{New or Increased Technical Capabilities}

Increased technical capability at the INEL as a result of this project was the broadening of the technical expertise of the INEL staff both in handling these types of materials and in spectral interpretation. Since agrochemicals are present in so many environmental samples, the information gleaned from this program strengthens the efforts of other programs involving characterization of environmental samples. Additionally, contacts were established with Idaho extension agents for future collaborations.

\section{Business Development Opportunities}

Presently, no new business developments were initiated as a result of this particular program, although other developments have been established related to the SIMS work at the INEL. Outside funding for further work toward application of SIMS technology to detection of agrochemicals is being pursued.

\author{
In Situ Biodegradation \\ of High-Energy Materials \\ LDRD 8403 \\ F. F. Roberto
}

The INEL and other DOE sites have explosives-contaminated areas ranging in size from small to extensive resulting from military ordnance testing and disposal (World War Il-era), and they produce and dispose of explosives resulting from DOE DPrelated missions. On the other hand, the Department of Defense, particularly the U.S. Army and its ordnance and munitions contractors, have a significant and expensive problem in the remediation of production facilities, proving grounds, arsenals, firing ranges, bombing ranges, and disposal facilities. In Europe and the Middle East, our allies continue to face longstanding problems resulting from military operations dating back as far as World War I, and face more contemporary actions, such as the Gulf War. The research and development encompassed within this project will result in the establishment of economical and efficient bioremediation strategies for dealing with the large volumes of soil and water contaminated with high explosives (HE), and with industrial process water containing potentially explosive nitroaromatics.

\section{Objectives}

- Expand microbial screening efforts to include Dupont proprietary microorganisms.

- Initiate screen for anaerobic bacteria capable of reducing ammonium nitrate levels present in Dupont industrial waste stream (contains about 40,000 ppm ammonium nitrate). Note that this effort was determined to be unnecessary after discussions with DuPont industrial process engineers, who indicated that ammonia concentrations can be reduced through process modifications.
- Continue mechanistic studies with Dupont microbes (at Dupont) for strain and process improvement. Note that owing to delays in obtaining full funding authorization (February 1995), DuPont opted to have INEL expand bioprocess development work at INEL instead.

- Expand bioprocess design and development work beyond logic diagram stage achieved in FY-94.

- Perform bench-scale experiment with INEL HE-contaminated materials to explore performance of biodegradation with chunk HE and amended soils.

The technical effort in FY-95 focused on establishing an immobilized cell bioreactor to treat nitroaromatic-contaminated aqueous waste streams. In FY-94, a suite of microorganisms were identified from explosives-contaminated soils at the INEL, and through an memorandum of understanding with DuPont, access to additional microbes with demonstrated capacity to degrade the nitroaromatic, picric acid, was obtained.

Batch studies had demonstrated complete biodegradation of picric acid at concentrations up to $150 \mathrm{mg} / \mathrm{L}$. Our primary goal was to increase available biocatalyst through the immobilization of cells to appropriate biocarriers. This was accomplished through growth of biomass in a picric acid containing medium, followed by passage of the aqueous cell suspensions through a column containing suitable biocarriers.

Initial operation of the bioreactor was studied with an influent picrate concentration of $300 \mathrm{ppm}$. Through the course of the year, the level of influent picrate was gradually ramped up to $1000 \mathrm{ppm}$ (Sep- 
tember 1995). With a residence time of about 24 hours, virtually all influent nitroaromatic is destroyed (see the figure). Analysis of effluents reveals that depending on the nature of the medium, byproducts indicative of alternative degradative metabolic pathways can be detected, indicating that medium composition is critical to optimal performance of the bioreactor.

Small-scale bench experiments were performed to examine the capacity of selected organisms to degrade TNT in soils. We have been able to demonstrate up to $40 \%$ degradation of this compound, but further studies have been hampered by problems in obtaining additional contaminated soil from the Site.

\section{New or Increased Technical Capabilities}

Explosives- or ordnancecontaminated soils and groundwater have been described as the single largest environmental problem facing the Department of Defense. Owing to the large geographic areas that potentially must be remediated, biotechnologies have received substantial attention by DOD, EPA, states, and private industry interested in attacking this problem. Three biotechnologies have now been demonstrated at pilot scale and accepted by the EPA for use in the remediation of TNT-contaminated soils. The INEL has a number of locations contaminated with explosives and ordnance. A unique aspect of the INEL problem is the presence of large chunks of explosive. These chunks pose a serious hazard for handling of these soils, and a challenge for the existing biotechnologies. INEL development of a bioprocess to deal with these soils will establish leadership in dealing with this particular problem.

\section{Business Development Opportunities}

A memorandum of understanding has existed since 1994 with DuPont, and this project has been one of several in which INEL and DuPont scientists have worked cooperatively to use INEL capabilities. In FY-95, $\$ 40 \mathrm{~K}$ of project funds were to support a postgraduate researcher resident at DuPont. However, delays in obtaining full funding authorization from Lockheed Idaho management resulted in that $\$ 40 \mathrm{~K}$ being redirected to support that work at INEL. At

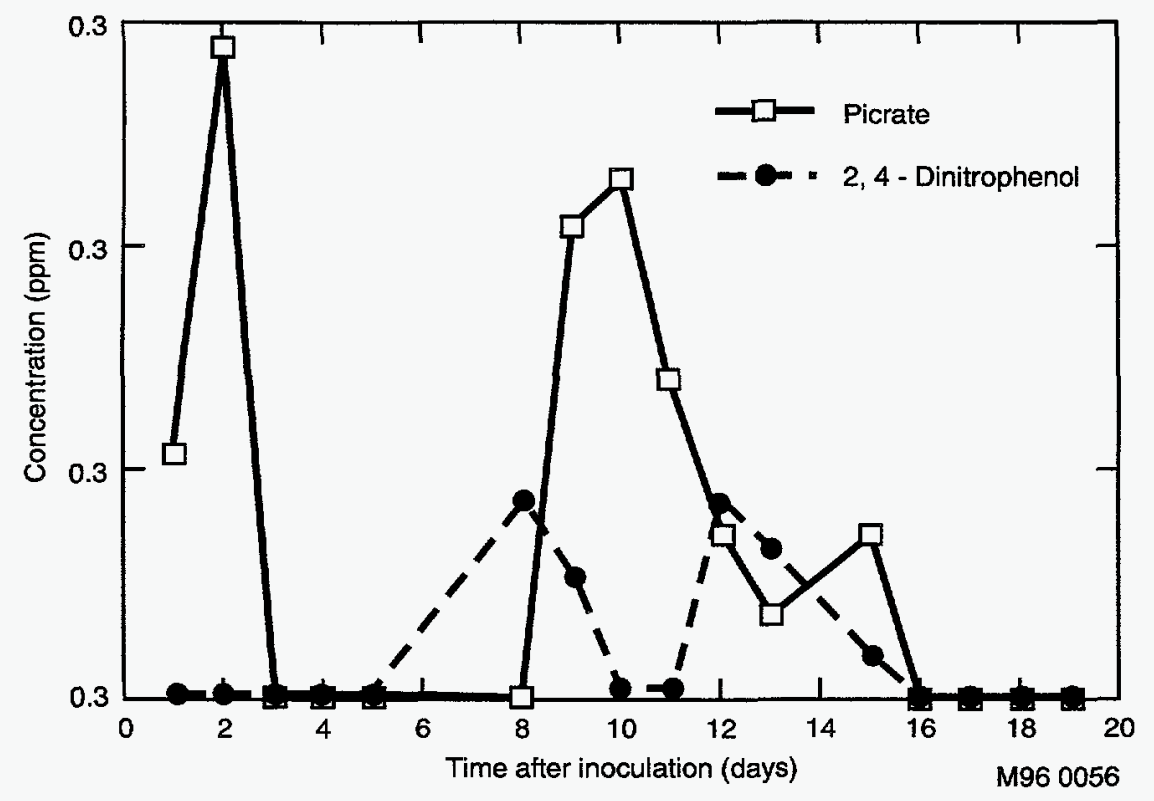

Dynamics of Picrate and Dinitrophenol Detected in Immobilized Cell Bioreactor Effluent upon Pulsing with 700 ppm Picric Acid

present, given the promising results emanating from the project, DuPont researchers are making numerous inquiries as to specific details of the INEL bioprocess. This may be an opportune time to request that DuPont indicate their continued interest by direct funding of part of this work.

\section{Rapid Laser Assay of Surface Contamination during Decontamination and Decommissioning RDRD 8409}

\section{T. E. Repetti, J. R. Fincke}

The deactivation and decommissioning of surplus facilities that contain radionuclide, hazardous, mixed, and special contaminants requires near real-time field characterization of contamination on concrete and metal building surfaces, soils adjacent to those buildings, and equipment such as glove boxes and other process hardware contained within. Rapid assay techniques, conducted in situ and in real time, would greatly facilitate both the initial survey and remediation phases of facility deactivation and decommissioning. This project will demonstrate that the proven technique of Laser Induced Breakdown Spectroscopy (LIBS) can meet the requirement for a real-time onsite, in situ, rapid assay of hazardous contamination during the deactivation and decommissioning of facilities. This technique is capable of identifying the presence of and quantifying Resource Conservation and Recovery Act (RCRA) metals, radionuclides that are difficult to identify because of their low rate of decay, and it can also be used ato identify the presence of solvents containing fluorine or chlorine. 


\section{Objectives}

- Demonstrate that the proven technique of LIBS can meet requirements for real-time in situ rapid assay of hazardous contamination for decontamination and decommissioning of facilities.

- Develop a database of LIBS identification lines for RCRA metals, Uranium, Plutonium, lead-based paint, and solvents containing fluorine and chlorine. Substrates include stainless steel, carbon steel, concrete, and soil.

\section{Accomplishments}

A database of LIBS identification lines was begun with analysis of data taken during preliminary scoping studies in FY-94. One hundred forty good LIBS lines were identified from nine elements (aluminum, cadmium, cerium, chromium, copper, iron, mercury, lead, and uranium) on various substrates. These lines were compared to analytical identification lines reported in many sources in the LIBS and Inductively Coupled Plasma - Atomic Emission Spectroscopy (ICP-AES) literature, with varying degrees of overlap. This is not surprising considering that the LIBS and ICP-AES processes excite atoms in different ways.

Preliminary contact was made with Atomic Engineering Corporation (AEC) of Gaithersburg, MD, which has developed a product called Multi-spectral Chemical Characterization System (MCCS) for automated identification of elements. MCCS is a computer database and spectral line identification system that can be hooked up to a customer's spectroscopy system to acquire and analyze spectroscopic data gathered by that system. An LIBS database would be a natural add-on to the MCCS product.
Work on this LDRD was halted after midyear when it became apparent that researchers at Los Alamos National Laboratory (LANL) have also been pursuing this research and have roughly a 2year head start (Photonics Spectra, December 1994, page 50). The primary point of contact at LANL is Dr. David Cremers (Group CST-1); discussions with Dr. Cremers indicated that LANL has already developed several systems, including a field-portable LIBS device and LIBS database, and has begun discussion with the AEC on integrating their LIBS database into a commercial system. In addition, LANL is pursuing a patent on remote standoff soil analysis from intermediate $(10-\mathrm{m})$ distances. These accomplishments were to be milestones of this LDRD during FY-96 (next year). For these reasons, further work on this LDRD was halted and roughly $\$ 35 \mathrm{~K}$ of funds was returned to the funding source via $\mathrm{CCB}$.

\section{New or Increased Technical Capabilities}

We have demonstrated the ability to perform LIBS experiments at the INEL and understand the basic issues involved in analyzing the data.

\section{Business Development Opportunities}

The primary intellectual properties to be developed from this project were the portable LIBS analyzer itself and the LIBS database. With a two-year lag in development time, it is doubtful that INEL could develop a portable LIBS analyzer to compete with LANL systems already in use. LANL has also developed an LIBS spectral line database and is proceeding with plans to market it in conjunction with AEC's MCCS system. Overcoming LANL's long lead time in this research would not be cost-effective.

\section{Measuring the Component Ratio in Oil/Water/Gas Mixtures and Determining Flow Regime \\ LDRD 8414}

J. R. Fincke

\begin{abstract}
Measurements of the individual flow rates of oil, gas, and water from individual wells in a field are required to develop reservoir management strategies for optimization of total productions over the life of the field. The conventional method involves massive equipment and is costly. Because of the potential savings and increased production, oil companies are beginning to address the issues of measuring multiphase flow at the well head.
\end{abstract}

\section{Objectives}

The final objective is the design, construction, and characterization of a rugged, accurate device for metering high void fraction $(95 \%)$ oil-gas-water flows in the gas field production environment.

\section{Accomplishments}

A flow section and flow sensing device (see the figure on page 64) were designed, constructed, and characterized. A specially designed venturi tube was required which allows the flow measurement of the predominant gas phase, which contains entrained particles. Pressure drop measurements are made between the upstream entrance and the exit of the extended throat, and between the extended throat exit and the exit of the diffuser section. The primary parameters that govern high void fraction gas-liquid flows were determined. These allow the mass flow rates of each phase of the gas and liquid mixture to be 


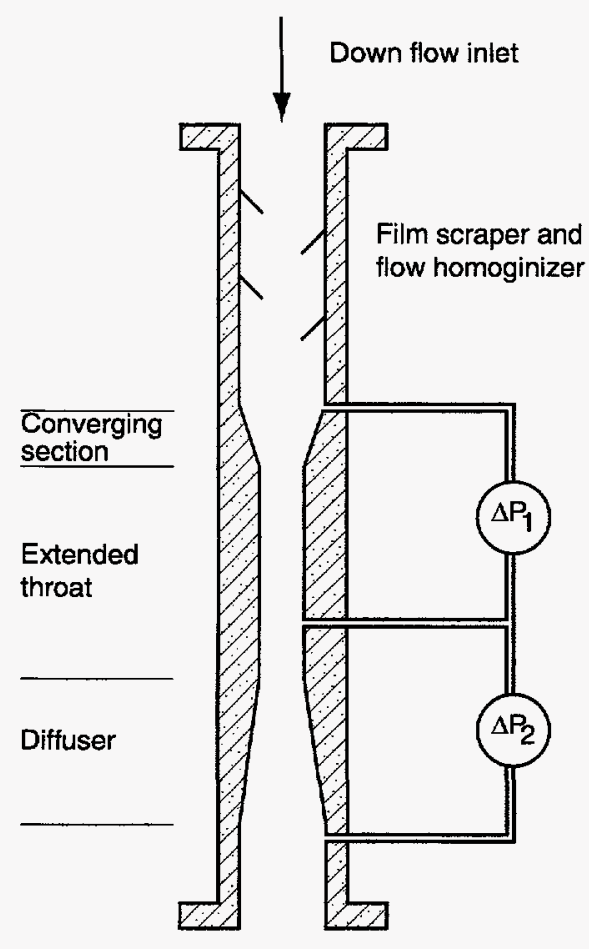

determined. Calibration was accomplished by characterizing the pressure drop delta-P1 versus pressure rise delta-P2 through the device for gas flow alone, and for the expected range of loading ratios.

\section{New or Increased Technical Capabilities}

The INEL has had expertise in liquid gas hydrodynamics over many years. This extension to a specific and valuable measurement technique increases the value of the INEL through the contribution of hydrodynamic sensing devices in the petroleum and natural gas industry.

\section{Business Development Opportunities}

This project has received both interest and monetary support $(\$ 20 \mathrm{~K})$ from CONOCO because of their interest and belief that this gas-liquid flow measurement system will be of value. They have stated that they are willing to cooperate with this INEL project in the development phase with another $\$ 100 \mathrm{~K}$. If this sensor device behaves as expected, it will represent a valu- able contribution to many aspects of oil production and refining.

\section{Polyethylene Sulfur Cement: Feasibility as a Waste Encapsulation Material for Low-Level and Mixed Wastes}

LDRD 8509

\section{A. W. Erickson, M. H. O'Brien}

This project is determining the feasibility of substituting low-cost recycled low-density polyethylene (LDPE) for the polymer phase in sulfur polymer cement (SPC). Polymers must be added to sulfur to accommodate the destructive crystallographic transform that occurs when sulfur solidifies after melting. The primary benefit of this substitution is to use LDPE, which accounts for a large percentage of unrecycled plastic and takes up valuable land fill space, and turn it into an inexpensive waste form. The LDPE is combined with inexpensive sulfur, often an industrial byproduct and lowlevel mixed waste or toxic metal waste such as fly ash. The mixture is stirred and heated to $150^{\circ} \mathrm{C}$ and, upon thorough mixing, becomes a thermoplastic waste encapsulation material called PES (polyethylene sulfur), which is similar to SPC.

\section{Objectives}

- Examine the microstructure of PES using sublimation techniques and the SEM (scanning electron microscope).

- Use waste form performance criteria with tests that are likely to become the basis for lowlevel and mixed waste form acceptance.

- The material test matrix contains LDPE with different melt viscosities and likely contaminants such as other recycled plastics, dyes, coatings, and plasticizers.

\section{Accomplishments}

The microstructure of PES was observed. PES is primarily a composite material consisting of polyethylene filaments that ball up as the sulfur is sublimed away. This is quite different from the dicyclopentadiene and cyclopentadiene oligimers used in SPC which chemically react with the sulfur rings to stabilize the material.

Testing of PES followed methods outlined in the Proposed Waste Form Performance Criteria and Testing Methods for Low Level Mixed Waste. PES and SPC were tested simultaneously in a two-tier format where waste form material must first pass two leachability tests and then pass several more tests for integrity under stress. The toxicity characteristic leaching procedure (TCLP) and all other testing were performed on $40 \%$ ash waste loaded SPC and PES, both of which contained 5\% polymer. The PES had small amounts of high-density polyethlene (HDPE), polyvinyl chloride (PVC), polypropylene (PP), and Polystyrene (PS). Results of the TCLP testing awaits ICP chemical analysis. The other leachability test is the Accelerated Leach Test (ALT). Two ALT tests were performed at different temperatures with the PES samples containing all of the above mentioned plastics. The leaching of cerium, cobalt, cesium, lead and strontium revealed that SPC was superior in this test. Two of the integrity under stress tests where performed.

Compressive Strength Testing showed SPC averaged 9956 psi with an acceptable standard deviation. The PES with all contaminants averaged 5,541 psi with a large standard deviation. The variation in strength of the PES seems to be related to the calculated density. NRC requires that this type of waste form be able to withstand 500 psi. A stability in water or $90-$ 
day immersion and compression test should be finished in early October. This test has sample groups of each plastic contaminant and measurements taken on the submersed samples show all less than the $3 \%$ dimensional change or show no spalling off of materials that would indicate failure. Compression testing of submersed samples compared to nonsubmersed compression testing must be no less than $75 \%$ of the regular compression test samples on the October deadline.

\section{New or Increased Technical Capabilities}

Using the new performance criteria has shown that at the previous test methods are irrelevant or there are problems in previous methods. The ALT has been performed for the first time at the INEL and results will be of value to the people studying SPC. This year's results will hopefully be significant enough that the IPC (intellectual properties committee) will go ahead with a patent either by Lockheed Idaho or DOE-ID.

\section{Business Development Opportunities}

The only business contact that was made was with a regional recycling consortium in an attempt to obtain information for an economic study.

\section{In-field Assay for Parts per Million Levels of Chromium in Environmental Media LDRD 8514}

\section{K. D. Watts, R. J. Gehrke}

Using a $0.38-\mathrm{mCi}{ }^{59} \mathrm{Ni}$ excitation source, an energy dispersive $x$-ray fluorescence ( $E D R X F)$ spectrometer for the analysis of low levels of chromium contamination in soils was developed and tested. Chromium concentrations of $\sim 10$ ppm were detectable in 1,000second counts using a $30-\mathrm{mm}^{2} \mathrm{Si}(\mathrm{Li})$ spectrometer. This represents a factor of 10 improvement over currently available commercial technology. By developing this spectrometer system for in-field use, a new level of control can be maintained at remediation sites where metals have been introduced into the soil from industrial processes.

\section{Objectives}

- Develop an in-field X-ray fluorescence spectrometer, using a $\mathrm{Ni}-59$ radioisotopic source, capable of analyzing Chromium (Cr) at ppm levels in environmental media.

This spectrometer employs a ${ }^{59} \mathrm{Ni}$ excitation source, an excellent source for the excitation of elements below iron $(Z<26)$ in the periodic table. ${ }^{59} \mathrm{Ni}$ emits cobalt $\mathrm{K} x$-rays (Ka $=6.92 \mathrm{keV}$ and $\mathrm{Kb}=7.65 \mathrm{keV}$ ) from the $100 \%$ electron capture decay to the ground state of ${ }^{59} \mathrm{Co}$. These $\mathrm{x}$ rays lie only $1 \mathrm{keV}$ above the $5.988 \mathrm{keV}$ Kabs energy of chromium so that they have a large fluorescence probability for $\mathrm{Cr}$.

The associated analysis program, modified from existing programs, measures the intensity of the x-ray peaks and convert this to a quantitative concentration in ppm. The quantity of $\mathrm{Cr}$ as measured by the spectrometer is printed automatically after the analysis is complete.

- Modify the shielding mechanism and software of a Gamma Products sample changer for use with a ${ }^{59} \mathrm{Ni}$ x-ray fluorescence spectrometer.

This modification allows up to 45 samples to be counted consecutively and with no operator interference (automatically). The final adjustment will be complete when a detector with the correct cryohead configuration is obtained and placed in the changer.

- Submit paper for refereed review and publication.
K. D. Watts and R. J. Gehrke, EDXRF Measurement of PPM Concentration Levels of Chromium in Soil Using ${ }^{59} \mathrm{Ni}$, Submitted to Radioactivity \& Radiochemistry, September 1995.

\section{Accomplishments}

In this study, the electroplated ${ }^{59} \mathrm{Ni}$ excitation source was used with a $30-\mathrm{mm}^{2} \times 3$-mm-thick lowenergy $\mathrm{Si}(\mathrm{Li})$ spectrometer to assay chromium contaminated soil by EDXRF. Several 11-gram samples of soil, each dried and sieved, were spiked with known amounts of chromium, covering a range of concentrations from 10 to 1,017 ppm. XRF spectra of these samples were acquired for 1,000 seconds (live time). The scaling factors obtained in the linear-least-squares fit (i.e., the factor with which the Cr component spectrum was multiplied to fit the sample spectrum) were used to develop a calibration curve as a function of chromium concentration. Scaling factors as a function of chromium concentration are shown in the figure on page 66 , along with a linear leastsquares fit through these scaling factors. As is seen, concentrations as low as $10 \mathrm{ppm}$ can be measured with an $11 \mathrm{~g}$ soil sample counted for $1000 \mathrm{~s}$. Since all soil samples had the same composition except for the chromium concentration, no sample matrix corrections were required.

Also, a set of blind soil samples were also prepared. These blind samples were counted to test the reliability and accuracy of the system. The measured results from counting these blinds indicate an error of $\sim 10 \%$ to $15 \%$ for an 1,800 -second count. 


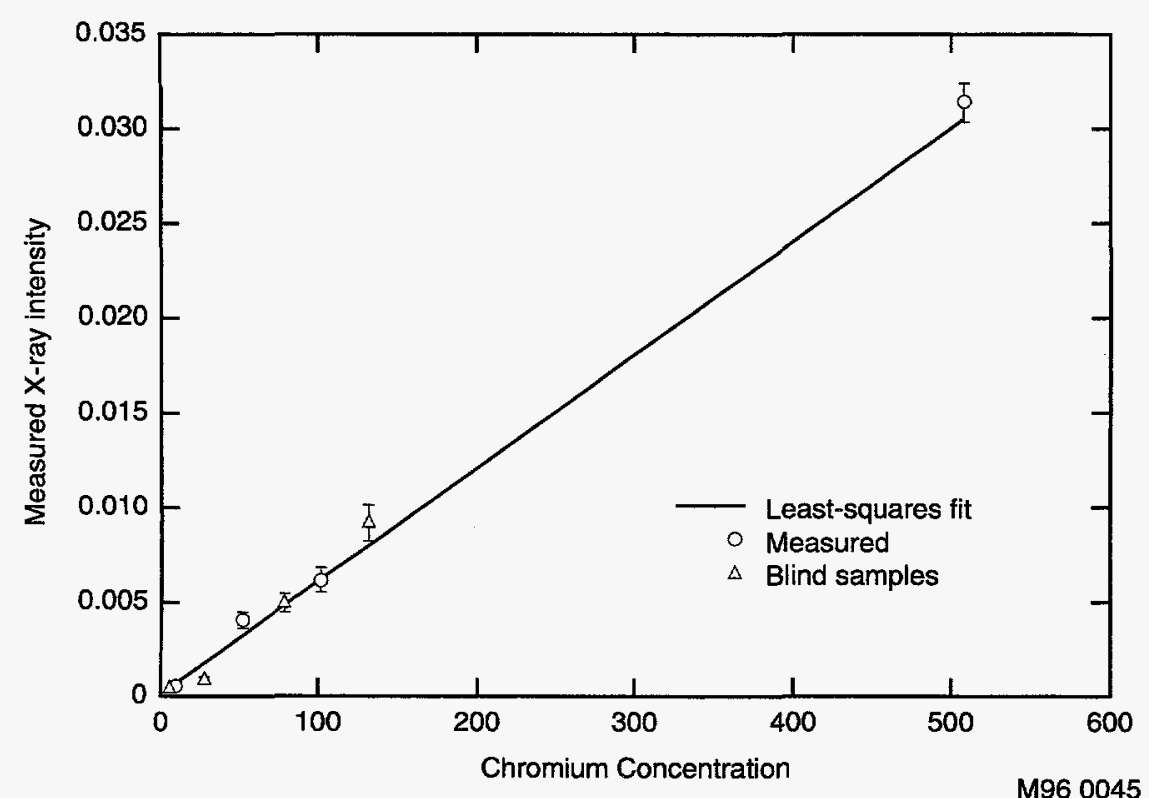

Measurement of PPM Levels of Chromium in Soil

Based on these present measurements, a $2-\mathrm{mC}{ }^{59} \mathrm{Ni}$ source could be used by an EDXRF spectrometer to detect chromium at a concentration of $5 \mathrm{ppm}$ with an uncertainty of $\sim 10 \%$ for a $200 \mathrm{~s}$ count.

\section{New or Increased Technical Capabilities}

This research demonstrates the potential value of the radionuclide, ${ }^{59} \mathrm{Ni}$, for use as an in-field EDXRF source for the assay of low (i.e., $<50 \mathrm{ppm}$ ) concentrations of chromium in soil. It is possible that the sensitivity for chromium will be sufficient to eliminate in many cases the need for collecting samples for laboratory analysis. Extension to assays of other environmental media is straightforward as is its extension to the assay of other elements in the $19<Z<25$ range, i.e. potassium to manganese. The development of the spectrometer as well as the feasibility of the ${ }^{59} \mathrm{Ni}$ excitation source for in-field applications gives the INEL a lead in this type of measurement.

\section{Business Development Opportunities}

We have received a letter of interest from Canberra Industries to possibly pursue this research.

\section{Low-temperature Reductive Catalytic Destruction of Halogenated Organics}

\section{LDRD 8615}

\section{S. K. Janikowski}

Halogenated organics represent some of the most versatile compounds used in our society. They include all organic materials with one or more fluorines, chlorines, bromines, or iodines as part of the molecular structure. They are used in cleaning, degreasing, paint stripping, as refrigerants, fire extinguishing media, aerosol propellants, oil and hydraulic fluid thinners, pesticides, antiseptics, medicines, PVC plastics, urethane foams, and as transformer and large capacitor fluids. in the nuclear industry, they are also used for cleaning hygroscopic metals (U, Pu, etc.) during reactor and weapons manufacturing and maintenance. The list is long because halogenated organics have many chemical and physical properties, which ideally suit them for hard duty applications. They are resistant to oxidation, reduction, thermal degradation, biological degradation, substitution, high energy field or radioactive degradation, etc. This robust nature is desirable for the intended application, however, when the halocarbons have completed their service and are released to the environment, they have adverse effects. They are toxic to humans and animals, and are responsible for destroying the ozone layer in the earth's upper atmosphere. These conditions plus the long life and difficult-to-treat aspects of halocarbons make them a nuisance of international proportion when they need to be destroyed. High-temperature incineration is currently the only approved method for destruction, however it has many severe drawbacks. It is energy intensive, has high initial capital costs, is difficult and time consuming to permit, and the process always discharges to the atmosphere compounds that are more hazardous than those fed into it. A process that mitigates the above mentioned drawbacks is one of today's most needed technologies. The titled process completely destroys halogenated organics under mild, nonoxidative conditions and is being developed as an environmentally safe alternative to incineration.

\section{Objectives}

- Design a continual flow reactor system.

- Construct the continual flow reactor system.

- Test the operation of the continual flow reactor system.

- Evaluate the reactor and dehalogenation process on several halogenated organics.

- Establish the operable temperature range for halocarbon destruction (at ambient pressure).

\section{Accomplishments}

A process to completely destroy halogenated organics 
under mild, nonoxidative conditions is being developed as an environmentally safe alternative to incineration. The proof-of-principle was demonstrated previously in a static $\mathrm{mL}$-size reactor, in which over 20 different hazardous and toxic, linear, cyclic and polycyclic, and aromatic and polyaromatic halogenated organics were converted to less hazardous, nonhalogenated organics. The present work was directed at (a) demonstrating the technology under continual process conditions, and (b) establishing the optimal temperature, at low pressure, under which a select group of halogenated organics could be destroyed. The process demonstrated complete destruction of the halogenated organics over the temperature range $250^{\circ} \mathrm{C}$ to $400^{\circ} \mathrm{C}$, with significant degradation down below $100^{\circ} \mathrm{C}$. The accompanying figure presents some of the results obtained recently in our continual flow reactor.

\section{New or Increased Technical Capability}

A patent titled Method and Apparatus for the Low Temperature Destruction of Halogenated Hydrocarbons has been applied for. The INEL will benefit from this intellectual property through licensing agreements with private industry, and through increased programmatic funding due to our increase in technical expertise. The INEL now has a niche in reductive catalytic processing. Aside from the novel halocarbon destruction technology we have developed, we now have access to an even larger oil industry.

\section{Business Development Opportunities}

The earlier results of this process have been presented to eight different companies, which have subsequently expressed an interest to partner with the INEL during the Field Demonstration phase. ETAS Corp. has submitted a Letter of Intent to CRADA with the INEL once the technology is mature enough to scale to pilot size. The ETAS application is primarily waste treatment and environmental remediation.

The other companies having interest in this technology have similar, but distinguishable applications, for which separate CRADA potential exists.

A large Government market also exists for this technology, which we now have specific expertise to contribute. Hundreds of thousands of tons of halogenated organics are used and discarded by the DOE and DOD systems each year, creating regulatory problems and costs in the millions of dollars. Furthermore, $21 \%$ of Superfund sites and 200-300 Resource Conservation and Recovery Act (RCRA) sites have $\mathrm{PCB}$ contamination with even greater percentages having other halocarbon contamination. The market for destroying halocarbons is hundreds of millions of dollars per year in the United States alone.

\section{Periphyton Communities for Environmental Monitoring LDRD 9510}

\section{R. M. Lehman}

We propose to advance development of a rapid, inexpensive and sensitive assay for detecting environmental contaminants in soil and waters, and monitoring the effectiveness of restoration activities. Developing this community-level bioassay (CLPP) would provide significant benefits in terms of cost savings, turnaround time, and sensitivity over existing baseline technologies for contaminant detection, e.g., direct chemical analysis for all compounds. The bioassay would be used for site characterization in association with environmental risk assessment. With respect to determining endpoints for environmental restoration, developing this bioas- say would offer the first rational criteria for declaring completion of restoration activities. At present, there is no universal solution to declaring that a site is sufficiently clean and resembles its unimpacted state. Developing the proposed technology would contribute to the forming criteria that are ecologically relevant for returning soils and waters to their original condition. The proposed project is a research and development effort that would focus on the use of a new, functionally based approach to estimating microbial community diversity and relating this index to environmental condition, e.g., the presence of toxic substances or other stress.

Incremental progression was made towards the ultimate goal of producing a novel and effective method (e.g., patentable) for environmental monitoring and assessment. We estimate that roughly 1 FTE of effort in FY-96 would lead to proof-ofprinciple, and 2 years of effort (at 1 FTE) would position the work to attract capital for a commercial software venture. We have an ongoing relationship with Barry Bochner, president of Biolog, Inc,. and a verbal agreement that he would be interested in commercializing any computer algorithms that we develop to manipulate the extensive community characterization data that are generated with this approach.

\section{Objectives}

- Analyze existing dataset and apply results to design of FY-95 field study.

- Design and construct Benthic Algae Substrate Sampler (BASS).

- Conduct FY-95 field experiment, perform analyses, and reduce data.

\section{Accomplishments}

The goal of this experiment was to demonstrate the use of microbial communities (algae and bacteria) to assess different environmental conditions in a freshwa- 
ter river in Idaho. The freshwater stream chosen was Iron Creek, which is 25 miles south of Salmon Idaho. It is a second order alpine stream impacted by mine drainage from an abandoned adit. This stream stretch is of interest to the Idaho Department of Environmental Quality.

Microbial communities are used to assess environmental stress by either collecting samples of the microbes from natural substrates or by placing artificial substrates in the natural environment and then collecting the samples off of the artificial substrates after a certain interval of time. During the summer of 1994, we used a 30-day colonization period for the artificial substrates. This was an adequate colonization period overall, but microbial development differed widely among different streams. Some substrates had very little microbial biomass, and other substrates had more than enough. Since Iron Creek is a relatively oligotrophic site and use of natural substrates have become more popular, we chose to design and construct a sampler Benthic Algal Substrate Sampler for this study. We sampled along an in-stream transect across locations that are pristine, impacted, and recovered. The choice of the number of samples collected from stream sites and the stream reaches to be sampled were based on the results of the 1994 stream study and other relevant studies. The biology of the periphyton and water grab replicate samples were analyzed by the Community-Level Physio logical Profile (CLPP), Phospholipid Fatty Acid Profile (PLFA) and algae community structure by light microscopy and point-counting techniques. General water chemistry analyses were conducted as well as metal content of the periphyton.

Principal components analysis (PCA) was applied to community- level physiological profiles (CLPP), which examine the heterotrophic microbial component of the periphyton. PCA and clustering algorithms were applied to phospholipid fatty acid profiles, which encompass structural characteristics of both prokaryotic and eukaryotic microbial communities. Algal communities were assessed by determining the abundance of the dominant species by direct observation in relation to the physical and chemical characteristics of the water. Canonical correspondence analysis was used to determine relationships between the abiotic characteristics of the water and the biotic measurements. This analysis is useful for identifying statistically significant correlations between water quality and population abundances. While CLPP data on the water correlate well with metal concentrations at the site, PLFA correlations are less conclusive.

\section{New or Increased Technical Capabilities}

This project will maintain INEL at the forefront of the development of novel methods for ecosystem monitoring, assessment, and restoration. We can provide technical support for remediation of local mining waste sites. And we are making technical progress toward proof of principle of an original idea with far-reaching practical applications continues.

\section{Business Development Opportunities}

The target commercial product for this research is a software package which would allow users to perform the complex data analysis and interpretation in a standardized fashion. The prime candidate for purchasing software licensing and marketing this product is Biolog, Inc., the manufacturer of the microtiter plates that the CLPP uses. Discussions with the president of Biolog (Barry Bochner) have resulted in verbal expression of interest in commercializing algo- rithms developed during this research.

Collaborator, Jay Garland, works for NASA contractor, Dynamach. Dynamach is eligible to compete for SBIR (Small Business Innovation Research) funds, which may fund the development of the software package. Phase 1 proposal was submitted by Dr. Garland. INEL researchers would continue to collaborate with Dr. Garland and would be positioned for subcontract from successful Phase 2 SBIR grant.

Boundaries of invention disclosure for CLPP applications are currently being negotiated with $D r$. Garland.

A patent disclosure is forthcoming for device, Benthic Algal Substrate Sampler (BASS), designed and constructed with FY-95 LDRD funds by university collaborator Dr. Genter.

Another potential industrial partner is Microbial Insights, Inc., which is a spinoff company from Oak Ridge National Laboratory that specializes in a structural assay with similar ER applications. We have recently published a paper comparing the results of our two assays (Lehman et al.; J. of Microbiological Methods 22:263-281, 1995). FY-95 funds were used to subcontract to Microbial Insights, Inc.

A seminar at Lawrence Berkeley Laboratory (9-95) has resulted in strong interest in a CLPP-based proposal from INEL for research in the emerging DOE program Natural and Accelerated In Situ Bioremediation. The solicitation for proposals expected in FY-96 is being coordinated by Lawrence Berkeley.

Effort in FY-96 will be directed (in part) to an EM site at the TAN injection well. Coordination with DOE EM and ER departments will occur. Locally, Keith Daum (ER) has expressed interest in this technology. 


\section{Tolo Lake Mammoth Excavation} LDRD 9513

S. J. Miller

The Tolo Lake Mammoth Project was a controlled excavation of mammoth fossils encountered unexpected$y$ in Tolo Lake sediments in the fall of 1994 and a scientific investigation of:

(a) the geologic age of the fossils,

(c) their fossilization history, which includes the present and past geologic and environmental setting, (c) clues to the natural history of mammoths present in the fossil deposit, and (d) detection of any possible association with early human cultures. The recovery and analysis of the mammoth fossils involved a cooperative multidisciplinary effort by participants from several scientific disciplines (e.g., paleontology, archaeology, geology, biology, chemistry, and remote sensing) from several institutions, and donated labor, materials, and logistical support from many community and agency sources.

\section{S. J. Miller (Lockheed Idaho} Technologies Company/INEL) served as excavation director in coordination with principal investigators $R$. Yohe (Idaho State Archaeologist/ldaho State Historical Society) and W. Akersten (Curator of Paleontology/laho Museum of Natural History). Regular public and school tours of the excavation-in-progress were provided and the project received media and popular attention (e.g., newspaper articles and television and radio coverage) throughout the Northwest.

Results of the excavation and analyses will be applied to studies of the prehistory and paleoecology of the INEL and management of its cultural and natural resources. The work at Tolo Lake will continue to be disseminated through presentations and lay and scientific publications.

\section{Objectives}

- To recover, for preservation, scientific study, and eventual museum exhibition, a portion of the mammoth fossils encountered at Tolo Lake.

- To gain, through systemic recovery of the fossils and associated geologic and environmental information, an understanding of the circumstances behind the occurrence of mammoths at Tolo Lake, the history of their preservation at the lake, documentation of the chronologic age of the fossils, and the past physical and biological environments of the mammoths.

\section{Accomplishments}

The Tolo Lake excavation focussed on two spatially and, probably, temporally related deposits of exceptionally wellpreserved mammoth and bison fossils. One area consisted of the remains of a large, adult male Columbian mammoth (Mammuthus columbi). This individual mammoth is represented by a nearly complete skeleton (over 140 elements were recovered) confined to a limited area and embedded in lake sediments. A nearby fossil deposit represented a scatter of broken and abraded skeletal elements (over 100 specimens recovered) of an estimated five additional mammoths and two extinct bison (Bison antiquus). These fossils were found in shallow lake-margin deposits on, in, and under rocks.

The recovery of a nearly complete mammoth skeleton for scientific study and exhibit will allow an understanding of the history of the death and fossilization of a single individual mammoth, and the fate of the skeleton under the special burial conditions of a shallow lake. The beach assemblage of fossils allows comparison of the relatively complete mammoth skeleton with a deposit of scattered, slightly eroded, partially-buried bones with a strikingly different depositional history.
No archaeological/cultural materials or features were detected in association with the fossils, now suspected to date back well within the Pleistocene or Ice Age.

In addition to directing the excavation, S. J. Miller will lead the study of the taphonomy of the mammoths and associated fossils. Taphonomy is the study of the events and biological and physical processes that lead to the creation and modification of a fossil assemblage from the time of death of organisms until their discovery and recovery as fossils. This involves the careful descriptive analysis of whole and partial fossils, recording their spatial distribution and condition, determining their geologic environments and age, identifying associated species, and recovering any information that can be gathered or inferred about the behavior and natural history of extinct species.

J. Tullis (Lockheed Idaho/AEDL) conducted a field survey of Tolo Lake geology and inspected the lake and nearby sediments. Her contribution to the study includes (a) a report based on field work, (b) inspecting photographs, maps, and literature, (c) interviewing State and Federal agency geologists, and (d) coordinating with University of Idaho researchers.

\section{New or Increased Technical Capabilities}

The Tolo Lake excavation offered the opportunity to: (a) acquire information on the geology and paleontology of the Quaternary events and time period, which dominate a landscape temporally and geologically similar to that of southern Idaho and the INEL, (b) apply paleontological and archaeological field, recovery, and analytical techniques, which would be useful in similar finds on the INEL, and (c) extrapolate the comparative climatic, paleoeco logical, and natural history information gained from this effort to cultural and natural resource 
predictive studies and management on the INEL and throughout the Northwest region.

\section{Business Development Opportunities}

Although no tangible business agreements resulted from the Tolo Lake research, active information flow and cooperation occurred between the project participants representing Lockheed Idaho Technologies Company/INEL, the Idaho Museum of Natural History, Idaho State Historic Preservation Office, State and Federal land management agencies, and the University of Idaho. Continuous discussion occurred about the project research design and future field and laboratory endeavors to answer general and specific scientific questions raised by the project; several of these cooperative approaches were implemented during the project and future interaction initiated between the researchers.

\section{Alternate Material for Stainless Steel-Lined Concrete LDRD W007 \\ P. A. Anderson}

Portland cement concrete (PCC), which is the most widely used masspoured construction material, is sometimes used in applications where it is not appropriate. For example, at the INEL numerous nuclear facilities are constructed with PCC. However, because of the need to decontaminate the facilities without affecting the durability of the material, stainless steel liners are added to the PCC structure. The liner is needed to compensate for the porous nature of $P C C$ and its lack of resistance to attack by the chemical environment prevalent in the facilities. There are other commercially available mass-poured construction materials that are relatively impermeable and are highly resistant to degradation in harsh chemical environments.
However, due to limited engineering information on the materials, their usage is limited. Two such materials are (1) ICOM, which is a composite concrete made from a proprietary blend of resins, corrosion-resistant fillers, and fine aggregates, and (2) sulfur concrete (SC) made from sulfur polymer cement (SPC). Both materials meet or exceed the mechanical and structural properties of PCC, with the added characteristic of impermeability. The purpose of this project is to expand the engineering information on these materials. Sufficient information would then exist for project engineers to specify the materials for applications where they are more appropriate than the costly combination of stainless steel lined PCC. The end result would be more cost-effective facilities with longer durability.

\section{Objectives}

- Complete chemical resistance screening tests.

- Conduct side-by-side chemical resistance testing on ICOM, SPC, ordinary Portland concrete (OPC), and a more durable mix design (DPC).

- Perform irradiation testing on the specimens.

- Perform decontaminatability testing.

- Perform permeability and porosity testing.

\section{Accomplishments}

Since the chemical resistance of the various formulations of ICOM was essentially untested, the screening tests were designed to establish the viability of the project. This was accomplished by screening each of the candidate materials against a variety of common corrosive chemicals at two different concentrations. The chemicals used were selected using the following general criteria:
- Commonly used by industry

- Commonly known to degrade PCC structures

- Commonly used decontamination solution.

The specimens were cast 1 -inch right cylinders. They were exposed to the chemicals for periods of 5, 50, and 100 days. At each interval the percent weight change was recorded along with any other pertinent observations. After the final exposure period, compressive strength tests were conducted. The results demonstrated that both ICOM and SPC are highly resistant to attack by the selected chemicals.

Based on the results of the screening tests, corrosion resistance testing was performed on 3-inch right cylinders and 36-inch by 6inch beams for all four materials. The beams were included to obtain modulus of rupture data in addition to compressive strength data. As illustrated by the attached figure, the percent change in compressive strength for the ICOM and SPC specimens was significantly less than that for the Portland cement based specimens. The decontaminability testing produced results comparable to stainless steel (98-99\% removal) of loose surface contamination. Additional testing is required for fixed contamination due to the difficulty experienced in fixing the simulated contamination to the surface of the specimens. The beam testing, irradiation testing, permeability, and porosity testing are still in process.

New or Increased Technical Capabilities

The results of this research will benefit the INEL by allowing the cost-effective use of these materials in new facilities where PCC has not performed well in the past. The enhanced durability of these materials makes them good candidates for use as engineered barriers 
in applications such as waste disposal facilities that require hundreds of years of service specifically from an engineered barrier. Additional testing should be geared to provide the data needed for the durability modeling of engineered barriers.

\section{Business Development Opportunities}

The two companies that produce the respective materials have collaborated on this project by providing the test specimens. They are keenly interested in the results of the research and stand ready to pursue expanded application of the materials made possible by this project.

\section{Oxidative Processes for the Remediation of Contaminated Soils} LDRD W016

\section{A. Atkinson}

This project studied the use of Fenton's reactions (iron catalyzed free radical reactions based on hydrogen peroxide) for the remediation of solvent-contaminated soils. This type of reaction allows for in situ remediation of a large variety of organic contaminants in less time and at a lower cost than a number of other remediation systems. This project focussed on the destruction of chlorinated solvents in soils through native mineral catalyzation of Fenton's reactions and the optimization of remediation conditions.

This work has benefits to both the INEL and the regional community. $A$ large number of dollars are spent in the United States on cleanup of contaminated soils. Remediating these sites is expensive, especially when cleanup occurs by excavation and incineration of the soils. Full-scale implementation of Fenton's based remediation would allow for a faster, more cost-effective method of in situ solvent-contaminated soil remediation. This work extends the use of traditional Fenton type systems to the use of native mineral catalyzed reactions, thus further reducing costs by eliminating the need to add catalyzing metal ion solutions to the soil.

\section{Objectives}

- Apply Fenton's chemistry to the remediation of chlorinated solvent-contaminated soils.

- Investigate the use of native mineral catalysis.

- Optimize remediation process variables to maximize treatment efficiency.

\section{Accomplishments}

Fenton's chemistry (iron/transition metal catalyzed hydrogen peroxide oxidation) was evaluated for use in the remediation of chlorinated solvent-contaminated soils. Solvents of interest to the INEL were studied: trichloroethylene, carbon tetrachloride, and 1,1,2trichloroethane. The soil remediation processes were studied in the laboratory using ex situ slurry reactors, however, the process is often used in situ, making implementation of this remediation system fairly easy and cost- effective. The research studied both systems catalyzed by the addition of soluble iron and native mineral catalyzed systems. The native mineral catalyzed scheme is particularly attractive, as it reduces costs by eliminating the need to add iron salts and the problems brought about by the precipitation of added iron salts.

Extensive degradation of the chlorinated solvents was achieved in both the iron salt and mineral catalyzed systems. The time involved for fairly complete $(>95 \%)$ remediation was nearly always $<100$ hours; this contrasts strongly with many bioremediation systems where weeks or months are needed to effect the destruction of the contaminant solvent at a greater cost. The figure illustrates the breakdown curve of trichloroethene (TCE) in a mineral catalyzed setup for both a pure geothite [a-FeOOH] system and a geothite/hematite mixture. Extensive destruction of TCE in both systems is evident. The rise in chloride concentration shown in the figure is most likely correlated with the breakdown of the TCE. The likely oxidation pathway is

TCE -> dichloroacetic acid --> $\mathrm{H}_{2} \mathrm{O}+\mathrm{CO}_{2}+\mathrm{HCl}$.

There is the potential that mineral catalyzed remediation reactions have additional oxidation pathways due to surface catalysis; this may account for enhanced degradations of highly substituted solvents such as carbon tetrachloride in mineral systems over conventional soluble iron systems. Since the INEL soil is fairly high in iron content and catalyzed native minerals, Fenton's remediation would be an ideal choice for site remediation problems.

\section{New or Increased Technical Capabilities}

This work gives the INEL an additional remediation technique for chlorinated solvent- contaminated sites, which in most cases will be quicker and cheaper than the traditional techniques of incineration and bioremediation. The Fenton's chemistry based system can be extended to other forms of organically contaminated soils also, such as hydrocarbons and explosives. With the large amount of soil remediation needed within the DOE complex, this work presents an advance that can be beneficial to environmental restoration activities.

\section{Business Development Opportunities}

Three companies have expressed interest in this work. IT 
Corporation, which is a large environmental remediation firm, has shown interest in this type of chemical remediation. In Situ Oxidative Technologies, which is a small environmental firm involved with chemical oxidative remediation, is also interested in this work. Both of these companies are users of remediation systems; their interest comprises possible use of such systems and proven applications. A third company, Parsons, at the INEL, is interested in this remediation system from a end user standpoint, with respect to remediation of chlorinated solvent-contaminated soils at Test Area North (TAN).

\section{Solid Phase Microextraction} LDRD W019

\section{J. L. James}

Solid phase microextraction (SPME) is a new method for extracting semivolatile organic compounds (SVOCs) from aqueous samples for analysis. This method eliminates the use of hazardous solvents required by conventional extraction methods. The sample volumes required for analysis at the $\mathrm{ng} / \mathrm{mL}$ level are reduced from $1 \mathrm{~L}$ to $10 \mathrm{~mL}$. The time required for the extraction is only 30 minutes and the extraction can be performed during the analysis of the preceding sample. A single analyst can easily perform the extraction while analyzing samples. This new method will decrease the time, labor, and expense of analyzing SVOCs and eliminate the solvent disposal problems.

Solid phase microextraction (SPME) is a very simple technique. $A$ fused silica capillary fiber covered with a coating designed to absorb the analytes of interest is dipped in the aqueous sample. After 30 minutes, the concentration of the analytes in the coating reaches equilibrium with the analytes in the sample. The fiber is then placed in the injector of the gas chromatograph/mass spectrometer
(GC/MS) and the analytes are thermally desorbed onto the chromatographic column for analysis.

This project has focused on developing a SPME method that meets the calibration requirements for the analytes of SW-846, Method 8270 and applying this method to environmental samples and highly radioactive waste samples. The quantitation limits have been met for most analytes. Matrix effects seem to be no different from the conventional method.

A remote method has been mostly developed for highly radioactive samples, although reproducibility between analytical runs is still a problem.

\section{Objectives}

- Generate a 5-point calibration curve starting at or below $10 \mathrm{ng} / \mathrm{mL}$ with \% relative standard deviations less than $30 \%$ and relative response factors greater than 0.05 .

- Expand the list of calibrated compounds to cover the Contract Laboratory Procedures (CLP) Target Compound List (TCL).

- Perform a comparison analysis between liquid-liquid extraction and SPME on actual environmental samples.

- Develop procedures and equipment for the remote use of SPME for the analysis of highly radioactive samples.

\section{Accomplishments}

The SPME conditions have been optimized for the TCL and calibration curves have been generated based on the new conditions. All compounds except the very polar compounds can be quantitated at $10 \mathrm{ng} / \mathrm{mL}$. Of the 73 calibrated compounds, 66 have relative response factors greater than 0.05 . Reproducibility is still a serious problem. Most of the calibrated compounds have percent relative standard deviations substantially greater than $30 \%$. Further research is required to determine if a better method for mixing the sample during sampling can be developed or if nonlinear calibration curves are inherent in the method.

The list of calibrated compounds covers most of the TCL. Further research is required to reliably analyze 2,4-dinitrophenol; 4,6-dinitro-2-methylphenol; 4-nitrophenol; hexachlorocyclopentadiene; and N-nitroso-di-n-propylamine.

A comparison extraction between the conventional analysis (separatory funnel liquid-liquid extraction followed with GC/MS analysis by SW-846 Method 8270) and SPME followed by GC/MS analysis was performed on an Idaho Chemical Processing Plant (ICPP) process equipment waste (PEW) condensate sample. The results of the analyses are attached. The results between the two methods are quite different, but the differences are within the normal variability of semivolatile organic analysis.

A disposable holder has been developed for the extraction fiber that will allow this method to be used remotely. A test extraction on a radioactive sample was performed to determine the magnitude of contamination of the fiber after contact with the sample. No contamination of the fiber could be detected.

New or Increased Technical Capabilities

This technique has great potential both for laboratory analysis and for field screening and sampling and is applicable to volatile and semivolatile organic analysis. The extension of this method for the extraction of inorganic compounds is also possible. Widespread use of this technique could dramatically reduce the costs of sampling and analysis. As yet, SPME is almost unknown. We are one of four organizations nation- 
wide doing any research and development in this area. The final steps toward developing a remote method that will cut hundreds of dollars per sample from the cost of analysis of highly radioactive samples are being performed. A method developed during this project will be submitted for inclusion in SW-846.

\section{Business Development Opportunities}

During FY-95, we have held discussions with Varian Instruments, Supelco, and the Environmental Protection Agency's (EPA's) Office of Solid Waste. We have received data and experimental materials from Varian and Supelco and have made an informal agreement with Varian and Supelco to cooperate in submitting an SPME method for inclusion in SW-846. Since the researcher responsible for development in the area of solid phase microextraction at Supelco is almost fully committed to production support and quality control, we have hopes of work-for-others opportunities doing development for the company. We have received a list of needed research from the EPA and are pursuing possible funding.

\section{Synthesis and Characterization of Pt/Pd Compounds for a Fiber Optic-Based Sensor for Volatile Organic Compounds LDRD W027 \\ S. D. Hartenstein}

The Idaho National Engineering Laboratory (INEL) has worked collaboratively with the University of Minnesota (UM) to synthesize platinum/palladium organometallic compounds. These compounds have a unique characteristic in that they change color reversibly (vapoc- hromism) when exposed to vapors of volatile organic compounds (VOCs). The focus of this laboratory directed research and development (LDRD) project was to evaluate these Pt/Pd compounds response to a variety of volatile gases. The objective of the research was to establish the potential of these materials to be integrated with appropriate transducers for future development of VOC sensor systems for environmental and air quality applications. The envisioned sensors are expected to detect VOCs at regulatory levels for DOE and industrial environmental remediation and monitoring activities.

\section{Objectives}

- Synthesize bulk quantities of three different $\mathrm{Pt} / \mathrm{Pd}$ vapochromic materials.

- Synthesize test quantities of oxidized/reduced $\mathrm{Pt} / \mathrm{Pd}$ vapochromic materials.

- Evaluate the response of $\mathrm{Pt} / \mathrm{Pd}$ vapochromic materials for at least five air quality gases.

- Evaluate the response of $\mathrm{Pt} / \mathrm{Pd}$ vapochromic materials using field effect transistors (FETs).

\section{Accomplishment}

A significant effort of this LDRD was synthesis of various forms of the $\mathrm{Pt} / \mathrm{Pd}$ vapochromic materials for continued evaluation of its potential sensor capabilities. Bulk quantities ( $>100 \mathrm{mg}$ ) of three materials (with varied metal-metal bond structure and varied alkyl chain lengths) were synthesized at the INEL. Aliquots of these materials were used for: (a) continued VOC sensor testing at the INEL; (b) synthesis of oxidized/reduced $\mathrm{Pt} / \mathrm{Pd}$ vapochromic materials; and (c) distribution to industrial and university collaborators. Materials were sent to GEO-CENTERS, Inc. to support of existing CRADA to develop an environmental screening sensor for VOCs. The University of Utah ( $U$ of $U$ ) received some materials to support FET sensor development as part of an INEL University Research Consortium (URC) collaboration. Other material was shipped to Honeywell to investigate their potential for process leak detectors.

Efforts to synthesize oxidized/reduced Pt/Pd materials were completed through a subcontract with Montana State University. The focus of this synthesis work was to create compounds with increased conductivity to make the materials more compatible with electrical-based transducers. During this effort, $\mathrm{Pt} / \mathrm{Pd}$ vapochromic materials were reacted with substoichemetric quantities of bromine and iodine. The results from these reactions were unsuccessful, in that the reaction products were not homogeneous and were not more conductive. However, the observation that some material did react with the bromine and iodine indicates that further experiments with more controlled conditions or with chlorine gas could improve the conductivity.

Several vapochromic materials were coated on quartz crystals and then evaluated for their adsorption characteristics to a variety of air quality gases using a quartz crystal microbalance. The various vapochromic materials were exposed to methane, nitric oxide, nitrogen dioxide, nitrous oxide, hydrogen cyanide, carbon monoxide, hydrogen sulfide, sulfur dioxide, BTEX (benzene, toluene, ethyl benzene, and xylene), carbon tetrachloride, and chloroform. The vapochromic materials showed very little ability to adsorb any of the gases except the BTEX and halogenated organics. The response for these gases confirmed previous observations that the vapochromes are selective for these gases with expected detection limits near 1 to 5 ppm. 
Efforts to evaluate the response characteristics of the vapochrome materials with FETs were performed to determine if a more sensitive transducer could be identified to take advantage of the vapochromic behavior of the materials. The characterization of the FET response was unsuccessful due to the inability of the $U$ of $U$ to deliver intact FETs. The effort to characterize FETS will continue under the FY-96 URC collaboration.

New or Increased Technical Capabilities

During completion of the research objectives, INEL investigators increased their technical capabilities in four areas.

They completed a safety review and job hazard analysis , which now creates an INEL facility for the routine evaluation of sensors for toxic gases at low concentrations.

While evaluating surface coating techniques for the quartz crystal microbalance (QCM) sensor system, the INEL investigators completed a fundamental evaluation of the principles of operation for the QCM. This evaluation has increased the understanding of how to make coated quartz crystals more efficiently, reduce their noise, and enhance the sensitivity.

For the FET evaluations, INEL investigators had to redesign an existing electronic control data acquisition system to specifically collect the electrical current response from the tested FETs. This system is now available for any future evaluations of FETbased sensors.

In synthesizing bulk quantities of the Pt/Pd materials, an INEL investigator developed improved material handling techniques that yielded higher purity materials. These techniques will be transferred to the UM, via a minitechnical exchange, to improve the quality of new materials UM will synthesize during its FY-96 INEL URC project.
Business Development Opportunities

These project supported ongoing URC collaborations with the UofU and UM to develop microsensors and additional vapochromic compounds, respectively. The fundamental chemical information garnered during the handling and reacting of the materials is supporting an existing CRADA with GEO-CENTERS, Inc. to develop a field screening fluorescence probe for VOCs.

\section{ICPP Testbed Framework and Field Tests}

LDRD w029

$$
\text { E. E. Filby }
$$

The overall purpose of this effort is to position the INEL as a place where new technologies can be field tested before final deployment, building on many years of less formal testing experience at the Site. The specific aim of this project was to develop an improved testbed infrastructure and to upgrade the skills of testbed personnel.

Because individual sensors, and integrated suites of sensors and control components are tested, the integration methodology is a key element of the infrastructure. The INEL has been funded separately by DOE (NN-20) to test the Modular Integrated Monitoring System (MIMS), which is an approach developed by Sandia National Laboratory (SNL). The SNL system uses nodes built around the Neuron chip from Echelon Corporation, and a custom-designed data logging station. Based on this effort and prior INEL experience with the Echelon LonWorkstm approach, we have chosen this product line as our core intelligent distributed control (IDC) technology. One aim of this project was to evaluate whether or not the customized SNL approach could meet the longterm needs of the INEL testbed.

Upgrading the testbed infrastructure will mean that a wider variety of test capabilities can be offered, thus providing more value to existing customers and enhancing our chance of attracting new customers. An expanded customer base should provide more opportunities for cost sharing on future tests, thus improving our overall competitiveness. Finally, increasing our skills and experience in this area should improve our ability to develop unique INEL products and systems.

\section{Objectives}

- Install components needed to test the Authenticated In-Plant Monitoring (AIPM) system.

- Expand infrastructure to thoroughly test the MIMS integration approach.

- Evaluate the MIMS network approach as the basic framework for the INEL sensor testbed.

- Procure a Lonworks network development workstation from Echelon Corporation.

- Provide training for additional personnel on the workstation.

- Add modules to handle more different kinds of sensors to the basic SNL network.

- Identify a better location for ICPP Test Control Room and transfer relevant equipment to it.

- Evaluate other facilities at the INEL for inclusion in the testbed infrastructure. The addition of RF capabilities to the network should help accomplish this task.

\section{Accomplishments}

The test of the AIPM was completed. This test produced one satisfied customer, provided more experience in formalized testing, and tested the testbed infrastructure. A test/demonstration of the MIMS (see the figure) was also performed, with observers from DOE Headquarters and a potential 
industrial partner. The test provided the first "data point" as to how well the MIMS might work as a general testbed network. This experience was used to develop the software needed to extract data for the testbed from the Idaho Chemical Processing Plant (ICPP) plant computer system. Further experience was provided by a test and demonstration for an AIL Inc. gamma-ray imaging system (GRIS).

An Echelon development workstation was procured and put into operation, and three individuals were trained in its use. This training and experience helped determine the components needed to expand the kinds of sensors used with the testbed network. Certain general-use items, such as a remote RF-linked video unit, were procured under this LDRD. Others items have been ordered using relevant programmatic funds; these include systems that can be linked via radio transmissions. The technical requirements for integrating National Oceanic and Atmospheric Administration (NOAA) meteorological data into the testbed system were examined. Although we can readily access their data for review, a fairly extensive effort would probably be required to integrate "live" data directly into a test protocol.

Because the data-review location for the early AIPM and MIMS tests proved to be unsuitable for long-term use, the equipment was relocated to a new ICPP Test Control Room. Means to transmit test data to this new location were designed and installed. Several other INEL sites, including the Test Reactor Area (TRA) and the Radioactive Waste Management Complex (RWMC), were evaluated for inclusion in the basic testbed network. Several areas seem promising; however, because our current customers are most inter- ested in the follow-on scenario being developed at the ICPP, none of these options were pursued any further. Planning is in progress for a common use scenario based on the spent fuel transfers taking place between the old basin in CPP-603 and the FAST storage basin. Common use means that systems from several different developers can be accommodated in the same test sequence.

Evaluation of the MIMS network approach as the testbed framework was essentially completed. While their nodes are suitable for certain kinds of tests, we need to expand well beyond the capabilities they provide. The customized data logger, while it meets the needs for which it was designed, is totally unsuitable as a testbed workstation. We need more flexibility and substantially better diagnostic capabilities.

New or Increased Technical Capabilities

We have broadened our experience in test planning and execution. The Echelon workstation substantially upgrades our ability to develop integrated IDC systems. (An older version of this system is available, but it is dedicated to a specific program and remains the property of the program sponsor.) New people have been trained, and are beginning to gain experience. Finally, new equipment has been procured to enhance future tests and demonstrations.

\section{Business Development Opportunities}

Raytheon and SAIC are interested in having their Corral Monitoring System tested. It appears likely this will happen early next fiscal year, but prospects for a longer-term relationship are unclear. Rockwell International would like to test and demonstrate a day-night video surveillance unit as part of our fuel-movement scenario. These potential customers are currently trying to identify funding for their part of the tests; the INEL would probably be funded through our ongoing program with the DOE Office of Arms Control and Nonproliferation (NN-20). Halberd Inc. (Salt Lake City) is currently in production to build six to eight RF-linked network nodes for outdoor sensor applications; these would be tested at the INEL. As noted above, we successfully tested the AIL Inc. GRIS here; discussions of a possible joint project to help adapt their equipment for new uses are progressing. Echelon Corporation has expressed interest in having a third-party site provide LonMarktm compatibility testingthis would be somewhat akin to the familiar UL Listing for product safety. These discussions are being kept very low key at this point; we need to gain more experience with this technology, and there are many sensitive issues that need evaluation before any commitments are even considered.

\section{Electrocatalytic Vapor Sensor} LDRD w032

\section{Carver, D. K. Kotter}

The electrocatalytic vapor sensor project is a joint collaboration between Lockheed Idaho Technologies and Argonne National Laboratory (ANL). Lockheed Idaho further developed the electrocatalytic vapor sensor built by $A N L$ by increasing the selectivity of the sensor through the use of polymers, membranes, and chemically modified electrodes. Polymers developed in house by the Inorganic Membrane Technology Research Program were used to increase the selectivity of the sensor. The electrocatalytic vapor sensor was a test bed for the Neural Network LDRD project. Test data for $\mathrm{SO}_{x}$ and $\mathrm{NO}_{x}$ were compiled 
and neural networks were built to identify the gas and the concentration of the gas. The neural network and the eleckocatalytic vapor sensor provide a complete sensor system capable of near real-time analysis of vapor effiuents.

\section{Objectives}

The milestones for FY-95 were focused on further characterizing the sensor and preparing the sensor for engineering applications, such as integrating the sensor with a biofilter to monitor the waste stream. Tests were conducted to determine the sensor's response in different environments and with different chemicals.

- Increase the selectivity of the sensor.

- Build a portable sensor system and integrate the system with the biofiltering project to determine long-term stability.

- Expand the library of sensor responses.

- Demonstrate that the sensor can be reused after being contaminated. Write proposals for FY-96 funding.

\section{Technical Accomplishment}

Increasing Sensor Selectivity.

Efforts to increase the selectivity of the electrocatalytic vapor sensor focus on a Lockheed Idaho internal collaboration conducted between the sensor product directorate and the Inorganic Membrane Technology Research Program (IMTRP). The IMTRP has developed many polymers and membranes that are selective for specific compounds or groups of compounds. This expertise was used by applying these polymers to the surface of the sensor. The sensor response was changed after the polymer was applied to the surface. There was also a change in sensor response to the presence of $\mathrm{NO}_{x}$ gas at varying concentrations. It is difficult to determine if the change in sensor response is due to the selectivity of the coating or if polymer coating was evaporated by the high operating temperature of the sensor. Further tests will be conducted to verify that the changes in the sensor response are due to the selective coating and not to other factors.

Build and Integrate Portable Sensor System with Biofiltering Project.

Other areas of the electrocatalytic vapor sensor project include building a portable sensor test system to be used with the biofiltering project. A collaboration with the biotechnology group was designed to integrate the electrocatalytic vapor sensor with a biofiltering project that removes hydrocarbons from waste streams. The sensor would monitor the levels of hydrocarbons in the waste stream over a period of several months. The current instrumentation for the sensor is bulky and cumbersome and cannot be integrated with the biofilter. A portable system has been built on a Toshiba computer with a D/A converter card. The data acquisition system has been configured using Lab Windows. This portable system will be used not only for the biofiltering project but also for laboratory and field demonstrations of the sensor technology. This portable system can also be used with other sensors.

Currently, the sensor has not been integrated into the biofilter because there are problems with sensor stability. The first sensor prototype tested has a yttria-stabilized zirconia matrix which separates the upper and lower electrode. It was determined that the sensor would drift from day to day and it was difficult to reproduce a baseline response. From tests conducted at ANL, it was concluded that the sensor would be more sta- ble if the matrix material were replace with tungsten bismuth oxide. Currently, tests are being conducted to reproduce ANL's results and determine if the new matrix material will provide a more stable sensor. Once the sensor stability problems have been solved, it will be integrated into the biofilter and the long term stability of the sensor will be monitored.

\section{Demonstrate a Reusable Sensor}

The durability of the sensor was tested by exposing the sensor to contaminates and testing the sensor response. The sensor was exposed to SOx and NOX. Over time a film would develop on the surface of the sensor which would dampen the sensor response. The effects of the contaminants on the sensor could be reversed by elevating the operating temperature of the sensor and burning off contaminants from the surface.

Increase Library of Sensor Responses This sensor project was also a test bed for the Neural Network LDRD. The sensor responses for SOx, NOX and mixtures of SOx and NOX were recorded with carrier gases of both air and nitrogen. Concentration levels were also varied from 5 to $100 \mathrm{ppm}$. The sensor produced unique signatures for each gas at each concentration level. The responses for the $\mathrm{NO}_{x}$ were more distinguishable than from the $\mathrm{SO}_{x}$. The test gas data were collected and sent to Lockheed Martin Research and Development Division. Don Specht who developed probabilistic neural network algorithms wrote neural networks for the $\mathrm{SO}_{\mathrm{x}}$ and $\mathrm{NO}_{\mathrm{x}}$ test gases. The ultimate goal is to reduce the neural networks to computer chips that can be installed on the portable computer for near real-time analysis of sensor test data in field demonstrations. 
Write Proposal for FY-96 Funding

Several proposals were written for funding with the Arms Control program. One proposal, written for the Advanced Concepts program, would increase sensor selectivity for compound that would detect nuclear proliferation activities. Another proposal would combine the neural network and the electrocatalytic vapor sensor project to build an entire sensor system to be used in the field for chemical weapons production or to detect nuclear proliferation activities. Currently, the Arms Control Office has has not awarded their proposals. Other funding areas and applications will be explored, such as environmental restoration and management.

\section{New or Increased Technical Capability}

The electrocatalytic vapor sensor has combined the talent within Lockheed Idaho and within the Lockheed Martin Corporation. The project uses Lockheed Idaho's expertise in polymers and membranes to increase sensor selectivity. This line of research will be expanded to test more polymers for increased selectivity to more gases. The sensor will also continue to support the neural network project, which brings the expertise of Lockheed Martin to the INEL.

\section{Business Development Opportunities}

Several proposals have been submitted to NN-20 for control of nuclear weapons. The electrocatalytic vanor sensor is ideal for testing of remote sites for treaty verification. ANL has been in contact with several commercial companies. One company is Terminex, which is interested in using the sensor for monitoring areas of pest infiltration. Currently, it is difficult to determine the exact location of pests but pests do release an off-gas that can be monitored to determine areas of infiltration.

\section{Characterization of Fuel Processing/ Waste Form Chemistry by High-Temperature Mass Spectrometry LDRD W043}

\section{A. Knecht, G. F. Kessinger}

A 10-yr-old high-temperature mass spectrometer, Nuclide Corporation Model 12-HT-90 which consists of a high-temperature Knudsen cell and a mass spectrometer, was procured from Westinghouse Corporate Research Center (available for the cost of shipping, set-up, and remaining instrument depreciation) and refurbished. The system will be used to develop new INEL capabilities for research and development of materials at up to $2000^{\circ} \mathrm{C}$ high temperatures, including capability for real-time off gas measurement, using the hightemperature Knudsen cell as a mini reactor to produce small quantities of candidate test materials. Installation and testing was completed, and operation of a high temperature mass spectrometer system was demonstrated. Specific tests were run on determining the off-gas composition of simulated residue of ICPP sodium-bearing waste, calcine, and recycled metals.

\section{Objectives}

- Complete installation and testing.

- Test fuel processing and waste form materials.

- Thermodynamic characterization.

- Conduct experimental studies

\section{Accomplishments}

Using personnel from a private vendor, the instrument was upgraded with expanded computer control of the magnet regulator. Delays were experienced in obtaining permanent power, power interruption of utilities, and intermittent water leaks into the laboratory due to steam system leaks. Fullsize chiller installation was com- pleted only at the end of the fiscal year, resulting in limited cooling capacity and a lower achievable temperature of $1500^{\circ} \mathrm{C}$ for the instrument. However, in spite of the above problems, testing and alignment of the mass spectrometer were completed with well characterized silver heated to $1400^{\circ} \mathrm{C}$ in the Knudsen cell.

The chemical, sodium nitrate, was tested to determine the temperature range of denitration and elemental sodium and sodium oxide vaporization. Denitration occurred in the temperature range of 300 to $600^{\circ} \mathrm{C}$, while sodium volatilization occurred above $900^{\circ} \mathrm{C}$.

A reference on thermodynamic characterization of the appropriate alkali nitrates was used to determine the chemical reactions which are applicable to waste form chemistry expected from the sodiumbearing wastes. Earlier studies of ICPP calcine denitration were also evaluated for applicable data to these experimental studies.

Experimental studies were run as check-out tests of the mass spectrometer system up to approximately $1500^{\circ} \mathrm{C}$. Some additional adjustment of the water cooling sequence and completion of the higher capacity cooler installation were completed to achieve higher temperature operation. Test samples were run of simulated ICPP sodium-bearing waste and HLW Fluorinel-sodium calcine, simulated aluminum spent fuel cladding which had been hydrated, and simulated contaminated recycled metal. In the simulated sodiumbearing waste and simulated Fluorinel-sodium calcine, the nitrates were decomposed in the temperature range of approximately $300-$ $800^{\circ} \mathrm{C}$, possibly because of the small 0.5 -g sample and high vacuum conditions not experienced in other laboratory and plant systems. At larger-scale conditions, the nitrates are decomposed at $600^{\circ} \mathrm{C}$. 
Elemental sodium and potassium and their oxides were observed after most of the nitrates were decomposed at 900 to $1200^{\circ} \mathrm{C}$. Some cesium was observed at approximately $900^{\circ} \mathrm{C}$. With the simulated Fluorinel-sodium calcine, there was no oxygen observed as a nitrate decomposition product at the lower temperature range compared with the simulated sodium waste where it was observed. At $1150^{\circ} \mathrm{C}$ the $\mathrm{NaO}$ dimer was observed. Analysis of the solid residues from the simulated fluorinel-sodium calcine were completed using $x$-Ray Diffraction and SEM with $x$-Ray Fluorescence. The residue in the crucible was analyzed to contain a calcium molybdenum oxide, calcium-stabilized zirconia, and a calcium-aluminumchromium-oxide phase. Crystals of a perovskite phase were found condensed on the lid of the Knudsen crucible.

New or Increased Technical Capabilities

Prior to completion of this project, there was no capability at the INEL for measuring the hightemperature vapor species from various materials. This LDRD project has demonstrated successful operation of such a system and potential application to HLW processing, spent fuel dry storage, and contaminated metal recycle. The high-temperature mass spectrometer capability will be included as one of the INEL user facilities for outside use.

\section{Business Development Opportunities}

No contacts with outside businesses or CRADAs have been explored for this project to date. The possibility may exist if determined from use by outside entities or in conjunction with other applications of materials development and testing.

\section{Telepresence for Mobile Robotics in Nuclear Environments} LDRD W089

$$
\text { M. D. McKay }
$$

A growing concern with the rapid advances in technology is that the robotic systems will become so complex that operators will be overwhelmed by the complexity and number of controls. Thus, there is a need within the remote and teleoperated robotic field for better man-machine interfaces. Telepresence attempts to bring real world senses to the operator, especially where the scale and orientation of the robot is so different from the scale of a human operator. This research identified and evaluated current developments in telepresence best suited for nuclear applications through a survey of national laboratories, universities, and evaluation of commercial products available in industry. The resulting telepresence system, VirtualwindoWTM, attempts to minimize the complexity of robot controls and to give the operator the feel of the environment without actually contacting items in the environment. Although it is of the opinion of the authors of this research that the Virtualwindo $W$ greatly reduces or perhaps eliminates the effects of vertigo and VOR corruption, Virtualwindow needs further study to determine the effects of prolonged usage of this technology in the work place. The authors of this report recommend that a prolonged usage study be conducted on the Virtualwindow to determine and benchmark the length of time users can be safely exposed to the technology. In addition, it is proposed that a stand alone system be developed which combines the existing multi-computer platform into a single telepresence platform. The stand alone system would provide a standard camera interface and allow the Virtualwindow to be ported to other telerobotic systems.

\section{Objectives}

The objective of this research and development project was to develop and integrate a tele-robotic system based on a mobile platform for the purpose of improving and studying telepresence for the use in nuclear environments.

- Produce an advanced teleoperated robot system for use in determining the benefits and uses in nuclear environments.

- Produce a transparent or immersive operator interface.

\section{Accomplishments}

Background and Introduction.

Robotics technology has played a key role in clean up and remote operations. While robotic systems are advancing, the human interface has not. The control station often includes controls for operating the robot manipulator, cameras, lights, gripper, deployment system (if applicable), and more. A growing concern with the rapid advances in technology is that the robotic systems will become so complex that operators will be overwhelmed by the complexity of controls. The solution to this problem is to make all controls as intuitive as possible. Telepresence attempts to bring real world senses to the operator, especially where the scale and orientation of the robot is so different from the scale of a human operator. The immersive experience that comes from telepresence, the feeling that you are actually in the environment performing the tasks yourself, is the most logical approach to making the controls as intuitive as possible. The robot does as you do, moves as you move, you see as it sees, hear as it hears.

State-of-the-art Technology Survey.

This research identified and evaluated current developments in telepresence best suited for nuclear applications through a survey of 
current technology and programs developed and researched at national laboratories, universities, and evaluation of commercial products available in industry.

Resulting Design and System Capabilities.

The Remotech multi-switch control box was replaced by a single joy stick for controlling the vehicle tracks and a Cyberman 6-D mouse for controlling the MK2 arm. The mono camera view and controls were replaced by the VirtualwindoW. The impression the user gets using the VirtualwindoW is that they are viewing the area through a real window rather than looking at a monitor or TV. The VirtualwindoW also incorporates a voice activated video switcher for commanding the system to change views from the overview cameras to the arm cameras. In addition to replacing the Remotech arm with the MK2 arm, several system additions were added to the vehicle in order to implement the VirtualwindoW. Two pairs of high resolution video cameras were mounted on the vehicle-one pair on the arm and the second pair mounted on a stem just behind the arm. The pan and tilt unit (PTU) is a high-speed unit which enables the VirtualwindoW to pan at a speed comfortable for the viewer. Two directional microphones are mounted on the overview cameras to provide the stereo audio. Haptic feedback is accomplished by using numerous pressure sensitive pads placed in strategic locations on the MK2 gripper. These sensors cause the haptic system to send an audible variable frequency to the user.

System Evaluation and Testing.

The industry system requires the operator to manipulate several toggle switches to control the arm, cameras, lights, gripper and tracks. Video is provided through a single mono monitor. In order to manip- ulate multiple functions at the same time the operator must be able to toggle more than one switch at a time. This can become difficult to do depending on the operation the user wishes to perform. The lack of depth perception necessitates the operator to feel their way around the environment which is unacceptable in nuclear environments. The telepresence system attempts to minimize the complexity of robot controls and to give the operator the feel of the environment without actually contacting items in the environment. The interface is designed so that the operator can sit in a chair much like sitting at a computer workstation. The operator then dons a pair of special head tracking stereo glasses. The angle by which the operator is looking through the window is read by a computer $s$ ystem and processed to determine the necessary commands required by the PTU to position the stereo cameras. All of the camera manipulation is done by the computer system, the operator only needs look at the desired object. The voice response system recognizes the users commands and issues the response to the video switcher. The robot tracks are controlled by manipulating a single joy stick with the left (or right) hand while the MK2 is controlled by the other hand. Functions like opening and closing the gripper, or stowing the arm have been incorporated into the mouse buttons.

Conclusions and Recommendations.

Although it is of the opinion of the authors of this research that the VirtualwindoW greatly reduces or perhaps eliminates the effects of vertigo and VOR corruption, the VirtualwindoW needs further study to determine the effects of prolonged usage of this technology in the work place. The authors of this report recommend that a pro- longed usage study be conducted on the VirtualwindoW to determine and benchmark the length of time users can be safely exposed to the technology. This will require human testing approval from DOD-HQ. In addition, it is proposed that a stand alone system be developed which combines the existing multi-computer platform into a single telepresence platform. The stand alone system would provide a standard camera interface and allow the VirtualwindoW to be ported to other telerobotic systems. To accomplish these recommendations further funding is requested.

New or Increased Technical Capabilities

The INEL has benefitted from this technology in two areas. First, the INEL has gained a new technology which can be applied to varying remote operations, not only in nuclear environments, but to all applications that require remote operations. Second, this research has positioned the INEL into the forefront of human interfaces such as telepresence or virtual reality.

\section{Business Development Opportunities}

Sufficient interest has been shown by universities, industry, and government to warrant continuing this research. Colorado School of Mines has been awarded research funding by Lockheed Idaho to develop range finding algorithms using the VirtualwindoW telepresence system to provide camera and vehicle position feedback. Also Dimension Technologies Inc. has written a Morgantown Energy Technology Center proposal which uses the telepresence interface to improve their 3-D interface. Finally various government laboratories have expressed interest in using the telepresence platform. 


\section{Sodium-Bearing Waste Treatment Technology for Separating the Nitrate Anion and Alkali Salts Using lon-Exchange \\ LDRD W097}

\section{W. Marshall}

\section{Some of the acidic aqueous} wastes at the Idaho Chemical Processing Plant (ICPP) are not immediately compatible with the fluidized-bed waste calcination process, because of the high alkali-metal salt content. Pretreatment of the waste to deacidify and remove alkali metals would increase the waste treatment options. We undertook a study to investigate the use of ion-exchange resins for deacidifying the waste and for selectively removing the alkali metals. Although the study did achieve the objectives, use of ion-exchange resins with simulated waste confirmed that it is not practical to remove the major constituents from a solution using ion exchange.

\section{Objectives}

Approximately 1.5 million gallons of radioactive, sodium-bearing acidic wastes at the Idaho Chemical Processing Plant (ICPP) were generated as an indirect result of nuclear fuels reprocessing, such as decontamination of process cells and equipment. This waste is not immediately compatible with the fluidized-bed waste calcination process, because of the high alkalimetal salt content. These wastes can only be calcined when diluted with other waste streams or cold chemicals, or when treated to separate the alkali metals. The high acidity limits the applicability of many candidate treatment technologies in lieu of or in addition to calcination. In an effort to minimize the mass and volume of solidified high-activity waste that will result from the stabilization of the ICPP sodium-bearing waste, we pursued the following objectives:

- Evaluate various anionic exchange resins for deacidifying simulated ICPP sodiumbearing waste (see major constituents in Table 1) up to $\mathrm{pH}$ = 4.0; quantify precipitate formation and regeneration capability, including regenerant volumes, regeneration efficiency, and resin poisoning.

Table. 1.

Major waste simulant constituents

\begin{tabular}{llll} 
Acid & $1.8 \mathrm{M}$ & $\mathrm{Na}$ & $1.25 \mathrm{M}$ \\
$\mathrm{Al}+3$ & $0.548 \mathrm{M}$ & $\mathrm{Cl}-$ & $0.022 \mathrm{M}$ \\
$\mathrm{B}+5$ & $0.016 \mathrm{M}$ & $\mathrm{F}-$ & $0.071 \mathrm{M}$ \\
$\mathrm{Ca}+2$ & $0.044 \mathrm{M}$ & PO4-3 & $0.010 \mathrm{M}$ \\
$\mathrm{Fe}+2$ & $0.025 \mathrm{M}$ & SO4-2 & $0.038 \mathrm{M}$ \\
$\mathrm{K}+$ & $0.144 \mathrm{M}$ & NO3- & $4.49 \mathrm{M}$ \\
\hline
\end{tabular}

- Evaluate various cationic exchange resins for removing alkali metals ( $\mathrm{Na} \& \mathrm{~K}$ ) from simulated ICPP sodium-bearing wastes, with or without prior deacidification; quantify the resin capacities and regeneration capability, including regenerant volumes, regeneration efficiency, and resin poisoning.

The ion-exchange resins that we evaluated in the course of the study are given in Table 2.

Table 2

Evaluated ion exchangers

\begin{tabular}{ll} 
Anion Exchangers & Cation Exchangers \\
\hline Amberlite IRA 35 & Amberlite IR 122 \\
Amberlite IRA 93 & Amberlite IRC 76 \\
Amberlite IRA 400 & Amberlite 200 \\
Amberlite IRA 910 & Diaion HPK 25 \\
Diaion PA 416 & Dowex 50x2 100 \\
Diaion SA 10a & Dowex 50x8 100 \\
Dowex SAR & Duolite C280 \\
Dowex SBR & Duolite C433 \\
Dowex 1x4 50 & \\
Dowex 1X8 50 & \\
Duolite A368 & \\
Duolite A7 & \\
\hline
\end{tabular}

\section{Accomplishments}

The twelve anion and eight cation exchangers were quickly evaluated based on their handling characteristics, product descriptions, and the distribution coefficient $(\mathrm{kd})$ values measured using simulated ICPP sodium-bearing waste. Several of the anion exchangers were difficult to handle because the resins became sticky, making it difficult to recover the exchanger for regeneration after a batch contact with the waste simulant. We tested the stability of the resins by cycling small quantities of each resin through ten adsorption/regeneration cycles. The anion and cation exchangers that have good handling characteristics and performance are Dowex SAR $(\mathrm{kd}=3.9 \times 104 \mathrm{ml} / \mathrm{g})$ and Amberlite IRC $76(\mathrm{kd}=0.67 \mathrm{ml} / \mathrm{g})$, respectively. These were selected for more detailed evaluation because of the good distribution coefficients and handling characteristics and because of the availability of independent data.

During initial batch deacidification tests, iron precipitates from the treated simulant began forming above $\mathrm{pH}=3.4$, regardless of the resin being tested. We conducted subsequent testing for alkali metal removal using deacidified simulant, with a $\mathrm{pH}=3.4$, to avoid potential problems of column plugging during regeneration.

We conducted sodium breakthrough tests on the Amberlite IRC 76 resin using untreated and also deacidified simulants. Simulants were injected into the column in 1-ml increments until $35 \mathrm{ml}(\sim 2.3$ bed volumes) had been added, followed by water to rinse the unsorbed cations from the bed. The breakthrough curves for the resin with the two simulants are virtually coincident, indicating that the sodium sorption is independent of acid concentration over the range tested. 
Although this study demonstrated deacidification of sodiumbearing waste simulant and removal of alkali metals using ionexchange, the high salt content reduces the efficiency of the resins for removing the target species. Ion-exchange capacities for the resins, when tested with the simulated ICPP sodium-bearing waste, were significantly lower than reported by the manufacturers. Furthermore, the high concentrations of target ions in the waste solution would require exceptionally large ion-exchange beds to achieve adequate treatment rates, and the solutions used to regenerate the resin beds would constitute a significant secondary waste.

\section{The Effect of Thermal Treatment of INEL Soil Prior to Cementitious Solidification} LDRD W104

\section{D. Siemer}

The purpose of the original project was to examine the possibility that cement kilns might be useful for processing waste \& contaminated soil at the INEL's burial ground. When all of the original funding not already subcontracted out was withdrawn, the scope of the project was reduced to seeing whether or not thermal treatment of INEL soil would improve its performance as a soilcrete barrierforming material. The Principal Investigator $(P I)$ decided to look into what one might hope to accomplish with pugmill-mixing, followed by vibratory compaction - not what might be achieved if pourable grouts were made with cement mixers (the latter approach would cost more to implement and would probably produce poorer-quality soilcretes because a higher water-to-cement ratio would be required). The laboratory work was done during a two-week visit by the PI to the laboratory of subcontractor Professor Barry Scheetz at Pennsylvania
State University's Material Research Laboratory. The most important conclusion drawn from this work was that raw INEL soil is already so pozzolanic that heat treatment is probably not worth doing if the object is simply to build soilcrete barriers.

\section{Accomplishments}

Approximately thirty different soilcrete formulations were prepared with various mixtures of INEL soil (both raw and calcined), chemical binding agents (e.g., lime, portland cement, sodium silicate), and water, pressed into pellets, cured, and then examined. The calcination temperatures investigated were 700 and $1000^{\circ} \mathrm{C}$. Duplicate samples were made of most of these mixes and strength testing was done on both wet and dry specimens. Four things became apparent. First, and most important, untreated INEL soil is quite pozzolanic (meaning that it reacts with lime to form a hydraulic cement; i.e., one that will set under water). This is good news because Roman concretes are generally recognized to be one of Mankind's most durable materials, and they, also, were made by mixing lime with natural pozzolans. Second, it appears that not much additional strength is gained by using more than about $10 \%$ binder. In fact, as little as $3.5 \%$ lime would convert raw INEL soil into a fairly strong (>1000 psi) soilcrete. Third, it appears that soilcrete specimens made with the calcined soil specimens lost a lower percentage of their dry strengths after soaking in water than did samples made from raw soil (by this measure, calcined soil is more pozzolanic than raw soil). However, their absolute wet-strengths were often less. This is because the calcined soils were considerably less compressible than the raw material, which means that soilcretes made from calcined soil (unground) are less dense/more porous. Fourth, soil- crete specimens made with soil that had been mixed with calcium carbonate before calcination were considerably weaker than were those to which an equivalent amount of lime binder had been added afterwards. (However, the $700^{\circ} \mathrm{C}$ calcination temperature used for that particular experiment may not have been high enough to convert all of the limestone to lime. The experiment needs to be repeated with a soil-limestone mix calcined at $\sim 900^{\circ} \mathrm{C}$, instead.)

While impressive compressive strength can be obtained with small amounts of cheap binding materials, those products do not have acceptable permeabilities. The better specimens evinced water permeabilities on the order of $200 \mu$ darcies-two or three orders of magnitude worse than those of the repository-sealing concretes developed at PSU a decade or so ago. This suggests that further work needs to be done. First, it would be a good idea to see if better permeabilities could be achieved if the soils were ground prior to being converted to soilcrete. One of the reasons that BFStype concretes are less permeable than OPC-based concretes is that the BFS binder is usually more finely-ground. Second, we could modify the chemistry. The other reason that BFS makes better radwaste-type concrete is that the ratios of the reactive cementitious agents in BFS are such that no freely soluble solid phases (such as portlandite) are left after a specimen has fully cured. Third, it's very likely that simply using a lot more binder (i.e., $30 \%$ instead of $\sim 5 \%$ ) will improve matters.

A more detailed report is available upon request.

New or Increased Technical Capabilities/ Business Development Opportunities

Since the amount of binder required to create a three-foot-thick 
soilcrete layer over RWMC is approximately 100,000 tons, it is reasonable to see if cheap, highvolume, materials such as the slag produced at FMC's Pocatello phosphate plant could be used for this purpose. Since that slag is already slightly radioactive, one may assume that the residents of the Pocatello area (and their representatives) might welcome an offer from DOE to put that material to beneficial use at INEL. Also, since the chemical composition of hotprocess phosphate slag is between that of blast furnace slag and portland cement clinker (it's closer to BFS), it is at least potentially cementitious. (The likely reason that it is not cementitious in its present form is that it was allowed to crystallize when it cooled. The PI obtained a sample of blue rock after returning from PSU, ground it to a fine powder, and then tried to make concrete of it; it would not set up, even when a sodium hydroxide activator solution was substituted for plain mix-water.) The slags from which commercial BFS cements are made have been quench-cooled (granulated) so that a good deal of the reactive metastable glass phase survives. To use FMC's slag for soilcreting, we would have to persuade FMC to install a suitable quench system and arrange for the granulated slag to be ground to a fine powder (perhaps at ASHGROVE's cement plant at Inkom). In any case, we would first have to establish whether or not this slag can be rendered cementitious.

Wet-process (phosphogypsum) slags such as those produced by SIMPLOT's Pocatello plant have been demonstrated to have cementious properties. Other possibilities include ASHGROVE cement plants's kiln dust and the Jim Bridger power plant's flyash.

\author{
Automatic Pressurized \\ Filtering of Environmental \\ Samples for Remote \\ Applications \\ LDRD 109 \\ R. M. Shurtliff
}

Several EPA-directed sample preparation methods specify that as part of the sample preparation procedure, liquids be separated from the solids portion of the sample. Specific instructions detail a pressurized filtering method to accomplish this. Industry has made available the apparatus to manually perform this laborious function in the laboratory. However, the apparatus is impractical to use in a remote environment such as a radioactive hot cell or glove box.

In consultation with INEL and Lockheed Corporate Analytical Chemistry personnel a pressurized filter unit for remote application was designed and constructed. Disposable Teflono liners were developed in conjunction with an outside vendor to eliminate cross-contamination/cleanup problems. A modular filter concept was also developed which simplifies the removal and installation of the filter remotely. An apparatus to hold the filter unit and rotate it was designed and constructed. Preliminary testing or the unit has been accomplished.

With the growing demands placed on laboratories internationally for environmental sample processing, this automatic pressurized filtering system will be of tremendous benefit to INEL and other facilities in terms of cost and time involved to process each sample. This technology, when thoroughly tested, will be transferred to private industry for commercialization.

\section{Objectives}

- Research several different types of materials for possible use as liners within the pressurized filter canister.

- Design an apparatus that could be operated remotely or with the aid of a robotic arm and that would be compatible with the requirements for hot cell and autonomous control applications.

- Work with industry to develop a disposable liner that could be used for sample preparations and minimize the amount of cleaning necessary to prevent sample cross-contamination.

- Build a prototype filtering unit that could be evaluated and compared to existing filtering technics used in the laboratory (see the figure to the right).

- Arrange to have INEL ICPP Analytical Chemistry department personnel evaluate the device and provide input and suggestions for improvement.

Accomplishments

Working with Massachusettsbased American Durafilm Company, disposable Teflon liners were developed for the sample canister. A few prototype liners were tested and evaluated with good results. A problem with the Dupont film used in the manufacture of the liners prevented American Durafilm from filling an order for twentyfive liners by the end of the fiscal year. There was a two month delay involved in determining the cause of the problem which has impacted the functional laboratory testing of the system by Analytical Chemistry personnel. More research into methods of manufacturing the canister liner needs yet to be done to assure reliability.

A modular borosilicate filter design was created which allows easy replacement in a remote hot cell environment. Further development is planned in conjunction with a filter manufacturing company to build this modular filter as a disposable unit. It is presently built of stainless steel. The sample canister is designed to allow the lid to be removed via three clamps which can be latched/unlatched 


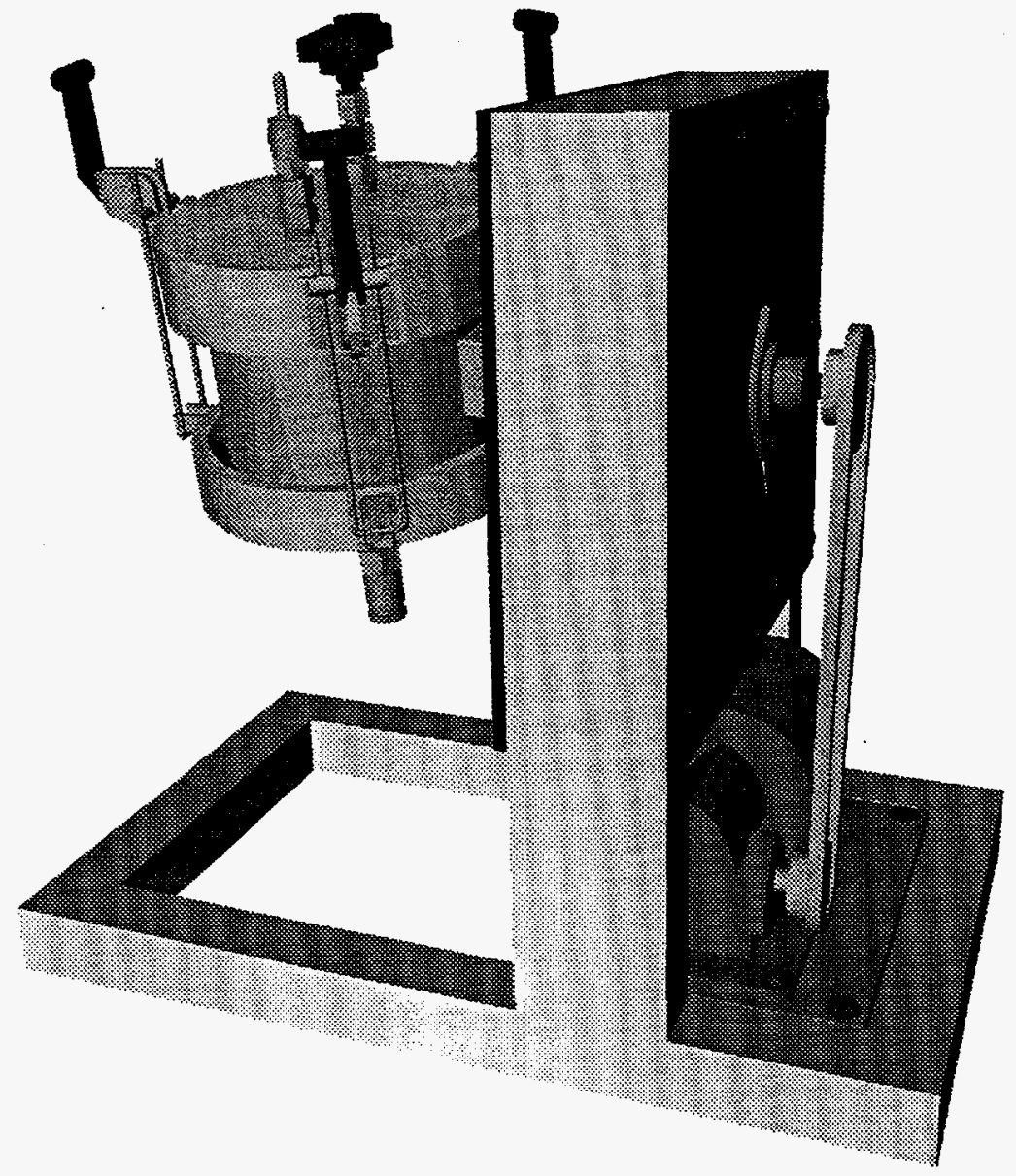

Pressurized Filter System

with remote master/slave manipulators. With the lid removed, the filter can be removed from or installed in the canister.

A prototype unit was machined from aluminum and Lexan making it possible to visually demonstrate the process of pressurized filtering. The filter unit is mounted on a unique rotator base which allows the sample to be filtered and rotated without moving the sample canister from one station to another.

New or Increased Technical Capabilities

An understanding of the leading edge technology of thermoforming Teflon film has been obtained. In the future a prototype of the pressurized filter will be installed in the Remote Analytical Laboratory (RAL) hot cell at the
ICPP for evaluation and testing. This will provide Lockheed-Martin with the first automated pressurized filtering system in the industry. It is far more advanced than the commercially available laboratory filtering systems which are manually operated and impossible to use in remote and automatic applications. FY-96 will be devoted to automating the pressurized filter system, building a prototype that can be used in the RAL, and pursuing commercialization of the system.

\section{Business Development Opportunities}

American Durafilm Company has helped in the development of the disposable Teflon liners, and should the automatic pressurized filter system be commercialized in the future, the market for these lin- ers could be significant. Following further development a disposable filter module could be manufactured with good market potential. The commercial market for an automatic pressurized filter system is significant. At least six vendors are marketing a manual laboratory version of a pressurized filter system.

\section{Position Tracking for Remote Subsurface Characterization Systems} LDRD W111

\section{W. T. Zollinger}

The objective of this FY-95 LDRD project was to perform preliminary work for the development of a remote real-time position sensor to continuously track the three-dimensional position of a subsurface characterization system as it moves underground. When developed, the positioning system will be located above ground and will passively listen for intentional emission of either acoustic or low frequency electromagnetic energy signals. Characteristics of different types of signal propagation through soil as well as different signal processing techniques was studied. This effort was in concert with an effort to develop a maneuverable remote subsurface characterization system (RSCS). Development of a maneuverable $R S C S$ is directed at the development of equipment to remotely characterize the waste without the risk of compromising containers, and without exposing personnel to unacceptable safety risks. The position sensor will be used not only for controlling the RSCS from above ground but for fusing position data with sensor data collected by the RSCS from underground. The technology may also be extended to INEL based needs in mapping activities on both a large and a small scale in hazardous and non-hazardous tasks. Potential customers include PNL, Savannah River, Rocky Flats, robotics, aerospace, and mining industries. 


\section{Objectives}

The goals of this effort were to demonstrate in the laboratory or on a scaled down model a remote real-time fault-tolerant position sensing technique for regional monitoring of the three dimensional position of a highly maneuverable RSCS. Preliminary studies on the most optimum source and type of energy for propagating through lossy soils needed to be performed. Acoustic and electromagnetic energy was to be considered. It was also an objective to study methods of transducing this energy from the RSCS and into the receiving sensors located above ground. An investigation was to be made into the selection of the appropriate signal, e.g., pulsed, continuous wave, or amplitude/frequency modulated. In developing the signal processing scheme, a position sensing algorithm developed at the INEL would be incorporated. The major milestones of this project were

- Complete the identification of techniques most probable for success of excitation, penetration and propagation through soils.

- Complete studies of energy loss and penetration through soil.

- Conceptual Design.

- Construct bench-top experimental system for emitter and sensor array.

- Test and evaluate system performance.

\section{Accomplishments}

Since the project commenced during the winter time and some preliminary conclusions needed to be made about attenuation in soil, a container was built in the labora- tory to hold some soil. The dimensions of the container were 4 -feet square by 2.5 -feet deep. The container was filled with clay soil which was representative of that found at the RWMC site. Antennas were placed in the soil so as to measure the propagation loss over several feet of soil. Because of the small limited size of the container and other reasons, inconclusive data were taken at this time. Later as the weather warmed up outside, additional data were taken with the antennas placed into a vertical position into the ground. The ground where the data were taken was made up of highly compacted clay with a small amount of soil moisture. Only electromagnetic energy was considered at this time. At $100 \mathrm{MHz}$, approximately 2-dB / foot to 3-dB/foot loss was observed. Therefore, if this is extrapolated to the 30-foot distance requirement for an RSCS system, an approximate $60-\mathrm{dB}$ to $90-\mathrm{dB}$ loss in the total signal power may be expected. If the source on the RSCS system was designed to emit a $20 \mathrm{dBm}$ power signal then $-40 \mathrm{dBm}$ to $-70 \mathrm{dBm}$ of power may be expected. This power level is well above the noise floor of most commercial radio receivers. Therefore, the conclusion is that for a subsurface RF emitting source, the propagating energy will be adequately strong in order to receive and detects its position.

Another accomplishment of the project included the investigation of the type of signal to be transmitted. The complexity of the transmitter and receiver hardware, the resultant inherent errors in position, the frequency bandwidth requirements, and signal processing complexity/performance were a few of considerations given. A conceptual design was made but not without the need for future changes

\section{New or Increased Technical Capabilities}

The bench top demonstration in the laboratory as hoped, was not completed due to unexpected difficulties. But much was learned about the conceptual design and what will be needed in the future in order to accomplish this task. We have become much more aware of the needed technologies be successful. A pseudorandom coded signal design needs to incorporated in future work. We have become more educated and smart in terms of the signal type and signal processing element requirements. More antenna work needs to be done with implementation on an actual RSCS considered.

New or Increased Technical Capabilities

Other than talking with vendors of current remote subsurface systems about their needs, additional collaboration with outside companies have not taken place this year.

\section{Evaluation of Delphi Oxidation for Activated Carbon LDRD W122 \\ D. Gombert}

This project studied the Delphi oxidation process (a wet $\mathrm{Fe}^{2+} / \mathrm{Fe}^{3+}$ system) for the revitalization of activated carbon used for organic compound adsorption. The potential benefits lie in the area of environmental remediation; activated carbon is used extensively for trapping organic vapors in pump and treat remediation systems. This activated carbon must then be dealt with, two common options are incineration and high temperature steam treatment. Both of these options are expensive, incineration is especially undesirable. The Delphi process offers a more cost effective 
system that allows reuse of the activated carbon after treatment. This work investigated the Delphi process for removal of organic contaminant from activated carbon and subsequent capacity reductions.

\section{Objective}

- Investigate the Delphi oxidation process for treatment of activated carbon used to trap organic compounds.

\section{Accomplishments}

The Delphi oxidation process uses a wet, catalyzed $\mathrm{Fe}^{2+} / \mathrm{Fe}^{3+}$ system to effect the oxidation of organic compounds. The process is carried out in tantulum lined reactors and has been proven in the past to be very effective in the destruction of organic compounds, both in the liquid and solid forms. Items such as waste solvents to latex gloves and wood have been destroyed with the Delphi process. There are a number of process variables such as temperature and reaction time than can be adjusted to control the severity of the reactor conditions and thus, the extent of destruction. The key to this pro- ject was to destroy the organic solvents adsorbed onto the activated carbon, but not greatly reduce the viability of the carbon for multiple uses as an adsorbant.

Carbon was loaded with toluene for the testing and run in duplicate under eight different process conditions:

1. $125^{\circ} \mathrm{C}, 1$ hour

2. $150^{\circ} \mathrm{C}, 3$ hours

3. $100^{\circ} \mathrm{C}, 8$ hours

4. $125^{\circ} \mathrm{C}, 8$ hours

5. $175^{\circ} \mathrm{C}, 1$ hour

6. $150^{\circ} \mathrm{C}, 8$ hours

7. $150^{\circ} \mathrm{C}, 1$ hour

8. $175^{\circ} \mathrm{C}, 3$ hours.

After loading and treatment, the carbons were reloaded and and the capacity again measured. In all cases, capacity was $>99 \%$ of original measured capacity. In the figure below, the capacity changes for each condition is illustrated. It is obvious that the Delphi process is successful in treating activated carbon for reuse, without degradation in the capacity of the carbon.

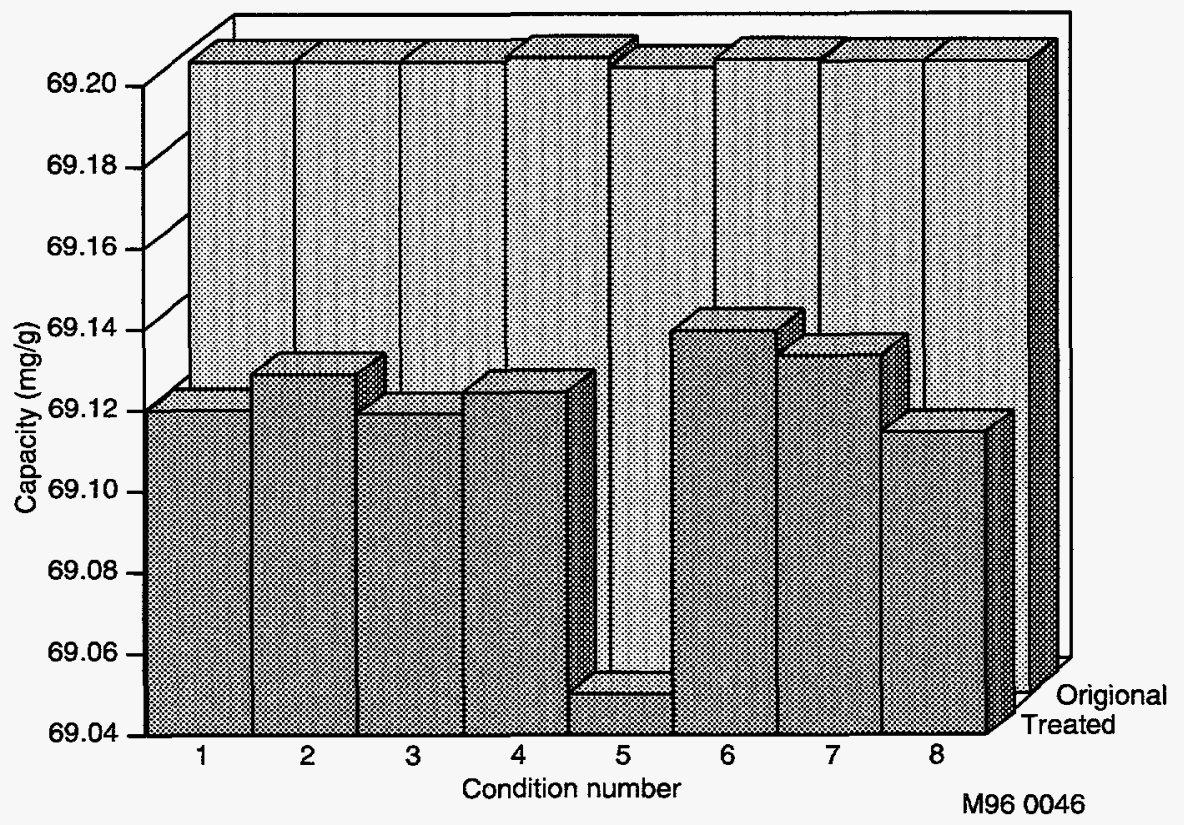

\section{Evaluation of EET Proprietary Decontamination} LDRD W123

\section{Gombert}

Decontamination of cesium bearing soils is an internationally unsolved problem. Treatability studies conducted at the INEL have used mineral acids, salt brines, and sequential extraction in unsuccessful attempts to remove cesium from the natural soil matrix. Proprietary technology developed by $E E T$, Inc. which has been used to successfully extract uranium and fission products from concrete at the Oak Ridge Reservation and the INEL, 
was tested on soil samples from the INEL. Personnel from EET did preliminary chemical tests with INEL soils and developed special procedures for adapting their technology to soil treatment. Two soil samples were fractioned into +10 and -10 mesh and treated in triplicate per instructions by EET. Data from a three-step extraction procedure indicated up to $30 \%$ decontamination was possible with the -10 mesh material, with dissolution of about $18 \%$ of the matrix. Results with +10 mesh material was less definitive.

\section{Objectives}

- Develop experimental procedures with EET, Inc. for adapting decontamination procedures for solid surfaces to soil particles.

- Conduct experiments at the Idaho Chemical Processing Plant with cesium contaminated soil samples collected from the INEL site.

- Review data from initial testing with EET, to determine if follow-on testing is warranted.

- Complete follow-on testing and analysis of results.

\section{Accomplishments}

All tests were completed, and support provided by EET personnel was exemplary. The data reported in Table I summarize the average results of the experiments. The initial test identified as Sample 1 was done in triplicate using the EET three-step extraction procedure. Owing to reagent viscosity and corrosion problems with laboratory filters, a follow-on test procedure was then developed with EET using only the first step extractant, but applying it twice to Sample II.

As can be seen from the data, the results with the -10 mesh material indicated $15-34 \%$ decontamination was attainable. Sample 1 , treated with the three-step extraction resulted in up to $34 \%$ decontamination also lost up to one quarter of the sample mass in dissolution. Sample 2 lost less than $1 \%$ of the sample mass during treatment, but decontamination was

decreased to $14-21 \%$.

The results from the +10 mesh samples are much more difficult to interpret. Earlier decontamination experience with the EET extractants at Oak Ridge and the INEL indicated that radionuclides could be initially brought to the surface (increasing the surface contamination measurement) requiring additional treatment to complete removal. This work was on solid surfaces such as concrete slabs which are surveyed by instruments which only detect surface or nearsurface contamination. Nuclides absorbed into the concrete beyond the depth of detection would be shielded from the instrument until the extractant brought them to the surface. This same increase seems to be apparent in the +10 mesh soil solids. However, in this case the sample is composed of particles much too small to possibly explain the apparent shielding. These anomalous data are therefore of questionable value and should not be used as an indication of decontamination effectiveness. The laboratory is currently dissolving the sample residuals for scintillation counting to verify the after treatment results.

The conclusions are as follows. Though the +10 mesh data will require more analysis pending sample dissolution and recounting, the results from the -10 mesh samples are adequate to discontinue further work for this application. Comparable decontamination results have been achieved with mineral acids which would be far less expensive for soil treatment.

New or Increased Technical Capabilities

This work was completed in the analytical laboratories at the ICPP. No new capability was developed.

\section{Business Development Opportunities}

No opportunities for soil treatment are envisioned at this time, however, the decontamination process developed by EET seems to be a major advance in technology for high-cost problematic situations. The support their personnel provided was exemplary and they should be kept in mind for future partnerships in this area of expertise. 


\section{Nuclear Operations and Nuclear Materials Dispositioning}

\section{Strategic Thrust}

$W_{\text {all mathematics, nor all logic, but it is somecial is not }}^{\text {e need imagination in science. It }}$ beauty and poetry.

-Maria Mitchell

American Astronomer, about 1860 


\section{ATR Radioisotope Production and Purification Study}

LDRD 1102

\section{J. D. Baker, J. E. Brasier, J. W. Rogers}

Several radioisotopes were identified in the ISIS Operation Systems Study (LDRD 7104) as likely candidates for production in ATR. This study focused on measuring the ATR production cross sections for several of the long lived radioisotopes and developing the chemical separation techniques necessary to purify the radioisotope of interest. The isotopes we evaluated are ${ }^{58} \mathrm{Co},{ }^{109} \mathrm{Cd},{ }^{89} \mathrm{Sr}$, and ${ }^{117} \mathrm{mSn}$. We have determined the ATR production cross sections and impurities produced for each of these radioisotopes. Chemical purification techniques were developed and tested where necessary. We produced and chemically purified $\sim 10 \mathrm{Ci}$ of ${ }^{58} \mathrm{Co}$. Several sources were electroplated, and positron emission studies were initiated at the University of Michigan. A CRADA is under negotiation with Positron Resources Inc. for joint development of positron sources for ultimate sale.

\section{Objectives}

- Fabricate targets of various stable isotopes, including neutron dosimeters for both thermal and fast neutrons.

- Irradiate the above targets for at least one ATR cycle.

- Develop chemical purification techniques, where required.

- Transfer the irradiated targets to a hot cell for disassembly, and gamma-ray count the neutron dosimeters and produced radioisotopes.
- Chemically purify the produced radioisotopes, if necessary because of impurities that are also produced.

- Determine the production amounts and costs for each radioisotope.

\section{Accomplishments}

This LDRD had two foci. The first evaluated the production, purification, and making of positron sources using ${ }^{58} \mathrm{Co}$. The second studied the production of $117 \mathrm{mSn}$ and level of impurities produced in ${ }^{117} \mathrm{Sn}$ from two different sources (US and USSR). Additionally, we measured the production rates for both the ${ }^{108} \mathrm{Cd}(\mathrm{n}, \gamma){ }^{109} \mathrm{Cd}$ and ${ }^{88} \mathrm{Sr}(\mathrm{n}, \gamma)^{89} \mathrm{Sr}$ reactions.

In collaboration with Positron Resources Inc. and the University of Michigan, we separated the ${ }^{58} \mathrm{Co}$ from all of the $\mathrm{Ni}$ dosimeter wires from one ATR cycle. These wires would normally be discarded as radioactive waste. Presently, 16 wires are used to monitor the fast neutron flux in various positions of the ATR core. This corresponds to $\sim 40 \mathrm{~g}$ of $\mathrm{Ni}$. In a hot cell, we dissolved the $\mathrm{Ni}$, chemically purified it, and analyzed for the amount of ${ }^{58} \mathrm{Co}$ recovered and its purity. We recovered $97 \%$ of the ${ }^{58} \mathrm{Co}$ activity, and it had a radiochemical purity of $>99.99 \%$. From the $40 \mathrm{~g}$ of $\mathrm{Ni}, \sim 9 \mathrm{Ci}$ of ${ }^{58} \mathrm{Co}$ was recovered. The ${ }^{58} \mathrm{Co}$ is a positron emitter and is used in positron experiments. Therefore, with the help of University of Michigan, we electroplated several sources of ${ }^{58} \mathrm{Co}$, which were then sent to the University of Michigan for evaluation as to the efficiency of positrons getting out of the plated source. They felt that the sources were excellent. Future work is necessary in the area of efficient electroplating and measuring the activity that resides on the positron source.

The results of the ${ }^{117} \operatorname{Sn}\left(n, n^{\prime}\right)$ $117 \mathrm{~m}$ Sn study are detailed in the report INEL-95/0164, Results of Neutron Radiation and Gamma-Ray Spectrometry Radioactivity Measurements Associated with the ${ }^{117} S n$ Sample Irradiation in ATR, by J. W. Rogers. Specifically, the production cross sections for epithermal and fast neutrons were measured to be 132 and $220 \mathrm{mb}$, respectively. The Russian ${ }^{117} \mathrm{Sn}$ appears to be a little more isotopically and chemically pure than the ORNL ${ }^{117} \mathrm{Sn}$.

Targets of ${ }^{88} \mathrm{Sr}$ and $108 \mathrm{Cd}$ were fabricated and irradiated. The ${ }^{88} \mathrm{Sr}$ target produced $1600 \mu \mathrm{Ci}$ of ${ }^{89} \mathrm{Sr}$, and the ${ }^{108} \mathrm{Cd}$ target produced $3550 \mu \mathrm{Ci}$ of ${ }^{109} \mathrm{Cd}$. We are still evaluating the production. cross sections.

\section{New or Increased Technical Capability}

Both Positron Resources and the University of Michigan would like to see the INEL build both the capability to produce positron sources for the positron users community as well as a facility that had higher levels of useable positrons, where users could come and perform experiments that required large numbers of positrons. We submitted an LDRD to initiate both of these capabilities.

\section{Business Development Opportunities}

We are negotiating a CRADA with Positron Resources Inc. where the INEL will help develop and produce positron sources of ${ }^{58}$ Co. Positron Resources will collaborate in the development of the sources and will sell them, initially, to researchers in the positron community. Ultimately, we envision a positron users facility at the INEL. 


\section{Neutron Delivery System for Fast-Neutron Radiotherapy}

LDRD 1171

D. W. Nigg

This project is directed toward the definition and development of specifications and initial conceptual design parameters for an advanced particleaccelerator-based neutron delivery system suitable for routine clinical deployment for the application of fastneutron radiotherapy for cancer with neutron capture augmentation. The workscope is in support of INEL commitments to a Cooperative Research and Development Agreement (CRADA) established in May 1995 between Ionix Corporation, INEL, and Washington State University (WSU).

\section{Objectives}

Currently operating neutron delivery systems for fast neutron therapy of cancer (there are three in the United States) are relatively large and/or expensive, and are located at major laboratories and teaching hospitals. To varying degrees, these systems are less than optimal as prototypes for widespread routine clinical deployment. Fast neutron therapy appears to be emerging as a favored modality for several malignant diseases. The market demand likely to develop for neutron therapy will considerably exceed the capacity of the current therapy centers. Thus a significant need for simple, reliable, relatively inexpensive, technicianoperable neutron delivery systems may very well materialize over the next few years. It is anticipated that the various activities being conducted under the current Ionix/Lockheed Idaho/WSU CRADA will lead to clinical application of fast neutron therapy with neutron capture therapy (NCT) augmentation under certain circumstances. None of the available fast neutron sources feature the ability to routinely produce the tunable fast neutron spectrum needed to take maximum advantage of NCT augmentation.

This project involves the specification and development of one or more preconceptual designs for advanced dual-purpose accelerator-based neutron delivery systems for fast neutron and NCT-enhanced fast neutron radiotherapy suitable for cost-effective clinical deployment. In FY-95 a modest effort was undertaken to establish the basic clinical and biophysical requirements that would define such a system and to identify one or more promising pre-conceptual facility designs. The specific objectives of the project for FY-95 were (a) to develop an initial top-level system specification using modern system engineering techniques, and (b) to begin to identify preconceptual system configurations. This is a prelude to a more comprehensive and detailed effort in FY-96, which will result in the completion of a system specification and preconceptual design to a level of detail that will allow solicitation of an industrial partner for actual prototype design and construction. In the years following FY-96 the effort has the potential to mature (under non-LDRD funding) into the construction of a prototype accelerator-based neutron delivery system for cancer radiotherapy, with licensing of the design and key component intellectual property to industry for commercial deployment.

\section{Accomplishments}

INEL recently established a Cooperative Research and Development Agreement (CRADA) with the Ionix Corporation (a biotech firm in Edmonds, Washington) and with Washington State University (WSU) to perform research and technology development directed toward the clinical implementation of certain advanced, very promising, forms of neutron radiotherapy and Boron Neutron Capture Therapy (BNCT) for cancer. One aspect of this CRADA (and its anticipated future extensions) involves the specification, design, development, and ultimate construction of advanced dual-purpose acceleratorbased neutron delivery systems for fast neutron and BNCT-enhanced fast neutron radiotherapy that are suitable for cost-effective clinical deployment.

This LDRD project was initiated in May 1995 to support our BNCT CRADA commitments. During the balance of FY-95 the workscope has involved a modest effort to establish the basic clinical, biophysical, and engineering requirements that would define such a system and to begin the process of identifying promising pre-conceptual facility configurations. A draft top-level system specification will be published at the end of FY-95. Dr. George Laramore, director of the Neutron Therapy Program at the University of Washington Medical School has agreed to work closely with INEL (at Ionix expense, as part of the CRADA) in order to provide appropriate clinical guidance for the project. A Team consisting of Dr. Laramore, as well as key INEL experts in neutron physics, accelerator applications, electrical engineering, systems engineering, and computer monitoring and control systems has been assembled and constitutes the working group for the study. This group will mature into an Integrated Product Team as the effort progresses, with addition of appropriate technical personnel as appropriate, in FY-96 and beyond. There will be a close collaboration with certain other key personnel at the University of Washington Medical School, which is the site of one of the currently-operating fast neu- 
tron delivery systems used for radiotherapy. Collaboration with Los Alamos in the area of accelerator systems to drive the neutron delivery system is also anticipated. At the appropriate time we will solicit the participation of one or more industrial partners with experience in the areas of accelerator and radiation delivery system manufacturing

New or Increased Technical Capability

There are several related activities at the INEL having to do with development of accelerator neutron sources for epithermal-neutron NCT. This proposed project is a new initiative to extend our activities into the transepithermal energy range. Because this will be a new system concept, we have taken the approach of using modern system engineering techniques to define the system requirements and specifications up front, from a clinical standpoint, with a view toward satisfying the needs of the anticipated market in the most cost-effective, safe, and reliable manner possible. We have incorporated computational studies based on well-established INEL technical strengths to more clearly define the system specifications. All of this activity will ultimately allow INEL to develop considerable technical expertise in the field of fast neutron sources for radiotherapy. Although we will not become an accelerator laboratory, we will develop expertise in the applications of accelerators to the production of neutron sources for medical uses.

Business Development Opportunities

The work is directed toward the objective of establishing a programatically funded collaborative INEL/university/industry program for the construction of a prototype accelerator-based neutron delivery system for cancer radio- therapy, with licensing of the design and key component intellectual property to industry and potential formation of a spinoff manufacturing business for commercial deployment.

\section{Real-time Measurement Dosimetry for Boron Neutron Capture Therapy LDRD 1173}

\section{Y. D. Harker, J. R. Venhuizen}

This project is directed toward the development of a dosimetry measurement system that (a) records the dose received by the patient, (b) reads out the dose in real time, and (c) has physically small sensors that do not perturb the radiation field nor the physical placement of the patient. This development is part of a commitment made by DOE in support of the CRADA established between INEL, IONIX Corporation, and Washington State University. Currently, doses to patients undergoing boron neutron capture therapy are determined using neutron activation wires or foils and TLDs (thermoluminescent dosimetry! dosimeters). Under this scheme, doses are not recorded in real time. The values are determined after treatment and serve as after-the-fact measures of the accumulative dose received by the patient. This method does not provide the physician any information as to whether or not the treatment is going according to plan and whether corrective action is necessary. Real-time monitoring systems currently used are only measuring the amount of neutron current being delivered to the patient area; they do not measure the dose received by the patient. The monitoring system being developed in this project will provide through small active sensors attached to the patient a measure of the neutron flux and gamma dose rate as the treatment progresses. The information will be displayed on a PC-based readout system. The real-time display will show the current measured values and compare them with corresponding values generated by the patient treatment planning program. An electronic record of the treatment will be maintained, and a hard copy of the data will be provided.

\section{Objectives}

- Test the fiber optic neutron detectors developed by Pacific Northwest Laboratory (PNL), in neutron fields typical of the fields encountered in BNCT treatments.

- Identify a small gamma sensor.

New or Increased Technical Capabilities

The fiber optic neutron detector developed at PNL was selected as a potential neutron monitor because its neutron sensitive isotope (lithium-6) has the same relative energy response as boron-10 and its physical dimensions are in millimeters. Through a cooperative arrangement between INEL, PNL, and WSU, the fiber optic neutron detectors were tested at the WSU research reactor. These measurements demonstrate that the fiber optic neutron detector has an approximate linear response with respect to neutron flux. The neutron-to-gamma response ratio is at least $8: 1$. The PNL fibers were developed for laboratory testing and had not been made rugged for use in medical applications. Further testing using more rugged versions of the PNL fibers are planned for FY-96.

While at WSU, INEL personnel became acquainted with Scott Jones of International Sensors Technology (IST) Corporation located in Pullman, Washington. With his cooperation, INEL was able to get valuable information about the use of laser readout TLD systems. During the tests involving the PNL fibers, IST was able to test their remote TLD readout system. The sensor part of the system is a small TLD phosphor 
attached to the end of a fiber optic transmission cable. Like the PNL fiber optic neutron detector, the IST sensor and cable are physically small and therefore easy to use when attaching to a patient.

Through fiber optic cable, the TLD is heated using laser light, and the resulting luminescence is transmitted back to a photo-multiplier tube. The photo-multiplier tube response is then converted into dose and output on a laptop PC. Results from ISTs testing show that this technique has definite possibilities as a part of the real-time monitoring system being developed by this project. Further work is planned for FY-96 to assess the capabilities of the IST remote readout system.

We have identified the core components of the sensing elements for the real-time patient monitoring system from the results obtained in FY-95. Further testing is planned to better characterize the responses of these sensing elements. If available resources are identified, a prototype multiple input readout system will be developed.

\section{New or Increased Technical Capabilities}

It is recognized at the INEL that an adequate dosimetry measurement system for use in human clinical trials involving BNCT does not exist. Development of this system is a key feature of the INEL BNCT program. Through the CRADA with WSU and IONIX, the first dosimetry measurement system will be installed in the WSU epithermal neutron beam facility.

\section{Business Development Opportunities}

This development is already linked with a CRADA involving INEL, IONIX Corporation, and Washington State University. Successful development of the dosimetry measurement system has application at other facilities involved in BNCT and also in other forms of radiation therapy. Because of its versatility, a dosimetry system of this type has definite commercialization potential. Once the key components have been tested and feasibility has been established, a patent disclosure application will be prepared.

\section{Mixed Oxide Fuels Testing in the Advanced Test Reactor LDRD 2108 \\ J. M. Ryskamp}

The safety of people throughout the world is seriously threatened by nuclear weapons stockpiles and by weapons-grade plutonium (WGPU) from nuclear weapons dismantled in arms-reduction programs. An intense worldwide effort is now under way to find ways to reduce this danger. One of the most attractive solutions would be to use WGPu as fuel in existing light water reactors (LWRs) in the form of mixed oxide (MOX) fuel, that is, plutonia $\left(\mathrm{PuO}_{2}\right)$ mixed with urania $\left(\mathrm{UO}_{2}\right)$. Numerous technical issues must be resolved before LWR operating licenses can be amended to allow the use of MOX fuel. The Advanced Test Reactor (ATR) at the Idaho National Engineering Laboratory (INEL) possesses many advantages for performing tests to resolve most of the issues identified in Table 1. It has ample core test volume, high neutron flux, test loops with cooling systems independent of the core coolant, and extensive support facilities. However, to be useful for MOX fuel testing, the ATR must be able to deliver a neutron flux of appropriate intensity and energy distribution to the MOX test specimens while simultaneously accommodating test requirements for other programs. We have performed calculations to show that the use of hafnium shrouds can produce spectrum adjustments that will bring the flux spectrum in ATR test loops into a good approximation to the spectrum anticipated in a commercial LWR containing MOX fuel while allowing operation of the test fuel assemblies near their optimum values of linear heat generation rate. The ATR would be a nearly ideal test bed for developing data needed to support applications to license LWRs for operation with MOX fuel made from weapons-grade plutonium.

Objectives

- Determine the feasibility of MOX fuel testing in ATR.

- Provide technical information for external proposals.

- Document this work in a technical report.

\section{Accomplishments}

This project has achieved its main objective by determining that it is feasible to test MOX fuel in the ATR. One technical summary and

Table 1

Issues and Appropriate Test Facility for MOX Fuel Qualification

\begin{tabular}{|c|c|}
\hline & Commercial \\
\hline
\end{tabular}

$\begin{array}{ll}\text { Yes } & \text { Yes } \\ \text { Partially } & \text { Yes } \\ \text { Yes } & \text { Yes } \\ \text { Yes } & \text { Yes } \\ \text { Yes } & \text { Yes } \\ \text { Yes } & \text { Yes } \\ \text { Yes } & \text { Yes } \\ \text { No } & \text { Yes } \\ \text { No } & \text { Yes } \\ \text { Partially } & \text { Yes } \\ \text { No } & \text { Yes } \\ \text { Yes } & \text { No } \\ \text { Yes } & \text { Yes }\end{array}$


one external report were published. A second external report documenting the entire LDRD project effort was published in September 1995.

We performed some preliminary analyses to resolve fundamental questions about the suitability of the ATR for a MOX fuel testing program. The test results from a MOX fuel testing program must give valid data from which to draw conclusions about MOX fuel performance in a commercial reactor. Also, the presence of MOX fuel test specimens in the ATR must not impair the ability of the reactor to perform simultaneous irradiations in other test locations, since only a relative few of the ATR's test locations would be occupied by MOX fuel specimens. Most important, the tests must not jeopardize the operating safety of the ATR.

We performed neutronics analyses with the code MCNP, using a full-core input model. A 21-pin MOX fuel assembly was put into the model's northeast loop test space. For an unshrouded 21-pin array of MOX fuel containing $7 \%$ plutonia, safety limits would require that the ATR quadrant power not exceed $7 \mathrm{MW}$. However, placing hafnium shrouds around the MOX fuel assembly would shield the MOX fuel so that higher quadrant power could be sustained. Use of the hafnium shroud allows the reactor to be operated in a normal regime of quadrant power. Fortunately, the same shroud thickness produces the proper neutron energy spectrum for MOX fuel testing. We also obtained results for other potential MOX fuel testing locations. A hafnium shroud slightly thicker than $1.524 \mathrm{~mm}$ (60 mils) is required in the large B-hole.

We performed thermohydraulics calculations to estimate fuel, cladding, and coolant tem- peratures in a MOX fuel testing installation in the ATR. These calculations used an analytical model of a pin with cladding in axial turbulent flow, with the heat transfer coefficient on the cladding surface given by the Dittus-Boelter heat transfer correlation. The maximum calculated fuel centerline temperature in the ATR test specimens is less than that in a Combustion Engineering pressurized water reactor (PWR). Even though the peak linear heat generation rate (LHGR) is slightly higher in the ATR, the much greater length of the PWR fuel pins and the much higher PWR coolant inlet temperature $(574 \mathrm{~K}$, as opposed to $325 \mathrm{~K}$ in the ATR) cause higher temperatures in the PWR. Therefore, it can be concluded that the MOX fuel tests can be conducted safely in the ATR at the desired LHGR values.

New or Increased Technical Capability

This project has established the feasibility of MOX fuels testing in the ATR. We are now aware of the technical aspects and issues associated with this area. Many technical questions have been answered; some have not. The INEL staff involved in this project are now prepared technically to perform a much larger, externallyfunded MOX fuels testing program in the ATR. Furthermore, through publications, presentations, and communication related to this project, our technical expertise is now known within the plutonium disposition community.

\section{Business Development Opportunities}

The record of decision (ROD) on the plutonium disposition options is scheduled for August 1996. Burning MOX fuel in existing LWRs is currently one of the best options. If this option is selected, MOX testing in the ATR is very likely. Funding from the
DOE Office of Fissile Materials Dispositioning (MD-4) could be available in FY-96. This funding would be passed to INEL through ORNL, which is currently the lead lab for the reactor-dispositioning option. Funding related to the triple play option could be passed directly to INEL from DOEDefense Programs. A current Defense Authorization bill for MOX and tritium target testing will most likely be approved to a level of \$5M for INEL in FY-96. A major test program involving ATR loops costs $\$ 10$ to $\$ 30 \mathrm{M}$. The INEL must develop a proposal for MOX testing before the record of decision for submittal to DOE-MD, DOE-DP, or ORNL.

\section{General Purpose Time-Dependent Particle Transport with Isotope Generation and Depletion LDRD 2111}

\author{
R. L. Moore
}

The overall objective for this project is to implement a new set of enhancements for the MOCUP computer code. These enhancements will strengthen the key capabilities of the INEL for the analysis of materials irradiation and isotope production. MOCUP provides the unique and seamless capability to do Monte Carlo particle transport calculations with depletion, by coupling the MCNP Monte Carlo transport code to ORIGEN2 isotope generation and depletion code. MOCUP is a system of extemal processors that allow for a limited treatment of the temporal composition of the user-selected MCNP cells in a time dependent flux environment. The ORIGEN2 code computes the time-dependent behavior of these individually selected MCNP cells. All data communication between the two codes is accomplished through MOCUP using the MCNP and ORIGEN2 input/output 
files, the MOCUP Processor Output files, and two user supplied tables. MOCUP is either command line or interactively driven. The interactive interface is based on the portable $\times 11$ window environment and the Motif tool kit. MOCUP was constructed so that no modifications to either MCNP or ORIGEN2 were necessary.

A new set of key features was added to the MOCUP utility program. These new features allow for better evaluation of the Pu-238 and tritium production capacity in the ATR, and for the evaluation of the use of nonfertile plutonium fuel for the disposition of weapons material in power reactors. Because of the enhanced capabilities added to MOCUP, the reactor community (national and international) has expressed interest in purchasing MOCUP from the INEL for a nominal fee.

\section{Objectives}

The purpose of the effort was to enhance the MOCUP code including the automation of certain manual tasks for increased efficiency. Specific technical tasks involved in the effort considered the following:

- ORIGEN2 IRP command tolerance

- Light element cross section updates

- Total atom density calculations

- Gram and mole mass specification consistency

- MCNP material ordering tolerance

- Isotope ordering in MCNP restart

- MCNP tally tolerance

- Preservation of ORIGEN2 $(n, 2 n)$ and $(n, 3 n)$ cross sections

- Preservation of $(n, \gamma)$ and $(n, 2 n)$

- Multiple MCNP tally sets and ORIGEN2 skeletal files
- Fission product cross section library access

- CNP material specification tolerance

- MOCUP manual.

\section{Accomplishments}

Enhancements addressing the issues listed above have been implemented into the MOCUP code. The code with the improvements has been validated against previous in-house results. The validation effort found no deviations from the previous results. Therefore, the improvements to the code have generated no new coding errors. The code is also being checked by Paul Chodak III, a graduate student at MIT. So far he has found nothing to suggest the code is not performing as it was intended.

\section{New or Increased Technical Capability}

This project has provided the INEL analyst and reactor physics community in general with a computer tool that will substantially reduce the labor and computer time required to perform fuel depletion and isotope production calculations using MCNP and ORIGEN-2.1. Thus, fuel depletion and isotope production calculations can now be done in generalized threedimensional geometry with continuous energy nuclear data and accurate transport theory.

\section{Business Development Opportunities}

After presentations on a preliminary version of MOCUP, we have received many expressions of interest. MIT and ABB-CE are interested in using MOCUP for plutonium disposition activities. Atom Analysis is interested in MOCUP as a tool for university research reactors. Researchers in South Korea have expressed a strong interest in obtaining MOCUP. We are in the process of trying to licensing MOCUP to the South Koreans for $\$ 15,000$. They are in the process of reviewing the contract.

\section{Development of a} Simplified Version of SCDAP/RELAP5 for Simulator Applications LDRD 2205

\section{R. J. Beelman}

This project is developing a realtime version of our RELAP5/MOD3 thermal-hydraulic systems simulation code in support of Lockheed Idaho's proposed technical contribution to our impending CRADA with

Science Applications International Corporation (SAIC). The objective of the CRADA is to demonstrate RELAP5 real-time core neutronic/ thermal-hydraulic simulation capabilities on a full-scope, beta-test, commercial nuclear plant training simulator as proof-of-concept. The current generation of nuclear plant training simulators runs simplified thermalhydraulic simulation codes designed to drive the full scope mock-up in realtime. The need for these simplifications arose because of the limitations of affordable, current-day computer hardware in meeting the real-time requirement. These simplifications have compromised training effectiveness with regard to the fidelity and reliability of these special purpose codes over the range of conditions encountered during full scope simulation. Recent quantum advances in prospective simulator host computers have enabled real-time RELAP5 execution on cost-effective hardware. Historically, the emphasis with regard to RELAP5 code development has been placed on the accuracy of the simulation. In order to achieve the CRADA objective, modifications to RELAP5/MOD3 are required to improve code reliability, robustness, and runtime performance to achieve sustained real-time simulation without loss of accuracy. Upon successful 
proof-of-concept demonstration, Lockheed Idaho will license the realtime RELAP5/MOD3 software to SAIC for commercialization as a final simulator upgrade solution to the probiems that have perennially plagued nuclear plant training simulators due to the deficiencies of simplified approaches.

\section{Objectives}

- Modify an existing RELAP5/ MOD3 input model to baseline code runtime performance.

- Investigate/implement RELAP5/MOD3 parallelization to achieve required runtime performance.

- Perfect existing nearly-implicit solution scheme.

- Identify/repair constitutive package numerical discontinuities.

- Remove border profiled lower-upper (BPLU) solver matrix transcription dependence.

- Restore the reflood model to operability.

\section{Accomplishments}

Initially, the beta-test site was identified to be a pressurized water reactor (PWR). An existing 4-loop PWR model was used to baseline RELAP5/MOD3 performance. Results indicated execution performance was about four times slower than required. Recently, the beta-test site was identified to have become a boiling water reactor (BWR). An existing BWR model achieved near real-time execution performance. The objective is twice realtime performance to accommodate simulator postprocessing. It was also observed that the number of repeated time steps was excessive and would have resulted in simulator failure owing to loss of realtime synchronization.
Efforts to parallelize RELAP5/ MOD3 to achieve the necessary code speed-up began with a survey of contemporaneous multiprocessing platforms. An existing parallel version of the now obsolete RELAP5/MOD2 release was useful in elucidating the capabilities of the various platforms. Cost-effectiveness considerations limited prospective suppliers to workstation vendors. The Digital Equipment Corporation (DEC) model 2100 , accompanied by the Kuck and Associates preprocessor (KAP) parallelizing software, appears to be the platform preferred by our CRADA partner. The RELAP5 nearly implicit fluid velocity solution methodology was successfully parallelized on the discontinued but proven Kubota Pacific Computer Corporation vector/parallel Titan 750 . A 15\% performance increase was noted on the Titan 750. Implementation to the ten-fold-faster DEC platform is proceeding, though not yet successfully accomplished. The effort to parallelize RELAP5/MOD3 will continue subject to availability of follow-on funding in FY-96.

In order to overcome the restrictions of Courant-limited time step size, we initiated a major effort to perfect the nearly implicit hydrodynamic solution scheme. A Courant-violating time step ratio of two is desired. Initial baseline studies revealed several problems. Originally, during the initial development of the nearly implicit solution scheme, inclusion of artificial viscosity in the formulation of the momentum flux terms of the discretized momentum conservation equations was considered unnecessary. This hypothesis was disproved, and implicit artificial viscosity was implemented and is still being tested. An inconsistent implementation of the boron transport equation was also detected, and the equation was rewritten in implicit form. A recent improvement to the semi-implicit solution scheme involving resolution of fluid temperature perturbations as noncondensibles appear was also retrofitted into the nearly implicit scheme. This retrofit is currently being tested and will be completed subject to availability of followon funding in FY-96.

Code failures and advancement repeats attributed to numerical discontinuities must be eliminated. In that regard, a two-phase approach was initiated: first, incorporate added implicitness; second, systematically remove discontinuities. The first phase was initiated this year. A Taylor-series expansion of the interphasic momentum drag was successfully implemented in the nearly implicit scheme. The second phase will be initiated next year, subject to funding availability.

Although the Courant-violating nearly implicit time step size offsets the increased computational time relative to semi-implicit advancement, real-time considerations dictated selection of the border-profiled lower-upper (BPLU) direct linear equation solver. Previously, BPLU relied on the sparse matrix solver to compose the solution matrix. BPLU then transcribed the matrix to its desired format. We have completed code architectural modifications to eliminate the need for matrix transcription and are currently testing them.

A two-dimensional reflood heat transfer model was incorporated into early versions of RELAP5 to track quench front propagation during core reflood/rewet following a large break loss-of-coolant accident (LBLOCA). Owing to NRC indifference toward RELAP5 as an LBLOCA code, the model had fallen into disrepair. The model was restored to operability and 
brought into conformity with other modeling improvements in the areas of heat transfer correlations, flashing and condensation at the wall, and implicit coupling between heat transfer and hydrodynamic solutions.

The project objective of realtime RELAP5 simulation is expected to be realized in FY-96, subject to the availability of follow-on funding.

New or Increased Technical Capabilities

This LDRD is consistent with the INEL's mission in derived-use technology transfer. The commercialization of RELAP5 to nuclear plant training simulators will allow the consolidation of the computational basis for plant licensing and operator training. Full-scope RELAP5 simulation will not only enlarge Laboratory visibility in the commercial nuclear industry, but a real-time RELAP5 analysis capability will also enhance cost-competitiveness with traditional governmental sponsors.

Business Development Opportunities

Upon CRADA completion, Lockheed Idaho will license RELAP5 to SAIC for nuclear plant simulation field-of-use applications. SAIC will market/install RELAP5 on full-scope nuclear plant training simulators with time and materials support from the INEL. It is anticipated that fees derived from membership subscriptions to a proposed Users Group will support the expansion of the business and the services of a full-time Laboratory-based code developer. End-user software support subcontracts through SAIC are a possibility. Eventually, this may expand to include maintenance and operation subcontract support through SAIC. A spin-off company is a another possibility.

\section{Nonfertile Fuel Fabrication Development and Irradiation Performance Assessment} LDRD 2207

$$
\text { G. S. Chang }
$$

The United States and Russia expect to have a surplus of approximately 150 metric tons ( $t$ ) of weaponsgrade plutonium (WGP) and $1000 t$ of weapons-grade uranium (WGU) (National Academy of Sciences Committee on International Security and Arms Control, Management and Disposition of Excess Weapons Plutonium, National Academy Press, 1994). One of the most favored candidate methods for disposing of the WGP is to blend it with natural oar depleted uranium down to commercial grade (up to 7 weight percent WGP) for light water reactor (LWR) fuel pellet fabrication. However, this approach, with a conversion ratio of 0.6 , will produce a lot of plutonium and other actinides in the spent fuel. This process only transforms the weapons-grade fissile materials to civilian-grade plutonium, which is still usable for fission weapons, so it does not completely solve the plutonium disposition problem. Disposition of weapons-grade plutonium in reactors without fertile material has been proposed by industry and national laboratories. This study describes a new actinide-reduced plutonium fuel (ARPF) that would use WGP mixed with medium-enrichment $\mathrm{UO}_{2}$ and the nonfertile material tungsten to achieve conversion ratio less than 0.1. ARPF can meet the WGP disposal goal while minimizing the plutonium production. Its physics and burnup characteristics are analyzed and the results are compared with $\mathrm{LWR} \mathrm{UO}_{2}$ and mixed-oxide fuel.

To maximize the use of current LWR technology, the design goals for $A R P F$ are to retain the lattice configuration of a Westinghouse PWR (a 17 $x 17$ fuel-element array), and to achieve a neutron energy spectrum and boron worth similar to those of $L W R \mathrm{UO}_{2}$ fuel. The ARPF is designed to achieve a conversion ratio $<0.1$. The design criteria for the advanced WGU/Pu-A/ $\mathrm{O}_{2} \mathrm{O}_{3}+\mathrm{W}$ fuels are (a) the volume fractions of tungsten and WGP should be adjusted such that the $k_{\infty}$ of lattices are close to the reference cases, (b) the neutron flux spectrum in the advanced $W G$ fuels should have a shape similar to those in the reference cases, (c) the boron reactivity worth should not be significantly different from that in the reference cases, (d) the $240 \mathrm{Pu} / \mathrm{Pu}$ ratio at the end of fuel cycle should meet the spent fuel standard for WGP disposition, and, most importantly, (e) the plutonium production in the spent fuel should be as small as possible, so that the advanced WG fuels can be qualified as once-through, nonreprocessing fuels.

In this project, tungsten (W), which has the highest resonance absorption power (the ratio of resonance absorption to thermal absorption) among candidate materials, is proposed as a substitute for $238 U$. It has been shown that this substitution can achieve neutronic characteristics similar to the low enriched $\mathrm{UO}_{2}$ fuels as in commercial LWRs. The plutonium transmutation characteristics of the proposed ARPF in a mixed pressurized water reactor) PWR) core are investigated using a MCNP checkerboard model and the ORIGEN2 computer code. To meet the WGUMGP disposal goal, spent fuel standard, while minimizing plutonium production, reducing proliferation risk, and having a clean spent fuel, a once-through ARPF cycle is proposed using the WGP and nonfertile tungsten.

\section{Objectives}

- Demonstrate ARPF, including tungsten with zircaloy cladding, has neutronic characteristics similar to those in LWRs and meets the fuel design criteria. 
- Document the work in a technical report and submit it to Nuclear Technology for publication.

\section{Accomplishments}

A study using a checkerboard pin-by-pin fuel assembly model (CBFAM) to analyze the ARPF and $\mathrm{UO}_{2}$ fuels depletion indicates that a representative equilibrium fuel cycle can be developed in which a $\mathrm{UO}_{2}$ driver core occupies three quarters of the total core and an ARPF region occupies the remaining one quarter of the core. This reactor operates in a threeregion cycle mode in the $\mathrm{UO}_{2}$ fuel zone and a one-region cycle mode in the ARPF zone. for a typical PWR (3411 MW $\mathrm{MW}_{\mathrm{t}}$ ), the equivalent total neutron flux in the ARPF assembly is $2.88 \times 10^{14} \mathrm{n} / \mathrm{cm}^{2}-\mathrm{s}$. The normalized fission power ratios in zones $1,2,3$, and 4 are $0.879,1.043,1.132$, and 0.946, respectively. Using MCNP and ORIGEN $2, \mathrm{k}_{\infty}$ and the concentrations of important isotopes as a function of burnup have been calculated. For ${ }^{240} \mathrm{Pu}$, because of considerable self-shielding with increasing nuclide density toward end of cycle, the capture cross-section is much smaller. Because the ARPF fuel assembly only occupies one quarter of the reactor core, $k_{\infty}$ does not decrease as rapidly for this core as it does for most proposed nonfertile MOX fuel cases. If the ARPF fuel assembly occupies one half of the reactor core, $\mathrm{k}_{\infty}$ would decrease from 1.128 $( \pm 0.0015)$ to $1.0935( \pm 0.0016)$. It still can achieve an adequate equilibrium fuel cycle. An investigation of ARPF half-core loading is currently under way. The atomic density of plutonium isotopes and $240 \mathrm{Pu} / \mathrm{Pu}$ ratio as a function of burnup have also been calculated. At the end of fuel cycle, the ${ }^{240} \mathrm{Pu} / \mathrm{Pu}$ ratio in ARPF reaches $27.49 \%$, which meets the spent fuel standard in the WG-Pu disposition.

\section{New or Increased Technical Capabilities}

This project has established the technical feasibility of using the nonfertile plutonium fuel ina LWR. Furthermore, through publications and presentation related to this project, our technical expertise in now known within the plutonium nonfertile fuel $R \& D$ community.

\section{Business Development Opportunities}

The Monte-Carlo methodology to develop for mixed-core equilibrium fuel cycle analysis can enhance the capability of INEL to perform $\mathrm{LWR} \mathrm{UO}_{2}$ fuel cycle analysis with mixed oxide or nonfertile fuel for weapons-grade nuclear materials disposition (fission option), and triple-play reactor core design and analysis. Further development of MonteCarlo methods to reactor core analysis can be beneficial to the nuclear industry in the area of fuel reloading analysis.

\section{Photoneutron Studies for Radiotherapy LDRD 2302

$$
\text { D. W. Nigg }
$$

This project is directed toward establishing the basic feasibility of a novel approach to the realization of a practical accelerator-based neutron source for epithermal-neutron capture therapy using electron accelerator technology.

\section{Objectives}

A recent resurgence of interest in neutron radiotherapy for cancer (in the forms of fast neutron radiotherapy and epithermal neutron capture therapy (NCT)) has led to increased activity directed toward the development of reliable, costeffective accelerator-based neutron sources. An enormous amount of work worldwide has been focused on the achievement of useful medical neutron sources featuring the use of protons and other heavy particles impinging on various targets. Clinical sources for fastneutron therapy have been built using this approach and some low-intensity prototypes of epithermal-neutron sources for NCT have been demonstrated. The heavy-particle approach, especially in the case of NCT applications, is, however, subject to problems associated with generating the required source intensity, as well as to some rather difficult problems associated with target cooling. This project is directed toward proof of principal for an alternate approach to the realization of clinically useful neutron sources for medical therapy using standard electron linear accelerator technology rather than heavy particle accelerator technology. Electron accelerators offer the possibility of generating tunable neutron sources in a manner that reconciles the often-conflicting objectives of target cooling, neutron source intensity, and spectral purity by way of a two-stage process that involves the use of electron-beam-induced bremsstrahlung radiation directed onto a material having a low threshold and high cross section for photoneutron production.

\section{Accomplishments}

In an ongoing collaborative effort with Idaho State University (ISU), the INEL has been exploring the applications of electron accelerators in a variety of areas including arms control, nondestructive examination, nuclear waste characterization, and, with the effort described here, radiation 
oncology. A 4-MeV, four-chamber, side-coupled electron linear accelerator driven by a magnetron $\mathrm{RF}$ power supply is available at ISU. This was used for some of the initial photoneutron source measurements described below. In addition, INEL has installed a much more powerful and flexible Varian electron linear accelerator at ISU. This has been used in the project to provide additional, extensive, measured data on photoneutron source intensities and spectra produced in a heavy water conversion region with and without a surrounding epithermal neutron filtering assembly. Measurement techniques used include indium foil activation and threshold foil activation. Corresponding theoretical calculations are performed using the ACCEPT electron-photon cascade code, coupled by way of theoretical photoneutron production data to the MCNP Monte Carlo NeutronPhoton Transport code, as well as the DORT two-dimensional discreteordinates neutron and photon transport code.

We completed measurements and theoretical calculations of the unfiltered photoneutron source produced in a heavy water conversion region exposed to the bremsstrahlung field generated by the standard 4-MeV medical electron linear accelerator with a tungsten target. Later, we repeated these measurements using a tunable Varian electron linear accelerator in order to obtain more detailed neutron source intensity and spectral data for three bremsstrahlung cutoff energies (4-MeV, 6- MeV, and 8- MeV) and two conversion region volumes. In addition, appropriate quantities of an advanced epithermal-neutron filtering material composed of aluminum and aluminum trifluoride were obtained as part of an ongoing collaborative effort on medical neutron sources with the Technical
Research Centre of Finland. An epithermal filtering assembly based on this material was constructed for use with the Varian accelerator-based photoneutron production apparatus and measurements of filtered epithermal neutron spectra appropriate for NCT applications have been performed for various electron beam energies and filtering configurations. The results of all measurements are being compared with theoretical calculations. This provides the basic performance data needed for proof of principal of the epithermal photoneutron device described in EG\&G Patent Idea Record EGG-PI-675, which, in turn, served as the basis for a formal patent application for the concept that has been filed by the Lockheed Idaho investigators with the U.S. Patent Office (Docket 08-237504).

New or Increased Technical Capabilities

This project involves strong collaboration between INEL and Idaho State University (shared equipment, laboratory space, and personnel). We have gained much experience in measurement and analysis of this type of neutron source and have taken the steps needed to capture the intellectual property. When the time for scaleup comes, we will be in a unique position to perform the work.

\section{Business Development Opportunities}

There is currently informal collaboration with Atomic Energy of Canada, Ltd., for the purpose of exploring the feasibility of a follow-on scale-up program to realize a clinically useful full-scale device should the results of ongoing BNCT clinical research lead to where epithermal-neutron BNCT becomes a therapy of choice for primary brain tumors or, possibly, other malignancies. AECL manufactures large electron linear accelerators for food irradiation and instrument sterilization. They are very interested in becoming involved in the neutron therapy field. Other opportunities to scale up the device to clinical levels will present themselves, provided that a clinical demand for epithermalneutron BNCT itself materializes.

\section{Feasibility Study of Using INEL-Developed Transport Tools to Calculate Photodynamic Dosimetry LDRD 2303}

W. Y. Yoon, D. W. Nigg, F. J. Wheeler, L. M. Montierth

Photodynamic therapy (PDT) is the interoperative use of directed laser light and photosensitive drugs to selectively treat cancerous tumors and other tissue abnormalities. Pretreatment optical transport calculations are required to provide the optimal treatment, i.e., the tumor receives a maximum treatment dosage while exposure to normal tissue is minimized. Feasibility of using INEL-developed light transport tools and computational methodology to calculate photodynamic dosimetry has been assessed. We chose The PHOTRAN computer code developed for the INEL's nuclear pumped laser program and undertook major reprogramming to obtain not only the optical boundary effects such as reflection and refraction but also light scattering and absorption in the medium. This Monte Carlo code can now calculate the light flux and energy deposition distributions in threedimensional geometry for any shape of laser beam source. By having this computational capability, the INEL is positioned to provide a practical method for computational dosimetry of PDT. Future effort is in collecting relevant optical parameters in various media, and code validation and verification. Implementation of various variance-reduction techniques are also desirable. 


\section{Objectives}

- Perform a literature search.

- Develop a computational methodology.

- Identify a primary computational code.

- Implement the computational methodology into the code.

\section{Accomplishments}

Photodynamic therapy (PDT) is a new modality being studied for the treatment of local solid tumors. Patients receive an injection of a photosensitizer into a vein. After 12 hours, the photosensitizer is cleared from most healthy tissue, but is retained in the tumor, which is then exposed to activating light. The laser beam acts as a concentrated source of red light. The laser is, however, not used to heat the tumor or to vaporize the tumor like other more powerful lasers that are used in surgery for these purposes.

Pretreatment optical transport calculations are required to provide the optimal treatment, i.e., the tumor receives a maximum treatment dosage while exposure to normal tissue is minimized. Unfortunately, current methods used to calculate optical dosimetry are oversimplified making it difficult to correctly determine dosage.

Computational Methodology.

To serve the needs of photodynamic dosimetry calculations, a three-dimensional photon transport code must be capable of tracking light photons through an optical medium. The Monte Carlo method is the model of choice for simulating light transport. The prime issues in the light transport are the optical boundary effects (reflection and refraction) and light scattering and absorption in the optical medium. When a photon has an interaction in the medium, one of two events would occur, scattering or absorption. The probability of interaction depends on the local cross section of the medium. An absorption event requires that both the location and the amount of light absorbed be recorded, which determines the dose distribution in the medium.

The flow chart on the following page presents the three-dimensional Monte Carlo technique of light photon transport.

\section{Code Implementation.}

Our primary focus was on developing a practical method for computational dosimetry of PDT, specifically the application of the PHOTRAN computer code developed by the INEL. We implemented the solutions of Snell's law and Fresenel equations to obtain the direction and intensity of the reflected and refracted lights. We performed an experiment using the $\mathrm{He}-\mathrm{Ne}$ laser, and the code reproduced the experimental results within the experimental uncertainties. Since the scattering and absorption play a more important role in the PDT application, this part of optical transport is implemented in the program following the flowchart. The initial PHOTRAN program was inherited from RAFFLE, the general purpose Monte Carlo code for neutron and gamma transport and included many options and routines relating to neutron and gamma physics. A major cleanup of the program was undertaken to make the code specifically applicable to general light transport. The program now calculates light flux, current and energy deposition distribution in three-dimensional geometry for any shape of laser beam source and can be considered a general purpose light transport code. Using the typical optical cross sections (scattering and absorption) and a phase function in tissue, the code was successfully tested with a three-dimensional geometry and a disk laser beam source. Implementation of various variance reduction techniques are still needed to improve code efficiency. Code validation and verification also has to be performed for general availability.

New or Increased Technical Capabilities

There is opportunity for the INEL to provide this tool and opportunity to compliment the INEL Boron Neutron Capture Therapy program. The project supports continued INEL interests in exploring light transport applications.

\section{Business Development Opportunities}

The need for better methods to determine dosimetry was brought to our attention by Dr. Warren S. Grundfest, M.D., during his visit to the INEL. We need to establish collaboration with Dr. Grundfest and other researchers at the Cedars-Sinai Medical Center and learn as much as necessary to correctly calculate PDT dosimetry. This will include information on photosensitive pharmaceuticals, laser wavelengths of interest (determined by the photosensitizer used), treatment geometry, etc. Once the validation and verification of the code and applicability to PDT is established, we will propose a formal CRADA with Cedars-Sinai Center or another appropriate clinical partner.

\section{Kinetic Modeling of the ITER Dissipative Divertor LDRD 2304 \\ L. M. Montierth}

The purpose of this project is to develop a numerical simulation code to model the International Thermonuclear Experimental Reactor's (ITER) 


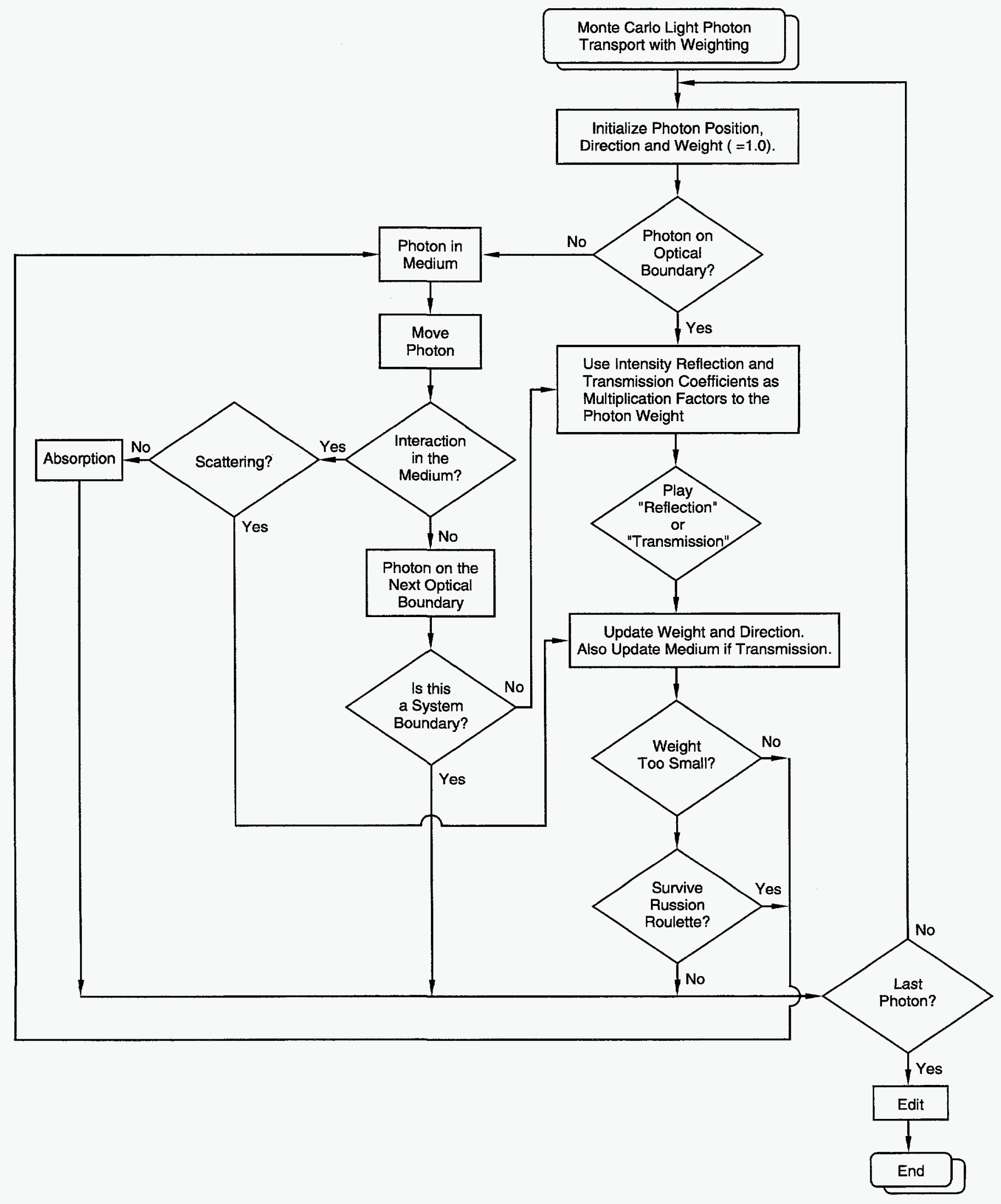

E96 0074 
dissipative divertor with a kinetic technique. ITER is a large international project funded jointly by the United States, Europe, Japan, and Russia, and the success of this project depends largely on the performance of the divertor. Since there will be no experiments at reactor geometry and power before the construction of the reactor, design of the divertor will depend heavily on numerical simulations. Because of the large mean-free paths of the particles in the reactor, kinetic modeling will be required to obtain accurate results. The project is designed to position the INEL to have a role in the design of the dissipative divertor for ITER.

\section{Objectives}

- Develop a two-dimensional kinetic code (one spatial dimension and one velocity dimension 1D1V) to model ion-electron interactions.

- Develop a three-dimensional (one spatial dimension and two velocity dimensions 1D2V) single particle code with advanced physics.

- Prepare an article for the Journal of Computational Physics describing our results.

Accomplishments

During this project, some results were obtained from the 1D2V code development results, but the numerical difficulties associated with the large problem size and the older numerical methods restricted progress. Because of these problems, more work was shifted to the 1D1V work.

For the 1D1V work, a new numerical technique was implemented and tested. The method implemented was a matrix-free implementation of the NewtonKrylov technique. This numerical method was originally tested on an ion only problem. The results were submitted to the Journal of
Computational Physics on March 10 , 1995 in an article titled Fully Implicit Kinetic Solution of Collisional Plasmas. The paper shows that this technique results in fast solutions that can be obtained on a workstation.

As work progressed toward a coupled ion-electron problem, it became apparent that more accurate differencing of the differential operators was necessary. These higher-order differencing methods were implemented and tested on simpler test problems, two species 0D1V, and single species Vlasov operator only.

This work is currently all being combined to solve a 1D1V ion-electron problem. The new difficulties of the coupled ion-electron problem are currently being worked.

New or Increased Technical Capabilities

The numerical integration technique, matrix-free NewtonKrylov, has shown promise for solving integro-differential equations, but further testing and development is needed. The higher order differencing schemes investigated in this project have enabled solutions that were not previously available.

\section{Business Development Opportunities}

Further development of the 1DIV ion-electron kinetic simulation code needs to be done before potential customers can be approached.

\begin{tabular}{l}
$\begin{array}{c}\text { Automated } \\
\text { Neutron Probe } \\
\text { Monitoring } \\
\text { LDRD } 5304 \\
\text { C. W. Bishop }\end{array}$ \\
\hline
\end{tabular}

Technology to automate the hand-held CPN Hydroprobe was developed. Neutron probe monitor- ing is used to determine moisture contents and changes in moisture levels in vadose zone soil and rock environments. Prior to the development of this technology, moisture monitoring involved the use of a manually operated system requiring manual feeding and retrieval of heavy cable (in some cases hundreds of feet) into and from the neutron probe access tubes installed into the subsurface. The manual system was slow, labor and time intensive, and subject to numerous operator errors. The prototype monitoring system, developed through $\angle D R D$ funding, largely eliminates the above drawbacks while permitting more frequent monitoring at reduced cost. The prototype well logging system features computer-controlled instrumentation that can be preprogrammed to operate and control the entire neutron logging operation. The system will allow preselection of logging intervals and storage of the information on disk. In the field, the system is located over the well to be logged, the disk placed in the computer, and the logging operation is initiated. From this point on, the entire operation is computer controlled. The computer raises and lowers the cable; takes, records, and stores the neutron counts; and finally signals the operator that the well has been logged. The operator can look at the plotted real-time data displayed in a second window and compare it with a background log, facilitating field-based decisions. Upon completion of the logging operation, the cart is moved to another well, and the process is repeated.

\section{Objectives}

- Design the automated hydroprobe cart. The cart was mechanically designed to be strong, easily mobile, and permit flexibility in placement of the probe and cable over the well.

- Develop the control system. A PC-based, stand-alone control system was developed to control the entire logging 
operation. The system includes data acquisition for both input and output data, customized displays, and automated well logging.

- Integration of the hardware and control systems. This includes (a) connecting a laptop computer on the cart, (b) installing an encoder wired to the computer, (c) installing a dc motor to operate the electric winch, (d) installing a sensor weight measuring device.

- Development of a two-way communication link between the hydroprobe and the computer. The link allows communication between the computer and the probe, and the probe and the computer.

- Field test the prototype.

\section{Accomplishments}

The hydroprobe cart was fashioned from a step ladder (see the figure). The step latter was selected as an initial frame because with the addition of wheels it is highly maneuverable and allows precise placement of the probe over the borehole. All the instrumentation, including the electronic winch, load cell, cable spool, encoder, and associated electronics are mounted on the frame. All the hardware was successfully integrated so that the technology functions as a unit. The control program has been written, which drives the hardware and permits communication between the probe and the computer. The program allows preselection of monitoring intervals in wells of any depth. Neutron counts are recorded on the computer hard drive, eliminating the necessity for recording raw counts in logbooks or entering them into the instrument storage. By looking at the main control screen, the operator can determine current logging depth, counts, and tension on the cable. The screen also shows the preselected parameters-borehole depth and count time. By opening a second window, a graphing capability can be accessed. The graphing capability permits easy, meaningful access to the data as they are being collected. The data can be compared with a baseline $\log$ to identify changes. Immediate access to the data in a usable format (counts or moisture contents) allows the operator to make decisions while still in the field and permits rapid identification of errors. Compared with the manual system, using this technology reduces logging time by about one third in a shallow borehole. Time savings for logging deep boreholes are even greater. Time saved is increased when data processing is considered. This technology allows much of the processing to be performed at the borehole site, whereas the manual system requires returning to the office, entering the data, and processing on the computer. The technology is currently performing as described above. During field testing, however, several problems were identified. We believe a modulator needs to be added to the motor to achieve desired accuracy. The work is ongoing. If we are allowed to purchase the required hardware and can field test the technology, we expect to have a fully functioning system that can be used to monitor deep boreholes at the INEL. An LDRD submitted for FY-96 funding proposes to adapt aspects of the technology for use in monitoring through horizontal

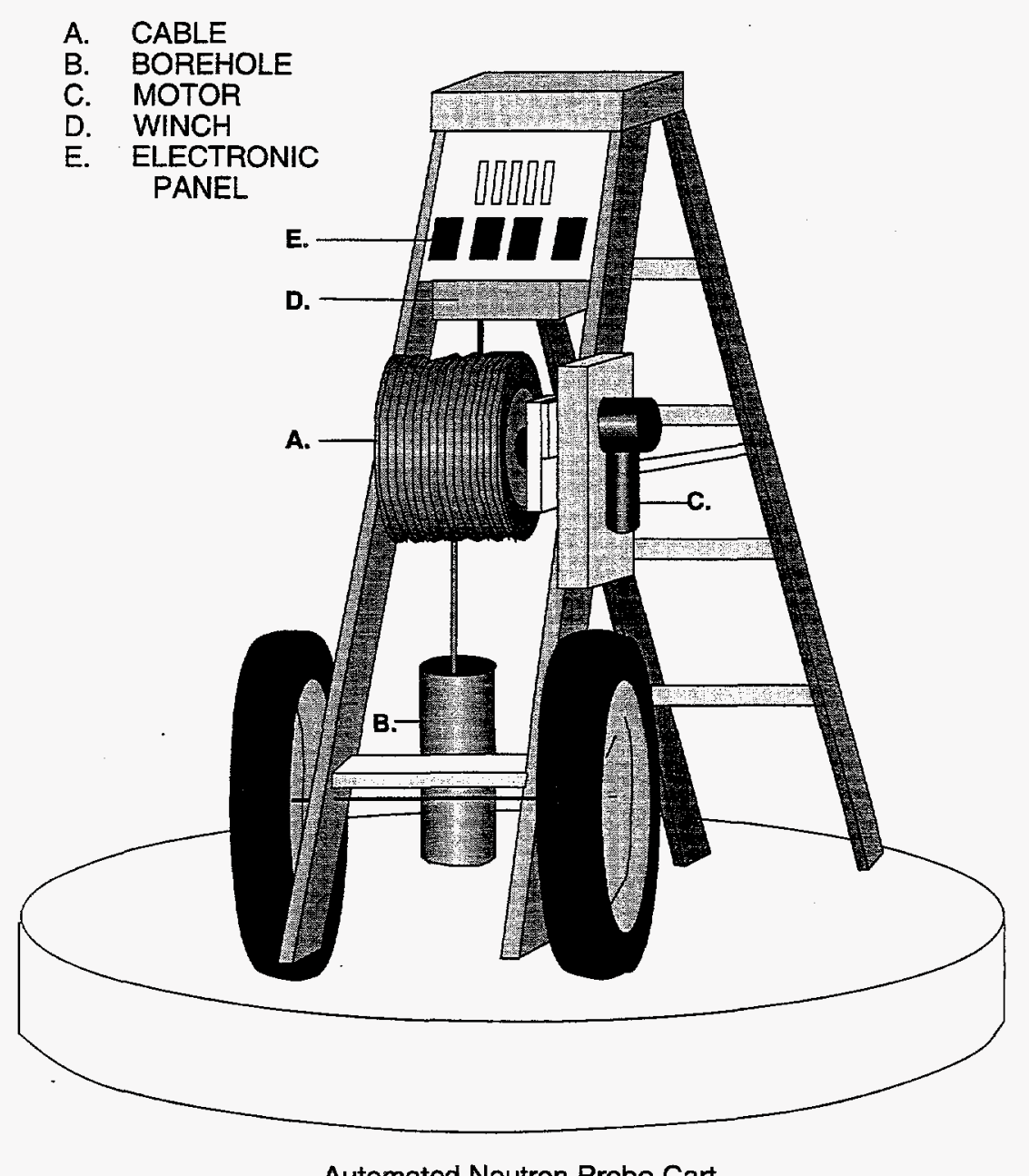

Automated Neutron Probe Cart 
boreholes or pipes. This is the basis for remote monitoring of moisture and is directly transferable for automated monitoring of any parameter for which small sensors are or will be available.

\section{New or Increased Technical Capabilities}

Development of this technology for small sensors in vertical boreholes in general and for the neutron probe in particular is new at the INEL, and anywhere else (as far as we can determine from the technical literature). This technology currently works, and when finished it will function with repeatable, millimeter accuracy. The control programming currently works flawlessly. This is the basis of automating the use of all small sensors that are used in vertical boreholes or pipes. We hope to adapt the program and other aspects of the technology for use with neutron probes and other small sensors in horizontal boreholes and pipes. The technology will be coupled with robotics technology to achieve these goals. Ultimately, we are working to develop an automated system that can be used in any borehole-like environment with any small sensor for remote monitoring, with the capability of transmitting data to the user over telephone lines.

This technology will catapult the INEL into a world leader position in automated small sensor monitoring. It also has application to work at the INEL in waste management, ER, agriculture, and other fields. Even though this technology will benefit INEL work, the industrial applications are far broader.

\section{Business Development Opportunities}

The investigators have not yet explored business opportunities. The focus has been to develop the technology to a marketable state. Boart Longyear Company has and continues to collaborate on development of this technology and are interested in it. The potential for licensing the technology and/or the development of a CRADA with Boart Longyear Company is real, but so far these opportunities have not been pursued. Opportunities are available with other industries as well, as evidenced by the interest shown in Denver at ER-95.

\section{Tritium Production by Irradiation of ${ }^{3} \mathrm{He}$ LDRD 7909}

\author{
R. A. Anderl, J. D. Baker, \\ J. W. Rogers, B. G. Schnitzler
}

The primary purpose of this project was to evaluate key issues that relate to production of tritium by irradiation of ${ }^{3} \mathrm{He}$ in a nuclear reactor, in particular, the Advanced Test Reactor (ATR) at the Idaho National Engineering Laboratory (INEL). Issues specifically addressed in this work were (a) tritium production rates and recovery yields and (b) reactor operational safety concerns resulting from an accidental loss of ${ }^{3} \mathrm{He}$ from an incore irradiation vessel. The emphasis of the work was on the first issue, with limited evaluation of the second. Both experimental and modeling/calculation efforts were undertaken. The experimental effort entailed irradiation of ${ }^{3} \mathrm{He}$-filled canisters and cobalt wires for neutron dosimetry in the ATR Critical reactor (ATRC) during FY-94 and postirradiation measurements and analyses in FY-95 to determine the quantities of tritium generated in each canister and to determine the thermal neutron fluence-rate (flux) profiles in the irradiation assemblies. A full-core, 3-dimensional model was developed to permit MCNP calculations of the neutron interaction rates in the ${ }^{3} \mathrm{He}$ and in the cobalt flux wires. Comparison of the experimental and calculational results indicate good consistency between global values for tritium production and dosimeter activation. Only a limit- ed evaluation of the operational safety issue was made, primarily by modeling/calculations. The results of this work are of particular significance to a novel approach for generating tritium by using a recirculating ${ }^{3} \mathrm{He}$ loop in a nuclear reactor or acceleratorbased tritium production facility.

\section{Objectives}

- Complete postirradiation measurements and analyses to determine the tritium contents of each irradiation canister.

- Complete postirradiation measurements and analyses for the cobalt neutron flux monitors to determine the neutron fluence rates for the experiment.

- Complete the model and perform calculations of the neutron interaction rates in the ${ }^{3} \mathrm{He}$ and in the cobalt flux wires.

- Evaluate the operational safety issue resulting from an accidental loss of ${ }^{3} \mathrm{He}$ from an incore irradiation vessel filled with ${ }^{3} \mathrm{He}$.

- Prepare a technical report that documents the work.

\section{Accomplishments}

The focus of effort during FY-95 was on completion of the postirradiation measurements and analyses and on neutronics modeling and calculations for six ${ }^{3} \mathrm{He}-$ filled canisters irradiated in the ATRC during FY-94. Measurement of the tritium contents of each canister required a laboratory setup to purge the canister contents with a $\mathrm{He} / \mathrm{H}_{2}$ gas mixture, heat the canister to release surfaceadsorbed tritium, and collect the tritium as the oxide in ethylene glycol. Liquid-scintillation assay of the ethylene glycol yielded the tritium content. After the cobalt flux wires were retrieved from the 
canisters, they were cut into sections, and each section was assayed radiometrically to determine the induced ${ }^{60} \mathrm{Co}$ activation product. Each canister was equipped with an axial cobalt wire and three circumferential wires at the top, middle and bottom parts of the canister wall. A spatial profile of the thermal flux distribution for each canister was determined from the measured activation data. A fullcore, 3-dimensional model was developed, with explicit detailing of the irradiation canister test trains. MCNP calculations were made to determine neutron interaction rates in the ${ }^{3} \mathrm{He}$ and in the cobalt flux wires. A comparison of the experimental and calculational results, shown in Tables 1 and 2, indicates good consistency between global values for tritium production and dosimeter activation. The results indicate a possible underprediction of neutron flux-depression effects. However, the results of these experiments do indicate that tritium production and recovery can be accurately quantified for ${ }^{3} \mathrm{He}$-filled canisters in the ATR.

A limited evaluation was made of the operational safety issue related to an accidental loss of ${ }^{3} \mathrm{He}$ from an in-core vessel. Based on MCNP calculations by $M$. Houts of Los Alamos National Laboratory (LANL), for a $3.64-\mathrm{cm}$ diameter canister located in a central, fluxtrapped hole of a TRIGA reactor, a canister filled with ${ }^{3} \mathrm{He}$ to 50 atmospheres has a reactivity worth of about two cents relative to a voided canister. Houts has demonstrated a novel means to reduce the positive reactivity insertion from loss of ${ }^{3} \mathrm{He}$ by incorporating a cruciform or spoked borated stainless steel assembly in the irradiation vessel. Owing to unavailability of appropriate personnel, experiments and model calculations were not made to evaluate this opera-

Table 1

Comparison of Calculated-to-Measured Tritium-Production Data

\begin{tabular}{|c|c|c|c|c|}
\hline Canister & $\begin{array}{c}{ }^{3} \mathrm{He} \text { Pressure } \\
\text { (Torr) }\end{array}$ & $\begin{array}{c}\text { Measured } \\
\text { (microcurie) }\end{array}$ & $\begin{array}{l}\text { Calculated } \\
\text { (microcurie) }\end{array}$ & $\begin{array}{l}\text { Calculated/ } \\
\text { Measured }\end{array}$ \\
\hline \multicolumn{5}{|c|}{1.5 liter canister } \\
\hline |-11 (top) & 2150 & 61 & 53.0 & 0.87 \\
\hline $1-11$ (mid) & 986 & 50 & 60.6 & 1.21 \\
\hline I-11 (bot) & 1500 & 54 & 56.4 & 1.04 \\
\hline \multicolumn{5}{|c|}{0.5 liter canister } \\
\hline $\mathrm{I}-13$ (top) & 2150 & 33 & 31.7 & 0.96 \\
\hline $1-13$ (mid) & 775 & 21 & 30.0 & 1.43 \\
\hline l-13 (bot) & 1505 & 30 & 32.8 & 1.09 \\
\hline
\end{tabular}

Table 2

Comparison of Calculated-to-Measured ${ }^{60} \mathrm{Co}$ Dosimeter Activation Data.

\begin{tabular}{|c|c|c|c|c|c|}
\hline Canister & $\begin{array}{c}{ }^{3} \text { He Pressure } \\
\text { (Torr) }\end{array}$ & Axial & $\begin{array}{l}\text { Lower } \\
\text { Circum. }\end{array}$ & $\begin{array}{l}\text { Middle } \\
\text { Circum. }\end{array}$ & $\begin{array}{l}\text { Upper } \\
\text { Circum. }\end{array}$ \\
\hline \multicolumn{6}{|c|}{1.5 liter canister } \\
\hline 1-11 (top) & 2150 & 1.31 & 1.07 & 1.07 & 0.90 \\
\hline $1-11$ (mid) & 986 & 1.07 & 1.14 & 1.09 & 0.95 \\
\hline $1-11$ (bot) & 1500 & 1.33 & 1.16 & 1.16 & 1.10 \\
\hline \multicolumn{6}{|c|}{0.5 liter canister } \\
\hline I-13 (top) & 2150 & 1.19 & 1.01 & 1.04 & 0.98 \\
\hline $1-13$ (mid) & 775 & 1.21 & 1.02 & 1.01 & 1.08 \\
\hline 1-13 (bot) & 1505 & 0.95 & 1.07 & 1.18 & 1.09 \\
\hline
\end{tabular}

tional issue specifically for the ATR. The work scope and expenditures were reduced accordingly.

A technical report, Tritium Generation by Irradiation of ${ }^{3} \mathrm{He}$ in the ATRC, INEL-95/0495, was prepared to document this work.

\section{New or Increased Technical Capabilities}

The work in this project is relevant to both reactor-based and accelerator-based tritium production (APT). DOE Defense Programs is developing a strategy to maintain a tritium stockpile appropriate to defense needs. Tritium production based on ${ }^{3} \mathrm{He}$ could serve as a very attractive interim supply approach for a fission reactor, and it is the target of choice for an accelerator- based tritium production system. The project has demonstrated experimental and computational capabilities and expertise at the INEL that can support new tritium production needs for the Defense Program.

\section{Business Development Opportunities}

In collaboration with $D$. Mecham of the ATR Reactor Programs business development, we have pursued DOE funding to conduct demonstration testing in support of the Defense Programs tritium strategy. The effort was pursued as an interlaboratory effort involving primarily INEL and LANL. Funding actions depend on tritium strategy developments. Presentations about this project were made at an APT workshop in January, 1995, and at the Tritium Focus Group Meeting in April, 1995, both at LANL. We have had discussions with Dr. J. Anderson, director of the APT tritium target and process system at LANL, regarding the ATRC ${ }^{3} \mathrm{He}$ experiments and INEL expertise in the development and use of metal getters to recover tritium from inert gas process streams. A strong possibility exists to obtain funding through LANL to pursue cooperative work in support of APT during FY-96. 


\section{Technetium Separation System \\ LDRD 9517 \\ R. G. Bennett}

This invention provides an environmentally benign system for producing high-purity technetium- $99 \mathrm{~m}$, widely used in nuclear medicine. Unlike reactor production of technetium-99m, this system uses accelerator technology to produce technetium-99m without spent nuclear fuel wastes. The system can produce quantities to supply an entire metropolitan area. A network of distributed production centers can deliver a far more reliable supply than a system based on a single nuclear reactor.

\section{Objectives}

A limited set of technical objectives was undertaken owing to the very short (2-month) duration of the project.

- Calculate expected production levels.

- Explore separation technologies and synergy with accelerator production.

- Define key experiments needed for future development.

- Peer review the production concept.

\section{Accomplishments}

The major technical accomplishments were a peer review of our concept conducted at the end of the fiscal year, a patent idea record prepared and nearing completion of reviews and approvals, a list of about two dozen experiments and analyses needed to achieve proof-of-concept, and a pilot demonstration of the system. These were delivered to the peer review team for comment.

In the effort to calculate production levels, we were able to verify one related experiment reported in the recent literature and to establish the feasibility of the desired production scale. It also helped to identify various nuclear cross sections that need to be known with more accuracy, especially for the further consideration of impurities in the product.

In the effort to survey chemical separation technology, we identified at least four candidate technologies: chromatographic (alumina), solvent extraction, sublimation, and chromatographic (gel) methods. We explored each of these at a reasonable level of detail and achieved a fairly indepth understanding of the various advantages and disadvantages of each. The synergy of each with respect to accelerator production was explored.

\section{New or Increased Technical Capabilities}

This project assembled an integrated product team with expertise in small accelerator applications, physical chemistry, radiochemistry, mechanical design, and systems engineering. While these are existing capabilities at the INEL, the value of this project has been to work collectively on a concept for product development that can potentially deliver a significant research project.

\section{On-Site \\ F-18 Production \\ LDRDW030 \\ J. L. Jones, W.Y. Yoon, J. F. Harmon}

An electron accelerator-based system has been assessed for the photonuclear production of the medically useful fluorine-18 isotope (a valuable, positron emitting radioisotope) at the end-user site (hospital) to support patient diagnostic medicine applications, especially for Positron
Emission Tomography (PET). Various natural fluorine-containing compounds were experimentally and numerically assessed as possible candidate production materials using the INEL 13-MeV Varitron linear electron accelerator. A separation method was selected that is directly compatible with existing, medically approved radiochemistry methods at PET centers. A liquid fluorocarbon in de-ionized water has proven to be the most promising for producing single patient $F-18$ treatment needs, using either a fully transportable (site-to-site) system, or for use at sites having existing $x$-ray therapy systems and basic radiochemistry capability. A conceptual transportable system design is presented. A market analysis of PET applications has projected world wide revenues over $\$ 350 \mathrm{M}$ per year as soon as 1998, and has indicated commercial viability for cost-effective, onsite fluorine-18 production. Potential business opportunity collaborations for continued efforts are identified. Future tasks must verify the complete radiochemistry from production to patient drug sample preparation using commercially-practical electron beam energies up to $24 \mathrm{MeV}$.

\section{Objectives}

- Identify primary candidate production materials.

- Identify separation approach.

- Experimental production evaluation.

- Perform market analysis.

- Generate basic conceptual system design.

Accomplishments

Introduction.

PET uses short-lived, positronemitting, radioisotopes to enable detailed in vivo organ visualization and diagnostics of various biomedical processes related to neurology, cardiology, psychiatry, and especially, oncology via the detection of the $0.511 \mathrm{MeV}$ annihi- 
lation radiation. Fluorine-18 (i.e., F-18) is one of several short-lived radioisotopes that are used in PET scans. Specifically, F-18 is used as the workhorse for oncology applications since, in the fluoro-2deoxy-D-glucose (FDG) form, it is well suited for quantitative determination of glucose and amino acid metabolism in cancerous cells. F-18, with its relatively longer halflife, permits adequate drug preparation times, enables a more optimal match to many physiological phenomena in living organisms, and, because of its reactiveness, allows tagging of various medically desirable compounds.

Current F-18 production methods provide multiple patient therapy needs (5-8 $\mathrm{mCi}$ per patient) with an initial bulk source production of $100-400 \mathrm{mCi}\left[{ }^{18} \mathrm{~F}\right]$. F-18 can be produced offsite in a nuclear reactor $\left(>10^{12} \mathrm{n}_{\text {thermal }} / \mathrm{cm}^{2} / \mathrm{s}\right)$ via a combined ${ }^{6} \mathrm{Li}(n, \alpha) \mathrm{t} \rightarrow{ }^{16} \mathrm{O}(\mathrm{t}, \mathrm{n})^{18} \mathrm{~F}$ reaction or by means of a charged particle (i.e., nonelectron) accelerator via the ${ }^{18} \mathrm{O}(\mathrm{p}, \mathrm{n})^{18} \mathrm{~F}$ or ${ }^{20} \mathrm{Ne}(\mathrm{d}, \mathrm{a})^{18} \mathrm{~F}$ reactions; the former is the more common accelerator method. While the reactor method generates higher activity F-18, it also generates as much as seven times higher tritium activity than that of the desired F-18, resulting in additional tritium separation requirements. Also, due to the remote locations of most nuclear reactor sites, a high activity delivery is required to a treatment site. The charged particle accelerator method (i.e., cyclotron) requires an onsite accelerator system with a dedicated operational facility, a costly target ( $\$ 225 / \mathrm{g}$ of $\mathrm{H}_{2}{ }^{18 \mathrm{O}}$ ) and enriched oxygen recovery system for the enriched oxygen method, and a high pressure target system for the neon method.

This study assesses an alternate, cost-effective, photoneutronbased method for onsite F-18 production using commercially available electron accelerators [either already at the medical site (enabling dual use applications: isotope production and $x$-ray therapy) or using ones transported to the medical site]. The latter approach enables expanded PET utilization to remote medical sites. In the photonuclear process the energetic electrons interact in an accelerator converter to generate energetic $x$-rays (bremsstrahlung radiation). The generated $x$-rays interact in a fluorine-19 $(100 \%$ natural abundance) production material to generate F-18 via the photonuclear reaction: ${ }^{19} \mathrm{~F}(\gamma, \mathrm{n}){ }^{18} \mathrm{~F}$ with a photon threshold energy of 10.4 $\mathrm{MeV}$. The resulting ${ }^{18} \mathrm{~F}$ ion will have sufficient recoil energy $(\sim 100$ $\mathrm{keV}$ ) to leave the interaction site and permit separation from the associated base production material.

In this assessment, effort was made to concentrate on inexpensive base production materials, to incorporate only minimal chemistry separation requirements, and use only commercially available products.

Candidate Production Materials.

A study was made of the Candidate production materials and the efficient separation method. Targets examined were $\mathrm{CaF}_{2} / \mathrm{H}_{2} \mathrm{O}$, Teflon $/ \mathrm{H}_{2} \mathrm{O}$ and a liquid fluorocarbon $/ \mathrm{H}_{2} \mathrm{O}$.

\section{Experimental Evaluation.}

Various experimental assessments have been performed at INEL with the primary candidate production materials at standard room temperature and pressure using the INEL Varitron at the Idaho State University Particle Beam Laboratory. While the maximum electron beam energy of this device is limited to $13-\mathrm{MeV}$, it has been quite adequate for this study and has permitted scale-up recommendations.

\section{Market Analysis.}

A market analysis was successfully performed. The overall results will be highlighted in the
Business Development Opportunities section below.

Conceptual System Design.

Based on a 5-8 $\mathrm{mCi}$ per patient drug sample requirement, an electron beam production system appears capable of generating single patient needs with a 1- to 2hour irradiation of a liquid fluorocarbon $/ \mathrm{H}_{2} \mathrm{O}$-based production assembly. Two systems are proposed: a transportable integrated system, and a dual-use F-18 production system. The transportable system is trailer-mounted and integrates the actual photonuclear production, radiopharmaceutical preparation, and (optionally) the PET scanning unit in a single system capable of site-to-site mobility. The second system includes incorporation of a simple, inexpensive liquid fluorocarbon/water assembly with existing high energy $x$-ray therapy systems to enable both radiation therapy and onsite F- 18 production. The application of the latter system will depend on associated onsite facilities to handle subsequent radiochemistry (drug) preparation and the PET scanning operation.

New or Increased Technical Capabilities

- Accelerator technology development supporting a nationally recognized need to provide cost-effectively F-18 for medical applications.

- Opportunity to enhance the INEL Boron Neutron Capture Therapy program by enabling better in vivo B-10 monitoring via F-18 labeling.

- Supports continued INEL interests in exploring the commercialization opportunities in the medical radiopharmaceutical markets.

\section{Business Development Opportunities}

PET is an expensive medical tool since it presently requires a charged particle accelerator (i.e., 
cyclotron) with costs up to $\$ 12 \mathrm{M}$ (excluding the building), a radiopharmaceutical laboratory, and a PET unit (\$1-2 M). An overall system cost reduction is critical to enhancing world wide PET marketability.

Market analysis (performed by the Idaho State University Business School) has shown that the market demand for onsite production of $\mathrm{F}-18$ is derived from the overall demand for PET. Current$1 y$, there are about 200 PET centers around the world ( $\sim 60$ in the U.S.) resulting in a $\$ 139 \mathrm{M} /$ year revenue stream. By 1998, world wide PETuse revenues are expected to be over $\$ 350 \mathrm{M}$ per year with the dominate application in the oncology area.

Because additional detailed design studies (based on a verified F-18 production-and-use cycle with a $18-25 \mathrm{MeV}$ electron accelerator) still need to be performed to verify the scaled-up process, no industry collaborations have currently been established for this effort. However, a potential radiochemistry collaboration with the University of Washington Medical School (Seattle, WA) has now been identified to assess feasibility of the patient sample (FDG) preparation process. Upon verification of the basic production and preparation feasibility, CRADAs with medical accelerator vendors, (Siemen's Inc., Varian Associates, Inc., GE, etc) and/or radiopharmaceutical companies should be initiated.

Finally, the economic benefits of this onsite production method can be greatly expanded if this method can also be made to produce other desirable PET radioisotopes.

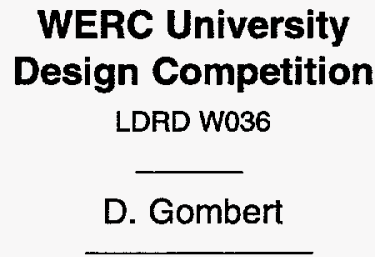

The Waste Management Education and Research Consortium (WERC) sponsored by the DOE holds an annual competition for universities nationwide to develop innovative engineering solutions to environmental problems. This year the WERC competition included two design problems: retrieval of the solid high-level waste saltcake in storage in the Hanford tank farm, and stabilization of the heavy metal contaminated pond sludges at Rocky Flats. The design team, selected from applicants at the University of Idaho, developed a creative robotic saltcake fracturing and retrieval system to remove the HLW from the underground storage tanks. The stabilization system using apatite, a naturally occurring mineral, was novel enough that the team was invited to Rocky Flats to discuss its use at that site. The University was then funded to do follow-on studies through the summer. The complete design reports are on file at the university and the Lockheed Idaho LDRD office (contact Dr. Debonny Shoaf). The University of Idaho contact is Dr. Woody Admassu, 208-885-8918.

\section{Objectives}

- Establish an interdisciplinary design team guided by faculty advisors to develop an engineering solution to the WERC problem statement.

- Analyze the problems and develop solutions based on library research, industrial contacts, and laboratory treatability studies.

- Develop a written proposal for technology implementation, including a technically defensible explanation of the approach, experimental data, cost analysis, and a bibliography of all contacts made and references used.

- Develop and present an oral discussion of the proposal, including working laboratoryscale demonstrations of the required technology.

\section{Accomplishments}

All tasks were completed on time and within budget. Of the 25 universities represented in the national competition, the University of Idaho team went on to win top honors for the best poster presentation, and third overall. In addition, they were invited to the Rocky Flats site to discuss their design under the belief that their concept could be more costeffectively applied than that of the current commercial vendor. This demonstrates the practicality of the university design.

The retrieval concept included remote drilling of salt cake, followed by injection of a cracking agent that expands on curing to break up the solid for retrieval by a robotic excavator. The stabilization proposal makes use of apatite, a naturally occurring calcium phosphate mineral, to reduce the solubility of the heavy metal contaminants. After the reviewers from Rocky Flats invited the design team to Colorado to discuss their presentation in greater depth, the Rocky Flats Operations Office funded the university to do follow-on research through the summer.

New or Increased Technical Capabilities

The INEL can and should continue to nurture and draw upon the emerging talent at our universities. This type of activity not only provides practical experience 
in the student curriculum, but involvement with the students is a fulfilling experience for the

INEL mentor. In addition, some of the best technical talent at the university are exposed to the type of environmental remediation work available in the DOE complex.

\section{Business Development Opportunities}

The university labor pool is talented, creative, and cost-effective. This year, the WERC competition is to use a groundwater cleanup problem from Test Area North at the INEL. By supporting this competition, we leverage our invest- ment by having 25 universities exploring possible solutions to reduce the cost of operating the INEL. The potential cost saving can make funds available for other work to attract revenues to the INEL. 


\title{
National Infrastructure Technology Test/Evaluation
}

\section{Strategic Thrust}

\author{
Cive me matter and motion, and I will \\ J construct the universe.
}

—Rene Descartes (1640) 


\section{Microstructural Influences on Corrosion \\ LDRD 5610}

\section{J. H. Wolfram}

Microbially influenced corrosion (MIC) is a well accepted phenomena in the scientific community. However, it is not well understood mechanistically, nor is it generally accepted by the engineering fields as a source contributing to the deterioration of metallic materials. The infrastructure of this country is deteriorating, often at a rate faster than current engineering models predict. Other phenomena than those considered in the model must be playing a role in the deterioration. Biological influences on materials are very seldom considered for the role they play in degrading materials. Only recently has the intrinsic microbial degradative capacity on hazardous organic compounds been shown to be a viable and valuable means of treatment.

This preliminary study is oriented to show that microorganisms in the fuel storage pool in the FAST building at CPP are able to attach to materials. Excessive pitting has been observed on the aluminum fuel storage cans. The FSA pool is lined with stainless steel, on which no has been observed as yet. In order to draw attention to the fact that the microbes present in the pool water could be attaching to stainless steel metallic surfaces, we wanted to develop a technique that shows attachment directly. This could lead to the initiation of an investigation into the possibility that pitting of stainless steel could be caused or accelerated by the presence of microorganisms. Very little effort has been given to the potential of accelerated deterioration of materials in the nuclear industry, whether it be in power generating facilities or in fuel storage and waste repositories.

\section{Objectives}

- Develop and test a technique to directly detect the presence of viable organisms on stainless steel coupons.
- Treat coupons thermally using the in-house Geeble apparatus to simulate field welds.

- Determine if microbes preferentially attach to thermal treated coupons.

\section{Accomplishments}

The stainless steel coupons were prepared at CPP and treated with the Geeble apparatus by Dr. Dennis Clark. The thermal treatments were varied so that some coupons were heated more than others. The setup included simulating the heat-affected zone of welds. Many case studies have shown that the predominate area of MIC occurs in this region. Our first approach, then, was to determine whether this is where most microbial attachment occurs.

To do this, we needed a way to show directly that microbes were attached to the metallic surface. In the past, many investigators simply smeared or removed the organisms from the surface that had been submerged in solution for a period of time. With this technique, the investigator could not say unequivocally that the organisms were attached or in the water film. This technique also does not allow one to associate the organism with a location on the metal's surface. In the future, it would be important to show that the organisms in a specific location are also where a pit occurred. The technique we developed uses a stain that attaches only to respiring cells. This informs us that at the time of staining the cells are actually alive; some stains indicate the DNA of live and dead cells. The coupons are removed from the test chamber and rinsed in sterile water to remove any cells not attached. The stain is then applied, excess stain removed, and the cells are fixed to the coupon for examination under a microscope. The microscope has to be adapted with an ultraviolet light source and the appropriate filters to allow the correct emission wavelengths to pass for viewing. After some trial and error, the technique has worked well and photographs are available. In the black-and-white photos, the cells show up as lighter spots. In color, the cell are red and stand out from any other background spots. The stain is CTC (5-cyano-2, 3-di-4-tolyl-tetrazolium chloride).

The thermal treatments were performed by the Welding Group at the IRC. The Geeble apparatus was used to apply a precise thermal treatment to each coupon. Each treated coupon reflected a portion of the heat-affected zone . The surface chemistry of the coupons with the highest thermal input $\left(1000^{\circ} \mathrm{C}\right)$ should have a surface chemistry similar to an actual weld without the addition of the weld metal. The coupons were sterilized, placed on hangers (plastic), suspended, and submerged in a media solution containing approximately 10E5 FAST basin organisms. These organisms were isolated from the interior of a CPP 666 mock-up fuel can. The coupons were pulled out over different time intervals in an attempt to study the attachment rate. These intervals spanned a time frame of several hours to a week in duration. Each coupon was counted using a grid system as shown in the figure at the top of the next page. The counts represent the number of individual viable organisms on a particular location based on the distance down a row from the machined hole in the coupon.

\section{New or Increased Technical Capabilities}

The INEL now has a technique for establishing a biofilm on metal coupons as a starting point to perform new business ventures in the area of biocorrosion studies, 


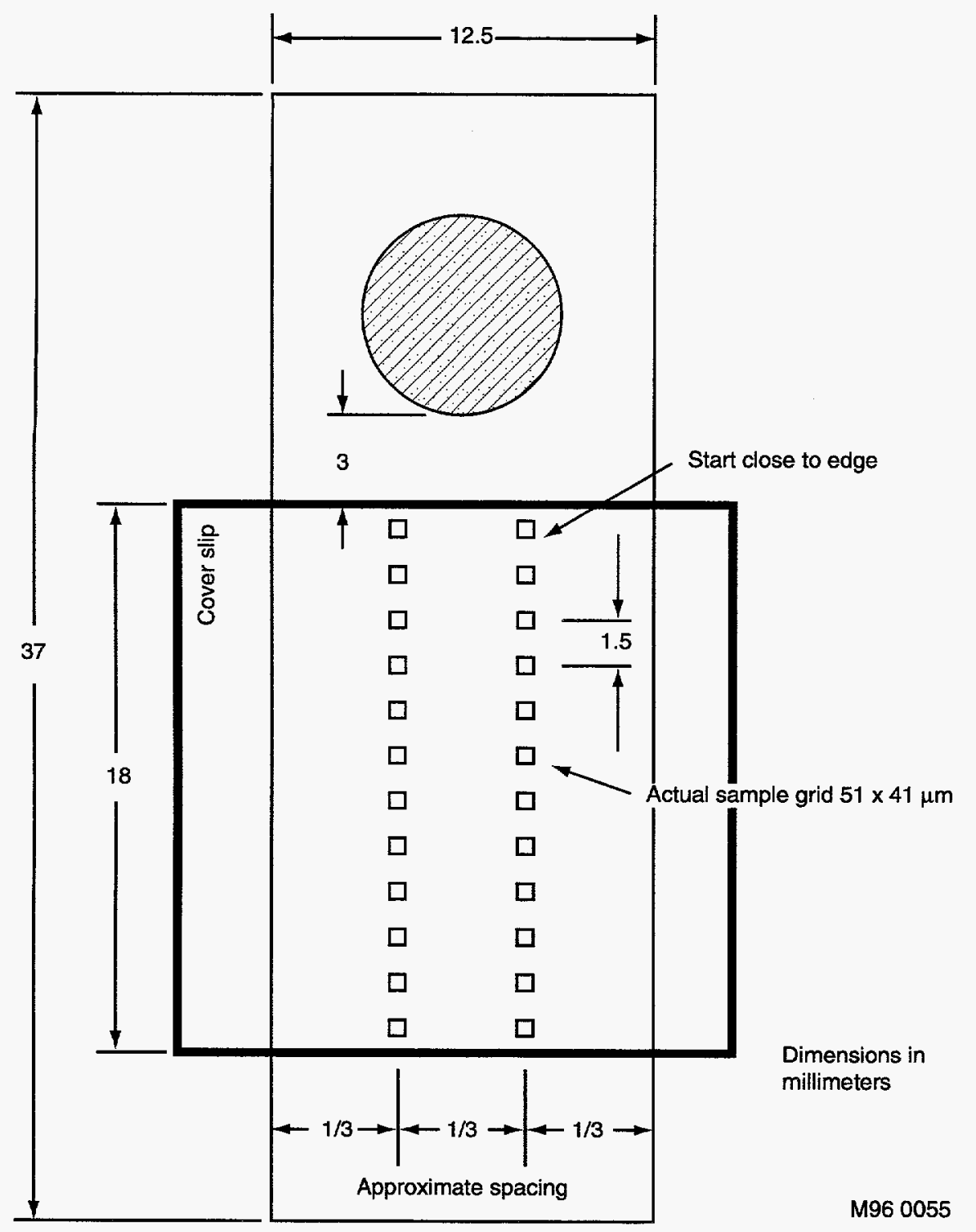

Biosampling Scheme for Gleeble Coupons

effectiveness of biocides on attached organisms, fouling of piping surfaces, etc. We have visualization of actively respiring organisms on attached surfaces. And though no trend in the attachment of organisms the Geeble-treated coupons was observed, it is a potential way of assessing the inducibility of MIC.

\section{Business Development Opportunities}

This effort was in support of developing a means of assessing the pitting potential for corrosion of stainless steel in the FAST basin at CPP. If surface attachment mechanisms can be understood as a result of further investigations, then the potential exists to develop new biocides that will prevent and cause detachment of the biofilms at the surface. This would have great commercial potential, since many industries are experiencing problems relating to surface/biofilm attachment. An long-term research initiative was written with a portion directed toward biocorrosion. A NATO-sponsored workshop is being organized by Wolfram on the subject of interim fuel storage pool deterioration as a result of microbial presence. This workshop is planned for 1996 and will be held in Hungary with participants from both NATO-member countries and the cooperating partner countries.

\section{Advanced Combined Environments Test Station Scoping Studies LDRD 7603}

\section{C. O'Brien}

This project defined and studied the feasibility of the Advanced Combined Environments Test Station (ACETS) concept. ACETS is visualized as a facility with the capability to conduct testing of full- or large-scale structures under severe natural and man-made loads such as earthquakes and hostile acts (terrorist bombings). The station is defined as a subsurface ground motion test area, shake table, and reaction wall for seismic testing; a hurricane and tornado simulation facility for wind and water damage testing; an aging facility to study accelerated natural material degradation; and a surface blast test area for hostile acts testing. The figure depicts the Station 10 years out.

ACETS is proposed as a major new mission for the INEL. Full-and large-scale structural testing has been identified by academia and industry alike as the missing element in life and property loss mitigation. By providing this service in an affordable and accessible form, INEL will fulfill its mission as an applied engineering laboratory by benefiting the American public through mitigation of losses from natural disasters. As a state-ofthe-art facility with size and capabilities unmatched in the world, the United States will re-establish itself as the leader in building technology.

As a new mission, ACETS will enhance established INEL capabilities in various areas such as large-scale 
test management, vibration testing, instrumentation, and modeling, and to define new areas of expertise. It will be a clean, constructive mission that will aid the local economy and provide tangible benefits on a national level.

\section{Objectives}

- Identify the need for seismic and wind test facilities.

- Define functional requirements of seismic and wind test facilities.

- Solicit and study ideas for unique mechanical drive technologies for seismic and wind simulation.

- Prepare a comprehensive report on task findings.

\section{Accomplishments}

Establishing the need for seismic and wind test facilities was perhaps the easiest task of the project. Not only were previous studies available, but support for full- and large-scale testing was readily obtainable from the structural engineering community. The team made extensive contacts with industry, academic, and agency professionals involved in hazard mitigation through conferences, arranged meetings, and the INEL Seismic Testing Workshop.

The INEL Seismic Testing Workshop was a two-day event held in June. Nine internationally recognized experts on seismic engineering convened to discuss loss mitigation strategies and the role of full- and large-scale testing. The workshop validated previous studies for needs and also proposed test facility functional requirements. The workshop findings are detailed in the Proceedings of the INEL Seismic Testing Workshop, which is included as an appendix of the comprehensive report.

We identified the functional requirements for a wind test facility through previous studies and meetings with the wind engineering leaders. Specifics are reported in the comprehensive report.

The magnitude and sophistication proposed of the seismic and wind test facilities requires performance beyond the levels possible with current testing technology. Consequently, a major task of the LDRD was to solicit and study ideas for unique mechanical drive technologies. We accomplished this by soliciting ideas on site through three brain storming sessions and assisting with the 1996 LDRD call. In addition, ideas for a unique shake table concept and hurricane simulation mechanisms were identified through the University Research Consortium. Other ideas evolved from professional contacts outside the company. A complete report on these findings is contained in the comprehensive report.

The outline for the comprehensive report, issued September 30 , 1995 is as follows:

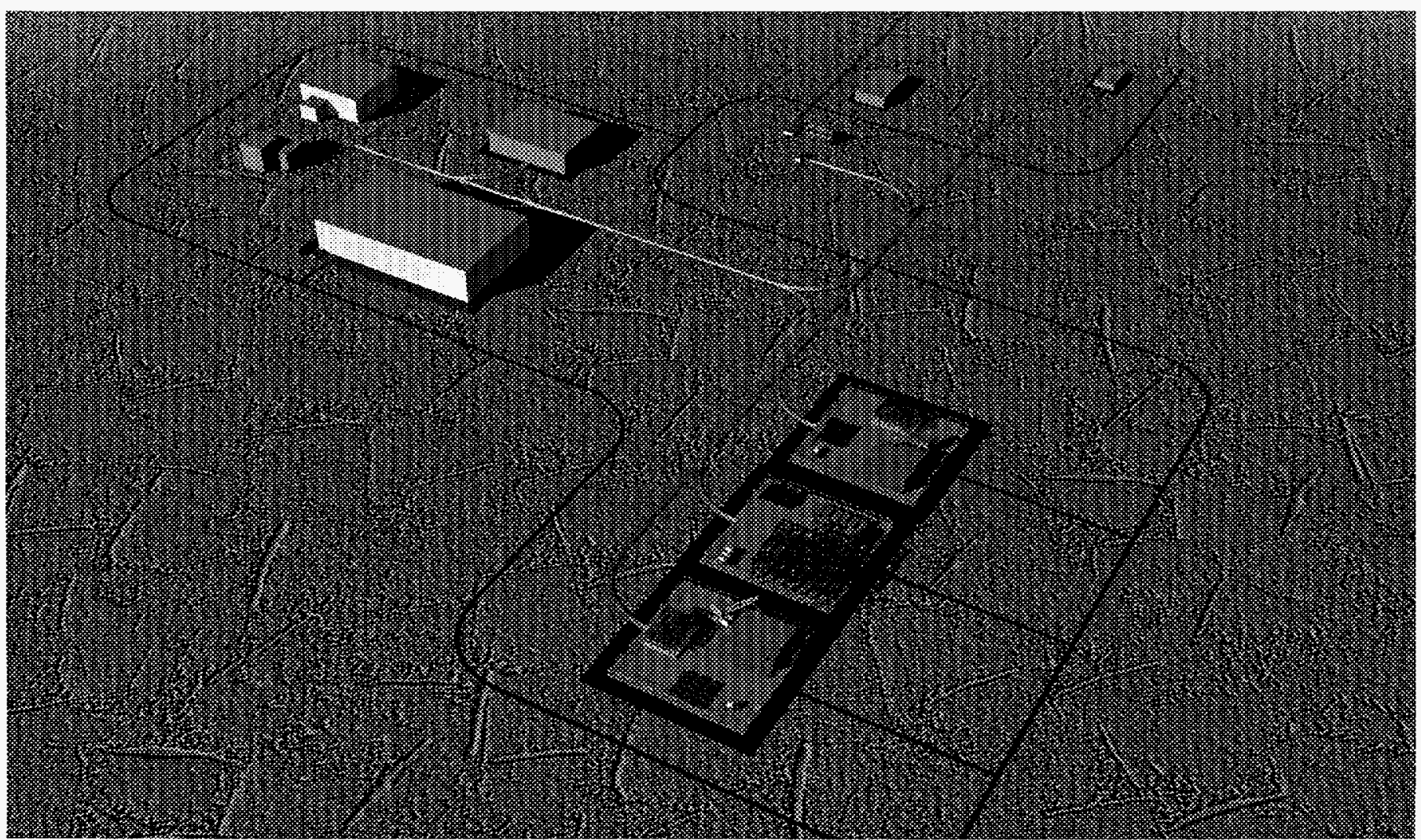

Advanced Combined Environments Test Station (ACETS) Concept 
Executive Summary

Highlights of Technical Findings

Marketing Interfaces and the Integrated Product Team

Future Needs/Plans/Recommendations

High-Level Milestones

LDRD Products

Appendices

Contact List

Library Reference List

Schedule/Milestones

LDRD Budget

Seismic Findings on Needs and

Existing Facilities

Proceedings of the INEL Seismic

Testing Workshop

Mechanical Devices for Wind

and Seismic Simulation

In situ Blast as a Seismic Simulator

Aquifer Issues

Blasting Contamination Issues

Blasting Contamination Issues

Aging

Wind Findings on Needs and

Existing Facilities

Operations Plan

INEL Seismic Testing Expertise

New or Increased Technical Capabilities

The INEL has raised its visibility as an applied engineering laboratory by proposing and investigating a mission of vital importance to the structural engineering community. Because of this visibility, the INEL was invited and participated in the Earthquake Engineering Research Institute workshop on U.S. seismic testing capabilities in July, and has been notified of several upcoming meetings and conferences.

\section{Business Development Opportunities}

The ACETS project included collaboration with MIT and a shake table manufacturer, MTS, through the URC program. MTS is quite interested in the drive technology under investigation by MIT and INEL.

Business opportunities identified for ACETS are considerable.
Key applications would include verification of computer modeling, testing of new structural techniques and materials for faster delivery to the market, and better understanding of structural behavior for more effective building codes and laws. In addition, spinoff technologies are probable from the technology that must be developed to build the facilities.

\section{Characterization of Aged Materials with NDE and Internal Friction Measurements LDRD 8501}

\section{W. G. Reuter}

There is a strong need in many U.S. industries (petrochemical, nuclear and fossil power generators, aircraft, etc.) and in the infrastructure (highway, bridges, sewers, and water lines, etc.) to extend the safe operating lifetime of structural components. This is needed to enhance the U.S. competitiveness in the world market because replacement of the components is expensive and often requires a couple of years or more before the replacement process is completed.

There are many factors that contribute to the deterioration of these structures during normal operating conditions. Some of these deterioration mechanisms include corrosion, fatigue, embrittlement (e.g., irradiation, thermal), etc. The Idaho National Engineering Laboratory (INEL) has had experience in extending the lifetime of structural components, in ATR, that experience irradiation embrittlement. Successful lifetime extension required that a team be developed that included expertise in fracture mechanics, NDE, and applied mechanics. This experience puts the INEL in a position to provide technology to industries that experience embrittlement regardless of the mechanism.

The purpose of this research is two fold:
- Methods (primarily based on NDE techniques, including subsized specimens) to quantify the embrittlement by measuring fracture toughness

- Models to predict the lifetime of structural components.

This process is to be verified using a material that experiences thermal embrittlement.

\section{Objective}

- Obtain test specimens from a material that has experienced thermal embrittlement.

- Develop methods (primarily based on NDE techniques or subsized specimens) to quantify the embrittlement at the time the measurement is made.

- Develop models to predict the lifetime of structural components. This requires the ability to predict the fracture toughness at the end of the design lifetime and to identify appropriate models for using fracture toughness data to predict a safe operating lifetime.

- Develop a plan to submit a proposal to DOE-BES.

\section{Accomplishments}

Develop Embrittled Specimens.

Because weldments are generally the source of failure in many structural components, weldments were fabricated from Alloy 718 and then embrittled. A comparison between the sensitivity of fracture toughness to exposure to $649^{\circ} \mathrm{C}$ for $1,000 \mathrm{~h}$ is made between Alloy 718 base metal and weld metal. The weld metal experiences more embrittlement than does the base metal.

Quantify Embrittlement Using Subsized Specimens or NDE.

It was possible to obtain reasonably useful estimates of fracture toughness using small specimens, 
but the size limits of applicability have not been defined. In addition, it has been possible to use NDE techniques to detect embrittlement, but the results are far from being conclusive. It is possible to suggest that qualitative capabilities exist for detecting and quantifying embrittlement, but additional research is required before these techniques will provide quantitative estimates of fracture toughness.

\section{Methods to Predict.}

We recognized at the start of the project that much time and money could be spent on attempting to identify the critical microstructural changes responsible for the embrittlement. This was why only a small effort has been made, to date, to identify the critical microstructures responsible for thermal embrittlement. It was not surprising to see that the findings from the open literature, from

AMES Laboratory, and from measurements made at INEL were inconclusive and contradictory. But it was necessary to identify some of the difficulties in identifying the significant microstructural changes responsible for embrittlement so that overly optimistic expectations would not develop. This is a path that needs to be continued but will be expensive and time-consuming, but could be significant. It is also necessary to continue to develop verified models for predicting the fracture process in structural components using test data generated from small specimens. Proposal Submitted.

A collaborative approach is in progress between S. David (ORNL) and researchers at the INEL to develop a proposal for a $2 \%$ initiative. This collaboration is expected to be powerful owing to significant, unique capabilities that exist at each of the two facilities.

New or Increased Technical Capabilities

We developed the ability to embrittle a structureal material simply by exposure to a nomal operating temperature and obtained an understanding of the difficulties associated with identifyng a critical microstructure responsible for embrittlement.

\section{Business Developm ent Opportunities}

We submitted a proposal to DOE for two percent initiative funding to study embrittlement of Alloy 718. A CRADA is being negotiated between Chevron Oil Company and the INEL to develop subsized test specimens to estimate fracture toughnbess for structural steels. 


\title{
National Derived Use Technology Transfer Center
}

\author{
Tf I have seen farther than others, it is by standing \\ Lon the shoulders of giants. \\ -An old saying \\ quoted by Isaac Newton
}




\section{Nondestructive Examination of Bottom-Crimped Seam on 55-gallon Drums \\ LDRD 230}

\section{C. Kunerth, G. D. Lassahn}

The Materials Physics Department is evaluating electromagnetic eddy-current techniques to determine the integrity of 55-gal drums based on wall thickness. The geometry of the bottom crimped seam is a test variable anticipated to present a problem. At best, a wall thickness measurement in the seam region is ill-defined, and an eddy-test response to material thinning by corrosion may be complicated by geometry variations resulting from manufacturing and handling. An approach to address this problem is to use a second inspection technique such as real-time $x$-ray radiography. Combining data from both techniques would help to ensure a more reliable determination of drum integrity. We propose that a real-time digital radiography technique be used with an eddy-current technique, and the combined data sets be examined in unison. We will adapt an existing prototype image analysis system being developed by the Optical and Plasma Physics Department to examine, jointly or separately as appropriate, radiographic image and eddy-current data to locate flaws. This system is useful in finding subtle patterns not readily apparent, particularly in the joint analysis of data from several disparate sensor types.

\section{Objectives}

Our objective is to develop a reliable tool for inspecting the integrity of corroded drums. Specifically, we plan to demonstrate the effectiveness of joint use of eddy-current and radiography inspection methods to verify drum integrity with particular attention to the difficult bottom crimp seam. Accomplishments

$X$-ray radiographic and eddycurrent data were acquired and evaluated for use with an existing image analysis and data fusion system. Digital radiographic techniques were used to acquire images in the region of interest on 55-gal drums. The digital images obtained were processed to obtain files compatible with the image analysis system. Similarly, eddy-current data were collected by a scanning system that could acquire, process, and produce two-dimensional data sets/images of barrel sections. The image analysis and data fusion system evaluated for use with these data has very general image analysis capabilities. Inherent in its operation is a data fusion capability that rests on optimization of a linear combination of features extracted from the several images input to the system. Joint analysis of eddycurrent and x-ray images is a natural application for the system. Information contained in the two images jointly but not available from either image separately would be easily extracted by the system.

However, in this particular application, we found that the system could not find in the available $x$-ray data any information relevant to the integrity of the bottom seam of a 55-gal drum, and combining the x-ray data with eddy-current data would not enhance the inspection of that seam.

The samples used contained no amount of rust to moderate amounts of rust. The geometry and the limited reduction in seam thickness makes acquisition of highcontrast digital $x$-ray images of the rusted seam areas difficult. We deem application of the technology to heavily rusted or defective seam areas not practical because of the obvious visual nature of the defective regions. However, it is, of course, still possible that other kinds of data could be advantageously combined by this method. New or Increased Technical Capability

Our efforts allowed the Materials Physics Department to develop improved NDE scanning capabili- ties that will directly contribute to existing and new programs.

\section{High Transition Temperature Superconducting Quantum Interference Devices for Nondestructive Examiniation LDRD 231}

\section{K. L. Telschow, K. K. Haulenbeek, F.W. Bruneel, C. H. Sellers, C. Snow}

New high transition temperature SQUID (superconducting quantum interference devices) are the most sensitive detectors known for measuring magnetic fields, having a sensitivity of $100 \mathrm{ft}$ at $77 \mathrm{~K}$. The new superconducting materials allow operation at liquid nitrogen temperature, which makes implementation into industrially usable instruments much simpler than their low $T_{c}$ cousins. This capability offers the potential for developing small, portable, perhaps hand-held, instrumentation that can be used for remote magnetic nondestructive evaluation (NDE) of materials. The small size will allow inspection of industrially important structures, such as pipes. Suitable NDE probes are correlations between microstructure and magnetic parameters, such as coercivity, remanence, and permeability in ferromagnetic materials; susceptibility in paramagnetic materials; and high penetration eddy currents in metals. All of these parameters reflect the underlying material microstructure and are measurable with SQUIDs. Two things are necessary for successful use of SQUIDs in NDE: (1) a means whereby unwanted external background magnetic fields (e.g., the earth $\sim 70 \mu T$ ) can be shielded or compensated for so that the SQUID can be used outside the laboratory, and (2) methodologies for transferring laboratory measurement techniques with their usually severe restrictions on sample size and shape to the local arbitrary geometry of samples found in industry. This project investigated some of the first uses of these new SQUIDs for NDE by researching answers to these questions. In particular, fatigue damage in stainless steel was quantitatively 
measured on large samples repeatedly stressed in varying degree to failure.

\section{Objectives}

- Purchase a high $\mathrm{T}_{C}$ SQUID sensor and associated drive electronics.

- Configure a sensor unit with appropriate shielding.

- Develop signal acquisition and analysis hardware and software to form a usable system.

- Begin measurements on identified applications for demonstration of capability.

\section{Accomplishments}

This project is based on using the new High $T_{C}$ SQUID thin-film technology developed and recently marketed by Conductus Corporation. These units are in bare sensor form, and their applicability to industrial environments is unproven. One of the first magnetometer configurations produced has been installed at the INEL. It consists of a single-loop squid system with a minimum detectable field of about $100 \mathrm{fT}$. The system has a maximum bandwidth of $20 \mathrm{KHz}$ and barely maintains stability in the laboratory background fields without shielding. A triplewall shielded container was obtained that contained an access port such that samples of less than 1 -in. width could be translated beneath the sensor for measurement. This system simulates the type of measurement when performing NDE measurements on industrial structures, except that the shielding problem has been eliminated. The figure shows the shielded apparatus that allows samples to be translated under the sensor with standoff distances of about 5 to $15 \mathrm{~mm}$. Without the shielding, very large background fields were observed that changed quickly, depending on the many external influences throughout the laboratory. Inside the shield, noise levels approaching the intrinsic level, as measured by Conductus, were recorded. The unit has a dynamic range of 108 , extending its measurement range from $100 \mathrm{fT}$ to $10 \mu \mathrm{T}$, while maintaining lock with a fixed number of flux quanta inside the sensor loop. It can operate in background fields as large as $100 \mu \mathrm{T}$, though the noise level rises in the larger fields. The minimum detectable magnetic moment of a sample passing underneath $1 \mathrm{~cm}$ from the sensor is about $108 \mathrm{Bohr}$ magnetons. This corresponds to a very small concentration of polarized magnetic atoms in a nonmagnetic host lattice.

The first investigations performed with the SQUID sensor were in collaboration with other projects: BES-Engineering Research program NDE of Superconductors, where flux penetration in tapes for local critical current measurement is being explored; LDRD 2501, Development of Nondestructive Fatigue Monitor, where fatigue damage in stainless steel is measured; and LDRD 8511, Lifetime Extension and Increased Reliability for Refinery Structures, where

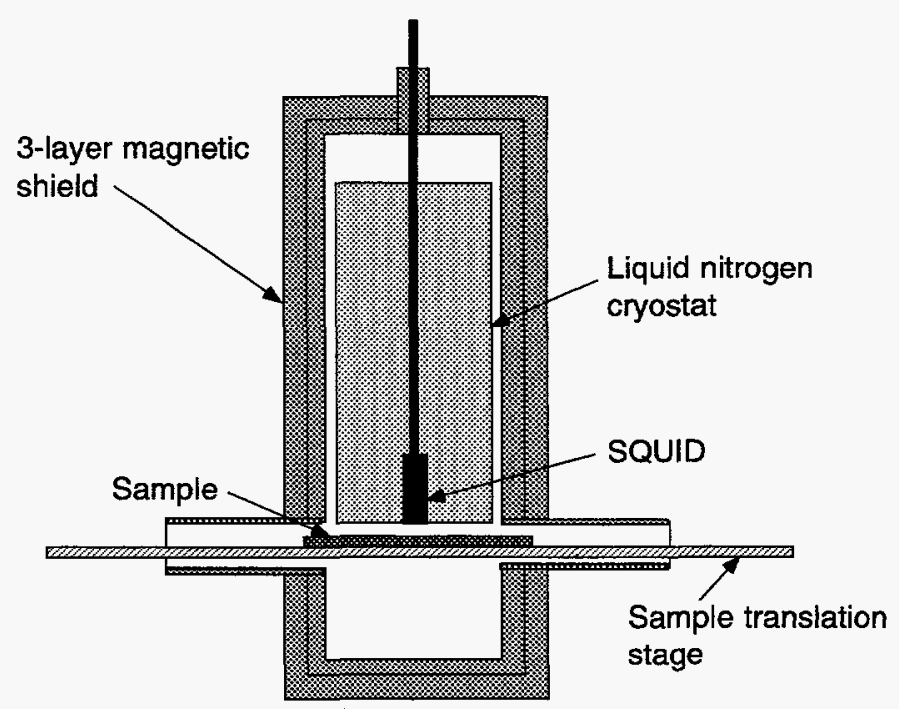

Three-layer Shielded SQUID Sensor Apparatus Used for Magnetic Moment Measurement in Long Sample of 250-mm-or-Less Width aging changes in microstructure are being probed magnetically.

Samples of stainless steel 304 were machined and subsequently cycled under stress by varying degree to full failure. Small cores were drilled, saturation magnetized, and their moments measured both in the vibrating sample magnetometer and with the SQUID. The results of both measurements agree and show a large increase in magnetic moment with position as the core sample gets closer to the failure point. Fatigue precipitates a magnetic phase in the originally nonmagnetic prepared stainless steel 304. Magnetic measurements record the amount of this secondary phase present. Contrary to the VSM, the SQUID can be used on larger geometries. The larger fatigued samples clearly show increased magnetic precipitate formation in the high fatigue region of the samples. The signal-to-noise level for these measurements is about 107 , indicating that very much smaller amounts of magnetic phase could be detected in a material with less background phase in its composition. These results illustrate the high resolving power of the SQUID. 
New designs for sensor arrays that compensate for background fields are being developed, and a gradiometer configuration has been purchased and is being evaluated. Ken Telschow attended the NATO Advanced Study Institute, SQUID Sensors: Fundamentals, Fabrication, and Applications, held in Maratea, Italy, June 18-30, 1995. There he interacted with experts in SQUID measurements from around the world and discussed shielding and compensation methods applicable to NDE. Ideas developed as a result of this interaction are being pursued at the INEL.

New or Increased Technical Capability

This project has established an entirely new key capability for the INEL. INEL has scientists experienced in the use of superconductors and electromagnetic sensors for NDE, as well as materials scientists who correlate NDE measurements with microstructure. This constitutes a very effective team for developing new NDE tools for using the SQUID in industry. Business Development Opportunities

Intermagnetics General Corporation Advanced Superconductor, (IGC-AS) of Waterbury, Connecticut have indicated that they would be interested in collaborating with the INEL to institute improvements in superconducting tape measurement methods using the new SQUIDs. Industrial partnerships with the nuclear and oil and gas industry are being established in collaboration with the other LDRD projects cited. Preliminary discussions have also been made between INEL and Conductus Corporation for developing new NDE applications of SQUIDs. Ken Telschow presented a seminar, HTS-SQUIDs for NDE, August 29, to orient others at the INEL to the possibilities of SQUID sensors. A new working relationship has been established between the INEL and

the U.S. Air Force, Office of Scientific Research, as a result of NATO institute participation.

\section{Robust Nondestructive Examination Using Photorefractive Nonlinear Optics \\ LDRD 232}

\section{K. L. Telschow, T. C. Chatters, S. Garwick}

Photorefractive crystals (PRC) have been employed to design a wide range of nonlinear optical applications, including phase conjugation, image amplification, optical computing, and adaptive interferometry. Research over the past 10 years has brought considerable understanding about the physical mechanism and capabilities of the photorefractive effect. Much work is under way developing this effect for applications in optical computing, image processing, and telecommunications. Nondestructive evaluation (NDE) of materials and processing is an application for photorefractivity that has been little explored, but could benefit greatly from this technology. Optical methods for the detection of ultrasonic and vibratory motion at material surfaces have been under development at the INEL for several years. The results of this work have contributed to the establishment of laser ultrasonics as a new noncontacting materials characterization technology useful for process monitoring and NDE. Optical methods of ultrasonic vibration detection use interferometry and are performed in industrial environments where such external influences as low-frequency vibrations and image distortions from temperature and wind gradients are present. Photorefractivity offers a means for applying the sensitivity of optical interferometry to industrial settings through self adaptive compensation. This project explored the use of photorefractivity in certain materials for development of a self-adaptive interferometric approach to the detection of ultrasonic motion in materials.

The project succeeded in demonstrating photorefractive optical ultrasonic detection immune to low-frequency disturbances. A new approach for high-sensitivity optical measurement of vibration modes of structures was also investiaated usina bhotorefractivitv.

\section{Objectives}

- Determine PRCs suitable for NDE applications using commercially available lasers

- Characterize PRC response with incident light power, orientation and phase modulation

- Implement PRC two-wave mixing in laser ultrasonic detection.

- Investigate PRC-phase modulation schemes for vibration mode structure analysis.

\section{Accomplishments}

The figure shows the experimental arrangement used for the two-wave mixing measurements of ultrasonic motion. The method mixes part of the laser beam scattered off the ultrasonically moving surface with the unshifted beam inside a PRC. The resulting diffraction grating contains all the low-frequency phase information of the two beams and produces a correlated output that is subsequently mixed and detected with photodiodes. The operation was analyzed theoretically, including the polarization effects of all beams, PRC properties, and postprocessing. The results then were compared with measurement as to mixing gain and signal response. Three crystals, in order of decreasing time constants, $\mathrm{BaTiO}_{2}$, $\mathrm{Bi}_{12} \mathrm{SiO}_{20}$ (BSO), and $\mathrm{GaAs}$ have been purchased, and the analysis of the two slower crystals have been completed. Barium titanate has the highest photorefractive gain and the slowest response (>1 sec). A contact piezoelectric trans- 


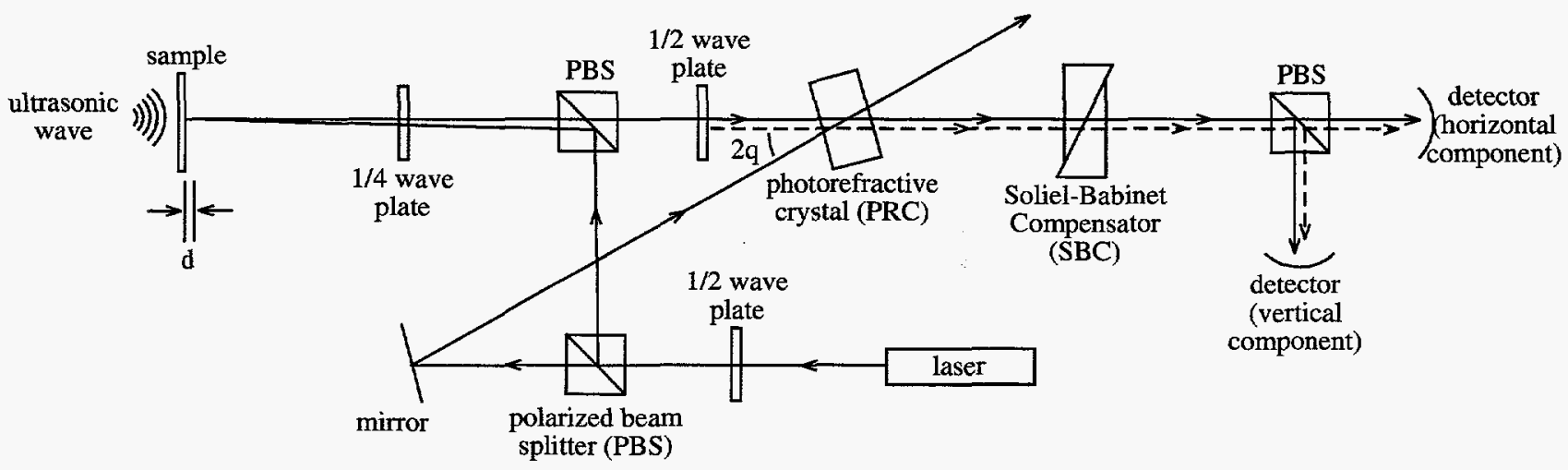

Experimental Setup for Detecting Ultrasonic Motion Using Two-Wave Mixing in a Photorefractive Crystal

ducer $(10 \mathrm{MHz})$ generated and detected ultrasonic waves in a plate of Inconel, 6-mm thick, from the back side. The signal-to-noise ratio using the $\mathrm{BaTiO}_{3}$ photorefractive crystal compares to that of the piezoelectric measurement. The slow response time of barium titanate makes this material ineffective for nonlaboratory measurements, as it is too slow to cancel low-frequency vibration. Results with BSO were much better owing to its faster time response $(\sim 0.01$ $\mathrm{sec}$ ); however, this material exhibits very low gain and correspondingly low-signal-to-noise ratio. The work with $\mathrm{GaAs}$, which has a very fast time response $(\sim 10$ $\mu$ sec) but operable only in the near infrared, is still in progress. Other possible photorefractive materials have been identified and will be explored if future funding becomes available. The results from this project are very exciting, showing that the photorefractive method is a solution to many of the difficulties encountered in optically measuring ultrasonic motion. Successful implementation of this technique could provide an optical ultrasonic transducer that is as easy to use as the contact piezoelectric.

The project also investigated a new idea that could advance measurement of low-frequency vibration modes of structures. It is called optical lock-in detection of vibration and uses the dynamic mixing properties of the photorefractive effect to discriminate between phase modulations occurring at different frequencies, as would be encountered in light scattered from a structure vibrating in several modes simultaneously. In addition to the sample beam being modulated by the vibrating surface (simulated by the electro-optic modulator), the pump beam is modulated by a moveable mirror. Synchronization occurs when the two modulation frequencies are equal and discrimination occurs, except within the bandwidth of the PRC. In operation, this device would perform as a noncontacting vibration spectrum analyzer with subnanometer sensitivity. Some of the operating characteristics have been measured in the laboratory, and the operation has been modeled theoretically. The results form the basis of a conference presentation and publication, T. C. Chatters and K. L. Telschow, Optical Lock-in Vibration Detection using Photorefractive Four-Wave Mixing, published in the Reviews of Progress in Quantitative NDE, (Plenum Press, New York, 1995).

New or Increased Technical Capability

Photorefractive adaptive interferometry is a new capability for the INEL. It offers new possibilities for unique noncontacting (optical) sensors for vibration and ultra- sonic motion detection. Its adaptive nature is especially important because it allows adjustment to external undesirable influences automatically, thereby producing sensors compatible with the industrial environment. Experience gained on this project will allow consideration of photorefractivity for other sensor measurement possibilities in the future.

\section{Business Development Opportunities}

Contacts have been made with Hughes Research Laboratories concerning photorefractivity for ultrasonic detection. Future work will involve developing a material suitably optimized in time response and gain for laser ultrasonic detection. The results of the optical lockin technique have been presented at the conference above and submitted for publication. An invention disclosure is also being pursued for the optical lock-in vibration detection.

\section{Polymers - Water Separations, Ozone/Oxygen Separations, Platform, and Laser-Assisted Polymerization LDRD 0248}

$$
\text { M. L. Stone }
$$

Research was conducted relating to various aspects of phosphazene 
polymer chemistry and properties. Four separate tasks afforded the opportunity to exploit the use of these and related polymers in industry application of interest to the INEL.

\section{Objectives}

- Develop new polymers that will function as water passing semipermeable membranes that can survive harsh operating conditions.

- Develop polymers that can function to separate ozone from oxygen.

- Develop polymers that have increased cyclic, crosslinked character, or both, that will provide the basis for new applications capabilities at the INEL.

- Explore methods for producing conducting polymers with improved properties and morphologies by exposing the polymer to laser light during electropolymerization.

\section{Accomplishments}

Water passing polymers. During FY-95, we achieved an increase in the efficiency of our water-passing polymers by one order of magnitude. This was accomplished by the custom synthesis of mixed substituent polyphosphazenes. One substituent was hydrophobic, with good film forming characteristics, while the other is hydrophilic. Four general classes of hydrophilic substituents were examined: methyl amine, mannitol (a sugar), sulfanilate salts, and polyethers.

The most promising materials were derived from polyethers, particularly diethylene glycol monomethyl ether (DEG). This material was attached in varying percentages to the polyphosphazene backbone in conjunction with either trifluoroethanol or cyclohexanol. Some of these polymers have excellent solubility in solvent and good film forming characteristics. Measured fluxes were an order of magnitude greater than other polyphosphazenes in our inventory.

Other promising materials are those using mannitol as the hydrophilic component. Mixtures of mannitol with trifluoroethanol were found to give good films and swell in water readily. Several methyl amine containing polymers were synthesized, but we view their suitability as water passing membranes to be limited owing to inherent shattering behavior in water. In addition, initial experiments using sulfanilate salts as the hydrophilic substituent were unsuccessful because we could not attach this material to the polymer backbone.

\section{Ozone/Oxygen Interactions}

Polyphosphazene membranes were cast and exposed to a mixture of ozone in oxygen. Analyses were performed on the feeds and permeates. The polymers tested did not show high selectivities for either component. However, perhaps what is more important is that the polymers survived very well in the ozone/oxygen environment. This unusually good chemical resistance will be substantiated in the future.

Platform Polymers. Research this year was aimed at creating what is known as a cyclolinear polymeric material. This is where cyclic phosphazenes are joined to each other using a spacer molecule.

This required the compound $\mathrm{N}_{3} \mathrm{P}_{3} \mathrm{Cl}_{2}(\mathrm{R})_{4}$ to be synthesized. Once this tetrasubstituted trimer was synthesized, it could be made into a cyclolinear polymer by reaction of the remaining chlorides with a dimeric amine. We have synthesized the tetrasubstituted trimeric compound above and have polymerized it with a diamine. In two separate reactions, the resulting material had the properties of a low molecularweight polymer and was not usable as membrane material. In both reactions, addition of the reagents was performed in anticipation of requiring exact stoichiometry, but the results seem to indicate a lack of high molecular weight material being formed.

We discovered why the synthesis of this type of polymer is extremely difficult. It requires scrupulously pure ingredients to build a polymer with an acceptable molecular weight. Owing to the stoichiometry of the polymerization, the first challenge was to synthesize a cyclic trimer that is tetrasubstituted. The synthesis of tetrasubstituted materials is difficult since a product distribution always results. In the case of tetrasubstituted stoichiometry, trithrough hexa-substituted products are all formed in the reaction. It then becomes necessary to separate the desired fraction through column chromatography. Once the pure material is obtained, it becomes solid. Any impurities in the tetrasubstituted material results in defects in the cyclolinear growth process. For instance, any tri-substituted compound will form crosslinks and will branch cyclolinear chains. Any pentasubstituted impurity will form a chain end and will effectively lower the molecular weight of the material. Even with a pure starting material, the addition of linking agents must also be stoichiometric in order to grow lengthy polymeric chains. Any excess of diamine will cause end-capping of the polymer chain; likewise, any excess of tetrasubstituted trimer will also function to end-cap the polymer chains.

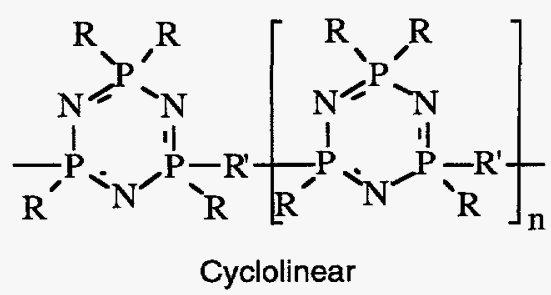


Laser assisted electropolymerization.

Experiments were performed using both UV and IR lasers. In both cases, the laser had a dramatic effect on the polymer produced. The UV laser, under the existing experimental conditions, inhibited the growth of the polymer. In one case, the IR laser had the same effect. However, in another case, polymer was only produced when exposed to the IR laser. This technique has the potential of controllably producing unique materials.

New or Increased Technical Capability

This project was very productive. It built upon a polymer synthesis capability started during FY-94. More than 25 new water-passing polymers were attempted and produced than ever before. Many of the polymers have good potential as membranes. Others should be useful in other applications. One of the potentially important features of the phosphazene polymers is the ability to design and synthesize materials with desired attributes. This project has demonstrated that to be true and opens doors for many applications in the future if these kinds of programs continue.

\section{Business Development Opportunities}

The results obtained through the work on the development and testing of water passing polyphosphazene membranes has led to a CRADA with a chemical company.

\section{Iron Aluminide Filters for Improved Efficiency Fossil Energy Conversion 0251}

\section{B. H. Rabin}

The objective of this project has been to conduct a cooperative $R \& D$ effort involving the Idaho National Engineering Laboratory, an industrial partner, and the Department of Energy
(DOE) Pittsburgh Energy Technology

Center to fabricate and field test porous iron aluminide filters produced using a novel, patented powder processing method developed at the INEL. Iron aluminides are proposed to increase the performance of gaseous filter applications in fossil fuel power plants owing to their high strength, wear resistance, and notable sulfidation resistance; however, it is well known that fabrication of suitable controlled porosity structures by conventional powder processing methods is extremely difficult. It is expected that this work will demonstrate the viability of the unique INEL processing technology as a low-cost manufacturing method, having significant market potential. If successful, the planned follow-on technology transfer efforts will involve either licensing or the creation of a regional spin-off business.

\section{Objectives}

- Fabricate and test porous iron aluminide filters using the patented INEL powder processing method.

- Perform collaborative work to optimize processing variables and improve material characteristics and performance.

- Demonstrate the commercial viability of this low-cost manufacturing method.

- Conduct market studies to evaluate the commercial value of this technology.

- Develop a plan for follow-on technology transfer.

\section{Technical Accomplishments}

Throughout the course of this study, a large number of $\mathrm{Fe}_{3} \mathrm{Al}$ (Fe-28 at.\% Al) samples were fabricated using the novel combustion synthesis elemental powder processing method developed previously at INEL and described in U.S. Patent 5,269,830. These samples were then characterized at the INEL using optical metallography, scanning electron microscopy, $x$ ray diffraction, and porosimetry. Experimental variables such as powder particle sizes, binder content, compaction pressure, sintering atmosphere, and annealing time were optimized in order to produce high-quality materials suitable for filtration performance testing. The testing was performed by our industrial partner, Pall Corp., Cortland, New York, the largest manufacturer of porous metal filters in the United States.

A preliminary market analysis for porous iron aluminide filters was conducted with the assistance of Thermo Technology Ventures. We concluded that no current market exists since use of these materials in conventional coal fired power plants is precluded by their high cost relative to current cloth bag filtration technology. This situation is unlikely to change without a change in air pollution control (APC) regulations, though there are possible opportunities in eastern Europe where APC regulations are more stringent, and large capital expenditures for new APC equipment may be justified. We believe the market for iron aluminide filters to be for use within pressurized fluidized bed combustors (PFBC) and coal gasification combined cycle (CGCC) power plants where requirements will definitely exist for elevated temperature high sulfur gaseous filtration. This market could reach $\$ 200 \mathrm{M}$ per year, though the timing for introduction of these technologies into the commercial power generation industry particularly depends on continued R\&D funding by the Department of Energy.

Samples were sent to Pall Corporation for evaluation using their standard testing procedures. The goal was to demonstrate the viability of the INEL processing method, and to compare the performance of the INEL material with that of 
material having similar composition being produced, with considerable difficulty, by Pall's standard powder technology involving sintering of prealloyed powders. After dialogue and several iterations of new samples to Pall for evaluation, we were able to produce samples that were comparable to the standard Pall material in terms of pore size and pressure drop characteristics. Since corrosion resistance is an important performance criterion, Pall then evaluated the corrosion resistance of the INEL material in an accelerated coal combustion environment. The initial results show the INEL samples to exhibit significantly lower corrosion resistance than Pall's samples made from prealloyed powder. Subsequent improvements in our ability to control sintering atmosphere resulted in samples that exhibited comparable corrosion performance in the simulated tests. Pall subsequently informed us that they had essentially solved the technical problems they were experiencing with fabrication of these materials using their standard processing technique, and therefore they had little need to continue pursuing the INEL technology as an alternative.

In addition to our relationship with Pall Corporation, we received a request late in our project from Westinghouse Electric Corporation's Science and Technology Center in Pittsburgh, Pennsylvania, to evaluate some of our porous samples in their test facility. Westinghouse is a potential end user of this technology. The evaluations are still in progress at this time.

\section{New or Increased Technical Capability}

The INEL has gained considerable experience in the area of powder fabrication and characterization of controlled porosity metallic materials. These capabilities can be applied to a wide variety of applications in addition to porous metals for gaseous filtration, and can be readily extended to different materials systems.

\section{Business Development Opportunities}

The relationship with Pall Corporation described above has essentially ended as a result of their ability to solve their fabrication problems internally. Our relationship with Westinghouse is continuing in the hope that favorable test results will allow us to approach other porous metal manufacturers, which have already been identified, to serve as partners in the commercialization of this technology.

\section{Wear Coatings for Aluminum Automobile Engine Components \\ LDRD 252}

\section{R. N. Wright}

A miniature plasma spray system has been developed to deposit highly defined (approximately 2-mm width) metallic coatings. The plasma spray gun is wire fed and operates at approximately 10-kW power. Carbon steel and monel coatings have been successfully deposited on aluminum substrates using this system. The deposition rates, power levels, particle size distribution and particle temperature have been characterized under a variety of operating conditions using monel wire as the feedstock.

Work has been carried out collaboratively with Ford Motor Company and the National Center for Manufacturing Sciences to develop wear coatings for valve seats on aluminum automobile engine heads, one anticipated application of this technology. Low-cost, reliable methods for producing wear and corrosion coatings on aluminum heads is a technology that will enable widespread application of aluminum alloys, rather than conventional cast iron, which will result in weight reduction and improved fuel efficiency.

\section{Objectives}

- Design prototype miniature plasma spray gun

- Manufacture miniature gun and integrate it with conventional wire-feed system

- Test system and determine relationship of operating conditions (current, gas flow, and wire feed rate) on gun performance and particle characteristics.

- Evaluate microstructure and bonding of monel coatings on aluminum substrates.

\section{Accomplishments}

A miniature plasma spray gun with and designed and built having an outside diameter of approximately $50 \mathrm{~mm}$ and a length of approximately $150 \mathrm{~mm}$. The gun operated at a power of approximately $10 \mathrm{~kW}$ and used commercially available power supplies, mass flow controllers, and wire a feeder. Deposits with a width of approximately $2 \mathrm{~mm}$ were produced on aluminum substrates using either carbon steel or monel as the feedstock. Monel is one of the materials that Ford Motor is considering for valve seat cladding. The $10-\mathrm{kW}$ gun was easily able to produce coatings at wire feed rates suitable for production scale application.

The plasma gun was found to operate successfully over a range of 150 to 200 amps, with gas flow rates of approximately 30 to $80 \mathrm{scfm}$. Advanced diagnostic instruments developed at the INEL were used to characterize the resulting particle temperature, size, and velocity in flight. Over the range of currents and gas flow rates noted above, we determined that the average droplet size varied from approximately 38 to $75 \mu \mathrm{m}$, and the average particle temperature varied from 1570 to $1940^{\circ} \mathrm{C}$. On average, the particles were well above the melting point of the monel 
alloy, approximately $1350^{\circ} \mathrm{C}$. Particle velocities were found to vary from approximately 60 to $150 \mathrm{~m} / \mathrm{s}$. The measured particle temperatures and velocities are very similar to those expected for spraying nickel-based alloys using commercially available plasma spray systems; however, the width of the spray pattern using the miniature gun is a minimum of a factor of 50 less.

Optical metallography of steel and monel coatings formed using the miniature gun indicate that essentially $100 \%$ of the particles were molten at impact and the coating density was above $99 \%$ of the theoretical value. Scanning electron microscopy and energy dispersive $x$-ray microanalysis indicate that with the monel alloy a thin reaction layer, presumed to be a nickel-aluminide, formed between the coating and the aluminum substrate. The coatings displayed reasonable adhesion; however, the magnitude of adhesion is very difficult to quantify with the limited material available from the narrow deposit.

A smaller plasma micro-torch was designed and built based on the performance of the miniature gun. The micro-gun had an outside diameter of $25 \mathrm{~mm}$ and an overall length of $100 \mathrm{~mm}$, which may make it more amenable to robotic control compared to the mini-gun. The torch was tested and found to operate at a power of approximately $5 \mathrm{~kW}$ with a spray pattern very similar in spatial resolution to the mini-gun.

New or Increased Technical Capability

We have demonstrated the ability to design and build a unique plasma spray system for application in producing highly defined coatings. Real-time process sensors previously developed at the INEL, and unique to this laboratory, have been used to quantify the performance of the plasma spray system, and modifications to the gun have been intelligently made based on these results.

The system developed has been tested specifically on a materials system of interest to the automotive community; however, the miniature and micro guns may have much more broad application. There is possibility for directwrite metallization of copper interconnects on circuit boards, rather than conventional lithographic methods. We are also pursuing application of high-definition (that is without using masks) coating technology to refurbish aircraft engine turbine blades, where currently a great deal of labor and materials are used to cover those portions of the blade where deposition of coating is not desired.

\section{Business Development Opportunities}

A draft statement of work has been prepared for funding from the National Center for Manufacturing Sciences to further develop this technology in collaboration with Ford Motor Company. The tasks outlined in the statement of work are specific to examining issues of thermal modification of the substrate and coating adhesion, with the eventual goal of engine testing wear coatings on valve seats. Funding is also being pursued from the Navy for refurbishment of thermal barrier coatings on turbine blades for military engines.

\section{Factors That Determine the Microstructure of Spray Deposited Materials LDRD 0253}

\section{R. N. Wright, J. R.Fincke}

Developing coatings by spray deposition processes is currently carried out in an Edisonian manner. Real-time process sensors recently developed at the INEL now make it possible to determine the size distribution, average temperature, and average velocity of particles in flight. This project determined the influence of particle characteristics on the microstructure of the coatings that result from spray deposition. A simple model system, commercial purity nickel on carbon steel substrates, was examined because the materials are well characterized in bulk form and are free of complicating factors such as phase transformations in the temperature range of interest. Determining the influence of fundamental particle characteristics on the development of coating microstructure will form the basis for scientific design of coatings and real-time control of the coating process.

\section{Objectives}

- Design and build a constant temperature sample holder for spray deposition processes.

- Model heat transfer and residual stress state for nickel coatings sprayed so that widely variable particle characteristics give rise to different temperature gradients in the coatingsubstrate system.

- Validate models by experiment.

- Spray nickel coatings on carbon steel substrates using the Plazjet plasma spray system over a wide range of process conditions. Evaluate coating microstructure using optical and scanning electron microscopies.

- Relate observed microstructures to measured particle size distributions, temperatures, and velocities.

\section{Accomplishments}

Finite element models have been developed that simulate the development of residual stresses during coating deposition and subsequent cooling to ambient tempera- 
ture in the nickel coating on carbon steel substrate system. Simulations suggest that there are three contributions to residual stresses in the system studied: thermal expansion mismatch between the materials, the thermal expansion and resulting plastic deformation associated with rastering the torch across the substrate, and nonuniform cooling of the system associated with fixturing required to hold the substrate during thermal spraying. Experiments have been used to validate the models. Coupons sprayed using a variety of process conditions have exhibited the type of gross deformation predicted by the finite element simulations.

A water-cooled constant temperature substrate fixture has been designed and built that allows computer control of the translation of the coupon in the plume. Experiments were conducted to demonstrate substrate temperature control using this fixture. Control of the substrate temperature allows control over one of the potentially important process variables that may influence microstructure formation.

Experiments were carried out with conventional (uncontrolled) substrate fixturing and with the controlled temperature stage to examine the influence of particle characteristics on the microstructure of nickel coatings on carbon steel substrates. Little first-order influence was found of temperature on the microstructure: however, the finite element simulations showed a significant influence on development of residual stresses. Nickel particles with in-flight average temperatures of either 1650 or $2050^{\circ} \mathrm{C}$ were deposited with a range of velocities. We determined that in general the influence of particle velocity on coating microstructure was minimal at the higher temperature. At $1650^{\circ} \mathrm{C}$, higher velocities were found to increase the density of the coating, which is generally desirable. However, the relative deposition efficiency (indicated by measurement of the coating thickness for the fixed spray conditions) decreased with increasing velocity, as did the fraction of particles that were unmelted. Both of these characteristics are undesirable. The relative deposition efficiency is also considerably higher for the lower temperature particles for a given velocity. The coating thickness measurements as a function of velocity are shown in the figure. The fraction of unmelted particles as a function of velocity was also studied. For this coating system, it appears that the optimum microstructures are formed using relatively low power and mass flow rates (corresponding to lower temperatures and velocities), which is counter to the industry rule of thumb that higher power yields better coatings.

New or Increased Technical Capability

The results of this work have increased the INEL core capability in the area of intelligent design and control of thermal spray processes. The process sensors used in the work are unique to this laboratory, and the ability to specify the particle characteristics necessary to produce

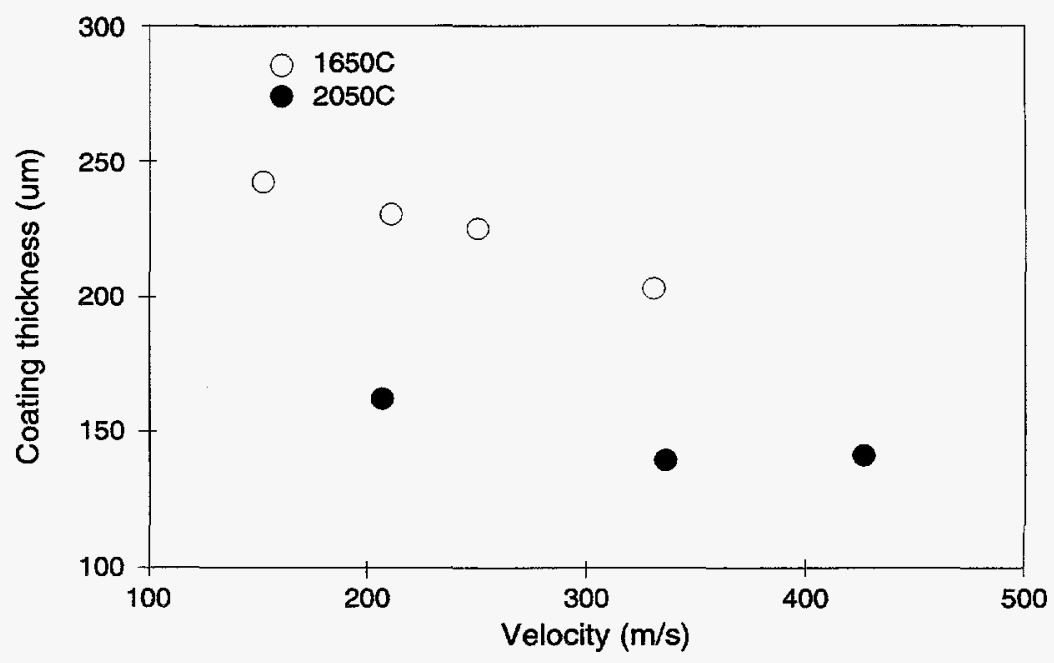

Coating thickness (a measure of the relative deposition efficiency) as a function of particle velocity for nickel coatings sprayed at 1650 and $2050^{\circ} \mathrm{C}$ optimum coating microstructures could only have been developed at the INEL. The finite element methods developed have also found application in programs developing thin diamond films and in developing a scientific understanding of the behavior of protective oxides on advanced alloys of interest to DOE.

\section{Business Development Activities}

The results of this work and a University Research Consortium program with State University of New York at Stony Brook have lead to a collaborative research effort with Caterpillar to develop graded thermal barrier coatings for diesel engines. While further work is necessary to develop this technology, Caterpillar is expecting to use methods developed here in production. We are also working with Lehigh University to develop intelligent methods to apply wearand erosion-resistant coatings for fossil energy applications. We anticipate submitting a proposal to DOE, Office of Fossil Energy, or Energy Efficiency, to carry out this work with Lehigh and a consortium of power producers. 


\section{Microbiology of Sugar Plant Flume Systems}

LDRD 305

\section{R. S. Cherry}

This project has characterized the growth and metabolic activity of the bacteria in the flume system and ponds of Amalgamated Sugar Co.'s Twin Falls sugar beet processing plant, one typical of the industry. The flume system floats beets from the unloading station into the processing plant. Under some conditions, this water system generates highly objectionable odors. We characterized the bacteria at key points in the water circulation loop and, to understand what determines their growth and odorcausing behavior, we tracked the sources and disposition of carbon, sulfur, and dissolved oxygen in the system. The major cause of odors is the bacterial conversion of sulfate ions from a flue gas scrubber and organic materials derived from sugar beets into hydrogen sulfide. Poor control of $\mathrm{pH}$ in the flume system leads to the intermittent evolution of this $\mathrm{H}_{2} \mathrm{~S}$ from the flume water. Amalgamated plans to improve the flume system $\mathrm{pH}$ control, perhaps with an automated control system, and to test revised operating procedures in the scrubber.

\section{Objectives}

- Prepare flowsheets and material balances of carbon, sulfur, and oxygen in the flume system at three different times (September, November, and February) during the sixmonth-long beet processing campaign. Log microbially significant operating conditions at these times.

- Characterize the bacteria in the flume system by numbers, type, and metabolic activity.

- Identify mechanisms of odor formation and recommend operating or equipment changes to control them.

- Present results at the 28 th General Meeting of the Association of Sugar Beet Technologists in New Orleans, March 1995.

\section{Accomplishments}

The closed-circuit flume system is used to transport the beets and wash soil from them prior to processing (see the figure below). The ponds have a total volume of six million gallons; the water is circulated through the flume at a rate of roughly $6000 \mathrm{gpm}$.

Microbial sampling indicated the presence of aerobic, heterotrophic bacteria at all sites at $10^{7}$ to $10^{8}$ colony forming units $/ \mathrm{ml}$, except for the scrubber effluent, which was approximately $10^{4}$ $\mathrm{CFU} / \mathrm{ml}$. These are significant bacterial loadings, as expected. The varied colony morphologies observed in these platings indicate a consortium of bacteria. Significant amounts of bacterial sulfate reduction activity were observed in every sample tested.

The ponds where most bacterial activity occurs were anaerobic, with oxygen values at or below $1 \mathrm{mg} / \mathrm{L}$. Oxygen concentrations increase in the flumes where there is turbulent flow and good air contacting, but aerobic bacteria consume this oxygen in the clarifier. The redox data confirm that most of the system was anaerobic ( -150 to $-300 \mathrm{mV}$ ).

Between the September and November collections, there was a significant increase in formic, lactic, and acetic acids, especially the latter two, whereas butyric acid decreased. These changes indicate a shift in the bacterial species present and the environmental conditions in the flume water, which can affect the end products made by any particular species.

In September, the flume $\mathrm{pH}$ was maintained at approximately 7 . It was increased to $\mathrm{pH} \mathrm{9-11} \mathrm{in}$ mid-November. Although these averages do not show the poor hourly $\mathrm{pH}$ control, it was clearly difficult to maintain a constant $\mathrm{pH}$ at the clarifier. Swings of two $\mathrm{pH}$ points were common at the east manhole, the clarifier inlet, the 3-acre pond, and the mud pond return. These swings can affect $\mathrm{H}_{2} \mathrm{~S}$ release. The equilibrium between the volatile undissociated $\left(\mathrm{H}_{2} \mathrm{~S}\right)$ and the nonvolatile dissociated (HS-) forms of hydrogen sulfide appears to be quite important in this system. As an acid-base reaction, the dissociation is very rapid, so the relative amounts of these species shift almost instantaneously as the $\mathrm{pH}$ changes. This can lead to rapid, large changes in the amount of the volatile $\mathrm{H}_{2} \mathrm{~S}$ form, hence the sudden evolution of odors from the flume system.

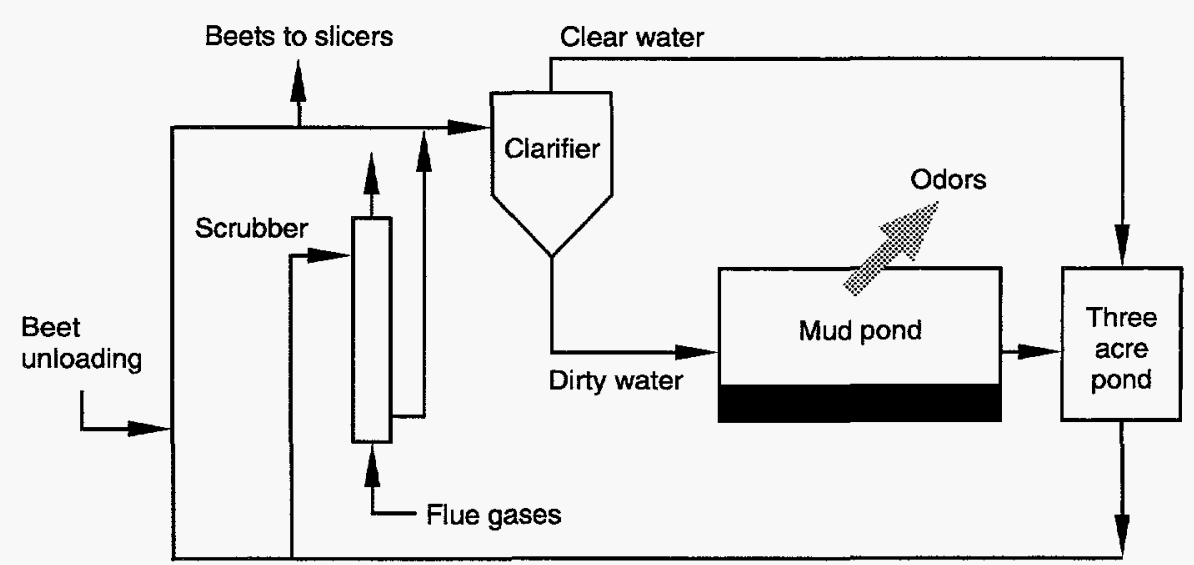

Sugar Beet Plant Flume System 
This $\mathrm{pH}$ problem is particularly acute in the dryer scrubber. At the scrubber effluent conditions, the hydrogen sulfide is about $75 \%$ in the volatile $\mathrm{H}_{2} \mathrm{~S}$ form. In addition, the high temperature there increases the volatility of this hydrogen sulfide, so it would tend to be stripped into the scrubber effluent gas. This hydrogen sulfide would rise with the plume and not be detected at ground level at Amalgamated's plant site. However, as the plume disperses in the atmosphere, odors might be noticeable well downwind of the plant.

Based on our work, we believe Amalgamated should operate the flume system with a $\mathrm{pH}$ near 9 throughout the entire campaign. An automatic control system using an on-line $\mathrm{pH}$ probe with feedback control of lime addition would be appropriate. A higher effluent $\mathrm{pH}$ in scrubber might help address the stripping problem there.

As a longer-term, more expensive option, if the other recommendations do not adequately address the odor problem, Amalgamated could consider adding an aeration step to the flume system. This aeration would operate continuously with the intent of microbially oxidizing organic acids that were formed elsewhere in the system. Removing them this way would greatly reduce the amount of substrate available to sulfate-reducing bacteria that make the $\mathrm{H}_{2} \mathrm{~S}$.

\section{New or Increased Technical Capability}

This project has allowed several of the staff in the Biotechnology group to become familiar with the issues and problems of factory operations in addition to the laboratory research work.

\section{Business Development Opportunities}

Sugar beet farming and processing is a major industry in Idaho and other northern states. This project established a close working relationship between
INEL and Amalgamated Sugar Co., the technical leader in the sugar beet industry, in which we cooperated to analyze a great number of samples at both locations. INEL also learned of a major problem in the beet sugar industry, loss of sugar to bacteria in the refining process, which represents a major cost to producers. Our experience on the odor problem puts us in a good position to develop a partnership with the industry to address this problem.

\section{Alkylation Catalysts for Operation at Supercritical Fluid Conditions LDRD 1206 \\ B. D. Lee}

This $L D R D$ is developing a solid catalyst supercritical fluid (SCF) isobutane alkylation process to replace the current liquid-acid catalyzed process used in the petroleum refining industry for gasoline manufacturing. The technology utilizes the unique solvent extraction properties of SCFs to simultaneously extract coke from porous catalysts while providing optimum reaction conditions, thereby yielding a process with increased reactivity, long catalyst lifetimes and improved process efficiency.

Motor fuel alkylation couples high vapor pressure isoparaffins and olefins to yield a low vapor pressure, high octane gasoline blend stock low in environmentally undesired components. Currently, alkylation employs a liquid acid catalyzed process using either highly concentrated sulfuric or hydrofluoric acid. These processes pose a serious safety and environmental threat through the transport, storage and processing of concentrated acids as well as in the disposal of acid-oil sludges. In addition, hydrofluoric acid alkylation has come under strong environmental regulatory pres. sure. For these reasons, it is quite desirable to substitute a solid catalyst for the liquid acids. Solid catalysts could replace liquid acids and eliminate the safety and environmental process concerns; however, solid catalysts deactivate rapidly due to the buildup of coke. Operation of a solid catalytic process at supercritical fluid conditions is being explored to control catalyst deactivation by the continuous extraction of coke from the catalyst surface and thereby produce a process that reduces refinery costs.

\section{Objectives}

- Develop a bench-scale, continuous flow, fixed-bed catalytic reactor unit capable of studying the alkylation reaction at supercritical temperatures and pressures.

- Develop a solid catalyzed, supercritical fluid (SCF) isobutane alkylation process.

- Identify and test solid acid catalyst for alkylation.

- Demonstrate efficient reaction at SCF conditions.

- Control catalyst deactivation by SCF extraction of coke forming compounds.

\section{Accomplishments}

The alkylation reaction over solid acid catalysts at SCF conditions was explored for the purpose of developing a fixed bed, solid catalyst alkylation process with significant catalyst longevity. By mixing various compounds with the reactants, a SCF reaction mixture is produced that can be tuned to provide optimal reaction conditions for high rates of olefin conversion, high octane alkylate product, and catalyst longevity.

We built a bench-scale, continuous flow experimental reactor unit that enabled us to study the solid catalyst reaction at both liquid and supercritical conditions. The reaction unit can be used to study reaction temperatures up to $350^{\circ} \mathrm{C}$ and pressures from ambient to 6000 psig. 
SCF reaction feeds were produced using isobutane, trans-2butene, and an added inert compound. Critical temperatures and pressures for the reaction mixtures with and without reaction products were predicted using the ASPEN/PLUS process simulation code.

Two classes of catalysts were studied, ultra stable $Y$ (USY) zeolites, and a class of solid super acid catalyst; sulfated mixed metal oxides. The USY zeolites were obtained commercially while the sulfated mixed metal oxides were produced in-house. Sulfated mixed metal oxides were produced through precipitation, dehydration, sulfanation, and calcination techniques. Variations in metal concentrations and levels of sulfanation were explored.

One USY zeolite was found to catalyze the alkylation reaction. However, in addition to producing the desired $\mathrm{C} 8$ hydrocarbons, the zeolite catalyzed cracking reactions, producing $\mathrm{C} 5$ to $\mathrm{C} 7$ hydrocarbons; catalyzed oligomerization reactions, producing C9 and heavier hydrocarbons; and produced significant amounts of coke. The catalyst deactivated rapidly, within 1 to 2 hours. The USY zeolites were determined to be poor catalyst for the alkylation reaction.

The sulfated mixed metal oxide catalysts were found to be better alkylation catalysts at SCF conditions. One catalyst was found to have reasonable reaction rates, produce primarily $\mathrm{C} 8$ hydrocarbons, and show good catalyst stability, even up to 8 hours time on line. A comparison of SCF operation to liquid operation shows that the reaction rates compared very well, but the deactivation rate at SCF conditions was over three times better than at liquid conditions. However, at both the SCF and liquid conditions, mass spectrometry analysis shows that the products were primarily olefins instead of the desired isoparaffins. Work is continuing to explore variations in the catalyst composition and process conditions that would improve the isoparaffin yields.

New or Increased Technical Capability

New or increased technical capabilities at the INEL produced by this LDRD are as follows:

- An experimental unit capable of studying continuous flow catalytic reactions in a fixedbed reactor in either gas, liquid, or supercritical phases at temperatures up to $350^{\circ} \mathrm{C}$ and pressures up to 6,000 psig is now available at the INEL.

- Control of catalyst deactivation at SCF conditions has been shown for a solid alkylation catalyst.

- Experience has been gained in the study of solid catalysts for isobutane alkylation.

- Experience has been gained in the production of sulfated mixed metal oxide solid super acid catalysts.

- Expertise studying catalytic reactions in supercritical fluids has been increased.

\section{Business Development Opportunities}

The industrial application of this process can be highly significant if the production of isoparaffins can be improved. In the U.S., Europe, and Asia/Pacific region, alkylation production capacity is currently at 1.2 million barrels per day, with expectations of $2.7 \mathrm{mil}-$ lion barrels per day by the year 2000. Investments in new plants and profits from the increased alkylation capacity are expected to be in the area of 2 billion dollars per year over the next ten years. Companies identified to have an interest in a finial product are Universal Oil Products, Inc., Mobile Oil Co., and Ashland Petroleum Company.

\section{Mixed Microbial Encapsulated Fertilizer Amendments LDRD 1241 \\ P. A. Pryfogle, R. D. Rogers}

The scope of this investigation was primarily directed at determining the efficacy of the immobilized phosphate ore and microorganisms in a pellet providing sufficient nutrients to enhance plant growth. This scope includes several tasks that were completed in a logical order to properly evaluate the pellet's potential.

\section{Objectives}

- The following tasks represent the objectives directing the research that was scheduled for FY95. They were broken down into seven activities.

- Incorporation of basic materials into pellets to support bacterial metabolic requirements and initiate their activity.

- Select crop-plants for use in the plant growth experiments.

- Comparison of immobilized pellet material to commercial fertilizer.

- Establish a logical experimental plan using a step-wise progression leading to plot or field study scenarios.

- Evaluate economics of production and handling, examine market potential, and plan technology transfer.

- Develop requests for patents on any products, procedures, or ideas deemed potentially patentable. 
- Write and submit summary papers to refereed journals.

\section{Accomplishments}

Overall the research was successful in providing information supporting the ability of the immobilized materials enhance plant growth. The development of market potential and technology transfer suffered some since there was some uncertainty associated with the proprietary ownership of inventions and products developed during this research and tied to CRADA documentation. The details of this problem are currently under consideration by both Lockheed Idaho Technologies Company (Lockheed Idaho) and J. R. Simplot, Pocatello, Idaho.

Barley was selected as the test plant for our research. This plant has demonstrated a particular sensitivity to phosphorus deficiency which is revealed as blue-edges on leaf blades and stems under extreme deficiency. Plant growth and development are also means of expression for nutrient or physiological stress. While blue edges were never observed in our test plots, leaf necrosis and differences in plant growth patterns were evident.

Three different experiments were carried out over the course of the year. Two of the tests were conducted using sand. The sand was washed with an acid solution to remove possible soluble contaminants (mainly phosphorus), and rinsed to a neutral $\mathrm{pH}$. The first experiment with sand proved to be a learning experience that provided many lessons on handling seedlings, growing, and harvesting conditions. The second sand experiment was determined to be successful in demonstrating the ability of the immobilized phosphate ore and bacteria to support plant growth. In fact, the immobilized material appeared to be bet- ter than the commercial fertilizer in the sand plots. There appeared to be a problem in the provision of secondary nutrients in the sand, so a third test was conducted using a typical desert soil, low in phosphorus content. This test confirmed previous results and indicated that the immobilized material did support luxurious plant growth, not only vegetative structure but grain production, too.

The next series of tests are projected to be completed in soil test plots outside the confines of a greenhouse. This would permit a more natural evaluation the plant growth potential for the immobilized materials. It is anticipated that as soon as the details are clear on the proprietary nature of this work this technology will be transferred to an industrial partner.

And, the plot tests will be a priority of interest and the responsibility of the technology union.

Several areas of this work are under consideration for publication. Due to the CRADA review process and other uncertainties at this time, these reports have not been submitted outside the research team.

\section{New or Increased Technical Capabilities}

This LDRD research provided an opportunity to develop technical expertise for constructing multiple component immobilized structures to perform complex biologically derived tasks. I believe one term used to describe the beads was "micro-scale bioreactor." This concept has many potential applications since it may be possible to control microbial activity through specific inclusion (or exclusion) of materials added to the immobilization matrix. This in particular may be of interest to degradation applications where contaminant breakdown is a secondary activity dependent upon the presence of a primary nutrient.
Other avenues for the utilization of this technique are already under design and testing. And the success of this research should place our scientists in a favorable position for technology transfer once the intricacies have been resolved on the proprietary nature of the LITCO/Simplot CRADA.

Business Development Opportunities

Business opportunities have been identified, however the exact actions are pending legal review of the nature of proprietary rights between the CRADA partners J. $R$. Simplot and the INEL. In addition to Simplot's interest, Monsanto and Carghil have expressed an interest in this research and have indicated that some research monies would be available, depending upon the outcome of the CRADA review.

\section{Sensors for Control of Thermal Spray Processes} LDRD 1301

W. D. Swank, J.R. Fincke

The thermal spray coating process is used extensively in the jet engine manufacturing and refurbishing industry, which represents only a fraction of the potential applications for thermally applied coatings. Other applications relate to assembly line manufacturing, for which it is necessary to continuously spray coatings of consistent quality. Uncontrollable variables in the process, such as nozzle erosion and fuel heat content, have made it nearly impossible to do this without some kind of active control. The lack of sensors that could detect variables directly related to the product quality is the reason active control has not been developed for thermal spray coating processes. This project is developing two sensors that can be used in real-time feed back control of spray forming and spray coating systems. The first is a particle spray position sensor. The position of 
the particles and their flight path through the hot gas determines their thermal condition prior to impacting the substrate. Their position is detected with the combination of light collection optics and a position sensitive silicon chip. The second sensor under development will measure particle velocity. It is described as LDRD 4082.

\section{Objectives}

- Design optical, electrical, and mechanical components of the position sensor concepts.

- Build bench-top prototypes of both sensors.

- Test both sensors for applicability and limitations on a thermal spray coating system.

- Present he capabilities and limitations of both sensors to potential industrial collaborators.

- Design, fabricate, and test industrial, hardened versions of the sensors.

\section{Accomplishments}

A particle position sensor has been developed, fabricated, and demonstrated, consisting of a sensor head and associated electronics. The compact optical head of the position sensor consists of optics that image the particle spray field on a position-sensitive silicon chip. Optical filtering minimizes background light interference. Integral to the measurement head is an iris that, when used with the level meter on the front panel of the electronics, provides a ready means for optimizing signal levels. The particle position sensor is insensitive to particle loading (particle feed rate), particle composition, and particle size distribution. A digital front panel display visually indicates spray pattern position. Through the use of a zero adjust feature, the readout may be zeroed for the baseline spray pat- tern trajectory. Deviations are read as a positive or negative delta in the spray field position. A 10-volt analog output, proportional to spray pattern position, inputs to data acquisition and controllers.

The particle position sensor senses the position of the spray pattern centroid relative to the torch centerline or other reference data. The figure shows changes in the particle spray field position as the particle temperature is being controlled over a $100^{\circ} \mathrm{C}$ interval. In this case, the particle position varies over a range of $4 \mathrm{~mm}$. Even small variations such as this can mean a significant amount of over spray and are a clear indication of torch malfunctions such as nozzle loading. When spray position sensing is available, changes in spray pattern trajectory can be easily compensated for by altering particle injection velocity, optimizing spray gun trajectory, or both.

\section{New or Increased Technical Capabilities}

The thermal spray coating process is unpredictable from one run to the next, resulting in coated parts that do not meet specifications. Then, at great expense, these parts must be either refurbished or scraped. This project has the potential to reduce the cost and improve the quality and reliability of thermally sprayed coatings. Also, with increased reliability comes an increase in applications for thermally sprayed coatings. Expanded use in the automotive industry could easily increase the thermal spray market by $50 \%$, while making many automotive manufacturing processes significantly more cost effective (Thermal Spray Coating, State-of-the-Art Assessment, unpublished, The National Center for Manufacturing Sciences).

\section{Business Development Opportunities}

December FY-95 will bring to a close a CRADA with Tafa Inc., in which an industrial, hardened inflight particle pyrometer (IPP) was developed. Tafa has developed and markets a computer-based robot/process controller (HAWCS II) that has the capability to accept input from sensors and deliver output to process control consoles. This computer controller has not been used to its fullest potential owing to the lack of suitable sensors. With the development of the $\mathrm{IPP}$ and the particle position sensor, Tafa has expressed intent to

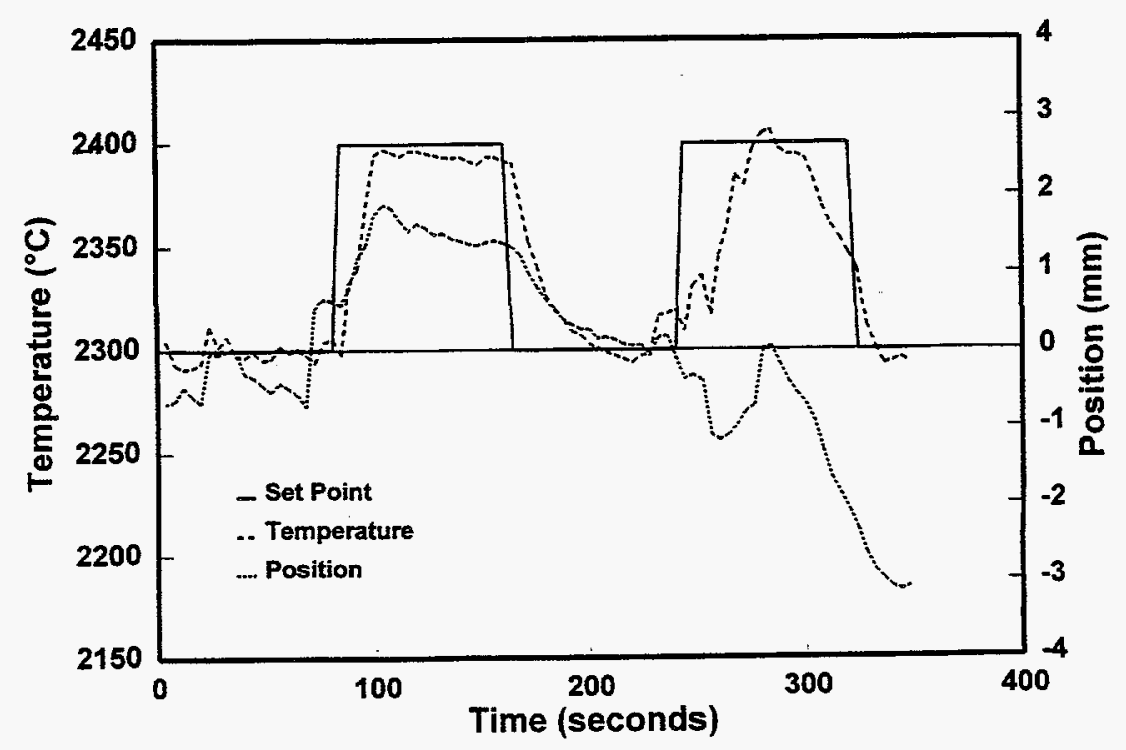

Changes in Spray Position as the Particle Temperature is Controlled 
continue its advancement of a control system for thermal spray processes.

Although no formal agreements are in place, end users of thermal spray coating processes, such as Pratt \& Whitney, General Electric, and Ford Motor Company have expressed interest in sprayed particle sensors and controls.

\section{Heat Transfer Through Solid Cryogen}

LDRD 1302

\section{A. S. Siahpush}

We are investigating the storage of hydrogen in a solid phase. In order to provide uniform cooling/freezing of the cryogenic material, an aluminum foam can be positioned in the tank. The foam provides dynamic stability during transportation and uniformly conducts the heat away from the cryogenic material during the freezing process. There has been no research performed on this new technique to determine the effective thermal conductivity and system efficiency of the cryogenic process.

\section{Objectives}

The scope of this project includes concept development of new and unique methods of storing hydrogen for either utility or transportation system use.

- Study the concept of a refreezable solid cryogen.

- Define an envelope of cryogenic requirements that will bound parametric trade-off studies of viable options and the basis of a conceptual design.

- Perform parametric trade studies for a variety of liquid and solid hydrogen storage options. The studies will include the use of aluminum foam, multi-layer insulation blankets, vapor-cooled shielding, and radiative cooling.

- Identify the key technology drivers associated with long-term hydrogen storage, such as thermal isolation materials, lowtemperature radiators, and the systems required for both liquid and solid cryogenic storage.

- Develop a baseline design concept of a long-term hydrogen storage system.

Business Development Opportunities

This new technology has attracted several potential customers. The Materials and Aerospace Corporation is very interested in cooperating with INEL in this research.

\section{Concentration Technologies LDRD 1303$$
\text { T. D. Turner }
$$

The Contaminant Analysis Automation project developed a concept for improving results during concentration of liquids. Typically, contaminants to be analyzed are dissolved in organic solvents, and they are often in low concentrations. Our concept uses ultrasonic energy to increase the concentrations of the solute, and therefore the recovery of the compounds of interest. This project investigated the possibilities of using this technology in a number of industries, though we found the petrochemical industry to have a large number of processes that involve concentration, and our efforts were directed toward that market. Several tests were run to decide if the ultrasound methods could be applied. Test data have shown some positive gains using ultrasonics. However, owing to the limitations of time and scope these positive results are only indicators of the improvements that could be realized.

At this time it is still difficult to determine the total impact the ultrasonic concept could have. There is substantial need for improved concentration technologies in many markets, i.e., perfume, pharmaceutical, environmental, and the petrochemical markets. For example, in the petrochemical market approximately $90 \%$ of all refinery processes involve some type of concentration or distillation. In addition, approximately $90 \%$ of the energy cost of producing petrochemical products are in these processes. Improving the concentration or distillation process by only a small percent, could result in large savings to the industry. We anticipate that similar needs and conditions could be found in the other industries.

\section{Objectives}

- Investigate the petrochemical market to determine if there is a need for improved concentration and distillation.

- Test to assess the application of this technology in some area of the petrochemical market.

- Consult with experts in the fields of interest to determine their assessment of the technology.

\section{Accomplishments}

The application of ultrasonics was part of a concept to automate the solvent concentration procedure in the environmental laboratory, primarily the Kuderna-Danish, Snyder ball concentrator. Testing has shown small improvements in this process, but enough testing to optimize the process has not been done. Time limitations during the design of the automated instrument required the suspension of the ultrasonic testing and all efforts were put into development of the concentration instrument. 
The first step was identifying other industries that concentrate liquids as part of their process. An obvious field was the environmental area, and a prototype instrument was built. Investigation showed that the petrochemical and perfume industries also used similar concentration methods to produce their products.

A petrochemical engineer at the INEL was contacted, and the technology presented to him. He believes there is a substantial potential in the industry and agreed to do some basic testing that would provide a baseline of information. The following excerpts are from his final report.

"Distillation is the primary method used to separate compounds in the petroleum refining and chemical industries. In petroleum refining industry, distillation is very capital and energy intensive. Approximately $90 \%$ of a plant's capital costs and $90 \%$ of the energy used operations are associated with distillation. Distillation columns employ either trays or packing to provide contact between the liquid and vapor phases....

"Ultrasonics were explored as an alternative method to increase the interaction between liquid and vapor. By providing ultrasonic waves within a distillation column, nucleation sites for liquid condensation could be provided. If the use of ultrasonics could replace trays or packing or reduce the spacing between trays or the HEPT (theoretical plate) of packing, the capital costs associated with distillation could be reduced. Additionally, if more theoretical plates could be achieved within a column, the reflux ratio required for separation could be reduced, decreasing distillation energy consumption."

Several tests were run to determine some basic base line data for ultrasonics in the petrochemical industry. Although several tests showed little or no effect, the use of the ultrasonic transducer did improve distillation efficiency in the experiment when the heat effects of the transducer were compensated for.

Our investigation also included an outside chemist consultant. He believed that based on the preliminary and limited test data, there are indications that ultrasonic energy applied at the reflux column can provide better results, even though the effect is very small under the current configuration.

\section{New or Increased Technical Capabilities}

We have been able to increase our understanding of how ultrasonic energy can assist in improving the separation in concentration and distillation operations. Test methods have been improved, and some problems have surfaced that will allow us to redirect further testing in order to improve the separation processes. We have determined that, even though the effects are small at this time, optimization of the process may provide a new and valuable method in concentration and distillation technologies.

\section{Business Development Opportunities}

Our initial results, while showing a small effect at this time, are encouraging. Based on our discussions with petroleum engineers and representatives from other industries, this may well be a viable solute concentrating method. With the improvement of this technology, there are many industrial markets that could benefit from improved concentration and distillation.

\section{Site-Specific Technologies for Agriculture: Pilot Project LDRD 2278}

\section{R. L. Hoskinson}

Agriculture today faces two major problems: declining profits and the adverse impact of agricultural practices on the environment. Both of these problems can be addressed by site-specific technologies for agriculture (SST4Ag). The objective of these technologies is to use sensors, instrumentation, controls, robotics, image analysis, computer software, and decision support systems tp apply agriculture inputs only when and where necessary. Instead of treating a field as a uniform piece of land, SST4Ag treats each part of the field according to the variations of soil conditions and crop status. By excluding unneeded

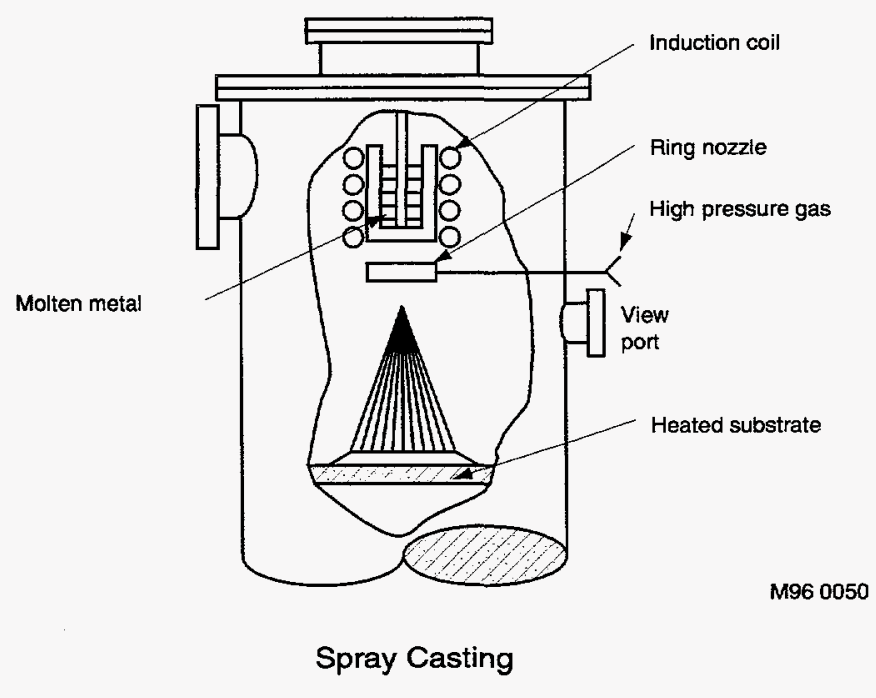


chemicals and energy inputs, farmers save costs and avoid polluting the environment. SST4Ag aims to combine recent advances in science and technology to make site-specific farming available, affordable, and flexible enough to be used throughout the United States, and eventually the world

\section{Objectives}

- Database Systems-GIS (prescription algorithm), relational, and model interfaces:

- Calculate GIS optimization and analysis routines for maximum production efficiency.

- Develop 1995 series of independent coverage maps.

- Integrate independent coverage maps into a combined field characterization map.

- Develop digital interface between University of Idaho crop model and GIS.

- Develop digital GIS output based on University of Idaho crop model prescriptions for site-specific application by smart machines.

- Develop 1995 site-specific yield maps.

- Initiate software development CRADA and technology transfer.

- Crop and Field Information Management Network:

- Evaluate preliminary bench and field test data.

- Enhance and improve developed hardware systems, based on field tests.

- Field test improved hardware systems (site-specific delivery of model's prescription).

- Evaluate results of testing.

- Document and report results.

- Initiate technology transfer.
- Sensors and Controls Engineering:

- Support engineering for interfacing a regional positioning system into the SST4Ag.

- Support industry partners in hardware and sensor design for smart machines.

\section{Technical Accomplishments}

The following activities were accomplished in FY-95:

- Conducted complete soil nutrient sampling and mapping.

- Collected irrigation seasonal data, including soil moisture sensors and rain gauges.

- Initiated environmental baselining of agricultural ecosystem.

- Conducted soil microbial assessment sampling collecting.

- Conducted field assessment of GPS accuracy.

- Designed and built soil physics mapping sulky.

- Conducted complete field soil physics mapping using INEL sulky.

- Installed two meteorological weather monitoring stations.

- Developed prototype SST4Ag computer workstation environment.

- Collected intensive GPS data defining features in and around research fields.

- Supported INEL mapping at Ricks College farm.

- Conducted INEL/Washington State University/USDA-ARS grain monitoring.

- Collaborated with University of Idaho and USDA on Mississippi soybean yield research.

- Monitored harvest yield.
- Acquired and developed SST4Ag workstation environment.

- Built and demonstrated prototype information management system IMS4Ag.

- Developed a series of independent GIS coverage maps for baseline data.

- Conducted a briefing and review meeting for nationwide agriculture industry.

- Participated in USDA national field day for site-specific farming.

\section{New or Increased Technical Capability}

The National SST4Ag project has been in progress for the past two years, during which the SST4Ag project has gained national recognition for Lockheed Idaho Technologies and the Idaho National Engineering Laboratory as a leader in site-specific technologies for agriculture. The project has produced a significant amount of intellectual property for Lockheed Idaho and the INEL.

Business Development Opportunities

The following CRADAs and potential license opportunities for LMIT have resulted:

- A CRADA is near signing with ESRI, which includes development of software that will be licensed (an invention disclosure has been filed).

- A CRADA with Micro-Trak is in progress.

- A CRADA with Trimble Navigation is in progress.

- An information management system prototype called IMS4Ag has been developed, which has strong potential to be licensed (three invention disclosures have been filed).

- A field-deployed geophysical sensor system, which includes 
the sulky, has been built and tested, and has potential as a licensed or marketed system.

\section{Development of Nondestructive Fatigue Monitor}

LDRD 2501

\author{
P. E. MacDonald, V. N. Shah, \\ D. W. Akers, C. H. Sellers, \\ K. L. Telschow
}

The project is developing nondestructive evaluation (NDE) methods to characterize microstructural changes caused by three major degradation mechanisms active in light water reactors: fatigue, radiation embrittlement, and thermal aging. The methods will detect and correlate the microstructural changes that are precursors of fatigue cracking and that are associated with radiation embrittiement and thermal aging, so that remedial measures can be timely and economically taken. Quantitative characterization of nuclear power plant material damage will help justify the current operation and any life extension of nuclear power plants, which would result in significant savings.

The project was organized in two activities: (1) assessment of NDE methods for characterization of microstructural changes associated with aging of nuclear power plant materials, and (2) development of NDE methods for in situ characterization of fatigue damage. A state-ofthe-art report on microstructural changes associated with fatigue, radiation embrittlement, and thermal aging was prepared. The report identifies the appropriate nondestructive examination techniques for characterization of these changes.

Two nondestructive methods for in situ characterization of fatigue damage in austenitic stainless steel components such as PWR surge lines and safety injection lines are being developed during FY 1995: positron annihilation, and magnetics using a hightemperature superconducting quantum interference device (SQUID). Ini- tial test results show that these methods can detect microstructural changes at a very early stage prior to fatigue crack initiation; as little as $5 \%$ fatigue damage can be detected.

\section{Objectives}

- Develop indepth understanding of microstructural changes in nuclear power plant materials caused by fatigue, radiation embrittlement, and thermal aging. Identify appropriate NDE techniques to characterize these changes.

- Design, fabricate, and fatigue test austenitic stainless steel specimens.

- Perform positron annihilation measurements on the fatigued specimens, and develop a methodology to correlate the measurements with the estimated fatigue damage.

- Configure a SQUID sensor for measuring the magnetic field of specimens placed in an extremely low ambient magnetic field (in a shielded enclosure).

- Develop methodologies for SQUID measurement of the remanent magnetic field of Type 304 stainless steel samples with small concentrations of ferromagnetic phase such as martensite. Correlate these measurements with fatigue damage and also with the martensite contents measured using another magnetic technique.

\section{Technical Accomplishments}

We performed an indepth assessment of advanced NDE techniques for quantitative characterization of the microstructural changes in light water reactor structural materials caused by three different degradation mecha- nisms: fatigue, radiation embrittlement, and thermal aging. Two NDE techniques were identified for characterization of fatigue damage: positron annihilation and magnetic measurements with a SQUID sensor.

Preliminary test results show that the positron annihilation results and SQUID measurements can be correlated to fatigue damage in Type 304 stainless steel specimens. The positron annihilation spectra show different live shapes as a function of fatigue. These shapes were measured, characterized, and compared for differing amounts of fatigue, including zero fatigue. The positron annihilation method was shown to be a viable method for examing metal fatigue.

The austenite-to-martensite transformation in austenitic stainless steels primarily takes place on cooling, but it can be induced by plastic deformation associated with fatigue. Because martensite is ferromagnetic and austenite is not, the amount of martensite can be estimated by measuring the remanent magnetization of a specimen. High-temperature SQUID was used to measure the remanent magnetization of a number of specimens having varying levels of fatigue damage. The maximum of the magnetization is present at the midpoint of the sample, and it increases with the increase in the fatigue damage. A correlation exists between the remanent magnetization and the martensite content measured with vibrating sample magnetometry. Several factors affect the martensite transformation: temperature, chemical composition, deformation rate, and cold work. Their effect on magnetic measurements needs to be evaluated before this technique can be applied in the field.

New Technical Capability

Further development of the NDE methods being evaluated in 
this project could provide new technical capabilities for aging assessment and life extension of nuclear power plant components. A patent application for the positron annihilation method is being prepared. Capability is developed for the measurement of the magnetic properties along the length of samples using a shielded $77 \mathrm{~K}$ SQUID sensor.

\section{Business Development Opportunities}

Potential collaborations exist with Conductus Corporation. for development of SQUID sensors and with electric utilities for field application of the NDE sensors.

\section{Advanced Barcode (Holotag) Reader} LDRD 3302

\section{Vance A. Deason}

The INEL advanced barcode is a new concept in medium density (a few megabytes) data storage in situations where bar codes have normally been used but where data bases much larger than bar codes now allow are desirable. Not truly a bar code, our concept is an optical device an inch or two across and a few tenths of a $\mathrm{mm}$ thick that contains a record of any of several types of data: images, graphics, text, conventional bar codes, digital data. Some of the data can be read by the naked eye; other types require a reader and an associated data system to acquire and interpret the data.

\section{Objectives}

Our goal this year was to complete the evaluation of the design criteria for the holotag reader. Based on this information, we would finalize the design of the reader and build a prototype unit. We also wanted to continue the evaluation of recording materials and methods for writing data to the tag.

\section{Technical Accomplishments}

We rebuilt the grating maker (originally developed under LDRD funding and patented as U.S. Patent No. 5,016,951, issued 5-2191), to match current needs and standards for writing the tags. This has been the workhorse system for making all kinds of gratings and tags.

\section{The Advanced Barcode Writer System}

We completed the integration of a Spatial Light Modulator (SLM) assembly and adapted it to store the contents of several $(>10)$ successive $640 \times 480$ pixel computer screens onto a single tag. This SLM is somewhat lacking in resolution, but was the highest resolution device we could afford. It is also very inefficient in its use of light, so our laser is barely adequate to write the tag using the SLM. In principle, and to some extent in practice, we can transfer screen images of text, graphics, and images to the tag in a very simple and convenient manner. This system constitutes the tag writer.

We have identified and tested a class of recording materials we believe is suitable for recording the tag, and which could make the transition to commercialization. These materials have adequate resolution, are very thin, are advertised as having reasonable environmental stability, and, while expensive in small quantities, could be reasonably priced in production quantities.

We modified the optical arrangement of the tag writer to produce tags that could be more conveniently read. With this new arrangement, one can read the tags by eye under the proper circumstances. A more convenient reader has been designed that would be relatively easy and inexpensive to make. This was possible by using appropriate lensing during formation to match the expected optical configuration of the reader. A prototype version of the reader is under construction and will be tested this fiscal year.

\section{New or Increased Technical Capability}

- Introduced the use of optically written SLMs to the INEL.

- Improved and upgraded the tag writer.

- Clarified the issues involved in the tag reader design and the techniques for writing a tag compatible with a simple reader.

- Built a prototype tag reader.

\section{Business Development Opportunities}

Symbol Technologies has expressed interest in this concept. We hope to have a CRADA in place soon to develop auto ID tags (i.e., tags containing information rather than simply being pointers to a data base). These might be a hybrid of our tag for archival information and an electronic tag for rewritability and updating.

Edward Merewether of Fluor Daniel requested information and received a general description of the technique that is being circulated among potential investors.

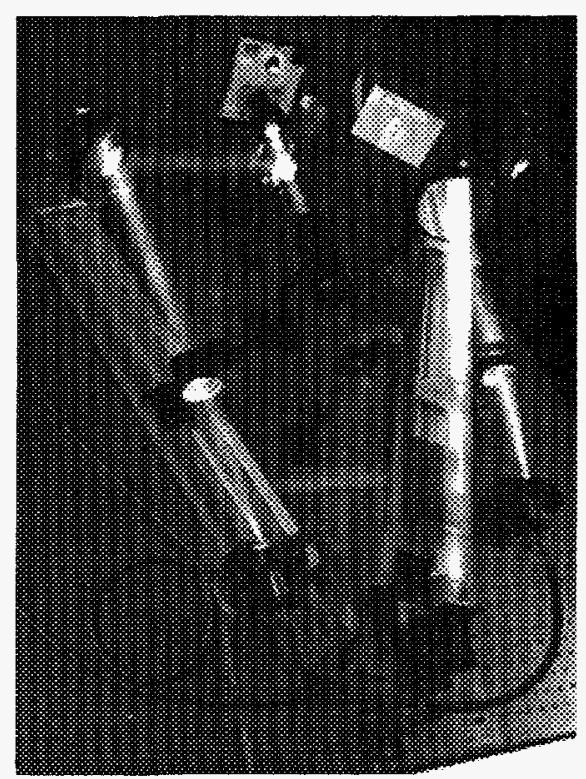




\section{Transportation Network Core Software}

LDRD 3601

\section{J. Kurkowski}

The Transportation Network Core Software project is designed to harvest software integration technology, previously developed by the INEL on a military project, for broad application to other potential customers in both the public and private sectors. The software provides an open, layered architecture, based on industry and government standards, used for distributed system integration. Transportation initiatives offer an ideal testbed for demonstrating the technology under real world situations.

Potential benefits to the INEL include solidifying our presence in the transportation industry as the nation moves toward intelligent transportation systems (ITS). The pervasive use of software and software integration of a variety of data sources (including sensor output and databases) are required for ITS to be able to distribute information to those who need it, when they need it. ITS is now in the preliminary stages of development, with most activities nationwide in the formative stages of specification and design. By providing a working demonstration of an information integration prototype, the INEL can influence the development of standards, build laboratory expertise, and provide a potential product available for deployment of the transportation systems of the future.

The software technology is also part of a proposed project for building the INEL transportation testbed. Here, numerous new sensors might be tested by INEL and industry engineers with control and data distribution delivered worldwide using this technology integrated with the emerging technologies of the Internet.

\section{Objectives}

- Update the operating system and database engine to current industry releases.
- Convert needed portions of existing software to the updated environment.

- Replace military-specific features and terminology with generic or tailored ones.

- Place the updated software items under configuration management.

- Prepare a demonstration using the Out-of-service Verification Field Operational Test prototype application in a laboratory environment.

- Analyze the viability of this technology to support transportation and general integration requirements. If favorable, pursue copyright of the software.

\section{Technical Accomplishments}

The operating system has been upgraded to Solaris 2.4, and the database engine has been upgraded to Oracle 7.1.4. Numerous hardware problems with the existing Axil computers (Sun workstation equivalents) were repaired. The source code from the Contingency Theater Air Control System Automated Planning System (CTAPS) was moved to the new target environment and placed under configuration management. A tool has been installed to assist in modifying the source code for operation in the new environment. Programming changes are under way to make the basic user interface and system administration features operable. Owing to software and hardware problems encountered during the upgrade, and staffing problems that were resolved midsummer, the desired changes have not been completed as of this writing. It is unclear whether the demonstration environment for the Out-of-Service project will be finalized before fiscal year end.
From collaboration with the Out-of-Service project, it became clear that the Network Core Software must fit into the national transportation system architecture currently under development and known as Commercial Vehicle Information Systems Network (CVISN).

We are working with the architects at the Applied Physics Laboratory at Johns-Hopkins University for specification of standards for CVISN.

New or Increased Technical Capability

The project has increased the technical capability of laboratory personnel in the use of current industry standard software environments and has set the stage for demonstration of a powerful integration environment for transportation systems. Collaboration with the CVISN architects places the INEL in an influential position for standards development.

\section{Business Development Opportunities}

Internally, the project has been pursued with INEL's Transportation Infrastructure Department. Through them, this work is being made known to the Idaho Transportation Consortium made up of the Idaho Transportation Department, University of Idaho, Federal Highway Administration, as well as the INEL. The consortium has vested interest in seeing how this software can integrate the Out-ofService Verification Field Operational Test with other systems at the East Boise Port of Entry.

This software is proposed as the integration environment for the proposed Transportation Testbed LDRD beginning in fiscal year 1996. The project will be the proving ground for new and innovative approaches to merge sensor data streams, communication, and distributed data networks.

Also, CVISN contacts provide a showcase for our products. 


\section{Optical Sensor Development for High-Voltage Metering and Protection Applications}

LDRD 3711

\section{G. K. Woods}

Accurate means for measuring electric power is a fundamental and indispensable requirement in the world-wide business of energy resource distribution. Power flow through a transmission line is calculated from direct measurements of voltage and current on the line of interest. Traditionally, voltage has been ascertained using outdated ferro-magnetic potential transformer technology, which suffers from a host of liabilities, including size, weight, low bandwidth, nonlinearity, electric burden, poor isolation, and high cost. The focus of this project has been to develop an optics-based alternative voltage measurement sensor free of these liabilities. By solving the problems of prior technology, it is possible to literally revolutionize the power industry by making it feasible for a substantially greater number of voltage measurement devices to be used at the same cost with virtually no impact on substation real estate. The presence of additional measurement points in a transmission and distribution system enables supervisory control and data acquisition (SCADA) operators to match supply and demand at higher levels of optimization and efficiency. In short, the ability to effectively control the allocation of electric power depends heavily upon the extent to which real-time information can be gathered.

This project sought out to address this need for information by designing a miniature, low-cost, passive, optical voltage sensor that could be economically used in large numbers to gather a complete profile of data on a transmission grid or distribution systemdata that could be relayed at the speed of light back to substation SCADA systems using fiber optic cable. By offering such a fundamental and economically powerful improvement to the electric power industry, the sensor developed herein holds great potential to bring national recognition and royalty revenue to the INEL through commercialization.

\section{Objectives}

- Investigate viable optical technologies for implementation of voltage, current sensors, or both.

- Perform a market analysis; further assess specific commercial demands in power measurement.

- Develop a concept for a lowcost, passive, optical sensor to fit one or more of the many niches for high-performance real-time measurement instruments in the power industry.

- Design and build an operational prototype for proof of principle, if time and funds permit.

\section{Technical Accomplishments}

Objectives were not only met this year but exceeded. We have developed an optical high-voltage sensor that can be integrated into numerous varieties of existing power transmission and distribution apparatus. It clearly surpasses prior attempts at optic voltage measurement by eliminating the need for costly voltage division hardware that nullifies many of the advantages of optical technology. The developed sensor eliminates the need for large stand-alone voltage measurement instruments that until now have occupied precious space in power substations. The system was designed at the concept level and was subsequently implemented in a fully functional prototype system that has been successfully tested to 3000 voltsrms L-N. A sample output waveform from the sensor is shown in the figure. A patent application for the sensor is in the final stages of revi- sion by the Thorpe, North \& Western Law Firm, and will be filed with the U.S. Patent Office in a matter of weeks.

The system comprises three major components: transducer, sensor head, and signal processor. Laser light delivered by optic fiber is polarized and collimated by the sensor head and then transmitted through the transducer, which is mounted within the sensor head. An optic polarization signal representing voltage is induced upon the beam by the transducer and is then converted into dual complimentary intensity signals for transmission to the signal processor. The sensor head is designed to be integrated into a variety of widely used electric power transmission and distribution equipment. The signal processor derives a voltage measurement based on the two intensity signals transmitted from the sensor head by fiber optic cable. A custom-designed signal processing scheme uses the two complimentary signals to reject common mode noise such as laser intensity fluctuation, and to minimize the effects of temperaturedependent intrinsic birefringence (a slow drifting of the refractive indices with temperature) on the over-all voltage measurement accuracy.

Substantial strides were made by this sensor in terms of safety and performance. The sensor is designed to operate without contacting the energized conductoran obvious advantage over traditional PTs, which are by definition capable of transmitting up-stream fault energy down to vulnerable instrumentation and personnel. Additional protection and noise immunity is offered by the optic system because the sensor's information is transported to the signal processor by fiber optic cable rather than conductive wire. The superior bandwidth of the optic 
sensor makes it suitable for highspeed relaying and fault protection applications that will make possible unprecedented standards of uninterrupted power delivery and consumer satisfaction.

Efforts under the FY-95 project have yielded a highly capable sensor that can be marketed as either a retrofit product or as a standard option on new orders of commercial transmission and distribution equipment. Having proven and demonstrated the principle, plans have been made to proceed with commercialization in an LDRD project submitted for FY-96. In the interest of brevity and protection of intellectual property, full details have been withheld from this report. A thoroughly disclosing discussion will be submitted in the project's comprehensive report.

\section{New Technical Capability}

The successful development of this product represents a substantial milestone in the INEL's recent endeavors to pursue research in areas that facilitate direct transfer of technology to the private sector. Following commercialization, the INEL will be the originator of a sensor whose market potential is highly attractive and growing even greater as the demands for electrical power transmission and distribution control, real-time load monitoring, and high-speed protective relaying continue their upward trends. By minimizing the cost of the voltage sensor even further, the number of potential spinoffs is maximized. One such spinoff that is planned for investigation in the proposed FY-96 project is an optic voltage sensor adapted for electric vehicle applications.

Business Development Opportunities

A working relationship was formed with Conoptics Inc. of Danbury, Connecticut. Working from our design specifications and an extensive series of telephone dia- logues, Conoptics engineers fabricated the custom sensor head for the proof-of-principle prototype sensor that was constructed and successfully tested under this project. Conoptics has expressed a strong interest in continuing this type of cooperation in future ventures. The Associated Western Universities and the University of Idaho also contributed to the success of this project by providing a vehicle for retaining the services of a graduate-level research associate. Contact has been made with a representative of the General Electric Company regarding this project. Prototype demonstrations for GE and other interested companies are planned for FY-96, at which time CRADA, licensing, and marketing opportunities will be examined and pursued in the interest of expediting transfer of the developed system to the lucrative market that awaits cheaper, safer, more capable technology.

\section{Development of Chemically Selective Surfaces for Adsorption and Detection of Gas-phase Contaminants}

LDRD 4061

Gary S. Groenewold, R. L. Cowan, J. C. Ingram, James E. Delmore, and Anthony D. Appelhans

Empirical observations in the laboratory indicate that certain materials promote efficient and selective adsorption of gas-phase contaminants. The goal of this project has been to identify materials that display selectivity for adsorption of specific contaminants. Phosphazene polymers, alkali and alkaline earth hydroxides, and transition metal oxides were tested in this capacity. Subsequent to adsorption, contaminant detection was achieved using secondary ion mass spectrometry (SIMS). Efficient adsorption for an organophosphate, an amine, degradation products of military compounds, and pesticides were demonstrated on different phosphazene polymers. Efficient adsorption of gaseous acids on solid basic materials was also demonstrated. The research has led to follow-on work with two sponsors, two draft manuscripts, and strong potential for patents.

\section{Objectives}

- Identify contaminant.

- Identify or develop an adsorptive media.

- Identify support materials (for adsorptive media.

- SIMS test contaminants, adsorptive media, and support materials.

- Fiber optic test adsorptive media (performed by Naval Research Laboratory).

- Develop and evaluate end user.

\section{Milestones}

- March FY-96: Adsorptive media/support combinations have been identified that are appropriate for detection of significant contaminants. This was completed on schedule with development of coating process for polyphosphazenes on steel SIMS targets.

- September FY-96: External funding for FY-97 and appropriate technology end-users will be identified. This was completed ahead of schedule (see Business Development Opportunities below).

\section{Technical Accomplishments}

Contaminants were identified based on requirements of past and anticipated sponsors. Tri-n-butyl phosphate (TBP) is a compound having applicability in uranium processing. Methyl phosphonic 
acids (isopropyl, pinacolyl, and unsubstituted) are degradation products of military compounds. Glyphosate and paraquat are pesticides of agricultural significance. Cyclohexyl-amine (CHA) and small organic acids were also tested in order to evaluate the effect of acidity and basicity on contaminant adsorption.

Adsorbent materials selected for testing were divided into two classes: phosphazene polymers and metal oxides/hydroxides. These materials were chosen because prior anecdotal evidence strongly indicate that they are capable of adsorption selectivity and efficiency. The polyphosphazenes have experienced extensive testing at INEL for membrane separations and are attractive because of their selective interactions with contaminants, and because they can be easily derivatized after polymerization. This latter feature makes a wide range of derivatives available; acidic, basic, ester, ether, polyether, and fluorinated ether polymers were examined. The metal oxides were selected on the basis of extensive catalyst and surface detection experience, which showed selective adsorption for a variety of organics.

Adsorption by Phosphazene Polymers. Thin layers of phosphazene polymers could be deposited on stainless steel targets (about 5 to 6 $\mathrm{mm}^{2}$ ) by placing up to several $\mathrm{ml}$ of THF or dioxane solutions on the targets and allowing the solvent to evaporate. The coated targets were then exposed to gaseous contaminants and analyzed using static SIMS. These experiments reveal that the different polymer derivatives varied greatly in terms of their ability to adsorb contaminants. Only the acidic $p-\mathrm{HO}_{2} \mathrm{C}$ PPOP derivative was capable of adsorbing the basic CHA; none of the other derivatives were capable of this. TBP was adsorbed by the $p$ - $\mathrm{HO}_{2} \mathrm{C}-\mathrm{PPOP}, p-\mathrm{EtO}_{2} \mathrm{C}-\mathrm{PPOP}$, and
$\mathrm{CF}_{3} \mathrm{CH}_{2} \mathrm{O}$-derivative, but not by the other polymers.

The study of the polymers was expanded by exposing the polymers to aqueous solutions of selected contaminant molecules. These experiments were pursued to evaluate the ability of the polymercoated targets to function as passive samplers in aqueous environments. The most intriguing result was the observation of isopropylmethylphosphonic acid (IMPA) adsorbed to $\mathrm{p}-\mathrm{MeO}-\mathrm{PPOP}$ from a $100 \mathrm{ppm}$ solution. IMPA is the principal degradation product from the nerve agent sarin, used in the Tokyo subway attack. None of the other polymers adsorbed IMPA, and the $p$-MeO-PPOP did not adsorb the related compounds PMPA and MPA. This indicates that $\mathrm{p}-\mathrm{MeO}-\mathrm{PPOP}$ is selective for IMPA.

The pesticide paraquat also adsorbed from aqueous solutions, though no selectivity was observed by comparing polymers. In contrast, no adsorption was observed for the pesticide glyphosate. These results again emphasize that adsorption is controlled by relatively subtle interactions that have a major impact on the process.

Metal Oxides, Hydroxides. The ability of Group I and II hydroxides to adsorb gaseous organic acids was probed by coating SIMS targets with the hydroxides and then exposing these to the acids. As expected, the hydroxides strongly adsorbed formic, acetic, and propionic acids. The solid bases also adsorbed $\mathrm{CO}_{2}$ from the atmosphere, which was observed as $\mathrm{M}_{3} \mathrm{CO}_{3}+$ in the SIMS spectra of the Group I hydroxides. The use of the weaker base AgO failed to adsorb $\mathrm{CO}_{2}$, but was effective for the small organic acids.

A large number of adsorption experiments using transition metal oxides were also performed, but the bulk of these data has not been reduced at the time of this writing.

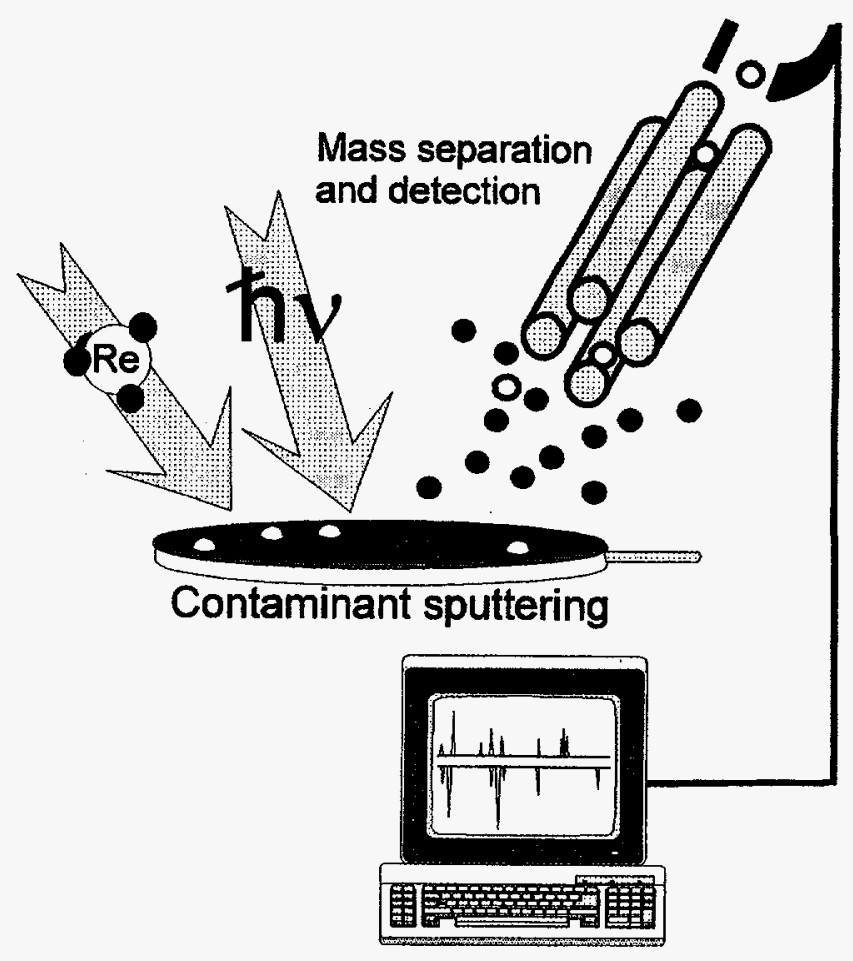

Chemically Selective Surfaces for Passive Sampling and Concentration 
New or Increased Technical Capability

The research demonstrated the feasibility of using polyphosphazene and metal oxide materials generated at the INEL for adsorption of selected contaminants from both airborne and aqueous media. Specifically, the following advances were made:

- A polymer specific for the adsorption of organic bases from air was identified.

- A polymer specific for the adsorption of IMPA from water was identified.

- Polymers effective for the adsorption of paraquat from water were identified.

- Gaseous acids could be fixed from the atmosphere using Group I and II hydroxide bases.

\section{Business Development Opportunities}

The research attracted attention from an outside funding source within the first six months of the project. The Naval Research Laboratory offered to fund a $\$ 50 \mathrm{~K}$ program for FY-96 to provide polymers for fiber optic detection testing. We were also invited by a classified sponsor to expand a 1995 proposal for FY-96 funding. The invitation was in response to a white paper that described some of the tailored surface chemistry research.

\section{Alloys by Design LDRD 4073}

\section{G. E. Korth, D. F. Glenn}

Two stainless steel alloys (one, a nickel-base, and the other an austenitic) were developed by rapidly solidified processing (RSP) under a DOE-OIT program and had advanced to the state where additional processing/properties were necessary to establish their superiority and competitiveness with current commercial counterparts. These additional investigations were beyond the scope of the DOE program, which was being phased out. The purpose of this midyear project was to explore an alternate processing technique (spray casting), thermal stability of tensile properties after aging, and hot aqueous corrosion of the two alloys. The spray casting processing demonstration was to eliminate the canning/decanning steps of processing the RSP metal powder and thereby reduce the cost of producing these superior alloys. Aging at 1000 hours at three temperatures representing extremes in service conditions would further demonstrate their superiority by permitting higher temperature applications. The hot aqueous corrosion tests were necessary to show at least equivalence and, hopefully, superiority in corrosion properties with commercial counterparts. This project represents, therefore, the next step in commercialization of the RSP technology and, in particular, two stainless steels.

\section{Objectives}

- Demonstrate spray casting of two alloys developed by RSP powder metallurgy techniques so as to eliminate the canning/decanning steps and decrease the cost of production dramatically.

- Determine thermal stability on the two alloys (actually four alloys, because each had a high and low nitrogen version) by conducting tensile tests of material that had been aged at 600,700 , or $800^{\circ} \mathrm{C}$ for 1000 hours.

- Conduct hot aqueous corrosion tests of the two alloys (four with the nitrogen variations) and compare corrosion properties with commercial counterparts.

\section{Technical Accomplishments}

Spray Casting. This processing technique involves placing a substrate in the path of the molten droplets being sprayed with the inert gas atomizer. Therefore, the droplets are not allowed to solidify into powder particles, but solidify on the substrate as a casting with a very fine microstructure. The spray casting was initiated, but initial results show that several processing parameters were necessary to optimize the casting to obtain properties equivalent to hot extruded powder. The temperature of the substrate, geometry of the substrate to facilitate a smooth two-phase flow, distance of the substrate from the spraying nozzle, and molten droplet velocity appeared to be interrelated process parameters that would have to be optimized in order to produce a casting that was free of porosity and retain the fine microstructure of the rapidly solidifying particles. Because the substrate heater and positioning device were being modified, the gas atomizer was turned over to another project. A total of four projects (both DOEfunded and LDRD-supported) depended on the gas atomizer to produce metal powders or castings for their investigations. Technical and equipment problems plagued two of the other projects, and this project never did get another turn in the atomizer to further develop the spray casting techniques.

Therefore, this task will have to be continued in another year, but this year's results did show the feasibility of spray casting alloys of these kinds.

Thermal Stability. Tensile bars fabricated from hot extrusions of the four alloys were aged in quartz capsules for 1000 hours at 600,700 , or $800^{\circ} \mathrm{C}$ and then tested at room temperature. Alloy 4 is the austenitic stainless steel, with B 
and $\mathrm{D}$ designating the low and high nitrogen, respectively. Alloy 5 is the Ni-base alloy, with B and D having the same meaning. Results of these tests were then compared with RSP material that had not been aged (see figure). The results show little or no detrimental effects on the ultimate and yield strength owing to thermal aging; in fact, significant increases in strength are shown in $4 \mathrm{D}$ alloy after $700^{\circ} \mathrm{C}$ aging. However, Alloy-4 shows a marked decrease in ductility with aging at 700 or $800^{\circ} \mathrm{C}$. Although it has not been verified, the formation of the brittle sigma phase is suspected to have caused this drop in ductility. Based on these results, two additional alloys were purchased with the same chemistry as Alloy-4, except the Mo content was reduced, to mitigate the sigma phase formation. Evaluation of these modified alloys will be performed in future years after they are atomized and consolidated. Even though the Ni-base (Alloy-5) shows slight reductions in ductility with aging, the levels are still well within the range to classify this material a suitable engineering alloy with enhanced applications up to $800^{\circ} \mathrm{C}$.

Hot aqueous corrosion. Corrosion tests were performed on RSP materials Alloy-4 and Alloy-5, which were produced earlier in FY 1995. Alloy-4 is an iron-based, high-chromium, high-nickel, highmolybdenum alloy that is nitrogen strengthened. It is highly resistant to salt water and pulp and paper industry environments. Alloy- 5 is a nickel-based, high-chromium, high-molybdenum alloy that is nitrogen strengthened. It withstands environments that contain reducing and oxidizing compounds. The polarization tests in room temperature $5 \mathrm{M} \mathrm{HCl}$ showed that the RSP alloys passivate under anodic polarizing current.
In comparison to their wrought competitors, the corrosion resistance of the RSP alloys showed much variation; sometimes it was superior, but for the most part it was not. There are long pits in Alloy-4B. More work will be needed on the study of the process variables.

New or Increased Technical Capability

The INEL has benefited from this project through an increased understanding of the spray casting process. Even though we have not perfected the process by any means, we have an understanding of what process parameters are important and what experiments to perform to solve the problems. The other tests (tensile and corrosion) and examination techniques used are routine procedures that have not substantially added to the technical capability of the INEL.

\section{Business Development Opportunities}

The only direct business interest in this project was by the U.S. Navy at KAPL, but their interest was mainly associated with the spray casting, which did not progress very far in the current year. Contacts will be maintained as the work continues.

\section{Improved Process Control Thermocouples LDRD 4076}

\section{R. N. Wright, J. R. Fincke}

This project is Phase I of development work aimed at producing industrial process control thermocouples that have improved long-term stability at high temperatures (and hence improved measurement accuracy). Contact with the glass industry served as the starting point for the proposed work. Classical platinum/rhodium thermocouples are standard control instruments in glass production but suffer calibration drift as a result of impurity contamination from even the highest quality insulating materials. The consequences to the glass industry are poor process control and product quality, excessive rejection and rework, and high energy costs. Newly-developed proprietary insulating materials have been used on a demonstration basis to produce significantly improved stability in Chromel/Alumel thermocouples at temperatures near their maximum operating limit. The similarity in platinum/rhodium and Chromel/Alumel calibration drift processes suggests that at least one of the new insulating materials may produce similar improvement in platinum/rhodium thermocouples. Successful demonstration of greater stability has immediate implications for better process control and enormous annual savings, not only for the glass industry but also other U.S. industries that employ platinum/rhodium control thermocouples, including the petrochemical and refinery, chemical, aerospace, and heat treating areas. AccuTru International Corporation agreed to supply proprietary insulating materials to permit side-by-side comparative laboratory testing of platinum/ modium thermocouples insulated with newly developed materials, and standard platinum/rhodium thermocouples insulated with classical materials. Attention is focused on Type $S$ and Type $B$ thermocouples in tests at two or three temperatures typical of key stages in the glass production process. Archive samples of the thermocouples tested will be retained for SEM (scanning electron microscope) examination at a time when resources allow, with the intent of determining the drift mechanism on a microscopic level. Future work in cooperation with AccuTru International is expected to include development of additional insulation materials tailored to mitigate the specific drift mechanisms found to be responsible for long-term calibration drift in industrial platinum/rhodium thermocouples.

\section{Objectives}

- Procure a tube furnace capable of operating in air at tem- 
peratures up to $1700^{\circ} \mathrm{C}$ for simulating conditions in glass manufacturing.

- Procure thermocouple materials for testing, including standard industrial thermocouple wires and insulating materials, standard thermocouple assemblies as used in the glass industry, and new insulating materials.

- Assemble data acquisition system for automatic collection, recording, and display of $\mathrm{Pt} / \mathrm{Rh}$ thermocouple test data during long-term testing.

- Perform side-by-side tests to compare the long-term (2000 hours) stability of standard industrial $\mathrm{Pt} / \mathrm{Rh}$ thermocouples with those having new, proprietary insulating materials supplied by AccuTru International.

\section{Technical Accomplishments}

All thermocouple materials required for the anticipated sideby-side testing of standard industrial $\mathrm{Pt} / \mathrm{Rh}$ thermocouples and thermocouples insulated with new, proprietary materials were procured. Industrial thermocouple assemblies of the type used in glass manufacture were also procured for comparative testing.

A PC-based data acquisition system based on LabView software was assembled, refined, and tested on dummy thermocouple assemblies. The system allows signals from up to ten Type S or Type B thermocouples to be recorded at a selectable rate, converted to temperature, and displayed either realtime or by selecting a data file. All ten measurements can be displayed simultaneously, but doing so results in a cluttered graph; consequently, comparison of two or three thermocouples with a reference measurement or the furnace control thermocouple is more easily interpreted.
Major problems arose in procurement of the tube furnace for this project. To simulate conditions in the melter during glass manufacturing operations, a tube furnace capable of operating at $1700^{\circ} \mathrm{C}$ in air was required for the proposed thermocouple tests. Quotes from furnace suppliers were obtained during February 1995, the Form 52 for Capital Equipment Request was submitted on February 21, and after word of approval was received the purchase order was submitted to Business Management Services for a charge number on March 14. For unexplained reasons, the purchase order was not received by Purchasing until May 4, and the order was not placed until June 1. The projected eight-week delivery stretched to twelve weeks, with the furnace arriving at the North Holmes laboratory facility August 25. Unfortunately, the furnace suffered major damage in shipping, with fully half of the insulation collapsed into a jumbled heap in the furnace cavity. Photos and diagrams documenting the damage were sent to the manufacturer, Carbolite, Inc., who will dispatched a service specialist during the week of September 18 to replace the damaged furnace insulation and assist in making the furnace operational. The consequences resulting from delays in ordering the furnace and from the shipping damage are obvious: the three-month period needed and anticipated for a 2000-hour comparative test was reduced to one week during FY-95.

Although the anticipated sideby-side thermocouple testing was not completed during this fiscal year, activities have resulted in several additional business development opportunities, as noted below.

New or Increased Technical Capability

AccuTru International was referred to the INEL as a source of thermocouple expertise by personnel at NIST. As a minimum, the complement of development and test equipment has been increased by the addition of a test furnace capable of operating at temperatures up to $1700^{\circ} \mathrm{C}$ in air. Work with the glass industry, in cooperation with AccuTru, in preparation for these tests has positioned the INEL in the forefront for work directed toward the Glass Industry Vision of the Future sponsored by DOE-OIT.

Business Development Opportunities

A CRADA is pending with AccuTru, who has been awarded $\$ 250 \mathrm{~K}$ by DOE-OTT, for continuation of the work initiated by this project. Agreements have been reached with Corning, Inc., Anchor Container Glass Corporation, and Libbey-Owens-Ford Company. for in-kind field testing facilities, as well as potential cash contributions to develop additional thermocouple materials. Negotiations are continuing with AccuTru for testing at the INEL of metal-sheathed Type $\mathrm{K}$ thermocouples having new insulation materials. A joint INEL/AccuTru proposal for developing a temperature control system based on sensors developed under the CRADA referred to above has been submitted to to DOE., and discussions are under way for modeling of the glass melting process to be performed at the INEL.

\section{National Infrastructure Renewal LDRD 4080 \\ Nancy M. Carlson}

U.S. industries involved in petroleum refining, chemicals, pulp and paper, power production, steel, aluminum, foundries, and glass have strong economic and environmental incentives to extend plant life, while 
conserving national resources and protecting the public and work force. These industries are vital to the U.S. economy, employing nearly two million people directly and providing basic materials essential for the entire U.S. manufacturing infrastructure. Degradation of structural integrity by corrosion, stress corrosion cracking, corrosion fatigue, thermal embrittlement, and creep are major considerations in extending plant life. Developing and validating predictive models of potential degradation mechanisms, monitoring critical process steps using on-line sensors, on-line monitoring of critical components and weldments, quantifying material degradation, developing new materials with enhanced materials properties, predicting end-of-life materials properties, developing predictive databases using past failures and failure modes, and developing simple rules for aging assessment are all key elements for developing a risk-based approach to plant life extension and for improving efficiency and environmental performance of industrial processes. The alternative to plant life extension for the refinery and chemical industry is locating plants outside of the United States, because the time and cost of establishing a new facility in compliance with all the current regulations in the United States is prohibitive.

\section{Objectives}

- In collaboration with MIT, develop critical flaw size code for both base metal and weldments in base metal with code validation experiments conducted at the INEL.

- In collaboration with Shell Oil, prepare a test plan for an inservice structure and acquire acoustic emission data for analysis using INEL-developed code. In collaboration with Iowa State University, evaluate nonlinear acoustic to determine potential as lifetime extension NDE technique.

- Complete preliminary tests to determine appropriate testing conditions to evaluate the stress corrosion characteristics of new INEL RSP alloys, to determine their potential for use in more corrosion resistant structures.

- Assemble an enhanced data acquisition system based on LabVIEW software for use in collection and display of test data during extensive thermocouple testing directed at improved temperature control in manufacturing processes.

- Produce metallic aluminum gratings on glass and bulk aluminum samples. Test gratings for durability and stability during temperature cycling for diffraction moire interferometry.

\section{Technical Accomplishments}

The MIT critical flaw size code has been completed, accompanied by a draft final report. An INEL experimental plan was developed based on the MIT code, and test specimens were fabricated. Testing of these samples is nearly complete. The final phase of the FY-95 task will use experimental data to validate the MIT model. Shell Oil and the INEL developed an experimental plan that required the location of a fluid-filled large structure to verify that the INEL acoustic emission code used on fracture samples is applicable to large structures. An appropriate structure, a diesel storage tank, was located at the IRC. Code modifications were made to acquire acoustic emission data from the structure with data analysis currently being completed. Nonlinear acoustics shows promise as a laboratory tool based on the results of work completed at Iowa State University, presented in their final report. In the corrosion task, the corrosion resistance of the RSP alloys was determined to be equal or superior to wrought alloys con- sidered to be the main competition to the INEL RSP alloys. The appropriate solution for the stress corrosion tests will be determined based on the corrosion resistance results. The data acquisition system for conducting thermocouple tests is completed, and discussions are under way with AccuTru International Corporation to establish testing of the long-term stability of newly-developed insulating materials for potential use by the glass industry. The final task, development of high temperature gratings for diffraction moire interferometry, has resulted in the production of metallic aluminum gratings on glass and bulk aluminum samples. Temperature cycling on the gratings in currently under way to determine grating durability and stability. The testing will include measurements of the grating at temperature to establish the applicability of the technique for use on in-service vessel and piping operating at plant processing temperatures.

New or Increased Technical Capability

A critical flaw size code has been developed and is being validated. This code will allow the INEL to develop enhanced predictive models for use in fitness-forservice tools as part of the INEL national infrastructure strategic thrust area. NDE techniques for testing and data analysis of large structures provides the INEL with expertise critical to developing collaborative relationships with U.S. industry for use in plant lifetime extension activities. We anticipate that a thermocouple testing facility will give the INEL the capability to generate money-in arrangements for the independent testing of thermocouple designs as well as new insulating materials. High-temperature moire capabilities will provide the INEL with potential patent as well as a new NDE technique to monitor local stresses in 
critical locations in an operating structure.

\section{Business Development Opportunities}

The critical flaw size code will be used as part of a proposed CRADA activity with Chevron, Shell, Exxon, Amoco, Arco, CTI Inc., Wesdyne, Subsea Systems, DNV, Johns Hopkins University, and Ames Laboratory at Iowa State. The proposal has been presented to Dan Wiley of DOE/EEOIT for potential FY-96 funding as part of the Refineries of the Future program. Shell Oil is very interested in pursuing the acoustic emission activity, as on-stream monitoring is vital to extending plant lifetime of refinery structures. This activity requires additional internal funding before a CRADA can be developed; therefore, an FY-96 LDRD task was proposed as part of a larger refinery LDRD submitted by Dick Rice. Talks are under way with AccuTru International Corporation to use the thermocouple testing capabilities developed under this LDRD. The arrangements will involve the services of the INEL to complete independent testing of new insulating materials. The exact terms are to be negotiated in the next few months.

\section{Optical Measurement Technology LDRD 4081}

\section{R. D. MacDowall}

New optical measurement techniques offer high-leverage opportunities in the INEL mission areas of environmental and infrastructural monitoring. These opportunities result from the continued rapid development of solidstate diode lasers and fiber optic technology in the communications and military electro-optics industries, providing new tools for application in the measurement of chemical and physi- cal properties. Techniques explored in this work include the use of nonlinear materials for improved nondestructive testing of materials, waveguide sensor arrays with speciality coatings for on-line contaminant monitoring, and ultra narrowband optical fiters for the standoff detection of chemical species.

\section{Objectives}

- Develop a nonlinear optical sensor.

- Evaluate a polymeric coating for use in a waveguide sensor for an on-line water contaminant monitor.

- Design an ultra narrow band optical filter.

\section{Accomplishments}

Photorefractive Lock-in Holographic Interferometer: Full-field Continuous Detection of Vibration.

This effort has been to develop advanced nondestructive test instruments based on photorefractive crystals. In particular, our goal has been to develop full-field detection-of-surface vibration using a four-wave mixing process in a photorefractive medium. Fullfield measurements are a significant improvement over the more normal point detection schemes. Our proposed method has the tremendous advantage of being insensitive to environmental vibrations, which destroy the effectiveness of most competing techniques.

In this method, an object is subjected to a continuous acoustic vibration that causes subsurface defects such as delaminations to be displaced relative to adjacent areas. The surface displacements caused by this process can be sensed and used to identify the defect. Optical lock-in vibration detection is performed by mixing a full-field image of the vibrating surface with a reference beam (which is syn- chronized with the acoustic excitation) in a photorefractive medium. When a read-out beam, counter propagating to the reference beam, is brought into the medium, a second (diffracted) image is generated. The spatially distributed intensity of this diffracted image depends on the relative displacements of the object image. This dependence of the interferometer output was demonstrated, as was its application to vibrational displacement detection and discrimination, and a model for the minimum detectable displacement was developed. In addition, if the input image and reference wavefronts are mixed with slightly different frequencies, the output image can be seen to intensity modulate (blink) at a rate equal to the difference frequency. This heterodyne operation is a visual aid in the subjective identification of subsurface defects. Automated quantitative data can also be derived. As in conventional electronic analogs to this fully optical process, both multiplication and low-pass filtering are realized in this optical lock-in device. In addition, the frequency matching character of this photorefractive holographic interferometer offers highsensitivity, full-field displacement detection at the subnanometer range. Furthermore, the interferometer exhibits extreme environmental noise insensitivity, indicating its potential for improved defect detection in industrial nondestructive evaluation applications.

On-line Contaminant Monitor.

This task is to evaluate a mechanochemical polymer for use as a transducer in a waveguide sensor array to measure heavy metal contamination. The work builds on a patent developed for the Department of Energy by Bend Research, Inc., of Bend, Oregon. The work is based upon the fact that many polymers are capable of exhibiting a physical response, 
expansion or contraction, in response to changes in the surrounding environment. In particular, polymethacrylic acid (PMAA), cross-linked with divinylbenzene is known to undergo volume expansions of up to $300 \%$ on conversion from the acid to the polyanion form. This expansion can be explained on the basis of conformational changes that occur in the polymer as it is converted from a tightly coiled neutral polyacid to the polyanion form. It is theorized that electrostatic repulsion between negatively carboxylate groups is responsible for this expansion. In this project, a sample of polymer reported to contract in the presence of metallic ions, was acquired.

Two sections of optical fiber were placed in a splice tube and secured with epoxy to a sheet of the polymer such that as the polymer expanded or contracted the fiber ends would move, modulating the light intensity through the fiber. One study examined the response of the transducer to water. It was noted that polymer expanded rapidly, causing a reduction in light intensity, and as the polymer dried it contracted, bringing the optical signal back to its initial level. In additional testing, the transducer was immersed in deionized water, and then a copper sulfate solution was added. While the polymer swelled approximately $17 \%$ in response to the water, little contraction, $3 \%$, was noted with the addition of the copper ions. In its present configuration, the transducer appears to be an excellent moisture sensor; however, it does not appear to have the sensitivity to ions needed for a successful online water contaminant monitor.

Optical Filter. This task studied the feasibility of developing an electronically dithered optical filter (EDOF), using the Pockel's effect to externally tune the incoming light passing through a reference gas cell. The external tuning capabilities would also have further applications in Faraday anomalous dispersion optical filter techniques. This would allow development of new spectroscopic techniques for passive, covert, remote detection of airborne chemicals with greatly simplified optical instrumentation.

The EDOF technique is an extension of conventional gas filter correlation radiometry (GFCR). Our calculations show it will be at least two orders of magnitude more sensitive than GFCR however. The key to the concept is an efficient means to determine if spectral correlations exist between the incoming spectrum from a scene of interest and the reference gas cell. Very simply, if the incoming spectra and reference gas spectra have spectral lines in common, then the scene will likely contain the same species as the reference gas. Incoming light is passed through an electro-optical phase modulator (EOPM), which frequency modulates (or equivalently wavelength shifts) the light passing through it. This wavelength-shifted light is then passed through the reference cell containing a gas of interest. The actual measurement, i.e., determining the degree of spectral correlation, is based on the intensity of the light exiting the reference cell. The purpose of wavelength shifting the incoming light is so that the incoming spectral lines can be periodically moved back and forth (in wavelength) across the fixed wavelength lines of the reference gas. The result of this is that the intensity of the light exiting the reference cell is amplitude modulated. This is similar to sliding one picket fence over another and observing modulation of the light transmitted through both fences. This amplitude modulation is easily detected because it is synchro- nized to the electrically-driven electro-optical phase modulator and is at twice the frequency of the its driving frequency.

We anticipate that this type of instrument would be useful in covertly detecting and observing chemical agent signatures of various weapons of mass destruction proliferation activities, and thus it would be useful for arms control and treaty verification. It could also find uses in remote environmental monitoring for EPA, NASA, and industrial applications

\section{New or Increased Technical Capability}

The application of photorefractive nonlinear processes to nondestructive testing is a new and active field, building on a strong INEL background in optics, interferometry, and noncontacting laser ultrasound detection. We anticipate advanced NDT systems with strong market potential. The online water contaminant task did not produce a useful short-term result; however, the work is a continuation of a multi-year effort at the INEL to design fiberopticbased chemical sensors, which has led to a half dozen patents over the past few years. The ultra-narrow band filter work has led to the filing of a patent application.

\section{Business Development Opportunities}

Customer interest in both the nonlinear NDE sensor and the narrow-band filter effort are high. The three tasks have lead to discussions, including possible CRADA opportunities, with a number of companies, including Hercules, Pratt \& Whitney, Atlantic Research Corp., Kennametal, Ceracon, Lanxide, Simplex Technologies, National Institute of Standards, Howmet and PCC Airfoils, GM, Ford, Chrysler, Harvest Technology, Thiokol Corp., Phillips Petroleum, Bend Research, Pall RAI Manufacturing, and Shay International. 


\section{Velocity Sensor and Control of Thermal Spray}

LDRD 4082

\section{J. R. Fincke, W. D. Swank}

Real-time, closed-loop, feedback control of the plasma spray process has the potential to improve final product quality, and increase the repeatability of the spray process, all by lowering the sensitivity of the process to inherent variations in spray parameters. As coating performance and quality requirements increase, the need for real-time process control becomes more necessary. In addition to deposition control, the ability to independently vary particle temperature and velocity during process parameter development is also a desirable feature.

Controllers for the thermal spray process may be required to act as regulators, whose purpose is to maintain conditions at an infrequently changed set point, and as servo or follow-up systems, where the controlled variables may be ramped through a sequence. The applications of the regulator mode are obvious. The servo mode may have application in advanced processes, such as the spraying of graded materials where it is desired to vary particle temperature and velocity over the thickness of the coating or even over different regions of the part as the composition of the coating is varied. In this project a particle velocity and temperature controller for the plasma thermal spray process was developed and its characteristics demonstrated.

\section{Objectives}

- Mock up and test Velocity sensor.

- Build spray system model.

- Develop control algorithm.

- Assemble prototype controller.

- Demonstrate controller.
Technical Accomplishments

The implementation of closedloop control requires the direct, real-time monitoring of process performance, via real-time sensing of particle temperature and velocity and the taking of corrective actions rather than the traditional approach of setting process variables and postprocess examination. A real-time digital controller capable of setting and maintaining particle velocity and temperature at designated set points was developed and demonstrated on a subsonic plasma spray process. The controller was designed using an accepted discrete digital control approach. In general, we have found that traditional proportionalintegral-differential (PID) controllers yield stable, robust performance with good steady-state response for the control of temperature and velocity, either alone or simultaneously in the plasma spray process. PID control is also easily implemented digitally using common PCS. Measurements of the tracking efficiency of the controller following a temperature and velocity ramp were made. For one measurement the velocity was held constant and the temperature set point was varied over a $600-\mathrm{K}$ range. The high-end response was limited by the system-imposed maximum gun current. Noise in the low-end response was observed owing to the fact that these conditions are somewhat outside the optimum operating range of the plasma gun. A second set of measurements was made where the temperature was held constant and the velocity set point was varied between 80 and $125 \mathrm{~m} / \mathrm{s}$.

\section{New or Increased Technical Capability}

The thermal spray coating process is unpredictable from one run to the next, resulting in coated parts that do not meet specifications. Then, at great expense, these parts must be either refurbished or scraped. This project has the potential to reduce the cost and improve the quality and reliability of thermally sprayed coatings. Also, with increased reliability comes an increase in the number of applications for thermally sprayed coatings. Expanded use in the automotive industry could easily increase the thermal spray market by $50 \%$, while making many automotive manufacturing processes significantly more cost effective (source, Thermal Spray Coating, State-of-the-Art Assessment, unpublished, The National Center for Manufacturing Sciences).

Business Development Opportunities

December FY-95 ended a CRADA with Tafa Inc., in which an industrial, hardened, in-flight particle pyrometer (IPP) was developed. Tafa Inc. has developed and markets a computer-based robot/process controller (HAWCS II) that has the capability to accept input from sensors and deliver output to process control consoles. This computer controller has not been used to its fullest potential owing to the lack of suitable sensors. With the development of the IPP, Tafa has expressed the intent to continue the advancement of a control system for thermal spray processes.

Although no formal agreements are in place, end users of thermal spray coating processes, such as Pratt \& Whitney, General Electric, and Ford Motor Company have expressed an interest in sprayed particle temperature, velocity and position sensors and controls. Tafa is interested in demonstrating and testing the control system at an end user's facility. To further broaden the technology transfer, the INEL will pursue these opportunities. 


\section{Hybrid Electric Vehicle Evaluation \\ LDRD 4103}

\section{G. H. Cole}

The purpose of this project is to develop and demonstrate the capability within the Idaho National Engineering Laboratory to measure time-based exhaust gas species and concentration from automobiles powered by fossil fuels. While bag measurements are routinely done throughout the automotive industry, research programs strongly need time-based measurements to determine optimum system designs. Development of this capability places the INEL in a unique position to aid automotive researchers working on reducing automobile emissions. This new capability combined with the existing expertise in performing tests on electric vehicles positions the INEL as the only Department of Energy Laboratory capable of performing complete systems tests on hybrid/electric vehicles currently being developed by the DOE and industry. Additionally, this capability improves the U. S. economic competitiveness by way of the President's program for the Partnership for a New Generation of Vehicles.

A dual-fueled (Liquefied Natural Gas (LNG) and gasoline) Dodge Dakota pickup truck was tested in the INEL Hybrid/Electric Vehicle Laboratory. During the testing, the vehicle was driven on a chassis dynamometer using the Federal Urban Dynamometer Driving Schedule. Measurements of the vehicle's tailpipe emissions were made using a Fourier Transform Infrared (FTIR) analyzer at one-second intervals, which simultaneously identified and quantified the exhaust gas constituents. The test was performed for both fuel types and the results of each were compared.

Measurements of regulated pollutants show a significant difference in the carbon monoxide constituent of the exhaust gases. Differences between oxides of nitrogen and hydrocarbons were less pronounced.

\section{Objectives}

- Identify and implement required safety processes for this type of activity.

- Investigate processes and procedures for testing fossil-fueled vehicles.

- Obtain time-based exhaust gas measurements using an FTIR analyzer.

\section{Technical Accomplishments}

We performed a code study to identify the safety issues and determine what lab modifications or operational (experimental setups) constraints were required to bring the vehicle into the INEL Research Center. A safety evaluation report was written and approved that addressed all new safety, environmental, equipment, and test issues, including a solution that enabled the engineers to perform the tests without imposing any restrictive operational constraints.

Hydrocarbon and carbon monoxide sensors were placed within the laboratory to monitor these gases and alarm upon detecting hazardous levels. Output from the sensor equipment would shut down any source of ignition within the dynamometer pit.

A vent line was routed from the LNG relief valves to the outside allowing the vehicle to remain in the laboratory for the entire test.

Test processes and procedures were developed based on the Federal Test Procedure found in Section 40 of the Code of Federal Regulations (40 CFR 86) and implemented for this experiment.

Time-based exhaust gas measurements were obtained for each of two fuels using the Federal Test Procedure cold and hot start specifications. Twenty-three exhaust gas constituents were measured over the 2744-second, $24-\mathrm{km}$ driving cycle. Detailed data were obtained as a function of time (0-10 $\mathrm{min})$ for carbon monoxide (cold start and hot start), $\mathrm{NO}_{\mathrm{x}}$ (cold start and hot start), and total hydrocarbons (cold start and hot start).

New or Increased Technical Capability

As a result of this research, the capability to test fossil-fueled vehicles and measure the exhaust gasses in the Hybrid/Electric Vehicle Laboratory at the INEL Research Center was demonstrated. The INEL is the only laboratory in the Department of Energy complex having the capability of testing any type of automobile presently contemplated to replace gasoline powered vehicles.

\section{Business Development Opportunities}

The Automotive Systems Technology Department is currently working under a cooperative research and development agreement with the California Air Resources Board. This CRADA presently focuses on the test and evaluation of electric vehicles. Discussions with the Board indicates strong interest in expanding the CRADA to include hybrid vehicles.

The vehicle tested was borrowed from the INEL fleet. Discussions with INEL personnel involved with the LNG-fueled vehicle programs and the Federal Fleet Conversion Program have indicated that the capability demonstrated by this LDRD can be used to enhance the INEL alternative fuel vehicle fleet program by accurately monitoring emissions over the life of the vehicles.

Each of the major U.S. automobile companies is expected to deliver prototype hybrid vehicles under Department of Energy's \$200 million Hybrid Vehicle Development Program within the next two years. Since the INEL is the only independent laboratory capable of performing complete tests and evaluations of these vehicles, there is strong likelihood that these services will be provided by the INEL. 


\section{Demand Side Management Control System for Electric Vehicles \\ LDRD 4105}

\author{
G. L. Hunt
}

This project was intended to evaluate a prototype electric vehicle battery charge controller incorporating adaptive control strategies for use with several types of EV batteries. The intelligent controller concept, previously developed by one of the project coprincipal Investigators, is capable of predicting the time, energy and power required to charge an electric vehicle battery based on a past history of vehicle operation and recharging behavior. The benefit of this approach is that it allows a vehicle owner to avoid utility peak demand charges for electricity while still assuring that the vehicle will be fully charged when required The project could not be completed during FY-95 due to limited avallability of some of the batteries which were planned to be used. However, a complete test sequence was able to be performed using an advanced nickel-cadmium battery system. Results of this testing are discussed and the economic potential for this concept is briefly discussed.

\section{Objectives}

- Develop a test plan for an intelligent battery charge controller which is adequate to establish its performance with several types of advanced electric vehicle batteries. The performance features of interest include (a) the controller's ability to accurately predict the time (and energy and power) required to recharge each of the batteries used after they have been subjected to a variety of discharges (i.e., simulated vehicle driving), (b) the stability of controller behavior, and (c) any identified anomalies needing correction.
- Perform a series of tests with each of three batteries using the test plan developed in (1). The batteries planned for use were: (a) nickel-cadmium; (b) valve-regulated (i.e., sealed) lead-acid; and (c) nickel metal hydride.

- Evaluate the controller performance, determine any needed improvements, and modify the controller software if applicable.

- Place the controller in field use with some potential user organization, most likely an electric utility, to further evaluate its potential for commercialization.

Accomplishments

The test plan for each of the three candidate batteries was based on the following sequence of events: (a) condition the battery to a stable capacity; (b) perform a series of discharge/charge tests to train the controller; (c) perform a series of discharge/charge tests using a simulated driving profile, with the battery recharged in an uninterrupted fashion to correspond to on-peak charging; and (d) perform a similar series of discharge/charge tests with battery recharge interrupted by simulated utility peak time intervals.

The first battery available for testing was an advanced nickelcadmium EV battery pack which was lent to INEL by a Work-forOthers sponsor after the sponsor's planned testing was complete. This battery was selected in part because its behavior differs significantly from the flooded lead acid technology on which the original controller design was based, particularly the way in which it is recharged. Termination of charging was controlled by a $10^{\circ} \mathrm{C}$ temperature rise, a condition which the controller was not designed to be able to detect directly but rather had to infer from other variables.

A series of 30 discharge/charge cycles was performed on the battery. The discharge cycles were a seudorandom sequence of tests at different power levels and depths of discharge, based on the U.S. Advanced Battery Consortium Dynamic Stress Test for electric vehicle batteries. The resulting charge behavior is summarized in the figure, which illustrates the actual recharge time resulting after each discharge. The solid line in this figure represents the maximum recharge time predicted by the controller after Cycle 64, about midway through the on-peak test cycles. (This curve includes an arbitrary programmed 0.5 hour 'finishing time' allowance, which has been subtracted out in the dotted line.)

In general the controller's predictive behavior for this battery is clearly adequate to assure that a user's vehicle would be charged when desired, while avoiding peak electric rates whenever sufficient time was available to make this possible. To measure the controller's ability to adapt to variability in the battery discharge behavior three cases were measured during the testing: (a) after the completion of training; (b) later during the on-peak testing; and (c) at the end of the off-peak tests. The controller stability was observed good throughout this test sequence.

Testing on a valve-regulated lead-acid battery is still in progress at year end. This data will not be presented in this report. This second test series did identify several instances of unexpected behavior that need to be addressed for reliability reasons, although none of these is fundamental to its operating principles. The most important problem occurred after a power failure, when the controller's nonvolatile memory kept it apparently working but several discharge/ charge tests were later found to be invalid because of a software malfunction. Some cases were also 
encountered where the controller energized its output (which turns on the actual battery charger) without having been requested to do so.

The third (nickel metal hydride) battery planned for testing was not available for purchase until September 1995 and has not yet been tested.

\section{New or Increased Technical Capabilities}

This project represents a first instance of INEL evaluation of EV battery charge control concepts. These concepts are generally at a much lower level of development than the battery technologies themselves, which have been the primary focus of the INEL Energy Storage Technologies Laboratory to date. Such control concepts and the electronic systems needed to implement them are very much open to technology improvements in areas where INEL and Lockheed Martin have strong capabilities.

\section{Business Development Opportunities}

The economic potential for this EV charge control concept is based on the avoidance of higher costs per kilowatt hour during peak utility periods, as well as the imposition of peak demand charges in some regions. Preliminary indica- tion is that EV on-peak rates will be at least double off-peak rates, with differentials as much as $\$ 0.15$ per $\mathrm{kWh}$. In states such as California, where the average residential electric cost was $\$ 0.11$ per $\mathrm{kWh}$ in 1993, this could equate to an avoided cost up to a dollar per day, which in turn could justify a cost of up to several hundred dollars to implement this capability for EV owners. Other utilities may choose to use a peak demand charge based on the power used by the charger, which would be on the order of $\$ 1.00$ to $\$ 1.50$ per day for typical EV chargers. This control capability could be realized by stand alone hardware for early EVs using dumb chargers, but implementation as an option in intelligent chargers is a more likely mid-term opportunity.

\section{Development of Intelligent, Cooperative Miniature Robot Systems} LDRD 4151

M. D. McKay

This project is a multi-year effort to develop miniature intelligently controlled robots for characterizing areas

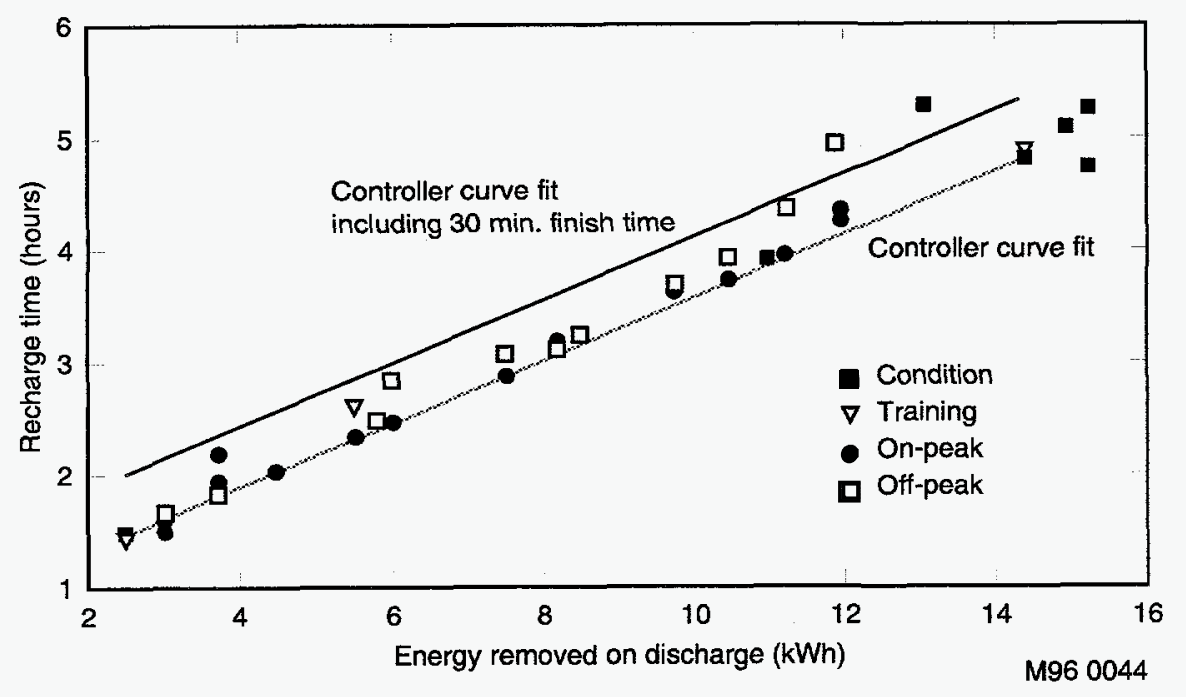

Nickel Cadmium Battery Pack Recharge Behavior inaccessible because of hazard or restrictive size. Emphasis is being given to developing an intelligent control architecture for self-guided tasks and cooperative interaction between multiple miniature robots. At the INEL, many areas are too congested for the use of conventional robots. The characterization of these areas requires simple miniature robots that can perform complex tasks. During FY-95, a six-wheeled vehicle was developed through a cooperative effort with Utah State University. The intelligent control system for the robot was developed at the INEL. The control system was designed to be portable and adaptable to diverse robot platforms and at the same time allow the different robots to act cooperatively. The system is also easily adaptable to carry various sensor packages as characterization payloads.

Utah State University is working closely with the INEL to develop this technology, both hardware and sottware. They have been given a grant through the MIT consortium to pursue development efforts to meet INEL needs. Through USU, Caterpillar Inc. has expressed the desire to conduct collaborative investigations which will lead to commercialization of products resulting from this research.

\section{Objectives}

- Research current intelligent control architectures.

- Procure a vehicle and control system hardware.

- Develop and integrate an advanced control system.

- Test the control system on the vehicle platform.

- Publish a final report.

All milestones were met on schedule.

\section{Accomplishments}

Our research of current intelligent control architectures focused on the applicability of various control systems and computational 
techniques to INEL-specific needs. We selected a hybrid control system that uses a real-time, multitasking operating system as a base and provides a combination of subsumption-based behavioral controls and more traditional sense-compute-act controls.

Procurement of the robot vehicle was accomplished as a cooperative effort with Utah State University (USU). Using a design for a proposed Mars rover, USU developed a small six-wheeled vehicle to integrate with the INEL-developed control system. This unique vehicle measures $12 \times 16 \times 8$ inches (WDH). It has six independently driven and steered wheels mounted on a suspension system that allows minimal displacement of the robot body while going over small obstacles. The vehicle is nontethered, relying on rechargeable batteries for power. The batteries and drive motors are mounted inside the wheels, which lowers the center of gravity and increases traction. Each wheel is independently steered, allowing the vehicle to move in any direction at any orientation. The electronics to control the motors are housed in an onboard aluminum enclosure with interconnections to motors by ribbon cables. The vehicle as delivered has nine simple, coordinated motions: forward, reverse, forward or reverse ackerman turns(left and right), spin right, spin left, and stop. These motions are activated by sending ascii commands to the motor controller.

The computer hardware selected for the INEL-developed intelligent control system was a single-board processor from Real Time Products. Also purchased for the project were the following PC-104 boards: a data module providing analog and digital I/O capabilities, timers, etc.; a SVGA video and flat panel driver board; and a PCMCIA board, providing one type II slot.
This hardware package was selected because of its size and capabilities and because it supports the QNX real-time operating system selected as the base for the control system.

Using a $\mathrm{QNX}$ base, a reactive or behavioral type control language was developed. This control system was written in $\mathrm{C}++$ and includes headers, macros, and libraries with many of the behavioral language functions described by Dr. Brooks (MIT). Behaviors that control the robots actions are written using these functions. Currently, a limited number of behaviors have been implemented to test the integrated system. These include reading sensors, displaying sensor data, and driving the vehicle in a simple structured pattern.

At this point, sensors on the vehicle include eight ultrasonic distance sensors, an electronic compass and inclinometer, and wheel and steering motor encoder readings. A board was developed at the INEL to allow the multiplexing of the eight ultrasonic sensors. This board greatly reduced the amount of on-board circuitry, increasing the system reliability and making the software much simpler. Under consideration are infrared sensors and bump sensors. Additionally, a rechargeable battery system was developed to allow up to one-hour operation on a charge and allows for replacement of battery packs without turning off the system.

Because of problems with the steering motors and the motor controller boards, we returned the vehicle to USU for upgrade. This precluded extended testing of the integrated system. However, the control system and its simple behaviors have been used to control all the sensors, run the vehicle in a simple pattern, and display data obtained by the sensors on an operator's console. This testing occurred with the vehicle being driven over a serial cable by the control processor sitting on a work bench.

New or Increased Technical Capabilities

The INEL has had a strong program in robotics over the past years and this is a new and important application of the technology acquired. In working with the Utah State University which has hardware experience in rover like robotics we have expanded our capabilities to work on a variety of performing robot systems.

\section{Business Development Opportunities}

While it is early in the development of these practical robotic systems companies such as Caterpillar have already discussed the desire to conduct collaborative investigations. The development of the software which operate actual robots has many applications in the industrial arena.

\section{Natural Gas Locomotives and Transportable Power LDRD 4157}

\section{J. A. Dearien, J. I. Mills}

This LDRD activity evaluated the technical, economic, and market potential of a mobile, high-power (20to $40-M W$ ) electrical generation device. The device is a natural-gasfired, turbine-generator set mounted on a railroad vehicle frame.

This LDRD project evaluated use of this device as a power supply for a railroad operation (e.g., pulling a train or supplying power to a railroad operation) and as a mobile power unit that is transported to the point of use over rail lines.

The technical parameters required to produce and operate such a device were evaluated, and the economics of both production and operation were explored. Potential general areas of application and markets for such a concentrated source of mobile 
power were identified and discussed. In the process of this research, contacts were made with experts in industry and government about the technical, economic, and market aspects of this device. These contacts included rail equipment manufacturers, power equipment manufacturers, electrical utilities, railroad operators, and electric power regulation experts.

\section{Objectives}

- Evaluate the technical feasibility of the concept (i.e., can it be physically built and operated).

- Perform a preliminary economic analysis of the operation of this concept (i.e., can power be produced by such a device at a cost that is within the range of present power production techniques).

- Perform a preliminary market assessment of this device (i.e., assuming that it is technically feasible and economically viable, is there a use for such a device now or in the future).

\section{Accomplishments}

This project accomplished all of its objectives in varying degrees.

The concept is definitely feasible from a technical standpoint. We contacted several entities in industry, and we performed sufficient engineering work within INEL, to indicate that a 20- to $40-$ MW electrical generating plant can be placed on a rail platform and moved about the country for rapid set-up and electrical production. We did not evaluate the potential for operating the plant while it was in motion, although no inherent obstacles were identified that would prevent operating the unit while in motion, given that a reason can be found for doing so (see market evaluation).

A preliminary economic analysis of the concept resulted in a predicted operating cost of between
$\$ 0.06$ and $\$ 0.12$ per $\mathrm{kwhr}$, which is within the range of power now being produced in several sections of the United States. For those regions of the United States (and the world for that matter) where the average production cost of electricity is below the $\$ 0.06$ to $\$ 0.12$ per $\mathrm{kwhr}$ cost, there are situations (peaking requirements, seasonal variations, load development, short-term industrial needs, etc.) where the higher priced electricity would be purchased for some period of time-thus, the need for a mobile power source that can be moved after the need for it ceases.

A preliminary market assessment of the technology indicated that a definite market is developing for this type of device. Market development is due to the rapidly changing regulatory environment, and utilities are currently positioning themselves to operate in this new market. The size, both breadth and depth, of this market could not be assessed, but utilities and power-related entities could see a market of some size for this technology.

A waiting market was not identified for in-motion use of the concept, i.e., as a power source for a moving train or for any other function that would require, or could use, power source operation while in motion. Potential developable applications were identified, such as in-transit processing, manufacturing, and waste remediation, but no looming market as was identified for stationary operation of a mobile power source.

\section{New or Increased Technical Capabilities}

The INEL has significant expertise in electrical power generation, control, and delivery. The INEL also has significant expertise in systems analysis/engineering, electrical, mechanical, and project engineering. All of these functions are needed for evaluation and development of the mobile power generator concept defined by this LDRD activity. It is expected that the above capabilities at the INEL would be expanded significantly as this concept advances toward commercial development.

Business Development Opportunities

As part of this LDRD activity and evaluating the mobile power generating concept, the team members met with electrical power utilities (Idaho Power Company), component manufacturing companies (General Electric, Stewart \& Stevenson, Morrison \& Knudsen), railroad entities (Burlington Northern Railroad, American Association of Railroads), and consultants to the power industry (E3, Inc., Energy International, Inc.). All of these companies indicated an interest in some form of cooperative association in developing this concept. Further definition of the concept and market is required before specific cooperative details can be proposed.

\section{Loom Feedback Controls LDRD 4184}

$$
\text { M. R. Stacey }
$$

The purpose of this project was to investigate the current role played by feedback control technology in the operation of weaving looms and assess the feasibility of adding on-loom feedback controls to looms. If adding feedback controls proved feasible, we were to identify one area of loom operation where feedback control technology could improve the quality and production in textile manufacturing and develop a conceptual design to implement this improvement.

This research project focused on the use of feedback controls on the looms. Two main processes that would benefit from feedback control were identified through research; one deals with remediation of broken yams 
(an extremely frequent and laborintensive occurrence), and the other deals with integrating the operation of the loom subsystems to variably control the tension of the warp threads (a conceptual design was developed for this latter process).

The main benefit hoped for from this research project was to offer the American textile industry a competitive advantage in the international market. The production of textiles is a laborintensive process that has resulted in a weakened market for the American textile industry relative to their international competitors, who have access to lower-cost labor. As feedback control technology is developed for the textile industry, American manufacturers can begin to regain their lead in the international market. The direct benefit for the INEL is that we are now in a lead position to participate with the AMTEX CRADA in the area of feedback control as the AMTEX research begins to migrate to this area.

\section{Objectives}

- Update the research team's knowledge about the use of feedback controls in the textile industry, with emphasis on loom operations.

- Evaluate the feasibility of implementing feedback controls on the looms, and select one specific area that would benefit from feedback controls.

- Develop a conceptual design to improve the operation of the loom, or the quality of its product, by automating operations using feedback controls.

- Document findings, and make recommendations for followon research.

\section{Accomplishments}

As the research developed, it became apparent that the textile industry in our country uses a wide spectrum of technologies, from the very primitive to the very sophisticated. At the most sophisticated end, the mills use looms that indeed have evolved to take advantage of the benefits of feedback controls, however, its applications are limited to individual subsystems, as opposed to an integrated use. Although there are several areas in the loom operation that would benefit from the development of customized or local feedback control improvement, we selected two main areas. The research pointed out, very clearly, that there are serious quality problems in the areas of (1) tension control in the warp beam, and (2) repairing broken yarns on-the-fly. Both problems are somewhat interrelated.

Our research focused in the area of tension control because it presented an opportunity to develop a preventive rather than corrective kind of technology, as would be the case in the yarn repair area.

A thorough patent search was conducted to confirm that the proposed technology was not, indeed, available, and one textile industry collaborator was contacted to verify the usefulness and appropriateness of the proposed technology. The patent search confirmed that the issue of tension control for the warp beam is indeed of serious concern to the industry, however, no one has taken advantage of feedback controls technology to integrate the operation of the entire loom. The industry collaborator also confirmed the need for integrated tension control, and he added the need for variability of the tension control across the width of the fabric (technology that has not been developed, either).

A conceptual design was developed to meet the following general design requirements: integrated tension control, variable tension control across the width of the fabric, and ability to input environmental and yarn properties data to the control system. The design includes, as some of its features: spatial actuation, real-time environment and product information, and real-time machine parameters.

This project should progress to design and test a prototype system in-situ. During the first phase of our research, we were offered the testing and learning facilities at the Philadelphia School of Textiles, as well as other production plants to install and test a prototype. It is our recommendation to organize a formal CRADA partnership with selected industry partners and prove the technology, and then transfer it to the private sector.

An Invention Disclosure Record is being filed with Lockheed Martin Idaho Technologies Office of Technology Transfer to document the proposed design. The principal investigator for this project is listed as one of the inventors; the other inventor is Kevin L. Moore, Ph.D., P.E., of System Analysis and Control Company, Pocatello, Idaho. His work is documented in final report: On-Loom Feedback Control Systems.

\section{New or Increased Technical Capabilities}

The next logical, and inevitable, step for the AMTEX CRADA research is to use feedback controls to integrate the operation of the loom during the weaving process. INEL is now in a prime position to contribute to the progress of the AMTEX CRADA in the research of feedback control. INEL knows what the industry status is now, where many of the deficiencies are, and where feedback controls can best benefit the industry.

Also, the industry collaborators for this LDRD are aware of the INEL's capabilities, and we have 


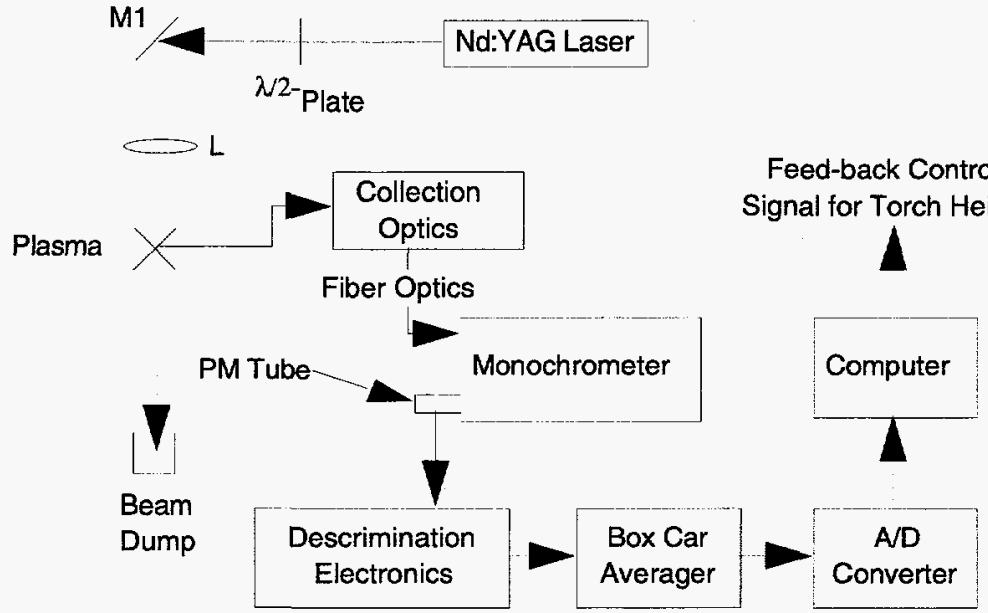

Laser-Integrated Scattering Technique

the opportunity to continue to develop this technology with the benefit of their process knowledge. As this technology develops, less woven fabric will be wasted (due to product defects), and therefore, the raw materials (cotton, wool, etc.) can be better used in the industry.

\section{Business Development Opportunities}

- John Pfeiffer, Pendleton Woolen Mills, Washougal, Washington: industry knowledge, supports need for technology, offered site for in situ testing, offered in-kind contribution.

- Robert Sadler, Baron's Rocky Mountain Wool Corporation, Brigham City, Utah: industry knowledge, supports need for technology, offered site for in situ testing, offered in-kind contribution.

- Martin Heilman, Wayne Mills Company, Inc., Philadelphia, Pennsylvania: industry knowledge, supports need for technology, offered site for in situ testing, offered in-kind contribution.

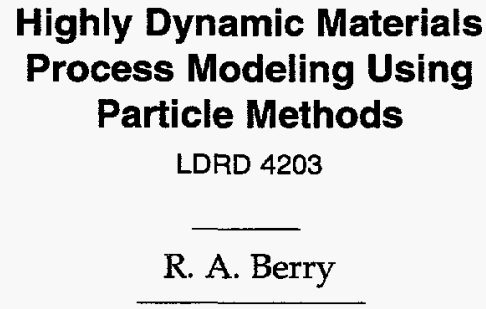

Advanced materials processing
research has increasingly depended
on numerical simulations to integrate
theory, experimentation, and efficiently
engineered production. Such
processes are frequently character-
ized by large deformations and high
heat transfer rates with nonequilibrium
solidification, requiring simulation
methods that provide complete history
information and the ability to track
details of the deformation process.
Tradition Eulerian and Lagrangian
techniques (as embodied in finite dif-
ference, finite volume, and finite ele-
ment methods) lack the ability to
simultaneously handle these require-
ments. This project investigates some
of the particle methods that have
appeared recently (mainly in other
contexts, e.g., astrophysics). The
methods examined are Lagrangian,
with computational points (particles)
carrying all of the material information,
so material histories are produced but
are gridless (or nearly so) so that grid
distortion is not an issue for large
material deformations. In the
research, some of the schemes are
tested on representative problems and

we conclude that the methods are definitely applicable and should be further pursued and used in the advanced materials process modeling area.

\section{Objectives}

Future advancement and competitiveness in advanced materials processing research requires that a shift be made toward a stronger emphasis on numerical simulation, forming a tightly coupled relationship between theory, mathematical modeling, and experimentation. Use of numerical simulation has lagged because many such processes are characterized by large deformations and rates and high heat transfer/solidification rates, and traditional methods of analysis (finite element, finite difference, or finite volume) cannot be appropriately applied to these problems. This project investigates alternative methods for approaching these problems-grid-free or nearly gridfree methods in which the reliance on an underlying grid is minimized.

\section{Technical Accomplishments}

Though quite new, grid free methods appear to offer tremendous potential for the solution of such complex processes. During the course of our research, we introduced several grid free (or nearly grid free) methods, including multi-directional finite difference (MDFE) methods, moving weighted least squares (MWLS) methods, reproducing kernel particle methods (RKPM), the elementfree Galerkin method (EFGM), and the fully Lagrangian FLIP scheme for solids. Others have been introduced over the past several years, including smoothed particle hydrodynamics (SPH) methods, particle and force (PAF) method, diffuse element methods (DEM), the neighbor node method (NABOR), the FLIP particle-in-cell method, and the quasi-particle (QP) method. 
We evaluated all these methods, giving in-depth examination to the smoothed particle hydrodynamics (SPH), the fully Lagrangian FLIP scheme for solids, and the quasi-particle scheme (QP). Our research focused on heat transfer, solidification, and droplet splat dynamics (liquid), as representative test cases that demonstrate wide applicability to many materials processing problems. During the course of our investigation, over twenty small computer programs were written to test various aspects of these methods.

The smoothed particle method is applicable to large deformations. Coding for this Lagrangian method can be intricate; permitting each particle to interact with all other particles is too expensive, so nearest neighbor interactions are sought. However, a particle's neighbors are continually changing, and efficient data handling methods are necessary. SPH was found to be a very appealing and valuable computational tool, especially for high-deformation events such as impact. We were also able to perform conduction and solidification problems with SPH. However, we found the SPH method still has a few technical barriers to overcome before becoming a widely useful tool in computational mechanics. Instabilities from a numerical artifact sometimes occurred. We could see no robust solutions to this problem and postponed further work on SPH (leaving this a topic of further research) and directed our efforts toward the fully Lagrangian FLIP scheme for solids.

With the fully Lagrangian FLIP scheme (a full-particle PIC scheme) each particle carries all of the properties of the fluid (or solid). It employs a simple background grid to form gradients of certain quantities by projecting information from the particles to the grid points, and then computing the gradient at the particles' locations as an interpolated integration by parts. The background grid can be quite regular even though the material is undergoing large deformations as represented by the Lagrangian particle motions. The particle data describe the fluid/solid completely from cycle to cycle (with low dissipation); the particle data are updated each cycle from the grid solution, not replaced. Constructing and modifying algorithms or codes using this technique were easily accomplished and were similar in many ways to finite element construction methods. Solutions were found, however, to be sensitive to the ordering or sequence of the calculations. We also found that while linear interpolation was fine for some variables, noisy pressures and densities were obtained for liquids and solids with barotropic equations of state (small changes in density produced large changes in pressure; see the figure right), indicating (we believe) that a quadratic or higher interpolation should be used for these variables. We are very optimistic that this method will have numerous applications. It appears to effortlessly handle solid-solid and solid-fluid interfaces and, since material points are followed, history-dependent variables such as those of plastic strain and nonequilibrium solidification are easily tabulated. Further work is necessary to include anisotropic features and a general slip condition at interfaces.

\section{New or Increased Technical Capability}

This project has clearly demonstrated the power of these grid-free methods to serve as a basis for advanced materials processing simulations where large material deformations occur and/or where it is necessary to keep accurate histories of the events associated with each material particle. The project has placed us now at the point where we can efficiently use these techniques (of course, with some specific enhancement features for specific problems at hand) in the development of simulation software for a whole new class of advanced materials processing problems. We are now further aware of several of the nuances of these methods. While significant effort was spent deriving and manipulating the theory behind these methods, even greater time was spent investigating these methods from a coding or implementational viewpoint. This latter is often overlooked by researchers, but, in reality, it is where a larger share of time is spent. We recommended that these methods be further pursued with research directed at removing the respective shortcomings, as listed, along with the MWLS and RKPM methods (which have appeared only in the past year) mentioned above.

\section{Business Development Opportunities}

Numerical methods development for advanced materials processing is difficult to sell as a stand alone product to an outside customer. It can, however, as is being demonstrated at an ever increasing level at the INEL, be the key link in forming a tightly coupled relationship between theory, experimentation, and efficient engineering implementation of advanced materials process development. Its real benefit in the business arena is to help, and even lead, in the selling of integrated, complete research programs to prospective customers.

\section{Advanced Plasma Processes for Black Liquor Gasification LDRD 4205}

\author{
P. C. Kong, J.D. Grandy, \\ A. D. Watkins
}

The pulp and paper industry has a need to develop technologies that 


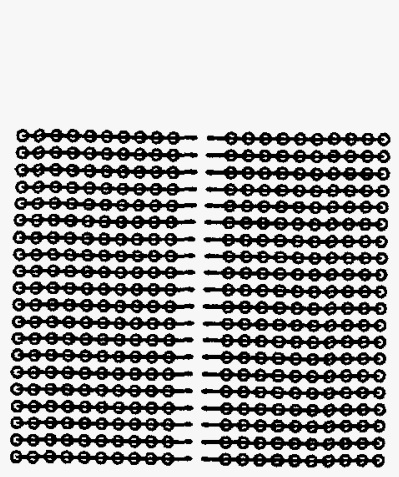

(a)

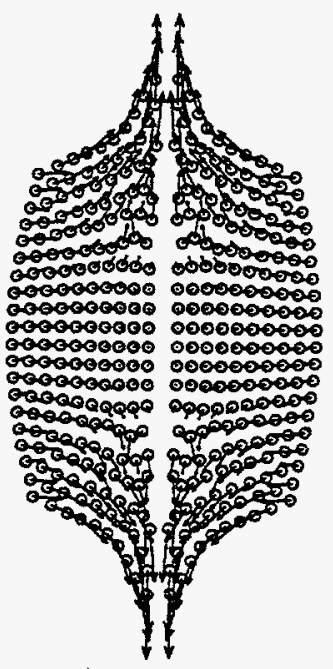

(c)

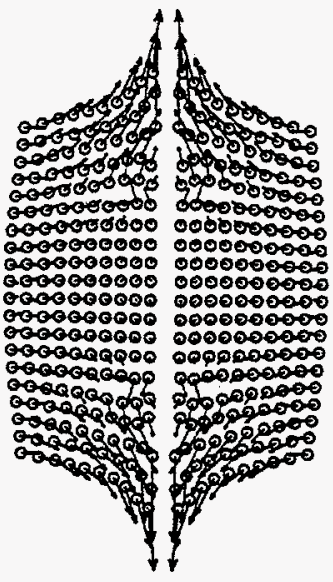

(b)

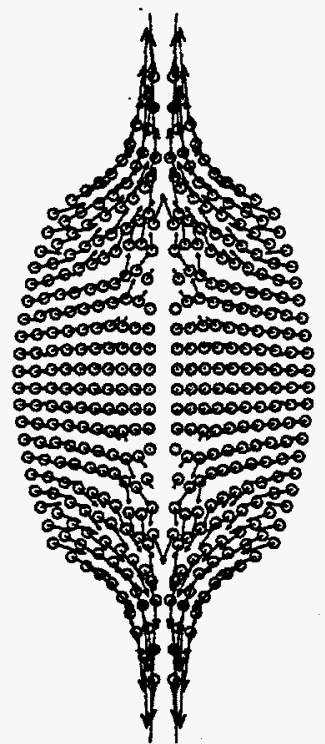

(d)

Computational Example of Full Particle PIC Scheme. Collision Sequence of Liquid Metal (Rectanguiar) Droplets, Each with $10 \mathrm{~m} / \mathrm{s}$ Velocity in Opposing Direction.

(Successive letter indicates increasing times.)

will allow them to incrementally increase production capacity by ten to fifteen percent at paper mills. At present, a bottleneck exists in the standard Kraft process for paper production once the capacity of the Tomlinson boiler is reached. A Tomlinson boiler costs $\$ 100 \mathrm{M}$. It is used to burn black liquor to recover energy and chemicals, which are then recycled back into the wood pulping process. Black liquor consists of organic residues and inorganic chemicals (sodium and sulfur) left over from the pulping process and has a high ener- gy content. Owing to economy of scale, manufacturing small boilers to accomplish a $15 \%$ increase in production is not cost effective.

The industry considers gasification as a promising method to provide incremental capacity and to generate electricity as modern paper mills become more electricity based. Three technologies under development are partial combustion in entrained flow reactors or fluidized beds, and steam gasification in a fluidized bed. A fourth alternative is steam reforming in a plasma reactor. All these gasifica- tion technologies produce syngas $\left(\mathrm{H}_{2}\right.$ and $\mathrm{CO}$ ), which can be burned in gas turbines to produce electricity. While steam plasma gasification will be a net user of electricity, an advantage over the other technologies is direct chemical recovery, which will eliminate causticizing of the recovered chemicals. This will result in reduced capital and operating costs and environmental concerns associated with causticizing. The capital costs of a plasma gasification system are expected to be considerably lower than for the other gasification methods.

\section{Objectives}

The technical steps toward developing a plasma gasification process using FY-95 LDRD funding included the following:

- Formulate and develop the hybrid steam plasma concept.

- Design and construct a benchscale hybrid plasma prototype for laboratory tests.

- Perform technology feasibility tests for black liquor gasification and inorganic chemicals recovery.

\section{Technical Accomplishments}

All of the technical goals listed above for FY-95 have been achieved; however, owing to a mid-year cutback in funding, the scope of the gasification testing was cut back considerably.

The hybrid steam plasma concept as it applies to black liquor gasification is to have a steam plasma torch operating in tandem with a second plasma torch (see the figure). Black liquor is injected into the steam plasma plume of the first plasma torch where initial heating and steam reforming reactions take place. The second torch allows the material to pass through the arc generation region where it is super heated to plasma temperatures. The high amount of sodium in the black liquor reduces the required operating power of the second torch by almost half, reducing the 


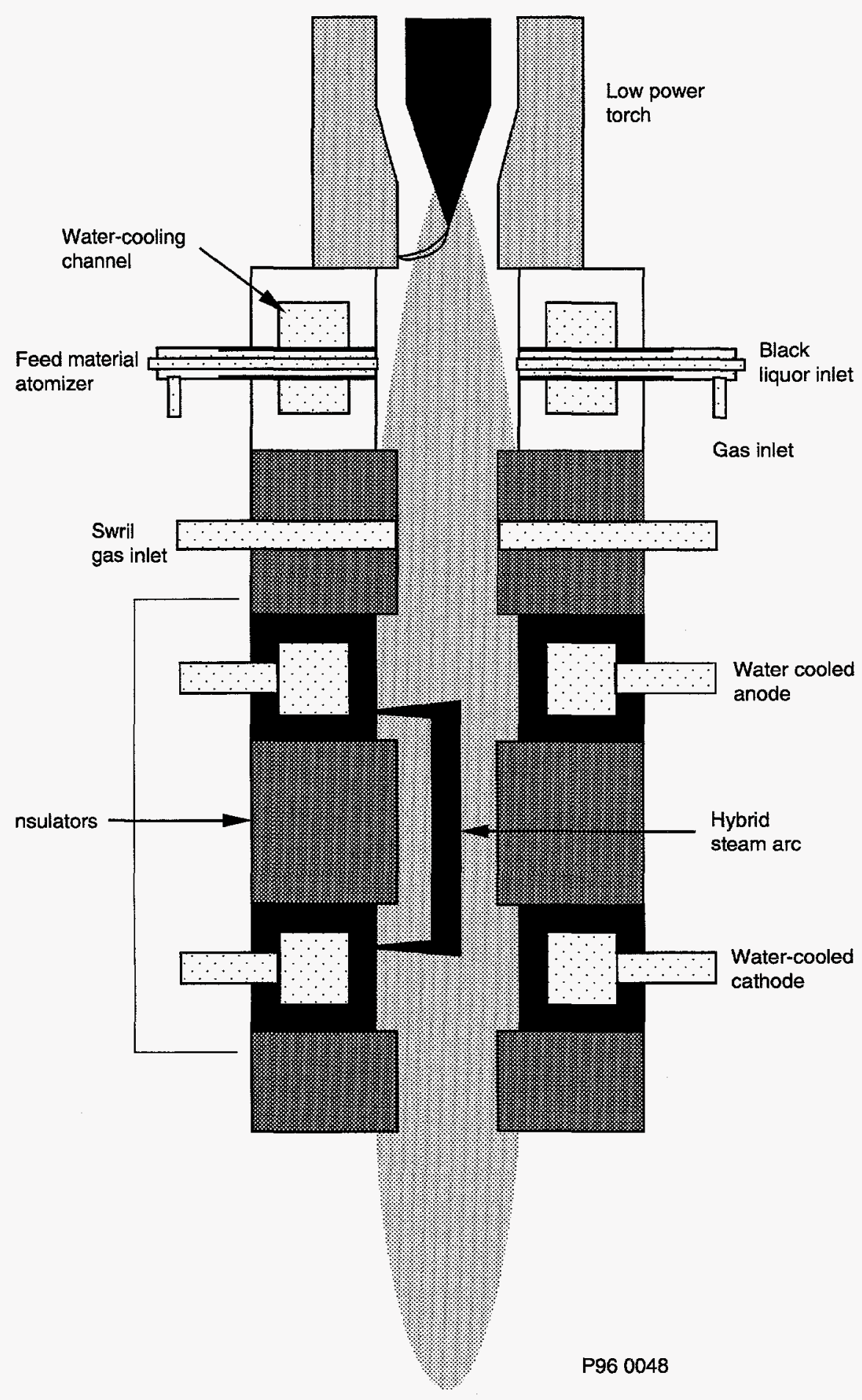

operating voltage from 80 to $45 \mathrm{~V}$. This is due to the very low ionization potential of sodium $(5.1 \mathrm{eV})$, creating plasma conditions at much lower temperatures, $=7000 \mathrm{~K}$ versus $12,000 \mathrm{~K}$ in an argon plas- $\mathrm{ma}$, in which the ionization potential for argon is $15.8 \mathrm{eV}$.

In this bench scale prototype, the first torch was a standard plasma spray torch operating on argon only. This was done for conve- nience and because the upstream steam plasma torch has not been developed. This is a critical development need for FY-96. The steam plasma was generated in the second torch by adding sufficient water to the black liquor.

Several black liquor processing runs have been conducted in the latter part of FY-95. Product gas samples were taken and analyzed with a standard mass spectrometer and an ICP mass spectrometer. Filtered particles were subjected to $x$ ray diffraction (XRD) analysis and energy dispersive $x$-ray spectroscopy (EDXS). The mass spectral results are qualitative only but show production of syn-gas $\left(\mathrm{H}_{2}\right.$ and $\mathrm{CO}$ ) and some $\mathrm{CO}_{2}$, as would be expected from the water gas shift reaction between $\mathrm{CO}$ and $\mathrm{H}_{2} \mathrm{O}$. Significant amounts of $\mathrm{N}_{2}$ and $\mathrm{O}_{2}$ were also found, indicating air contamination either during sampling or simply left over from the air originally in the reactor. Notably, there was no $\mathrm{H}_{2} \mathrm{~S}$ in the exhaust gases. This indicates the sulfur is reacting preferably with the sodium vapor at high temperatures. Also, $\mathrm{H}_{2} \mathrm{~S}$ is unstable at high temperatures and would dissociate to $\mathrm{H}_{2}$ and $\mathrm{S}$ in a rapidly quenching thermal plasma. The ICP mass spectral produces a quantitative analysis. Ignoring the $\mathrm{N}_{2}$ and $\mathrm{O}_{2}$, a mass balance can be done for the exhaust, comparing the theoretical amounts of syngas generated versus the measured, renormalized values, as seen in Table 1 . The XRD analysis of the particles was somewhat enigmatic as it did not identify any compounds of $\mathrm{Na}, \mathrm{S}$, $\mathrm{O}, \mathrm{C}$, or $\mathrm{H}$ with a high probability. The compounds matched include $\mathrm{Na}_{2} \mathrm{~S}, \mathrm{NaHS}, \mathrm{NaOH}$, and various sodium oxide phases but with a low correlation factor. EDXS results of the particles are still pending and should provide a more definitive identification of the compounds. 
Table 1

Theoretical exhaust gas composition versus experimental composition.

(Experimental values have been renormalized after subtracting $\mathrm{N}_{2}$ and $\mathrm{O}_{2}$. Note the $\mathrm{CO}$ forms even though there is enough water to theoretically convert all the carbon to $\mathrm{CO}_{2}$.)

Theoretical Experimental (\%) (\%)

\begin{tabular}{lrr}
\hline $\mathrm{Ar}$ & 77.0 & 83.0 \\
$\mathrm{H}_{2}$ & 16.0 & 11.0 \\
$\mathrm{CO}$ & 0.0 & 4.0 \\
$\mathrm{Co}_{2}$ & 7.0 & 2.0 \\
\hline
\end{tabular}

The overall results of the project indicate that the steam reforming reaction is occurring and converting the black liquor into syngas and sodium compounds that can be used to directly form a caustic solution (white liquor) for the pulping process.

\section{New or Increased Technical Capability}

This project has allowed further development of plasma technologies and plasma chemical processes, which will establish the INEL as a leader in these fields. The hybrid steam plasma technology finds wide applications in hazardous waste destruction, inorganic materials synthesis, coating deposition, chemical synthesis, and energy conversion, which are of vital strategic and economic interest to the United States.

\section{Business Development Opportunities}

Many industry contacts were made during the year by travel to the Institute of Paper Science and Technology (IPST) and the Chemical Recovery Conference in Toronto, as well as through phone conversations. Union Camp, Weyerhauser, and Consolidated Papers are very interested in this plasma gasification technology. They are watching the technology development very closely, but they are extremely reluctant to invest money at this early stage.

The pulp and paper industry is very conservative and suffers from small profit margins. The plasma gasification must prove autocausticizing chemicals recovery and have a persuasive analysis for energy and cost benefits before the industry will consider a partnership where money is invested.

\section{Joining and Testing of Small-Scale Automotive Components LDRD 4206}

$$
\text { D. E. Clark }
$$

This project was designed to explore some issues that arose out of our continuing contacts with the automotive industry. In particular, the stabilization of crimped joints for hightemperature service, such as oxygen sensors, involves welding and soldering processes; these processes are sometimes a source of defects, and their control is essential to efficient production. The INEL has had significant experience in the diagnostics and control of welding processes and has been working on CRADAs in several areas with USCAR and with individual American car makers.

The project has addressed two major areas of interest to the automotive industry, with the aim of positioning the INEL for further work and program development in the relatively unexplored area of these small-scale automotive components. One task involves the development of a small scale machine similar to a Gleeble, a hydraulically powered thermomechanical testing machine typically used for much larger specimens; the present machine as developed allows the exploration of mechanical property and liquid metal embrittlement issues on the small scales encountered in the new generation of very small automotive connectors. The other task addresses the peculiarities of the solidification mechanics of the very small welds typical of these connectors, in particular, how copper wires can be welded to stainless steel crimped connectors without the hot cracking usually seen with copper/iron dissimilar metal welds.

\section{Objectives}

Elevated temperature small-scale testing device.

- Determine specimen size, desired loads, and resulting mechanical design of apparatus.

- Determine data acquisition requirements.

- Obtain mechanical components and construct apparatus.

- Obtain appropriate data acquisition (DAQ) hardware and software.

- Obtain appropriate initial test specimens from industrial partners.

- Design test matrix and characterize hot ductility of specimens.

- Evaluate performance of apparatus and inform industrial partners.

Microscopic examination of lowcurrent Gas Tungsten Arc spot welds.

- Examine existing welds metallographically and with SEM/Auger as appropriate.

- Use existing industrial equipment to make welds across a typical parameter space.

- Examine parameter space welds for changes in metallurgical phenomena.

- Quantify effects and develop phenomenology for lowcurrent GTA spot welds.

- Inform industrial partners of results.

\section{Accomplishments}

Elevated temperature small-scale testing device.

The newest generation of automotive crimped connectors is 
formed from material as thin as 0.010 in., yielding cross sections of the order of $0.001^{2}$ inches for a typical connector. In testing for liquid metal embrittlement, the loads when embrittlement occurs are very low, approaching zero load and nil ductility. We selected an electrically driven (brushless dc) actuator for loading purposes; with a maximum capacity of $1000 \mathrm{lbf}$, it overlaps the lower end of the range of the hydraulic Gleeble. Cells of 1000- and 100-lbf load were obtained, kept external for easy interchangeability. The resolution of the DAQ systems allows small fractions of a lbf to be measured. A 300A laboratory dc power supply was chosen because it already existed in the laboratory, and because testing showed that it could heat small specimens at adequate rates of several hundred $\mathrm{K} / \mathrm{s}$ without causing noise in the DAQ system. The DAQ system selected was LabView software running on a laptop 486 computer, used because available.

The actual control of load and motion was accomplished by a controller furnished with the linear actuator. Although the linear actuator has an encoder, an LVDT was added to the system to provide independent measurement of displacement. A spare control axis within this controller was used to control temperature. A user interface was developed that allows the user to graphically specify load, time, and temperature, and control either load or stroke.

Appropriate test specimens were not available from the industrial partners at this time, nor were the particular combinations of base material and solder alloys required for LME characterizations, so sheets of brass and bronze materials typically used in such connectors were obtained from vendors. It was determined that the apparatus functioned as expected. The machine was built into a custom, desk-sized unit.

\section{Microscopic examination of low-} current GTA spot welds.

The specific results obtained in this work are based to a large extent on proprietary materials and designs supplied by the industrial partners and cannot be discussed in detail at this time. The task of clearing some of this work for publication in the open literature is being pursued.

The general phenomena associated with GTA welds of this size indicate that the molten copper of the wires and the stainless steel of the crimp, though melted into a continuous pool, do not alloy. The duration of the welds is quite short; the impinging surfaces of the two materials are not atomically clean; and they thus remain immiscible. This can be seen in many cases because of the color differences between copper and stainless steel under metallographic exami- nation; although it is possible for copper to alloy in the iron-based material without causing a substantial color change, SEM x-ray mapping, shown in the figure below, verified the segregation in all cases where it was applied. New or Increased Technical Capabilities

The small scale testing machine is functional, practical, and easy to use. As far as we have been able to determine, it is unique.

The technical capability represented by the solidification mechanics study is somewhat more abstract; it is not well represented in the technical literature. To some extent, we know about it because we happened to be working with industrial partners in these particular areas. The concept of rapidly solidifying dissimilar materials in this context, to avoid alloy incompatibility in structural welds, bears some further thought.

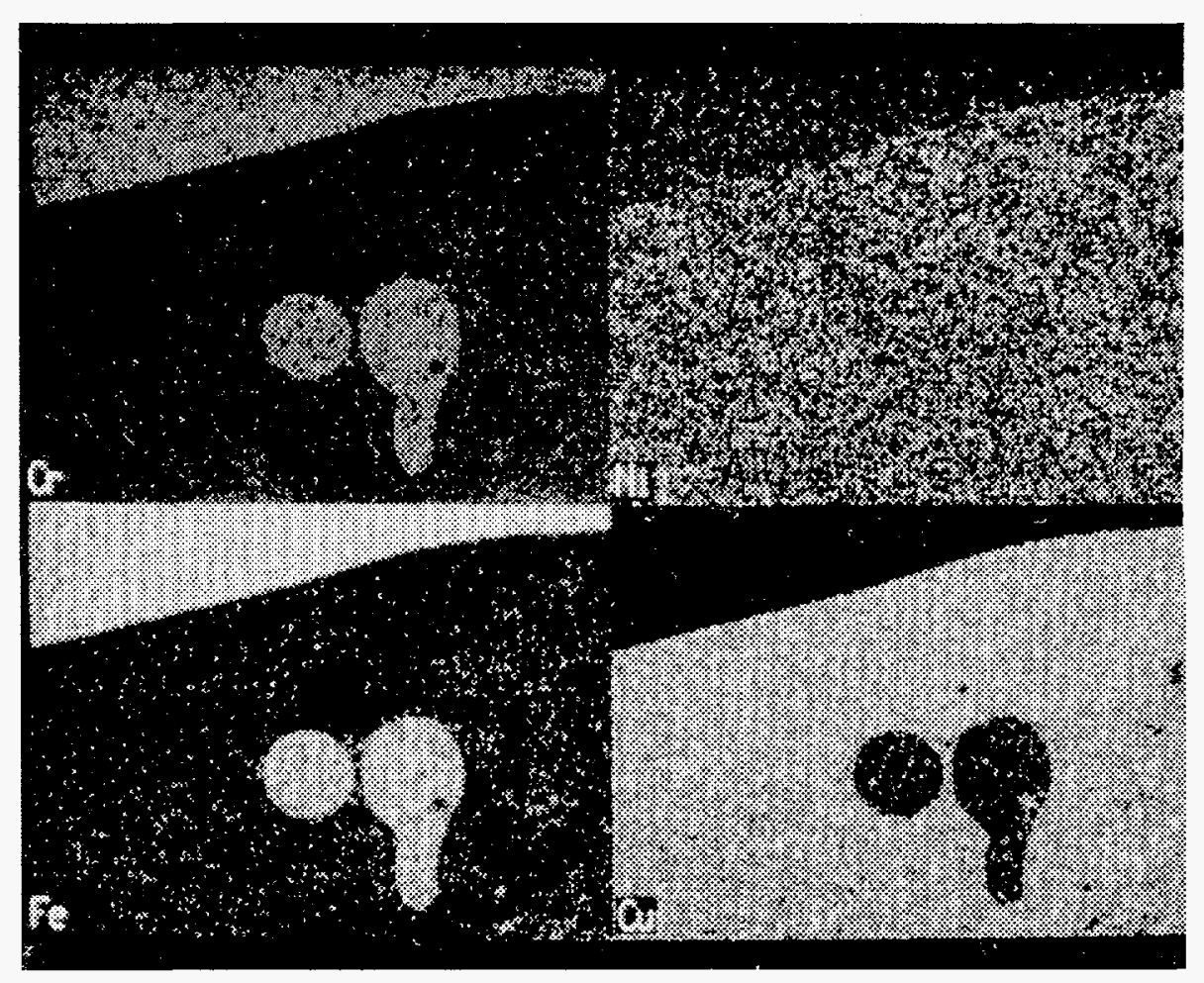

X-ray Map of Small Area of Weld Between Copper Wire and Stainless Steel Crimp, Showing Lack of Mixing of Materials 


\section{Business Development Opportunities}

Industrial CRADA partners were informed of the development of the small-scale Gleeble during a series of visits to the INEL in September of 1995 . There was considerable interest in the capabilities of the machine, and in its usefulness as a screening tool for alloy combinations.

There is a window of opportunity at this point. The small-scale testing machine was inspired by real problems in automotive connector joining, and its construction on a relatively small budget was made possible by borrowing from existing hardware and ongoing programs, and by advances in microcomputers and data acquisition software. A conference on thermomechanical simulation is scheduled for June 1996 in Columbus, Ohio; many academic and commercial organizations will be represented there. And an abstract has been submitted for internal patent clearance, so we can describe the machine to the academic community and also determine if there is any outside commercial interest. In the meantime, continuing contacts with our CRADA partners may turn up intermediate work in the laboratory that will allow the practical limits of the machine to be tested.

\section{Process Control Development for Ultracapacitor Electrodes by Liquid Injection Plasma Deposition LDRD 4208}

\section{A. D. Watkins, S. C. Snyder, P. C. Kong}

Atmospheric plasma chemical vapor deposition (CVD) has been identified as an enhancing technology for the INEL hybrid electric vehicle concept, where the power plant is a combination of solid oxide fuel cells (SOFC), batteries, and supercapacitors. This technique is used to deposit thin, dense, solid oxide electrolyte layers, electronic conducting ceramic seal layers, and porous electrodes for the SOFC. This process is also an enabling technology to deposit ultra-high surface ceramic electrodes for supercapacitors. Consistent deposition of high-quality coatings on an industrial scale requires the development of real-time process control of the substrate temperature. The main objective of this work is to develop a real-time, nonintrusive technique to measure plasma temperatures above the substrate surface and to investigate the feasibility of using this sensor for feed-back control.

Using a frequency integrated laser scattering technique, plasma gas temperatures as a function of axial position were measured in a pure argon plasma to obtain base-line data for atmospheric plasma CVD process control. The frequency integrated laser scattering technique was enhanced to measure temperature in a lightly loaded plasma and investigated for use as a real-time sensor.

\section{Objectives}

- Investigate Liquid Injection Plasma Deposition (LIPD) using Fabry-Perot monochromator to determine plasma velocity and temperature.

- Modify LIPD and monochromator equipment.
- Investigate use of frequencyintegrated laser scattering technique as a real time sensor.

- Evaluate the effect of plasma temperatures on coating deposition.

\section{Technical Accomplishments}

The velocity and temperature measurements of a plasma vary along the axial distance from the torch, allowing the feasibility of controlling substrate temperature by changing axial distance of the torch from the substrate. For distances greater than 0.5 in., the process operating temperatures are well within the regime where Rayleigh scattering dominates, indicating that integrated laser scattering is a viable technique to measure temperatures at the substrate providing that particle nucleation has not occurred, or the particle density is very low.

The figure indicates the overall experimental arrangement to implement the integrated laser scattering technique and the LIPD system. The plasma torch used was a Miller model SG-100 spray torch operated at a current of approximately $500 \mathrm{~A}$, with an argon flow rate of $35.4 \mathrm{~L} \mathrm{~min}^{-1}$. The laser source was a pulsed neodymium-doped yttrium aluminum garnet (Nd:YAG) laser operating at $532 \mathrm{~nm}$ and $20 \mathrm{~mJ}$

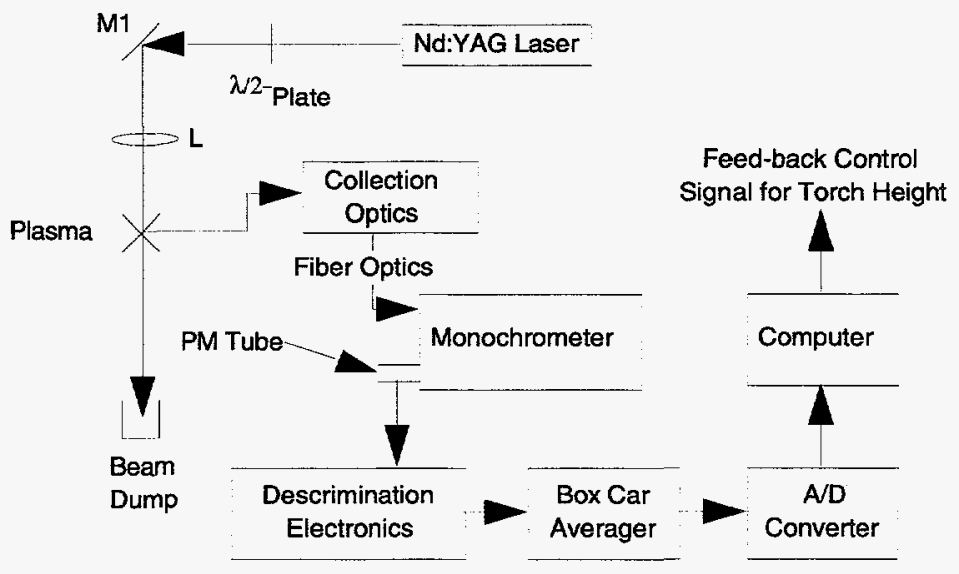

Laser-Integrated Scattering Technique 
with a pulse width of $10 \mathrm{~ns}$ and pulse rate of $10 \mathrm{~Hz}$. The scattered laser light was collected by fiber optics and focused onto the entrance slit of a 0.33-m monochromator. The entrance and exit slits of the monochromator were $150-\mu \mathrm{m}$ wide. In addition, the entrance slit was masked with a horizontal slit $150-\mu \mathrm{m}$ wide to form a square entrance aperture. The scattered laser light signal was detected using a photomultiplier tube (PMT), and its output was integrated using a gated boxcar averager. The analog output of the boxcar averager is proportional to the gas temperature.

The plasma torch and injection system deposits material onto a substrate, where the plasma torch is mounted vertically above the substrate. Material was injected into the plasma injection chamber, vaporized by the plasma, and deposited onto a quartz disc substrate. Adjustment of the distance between the substrate and the injection chamber to control temperature is physically performed by a computer-controlled linear slide assembly. The slide assembly is equipped with a high-resolution encoder to verify and transmit to the computer the plasma torch position relative to the substrate. Computer control is accomplished through a 486 microprocessor and a bus-based stepper motor control board. The computer control system uses Labview as an operator graphical interface with virtual instrument software package supplied by the linear slide vendor to interface the graphical interface with the motor control board.

Liquid precursors (e.g., water), $0.01 \mathrm{M}$ nickel nitrate, and $0.001 \mathrm{M}$ nickel nitrate were injected into the plasma to deposit a thin film of nickel oxide on the surface of a sub- strate. A 45-min time-temperature experimental run was made where precursors were injected into the plasma and the temperature was measured using the abovedescribed equipment. The temperature measurement signal was stable and reproducible for different process parameters, an indication that the process can be controlled through feed-back control. The temperature of the plasma changes as the injection rate is changed. A difference of $200^{\circ} \mathrm{C}$ is shown for injection rates of $5 \mathrm{ml} / \mathrm{min}$ and $10 \mathrm{ml} / \mathrm{min}$., which is a clear indication of the sensitivity of the control technique.

Evaluation of substrate temperature on the deposition of porous materials is continuing.

New or Increased Capability

Control of the liquid injection plasma deposition using a frequencyintegrated laser scattering technique is a new capability at the INEL. The process and technique allows for the consistent deposition of thin films that have been predominately done in the past by chemical vapor deposition. Experience gained on this project will allow consideration of this process and technique for the deposition of barrier, functionally dense, functionally porous coatings.

\section{Business Development Opportunities}

Contacts have been made with Willie Hendrickson of Aveka, Inc. (a 3M spinoff). Future work will involve development of high surface area porous electrodes; thin, dense zirconia and cerium oxide coatings for solid oxide fuels cells and oxygen sensors; and the investigation of multi-laminates materials. An invention disclosure is being pursued for this measurement technique.

\section{Miniature Alternative Power Supply Solid Oxide Fuel Cell \\ LDRD 4395}

\section{R. Barklund}

The Miniature Alternative Power Supply (MAPS) project purpose was to begin development of a power supply intended to replace conventional chemical batteries in portable devices such as cellular telephones, pagers, laptop computers, and similar products. At present, these devices are powered by a variety of (usually) rechargeable chemical cells. The most popular battery type in these applications is the nickel-cadmium (NiCd) cell. The NiCd cell has its drawbacks, though. It has a very low energy density, which provides very short operating time and requires frequent recharge. It can also develop a memory, which reduces its capacity, and it is considered a hazardous material when discarded at end of life.

The Solid Oxide Fuel Cell (SOFC) directly converts either gaseous or liquid fuel and air to direct current (dc) electricity. This conversion is effected within a ceramic catalyst that can be as thin as three microns. The desired output power is achieved by stacking several SOFCs together. The method to firmly attach these cells to each other with an electrically conductive means was one focus of the research, as was the optimum method to fabricate the cells themselves.

The operating time envisioned for an SOFC power supply in a typical application greatly exceeds that available from chemical batteries. The recharge time would be seconds, compared to hours, and the power supply would not present an environmental hazard upon disposal. The potential benefits to the nation's economic competitiveness, as well as to the consumer, is immense and obvious.

\section{Objectives}

- Determine the optimum method to use for thin-film deposition to fabricate the 
cells. The desired material density is critical for the ion exchange process to occur to generate the dc electricity.

- Fabricate and test a small (3-mm diameter, $1.5-\mathrm{mm}$ thick) singlecell SOFC. This size of cell was calculated to produce the current required for a 3-watt cellular phone application.

- Develop a means to stack several SOFCs together to achieve the desired voltage output. This adhesive must have good electrical conductivity and have a thermal expansion coefficient similar to the ceramic materials of the SOFC.

Accomplishments

As this LDRD was funded about mid-year, the work has been in progress for only six months. This has, of course, severely limited the accomplishments. Various compounds of lanthanum, manganese, and strontium oxides have been explored for use as the anode, cathode, and catalyst of the SOFC. There were primarily two methods investigated to form the thin-film layers desired. These methods were chemical vapor deposition and plasma spray. Additionally, an ion hammer was used on some samples in an effort to increase material density. This work has been somewhat successful in that cells have been fabricated, but SEM analysis has revealed there are still some voids larger than desired in the structure. A more consistent ceramic structure is desired, and it is expected given the results of work thus far.

A furnace in which to test the sample cells was devised and constructed. This furnace isolates the sample in a glass tube with an ID slightly larger than the cell OD. A ceramic sealer is used to render the cell plane gas-tight. When the glass tube with the cell inside is heated in the furnace to about $850^{\circ} \mathrm{C}$, hydrogen is introduced on one side of the cell with oxygen introduced on the other. The resultant reaction is an ion exchange that produces dc electricity and $\mathrm{H}_{2} \mathrm{O}$ vapor. The electrical output is extracted via platinum wires attached to the cell. The electrical characteristics of the cell can then be explored using sensitive test and measurement equipment.

The effort to develop an electrically conductive intercell adhesive with the proper thermal expansion coefficient has been less than satisfactory. Several compounds have been tested, but the major difficulty lies in the electrical conductivity. More development work in this area is indicated.

New or Increased Technical Capabilities

This project has enhanced the technical capability at the INEL in the ceramic materials processing area. The thin-film deposition portion of the research greatly expanded our knowledge and ability to produce thin films of consistent thickness with generally stable density. The electrical characteristics of the various ceramic compounds should be investigated further. This is a new research direction, since ceramics heretofore have been used primarily for their high-temperature operability characteristics. Research in this area could have unexpected benefits in the future.

\section{Miniature Alternative Power Supply Thermo-photovoltaic LDRD 4396}

\section{R. Barklund}

The Miniature Alternative Power Supply (MAPS) project was to begin development of a power supply intended to replace conventional, chemical batteries in portable devices such as cellular telephones, pagers, laptop computers and similar products. At present, these devices are powered by a variety of (usually) rechargeable chemical cells. The most popular battery type in these applications is the nickel-cadmium (NiCd) cell. The NiCd cell has its drawbacks, though. It has a very low energy density, which provides very short operating time and requires frequent recharge. It can also develop a memory, which reduces its capacity, and it is considered a hazardous material when discarded at end of life.

The Thermo-photovoltaic (TPV) device uses a dual-conversion methodology to generate dc electricity. A gaseous fuel, typically propane or butane, is mixed with air and burned in a chamber. A fibrous, rareearth, oxide mantle in the flame is heated to incandescence and emits a unique, narrow-band light in a nearvisible wavelength. This radiation is incident on a photovoltaic cell that has a sensitivity optimized for the emitter wavelength. This PV cell converts the incident photons to electricity.

The operating time envisioned for a TPV power supply in a typical application greatly exceeds that available from chemical batteries. The recharge time would be seconds, compared to hours, and the power supply would not present an environmental hazard upon disposal. The potential benefit to the nation's economic competitiveness, as well as to the consumer, is obvious and immense.

\section{Objectives}

- Develop thermal and optical recuperation methods and materials to increase TPV device efficiency. An existing TPV device converts fuel to electricity with a $2.8 \%$ efficiency.

- Find or develop a PV cell with high conversion efficiency and high sensitivity to the emitter wavelength. The commonly available photovoltaic (PV) cells have a broadband sensitivity, which acts to decrease 
sensitivity in any particular narrow bandwidth.

- Develop some means to minimize the thermal impact to users from the waste heat. Since the fuel is consumed in a flame, there are hot gases generated which must be exhausted externally. The product user must be protected from casual contact with these hot exhaust products.

- Develop a power conditioner to match the load characteristics to the TPV output. Most consumer electronic products require power in rapidly changing levels. The TPV device, however, would be most efficient operating at a steady power output.

Accomplishments

As this LDRD was funded about mid-year, the work began with only six months remaining in the fiscal year. A proposed design was carefully evaluated, and calculations performed based on information from the rare-earth emitter developer. The efficiency and sensitivity of the latest, high-performance PV cells were included in these calculations. The calculations show that the maximum efficiency attainable in the near future would be 9 to $10 \%$. This efficiency is the minimum necessary to effectively compete with chemical batteries in their typical applications. Additionally, the waste heat issue was problematic. With $90 \%$ of the energy rejected as heat, this power supply would be unsuitable for use in a hand-held device. With this information, the project was directed to stop development work after about two months. Eighty percent of the funds were returned. The project was, however, directed to develop a performance requirements summary document to outline, in basic terms, the parameters of interest in developing a power supply for portable consumer electronic devices, particularly cellular telephones. This document was generated and distributed as letter CRB-08-95.

\section{New or Increased Technical Capabilities}

This project has added to the INEL's knowledge of rare-earth materials and photovoltaic cell chemistry. This knowledge may prove useful in future research activities.

\section{Optimization Based SIMPLEV \\ LDRD 4468}

M. D. Landon, G. H. Cole

This project worked toward development of SIMPLEV version 4.0 by creating and implementing software tools to be able to find optimal designs for electric/hybrid vehicles. Optimization is a mathematical science that can be applied to engineering design to automatically modify existing designs to maximize performance. The software design includes the following features: run on a PC (with the $C / C++$ language), a windows-based graphical user interface, a database, graphics for visualization of results and report creation, and optimization algorithms and methodologies for optimal design search. The software package was not completed under this LDRD; however, the database was completed, most of the graphical user interface was completed, and the optimization design variables (i.e., parameters to be changed as the optimization algorithms search for a better design) and the criteria (i.e., the objective and constraints) were defined. Non-LDRD follow-on funding (carry-over to FY-96) was obtained to complete SIMPLEV 4.0.

\section{Objectives}

- Build a complete database for SIMPLEV 4.0. This database includes vehicle definitions (weight, drag, etc.), driveline components (motors, inverters/controllers, transmissions, batteries, engines, etc.), driving cycles, and road conditions (grade, wind speed and direction).

- Develop a graphical user interface. Powerbuilder, a PC/windows-based graphical user interface developer, was used to develop the $a$ windows, mouse-driven, point-and-click operation of (a) database population and modification, (b) vehicle type selection, (c) vehicle component definition, (d) driving cycle definition, (e) road condition definition, (f) links to the simulation calculations, (g) etc.

- Define optimization parameters. The design variables were defined (i.e., the parameters that the user/optimizer changes to achieve an improved vehicle design). The design functions were defined (i.e. , the criteria to define possible objective and constraint functions).

\section{Technical Accomplishments}

SIMPLEV 3.0 is a general purpose simulation program for performing parametric studies of electric and hybrid vehicles. It was written in the BASIC language and is being used by over 100 sites in the country by engineers who design electric/hybrid vehicles. Improvements, enhancements, and additional capabilities are needed to keep SIMPLEV as the lead code of choice. The accomplishments achieved under this mid-year project go a long way to reach the goal of keeping SIMPLEV in its leading role.

After the user has completed the definition of the vehicle components and the driving cycles, simulation results are ready to be computed. The necessary code that 
calculates the physics of the electric/hybrid simulation is partly complete. This work is under way and is being completed with followon funding. Graphing of results and report creation capabilities are also being developed. All of these capabilities are being developed to be compatible with the windows environment.

Once the simulation calculation is available, it will supply the necessary results based on the input vehicle definitions to be able to define the criteria for electric/ hybrid vehicle optimization. A result of this project was the definition of the optimization design variables and objective function and constraints.

The variables that will be available to perform a design optimization are vehicle weight, drag, frontal area, tire resistance, tire radius, wheel bearing torque drag, energy regeneration, transmission gear ratios and gear efficiencies, transmission output torque, motor speed, torque, efficiency, inverter efficiency, min voltage, max current, battery size, weight, number of cells, open circuit voltage and internal resistance versus depth of discharge, driving cycle, road grade, wind speed, wind direction, etc.

The resulting functions from the simulation that will be available for defining the objective function (the function that we want to minimize or maximize) and constraints (the functions that need to have limits imposed on them) are range of travel, total energy consumed, total vehicle weight, predicted pollutants, etc.

Several of the important optimization problems that will be able to be posed and solved are as follows: Find

vehicle variables

battery variables

motor variables

inverter variables

transmission variables
To

Or

maximize range of travel

minimize energy consumption

Subject to

constraints on total vehicle weight.

\section{New or Increased Technical Capability}

The capability to model electric/hybrid vehicle performance with a windows-based graphical user interface contributes to maintaining the INEL's position as the technical expertise leader in electric/hybrid vehicle simulation. The near-term completion of the total SIMPLEV version 4.0 (complete with optimization methodologies) will help position the INEL to remain a leader in electric/hybrid vehicle simulation and design.

\section{Business Development Opportunities}

This work helped procure follow-on funding from the Department of Energy to continue the development of the code. Approximately $\$ 128 \mathrm{~K}$ is available to complete SIMPLEV 4.0 and to continue incorporating design optimization capabilities. There are already over 100 licensed customers using SIMPLEV 3.0, who will be customers of version 4.0 . SIMPLEV 3.0 licenses sell for $\$ 795$. SIMPLEV 4.0 licenses will sell for $\$ 1500$. System engineers at NASALewis working with the PNGV consortium (Ford, General Motors and Chrysler) have agreed to use SIMPLEV to formulate electric/ hybrid vehicle system designs by the systems analysis team at NASA-Lewis and PNGV. user interface contributes to maintaining the INEL's position as the technical expertise leader in electric/hybrid vehicle simulation. The near-term completion of the total SIMPLEV version 4.0 (complete with optimization methodologies) will help position the
INEL to remain a leader in electric/hybrid vehicle simulation and design.

\section{Business Development Opportunities}

This work helped procure follow-on funding from the Department of Energy to continue the development of the code. Approximately $\$ 128 \mathrm{~K}$ is available to complete SIMPLEV 4.0 and to continue incorporating design optimization capabilities. There are already over 100 licensed customers using SIMPLEV 3.0, who will be customers of version 4.0 . SIMPLEV 3.0 licenses sell for $\$ 795$. SIMPLEV 4.0 licenses will sell for $\$ 1500$. System engineers at NASALewis working with the PNGV consortium (Ford, General Motors and Chrysler) have agreed to use SIMPLEV to formulate electric/ hybrid vehicle system designs by the systems analysis team at NASA-Lewis and PNGV.

\section{Hybrid Vehicle Component Hardware-in-the-Loop Feasibility Study} LDRD 4469

$$
\text { G. L. Hunt }
$$

This project was intended to establish the technical feasibility of using the INEL Energy Storage Test Laboratory high power battery testers as simulated energy storage devices. This would make it possible to accelerate the testing of subsystems and interface components for hybrid energy storage systems in cases where full size devices are not yet available. Specifically this project aimed to use an Energy Systems programmable battery tester, controlled by an external PC-based simulation program, to simulate a 150 volt ultracapacitor bank. A large resistor bank was used as the load for the simulated device. An actual capacitor bank was also discharged into this same load for comparison purposes. The test results 
presented suggest that the tester response is fast enough to make such a simulation useful, although software glitches were discovered in the tester which must be corrected prior to any practical use.

\section{Objectives}

- Develop a test plan and build the resistive load bank for the intended testing.

- Construct a PC-based realtime simulation of a capacitor to control the battery tester, and develop a battery tester program to vary its outputs in response to this control input.

- Discharge a Panasonic ultracapacitor bank into the load bank for baseline data.

- Discharge the simulated capacitor (PC controller-battery tester combination) into the load bank and compare the results to the actual capacitor discharge.

- Determine the transient response limits of the simulated capacitor.

\section{Accomplishments}

A load bank having a resistance of about $3.6 \Omega$ was used for all testing in this project; this provides a load of about 6,200 watts at 150 volts. The voltage decay curve into this load for an actual capacitor bank with a value of about 30 farads was measured. A capacitor model was then constructed using a real time version of ACSL (Advanced Continuous Simulation Language). This model was intended to measure the current and voltage across the load bank and generate a control signal to the tester to vary its output to match the capacitor characteristics. The simulated output from ACSL using this model (based on a lower resistance load, gives a shorter decay time but does not affect the shape of the curve).

Prior to coupling the PC controller to the Energy Systems tester, a series of response tests were run using a function generator to provide the control input signal. The Energy Systems tester outputs cannot be directly commanded in programmed mode; it is necessary to run the tester under control of a program which jumps to one of several (fixed) output steps based on an input voltage. This provides coarse output resolution but was the quickest way to evaluate the tester output behavior. The figure shows the resulting response to an updown ramp input, indicating both some output instability and a significant error in the achieved voltage. Viewing the output disclosed that this behavior was due to the tester output being with an oscilloscope reset to zero at the beginning of each internal programmed step for almost $40 \mathrm{~ms}$. This meant that the average output voltage was significantly less than requested because program steps were only $100 \mathrm{~ms}$ long as a result of this

(Program Simple6.tpf)

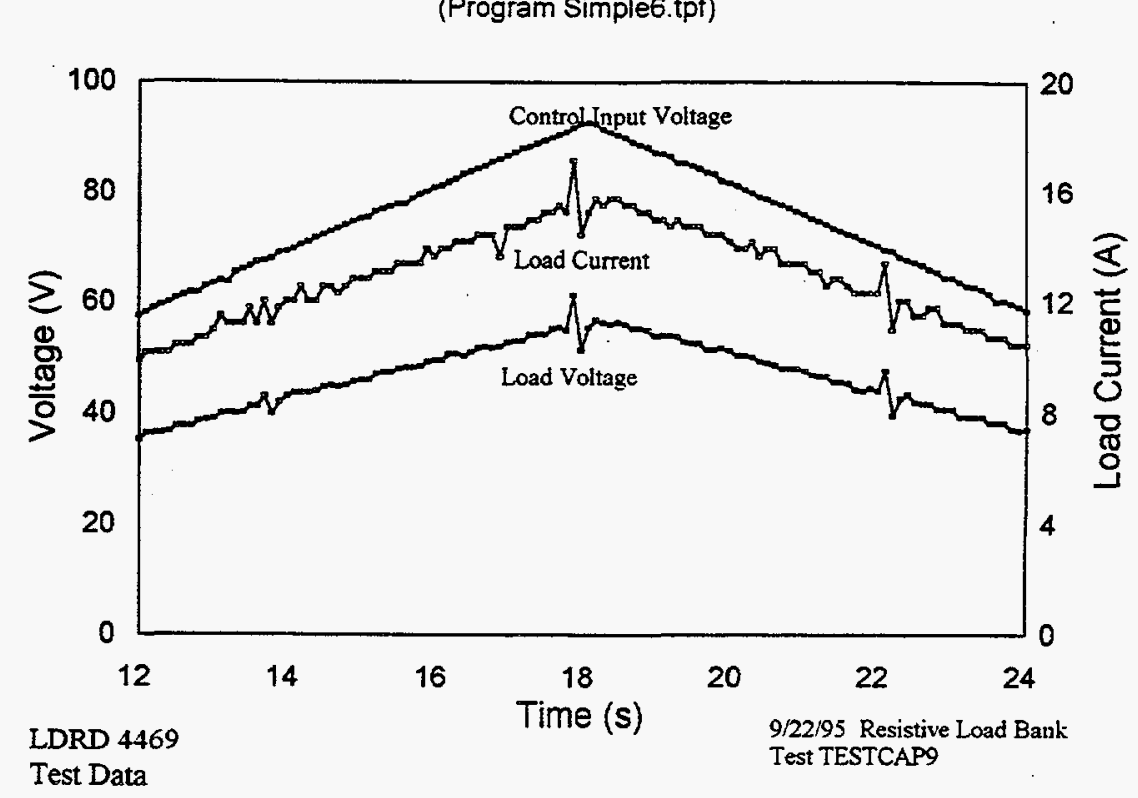

Energy Systems Tester Response to Up-Down Ramp Control Input programming approach. This anomaly has not been observed during previous use of the tester because its duration is too short to be seen by the data system, which samples synchronously with respect to the start of each program step. (Program steps are normally multiple seconds in length.)

The tester designer consequently confirmed that these outcorrective action is being planned. The PC controller has not yet been coupled to the tester because of this behavior. However, the oscilloscope observations indicate that the tester hardware removes and restores its output current across this particular load in less than $5 \mathrm{~ms}$, which is much faster than expected. Follow-on LDRD funding has been requested to continue demonstration of this capability, which will be done using the 'slave' mode of the Energy Systems tester. This mode, which has never been exercised in the laboratory, permits direct control of the tester outputs. This should provide significantly faster response than a stored program is capable put glitches are unintentional, and 
of, although the external controller has to assume responsibility for keeping the tester within its operating limits to avoid damage.

New or Increased Technical Capabilities

The goal of this very small project was to provide a minimal demonstration of the capability to use a high power battery tester as a simulated energy storage device under control of an external simulation program. Results of this effort support the contention that this is in fact feasible, although additional work and some minor tester modifications are required for a practical realization. A more detailed characterization of the dynamic characteristics of this software/hardware simulation is needed to establish that it can function over a wide range of outputs using nonresistive loads.

Business Development Opportunities

Establishment of this capability, which is believed to be unique at this time, is expected to lead to opportunities for testing large hybrid energy storage devices and subsystems for the U.S. auto companies and the Department of Energy.

\section{Software Metrics Program LDRD 4470}

K. D. Welker, C. S. Miller. R. H. Raymond, V. A. Erickson, K. R. Johnson, B. C. Hong, M. W. Snyder

Understanding INEL software development processes is essential to developing quality software. The Software Engineering Institute (SEI) has recognized the value of establishing a software metrics program. They have recommended software metrics as a key aspect of software processes capable of producing truly outstanding software. The basis behind this recommendation is the understanding that measurement is the cornerstone of process maturation. Currently, there is no unified, company-wide, INEL software metrics program. Many INEL software projects are deficient in collecting important metrics. Before a program can be established, some preliminary work must be performed. This LDRD evaluated the feasibility of applying software metrics technologies at the INEL.

\section{Objectives}

In an effort to help establish the foundation for a software metrics program at the INEL, this LDRD project investigated the feasibility of using software metrics as part of software process improvement initiatives for several pilot projects. The investigation for each pilot included:

- Analysis of the software project, products, and processes from a software engineering perspective, making an assessment as to the current state of the project, and providing recommendations for improving software quality.

- Established the feasibility of using software metrics to improve INEL software quality and improve software development processes.

- Developed a metrics implementation recommendation which can be followed over the software lifetime.

\section{Accomplishments}

The results of this research fall into two areas: first, the formal analysis of six pilot projects and the mini-analysis of twelve other software systems; second, the development and investigation into software tools which support software metrics and process improvement.
The engineering analysis focused on understanding and documenting the following aspects for each of the pilot projects:

- Business Goals

- Structural Integrity

- Functional Overview

- Database Assessment

- Operating Environment

- Software Maintenance

- Software Development Process

- Run-Time Performance

- Software Lifecycle

- User Interface

- Software Development Methodology

- Configuration Management

- Software Change Process

- Software Quality Assurance

- Documentation Suite

- Software Metrics Application

- Software Architecture

- Reengineering Assessment.

Pilot projects assessed include: Electronic Combat System Integration (ECSI), Reactor Loop Analysis program (RELAP), Occupational Medical Program (OMP), Radiological Waste Management Complex Portable Container Inspection System (RPCIS), Stored Waste Examination Pilot Plant Assay System (SAS), and Beryllium Reflector Lifetime Model (BRLM).

Some common threads derived from this effort include the following:

- All projects were collecting some meaningful metrics already and had performed some level of self-assessment.

- All projects investigated were at SEI maturity level one. A common deficiency was the lack of appropriate organizational infrastructure and standard processes.

- The use of a Maintainability Index metric found appropriate application across a large 
number of pilot projects in assessing not only maintainability, but also architectural degradation over time as a result of software change. Several project software change processes were modified as a result of analysis.

- Function point metrics have seen the most success in areas where they can be automatically extracted from the software system. Projects using function points are also able to measure productivity.

- General software engineering knowledge (technologies, processes, theories, and jargon) across pilot projects was fairly consistent, and was slightly above national averages.

- Defect/change tracking, software quality assurance and formal testing are general weaknesses. Projects cannot yet quantify how much system maintenance is costing them.

- Most projects would benefit from some level of reengineering.

In addition to the specific project assessment results, software metrics tools were evaluated and a proof-of-principle prototype for a three perspective maintenance measurement tool was constructed. Techniques for using the tools were investigated and applied.

New or Increased Technical Capabilities

As a result of this effort, four important capabilities have been strengthened:

- The pilot projects have established a foundation for performing meaningful project measurement. They have learned new techniques, which will improve software quality.
- The organizations that sponsor/support the pilot projects have important information that can help strengthen meaningful process improvement efforts.

- Metrics awareness, knowledge, and capabilities at the INEL have been advanced. This provides a metrics technology base that can be marketed beyond the INEL.

- A metrics assessment product has sufficient groundwork so as to be marketable.

New or Increased Technical Capabilities

The primary business development opportunity that emerged as a result of this effort was with SET Laboratories. SET Laboratories produces a family of metrics products. Ideas from this project have been incorporated into the SET Laboratories product baseline.

\section{Manufacturing Research LDRD 4479}

$$
\text { H. B. Smartt }
$$

This project is to leverage the existing INEL manufacturing research position into a rapid manufacturing program with associated technical capabilities to support the DOE need for a strong U.S. manufacturing industry, and to support the INEL Manufacturing Outreach Program to strengthen the regional economy. Critical missing elements of a general rapid manufacturing technology are to be acquired, while building a coordinated INEL-university-industry product development team. Task leaders have worked with university counterparts to coordinate proposals for the INEL University Research Consortium (including industrial participants) and to bring students and faculty to the INEL via the AWU program. The project would be the basis for a model of product development at the INEL.

\section{Objectives}

- Form a multidisciplinary technical team to provide the basis for future growth of INEL manufacturing $R \& D$ activities.

- Define an appropriate focus for the INEL manufacturing program consistent with industrial market needs and INEL technological capabilities.

- Develop a plan for acquiring funding for continuing the work and transfering the technology to suitable users and vendors.

- Develop low cost, PC-based soft tools for agile/rapid manufacturing.

- Develop processes to support manufacture of functional gradient materials-based products.

- Establish a framework for agile/rapid manufacturing to support INEL product development activities.

\section{Accomplishments}

This activity started mid-year. The initial activity was to formulate a hypothetical architecture for the materials processing and information management structure of a generic rapid manufacturing capability. During FY-95, significant portions of the basic modules in that architecture were identified, and major parts were either acquired or developed.

The Figure shows the information flow and associated processing needed for manufacture of low-cost, rapid tooling. A team of INEL scientists and engineers were assembled to form the core of an integrated product team to support this work. Contacts were also made with several universi- 


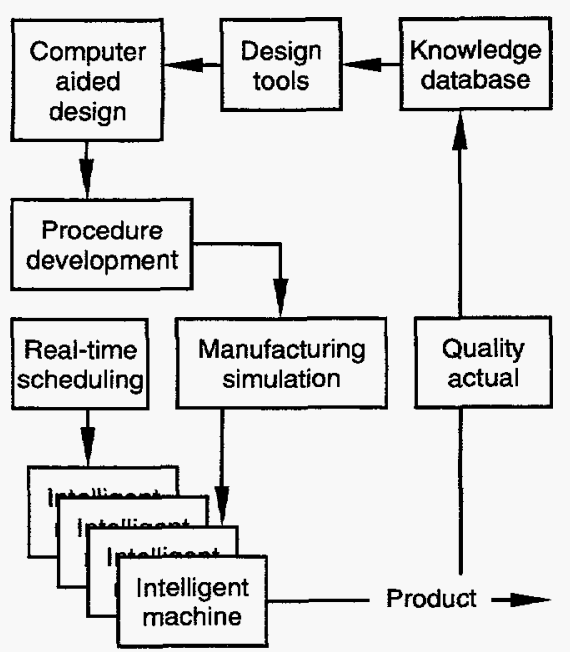

E96 0069

Rapid manufacturing system (With respect to this proposal, the product shown would be a specific piece of tooling.)

ties, and two INEL University Research Consortium proposals were funded for FY-95 supporting the needs of the work: Advanced Welding Control Technology (G22), Professor Kevin Moore, Idaho State University, and Plasma Processing of Functionally Gradient Materials (G05), Professor Herbert Herman, State University of New York at Stony Brook. Two additional university teams were invited to submit related proposals for potential FY-96 URC funding: An Adaptive, Real-Time Scheduling Tool for Small-Scale Manufacturing, Professor Steven Smith, Carnegie Mellon University, and Genetic Algorithms Applicable to Welding Cell Design and Specification, Professor Susan Carlson, University of Virginia.

Contacts were also made with Lockheed Martin Aeronautical Systems personnel, and with several additional companies that expressed interest in becoming involved with the work through CRADA or other arrangements.

New or Increased Technical Capability

During FY-95, a graphical user interface was developed for an existing robot cell based on a CRS open architecture, seven-axis, articulated robot that uses either textual or graphical off-line programming tools, which eliminates the necessity of writing conventional computer code for the robot. Work also started on a video sensing system for object identification for the cell and hooks necessary for integrating a voice recognition tool into the graphical user interface to make programming even simpler.

The team developed two process simulation tools. One is a robot motion visualization tool, which is still under development. The other is a welding procedure development tool that imports product geometry from a CAD file.

We also made several critical decisions about the information management infrastructure.

Based on industry feedback, we began developing technology for IBM-PC (or clone) platforms with Windows or Windows NT operating systems, using LabView for processing related software development. We developed an Oracle relational database, for storage of welding data, incorporating a Hyper Text Markup Language (HTML) Mosaic-like graphic user interface, TCP-IP network software, and ethernet hardware.

\section{Business Development Opportunities}

Manufacturing industries are moving toward a body of technologies referred to as rapid manufacturing. A roadmap for rapid manufacturing leads one from agile/flexible manufacturing through rapid prototyping to virtual prototyping to rapid manufacturing. However, most industries depend extensively on tooling for a large variety of unit operations in discrete product manufacturing. (Discrete products include such high-value items as automobiles and aircraft, both critical to the U.S. economy.) Lead times for tooling range from weeks to months to years, depending on the complexity and size of the product. It is typical in the aerospace and automobile industries for large tools to require well over a year for production. The solutions to the time problem are either to develop toolingless manufacturing processes, or to develop the methodologies to manufacture tooling rapidly. Ordinarily, either solution would be daunting. However, this past year during meetings between Lockheed Martin Aeronautical Systems Company (LMAS) personnel and Lockheed Idaho personnel, it was apparent that existing INEL capabilities could be combined with a unique tooling design concept being developed by LMAS to allow timely development of a rapid tooling manufacturing process years ahead of the roadmap.

Opportunities exist to establish CRADA relationships with Adept Technology, Inc., San Jose, California (a major U.S. manufacturer of open architecture robots) and CRS, Brantford Ontario, Canada (a major Canadian manufacturer of open architecture robots). Team members had worked previously with Adept as part of the Navy Programmable Automated Welding Systems project. We should pursue the CRS relationship through the Lockheed Martin offset program. We have been acting as an alpha software test site for CRS for several years and have a good working relationship with them.

Professor David Hardt, Codirector of the Leaders for Manufacturing Program at MTT, worked with us informally, giving advise on positioning of the technologies for maximum potential market penetration. Professor Hardt has 
been working on flexible tooling and toolingless manufacturing projects for several years. He and Jim Connell at Thermo Technology Ventures, Inc., (TTV) are working on obtaining technology transfer funds (passed from Lockheed Idaho through TTV to MIT for such activities) for Professor Hardt to continue to work with us.

We understand that Sandia National Laboratories has a membership in NCMS (National Center for Manufacturing Sciences, Ann Arbor, Michigan). Through that membership (which should extend to other Lockheed Martin companies), we should seek membership on appropriate NCMS committees. This will provide considerable insight into national activities in rapid manufacturing and will lead to additional opportunities for industrial interactions, as well as potential funds-in projects.

Gail Cordes visited various Lockheed Martin facilities as part of her duties in the Sensor Products Directorate. She provided the team with identification of several relevant contacts, products, and activities in various Lockheed Martin companies that present business development opportunities.

\section{Building Materials LDRD 4480 \\ L. L. Wiedert}

This project is researching the use of agricultural crop residues for the fabrication of building materials and looking at agriculturally derived adhesives to fabricate such materials. The project was to determine the feasibility of converting agricultural crop residues into a useful, economical, marketable resource. The effort started by completing a literature search and interacting with knowledgeable companies, universities, and individual persons. Sample pan- els were produced and laboratory testing was performed based on established ANSI standards. University partners were found and contracted the research and testing of new and innovative agriculturally derived adhesives to bind agricultural crop residues into building materials suitable for commercial markets.

\section{Objectives}

- Complete a literature search to determine the current status of the use of agricultural crop residues in the fabrication of building materials, specifically particleboard. Also, research the feasibility of using an agricultural derived adhesive to fabricate this particleboard.

- Construct particleboard samples using an agricultural crop residue and compare the results with ANSI standards as they pertain to the wood particleboard industry. Show how this product could have commercial application.

- Develop a University partnership to evaluate the use of agricultural derived adhesives in the fabrication of straw particleboard.

\section{Accomplishments}

A literature search was performed in March 1995 to determine the feasibility and current status of using agricultural crop residues in the fabrication of building materials. The research indicated that an interest in this area has existed for over thirty years and that fabrication of building materials has been attempted. The adhesive that appears to have the most promising properties for binding agricultural residues is diphenylmethane diisocynate (MDI), which is derived from naphtha. The search also lead to using soy and animal blood as adhesives that could potentially be used and are derived from agriculture.

As a result of previous research, Lockheed Idaho engineers had the capability to produce commercial grade particleboard fabricated from straw. An independent consultant heard of these capabilities, visited the INEL and expressed interest in particleboard fabricated from bagasse (sugar cane residue). The literature search indicated that bagasse was an agricultural residue that had promise for particleboard fabrication, and also had tremendous global implications. Based on this interest, and to demonstrate the feasibility of using agricultural residues, sample trials were established at Washington State University. The target for these trials was to produce high quality industrial particleboard made from bagasse and test according to ANSI wood particleboard standards.

After drying and hammermilling the bagasse, it was segregated into course and fine fibers. The processed fibers were blended with MDI with the intent of producing a strong, water-resistant, formaldehyde-free panel. Accordingly, two separate trials were designed to evaluate panels with MDI dosages of 5.5 and $8 \%$ by weight. Blending of premeasured amounts of processed fiber and MDI was achieved in a rotary drum via an atomized spray. Course and fine fibers were blended separately. The blended fibers were formed by hand in a box in such a way as to distribute the fibers evenly with a $25 \%$ fine/ $50 \%$ course $/ 25 \%$ fine layer.

Homogenous samples were also formed without layering. The size of the resulting mat was 16 by 20 inches with a target thickness of $3 / 4$ of an inch. The target density of all samples was 45 pounds per cubic foot.

The mat was then inserted into a computer-controlled press. 
The predetermined program involved parameters controlling time, temperature, pressure, and the staging of certain pressure steps. Six separate samples were produced.

Finished samples were analyzed and the results were compared against ANSI standard A208.1-1993 criteria. Several particleboard grades are contained in this standard. These various grades are required to meet various strength and performance criteria. For comparison to the bagasse trials, we compared internal bond, modulus of rupture, and modulus of elasticity. Table 1 below shows which existing particleboard grades were achieved by the samples, marked by a $\sqrt{ }$. A grade marked with a $\ddagger$ indicates when the samples were within $15 \%$ of specific properties.

University partners were identified at the University of Georgia, (Dr. Chia-Ming Chen), and at Iowa State University, (Dr. Deland Myers). Dr. Myers was investigating the feasibility of using soy and animal blood as potential adhesives in binding straw to produce particleboard. Dr. Chia-Ming Chen has developed agriculturally based adhesives using peanut husks and also pecan shells and has offered his time, laboratory, and chemicals to evaluate the feasibility of producing straw particleboard using this technology. Information should be available in early FY-96 as to their success.

New or Increased Technical Capability

Observation of sugar mill operators in India, using bagasse as a fuel to run the process, indi- cated that if a reverse osmosis process could be used for sugar extraction, fuel requirements could be reduced by $\approx 80 \%$. The INEL has the knowledge to design and fabricate the reverse osmosis system, thereby allowing sugar mills to have an excess supply of bagasse, instead of using most of it for fuel. Using this technology, coupled with the demonstrated capabilities of bagasse particleboard, the excess bagasse could be used to fabricate particleboard in a facility located next to the sugar mills. This concept has tremendous potential for global application in sugar producing countries.

Business Development Opportunities

As a result of this research, a letter is to be issued to potential interested parties such as the sugar growers associations both in the United States and abroad. There is tremendous opportunity to use INEL assets to redirect the sugar industry into a more efficient operation, with the opportunity to expand their business base and bring addition outside work to the INEL.

\section{Smart HVAC Systems Design LDRD 4481}

\section{R. I. Antill, E. P. Keating, \\ R. D. MacDowall, K. A. Church-Riddle}

This abstract summarizes the results of experiments in measuring, tracking, and modifying building air quality. The project ran from March

Table 1

\begin{tabular}{cccccccccc} 
Sample & $M-1$ & $M-2$ & $M-3$ & $M-S$ & LD-1 & LD-2 & PBU & D-2 & D-3 \\
\hline & & & & & & & & & \\
$1-A$ & $\sqrt{ }$ & $\ddagger$ & & $\sqrt{ }$ & $\sqrt{ }$ & $\sqrt{ }$ & $\sqrt{ }$ & \\
2-A & $\sqrt{ }$ & & & $\sqrt{ }$ & $\sqrt{ }$ & $\sqrt{ }$ & $\sqrt{ }$ & \\
$1-B$ & & & & & $\sqrt{ }$ & $\sqrt{ }$ & & \\
$1-C$ & $\sqrt{ }$ & & & $\ddagger$ & $\sqrt{ }$ & $\sqrt{ }$ & $\sqrt{ }$ & \\
$2-C$ & $\sqrt{ }$ & & & $\ddagger$ & $\sqrt{ }$ & $\sqrt{ }$ & $\sqrt{ }$ & \\
$1-D$ & $\sqrt{ }$ & $\sqrt{ }$ & $\ddagger$ & $\sqrt{ }$ & $\sqrt{ }$ & $\sqrt{ }$ & $\sqrt{ }$ & $\ddagger$
\end{tabular}

through September 1995. Research focused on the capability of an integrated sensor and microprocessor control system to monitor and control building air quality. Simply, the control system would monitor the contaminant level within a facility and adjust the amount of outside air brought into the supply air for the facility to offset the generated contaminates and ensure indoor air quality $(I A Q)$ is maintained at acceptable levels. Buildings generate and accumulate contaminants from many sources within. Typically, the most significant contributor in office buildings are the building's own occupants, who generate carbon dioxide $\left(\mathrm{CO}_{2}\right)$, humidity, odors, etc. Off-gas from carpets and furniture, especially formaldehyde, play a significant role since this offgas is a major contributor to occupant discomfort and sickness. Other contaminant sources include copiers, paint, plastic, heating, ventilation, and air conditioning (HVAC) equipment, and printers.

At present, the basic standard that designers and building owners use to ensure IAQ is ASHRAE 62-89. The methods and procedures for designing and determining acceptable IAQ are addressed within this standard. A principal focus of ASHRAE 62-89 is the amount of outside air to be introduced within a building to dilute the recirculated air to ensure adequate air quality. Determination of this amount is specifically addressed where two separate methods are outlined: Ventilation Rate Procedure (simple and most used), and the Indoor Air Quality Procedure. The Ventilation Rate prescribes 20 cubic feet per minute (cfm) of outside air per occupant is brought into the facility. The value is based on the $\mathrm{CO}_{2}$ level which is used as a general indicator of IAQ, encompassing body odors, clothing contribution, respired contaminants, etc.

As a HVAC mechanical engineer/designer will be quick to point out, the amount of wasted energy that is consumed by an HVAC system in conditioning the specified large amounts of outside air is very high. Additionally, heating and cooling equipment sizes increase dramatically to handle this high load. 
To lower energy costs and ensure building IAQ in compliance with ASHRAE 62-89, an integrated sensor and microprocessor control system was assembled to test the feasibility of tracking and modifying the building air quality. Equipment was obtained from commercial suppliers.

Initially, the sensor array underwent stringent gas testing in the field to determine, verify, and record the detecting capabilities of the sensor array, in particular, the mixed gas (MG) sensor for various gases and volatile organic compounds (VOC). Calibration gases were used for this testing. These data was used to corroborate and establish the baseline capabilities of the sensor array.

Results of the tests indicate that a single sensor could not be used to monitor the building IAQ. This result ran against industry's argument for using either a carbon dioxide or MG sensor as a sole determinant of the IAQ. Obviously, the dedicated $\mathrm{CO}_{2}$ sensor could not pick up other contaminants such as formaldehyde and other VOCs that are a problem. However, the MG sensor did not indicate a sensitivity to $\mathrm{CO}_{2}$. The lack of adequate sensitivity of the $M G$ sensor to $\mathrm{CO}_{2}$ means an inadequate representation of the building air quality.

\section{Objectives}

- Design and install an IAQ sensor array with microcontroller and data collection system in an existing HVAC system and monitor/track the air quality.

- Determine the adequacy of the sensor array and ability of system to modulate the outside/ return air dampers.

\section{Accomplishments}

The mechanical room at TAN602 of the INEL was chosen as the location for the experiment. The equipment included sensors as well as a microcontroller and data collection system. Along with a temperature probe, the following sensors were installed: sulfur dioxide $\left(\mathrm{SO}_{2}\right)$, carbon monoxide (CO), $\mathrm{CO}_{2}$, nitrogen dioxide $\left(\mathrm{NO}_{2}\right)$, and a mixed gas sensor in accordance with ASHRAE 62-89. The $\mathrm{MG}$ and $\mathrm{CO}_{2}$ sensors were mounted in the discharge of the return air fan. For the other sensors, plastic tubes were routed from the outlet of the sensor to the suction side of the return air fan. For the other sensors, plastic tubes were routed from the inlet of the sensors to the discharge side of the fan. Since the building dampers were arranged for recirculation, the sensors could pick up the air with added contaminants.

Damper actuators were also provided although they were not be used.

Signals from the various sensors were routed to the microcontroller and data collection system within the mechanical room for data storage and processing. The micro controller that was used for this experiment was a Octagon systems $2300 / \mathrm{H}$. The Octagon systems $2300 / \mathrm{H}$ uses a 64180 CPU, with a 12-bit A/D conversion. Programming was done in CAMBASIC II software, a basic programming language that has been modified for data acquisition and control applications. The algorithm was developed to store the data and process the signals from the sensors. The algorithm converted the 4-20 ma signal into a ppm value and compared this value with a set value in a go/no go manner.

Based on the data acquired, the mixed gas sensor does not apparently track the $\mathrm{CO} 2$ level of a building. Furthermore, gases

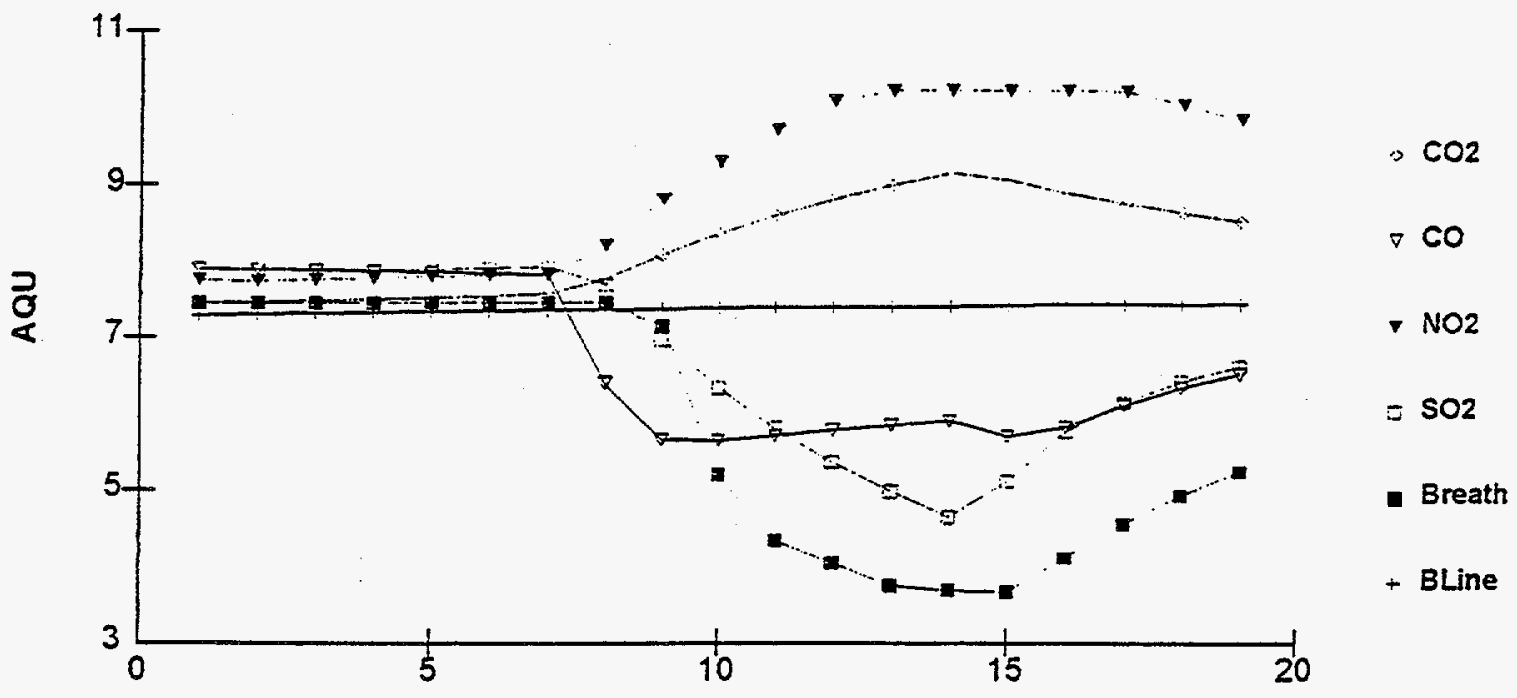

Time, each series $3 \mathrm{~min}$. time period, 9.5 seconds

Response of Mixed Gas Sensor to $\mathrm{CO}_{2}, \mathrm{CO}, \mathrm{NO}_{2}, \mathrm{SO}_{2}$, and Human Breath 
such as $\mathrm{NO}_{2}$ had little or opposing affects on the MG sensor. Per the figure, the response of the MG sensor to $\mathrm{CO}_{2}$ and $\mathrm{NO}_{2}$ showed an improvement in the quality of air, not a decrease. The concentration of $\mathrm{NO}_{2}$ was $5 \mathrm{ppm}$. For the $\mathrm{CO}_{2}$, the major component was air and a concentration of $\mathrm{CO}_{2}$ at 1000 ppm or $0.1 \%$, with approximately $2 \%$ minor components. The gas CO was $50 \mathrm{ppm}$ and used air with $5 \%$ minor components. The only gas that did not use air as its major component was $\mathrm{SO}_{2}$. Nitrogen (N2) as the major component with $10 \%$ minor components. If one examines the time period from 0 to 24 , there is no relationship between the output of the MG sensor and the $\mathrm{CO} 2$ sensor. From the 24th to the 32th time period, the two sensors seem to track each other, but not as they should. There seems to be no relationship, or it is opposite of what would be expected if the MG sensor could be used to track the $\mathrm{CO}_{2}$ level. The response of the MG sensor to $\mathrm{CO}_{2}$ indicates an increase in air quality as the level of $\mathrm{CO}_{2}$ increases.

The strength of the MG sensor is how it can respond to many gases. This is what makes it useful in determining the measurement of such gases as $\mathrm{CO}$ or formaldehyde. The MG sensor cannot be used to determine the level of $\mathrm{CO}_{2}$. In addition, since there are selected gases that can cause the MG sensor to increase its output reflecting better air quality, the mixed gas sensor cannot be used in isolation.

\section{New or Increased Technical Capability}

While industry tends toward simple and inexpensive solutions that can be accepted at face value, results of the tests indicate that a minimum of BOTH the $\mathrm{CO}_{2}$ and the MG sensor would be required to establish adequate tracking of IAQ. What this means for the
INEL is that our research tends toward a new direction in the IAQ knowledge bank that could develop toward establishing the INEL as a recognized research leader in IAQ. With no vested interest in any specific product, our research should be recognized as authoritative and substantial. Further sensor testing in conjunction with comparisons with a gas chromatography with mass spectrometry (GCMS) could establish a new baseline that other industries could not ignore.

As a direct business benefit to the INEL, a system using all of the above mentioned sensors would be developed along with a very cost effective and practical technique to track and modify IAQ within building spaces, which is the prime pursuit in industry at the moment. Our technique is designed.

\section{Business Development Opportunities}

There were no collaborative relationships with internal/external business units. While interest was expressed in experimental data and results in and from external business units, there were no possible relationships to build since the relationship was competitive.

\section{High-Temperature Porous Cermet Filters for the Paper and Pulp Industry LDRD 4482}

\section{P. C. Kong, P. A. Lessing}

This project develops catalytic high-temperature cermet filters for applications in particle filtration and $N O_{x}$ destruction for the pulp and paper industry. These filters remove corrosive fly ash, which currently fouls the pulp and paper industry black liquor recovery furnaces. Fouling of recovery boiler components during black liquor combustion is a significant problem for the paper industry. This project also tests the capability of these specific porous materials for catalytic decomposition of $\mathrm{NO}_{x}$ and $\mathrm{SO}_{x}$ generated in the black liquor recovery boilers. Successful development of these materials will likely solve these problems for the pulp and paper industry.

The catalytic porous filters are made of intermetallic aluminides and ceramic oxides into a net-shaped bulk structure by a self-propagating combustion synthesis (SHS) process using mixed powders. The cost of producing these cermet filters is very low.

These types of intermetallic cermet filters have interesting material properties. Some of the specific properties include high melting temperatures $\left(>1500^{\circ} \mathrm{C}\right)$; good thermal shock resistance (fracture toughness $\geq 22 \mathrm{mPa} . \mathrm{m}^{1 / 2}$ ); oxidation resistant to at least $1000^{\circ} \mathrm{C}$; catalytic-to-steam reforming and partial oxidation of hydrocarbons to syn-gas; may be sulfur resistant; controlled porosity; can be brazed to existing structural configuration during SHS; material compositions can be tailored to be catalytic to $\mathrm{NO}_{x}$ and $\mathrm{SO}_{x}$ decomposition; electrically conducting (internal heating capability); light weight materials, mechanically rather strong 17.5 to $23.2 \mathrm{kpsi}$; high heat capacities; and thermal expansion coefficient controllable. With these material properties, several potential applications for the cermet filters have been identified.

\section{Objectives}

- Develop a combustion synthesis process for near net shaped high temperature catalytic cermet filters.

- Test cermet compositions for catalytic $\mathrm{NO}_{x}$ destruction.

- Test cermet composition for catalytic steam reforming of hydrocarbons.

- Determine fine particle removal efficiency from the gas stream for the cermet filters. 


\section{Accomplishments}

The first three objectives have been completed. The last is determining the particle filtration efficiency.

The catalytic cermet filters are made of intermetallic aluminides and ceramic oxides into a netshaped bulk structure by a selfpropagating combustion synthesis process using mixed powders. The cost of producing these cermet filters is very low. Powders used included $\mathrm{Al}$, transition metals, and ceramic oxides. For example, $\mathrm{Al}$ and $\mathrm{Ni}$ powders in 1:1 mole ratio are mixed with an oxide powder ( $40 \mathrm{wt} \%$ ) to form a green disc compact. The disc compact was than fired in a furnace at about $800^{\circ} \mathrm{C}$ to initiate the combustion synthesis process, which continued until the entire powder compact was reacted and would retain the net shape of the green compact. The final product is a porous structure with ceramic powders dispersed in an open network of metal matrix. The asformed porous discs were tested for their catalytic properties for steam reforming of black liquor and for $\mathrm{NO}_{\mathrm{x}}$ destruction. Tables 1 and 2 show some of the $\mathrm{NO}_{\mathrm{x}}$ destruction test results. The results indicate that under a dry reducing atmosphere (i.e., no moisture) the $\mathrm{NiAl}$ intermetallic with iron oxides has $100 \%$ NO reduction at high temperatures. The NiAl intermetallic with $\mathrm{ZrSiO}_{4}$ shows only moderate NO reduction under the same test conditions. These cermet filters also show effectiveness in NO reduction under a moist $(10 \%$ water vapor) reducing atmosphere. However, the intermetallic, $\mathrm{MoSi}_{2}$, did not show any significant catalytic property to NO reduction at any temperature. When tested under dry or moist oxidizing conditions, these materials showed no

Table 1

NO reduction for the cermets under a reducing atmosphere (Test condition: $5 \% \mathrm{NO}+1.67 \% \mathrm{C}_{2} \mathrm{H}_{4}+93.33 \% \mathrm{He}$ [reducing])

$\%$ NO conversion (\% N2 Formed) under reducing atmosphere

Temperature $\left({ }^{\circ} \mathrm{C}\right) \quad \mathrm{NiAl}+\mathrm{ZrSiO}_{4} \quad \mathrm{NiAl}+$ iron oxides $\quad \mathrm{MoSi} 2$

\begin{tabular}{lccc}
\hline & 0 & 0 & 0 \\
400 & 0 & 2.5 & 0 \\
475 & 0 & 2.6 & 2 \\
550 & 0 & 4.7 & 2 \\
623 & 3.8 & 13.3 & 2 \\
696 & 11.3 & 43.1 & 3 \\
797 & 38.7 & 98.3 & 7 \\
904 & & 100 & \\
\hline
\end{tabular}

Table 2

No NO reduction for these cermets under an oxidizing atmosphere (Test condition: $5 \% \mathrm{NO}++95 \% \mathrm{He}$ [NO is oxidizing])

\begin{tabular}{|c|c|c|c|c|c|}
\hline Temperature $\left({ }^{\circ} \mathrm{C}\right)$ & \multicolumn{2}{|c|}{$\mathrm{NiAl}+\mathrm{ZrSiO} 4$} & \multicolumn{2}{|c|}{$\mathrm{NiAl}+$ iron oxides } & Temperature $\left({ }^{\circ} \mathrm{C}\right)$ \\
\hline 26 & $x$ & 0 & 0 & - & 73 \\
\hline 100 & $x$ & 0 & 0 & $\bar{x}$ & 203 \\
\hline 200 & $x$ & 0 & 0 & $x$ & 299 \\
\hline 300 & $x$ & 0 & 0 & $x$ & 302 \\
\hline 403 & $x$ & 0 & 0 & $x$ & 450 \\
\hline 501 & $x$ & 0 & 0 & $x$ & 601 \\
\hline 599 & $x$ & 0 & 0 & $x$ & 798 \\
\hline 695 & $x$ & 0 & & & \\
\hline
\end{tabular}

significant catalytic behavior to $\mathrm{NO}_{\mathrm{x}}$ destruction.

In the steam reforming of hydrocarbons to syn-gas, the $\mathrm{NiAl}$ cermets (with either $\mathrm{ZrSiO}_{4}$ or iron oxides) all showed catalytic activity. Comparison of the ratios of $\mathrm{H}_{2} / \mathrm{CO}$ in the steam reforming of hydrocarbons by these two cermet systems at the same temperature indicates that the $\mathrm{NiAl}+\mathrm{ZrSiO} 4$ system seems to produce a better $\mathrm{H}_{2} / \mathrm{CO}$ ratio.

Particle filtration efficiency is still under analysis at this reporting time. Update information on filtration efficiency tests will be sent to the LDRD office.

\section{New or Increased Technical Capability}

This project allows the plasma technology group to expand its technical capability beyond plasma science. Materials fabrication and processing, whether using plasma or nonplasma methods, has been an important area for the plasma processing group. This project has provided new and increased technical capability at the INEL to address problems critical to the paper industry. The technology can also be applied to other industries for offgas control.

Business Development Opportunities

Union Camp, Weyerhauser, and Consolidated Papers have been contacted about this technology, and they are interested in the progress of technology development. The Alberta Research Council contacted the principal investigator through the Lockheed Canadian Offset Program and inquired about potential development and application of the cermet technology to the Canadian paper industry. 


\section{Silent Discharge Plasma for Treatment of Automobile Exhaust}

LDRD 4483

\section{P. C. Kong, A. W. Erickson}

Automotive exhaust consists of unburned hydrocarbons, carbon dioxide and monoxide, nitrogen oxides, sulphur oxides, water vapor, and soot particles. The level of unburned hydrocarbons, carbon monooxide, nitrogen oxides, and sulphur oxides from automotive exhaust is tightly regulated by the U.S. Environmental Protection Agency (EPA). Current automotive exhaust control systems use catalytic converters to minimize the emission of these EPA-regulated gases, particularly the $N O_{x}$ level. In order to meet tight future EPA regulations, it is necessary to take additional measures to minimize automotive exhaust emissions. Installing a simple, reliable, rugged, and inexpensive auxiliary pollution control system after the catalytic converter will help minimize automotive exhaust emissions to below EPA-regulated levels.

Nonthermal plasma techniques involving corona discharges, silent discharges, and electron beam (e-beam) systems have been employed to remove pollutants in various applications. For example, pulsed corona discharges and ebeams have been tested for removal of $\mathrm{SO}_{x}, \mathrm{NO}_{x}$, and other trace hazardous hydrocarbons from stack gases. Silent discharges such as dielectric barrier discharge plasmas $(D B D P)$ is also being investigated for their ability to destroy stack gas pollutants. These techniques can be extended to the treatment of automotive exhaust. This project is assessing the feasibility of applying the $D B D P$ technology for the destruction of automotive exhaust pollutants.

\section{Objectives}

- Develop an operational benchscale DBDP device for engine exhaust treatment.
- Test the bench-scale DBDP device with bottled engine exhaust gases.

- Assess the performance of several transition metals as catalysts for $\mathrm{NO}_{\mathrm{x}}$ destruction in the device.

- Assess the performance of several transition metal oxides as catalysts for $\mathrm{NO}_{\mathrm{x}}$ destruction in the device.

\section{Accomplishments}

All the objectives of this project have been met, and the analysis of the DBDP-treated residue gas continues. Bottled lawnmower exhaust gas was used as the test gas, since the $\mathrm{NO}_{\mathrm{x}}$ level is much higher than any automobile exhaust.

The figure at the top of page 176 shows a schematic of the bench-scale test device. A series of experiments were performed using various electrodes at 4.14 $\mathrm{kV}$. A fourier transform infrared (FTIR) spectrometer was used in conjunction with gas chromatography (GC). The FTIR unit is equipped with a residual gas analyzer detector cell designed for an EPA exhaust gas analysis regime and real-time data collection required a flow of one liter per minute. This meant the use of teflon bags for gas storage before analysis could be performed. Data from GC and FTIR compared well during one stainless steel electrode test. However, the other tests results varied widely, and FTIR concentration errors indicate that the numbers are unreliable. Several calibration attempts on the FTIR were futile. A procurement embargo resulted in cancellation of calibration gases that probably would have allowed FTIR analysis of $\mathrm{NO}_{x}$ gases. Since the FTIR analysis is not reliable at present, a method is necessary to infer the destruction process occurring in the plasma. GC was used to mon- itor $\mathrm{N}_{2}$ concentration changes due to $\mathrm{NO}_{\mathrm{x}}$ dissociation to $\mathrm{N}_{2}$ and $\mathrm{O}_{2}$. Bottled lawn-mower exhaust was the test gas because the $\mathrm{NO}_{\mathrm{x}}$ concentration level would be much higher than regulated automobile exhaust. Cu and SS catalyst show nitrogen concentration increases after plasma treatment of the raw exhaust gas. The increase in $\mathrm{N}_{2}$ concentration in the treated gas is probably due to $\mathrm{NO}_{\mathrm{x}}$ dissociation. Co and Ni catalysts did not show this effect. The plasma-treated exhaust gas shows $\mathrm{CO}_{2}$ concentration increases using $\mathrm{Cu}, \mathrm{SS}$, and Ni catalysts. This could have resulted from hydrocarbon oxidation either with $\mathrm{O}_{2}, \mathrm{NO}_{x}$, or $\mathrm{H}_{2} \mathrm{O}$. The GC used for the gas analysis is not sensitive to hydrocarbons $>\mathrm{C}_{2}$. The hydrocarbon destruction results are not very consistent, and no preliminary conclusion should be drawn at this time. More tests should be performed in following years. The transition metal oxide catalysts are still under testing at this final reporting time. Update of the test results will be submitted to the LDRD office when they become available.

New or Increased Technical Capability

This project has allowed further development of plasma process technologies that will establish the INEL as a leader in these fields. The silent discharge plasma technology finds wide applications in off-gas pollutants control, air toxin destruction, chemical synthesis, and energy conversion, which are of vital strategic and economic interest to the United States.

\section{Business Development Opportunities}

Ford Motor Company visited at the INEL in December 1994 to look into what technology would have the potential to reduce automotive exhaust pollutants under both cold-start and lean-burn conditions. If the INEL technology 


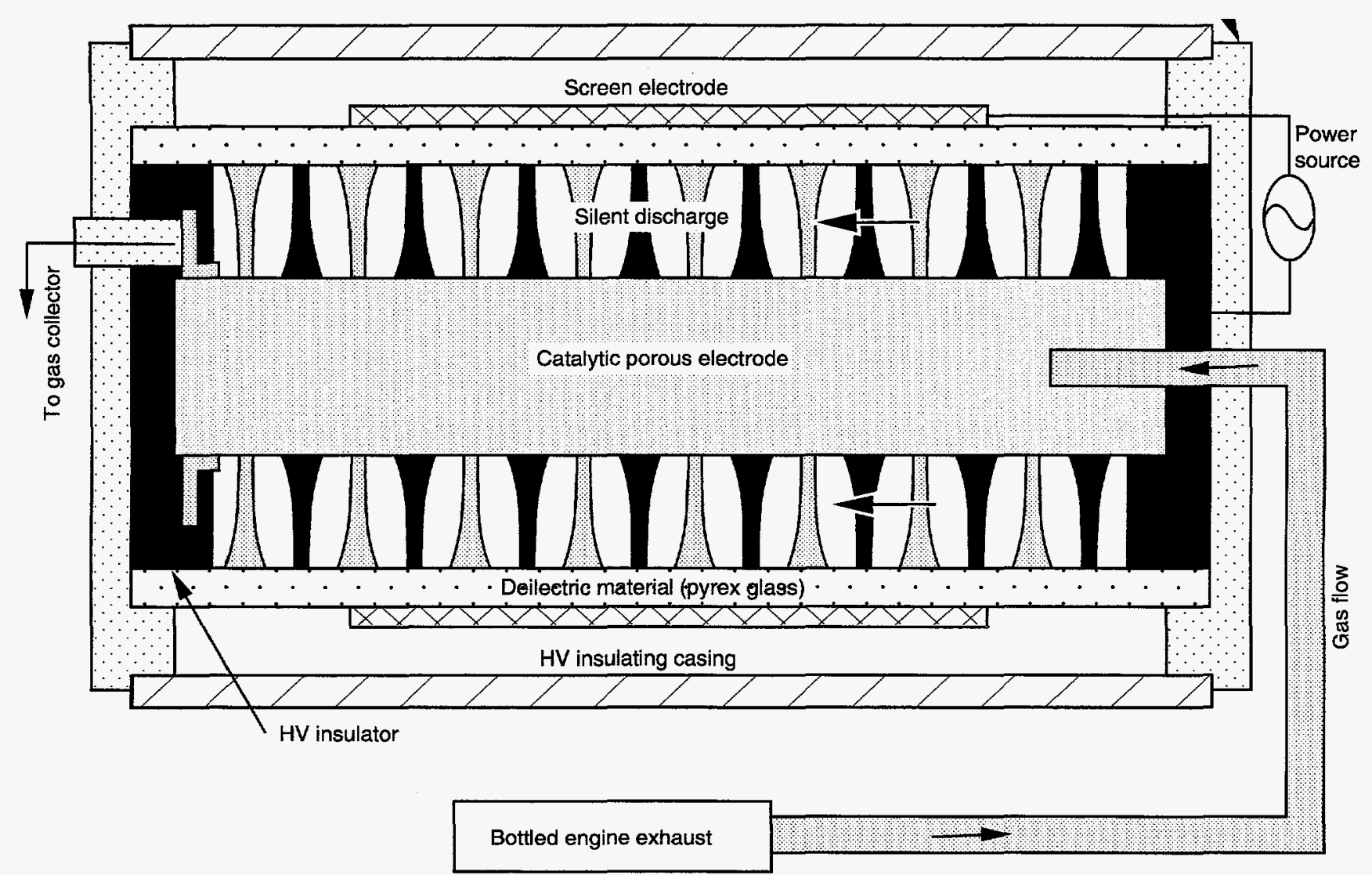

Schematic of the DBDP Offgas Treatment System

P.96 0049

can demonstrate technical feasibility and meet automobile application criteria (use not more than 1 to $2 \%$ of engine power), it should create business opportunities with the U.S. automotive companies. If the U.S. EPA extend the air emission standard in the future to small engine tools (e.g., lawn mowers, garden tractors) it will also create business opportunity for this technology.

\section{Evaluation of Liquid Metal Technologies Applicable to Radioactively Contaminated Scrap Metal Recycling LDRD 4485}

\section{G. F. Kessinger, T. E. Bechtold}

This project identified and evaluated metallurgical processing tech- nologies that could be used to support the conversion of radioactively contaminated scrap metal (RSM) stainless and carbon steels at the Idaho National Engineering Laboratory (INEL) into products appropriate for reuse within the DOE complex (specifically at the INEL), while using facilities already present at the INEL.

The results of this investigation show that the conversion of INEL RSM into a LLW disposal boxes for use at the Radioactive Waste Management Complex (RWMC) is technically feasible. Based on costs and facility requirements associated with production activities in the private sector and on estimated and projected RWMC disposal costs, we conclude that INEL RSM steel could be converted to product for production costs (not including equipment costs) that are comparable to the cost of dispositioning RSM to the RWMC.

The proposed product was identified during discussions with personnel from the Lockheed Idaho Environmental Operations branch. The choices of technologies to accom- plish material conversion were based on a number of considerations, including operating/production costs, technology maturity, and integration of equipment already present at the INEL (a four-high reversible rolling mill located the Test Area North-Specific Manufacturing Capability (TANSMC) facility) into the processing scheme.

These results should be instrumental in developing a path forward for future recycling activities at the INEL.

\section{Objectives}

- Identify a product that could be used at the INEL and that could be fabricated from INEL RSM.

- Identify facilities and capabilities at the INEL that could be used to perform the desired material conversion.

- Perform a literature search to identify technologies appro- 
priate for converting INEL RSM into the product of choice.

- Develop a process flowsheet for material conversions.

- Develop a spreadsheet to compute material throughputs and costs associated with material conversion.

- Write a final report documenting the results of the development activities (product, INEL facilities that could be used, results of the literature search, and flowsheet and spreadsheet of development and results).

- File an invention disclosure if the product developed is intellectual property in need of protection.

\section{Accomplishments}

The process flowsheet consists of the following unit operations and tasks: (a) sorting, (b) sizing, (c) melting, (d) molten metal refining, (e) casting, (f) rolling, (g) fabrication, and $(h)$ waste management and disposal. (See the block diagram figure below).

The spreadsheet calculations performed to identify costs associated with material conversion are based on private-sector production costs. Analysis of the spreadsheet/cost model results leads to

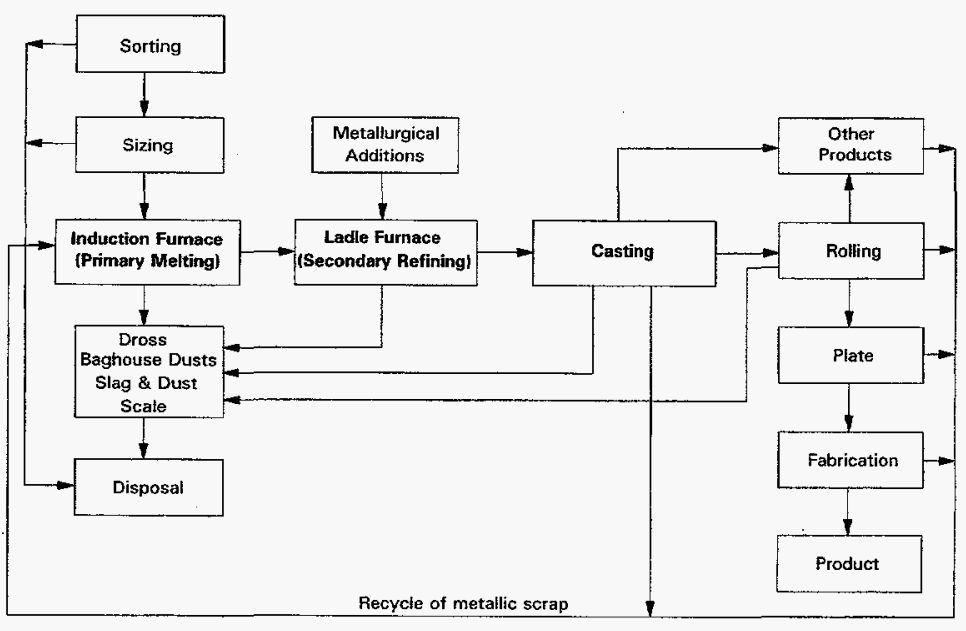

Radioactively Contaminated Scrap Metal Process Flowsheet the conclusion that the production costs associated with the conversion of RSM steel to LLW boxes are less than the costs associated with dispositioning RSM to the RWMC.

The costs of conversion of an on-site facility into a foundry facility capable of the ingot casting, rolling and fabrication activity described is in the $\$ 5,000,000$ to $\$ 10,000,000$ range.

\section{New or Increased Technical Capability}

The results of this investigation are important because they lay the groundwork for RSM beneficial reuse at the INEL. This study addressed the significant issues related to RSM recycle: identification of a product, identification of appropriate technologies and the development of a processing scheme for dealing with the materials that should be available, and use of facilities already present at the INEL.

The process flowsheet and spreadsheet allow the estimate of throughput and associated costs for three unique scenarios: production of carbon steel products from RSM carbon steel, production of stainless steel product from RSM carbon steel, and the production of stainless steel product from RSM stainless steel. The spreadsheet also computes the costs of direct disposal of RSM at the RWMC, and of direct disposal of melt-consolidated RSM (using the primary melting facility to volume reduce low density scrap into ingots that would be subsequently go to the RWMC for disposal).

\section{Business Development Opportunities}

None have been identified as a part of this activity.

\section{INEL Metal Recycle: Induction Melting LDRD 4486}

\section{T. E. Bechtold}

The disposition of radioactive wastes in the United States is becoming an increasing problem to the Department of Energy and to private utilities. To alleviate part of this problem, this project studied the vacuum induction melting (VIM) method to decontaminate stainless steel radioactive scrap metal ( $R S M$ ) for reuse. The project builds on previous refining work performed at the INEL using air induction melting (AIM). The technique effectively removed the surrogates but caused carbon pick-up in the melt. This is a problem because the product needed for waste storage products such as drums and nuclear fuel overpack containers is the low-carbon grade (304L) because of corrosion concerns. The goal of this research is to remove radioactive contaminants from stainless steel in a molten metal bath using the VIM process and lower the carbon content of the stainless steel bath by chemical additions or gas injection. The modified VIM melt technique may produce a highvalue $304 \mathrm{~L}$ steel product for reuse by the DOE and the commercial nuclear industry. A business could be developed where INEL and other scrap metal is melted at the INEL or elsewhere and rolled and formed into products at the SMC Facility. (See flowsheet on the previous page.) 


\section{Objectives}

- Develop a thermochemical model: the VIM system will be modeled to predict the thermodynamic distribution of species.

- Conduct VIM experimental studies: the calculated VIM parameters will be tested with metal containing radionuclide surrogates.

- Conduct a VIM melt using INEL RSM: a charge $(2,000 \mathrm{lb})$ of INEL stainless steel RSM will be processed at an outside vendor.

- Write a final report.

\section{Accomplishments}

The goal of this research is to demonstrate that the VIM process can remove radionuclide surrogates (cerium, lanthanum, neodymium) and reduce the carbon content of stainless steel to meet a low-carbon specification $(<0.03 \%)$. The VIM process was modeled (CSIRO/Monash calculational program) to predict the thermodynamic distribution of species between gaseous and condensed phases as a function of the system and test variables such as melt composition, test temperature, gas or chemical additions, impurity (surrogate) content, and gas/liquid/solid (furnace lining) interactions. The equilibrium calculations show that there is enough oxygen present in the system ( $\mathrm{Cr}_{2} \mathrm{O}_{3}$ oxide on stainless steel) to react with the surrogates and allow them to report to a slag phase. The calculations also show that the carbon level could be reduced by reacting it with a gaseous $\left(\mathrm{CO}_{2}\right)$ or solid (Magnetite, $\left.\mathrm{Fe}_{3} \mathrm{O}_{4}\right)$ addition. These additions will also provide oxygen for reaction with the surrogates.

The melt stock had surrogate levels of approximately 500 ppm and a carbon level of approxi- mately $350 \mathrm{ppm}$. The ingots were sampled for chemistry at the top and bottom. Test 1 was run with straight vacuum in the melt chamber. The test confirms the prediction that the surrogates will be oxidized and removed to the slag phase with the oxygen available in the system. Test 2 was designed to have an addition of $\mathrm{CO}_{2}(0.10 \mathrm{~atm})$ to the vacuum chamber. A leak occurred in the vacuum chamber sight glass, and the run was terminated before completion. Test 3 was designed to analyze the effect of a stoichiometric Magnetite $\left(\mathrm{Fe}_{3} \mathrm{O}_{4}\right)$ addition for carbon reduction. This amount of Magnetite had no measurable effect on the carbon level. Tests 4 and 5 looked at the effect of $\mathrm{CO}_{2}$ additions to the system. In both tests, the $\mathrm{CO}_{2}$ was added during the heat-up before the charge was molten. The carbon was not reduced in these tests; the reason is thought to be a high-temperature solid formation of metallic carbides on the surface of the charge, which would tend to increase the carbon level. Tests are continuing as follows: magnetite addition ( $2 X$ stoichiometric amount will be added); $\mathrm{CO}_{2}$ additions into the vacuum chamber (0.10 atm) and/or through a lance when the charge is molten.

A VIM melt (straight vacuum) of 2,000 pounds of INEL generated stainless steel RSM was conducted on September 1, 1995 at Manufacturing Science Corporation, Oak Ridge, Tennessee. The melt had to be terminated just before tapping owing to a furnace lining breach. The material was retrieved and will be remelted.

\section{New or Increased Technical Capability}

The data produced in this project (VIM refining) can be added to the INEL knowledge base developed in previous INEL RSM refining studies in air induction melting, vacuum arc melting, plas- ma cold hearth melting, and electroslag remelting to give a good understanding of various technologies that could be used for melt decontamination/refining.

Business Development Opportunities

The INEL has the capability (SMC Facility) to turn an ingot melted and refined/decontaminated from stainless steel RSM into a plate product that could be fabricated into useful products such as waste storage boxes or drums and long-term nuclear fuel storage containers. The INEL could recycle all of its RSM into these products, thereby avoiding the burial cost for the RSM and the purchase price for new material. A possible material flow would be to melt and refine the RSM at an outside vendor and bring the material back to the INEL for rolling/fabrication.

Scientific Ecology Group (SEG) and Manufacturing Sciences Corporation (MSC) have evaluated the INEL as a possible site for RSM recycling and have evaluated possible end products. MSC has expressed an interest in setting up a joint venture with Lockheed Idaho for an RSM recycling center at the INEL.

\section{Process Industries- Industrial Improvement Using Integrated Process Evaluation LDRD 4487}

\section{B. W. Brown}

The purpose of this project was to identify process improvements in the food processing, aluminum, and pulp and paper industries. Consultation with industry personnel, site inspections, and flowsheet modeling with ASPEN PLUS were used to identify the process improvements in each industry. For food processing, improvements were identified for recy- 
cling of noncontact process water, conversion of potato processing waste to value-added chemical products, and alterative waste-water treatment methods. For aluminum, an energysaving process improvement was identified using the INEL plasma quench reactor in carbothermic reduction of alumina. This process would potentially reduce the current two-step process to a single step, eliminate the production of caustic by-products, and produce saleable silicon and iron. Pulp and paper process improvements included possible elimination of the causticizing step through use of a hybrid plasma process, heat recovery and cogeneration from the gasification process, and use of ammonia to recover hydroxide ion. Each area of research resulted in follow-on work being identified, including work-for-others, FY-96 LDRD proposals, and possible CRADAS.

\section{Objectives}

The objectives of this LDRD were to investigate industrial processes and to suggest improvements to these processes. The approach was to target a specific industrial activity and model its operation as a series of unit operations using ASPEN PLUS, a process simulation program. Three industries were selected: food processing, pulp and paper, and aluminum. Specific objectives included the following:

- Develop simulation outputs such as process flowsheets, summary input/output tables, and associated plots used for analysis.

- Produce a project evaluation report summarizing the optimized integrated simulation and cost results.

- Develop a conceptual pilot plant and/or process change design for engineering development testing.

\section{Accomplishments}

Food Processing. Process assessment inspections were conducted at four potato processing facilities. Process improvements identified were presented to the Idaho Association of Commerce and Industry. Areas for process improvement include the following: (a ) water use minimization via cost-effective recycling of noncontact water, (b) research and development to develop a process for conversion of potato processing to value-added chemical products, and (c) evaluation of alternative waste-water treatment methods. Proposals were sent to, and subsequent discussions were held with, each company visited.

Aluminum. Efforts were directed at modeling the direct, carbothermic reduction of bauxite. This direct reduction would reduce the current two-step process into a single step, eliminate the production of caustic red mud, produce potentially useful iron and silicon byproducts, be a much simpler process, and result in an energy savings of approximately $25 \%$. The improved process is unlike other carbothermic processes investigated in that it would use the plasma quench reactor (PQR) developed at the INEL to effect a rapid quench of reaction products, thereby preventing back reaction to undesirable by-products. Calculations of the kinetics of the process suggest that rapid quench is the key to process success and is feasible using the INEL PQR.

Pulp and Paper. Three different focus areas were addressed: (1) ASPEN PLUS modeling of the gasification of black liquor using a hybrid plasma process showed that elimination of the causticizing step was possible, (2) heat recovery from the plasma torch and co-generation using a twin turbine approach on the gasifier effluent might be feasible, and (3) higher productivity or energy savings may result from an alterative process that uses ammonia rather than sodium to recover hydroxide ion.

\section{New or Increased Technical Capabilities}

Aluminum. Experimental work at the INEL is proposed for FY96 to confirm the feasibility of using the plasma quench reactor for the carbothermic reduction of alumina. If these experiments are successful, a new process for production of aluminum will be developed with INEL technology as the centerpiece.

Pulp and Paper. Modeling of the hybrid plasma process at the INEL increased the technical capability at the INEL and will serve as the basis for future modeling.

\section{Business Development Opportunities}

Food Processing. Discussions are ongoing with the J. R. Simplot Company on potential collaborative work on the three areas cited above. It is believed that the INEL is positioned to continue to build an alliance with this industry that has a good chance to develop into CRADA work.

Aluminum. A new process has been identified that may revolutionize the aluminum industry. Business development will be pursued after proposed tests are complete in FY-96.

Pulp and Paper. The research conducted generated the three new LDRD proposals for FY96 focused around actual industrial needs with tremendous economic potential:

- Sensors and controls development to detect nonprocess elements within the Kraft process.

- Design optimization of Kraft components. 
- Solubility studies and a possible alternate Kraft process.

\section{Depleted Uranium Fiywheels for Stationary Applications LDRD 4488}

\section{B. D. Hawkes}

Historically, flywheels were used primarily as momentum devices on applications such as steam engines or stamping presses. The level of research by private companies and universities has taken some major strides in recent times. The main emphasis in flywheel research has been in connection with alternativeenergy systems such as wind or solar energy. These intermittent power sources need an economical way of storing their power. Current research is focused on flywheels used as energy storage devices or Electro-Mechanical Batteries (EMB). The goal of this $\angle D R D$ was to investigate a flywheel rotor that would store $25 \mathrm{~kW}-\mathrm{hr}$ and spin at a maximum speed of 40,000 RPM (due to the electronics). This flywheel would be composed of altemate layers of an elastomer and a laminated composite wrapped around a steel shaft. Typical flywheels composed of a single isotropic material experience very high radial stresses toward the center of the spinning disk and tangential stresses toward the rim. The flywheel design being investigated shifts the high radial stresses to tangential stresses by allowing the elastomer between rings to stretch, causing the laminated rings to stretch tangentially. The laminates can be laid up so that they are strongest in the tangential direction. Also, the use of laminates allows the flywheel to store more energy per pound than an isotropic material such as steel.

\section{Objectives}

- Investigate the laminar composite design to shift the high radial stresses typically pro- duced toward the center of the spinning disk while shifting the tangential stresses toward the rim.

- Develop three-dimensional, finite-element methods to investigate the natural frequencies inherent in this design.

\section{Accomplishments}

The flywheel design we investigated used a steel shaft 2 in. in diameter and $26 \mathrm{in}$. long. This was surrounded by alternate layers of elastomer (.5 in thick) and laminate (1.3329 in. thick) to bring the diameter of the flywheel to 24 inches. This flywheel would store $25 \mathrm{~kW}$-hr of energy when spinning at 40,000 RPM. An axisymmetric, finite-element model of this design shows that the peak stresses in the laminate rings would be about 210,000 $\mathrm{psi}$, and the elastomer would experience up to 28,000 psi. Common, fiber-reinforced laminates have tensile strengths in excess of $210,000 \mathrm{psi}$.

A 3-D, finite-element model of the rotor was generated to investigate the natural frequencies of this design. The results show 5 natural frequencies in the operating range of $0-658 \mathrm{~Hz}(0-40,000 \mathrm{RPM})$ The design could be modified by making the shaft diameter larger and removing or altering the first layer of elastomer between the shaft and the inner ring of laminate.

\section{New or Increased Technical Capabilities}

The development of kinetic energy techniques, both in respect to computer codes and actual machine construction, adds to the mechanical engineering capabilities of the INEL. New projects that are under question, such as the ACET project involving response to earthquake and wind forces, can be aided with the understanding of kinetic motion gained in this project.
Business Development Opportunities

Flywheels and other kinetic devices are being reexamined by industry in general. The previously mentioned EMB application is one example. EMBs are being developed for stationary applications such as load balancing for a plant or facility, or to level out line surges caused by starting pumps or other electrical machinery. They are also being researched for mobile applications such as electric cars, portable power-packs, and space applications. Mobile applications have to deal with other aspects of design, such as weight and the gyroscopic effects of spinning masses, that are not problems with stationary applications. The understanding we gain through this work will be valuable to the industries considering the use of kinetic energy devices.

\section{Simulation-based Prototyping for Manufacturing-based Engineering LDRD 4758}

W. R. Nelson, W. Hawkes, W. Richins, T. Turner, B. Wilding

This project has assessed the need for enhanced software tools to support engineering design and to assess the suitability of currently available packages to form the basis for development of commercially viable software tools for engineering process enhancement. Currently, there are many opportunities for errors and inefficiencies in engineering design and product development processes. Currently available software tools primarily have been developed to automate current approaches for engineering design. A significant potential exists for enhanced tools that will fundamentally change the way product development is performed and to eliminate or reduce 
inefficiencies and errors. Such software tools could significantly improve the productivity of engineering teams across a broad range of American industries, reducing the time and expense required to bring a product to market and to reduce the likelihood that the product fails to meet customer and user requirements.

\section{Objectives}

- Identify requirements for software support to engineering processes to enhance the design/manufacturing interface and telepreneuring.

- Assess the suitability of GS Design to serve as the basis for a software product to support manufacturing based engineering and telepreneuring

- Develop recommendations for development of programs to support product development and application of lessons learned to enhance INEL engineering processes.

\section{Accomplishments}

A significant first step was taken toward understanding the product development process at a high level and highlighting those places in the process where software supoor tools could significantly enhance productivity, reduce design/manufacturing cycle time, and lessen the likelihood of errors that cause delays, increase costs, and degrade product performance. Leverage points were identified where new software tools could be applied to further enhance engineering processes (see the figure on the top of the next page). These include the design modification process, testing of the design for manufacturability during design rather than after it is complete, and facilitating communication among designers and reviewers. It also appears that additional process enhancements could be identified by fun- damental changes to the ways that engineering processes are performed, and perhaps redistribution of functional responsibilities among the members of the design team.

It was found that currently available software tools support many of the tasks and functions identified as part of the desired engineering design process, but that considerable room for improvement remains. Although the tools provide many features that make tasks easier for the engineering design team, in most cases there is still a requirement that the users provide the necessary ingredients to effectively use the capabilities, and the organization must provide an environment that encourages the design team to function optimally. In too many cases, software capabilities cannot be effectively used because organizations impose impediments. There is significant potential for new meta-tools to provide a higher level of support to the engineering design team and to make possible the effective functioning of the overall process. However, in most cases organizational changes will be required for effective use of such tools, so that a fundamental culture change within the organization may be required.

Although GS Design is a very capable solids modeling package, it lacks many of the features that would be necessary to realize the vision of the enhanced engineering team. Because we did not have access to the internal software structure of GS Design, we cannot at this time estimate how feasible it would be to bring GS Design to this state of development, or how well the fundamental engine of GS Design would support such a system.

This project has added to an embryonic effort at INEL to estab- lish a center for the development and application of methods and tools to support engineering processes. In order for the INEL to fulfill its role as the Department of Energy's premier applied engineering laboratory, and for the INEL Applied Engineering development Laboratory to fulfill its mission, it is necessary to develop a more complete understanding of engineering processes and to develop methods and tools for more effective processes. Past INEL efforts have focused on what is engineered rather than how engineering tasks are carried out. The establishment of the Center for Engineering and Human Performance Optimization has formalized Lockheed Idaho's intent to be a serious player in this field. Successful developments in this area could have a significant impact on the productivity of U.S. engineering and manufacturing organizations. This project has added to Lockheed Idaho's capabilities to be in the forefront of this important activity.

\section{Business Development Opportunities}

One of our objectives was to determine whether GS Design could serve as the basis for a Lockheed Idaho-led development program to develop new engineering process support software. We have determined that significant work would be needed to bring GS Design to the state where the desired enhancements could be simply added on. That is, significant modifications would be required so that GS Design could function as an engineering design package that is competitive with other available tools, before it would be possible to add next generation enhancements. This does not imply, however, that there is no opportunity for Lockheed Idaho to enter the field. An 


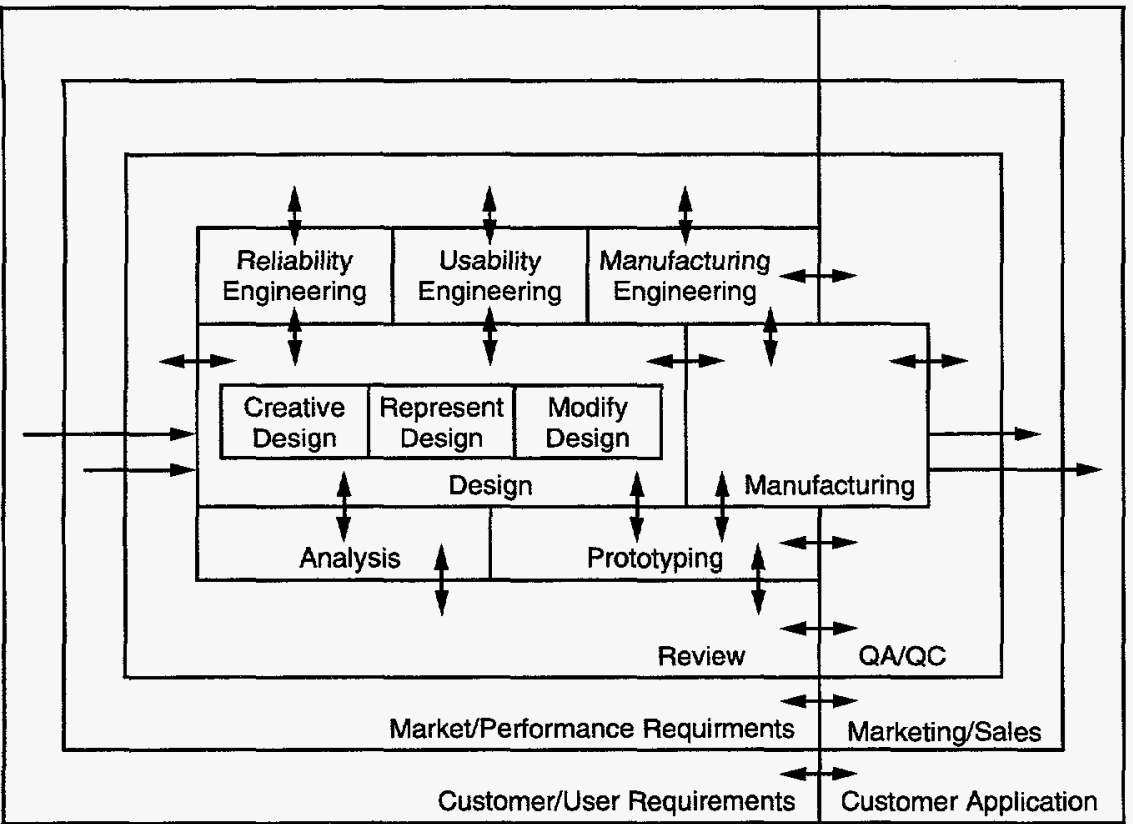

Product Development Process

appropriate partnership with one of the software vendors (whether Graphic Systems, SDRC, fjhsdfh, or kdjdj) could be developed to begin to seriously attack the inefficiencies in engineering processes identified in this study. There is still a market niche that needs to be filled, and Lockheed Idaho's center for Engineering and Human performance Optimization is positioned to serve as a catalyst to address it.

\section{Readiness}

LDRD 4759

\section{J. I. Mills, B. L. Kaplan}

This research investigated trends in thought about organizational and human readiness for success in the electronic age, with a view toward establishing requirements for computerized tools to support cooperative group work. A general set of requirements for distributed work support systems (DDWS) was developed, considering human-computer interaction and distributed group work issues. Key concepts were applied to a case study of an actual organiza- tional setting involving distributed group work.

Lessons learned for this work include: insights into the potential of new technologies to facilitate desired cooperative work behaviors; important distinctions between problems in information exchange and those involving collaboration; needs for information infrastructure, phased approaches, and for fitting DWSS tools to the specific work to be performed. Questions regarding distributed systems thinking and simulations are treated as subcategories or specific instances of DWSS, and specifications for a prototype DWSS were developed.

\section{Objectives}

- Address organizational and human readiness questions in the following areas: computersupported collaborative work tools; tools to support distributed systems thinking; games or simulations.

- Perform a case study on a real-world distributed work team.

- Develop specifications for a prototype distributed work support system.

\section{Accomplishments}

Organizational, individual, and technological factors, as well as currently available tools and technologies, were assessed relative to their applicability to the project's objectives. Based upon available tools and the identified general requirements, as well as the requirements of the selected actual organizational setting, a general set of requirements for a distributed work support system was developed, considering human-computer interaction and distributed group work issues. Key concepts were then applied to a case study of the critical Mixed Waste Focus Area. At the conclusion of the case study, prototype distributed work support system guidelines, generally applicable to all situations where organizational and human readiness are key, were developed.

New or Increased Technical Capabilities

In the course of this work, LITCO was able to integrate into its existing capabilities knowledge of and experience with a rapidly growing area of organizational and technology development that is important on two fronts. First, the competencies and capabilities explored and developed may be critical in keeping LITCO at the cutting edge in doing engineering development work in the years to come. These capabilities are quickly being recognized as a key part of engineering and human performance optimization. The Mixed Waste Focus Area was only one of many projects and programs at the INEL that are increasingly requiring support for distributed work. Secondly, the LDRD gave LITCO and the INEL an understanding of how existing INEL resources can be most efficiently applied to the enhancement of products and services. In addition, the foundation for licens- 
able management and organizational support tools was established.

\section{Business Development Opportunities}

Extensive opportunities for application of distributed work support systems have been identified in the private sector. Specifically, there exists a growing interest in the private sector in performance optimization. Performance optimization includes engineering, business, human and organizational. The foundation established by this LDRD will be directly supportive of, and complementary with, all on-going LITCO activities involving environmentally conscious manufacturing and general manufacturing management, leading to an ability of the private sector to more robustly respond, in an environmentally sustainable and profitable manner, to the growing challenges of the marketplace.

\section{Butterfat Management}

LDRD 4860

\section{J. L. Morrison}

The United States dairy industry is greater than 30 billion dollars per year. The major growth area in this industry is cheese (\$6B). The quantity and quality of cheese products from milk depend on the percent of butterfat and percent of casein protein, roughly 76 to $80 \%$ of the total protein in milk. Presently, instrumentation is able to measure the percent butterfat and percent total protein to an accuracy of $0.06 \%$ or less. These measurements use toxic reagents for sample preparation for an infra-red spectroscopy analysis. This technique has limited application to realtime process control (presently only butterfat). Clearly, there is a need, in this very large industry, for real-time process control to optimize the product yield and quality. The overall objective of this research is to develop this sensor technology and license it to U.S. industry.

\section{Objectives}

- Develop physical conceptual measurement techniques that might indicate a sensitivity to casein protein or butterfat in milk.

- Perform feasibility laboratory tests on those approaches that were compatible with the 6month schedule and available budget.

- Perform an initial system analysis to investigate and validate the applications and business opportunities for the proposed technology being developed.

- Demonstrate adequate success that would justify committing a full year to support the effort in FY-96.

\section{Accomplishments}

The accomplishments in FY-95 were significant, in spite of the short duration and funding constraints on this project:

- CRADA with Utah State University

- Initial development that led to five invention disclosures

- Preliminary results with three microwave techniques demonstrating a promising sensitivity to percent butterfat in control samples of milk.

\section{Concepts Lacking Sensitivity}

1. A capacitance probe: Lockheed Idaho Invention Disclosure Record, Dairy Butterfat Quantitative Sensor, Robert D. Dinsdale, February 15, 1995, to detect butterfat in milk. It has since been shown with measurements on a network analyzer that low- frequency electromagnetic sensing techniques, as this probe is, have little sensitivity to butterfat or protein in milk.

2. A differential capacitance probe: Lockheed Idaho Invention Disclosure, Differential Capacitance Probe for Process Control Involving Aqueous Dielectri Fluids, J. M. Svoboda, J. L. Morrison, March 22, 1995, to detect butterfat in milk. It is also a low-frequency electromagnetic concept and as such has inadequate sensitivity for butterfat or casein.

\section{Concepts that Appear to Perform}

3. An antibody-antigen detection system: Lockheed Idaho Invention Disclosure, Antigen Detection System Via Surface Plasmon Resonance Intensity Control System, Dr. Thomas Unger, Gary Bergeson, John Morrison, June 5, 1995 , to measure casein in milk. Could detect casein but is not tested.

4. An air-line coax sensor: Lockheed Idaho Invention Disclosure, Method for Measuring BF Content in Milk, J. Morrison, M. Fryer, July 31, 1995, to detect butterfat in milk. This method indicates sensitivity to butterfat.

5. A microwave resonant cavity: Lockheed Idaho Invention Disclosure, A Microwave Resonant Cavity Method for Measuring Butterfat Content in Milk, J. Morrison, M. Fryer, A. Hills, September 8, 1995, to detect butterfat in milk. This method indicates sensitivity to butterfat.

6. An open-ended coax to detect butterfat in milk. This method indicates sensitivity to butterfat.

Concept 3 was not tested this year.

Concept 4, a measurement technique with an air-line coax (ALC), yielded promising results with a sensitivity to butterfat. The absorption idea was tested against different milk samples with known levels of fat concentration. 
The absorption followed the fat concentration in the frequency band from $10 \mathrm{GHz}$ to $20 \mathrm{GHz}$ with a peak sensitivity at $15 \mathrm{GHz}$. (See the figure below.)

Concept 5 , the resonant cavity, also was sensitive to butterfat. The test results show a promising sensitivity to fat concentration above $15 \mathrm{GHz}$.

An additional concept, an enhancement based on the microwave resonant cavity, developed was a differential resonant cavity. It is similar in concept to the differential capacitor. The resonant cavity would be $Y$ shaped and have an unknown sample and a reference sample respectively in each branch of the Y. Excitation would be input at the base of the $Y$.

Concept 6 , the final area of electromagnetics investigated that was sensitive to butterfat, used the technique of sensing with an open ended coax. This structure is suitable for measuring liquids and semi-solid materials. The open end of the probe is placed in the milk while the other end of the probe is connected to a vector network analyzer that measures the microwave energy reflected from the milk. From this, we calculate the dielectric constant of the milk.

\section{FY-96 Research Areas}

- Further evaluate each of the three microwave butterfat sensing techniques and downselect to the best performer.

- Develop and test concepts such as the antibody-antigen concept to detect casein in milk.

- Continue the systems/business analysis to obtain overall focus and guiding requirements to the research.

\section{New or Increased Technical Capability}

During the course of this research, the INEL's technical expertise was enhanced in the following areas:

- Investigation of microwave technology to measure process milk properties has led to enhancements and development of new measurement techniques that could be applied to other real-time process control applications.

- Initial research may have resulted in a new microwave application to quantify butterfat in milk, which could replace a currently more expensive infra-red optical technology.

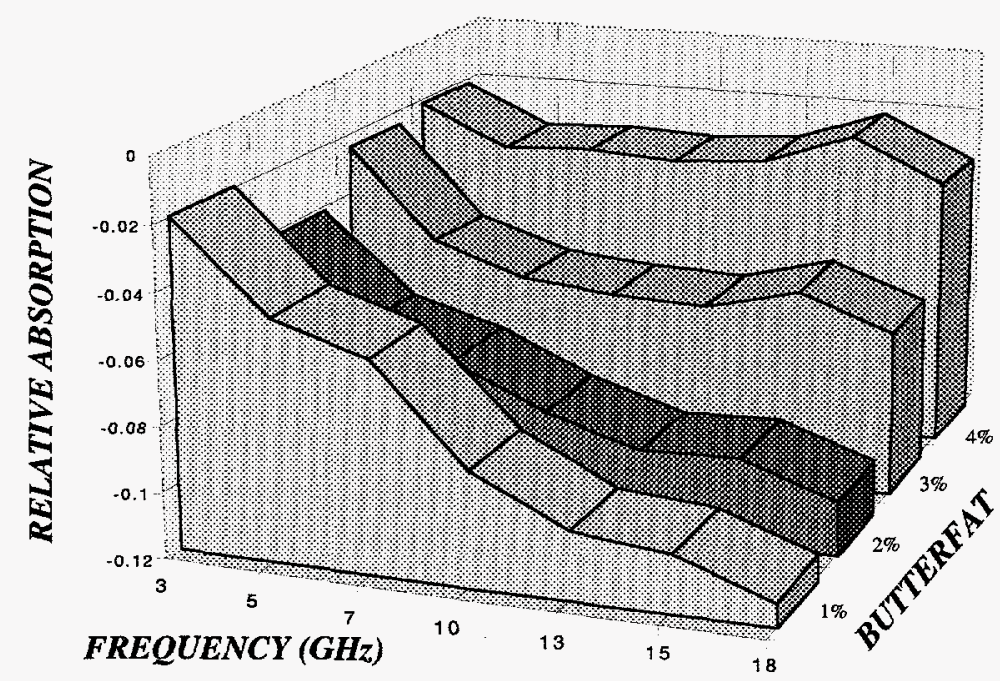

The initial results of the team's efforts led to new ideas for the direct measurement of proteins in milk. These ideas resulted in new capabilities and opportunities for the INEL:

- An INEL control systems and electronics development capability has been integrated with the antibody- antigen technology, and the result is a new sensor technology at the INEL in the area of bioelectronics.

- The systems investigation of the protein measurement problem in milk has shown that control system technology at the INEL has significant opportunity to enhance the food processing dairy industry to upgrade batch recipe production methods into realtime process control.

Business Development Opportunities

Our research and development has been aimed at developing measurement techniques that can detect casein or butterfat, or both, in process milk. The goal is identify sensors that will detect casein and improve the current percent fat accuracy measurements with a lower waste stream at lower cost. Our ultimate goal is to be able to license this technology so as to generate revenues for DOE-ID and the INEL. Our initial success this fiscal year has been in detecting butterfat. The focus to this stage has been with initial sensor synthesis and evaluation, and is strongly supported by the internal sensor product directorate. We also have in place a CRADA with one of the top three agricultural schools in the nation, Utah State University, and are collaborating with the internationally renowned dairy expert Dr. P. Savello. We have visited a number of processor plants where management has expressed a 
desire for improved measurement technology. We have also determined through discussions with Dr. Savello that any cost competitive device, whether real time or not, whether in line or not, would be of tremendous benefit to both producers and processors. This will ensure the opportunity to achieve our ultimate goal. We expect to focus next year's efforts on exploring total protein and casein protein detection sensors, enhancing the fat sensors, establishing points and methods of intervention in the chain of producer to final product, and evaluating a variety of possible system implementations of the sensors.

\section{Advanced Process Diagnostics System} LDRD 4862

\section{W. E. Austad}

Manufacturing processes are by their nature designed to be repetitive and precisely the same for each item produced. During production runs, tolerances drift, resulting from various factors such as tool bit wear, variability in part material, and, to a lesser extent, machine wear. Eventually, defective items are produced if tolerances become too large. Costly rework or waste can result from the machine degradation. But complicated machining patterns are difficult to analyze in a systematic and robust way. This project aims at one of the most basic industries in the manufacturing hierarchy, component machining with mills and lathes. The application area was chosen for its enormous potential market, the complexity of analyzing such signals with other techniques, and the relevance to cooperative commercial research at the INEL for robotics control and automotive manufacturing.

Our goal is to cooperatively develop an adaptive, data-based performance monitor for a milling machine that can detect subtle changes in tool wear and machine health. INEL and Clemson University are applying previous successes with $R K H$-space covariance techniques to industrial monitoring and control problems. Generic monitoring techniques produced by this research could be applied to many other application areas in advanced monitoring and control.

\section{Objectives}

- Identify industry application to investigate (M1-system selection completed).

- Collect sample of application data.

- Preprocess and analyze data with prototype algorithms (M2-validation of technology for industrial application).

- Report (M3-final report complete).

- Collaborate with industry and other INEL programs.

\section{Accomplishments}

Covariance algorithm background. Traditional parametric approaches to linear systems modeling require high-level analysis to form a model of a potentially nonlinear system. The number and types of relevant parameters are selected by a highly trained expert and/or by systematic reverse engineering of the process or component. The reproducing kernel Hilbert (RKH) space approach is based on low-level analysis of the actual system data to produce a discrete nonparametric model in terms of the input and output covariances. The resulting linearization is suitable for system state monitoring and control applications concerned with adapting to changes in system behavior.

This project investigated several potential application areas and selected computer-controlled milling machines as the initial class of applications to study. Simple but sensitive sensors were selected (piezoelectric pinducers) and analog test data were collected for variations in cutting tool depths and simulated material voids. Material voids were simulated by drilling small holes of controlled sizes in the material prior to actual milling. We used borrowed instruments for this experiment, so the data acquisition equipment and sensors were not ideal for a fielded industrial system. A commercializable effort requires a portable data acquisition system, more rugged sensors, and possibly a more orthogonal sensor suite, so sensor changes can be intelligently differentiated from actual machine changes. Collection of stroboscopic images of wear particles produced during machining was originally for physical verification and related research interests. But because of time constraints, these images and large suites of dull tool data have not yet been collected. Analysis of the mill data is expected at the end of FY-95 after the analog data have been digitized. However, prototypes of the covariant algorithms were tested using simulated noisy data generated by a chaotic process. The results seemed to confirm the validity of the algorithms, which successfully detected state transitions.

\section{Further Work}

\section{FY-96, Phase I. Software} should be implemented and tested on a full laboratory-scale prototype for one machine, one tool, and for a sequence of cutting operations. Design and procure portable laboratory-scale monitoring equipment. Formulate test patterns, collect data, and develop a method to communicate the current location in a cutting sequence. Provide physical verification of 
results with stroboscopic images of wear particles. Foster commercial and university relationships that can assist in data collection and successful industrial deployment. In collaboration with Clemson University, validate codes, establish criteria for machine performance, achieve sensitivity or robustness as appropriate, tune implementation, build graphical interface, and achieve portable " $\mathrm{C}$ " versions of the Matlab algorithms.

FY-97, Phase II. In Collaboration with Clemson and commercial partners, implement software on a more advanced commercial prototype for one machine, a sequence of tools, and a more complex sequence of operations. Formulate test patterns and collect data for multiple tool types. Develop a method to communicate what tool is currently loaded. Upgrade laboratory prototype.

FY-98, Phase III. In collaboration with commercial partners, define hardware and software to support integration with existing control systems. Explore methods to expand to plant level control. Design communications with possibly proprietary control systems from multiple machine venders or new open architecture for generic covariant control systems.

New or Increased Technical Capability

Dr. James Reneke, Associated Western Universities (AWU), from Clemson University delivered a preliminary version of RKH-space covariance algorithms in Matlab with documentation for general use at the INEL. We have gained additional experience with databased, covariant analysis, and the practical problems faced by metal cutting machine industries. Mike Fryer presented a paper titled System Health Monitoring at the 41st International Instrumentation
Symposium in Aurora, Colorado, in May 1995.

\section{Business Development Opportunities}

Internal Collaborations. Collaborating directorates included Bob Polk and Alan Anderson in Sensor Products (4800), whose interests are generic, adaptive control technologies and also potato water content data processing; Nancy Carlson in End Use, Energy Efficiency Products (4400), whose interest is intelligent control of welding and manufacturing processes; and Dennis Bingham in Robotics and Remote Systems (4800), whose interest is covariant control of cryogenic jets. Collaborating programs included Robotics and Cryogenic Cutting, DOE-OIT Industry, USCAR-LEP-ESSP Advanced Manufacturing CRADA, USCAR-LEP-ESSP Intelligent Welding of Thin Metal CRADA.

University Collaborations. Clemson University (URC Proposal and AWU program), Colorado School of Mines (by way of the Robotics Program).

Expected Industrial Collaborations. Cimetrix ZAWCAD CRADA, with interests in covariant and open systems control for robotics and CNC mills; Control Vision, an Idaho Falls company interested in applying stroboscopic imagers currently used for welding control to control metal cutting machines; and the National Center for Tooling and Precision Components (NCTPC), with interests in advanced machining technologies. Sandvik Tools also has a simple strain gage, level-based tool monitoring system that might be well suited to more advanced signal analysis algorithms.

\section{Miniature Tag and Covert Communicator LDRD 4866 \\ G. C. Bergeson}

Three major research objectives were successfully addressed.

$A$ radio frequency $(R F)$ tagging systems currently on the market was researched and characterized suitable for tracking farm vehicles and harvest operations to determine crop yield mapping and to support prescription farm methods. An RF system for demonstration research efforts at the INEL was selected and assembled.

We researched and characterized global positioning system (GPS)based tagging and tracking systems capable of pinpointing global coordinates of vehicles, animals, or people. We also selected and assembled a GPS-based system that can be miniaturized for covert implant and covert tracking of fleeing vehicles, drug smuggling vehicles, or military reconnaissance. The system was purchased for demonstration and for future agricultural, law enforcement, and national security programs research efforts at the INEL.

Research biological tagging techniques can be used for fast identification of mammals for tracking and positive identification. We also selected and demonstrated a technique suitable for tracing meat products from live animal to individual packaged product.

The INEL now has demonstration systems or documentation in all three areas, which will be used to support future INEL projects and missions.

\section{Objectives}

- Obtain an RF tracking capability with the following specifications:

- Small read/write tag that can be mounted in farm vehicles and trucks 
- Communicate over an RF link with a range of six feet or greater

- Data interface such as RS232 to link into a PC computer system

- Withstand environmental conditions normally found in a farm environment

- Obtain a GPS tagging and tracking capability with the following specifications:

- GPS coordinate readout,

- Range greater than 5 miles, radio LOS,

- Miniaturizable to $1 \times 1 \times 1$ in.,

- Frequency options for cellular telephone, mobile radio, and GPS interface,

- Receiver data suitable for mapping systems.

- Obtain a biological tagging and tracking technique with the following specifications:

a. The technique shall work with animals and humans

- It shall provide a high power of discrimination even between closely related or genetically identical individuals

- Rapid procedure (Less than one hour)

- Cost effective (Less than $\$ 3.00$ per individual test)

- Can be performed in the field or laboratory

- Will allow computer analysis of results, including archiving and database searches by pattern matching

\section{Accomplishments}

Within the three research topic areas, the project was divided into the following activities:
- Determine the needs of each system based on current INEL Sensor Products and Agricultural programs capture plans.

- Research the state-of-the-art products available which have the potential of meeting the INEL program needs.

- Select a system or assemble a system which best fits the INEL program needs.

- Demonstrate functionality of the system for its intended INEL use. Test the system per the initial needs and specifications.

- Make the system available to support INEL programs and missions as identified during the needs definition phase.

The above objectives were met as stated.

Currently, software is being developed to integrate this system into the pilot potato harvest system that will be used in the fall of 1995. Based on the results, the system could be developed into an integral part of the SST4AG program.

\section{New or Increased Technical Capabilities}

The three research topic areas were firmly rooted in INEL technical programs and each specifically addressed a deficiency in current programs as follows.

The INEL needed an RF tracking capability specifically for farming operations. The project has successfully filled that need and has even supplied a system to the agricultural program. The INEL needed a covert tracking system with sufficient range to support the National Institute of Justice (NIJ) proposal titled Fleeing Vehicle Tagging System. This program had jointly developed a tag emplacement system for fleeing vehicles such that law enforce- ment personnel could avoid dangerous high speed pursuits. However, the tag electronics were very weak in providing exact vehicle location and range. This project has solved this weak link for NIJ and other covert tagging and tracking applications. In addition, the system can be applied to many agricultural applications.

The Blue Ribbon Task Force formed by the National Livestock and Meat Board identified nine segments of the meat production chain from preharvest through consumption. Each segment has the common need to trace products back to their previous production step; ultimately achieving end product trace back to the live animal and growth/nutrition/ genetics environment. The INEL, in partnership with Miragen and potential future research with Arthur D. Little, Inc., now has the biotechnology to accomplish full trace back.

\section{Business Development Opportunities}

As stated, the INEL has established two CRADAs with Miragen, Inc., to further develop and refine the AbP technology for meat packing applications.

Recently, at the Sensors Expo95 in Chicago (9/11-14/95), the INEL initiated a relationship with Arthur D. Little, Inc., to assist in developing the biosensors required for beef grading and trace-back.

Lockheed Idaho Invention Disclosures:

- Antigen Detection System via Surface Plasmon Resonance Intensity Control System, Dr. Thomas Unger, Gary C. Bergeson, Dr. John L. Morrison, June 5, 1995.

- Fiber Optic Plasmon Resonance Sensor, Dr. John L. Morrison, September 11, 1995. 
- Antibody/Antigen Volume, Plasmon Sensor, Dr. John L. Morrison, September 11, 1995

- Antigen Filtration System, Dr. John L. Morrison, September 11, 1995.

\section{INEL Multimedia Object Database LDRD 4952 \\ K. W. Derr}

This project is developing a prototype application using object technology, specifically an object-oriented database (ODBMS), to provide access to islands of information at the INEL. Ideally, the information should be accessible over the Internet.

Generally, it is the need of applications to support the manipulation of complex data that instigates the use of an ODBMS. Prototype development first required selecting an appropriate problem domain area and then developing an object technology solution to address that problem domain. The problem domain selected was resource management because this domain provided us with opportunities to potentially connect to legacy databases, demonstrate the use of an ODBMS to represent complex data relationships, and capture multimedia objects (i.e., full-motion video, audio, and images). An architecture has been established that would allow us to access the information contained in the object database from a ubiquitous browser such as Netscape.

The benefit to the INEL resulting from this project is that we explore the use of object technology to address a general information access problem faced by all major corporations and government laboratories today. This exploration has resulted in the INEL beginning to learn how to apply object technology to reduce the cost of developing and maintaining software applications over their life cycle. An initial prototype application has been developed that requires fur- ther development to market, which can occur in the next phase.

\section{Objectives}

The FY95 LDRD proposal was intended to be the first phase of a multi-year proposal/research effort.

- Develop a prototype product for accessing and managing multimedia objects.

- Perform an analysis of the object-oriented database (ODBMS) marketplace.

- Build a skill set in the ODBMS technology.

- Develop a paradigm for solving existing storage and access problems.

- Perform a market search with the assistance of ORTA to determine the probability of success for transferring or licensing the product to industry.

\section{Accomplishments}

Several ODBMS vendors were brought in house to discuss and demonstrate their products. One ODBMS vendor was selected and the procurement was issued. The procurement was delayed for approximately 6 to 8 weeks owing to indemnification issues.

No alliances were formed with universities or industry. This project did not start until March, and then only on a part-time basis, and time was insufficient to initiate alliances with universities or ODBMS vendors. However, conversations with the University of Idaho and industry did occur concerning technical collaborations. Collaborations are expected to occur in the FY-96 phase of the project.

A set of criteria was first established to evaluate potential problem domain areas and to facilitate selecting a particular problem domain area. Several potential areas were identified, and one was selected based on the evaluation criteria.

The first step in developing an object-oriented application that addresses the selected problem domain is to understand the requirements by developing a real-world model of the problem domain. Developing the analysis model involved determining the use cases or patterns of usage for the system, identifying the potential actors/entities that would interact with the system, and conducting a domain analysis. This model was developed and reviewed with potential users to ensure that the problem domain was well understood and represented a solution with which the users agree. Additional details were added to the analysis model to address how to solve the problem, forming the basis for the design model. The design model represents how the solution will be implemented to address the specific problem. Multimedia objects and capabilities were designed into the program; i.e., the ability to play full-motion video files and audio files, and to display images.

The basic design model was implemented using object-oriented programming techniques. The first prototype developed was a standalone application. Upon invocation, this prototype assembled objects by reading data from files. The objects were disassembled and written back out to files upon exiting the program. This prototype has been demonstrated to management and members of the user community who helped with identifying initial requirements. A second prototype is now under development that integrates the application with the object-oriented database. 
Our goal was to present a paper at the INEL Computing Symposium this year. The symposium has been canceled and no paper was written.

New or Increased Technical Capability

As a result of this project, the INEL has gained experience with multimedia technologies, object database, and object-oriented programming technologies. This environment was found to provide true binary portability across multiple platforms (PC, Macintosh, UNIX) without any recompiling or reprogramming effort.

\section{Business Development Opportunities}

A basic prototype of an automated resource management system has been developed. The capabilities offered by this type of system are needed in any sizable company that runs multiple projects; i.e., tracking projects, people, job requirements, and matching resources to job requirements.

\section{General User Interface Development Environment LDRD 4953}

\section{A. E. Egger}

\section{A Graphical User Interface} Development Environment (GUIDE) includes software tools to implement Graphical User Interfaces (GUIs) for any hardware platform and includes a repository of information to assist software developers in implementing GUls. The goal of the project is to establish the environment and then to demonstrate its effectiveness with sample projects.

We surveyed INEL GUI developers as part of gathering requirements, surveyed the market for tools, and, based on preliminary evaluations, recommended software tools for purchase. However, the recommendation was made several months behind schedule, and owing due to manpower shortages the requisitions for these tools were canceled as part of a company-wide LDRD spending freeze.

Sample projects were performed with tools already in-house. Human Factors staff interacted with software staff during implementation and developed a questionnaire to use in interviewing the software staff about the tools.

The written deliverables for this project include a summary of the developer survey, tool evaluations, recommendations for purchasing books and journals, a description of the tool survey that led to recommendations, and the tool evaluation questionnaire. Copies of these documents are available from the principal investigator.

On-line access to the GUIDE repository and the project results is available via the Advanced Computer Interface Products department internal World-Wide Web (WWW) page.

\section{Objectives}

- Survey INEL GUI developers.

- Survey marketplace for GUI development tools.

- Evaluate tools and end products.

- Gather information useful to GUI developers (bibliography, book list, journal list, vendor list, related URLs).

- Report LDRD results via a Web page internal to the INEL.

\section{Accomplishments}

We conducted a survey asking what GUI developers at the INEL are doing and what they want in relation to GUI tools and support. The survey revealed that many GUI developers are isolated from their colleagues but would like a way to share information and interact with other GUI developers. Using the responses to the developer survey and recommendations from ACIP personnel, we established requirements for desired GUI development tools.

We started surveying the market by purchasing the report Ovum Evaluates: GUI Builders by Ovum Ltd. Based on the vendors listed in his report, we requested information on over 100 products. We continued the survey by browsing related World Wide Web (WWW) pages, Usenet newsgroups, and Internet Frequently Asked Questions documents (FAQs). From this information we identified promising tools for further investigation.

We acquired and evaluated available tool demonstrations, and then technically evaluated seven tools. Each of the seven technical evaluations was followed by a human factors evaluation. We used the results of these evaluations to recommend GUI development tools for purchase.

The Human Factors staff on the project developed a questionnaire for interviewing the developers after the developers technically evaluated a tool. An interview conducted with a questionnaire generates a thorough evaluation of a tool. This evaluation can be readily compared to similar evaluations of other tools. We recommend the questionnaire for generating future tool evaluations and that it be used to interview a developer rather than having the developer answer the questions alone. An interviewer can encourage fully explained answers.

One Human Factors specialist reviewed three real GUIs, and a clear pattern emerged from these reviews. The specialist helped guide the appropriate use of color. He also was sensitive to proportions and patterns, especially those that cause stress to the eyes. The specialist advocated on-line context-sensitive help and enduser confirmation for any act that could cause loss of data. The most 
important lesson learned is that software developers and human factors specialists must talk in terms of root problems, not in terms of implementation.

As we gathered information, we discovered the need for an on-line GUIDE repository to keep from repeatedly gathering the same material. WWW technology offered us an implementation solution. The ACIP page, internal to the INEL, was constructed to improve project collaboration, provide an on-line version of the GUIDE repository, and provide on-line access to all GUIDE LDRD results.

New or Increased Technical Capability

As a result of the research into the tools available for developing GUIs, it is apparent that no $X$-Windows to MS-Windows translator exists. Also, none of the available platform independent GUI (PIGUI) development tools are particularly easy to learn. But two of the better tools were identified and recommended for purchase:

- Zinc from Zinc Software Inc., a PIGUI tool with a moderate learning curve.

- TeleUSE from Thomson Software, a tool for developing $X /$ Motif applications and then hosting the applications on both UNIX systems and personal computers (PCs).

INEL software engineers involved with waste management have indicated that future software work in this area will be multiplatform and client/server based. Users will access applications most often from Macintoshes and PCs. For developing applications of this sort, we recommend investigating a tool like SNAP from Template Software, Inc. SNAP is a total software development environment for creating distributed multi-platform applications.
Business Development Opportunities

A number of internal people and organizations have expressed interest in using the results from this project. They include Lynn Dean (principal investigator of the Mediator LDRD), the documentation unit in Information Resource Management, the Environmental Management Integration organization, and Gus Caffrey in the Nuclear and Radiological Physics unit.

\section{Intelligent Data Access and Retrieval System LDRD 4954}

R. K. Fink

The objective of this research project is to develop an approach for retrieving multimedia, object data from a distributed, object, database environment via an intelligent, adaptive, human interface. The basic technology that will be applied to this problem will be case-based reasoning (CBR). Case-based reasoning has evolved over the past ten years as a key artificial intelligence technique for building intelligent systems. Unlike rule-based systems, which require sets of specific rules that are chained through sequentially, casebased systems contain sets of cases, or exemplars, which are addressed by selected indices. These cases represent a set of circumstances, or criteria, and a solution, or result. The indices can be used to select cases in an efficient fashion. In addition, a similarity measure can be used to indicate closeness of fit. When casebased reasoning is used for database retrieval, the approach permits fuzzy searching, which produces inexact, but qualified, matches. Research conducted by the Lockheed Palo Alto laboratory has shown case-based reasoning to be an effective tool for building database retrieval systems.

\section{Objectives}

- Recruit a principal investigator.

- Perform an assessment of CBR development tools and select one.

- Prototype a product for accessing and retrieving data from a multimedia database.

- Demonstrate the utility of the prototype in an INEL problem domain.

- Perform a market search to determine probability of success for transferring or licensing the product to industry.

\section{Accomplishments}

With technical assistance from Mr. Ralph Barletta, an internationally recognized expert in casebased reasoning applications development, a team of four engineers and computer scientists were trained in the use of casebased reasoning technology. An introductory seminar on casebased reasoning technology was attended by approximately twenty people from the LITCO software engineering organization.

Four INEL application domains were selected for development of initial prototypes. These domains were: (1) Analysis of Site-Specific Agriculture data, (2) Personnel skills matching, (3) Robotics control architecture, and (4) Portal monitoring for weapons detection. As of mid-September, prototypes were successfully demonstrated for the first two domains, and development was in progress for the robotics control prototype. The portal monitoring prototype was deferred due to unavailability of data from the project developing the monitoring system.

A prototype system for analysis and use of site-specific agriculture data was developed, and an Invention Disclosure Record (LIT- 
PI-167) was filed for this methodology. The prototype uses a combination of inductive classification and CBR techniques to identify significant patterns in crop yield data and make appropriate recommendations to increase crop yield. The Site-Specific Technology for Agriculture (SST4Ag) Project provided a test data set for analysis purposes; live data will not be available until later in calendar year 1995 (after field harvest is complete). The prototype was reviewed and well received by the SST4Ag project manager.

The personnel skills matching prototype uses detailed employee skills information for the Software and Electronics home organization (in the Chief Engineer's directorate) to provide a rank-ordered matching of employees best suited to fill a particular project assignment. The employee skills information is maintained in a relational database, and case-based retrieval techniques are used to provide nearest-neighbor weighing against a query for a desired skill set. A modification of the standard nearest-neighbor algorithms for list matching was developed to address shortcomings in these algorithms for this particular application. The structure of the skills data is hierarchical, many-to-one (each employee has many skills) rather than a single, flat record for each employee. Successfully working with this type of case structure, in an external database rather than within a CBR development tool's internal database, represents a significant and useful advance in capabilities for this technology. The demonstration prototype has been enthusiastically received by Software and Electronics management personnel.

\section{New or Increased Technical Capabilities}

We have developed a staff capability trained and experienced in the use of case-based reasoning technology.

The CBR analysis of site-specific agriculture data has created the ability to analyze data in a manner that complements traditional statistical methods and is ideally suited to the identification of conceptually important patterns that are typically difficult to discern in a statistical model.

The personnel skills selection prototype represents a highly useful decision support tool for resource management. We intend to continue development of this capability, performing integration into a multimedia object database environment in FY-96. The integration of CBR with relational databases is an important technology step.

\section{Business Development Opportunities}

The technology represented in the case-based reasoning for sitespecific agriculture represents a significant potential area of business. Pending DOE's completion of the Invention Disclosure Record review, the details of this technology have not been discussed with outside organizations. We expect that this will result in significant CRADA and/or licensing opportunities.

The capabilities demonstrated in the personnel skills selection prototype are directly relevant for management in other corporations, and could also be used with little or no modification by employment agencies and similar operations. With appropriate modifications, this technology would also be useful as a matchmaker, or information broker, for subcontracting skilled employees, consulting organizations, or component suppliers. We have had one meeting with General Motors Manufacturing Information Services representatives in which this technology has been discussed; they expressed interest in further information and potential collaborative development.

$$
\begin{aligned}
& \text { C++ Sage } \\
& \text { LDRD } 4955
\end{aligned}
$$

$$
\text { A. E. Egger }
$$

SAGE is an INEL/US government-owned software technology that allows software developers using Ada and Modula-2 to build applications rapidly. SAGE also provides a high-performance database engine. SAGE has been available in DOS and UNIX text versions.

The C++ SAGE LDRD provided funding to build $a \mathrm{C}$ and $\mathrm{C}++$ interface to the existing SAGE Modula-2 Dynamic Link Libraries (DLLs) for programs targeting WIN32 (Microsoft Windows NT/Intel) and written in C or $C_{++}$. Documentation on using these libraries is provided in the form of an on-line Windows help system. Included in the scope of this LDRD has been a development effort for the Low-Level Radioactive Waste Tracking System written in C++.

\section{Objectives}

- Design an ANSI C interface to SAGE.

- Design a $\mathrm{C}++$ interface to SAGE.

- Implement/test the ANSI C interface.

- Implement and test the $\mathrm{C}++$ interface.

- Develop a Windows help system.

- Conduct a joint development effort using CSAGE++.

Accomplishments

SAGE is an existing, INELowned, software development technology that allows rapid development of software systems. Prior to this LDRD, users of SAGE 
technology were limited to software developers using the Modula-2 or Ada programming languages. The purpose of this LDRD was to broaden the scope and availability of SAGE to $C$ and $\mathrm{C}++$ developers. In addition, the target operating system for the SAGE development system was Windows NT or Windows 95. This effort has been very successful, and the new CSAGE and CSAGE++ tools are being used within the INEL for Windows software development. This new technology allows developers of information systems, and engineering and scientific applications to use the INEL-owned and maintained tools in four different languages, instead of only two. It also allows $\mathrm{C} / \mathrm{C}++$ developers to benefit from the black-box, reuse technology that SAGE offers.

New or Increased Technical Capabilities

The CSAGE/CSAGE++ products allow rapid application development under Windows, and they allow application developers at the INEL to develop software that requires no licensing fee or royalty. This LDRD has shown the benefit of using Windows, 32-bit Dynamic Link Libraries (DLLs) to provide a cross language development solution. In addition it has provided invaluable training for INEL software developers in $\mathrm{C} / \mathrm{C}++$ and Windows development.

Business Development Opportunities

Current PC Software tool vendors (such as Borland and Microsoft) are starting to market the idea of a callable database engine that is bundled with development tools. There is also a large market in low- or no-cost database solutions. Market analysis to determine the feasibility of commercializing CSAGE/CSAGE++ in these areas is currently under way.

\section{Fractional Frame Augmentation \\ LDRD 4956}

\section{P. R. Schweider, D. G. Larsen}

This research project is intended to develop an approach for incorporating video/audio data transmissions into existing computer network communications topologies (primarily ethernet) thus allowing video teleconferencing over installed network cable plants. Although there are commercial systems available, they have two inherent problems: (1) data flow rates are not adequate to prevent video/audio distortion and (2) irregularities caused by contention with other data packets on the network cause choppy or broke images and sounds. Initial research conducted by the INEL has indicated that by using standard data compression techniques and current network protocols (TCP/IP), it should be possible to increase data flow rates to acceptable levels. The thrust of this project will be to continue to increase data flow rates to acceptably smooth the flow of data to the screen and speaker thereby avoiding the choppy or broken transmissions. The results of this $L D R D$ will provide areas for the INEL to develop relationships with commercial industry for new or enhanced video teleconferencing products.

\section{Objectives}

- Develop a software algorithm to create stamping of fragmented data.

- Create a technique for correlated insertion of changes into existing video frames

- Optimize data transmission and evaluate data compressions

Accomplishments

Early in the project it was determined that it would not be possible to use local area network (LAN) protocols and standard compressions techniques to achieve the data transmission integrity and speed to accomplish the goals of this project. The project was shut down at that time.

\section{Development of Prompt Gamma Assay Method for Mining of Phosphate Ore LDRD 5201}

\section{J. W. Mandler, S. J. Losinski}

In a single year, this LDRD progressed from a fundamental idea, to a laboratory proof-of-principle test, to prototype instrumentation, and to a field demonstration, with such success that licensing/CRADA negotiations with industry are currently in progress. In order to enhance cost effectiveness and competitiveness, the phosphate mining industry needs improved methods and instruments for more accurately locating ore beds and boundaries at a dig surface and for rapid assay of phosphate ore in the field and at various locations in process streams. The objective of this project was to investigate the use of nuclear techniques (e.g., prompt gamma-ray neutron activation analysis (PGNAA) and gross gamma scanning) as rapid assay and ore bed locating methods. Laboratory research on phosphate ore resulted in the development of critical measurement parameters required to adapt the nuclear techniques to the needs of the phosphate mining industry. The laboratory experiments also indicated that application of these techniques to the phosphate mining industry is feasible. A field demonstration carried out at a phosphate mine showed that: (a) gross gamma scanning can be used to rapidly and accurately locate ore beds and ore bed boundaries, even in highly altered regions where visual methods fail, and (b) PGNAA can be used in the field to assay for phosphorus and other elements of interest. 


\section{Objectives}

The overall objective of this LDRD was to investigate the potential of using nuclear techniques (PGNAA and gross gamma scanning) to meet the assay needs of the phosphate mining industry.

- Pursue patent applications for use of PGNAA to assay for phosphorus and other elements of interest to the phosphate mining industry and for the use of gross gamma scanning for accurately determining ore beds and ore bed boundaries at the dig face.

- Determine experimental parameters required to adapt both techniques to meet the needs of the phosphate mining industry.

- Determine quantitative assay capabilities of PGNAA for phosphorus and selected other elements of interest.

- Demonstrate the two techniques at a phosphate mining site.

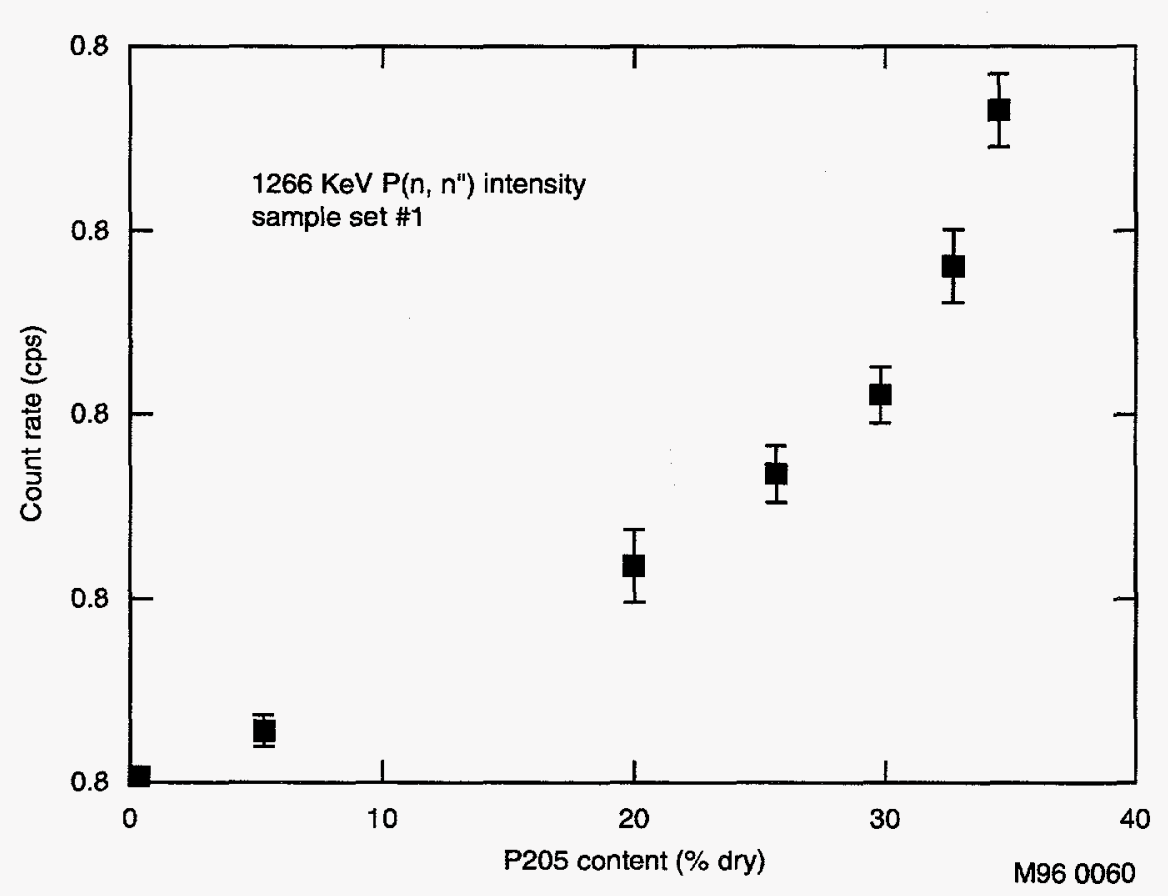

Correlation between PGNAA Count Rate and Phosphate Concentration
New or Increased Technical Capabilities

All technical objectives of this LDRD were successfully accomplished during FY-95. Laboratory research to investigate the feasibility of PGNAA and gross gamma scanning was performed at the INEL using phosphate ore samples obtained from FMC's Dry Valley Mine, located near Soda Springs, Idaho. Results were very positive and indicated that PGNAA can detect phosphorus and other elements of interest. Furthermore, correlations between gamma intensity and phosphate concentration (see the figure below for PGNAA results) indicated that PGNAA can be used for quantitative analysis. Preliminary values for experimental parameters (e.g., gamma energy regions, detector type and size, neutron source strength, count time) required to adapt both techniques to meet the needs of the phosphate mining industry were determined. A prototype gross gamma scanner was built and tested. 
tive tool and has shown that PGNAA is potentially capable of use in industrial process control.

\section{Business Development Opportunities}

As a result of this LDRD, several new business opportunities have evolved. FMC analyses have indicated potential multi-million dollar benefits associated with implementation of these techniques. Furthermore, applications outside of the phosphate mining industry also have significant business development potential. These include statistical process control, environmental monitoring, and material characterization. FMC's interest in both the PGNAA and gross gamma scan techniques have resulted in preliminary negotiations between FMC personnel (R. Conroy, manager of the Dry Valley Mine, and T. Fekete, director of technology for the Phosphate Chemicals Division), a Lockheed Idaho TTO representative ( $\mathrm{J}$. Alexander), and Lockheed Idaho research personnel (J. Mandler and S. Losinski) concerning potential business relationships. As of the date of this report, negotiations are in progress to determine the nature of this business relationship.

\section{Develop Intelligent Farm Management System Database Prototype} LDRD 5204

\section{R. L. Hoskinson, K. C. Mousseau}

The objective of this $\angle D R D$ was to research existing statistical, linear programming, and artificial intelligence systems, and to develop the interfaces between them and a Geographic Information System (GIS), and between the GIS and the relational database. The benefit of this effort is in developing the core competency in the technology necessary to interface the modules of the Information Management System for Agriculture (IMS4Ag).

\section{Objectives}

- Research present linear programming and relational database management systems software.

- Develop the relational database to store historical information.

- Research and develop an interface between the linear system and the Yield Mapping GIS model.

- Research and develop an interface between GIS and the relational database.

Accomplishments

The IMS4Ag prototype has been successfully developed and demonstrated. This system has interfaces allowing integration of relational database information with spatial database information from the GIS. Additional input system forms have been developed to provide for data input and management of non-spatial data.

The IMS4Ag will continue to be developed; it is the information management system for the SiteSpecific Technologies for Agriculture (SST4Ag) project.

\section{New or Increased Technical Capabilities}

This prototype has given the INEL's SST4Ag project a leadership position in site-specific farming technologies development.

\section{Business Development Opportunities}

This IMS4Ag has the potential to become a useful tool to major agricultural companies that are moving towards site-specific farming service and support for farmers and, therefore, has a potential for spinoff or licensing. A CRADA has been formed with the GIS company within the overall SST4Ag project.

\section{Measurement of Potato Water Content LDRD 5306}

\section{G. L. Hawkes}

The objective of this project was to develop and demonstrate in the laboratory an inexpensive, easy to use, and portable sensor for rapid measurement of water content in freshly dug field samples of potatoes. It is well known that the tuber dehydration level is the major determinant in the kind of bruising that may occur in potato handling. Bruising is the most important factor in lowering the quality of fresh potatoes and increasing rot in potato storage. This technology may be used during the growing season, or at potato harvest time, to select the best conditions, or with additional irrigation techniques, improve the potato moisture conditions. Several potential techniques for sensor development were analyzed for their merits and weaknesses. In future work, the sensor output response will be converted to a userfriendly and understandable display of optimum moisture using pass/fail indicators, or scale, depending on industry requirements. Producers such as private and corporate farmers, state extension services, consut tants, and irrigation technology companies are but a few potential industry partners.

\section{Objectives}

The objective of this FY-95 LDRD project is to develop the technology and conduct a laboratory demonstration of an inexpensive, fieldable sensor for the rapid measurement of water content in potatoes. Several potential techniques for the sensor development were analyzed for their merits and 
weaknesses. Another objective of this effort is to maintain simplicity and ease of use in the sensor design. Sensor cost must remain below approximately one to two thousand dollars, and it will operate from a small battery supply with user-friendly controls and read-out display. Several ideas, along with those generated by outside published papers on dielectric and moisture measurements, were considered.

- Closely evaluate current techniques in dielectric, soil, and grain moisture measurements, and identify any existing technology that will facilitate the conceptual design of the potato moisture sensor.

- Develop a conceptual design of the moisture sensor.

- Design and fabricate a laboratory experimental apparatus following the conceptual design.

- Test and evaluate the laboratory apparatus. Compare the experimental apparatus data with data measured by laboratory instrumentation techniques.

- Test and evaluate the final experimental design.

- Demonstrate the experimental design.

- Identify potential industry partners for technology transfer and initiate contacts.

\section{Accomplishments}

Several ideas have been considered for developing a fieldable and inexpensive sensor for measuring the water content of potatoes. Various techniques currently used for measuring complex permittivity were evaluated to determine their merits in terms of the objectives of this work. The litera- ture was searched to obtain any other ideas that might contribute significantly to the project. Moisture sensors currently marketed and used in the agricultural industry were also surveyed. It was decided early-on that a sensor that relies upon time-varying, highfrequency, electromagnetic energy provides the greatest potential for success.

A probe that relied upon the capacitance between electrodes was tested. A temperature dependence was observed, and the repeatability of measurements was poor. Sensor probes that operate on the principle of capacitance, radiation, and resonance were tried. These probes were based on coaxial transmission line, microstrip, and waveguide theory. Potato surface contact, sample extraction/preparation, and surface penetration were a few of the concepts considered.

After much time and effort, a successful test apparatus was designed and constructed, and a demonstration was performed. Instrumentation located in the NHL facility was used to collect data and perform the demonstration. The demonstration consisted of collecting sensor data from various samples of potatoes. The latest samples included freshly dug potatoes from selected fields. The sensor data was not calibrated with actual percent-by-weight water content. However, steps were taken to provide a high degree of confidence in the observed sensitivity of the technology to changes in water content. Since abundant amounts of data will be taken, various output format options were considered. As one option, a mathematical model developed by Dr. James Reneke of Clemson University was used to provide a single valued numerical output which provided a comparison to similar data collected from water. The technique appeared to work extremely well. With some modifications, the technology may provide an easy way of sending a user-friendly output to the operator. In order to protect intellectual property, the details of the final design, apparatus, and technology description will not be given here. Suffice it to say that a demonstration of the laboratory-developed sensor performance was given. It was shown that less than one percent change in the water content of a potato sample was detectable using the technology in the laboratory.

A patent disclosure was submitted and other patents may be applied for as a result of this activity.

\section{New or Increased Technical Capabilities}

This effort was extremely successful, providing needed information in order to design a prototype in future work. The ability to sense water content has been demonstrated, and the conceptual design for a prototype has been partially completed. Much was learned about the conceptual design and what will be needed in the future in order to accomplish the task of a first generation prototype for the sensor.

\section{Business Development Opportunities}

Contacts were made with Farmex, Milestone Inc., Simplot, University of Idaho, and Clemsen University. We met with the owner of Milestone, Inc., located in Blackfoot, Idaho. He expressed his desire to collaborate in a joint effort in future work. He would be willing to make a financial contribution towards the effort. However, it must be emphasized that much more work needs to take place before this company or any other will be able to use the technology. Simplot and others have made other favorable comments. 
Milestone Blackfoot is waiting for Lockheed to initiate a CRADA and to provide additional developments. A patent or multiple patents will definitely be applied for as a result of this activity

\section{Numerical Investigations of Dynamic Pressure Seals in Geologically Active Basins LDRD 5308}

\section{G. M. Shook, J. L. Renner}

A numerical model for the formation of an overpressured zone in homogeneous porous media has been developed. The model incorporates various features of the conceptual model provided by Conoco, but, has also identified relevant physical phenomena that support that conceptual model. The significant features of the numerical model are: (a) a nonuniform heat flux at the base of the domain, (b) approximately constant fluid flow in the vertical direction, (c) a methane-saturated aqueous phase flowing from source rock into the domain, and (d) a minimum in methane solubility in water. As methane-rich brine rises (from buoyancy), gas solubility reaches a minimum, and gas exsolves from solution. This reduces the relative permeability to brine. Because of the constant flux condition at the bottom of the numerical model, a decrease in relative permeability results in an increase in the pressure gradient locally (the classic definition of overpressure). This pressure seal is leaky, and brine continues to flow through the seal, albeit at higher pressures.

\section{Objectives}

- Reproduce the original laboratory experiments numerically. Data for this step was provided by Conoco.

- Develop a conceptual model from the Conoco experiments. Identify physical phenomena that would support the conceptual model at the basin scale.

- Develop a numerical model at the basin scale to determine the validity of the conceptual model at that scale.

\section{Accomplishments}

Through a literature search, evidence that supported the conceptual model was found.

Methane and $\mathrm{CO} 2$ exhibit minima in solubility in water. These mimina occur at $72^{\circ} \mathrm{C}$ and $125^{\circ}$. Converting these temperatures to depths (using typical Gulf of Mexico temperature gradients) suggests that the solubility minima occur at $2200 \mathrm{~m}$ and 4080 . These are approximate depths to known geopressure in the Gulf of Mexico; therefore, this solubility data lends support for our conceptual model.

A numerical model was developed that included data from the Conoco experiments and field observations on geopressure in the Gulf of Mexico. This data included: gas generation taking place contemporaneously at depth (i.e., an active basin), gas-saturated brine moving upward due to buoyancy, essentially constant fluid flow vertically from below the model domain, and variable heat flow at depth. The model is shown schematically in the figure below.

This model was set up and run for a simulated time of 750,000 years. In the simulation, the temperature field came quickly to a steady state condition. The effect of the nonuniform heating from

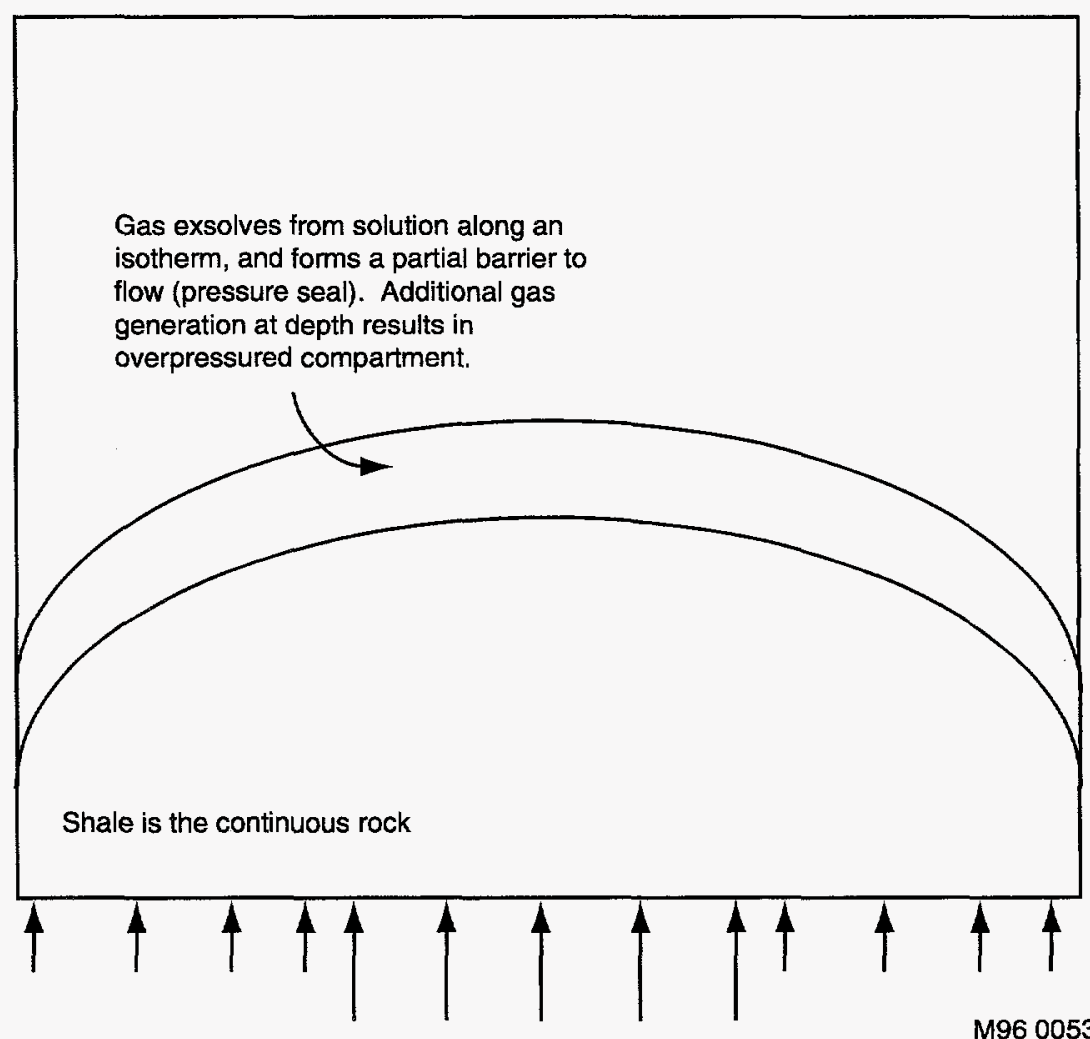

Variable heat and fluid flux from below. Heat flux is $50 \mathrm{mWm}^{2}$ on either side, $149 \mathrm{mWm}^{2}$ in the middle of the model. Fluid flux is $10^{-6}$ in the middle. Brine is fully saturated with methane.

Conceptual Model for the Formation of a Pressure Seal in Active Basins 
below is dampened a short distance away from the bottom of the domain, and temperature gradients, while not constant, are similar everywhere in the domain. This is consistent with field observations in the Gulf of Mexico.

As was expected from the gas solubility data, methane does exsolve along the $72^{\circ} \mathrm{C}$ isotherm, and gas saturations increase to about $20 \%$ by pore volume. This reduced the relative permeability to brine significantly. Because a constant fluid flux was imposed at the base of the model, this reduction in relative permeability leads to a substantial increase in pressure gradient. The pressure profile at $t=750,000$ years was calculated. Of particular interest is to consider a hypothetical, directionally-drilled well, drilled above the crest of the abnormal pressure: depending on the direction (and angle) the well is drilled, one might encounter either of two extremes in pressure gradient. If the well is drilled perpendicular to the pressure crest, the pressure gradient would exceed $40 \mathrm{kPa} / \mathrm{m}$ $(1.75 \mathrm{psi} / \mathrm{ft})$. If the well is drilled parallel to the pressure crest, a pressure gradient of zero would be encountered, even as the well is drilled to greater depth.

Hypothetical vertical well traces were calculated. These traces are pressures in grid blocks in (a) the first column of the domain (the flank of the seal), (b) a column $2 / 3$ the distance to the mid-point of the model (the shoulder of the seal), and (c) the middle column of the model (the crest). The maximum pressure gradient is on the crest of the geopressure. That pressure gradient is $9.6 \mathrm{kPa} / \mathrm{m},(1.75 \mathrm{psi} / \mathrm{ft})$ well in excess of a lithostatic gradient. However, the pressure gradient on the flank is only slightly greater than hydrostatic, consistent with upward fluid flow.

In summary, the numerical model developed on the basis of the Conoco conceptual model was shown to develop abnormal pressures. The most significant feature of the numerical model is the exsolution of methane at approximately $72^{\circ} \mathrm{C}$. In response to the increase in gas saturation, relative permeability to brine decreases. Because of the constant flux condition imposed, a decrease in relative permeability results in an increase in the pressure gradient.

New or Increased Technical Capabilities

This LDRD assisted greatly in obtaining funding for an ACTI project, and we are still in the process of identifying relevant geopressure characteristics. Of most interest in the ACTI project is an extension of this LDRD; that is, to identify the mechanism for charging a hydrocarbon reservoir within the pressure seal. Our ability to do so will greatly improve our ability to identify geopressured resources in the Gulf of Mexico; thereby, reducing our dependence on foreign oil, and significantly reducing the cost of exploration in active young basins.

Business Development Opportunities

Because of this LDRD, funding for an ACTI proposal was obtained. The ACTI project currently has four oil companies funding part of that work, as well as additional researchers at New Mexico Tech and Colorado School of Mines. They are Oryx Energy, Conoco, VASTAR, and Phillips Petroleum

\section{Evaluation of Potential Antimicrobial Properties of Malted Barley and Its Agricultural Applications LDRD 5604}

\section{K. B. Barrett, M. A. Hamilton}

This $L D R D$ responds to an agricultural necessity by developing a biological biocide used to control potato pathogens. This represents a nontraditional use of a renewable agricultural product such as barley to sustain potato production practices. In addition, the use of a biological biocide contributes to the conservation of soil and water by minimizing use of chemicals before, during, and after potato production, improves crop yields, and enhances foodprocessing technologies. The proposed project involves the development of a barley-based product that can be used to control pathogens that cause considerable damage to potato crops. To date, there are no registered antibacterial agents that can be applied to control the detrimental effects of potato pathogens. If the antimicrobial properties of malted barley can be successfully characterized and developed, storage life of seed potatoes or commercial potatoes will be markedly improved.

This research identifies an antimicrobial, barley-based product that can be used to control pathogens that cause considerable damage to potato crops. It is estimated that approximately $\$ 5$ million are lost to soft rot annually in Idaho. Idaho produces approximately $30 \%$ of the gross potato crop in the United States; therefore, the economic impact is considerable. The antimicrobial product could be used to protect potatoes from the effects of soft rot while in storage, while being cut for planting, or during process operations. This technology, to date, includes no credits for application to other agricultural crops or for development of related products.

\section{Objectives}

- Evaluate the antimicrobial effects on a variety of potato pathogens such as Erwinia and Silver Scruff.

- Design and conduct experiments to evaluate the antimicrobial efficacy of malted barley.

- Determine the effects of physical variables such as moisture, 
temperature, and oxygen concentrations on the antimicrobial properties associated with malted barley.

- Conduct experiments to evaluate the longevity of the antimicrobial product with emphasis on the feasibility of commercial applications.

\section{Accomplishments}

Prior research conducted at the INEL has revealed that malted barley has striking antimicrobial properties. Experiments were designed, in conjunction with the University of Idaho Agricultural Extension Office, to assess the competence of the barley-based product. Several fungal strains, including Silver Scruff and two bacterial strains (Erwinia carotovora and Erwinia atoseptica) were evaluated. A rapid procedure for determining the degree of sensitivity or resistance to the product was used to screen the potato pathogens described above. Definitive zones of inhibition were characteristic for the Erwinia strains. The fungal cultures were resistant to the product. To further evaluate the efficacy of the product, in situ box tests were set up. Russet Burbank seed potatoes were cut and infected with both of the Erwinia strains and subsequently treated with the product, then placed in potato boxes $(12 \times$ 19 inches) containing $13 \mathrm{~L}$ of a special soil-compost mixture. The components were watered and moisture content measured. The potatoes were incubated at ambient temperature for approximately four days, at which time the seed pieces were dug and measured for degree of rot. The experiment was set up using uninoculated controls and four replications of each subset. After recording the degree of rot, the results were analyzed sta- tistically; results indicate a significant improvement (less rot) for the potatoes that had been treated with the barley-based product.

Time constraints allowed only one screen to address the longevity of the product. A previously prepared extract of the product was refrigerated at $4^{\circ} \mathrm{C}$ for two and a half weeks, at which time an in situ experiment similar to the one described above was performed. Results were statistically analyzed. They indicated a decrease in the efficacy of the product. More research is needed to optimize storage requirements for maintaining maximum efficacy of the product.

\section{New or Increased Technical Capabilities}

This research has broadened and improved the capability of applied biotechnology for the INEL. A professional working relationship has been established with the University of Idaho Agricultural Extension Office, local potato growers, and Bush Agricultural Resources Inc., located in Idaho Falls, Idaho, and Fort Collings, Colorado. We expect that the INEL will be a resource for the agricultural communities in developing methods and products to abate or control plant pathogens.

\section{Business Development Opportunities}

The successful development of environmentally friendly biological biocide is the only way to control the detrimental effects of pathogens in food products such as potatoes. A spin-off company could be formed to produce and market the developed antimicrobial product. In addition, further development of the product could be funded through co-operative research and development agreements (CRADAs).

\section{Biological System for Organic Acid Removal with Minimal Effluent \\ LDRD 5606}

\section{R. S. Cherry, K. B. Barrett}

We have demonstrated, at the laboratory scale, a novel process for removing organic acids from organic liquids such as petroleum refinery or chemical plant intermediate streams. This process could replace the current once-through use of sodium hydroxide solutions that are now disposed of by deep-well injection. Because deep-well injection is likely to be banned in the foreseeable future, many chemical companies are seeking an alternative, but no current technology is fully acceptable. This new process uses alkaline solution to extract the acids; bacteria tolerant of this solution convert the acids to carbon dioxide, allowing the solution to be recycled many times. The amount of liquid that must be purged is greatly reduced, both in quantity and in difficulty of disposal, and this process can be retrofit into the existing process equipment.

\section{Objectives}

- Order alkalophilic bacteria from culture collections and isolate mixtures of them from natural samples.

- Characterize the bacteria's ability to degrade mixtures of formic, acetic, and propionic acids.

- Demonstrate simulated closed-loop, continuous operation extracting acids from hexane.

\section{Accomplishments}

The first effort in this research was to screen a variety of alkalophilic bacteria for their ability to rapidly degrade a mixture of formic, acetic, and propionic organic acids, a mixture represen- 
tative of chemical plant xidationo by-products. Various strains were aerobically cultured on acetic acid, an acid commonly metabolized by bacteria, to see which best tolerate the conditions of the extraction medium. A variety of species were tested, including several salt-tolerant Natronobacter species obtained from Professor Yuderian at the University of Idaho, two alkalophilic species obtained from the American Type Culture Collection, and a consortium of unidentified species cultured from dirt taken from a rhubarb patch (rhubarb produces large amounts of oxalic acid, a related small acid). Sulfate-reducing bacteria were also to be tested, but were decided against after realizing that, although they would consume acids, they would produce hydrogen sulfide as a noxious product. The rhubarb consortium and Bacillus alcalophilus halodurans (ATCC 27557) both grew well on acetic acid at high $\mathrm{pH}$. They were then weaned onto a mixture of $3.75 \mathrm{~g} / \mathrm{L}$ each formic, acetic, and propionic acids in a minimal salts medium to verify that those are each consumed. After brief initial work, the B. alcalophilus halodurans and the rhubarb consortium were mixed to produce a new consortium for all future work.

The second stage of work was to grow this consortium in continuous stirred tank reactors (CSTRs) to measure their rates of conversion of formic, acetic, and propionic acids under various conditions. Five parallel CSTRs were set up and run under various conditions of temperature and residence time. In all cases the feed contained $3.75 \mathrm{~g} / \mathrm{L}$ of each of the acids, titrated to a standard high $\mathrm{pH}$. The consortium had a broad temperature tolerance, growing best at $40^{\circ} \mathrm{C}$ but doing acceptably well at all temperatures between
30 and $50^{\circ} \mathrm{C}$. All three acids were degraded in parallel, with formic having conversions near $100 \%$, acetic in the range of 50 to $90 \%$, and propionic in the range of 10 to $30 \%$. There was some correlation between the conversions of the three acids, so if the conditions were good for the conversion of one, the other two were converted well also.

The final stage of work on this project was to demonstrate that the bacterial consortium could operate in conditions similar to those of the process. Hexane solvent was loaded with formic, acetic, and propionic acids as a simulated process stream to be treated. Spent fermentation broth from one of the previous studies was used to extract the acids from the hexane in a batch operation. The broth was then fed to a CSTR to mineralize the acids. The hexane was again loaded with acids, and once the extraction broth had been completely fed through the CSTR, it was used, complete with suspended bacteria, to extract more acids from the hexane. This was repeated five times, with no unusual problems with bacterial viability or, importantly, with formation of an emulsion in the batch extraction step despite the continuing presence of bacteria and the many possible molecules they contain that have surfactant properties.

Overall, this work successfully demonstrated that this process concept can work and that further work can be justified.

\section{New or Increased Technical Capabilities}

This project has extended INEL's microbiological experience into the area of alkalophilic bacteria. Also, it has given some microbiologists an introduction to the preliminary stages of process $R \& D$, as distinct from classical microbiology.
Business Development Opportunities

Caustic washing, or extraction of organic acids from an organic stream in a chemical plant or a petroleum refinery into an aqueous solution of sodium hydroxide, is a commonly used unit operation in these industries. (Nuclear materials handling does not require processing many organic liquids so this process operation is not found at DOE sites, despite its general utility and ubiquity.) The spent caustic is a hazardous, aqueous waste of $\mathrm{pH} 12-13$ containing 1 to $3 \mathrm{wt} \%$ sodium hydroxide and the sodium salts of neutralized acids such as formic, acetic, and benzoic acids and phenol. It is typically disposed of by injection into deep-wells and constitutes a large fraction of the 6.6 million tons of hazardous waste disposed of this way in 1989 (National Biennial RCRA Hazardous Waste Report, EPA, February 1993). The expectation in the industry is that this option will not be allowed much longer. However, no alternative ways of disposing of this stream, including incineration, plasma arc treatment, and dilution into existing waste treatment plants, look especially promising, either technically or economically. This biological process, with further development, should be of interest to these industries.

\section{Isolation of Gossypol Degrading Bacteria LDRD 5609}

\section{T. E. Ward}

The goal of this project is to isolate bacteria capable of degrading gossypol, a toxic compound present in cottonseeds, and eventually to develop a process for removing gossypol from cottonseed meal using these bacteria. Cotton is one of the 
main natural-fiber inputs of the American textile industry. Cotton growers seek to maximize their financial return and use as much of their plant crop as possible. Thus, cottonseed oil and an animal feed meal are produced from cottonseeds. A limitation on these products is the toxic compound gossypol. Cotton growers seek methods to reduce or eliminate gossypol, either in the seeds before harvest, or in the processed products. Gossypol-degrading microorganisms, or enzymes isolated from them, could be used in the processes of manufacturing value-added products, especially cottonseed meal, from cottonseeds. Alternatively, and longer term, genes specifying enzymes for gossypol degradation could be isolated and introduced into cotton plants, leading to destruction of gossypol in the seeds. This is a specific goal of Cotton Incorporated. This research will strengthen the economic position of U.S. cotton growers. Removal of the gossypol from cottonseed meal would significantly increase the value of this secondary product, which is going to be produced anyway, and strengthen the growers' overall financial position. This work supports the Agriculture Initiative and the AMTEX/Textiles Initiative at the INEL, and should lead to patentable and/or licensable technology and a royalty stream.

\section{Objectives}

- Obtain environmental samples from various sources.

- Develop assay procedures for gossypol-colorometric, scanning of spectra on the spectrophotometer, or HPLC.

- Search for microbes able to use gossypol as a sole source of carbon and energy.

- Alternatively, identify potential gossypol-degrading microbes by zones of clearing around colonies on plates containing gossypol.

- Characterize the gossypoldegrading activity in candi- date bacteria-investigate the effects of variations in medium, strain, length of incubation, presence of potential inducers, etc.

- Quantify gossypol remaining after incubation with cells under these various conditions.

Accomplishments

The first assay for gossypol investigated was a colorometric assay for phenols using the Gibb's reagent, 2,6-dichloroquinone-4chloroimide. This yielded strong color development with gossypol, but was not specific enough, and reacted with a number of compounds present in microbiological medium. Thus, it was not suitable for our purposes. Extracting the gossypol remaining after incubation with cells and taking the spectrum with a spectrophotometer showed promise. There was no interference from either bacterial medium or cells, and the height of the peaks did decrease under certain conditions (as expected), but the spectra still represent the sum of all molecules present in the sample. This is a particular problem when inducers are added, since they tend to be aromatic, organic molecules that are extracted with the gossypol and contribute to the UV spectrum. HPLC is the most definitive analysis technique, since it separates the sample into its individual components.

The first samples containing potential gossypol-degrading microbes were obtained from a cottonseed oil mill where the material had been exposed to crude cottonseed oil containing gossypol. Microorganisms were isolated from these environmental samples and inoculated under a variety of conditions. We first searched for microbes that could use gossypol as a sole source of carbon and energy. This was done by incubating cells from the samples in both liquid and solid defined medium containing gossypol as the only source of carbon and energy. Any cell that is able to grow under these conditions should be able to degrade gossypol. Unfortunately, no isolates that grew reliably under these conditions were ever identified.

A second set of samples containing potential gossypol degraders was then obtained. These samples came from the intestines of pink bollworms, which are insect pests able to eat cottonseeds containing gossypol. The hypothesis is that their tolerance of gossypol, which is toxic to most insects, might be due to the presence of bacteria in their digestive systems that can degrade or otherwise inactivate gossypol. We also adopted an alternative strategy for searching for potential gossypol degrading microbes. This involved searching on rich, solid medium for colonies that were surrounded by zones of clearing of the yellow, translucent gossypol, which would indicate degradation, or at least removal, of the gossypol. Bacteria producing such zones of clearing were identified, grown up, and used in quantitative gossypol degradation experiments, in which cells were incubated in growth medium with a defined amount of gossypol under various conditions. Variables included the length of the incubation, various strains, various media, different concentrations of gossypol, and the presence or absence of various potential inducers (compounds that activate degradation genes). The gossypol remaining was analyzed using HPLC. Under the best conditions of strain, medium, and inducer, up to $50 \%$ of the gossypol originally present could be degraded in a one week incuba- 
tion. (See the figure below: $\mathrm{A}=$ control, no cells; $\mathrm{B}=$ Strain 5 , no inducer; $C=$ Strain 5 , with inducer; $\mathrm{D}=\mathrm{Strain} 3$, with inducer).

New or Increased Technical Capabilities

Bacteria capable of degrading gossypol, which should benefit cotton growers nationwide and provide licensable technology for LITCO, have been isolated. We have increased our expertise in isolating, identifying, and studying bacteria that can degrade complex organic molecules, and have developed techniques and capability in analyzing such molecules using HPLC. In addition, a staining procedure that discriminates between live and dead cells, which was initially used on this project, has found very wide use in other projects in biotechnology.

\section{Business Development Opportunities}

This project supports the Agriculture Initiative and the Textiles Initiative at the INEL, as this research should improve the financial position of cotton growers. Extensive discussions have been held with Dr. Norma Trolin- der of South Plains Biotechnology, Inc. She is interested in genetically engineering cotton to produce gossypol-free seeds. Genes isolated from one of these bacterial strains would be well suited for use in her work. Since other researchers at the INEL are already collaborating with her, we should also be able to collaborate with her on this work. Cotton Incorporated is also very interested in this line of research. They are funding Dr. Trolinder. We might be able to get funding from them directly, or they might be able to influence AMTEX to provide funds to us.

\section{System for Growing Conifer Embryos LDRD 5615 \\ R. S. Cherry}

This project continued the development of an automated system for culturing plant embryos into plantable seeds or seedlings. INEL worked cooperatively with Weyerhaeuser

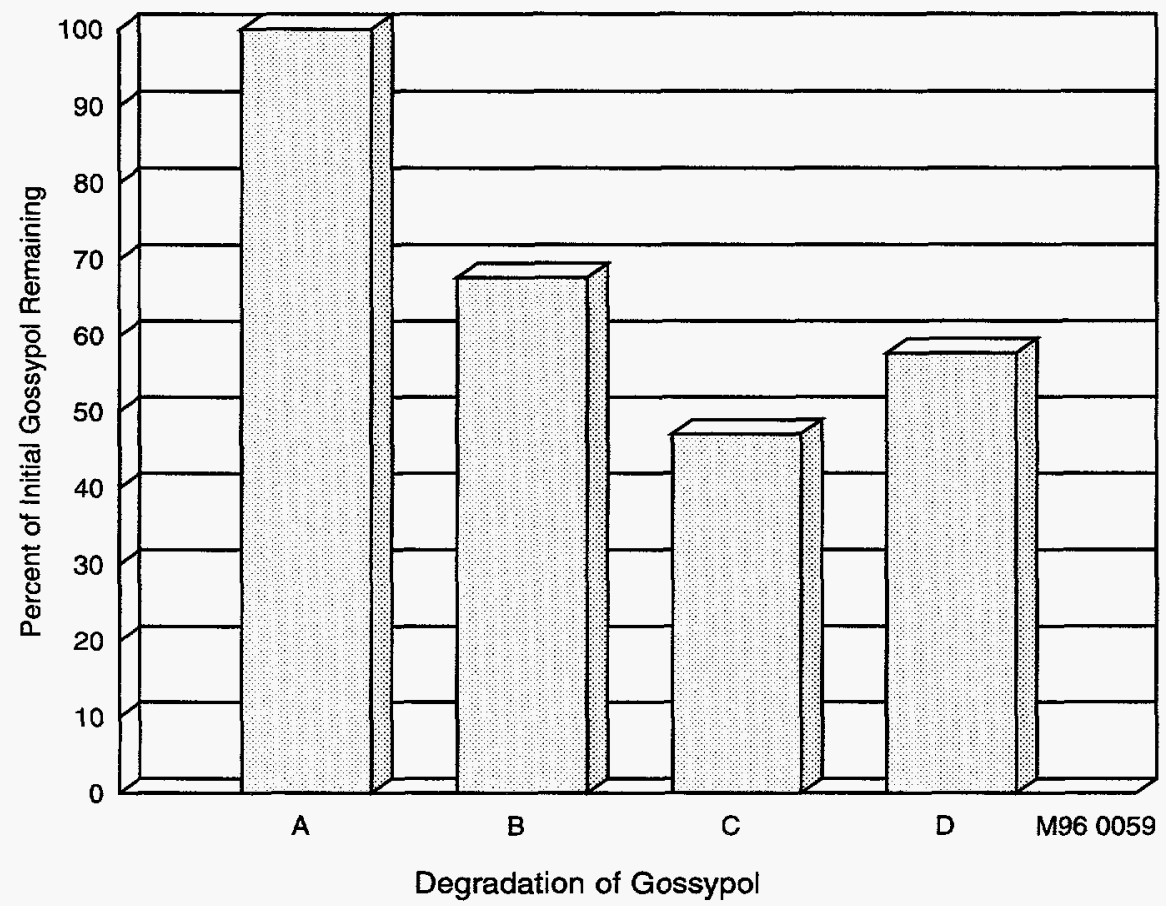

Company to do Douglas fir embryos, though the technology will be broadly applicable. The approach was to build a computer-controlled, bioreactor system to supply the embryos with the nutrient and hormonal signals they need for normal development. Two devices were built: one for suspension culture of embryos, the other for the later plating stage when the embryos grow only if not immersed. The result of this work will be a device and procedure that can be applied to many other plant species, either as a development tool for cloning better strains or as a production tool for reproducing large quantities of plants such as Douglas fir.

\section{Objectives}

- Develop a bioreactor system that incorporates Weyerhaeuser's operating conditions and allows the time-dependent adjustment of nutrient and hormone levels to control the development of early-stage embryos in suspension culture.

- Develop a second bioreactor with similar capabilities, but this one will be for growing early-stage embryos on a solid surface to form fully differentiated, mature embryos capable of germination.

- Deliver both bioreactors to Weyerhaeuser for testing over the next 1 to 2 years.

\section{New or Increased Technical Capabilities}

To improve the culture system over that built in the last year for use with wheat at Utah State University, we designed and tested an electrolytic approach for controlling $\mathrm{pH}$ in the system. One inert, gold electrode is placed in the growth chamber, the other in an external tank connected to the chamber by a salt bridge. By passing a current through the electrodes, water in the growth medium is electrolyzed to form 
hydroxide ions at one electrode and protons at the other. These ions respectively raise and lower $\mathrm{pH}$, so by adjusting which way and how often current flows, the $\mathrm{pH}$ in the embryo chamber can be controlled without the addition of external fluids.

The first major accomplishment was to build a bioreactor system for suspension cultures that will automatically adjust the physical conditions and the nutrient and hormone concentrations on a predetermined schedule to mimic the changing hormonal environment of an embryo in ovulo. This bioreactor system has four stirred culture vessels of $500-\mathrm{ml}$ volume each, with dissolved oxygen, $\mathrm{pH}$, and hormone addition control on each one. A single computer will control them and collect and log operating data. These vessels were custom fabricated by a glassware vendor (Bellco)

The second task was to build another bioreactor to grow the second stage embryos to maturity. These embryos are 2 to $3-\mathrm{mm}$ cubes of tissue that grow on plates in an environment where the hormone concentrations are quite dynamic. The development of this chamber required greater interaction with Weyerhaeuser to understand the particular conditions that Douglas fir embryos require to grow well. We have incorporated some of their methods into this device, so it will be able to better implement the procedures that are known to work well.

New or Increased Technical Capabilities

Working with Weyerhaeuser has provided the Biotechnology group at INEL with greater experience in what is required to successfully grow plant embryos. This experience will be useful, as this device is applied to other crops such as wheat, cotton, etc
Business Development Opportunities

During this year we began CRADA negotiations with Weyerhaeuser, but ultimately decided that at this point a cooperative testing program done under a nondisclosure agreement was adequate. A future CRADA is highly likely. In addition, it appears that this device will be useful in other work being done by INEL on producing genetically engineered cotton.

\section{Improved Transformation and Regeneration Techniques for Cotton} LDRD 6111

$$
\text { J. R. Hess }
$$

AMTEX industrial partners are strongly encouraging DOE to direct resources into cotton biotechnology, with their highest priorities aimed at improving efficiency of transformation and regeneration systems. Genotype restrictions prevent conventional culture procedures from regenerating many varieties of cotton. In the absence of an effective regeneration system, genetic transformation becomes more an academic than a practical approach to cotton improvement. Sophisticated in vitro systems that control the environmental and physicochemical parameters for the regeneration and multiplication of very young and genetically instable somatic embryos were constructed by previous years' LDRD efforts.

These systems have been tested, with results indicating that precise control of physicochemical parameters can be achieved, including hormonal profiles, toxin removal, and basic nutrient levels of the in vitro system. Cotton germplasm was grown and collected from field sources for final analysis of essential physicochemical parameters necessary to define medium and growth condition parameters for the in vitro system, and culture experiments are in progress to develop enzymatic iso- lation procedures of fertilized and unfertilized egg cells for culturing. Completion of the analyses and isolation/culture system design are very near (target date December 1995), but clear funding paths to completion are currently uncertain.

\section{Objectives}

- Produce germplasm.

- Analyze physicochemistry.

- Evaluate culture system.

- Test control hardware.

Accomplishments

Cotton bolls of commercial HS varieties were produced in Lubbock, Texas in field research plots. Bolls at various stages of development were harvested and shipped via overnight delivery to Utah State University for embryo extraction. Excised embryos were cultured and prepared for physicochemical analysis. Harvest of 1995 cotton germplasm was completed in late September 1995.

Using material collected in FY-94, preliminary analyses were completed, which suggested that (a) physicochemical mineral levels are similar to other systems (e.g., wheat); (b) Arabinose, Sucrose, Myoinositol, Glucose and Fructose are present at physiologically significant levels; and (c) Arabinose levels are several orders of magnitude higher than expected, which may be important since Arabinose is essential for cell wall formation. Hormones and amino acids analyses, along with detailed analyses of minerals and sugars, require large amounts of tissue grown under uniform conditions. These analyses will be completed with the FY-95 field grown germplasm, though funding to complete the analysis of the material produce in FY-95 is uncertain.

Several culture media systems/formulations were tested with cotton embryos to establish 
baseline culture responses to conventional and new culture systems. Tobacco phloem media regenerated 14-17 DAF embryos with large leaf growth and very little root growth. Plants did not survive transplanting. One-half strength Wheat-1 (formulated from physicochemical analysis of wheat endosperm) showed the best regenerative growth of embryos, but less than acceptable root growth. Plants survived transplanting and grew into $2-\mathrm{ft}$ plants before termination.

Murashige and Skoog plus IAA and Kinetin was most effective at promoting root growth. Improved root growth enhanced the regenerate plants' survival of transplanting. Plants survived transplanting and grew into 2-ft plants before termination. Accurate and nondamaging isolation of egg cells continues to be essential. Enzymatic hydrolysis of surrounding tissue for the purpose of isolating cotton egg cells is most promising, as this system has been proven to work with maize. Development of egg cell isolation and culture procedure is in progress, though funding to complete this work is uncertain.

Control hardware has undergone preliminary testing (e.g., to ensure sterility can be maintained, to test handling procedures and protocols, etc.). The control systems, including hardware and software, have been tested and found functional, and many improvements have been made to all systems. Efforts to date have identified several system design features needing development, including systems to remove phenolics, which cause tissue browning and death; tissue attachment mechanisms; and enhanced microenvironment control.

New or Increased Technical Capabilities

This work is very much aligned with the INEL strategic thrust areas, keeping in mind that the objectives of this project are to build fundamental tools for plant transformation and regeneration. Once the tools are proven effective, engineering plants for phytoremediation, water conservation, and reduced usage of fertilizers and chemicals to mention a few, will have a more significant positive impact on the environmental, energy, and economic competitiveness goals of INEL and the Department of Energy than any engineering solution conceived of to date. This project has significantly improved technical capabilities and expertise at INEL. The opportunity to further strengthen these capabilities through hiring new professionals was proposed in the FY-96 LDRD proposal.

\section{Business Development Opportunities}

We expect that through immediate continuation of this work, INEL and partners will benefit through licensing and royalties from transformation and regeneration technologies not only for cotton, but also for other commercial crops as well. Once the regeneration tools only are proven to work (target date December 1995, early 1996), funds in CRADAs are expected to be developed with Cotton Incorporated and/or AMTEX. Companies including Monsanto, Cargill, Delta Pine, Pioneer Hybrid and others are also interested in the technologies being developed in this project. As a result of this project being successful, funds in CRADAs are very likely with each or all of these companies to develop specific applications for the transformation and regeneration technologies. A sweeping patent on robust genetic engineering and tissue regeneration tools would put INEL and its partners in the position of granting licenses and receiving royalties on numerous applications ranging from the engineering of gossypol free cotton seed proposed herein to general crop improvement via genetic engineering. In a recent issue of "Genetic Engineering News" (July 1995), experts estimate the potential value of genetically engineered crops to exceed $\$ 500$ billion in North America alone and nearly three times that world-wide.

\section{Compressed Gas Safety Valve LDRD 6808}

\section{J. M. Hubbell}

This LDRD focused on designing, building, and testing a compressed gas safety valve to prevent the rapid decompression of compressed gas cylinders if the valve were broken off. Several working demonstration safety valves have been built. This device replaces the typical cylinder valve assembly on standard compressed gas cylinders or is an addition to existing valves. Compressed gas cylinders are used in nearly every industry with over 150 million gas cylinders in use in the United States. It is estimated that there are two cylinder accidents a day in the United States; however, exact numbers are not available. Current designs of valves for compressed gas cylinders do not incorporate safety features to shut off the valve should the valve be broken off. There is a great need in the compressed gas industry to produce devices that are safer than those currently available. Use of this valve may ultimately reduce stringent transportation requirements for the cylinders. Development of the safety valve may result in hundreds of new jobs in the manufacturing industry.

\section{Objectives}

The overall objective of this project was to develop a compressed gas safety valve. 
- Complete the engineering and design of the most promising compressed gas safety valve(s).

- Fabricate demonstration valves. Choose the best design(s) for the safety valve and have several fabricated for testing.

- Laboratory test the demonstration safety valves to refine the design. The demonstration valves were to be tested at low pressure in the laboratory and final versions at high pressure in the field.

- Prepare and submit a patent.

- Prepare a final report and contact industry representatives for licensing potential.

- Pursue joint cooperative efforts with industrial partners (CRADA).

\section{Accomplishments}

Funding for the compressed gas safety valve (CGSV) was eliminated at midyear FY-95 but $5 \mathrm{~K}$ was added to the budget to allow closeout of the project. Two designs of the CGSV were refined following tests in FY-94. One of these designs was used to build a prototype CGSV, which was successfully tested in the laboratory at low and high pressures. The second prototype design was built but not tested. Some refinements to this design are required before pressure testing.

The Technology Transfer Office rescinded its election to retain title to this invention due to insufficient programmatic interest. A patent search was conducted, indicating that this design is not covered by existing patents. The INEL-designed safety valve is simpler to construct than the other designs and incorporates several safety features, making this a bet- ter product than those covered by other patents. A patent application has not been filed. Task 5 was not completed because funding was discontinued.

An advertisement was placed in the Commerce Business Daily seeking a partner to work with the INEL on development and commercialization of the CGSV. Two companies replied to the advertisement. Discussions were ongoing with one of the firms as of October 1995.

\section{New or Increased Technical Capabilities}

The INEL has intellectual property rights for these compressed gas safety valve designs. Additional work has not been pursued because of differences in this work and the mission of the INEL. A patent will probably be filed by the DOE office in Chicago.

\section{Business Development Opportunities}

The INEL has made contacts with a patent holder for a compressed gas safety valve. The Compressed Gas Association is not interested in pursuing development of the safety valve at this time, presumably for liability reasons. This valve has a large potential for business development should the regulatory climate change, calling for additional safety measures for the use and transportation of compressed gas cylinders. The compressed gas industry needs to produce valves that enable cylinders to be used and transported more safely than those currently available. Unfortunately, data is not available to quantify the number of accidents involving gas cylinders. Since there are over 150 million compressed gas cylinders in operation in the United States, there is an extremely large potential market ( $\$ 2$ billion market). Most compressed gas cylinder users that we have discussed this concept with have shown interest in the valve, and have indicated that if this safety valve were available for their use, they would be willing to pay additional money to put this valve on their cylinders. Development of the safety valve may result in hundreds of new jobs in the industry manufacturing replacement valves.

\section{Internal-Reforming Solid Oxide Fuel Cell Thermo- Chemical Modeling LDRD 7105}

\section{G. Motloch}

Fuel cells offer high operating efficiency for electric power generation and are environmentally benign. The primary advantage of Solid Oxide Fuel Cells (SOFCs) is that it has no liquid electrolyte with its attendant material corrosion and electrolyte management problems. Also, the solid-state character of all SOFC components results in fewer restrictions on the cell configuration. However, no general-purpose SOFC analysis tool currently exists. Consequently, research and development of a cross-flow, internal-reforming SOFC computer model has been underway for 18 months at the Idaho National Engineering Laboratory (INEL). A one-dimensional model is being completed and extensions to two and three dimensions are planned. Efforts have focused on developing a thermo-chemical model that will help determine the scope and design of the INEL SOFC to address key questions about its performance. These include the calculation of the SOFC thermal distribution, fuel and electrical efficiency, and whether the SOFC becomes thermally quenched. Mass, heat, electrochemical, and chemical kinetic balance equations have been developed to represent the dynamic processes that occur within the SOFC. 


\section{Objectives}

The work is centered on two aspects. The first is to advance the state of SOFC computer modeling, and the second is to characterize the INEL SOFC behavior using the new model. The INEL SOFC model will calculate thermal and mass flow distributions throughout the SOFC; this includes the fuel and air channels and within the interconnect. It will also include full multi-region, multispecies diffusion in the porous anode, electrolyte, and cathode. The INEL SOFC model explicitly details each of these fuel cell regions. This is necessary to accurately model the reforming that occurs within the interconnect and anode in the INEL SOFC. This affects the rate-determining steps and the evolution and distribution of product species and heat. Explicit modeling of each of the species is also important because the interactions between them affect their diffusivities, which in turn affect the dynamic mass, energy, and electrochemical processes.

Constitutive models include (1) steam-methane reforming in the interconnect and in the anode, (2) oxidation of $\mathrm{H} 2$ and $\mathrm{CO}$ within the anode, (3) Joule heating within the solid structures, (4) electrical polarizations, and (5) electrical and thermal performance. Also, the model features temperaturedependent material properties including (6) heat capacities of $\mathrm{CH}_{4}, \mathrm{CO}, \mathrm{CO}_{2}, \mathrm{H}_{2}, \mathrm{H}_{2} \mathrm{O}, \mathrm{N}_{2}$, and $\mathrm{O} 2$; (7) thermal conductivities of air, fuel, $\mathrm{NiAl}, \mathrm{Ni}-\mathrm{ZrO}_{2}, \mathrm{ZrO}_{2}$, and $\mathrm{LaMnO}_{3}$; and (8) heat transfer coefficients for air and fuel. Additionally, (9) the Gibbs Free Energies and (10) the heats of formation are calculated for $\mathrm{CH} 4, \mathrm{CO}, \mathrm{CO}_{2}$, and $\mathrm{H}_{2} \mathrm{O}$; and (11) the relatives enthalpies are calculated for $\mathrm{CH}_{4}$, $\mathrm{CO}, \mathrm{CO}_{2}, \mathrm{H}_{2}, \mathrm{H} 2 \mathrm{O}, \mathrm{N}_{2}$, and $\mathrm{O}_{2}$.
The model input, output, and physical functions consist of a significant number of thermodynamic variables.

The technical objectives thus include completion of the thermochemical computer model of the INEL internal-reforming solid oxide fuel cell stack, incorporating the features described above. The model will then be used to address issues vital to INEL SOFC development, which include (1) whether partial or complete quenching of the cells occurs as a result of the internal reforming; (2) the practical limits of fuel utilization; (3) the advisability of alternative schemes for manifolding the stack; (4) characterization of test data from the SOFC stack demonstration funded by the Advanced Research Projects Agency (ARPA); and (5) analysis of various other internal-reforming SOFC system concepts, such as partial oxidation.

\section{Accomplishments}

A single-channel, coflow model of the SOFC air channel and fuel channel and the interconnect with steam-methane reforming has been completed. It enables the calculation of temperature, mass flow, and power distributions throughout those regions in the SOFC. The figure shows a typical SOFC cross-flow stack from which a mathematical SOFC unit cell is constructed, which is the building block for the SOFC model. A peer-reviewed paper based upon recent accomplishments titled Thermo-Chemical Modeling of Internal-Reforming Solid Oxide Fuel Cells at the INEL has been presented at the SOFC-IV Conference held in Yokohama, Japan in June 1995. Also, a copyright application has been submitted for the computer code.
New or Increased Technical Capabilities

The present model provides the new capability to calculate temperatures, mass flows, and power distributions within the SOFC based upon an assumed current density. Further model development will enable the calculation of all of these plus electrical and thermal performance based upon first principles, with very few simplifying assumptions. This will enable the performance of design and scoping studies to help optimize the INEL fuel cell. Additional enhancements would make the model applicable to other SOFC designs, as well.

Business Development Opportunities

Beyond using the model to support internal INEL SOFC design, a number of organizations have expressed an interest in the possibility of generalizing the model to their particular SOFC designs. The principal investigators at these organizations are Dr. Sunil Wijeyesekera, research leader in the Ceramics and

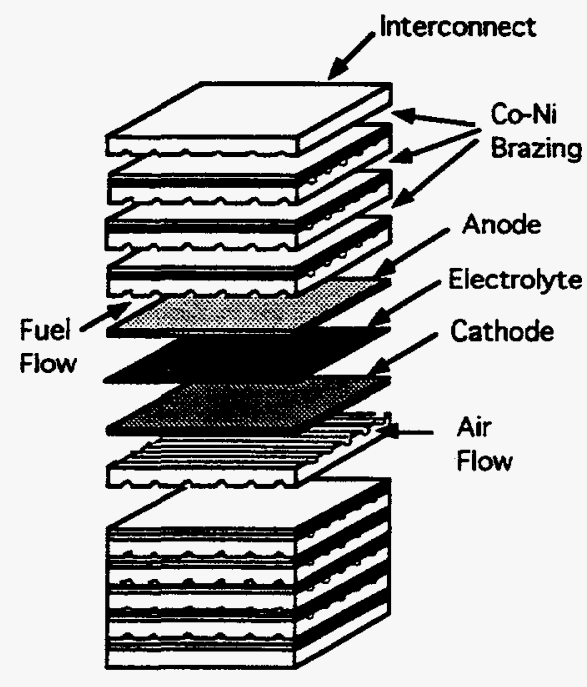

INEL SOFC Stack 
Advanced Materials Group at the Dow Chemical Company in Midland, Michigan; Dr. Harry Tuller, Professor of Ceramics and Electronic Materials in the Department of Materials Science \& Engineering at the Massachusetts Institute of Technology; and Dr. Harlan Anderson, Curator's Professor of Ceramic Engineering at the University of Missouri-Rolla.

Also, Dr. Ulf G. Bossel, a fuel cell consultant in Baden, Switzerland, has developed a bench-top $1 \mathrm{~kW}$ SOFC system. A three-party nondisclosure agreement is being prepared between INEL, Thermo Technology Ventures, Inc., and Dr. Bossel to investigate further development and commercialization of his SOFC prototype.

\section{Advanced Methods for Adaptive Control for Automated Traffic Management} LDRD 7203

\section{H. B. Smart, R. K. Fink}

The major objective of this LDRD is to apply INEL expertise in adaptive control methodologies to traffic management applications in order to expand the business base in federally supported R\&D in this area. Development of real-time adaptive control methods and techniques is being funded as specific $R \& D$ projects and operational tests as a part of the Intelligent Vehicle Highway System program. Several concepts will be explored in a two-phase approach applying neural networks, fuzzy logic, and symbolic rule-based adaptive control schemes to the real-time tratfic pattern recognition and control of green timing signals. Phase 1 will look at the methods and determine the feasibility of practical application using simulation and system analysis tools. Phase 2 will implement a field test on a small system. Collaborators on the project will be Idaho State University, the Ada County Traffic Management District, and the University of Idaho.

Objectives

- Obtain appropriate traffic network simulation software and install on INEL computer system-January 1.

- Define controller requirements, formulate architecture, and generate rules for intersection control-March 1.

- Conduct comprehensive literature review of application field-April 1.

- Investigate the application of artificial neural networks and fuzzy logic to the problem of traffic flow recognition-June 15.

- Form controller algorithmJuly 15 (Deliverable 1).

- Evaluate controller algorithmSept. 15 (Deliverable 2).

- Submit abstract/manuscript to suitable conference for presentation/publication-Sept. 15 (Deliverable 3).

- Develop plan for acquiring funding for continuation of the work-Sept 15. (Deliverable 4).

\section{Accomplishments}

During FY-95 we were able to outline a product definition for the traffic controller. It is envisioned to be small (about lunch- box-sized), low cost (about $\$ 2 \mathrm{~K}$ ), suitable for direct plug-in replacement of existing $d u m b$ intersection controllers, capable of local control at an intersection with cost-functionbased optimization, have continuously variable timing plan(s), incorporate fault detection and interrupts, be fail safe, and be capable of central override. The conceptual controller architecture is shown in the figure below. A timing plan generator is actually made up of two modules, one of which solves the conditional logic describing the control law and the second which handles the boolean logic function that ensures proper switching sequence of the lights in the traffic signal.

The conditional logic statements that generate the control law have been formulated. The statements are associated with control of a simple two street intersection with turn lanes. Details of this example can be obtained from the Principal Investigator. Cost is the calculated value of a cost function.

New or Increased Technical Capabilities

This work has increased our understanding of the operation of central computer controlled traffic signal systems. This work has also considerable increased our understanding of autonomous intelligent controller design.

\section{Business Development Opportunities}

Business development opportunities will be defined in FY-96, but included licensing of the technology to Traconex, Inc. the major manufacturer of traffic signal controllers.

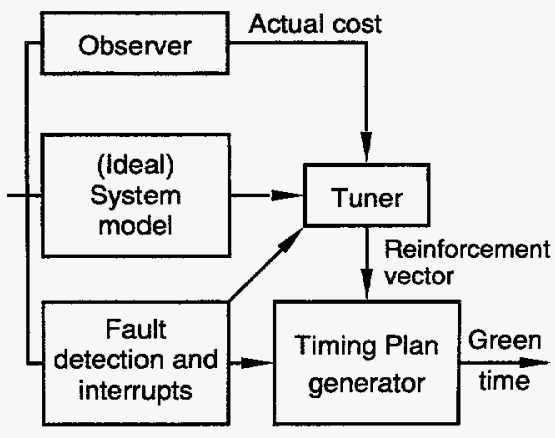

E96 0072

Controller Conceptual Architecture 


\section{System Dynamics Model of Intermodalism \\ LDRD 7204}

\section{E. Sebo, D. J. Kurkowski}

Intermodalism refers to interconnections among modes of transportation, e.g., road, rail, water, and air. Effective intermodal planning must cross boundaries between the public and private sectors as well as transportation modes. There is a requirement to identify the barriers to intermodal transportation and investigate the impact of proposed changes to policies and infrastructure to ensure an effective and efficient transportation system. A systems approach is necessary to adequately represent the interaction between the sometimes incompatible concerns of all stakeholders.

The analysis is a dual approach, describing (1) the system that drives an intermodal facility and architecture and (2) tools used to feed information to the system. A systems dynamics model of intermodalism at the Port of Lewiston has been developed. The model was designed to highlight leverage points, hidden assumptions, second-order effects resulting from feedback loops, and system drivers. The information model is used to provide insight into the systems that support current operations.

This work has identified issues both specific to the Port of Lewiston and general across intermodal transportation. The major issues specific to Lewiston were: the geographic location of Lewiston, including access infrastructure and volume flow imbalance; difficulty in obtaining empty containers; closure of the MoscowArrow rail gap; and salmon recovery issues. Overall intermodal issues involve sensors and information systems required to seamlessly track container location and make the data available at the right place at the right time.

\section{Objectives}

- A causal loop model addressing intermodal transportation issues with specific emphasis on the Port of Lewiston and the Columbia/Snake River system.

- A report identifying system leverage points, system drivers, and issues identified during model development.

- A report describing the current information environment supporting the Port of Lewiston.

\section{Accomplishments}

A series of causal loop diagrams or models have been developed for intermodalism at the Port of Lewiston. These loops consider the factors important to the development of an intermodal facility. Additional causal loop diagrams have been developed specifically to model intermodalism at the Port of Lewiston. These diagrams include a top-level Lewiston causal loop model to provide a high-level overview and emphasize the most important factors affecting Lewiston. Additional detailed submodels were developed for cost, facility volume flow, economic impacts, environmental issues, and information systems.

The major Lewiston-specific issues result directly from the geographic location of Lewiston. The issues include access infrastructure, volume flow imbalance, difficulty in obtaining empty containers, closure of the Moscow-Arrow rail gap, and salmon recovery issues. However, additional intermodal issues exist which are general across intermodal transportation concerning sensors and information systems were also identified during model development.

Tracking container status is a priority issue for intermodal transportation. This problem has two facets: data collection and data access. Data collection requires automatic equipment (container) identification and transmitting the data to appropriate databases. Tracking containers requires more than recording and storing data. It is necessary to be able to provide the right information to the right people at the right time. Standard electronic data interchange (EDI) can only solve part of this problem. A logical approach for providing a seamless container tracking system is a system that can access data as needed from individual databases. A distributed database would act as type of EDI on demand, similar to the TEXNET system under development at the INEL for the textile industry. A variety of other issues, including monitoring of hazardous cargo, border crossings, nonintrusive cargo inspections, and identification of overweight containers are additional issues that need to be addressed for efficient intermodal transportation. A white paper report summarizing the modeling effort has been written.

The information modeling effort involved a series of interviews with transportation companies and the Port Authority to investigate information systems in use at the Port of Lewiston. To determine the wider picture, discussions were held with the Idaho Transportation Department. The Pacific Rim TransTech Conference was attended for an international viewpoint.

Analysis of the information gathered shows that, like other intermodal facilities, only pockets of information exist causing information flow bottlenecks. Most entities operating at the Port of Lewiston operate independently. The infrastructure is disjointed, relying on adhoc information flows, phone, fax, etc. Lack of coordination in information flow results in lack of coordination in freight movement. The system is relatively inefficient. Although 
most participants realize additional electronic data interchange is needed, no infrastructure currently exists and there are apparently no coordinated efforts underway to meet this need. The results of this study are documented in an INEL white paper.

\section{New or Increased Technical Capabilities}

The major benefit to the INEL is an increased knowledge of the problems and issues facing intermodal transportation. In particular, these issues include information systems and sensors which are INEL core capabilities. This knowledge can be combined with ongoing transportation initiative efforts to provide new opportunities for transportation work at the INEL.

\section{Business Development Opportunities}

A number of private companies and the Lewiston Port Authority have participated in this LDRD by providing data and information essential for successful completion of this work. In addition, personnel at the Idaho Transportation Department (ITD) have followed model construction and provided assistance in the development of contacts within the transportation industry. There is additional potential for partnering with ITD and the Idaho Transportation Consortium for the extension and application of this work.

In addition, this LDRD has identified technical problems appropriate for the innovative approaches to merging sensor streams, communications, and distributed databases being developed in the proposed transportation infrastructure LDRD.

\section{Alternate Joining Techniques for Textiles \\ LDRD 8415}

\section{A. Deason, P. L. Taylor}

The U.S. textile industry has identified automation of garment production as one of the most important requirements for revitalizing this important sector of the economy. One aspect of this is the goal of socalled 3-D assembly methods, which implies fitting cloth panels to a 3-D mannequin/mandrel and sewing the garment into its final form. To achieve this, one must radically alter the current practice of garment assembly, which depends almost totally on the conventional sewing machine.

One of the greatest obstacles to use of the sewing machine in $3 D$ assembly processes is that the sewing machine requires a bobbin to hold the locking thread. This bobbin is located on the opposite side of the fabric from the needle position and can only be loaded with a short length of thread due to various mechanical limitations of the sewing process. Thus, to achieve true automated 3-D sewing, one must eliminate the bobbin. This may or may not eliminate thread as well.

We proposed investigating the potential for substituting a novel variation on adhesive assembly which might solve some of the problems encountered with the present techniques. Currently, a band of adhesive is applied along the seam and cured. This leaves a stiff, uncomfortable seam which causes customer rejection, except in rain gear and certain military garments. In this concept, one applies numerous small injected or applied dots of adhesive along the seam, which emulate the local nature of the thread stitch. Hopefully, this will be more conformable for the user.

\section{Objectives}

During FY-95, we planned to evaluate alternate fabric joining techniques, focusing especially on novel uses of adhesives for this purpose. The goal will be to develop a glued seam with the same feel or hand as a sewn seam. We will also attempt to evaluate the concept of an alternate, bobbin-less sewing system, perhaps incorporating a combination of thread and adhesive. This is intended to provide a system compatible with automated, delivery on demand garment fabrication methods.

\section{Accomplishments}

We made good progress in identifying potential candidates for adhesives based on discussion with Judd Early at TC2. These adhesives were developed for use by Levi Strauss to glue pockets in jeans. They produce a softer and stronger seam, while maintaining resistance to degradation by washing and dry cleaning.

We began the process of hiring a lab technician to assist in this and other projects early in FY-95, but progress was slow, and it was early summer before we succeeded. For this reason, we made little further progress in this project and a portion of the money was returned to the LDRD office.

New or Increased Technical Capabilities

This project was terminated before significant progress was made.

\section{Business Development Opportunities}

We still believe this is an important effort, and have resubmitted an expanded version of the project for FY-96. We believe a new concept in garment assembly is fundamental in insuring the 
success of the most advanced concepts in automation.

\section{Real-Time On-Loom Knit and Hand Characterization Using Diffractive Optical Methods} LDRD 8416

\section{T. M. Crawford, V. A. Deason, T. E. Repetti}

At various stages during the production and marketing of cloth, it is necessary to check the thread spacing and pattern in the cloth. This number (threads per inch) is related to the quality, strength, and shrinkage of the cloth. Current practice is to use a hand lens and to count threads per inch by eye. We have proposed using a video image processing technique to extract this information from the cloth and display the results in a convenient manner.

As part of this project, a variety of digital $C C D$ video cameras were evaluated for use in acquiring the cloth images for further processing. Several likely candidates were identified and two were procured which provided a relatively low cost option for acquiring the necessary data. One of these was incorporated in a simple prototype assembly which automatically positions the camera at the proper distance from the cloth for correct focus. A pistol type grip can be used to carry and position the device. Images are acquired, digitized and transferred to a computer or other data processing system for analysis, display and storage.

\section{Objectives}

- Investigate diffractive and Fourier optical techniques to develop sensors and instrumentation for real-time, onloom characterization of thread count and knit/hand parameters.

- Develop a compact device which can measure a fabric's thread count and enter the results into a database on a PDA (Personal Data Assistant) or other device.

\section{Accomplishments}

Hardware. We identified a suitable camera for the prototype, although final decisions on a camera for a production model are not appropriate at this time. We developed the necessary system support for acquisition, display, storage and analysis of the raw data. The resolution is adequate for the purpose, and might even be reduced if a cost benefit is evident. A color camera is a potentially valuable upgrade, where color information might be used to distinguish thread patterns from printed patterns. This might also be used for inexpensive color analysis of dyed or printed fabric, if a suitable color calibration scheme could be developed.
Software. We investigated various algorithms for analyzing thread count and concluded that the Fourier Transform method leads to a reliable and accurate measure of thread count for a surprisingly wide variety of fabric weaves. Issues of desired image size, signal to noise, rotation, and resolution were encountered and resolved, as well as issues involving efficiency of the thread count algorithm. We believe we can efficiently implement this algorithm in a handheld device for measuring thread count.

\section{New or Increased Technical Capabilities}

We improved our capability in the use of video techniques by evaluating and gaining experience with a variety of digital solid state video cameras. We improved our understanding and use of Fourier Transform methods in evaluating two dimensional patterned images. We better understand the

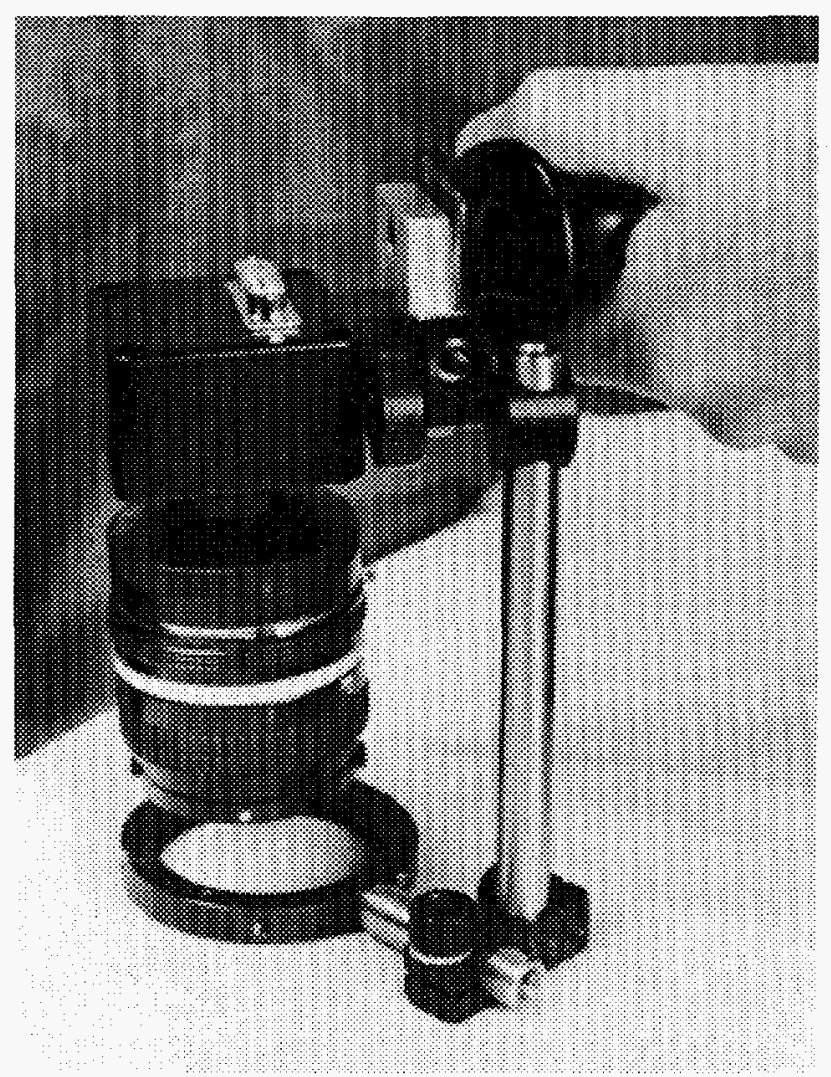

Thread Count Imaging Head 
variability and types of weaves seen in typical fabrics. We have developed a Fourier Transform method to measure thread count and weave pattern for various fabrics. Similar methods are envisioned for application to the determination of hand, which will be examined in an FY-96 AMTEX project.

\section{Business Development Opportunities}

A Patent Idea Record is in preparation; contact Vance Deason (6-2501) for details. There is strong potential for a thread count device to be developed into a product; we will be working with the appropriate Lockheed Idaho groups during FY-96 to see this through in conjunction with an FY-96 AMTEX LDRD which will develop an intelligent sensor system to measure hand (the qualitative properties of a fabric such as softness, texture, etc).

\section{Micromechanical Analysis Capability for Composite Materials LDRD 8506}

\section{R. L. Williamson}

\begin{abstract}
Metal matrix composites are typically produced at high temperature. During cooling to use temperatures, large thermal stresses develop between the constituent materials because of differences in thermal and mechanical properties. The effects of these stresses on composite integrity and strength are important considerations in composite design. In this project, micromechanical models have been developed to provide enhanced understanding of the effects of residual stresses and provide guidance in the composite design and optimization process. Model results indicate that, for certain cases, residual stresses provide an effective material strengthening in the elastic regime. Using the model, the
\end{abstract}

effects of fiber volume fraction and shape were considered. Additionally, the effects of a third material interlayer were investigated. Results suggest this layer can provide a method of controlling stresses and strains, thus providing an optimization parameter in composite design. Qualitative agreement exists between model predictions and experimental data.

\section{Objectives}

- Develop and demonstrate a micromechanical level modeling capability to predict processing-induced residual stresses in metal matrix composite materials.

- Investigate the effects of ceramic volume fraction and particle shape.

- Investigate novel techniques designed to reduce detrimental stresses and strains.

- Compare predicted stressstrain response of the composite with experimental data, if available.

\section{Accomplishments}

Metal matrix composites (MMC), composed of particles, fibers, or whiskers embedded in a metal matrix, are typically produced at high temperature. During cooling, large thermal stresses can develop between the constituent materials due to significant differences in thermal and mechanical properties. The effects of these stresses on composite integrity and strength are important considerations in MMC design. Micromechanical models are used to develop enhanced understanding and provide guidance in the design process.

A geometrical approach used to develop a MMC micromechanical model. By assuming a regular fiber arrangement, each fiber and a portion of the surrounding matrix material forms a symmetric hexagonal unit cell, which, for simplicity, is approximated as a cylindrical cell. With this approximation, the model becomes axisymmetric, consisting simply of a fiber cylinder encased in a larger matrix cylinder. The effects of neighboring fiber/matrix cells are included using periodic boundary conditions. The fiber and matrix are assumed to remain perfectly bonded and are initially stress-free at processing temperature. Various fiber volume fractions $(3,6$, 12 , and $19 \%$ ) were considered by holding the fiber size constant, and varying the matrix cylinder size.

Solutions to the basic equations of thermomechanics were obtained using standard finite element numerical (FEM) techniques. A typical computational mesh is shown in the figure to the right; relatively fine meshing is used near material interfaces to predict locally large stress and strain gradients. Note that symmetry permits simplification of the analysis to only one of the quadrants of Fig. 1(b). The selection of Si3N4 for the fiber and $\mathrm{Al}$ for the matrix were driven by a companion project (LDRD 8507), wherein squeeze casting techniques are used to produce composites based on novel fibers produced at the INEL. Also note that the mesh contains an interlayer region separating the fiber and matrix. To investigate interlayer effects on residual stresses and strains, as well as stress-strain behavior under loading, simulations were performed with either a highly compliant (half the yield strength of $\mathrm{Al}$ ) or a composite $\left(25 \% \mathrm{Si}_{3} \mathrm{~N}_{4}-75 \% \mathrm{Al}\right)$ interlayer material. Although the fiber was assumed to remain elastic, plasticity was permitted in the aluminum and both interlayer materials. Temperature-dependent mechanical and thermal 
property data were used for all materials. In addition to the geometrical model previously discussed, models were also developed for fibers having pointed rather than flat ends, providing an initial investigation of the effects of fiber shape.

Assumed stress-strain curves were plotted for the MMC parent materials as well as the predicted curves for four composites of varying fiber volume fraction. Data for the curves are generated by displacing the composite in the $z$ direction and monitoring the predicted stress-strain behavior (much like a standard tensile test). In one study, the effects of processing-induced residual stresses were neglected, and the composite behavior predicted is much like what would be expected intuitively; both the elastic and plastic regions are proportionally strengthened as the fiber volume fraction is increased. Later processing-induced residual stresses were included in the model, and it was apparent that residual stresses, at least for loading along the fiber axis, provide an increase in the elastic modulus, and thus an effective strengthening. Further note that this strengthening is more significant for the smaller fiber volume fractions.

As mentioned above, as one technique for controlling residual thermal stresses and strains, a third material interlayer was inserted between the matrix and fiber. Highly compliant interlayers resulted in significant strain increases with subsequent decreases in stress. The more rigid composite interlayer, however, had an opposite effect; strains were reduced with compensating increases in stress levels. Thus

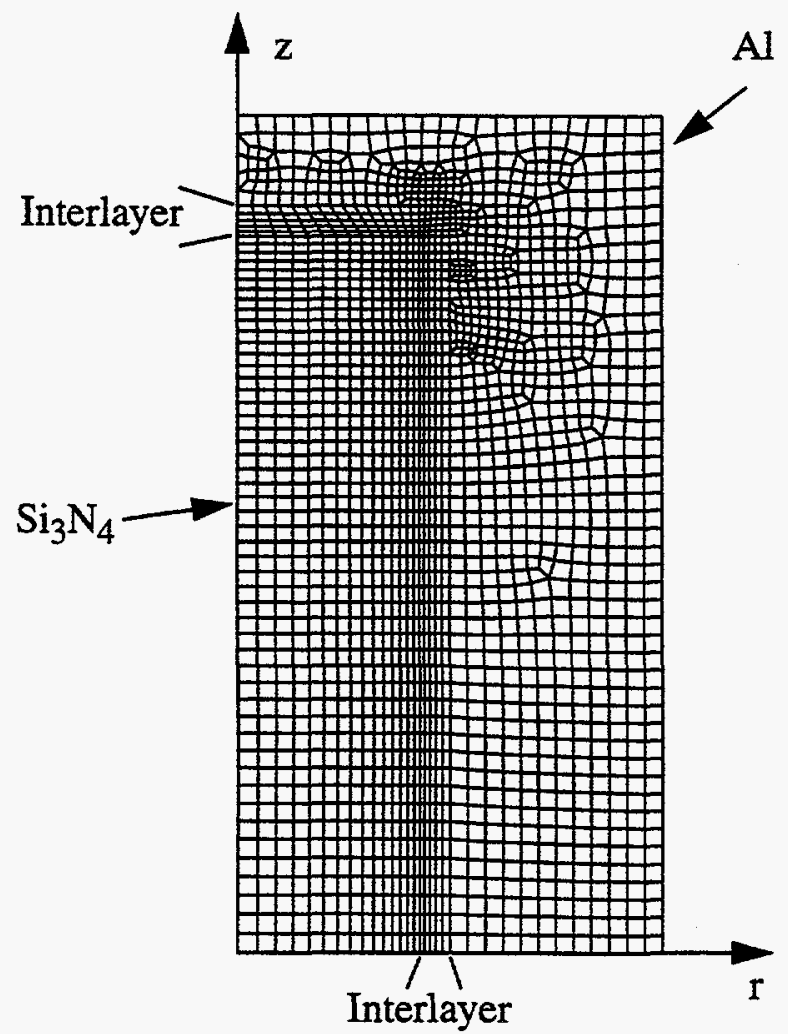

Typical Finite Element Computational Mesh Showing the Assumed Fiber and Matrix Materials. Interlayer Region Is Included to Study Its Effects on Residual Stresses and Strains. controllable trade-offs are predicted between stress and strain, indicating interlayers might provide an opportunity for design optimization.

We attempted to locate experimental data to corroborate the model predictions, but found no reliable data for the material system selected. Qualitative agreement exists between model predictions and available data for other material systems.

\section{New or Increased Technical Capabilities}

As a result of this project, we developed a micromechanical analysis capability for predicting residual thermal stresses between dissimilar material components in composite materials. Although for simplicity, a specific materials system and geometry were considered, the techniques and expertise developed are applicable to a wide range of materials and geometries. We acquired an increased understanding of the effects of residual stresses, and where they may prove beneficial or detrimental. Since the interest in and use of composites is rapidly expanding, possessing finite element models that can enhance understanding and provide valuable design and optimization information will be of benefit to INEL materials research programs.

\section{Business Development Opportunities}

This project was conducted jointly with LDRD project 8507 and involved collaboration with General Motors Corporation. Business development opportunities may exist directly with GM or jointly with other funding agencies. 


\section{Metal Matrix Composites from Novel Nitride Preforms LDRD 8507}

\author{
M. H. O'Brien, R. J. Heaps, \\ D. M. Garrard
}

The objective of this project is to investigate metal matrix composites using silicon nitride whisker "preforms" for potential application in advanced transportation systems. This project uses a proprietary process developed at INEL to fabricate silicon nitride whiskers from silicon carbide powder. The whiskers are formed in a boron nitride mold at high temperatures inside a furnace containing high pressure nitrogen. The whiskers are locked together to form a porous structure that can be easily shaped relative to a dense structural ceramic. Whisker preforms solve some health hazard and processing problems associated with loose whisker reinforcements. The near-net-shape capability of the preform approach is also a significant advantage.

This study emphasizes metal matrix composites (particularly aluminum) because of recent interest from several companies including General Motors, Garrett Turbine Engines, and General Electric Aircraft. An informal collaboration between the INEL and General Motors to investigate metal matrix composites started in FY-94. Preforms were made and characterized at the INEL. GM squeeze-cast an aluminum alloy into preforms and selected composite properties were measured. While feasibility and potential were demonstrated in FY-94, further development in whisker preform processing was necessary. In FY-95, preform processing improvements were investigated, specifically to increase whisker size and to create cleaner interface.

\section{Objectives}

- Investigate the effects of different chemical fluxes to enhance crystal growth.
- Examine the influence of temperature and pressure on preform cleanliness and whisker conversion.

- If significant improvements can be made in the preform, squeeze-cast at GM and measure the properties of the aluminum matrix composite.

\section{Accomplishments}

To summarize the accomplishments of FY-95, we investigated a number of processing variables, but noted no significant improvement in overall preform properties. More specifically, we observed that some properties could be improved, but at the cost of other desired properties. For example, larger diameter whiskers could be made, but the amount of conversion to whiskers and the cleanliness of whiskers suffered badly. Although the initial properties of these preform materials showed significant promise, after further study it is uncertain whether they have real commercial potential without further improvement. It is uncertain how to proceed, consequently no proposal for future studies has been submitted.

New or Increased Technical Capabilities

We learned several important and necessary attributes of whiskerized materials as a result of this project. For example, larger whisker size and clean interface between the whisker and the metal matrix were observed to be very important to the composite performance. These and other lessons learned will be a benefit to future composites projects. Metal matrix composites offer near-term commercial benefits in applications requiring light weight. Future proposals will probably also feature ambient pressure processing in order to reduce fabrication cost.

\section{Cooperative Industrial \\ Research with \\ General Electric \\ Lighting Products}

LDRD 8510

\section{J. S. Epstein, B. H. Rabin, R. L. Williamson}

The purpose of this project was to explore collaboration with General Electric (GE) Lighting Products, Cleveland, Ohio, and possible joint funding from the DOE Office of Lighting Technology. This collaboration is based upon a history of scientific collaboration (1988-1994) between J. Epstein and C. Scott at GE on the fracture of the Lucalox Alumina produced by GE Lighting for their Sodium Vapor Lamp housings.

\section{Objectives}

- Using INEL's phase shifting moire interferometry, determine the residual strain profiles in the end caps of the $250 \mathrm{~W}$ sodium vapor lamps.

- A visit of the INEL staff to GE Lighting Products Plant in Cleveland:

- To brief ceramic lamp housing residual strain results and additional computational work on IR thin film technology.

- Discuss with Fred Ahlgren, Director Industrial R\&D a joint research proposal to $\mathrm{DOE}$ and CRADA.

- Attend Lighting requirements workshop to have input on future $R \& D$ requirements for lamps.

\section{Accomplishments}

Application of phase shifting interferometry to analyze residual strains in a series of Sodium Vapor Lamps. This involved the development of a detailed experimental laboratory procedure started in the 
previous year to section the lamps at their end caps in order to release residuals strains. Phase shifting moire was applied to assess the strain profiles in these lamp end cap: to housing seals, in particular a comparison between a $250-\mathrm{W}$ Sodium Lamp made in the United States and by a GE subsidiary in Hungary.

Visit to GE Lighting Products.

INEL results were presented for the residual strain profiles in two designs of 250W Sodium Vapor lamps, one design produced in the United States by GE and another Hungarian design by a recent $\mathrm{GE}$ acquisition. It was shown that the Hungarian design produced lamp housings less prone to cracking owing to a series of end cap slots that act as expansion joints for thermal strains. Further work was discussed toward understanding a new single crystal lamp design.

Discussion took place on the INEL residual stress modeling of the IR reflective thin films used for incandescent and halogen lamps. The discussion could not cover detailed manufacturing issues owing to the proprietary nature of the GE IR thin-film CVD deposition process.

Epstein and Rabin attended the Lighting Workshop attended by industry, Government, and academia to define current priorities in lighting research.

New or Increased Technical Capabilities

A procedure was developed to map residual strain fields in exceedingly stiff and small industrial parts. Typically residual joining strains are the major cause of material cracking and failure during a manufacturing phase of an item. This procedure was developed fully under this LDRD.

\section{Business Development Opportunities}

Important contacts were developed in the lighting research indus- try (GE, Sylvania, and Phillips) and DOE (Dr. Lee Anderson) with the INEL. New business dollars as such were not brought into the laboratory for a number of reasons:

Dr. Fred Ahlgren, Director of GE's Industrial Lighting Development said that GE Lighting is making money without Government funds. He indicated that GE owns one third of the world market share in lighting and believes it is not in GE's benefit to team contractually with the Government in the form of CRADAs or reveal trade secrets developed solely by GE that are an important part of their market share.

Dr. Lee Anderson, of DOE, is willing to fund the introduction of new lighting products into the market and not applied R\&D. From the lighting workshop the current priorities are on LED flat panel lights being developed in Japan and by Hewlett Packard.

\section{Lifetime Extension and Increased Reliability for Refinery Structures LDRD 8511}

\section{N. M. Carlson, W. G. Reuter}

The need for lifetime extension tools is best explained by Chevron in a letter to DOE: "The refining and petroleum industry faces a problem relating to aging equipment that is similar to that which other industries are experiencing concerning aging infrastructure. In the past several years, our industry has considered several technologies that are crucial to address the reliability of aging equipment in our plants. Since most of these technologies are not considered core technologies by the industry, it has been difficult for any single company to justify an investment in developing these technologies alone. $A$ highly integrated program is needed to properly address the aging equipment issue. This integrated program should include a better understanding of structural failure mechanisms in refining and petrochemical systems, the development of analysis techniques and models for improved determination of remaining equipment life, and the development of on-line nondestructive inspection/monitoring tools." To address the identified lifetime extension needs of the refinery industry, this project focused on two tasks. In Task 1, a noncontacting ultrasonic system using the high-temperature EMAT developed at the INEL and a laser system developed at Johns Hopkins were integrated to provide a laboratory version of a potential on-line NDE system for monitoring defects in critical components. Task 2 focused on the measurement of fracture toughness in base and weld materials (including the heat affected zone) selected by the refinery industry and on identifying candidate NDE techniques to monitor fracture toughness.

\section{Objectives}

- Optimize operational hightemperature EMAT to work with the Johns Hopkins narrowband laser.

- Collaborate with Johns Hopkins to establish a laser hardening strategy and establish potential vendors for the laser/EMAT system.

- Correlate subsize fracture sample techniques to fracture toughness in aged materials.

- Evaluate selected NDE techniques to detect microstructure based on correlation with fracture mechanics results.

- Establish additional industry contacts to broaden the support base for plant aging issues.

- Develop collaborative relationship with Ames Laboratory on NDE techniques and MIT on applied mechanics. 


\section{Accomplishments}

Collaborative research between Johns Hopkins University and the INEL resulted in an enhanced EMAT design for use in high-temperature ultrasonics. The enhanced EMAT is tunable to match the output pulse frequency of the Hopkins laser. Testing of the laser/EMAT system was completed at Johns Hopkins, as the current laboratory version of the narrowband laser is not portable. The INEL is currently hardening the EMAT coil and magnet for use at temperature. Preliminary tests with the nonhardened EMAT were conducted to $450^{\circ} \mathrm{F}$. The INEL and Johns Hopkins were involved with discussions for hardening the laser and making the laser system more fieldable. Discussions with NDE vendors established that there is industrial interest in packaging the laser in a portable system as well as the high-temperature EMAT. The discussions centered around the NDE vendor providing system integration to ensure that the NDE product is marketable for field use. Fracture toughness testing was completed on full-size samples, both welded and unwelded. NDE samples were made from the same material as the fracture samples. Initially, five NDE techniques were evaluated for use in monitoring fracture toughness: harmonic generation, resistivity, temperature coefficient of resistance, SQUID, and subsize specimens. Of the techniques evaluated, harmonic generation, resistivity, and subsize specimens show the most promise for potential techniques for monitoring reduction in fracture toughness based on testing with a wellcharacterized set of thermally aged Inconel 718 samples. Subsize samples are being actively pursued as an NDE technique based on the refinery industry input. Currently, subsize sample results are being analyzed to determine the optimum sample size that provides reliable data. Positron annihilation is currently being evaluated on a few Inconel 718 samples and an additional potential NDE technique, as part of another LDRD funded from FY-95 funds. Collaborative efforts were established with both Massachusetts Institute of Technology and Ames Laboratory to leverage research dollars and determine the applicability of both code development and NDE techniques not resident at the INEL. Extensive efforts were conducted to establish collaboration with the refinery and NDE service industries of the United States (see Business Development Opportunities for details).

\section{New or Increased Technical Capabilities}

The high-temperature EMAT developed from this project will allow the INEL to pursue onstream monitoring of operating industrial systems at temperature. Subsize fracture toughness sample techniques capabilities of great interest to U.S. industry have been developed under this task. The INEL is actively involved in the DOE/EE-OIT Refinery of the Future in the area of lifetime extension, as well as currently being involved in establishing a national plan to address needed research in the area of lifetime extension with the Pressure Vessel Research Council, Materials Properties Council, NIST, EPRI, other national laboratories, DOE-BES, and utility, refinery, and chemical industries.

\section{Business Development Opportunities}

Currently, three business development opportunities are being actively pursued. A collaborative effort is progressing between Dr. Stan David of Oak Ridge

National Laboratory and researchers at the INEL to develop a proposal to submit to DOE/BES as part of the $2 \%$ initiative effort, with a proposal submittal slated for December 1995. A proposal for lifetime extension has been prepared, and industrial letters of endorsement for that joint industry/ national laboratory/university have been presented to Dan Wiley of DOE/EE-OIT for inclusion in the Refinery of the Future projects for possible FY-96 start. This project was developed in collaboration with Chevron, Shell, Exxon, Amoco, Arco, CTI, Inc., Wesdyne, Subsea International, Det Norske Veritas Industry, Johns Hopkins University, and Ames LaboratoryIowa State University. The INEL and Chevron are currently negotiating an industry funds-in project. The technical scope of work has been agreed upon by both the INEL and Chevron technical performers. Currently, the negotiations involve the most appropriate legal document process to follow to complete the agreement.

\section{Solar Bimodal Technologies Demonstration LDRD 8515}
B. G. Miller

The U.S. Air Force, Phillips Laboratory, has recently initiated an Integrated Solar Upper Stage (ISUS) Advanced Technology Demonstrator (ATD) program to evaluate and demonstrate the feasibility of a combined solar power and propulsion upper stage. As part of the ISUS ATD, the Idaho National Engineering Laboratory and NovaTech, in collaboration with Phillips Laboratory, have been tasked to design, fabricate, and experimentally test a near prototypic receiver cavity to demonstrate that a solar bimodal concept using sensible heat thermal storage can produce high specific impulse (800 seconds or better) at nominal thrust levels. 


\section{Objectives}

The primary objective of this project is to perform a comprehensive series of energy conversion tests on thermionic heat pipe modules (THPMs). The design, fabrication, and testing of a near prototypic solar bimodal receiver is one of the key milestones for the ISUS program.

\section{Accomplishments}

Very early in the design process, it became apparent that materials selection and performance would be the greatest challenge. For most of its operational life, the ISUS will operate at temperatures exceeding $2300 \mathrm{~K}$ for long periods of time in the hard vacuum of space. During this time, the testing events schedule (TES) material will undergo numerous thermal cycles as the satellite system moves into and out of the earth's eclipse. Hence, long-tern survivability is a critical concern. More challenging, however, are the requirements set forth by the thruster operation of the ISUS. In the end, graphite was selected as the TES material owing to its high melting temperature, high specific heat, low cost, and commercial availability. However, it is clear that graphite does not meet all of the necessary criteria. In particular, graphite is incompatible with hydrogen, and it has a rather significant vapor pressure at high temperatures. Over the lifetime of the ISUS, this high vapor pressure results in unacceptable mass loss. Therefore, while graphite meets most of the material requirements, it must be coated to eliminate hydrogen interaction and to minimize carbon evaporation.

Coating materials must meet most, if not all, of the same requirements as the TES material in addition to being chemically and physically compatible with graphite. Previous studies on solar rocket propulsion systems have shown that of the refractory metals, only tungsten and rhenium can readily meet the high-temperature material requirements outlined. Rhenium was chosen due to unique thermal and mechanical properties which allow it to possess high creep strength at high temperature and remain ductile even at room temperature. Equally important is that the coefficient of thermal expansion of rhenium closely matches that of the selected graphite. Rhenium is nonreactive in hot hydrogen and has a lower hydrogen permeation rate than that of tungsten. Finally, the vapor pressure of rhenium is only slightly higher than that of tungsten resulting in negligible mass loss over the life of the system.

Once the TES and coating materials were selected, the design and layout of the receiver began. In all, two receiver cavities have been fabricated. Each receiver represents half of the final design that would be used on the corresponding flight system. The other half would be the mirror image (mirrored about the rear face). Thermal hydraulic modeling of the baseline receiver design was performed using the computational fluid dynamics code FLUENT under nominal conditions of flow and temperature. A number of experimental studies have been, and continue to be, performed to evaluate the suitability of various coatings for use with the graphite TES ground test article. The bulk of this effort has centered on thermal plasma sprayed rhenium as the baseline design.

A first-generation high-temperature solar receiver cavity has been designed and fabricated to meet the unique functional requirements of a solar bimodal system using sensible heat thermal storage. The baseline design consists of a rhenium coated graphite cavity which approaches a near blackbody. Coating of the cavity/TES is accomplished using thermal plasma spray techniques to thicknesses of 20 to 30 mils over the entire exterior surface. Coupon studies have verified the feasibility and robustness of the rhenium coating to withstand repeated thermal cycles and internal propellant pressures at nominal temperature conditions without signs of failure. These coupon tests have also shown that diffusion of carbon through the rhenium coating occurs readily and matches closely the results of previous investigators based upon published rhenium-carbon phase diagrams. Further the rhenium-carbon eutectic with a melt point of $2778 \mathrm{~K}$ represents a hard upper temperature limit for the baseline ISUS ATD design. Based upon the results of the program to date, the ground test portion of this program will begin in early June with on-sun testing at the PL/E Solar Facility.

\section{New or Increased Technical Capabilities}

Results of the tests indicate that the plasma sprayed rhenium exhibits good bond strength which is unaffected by thermal cycling from room temperature to $2500 \mathrm{~K}$ and back at heating/cooling rates of $15 \mathrm{~K} / \mathrm{sec}$. INEL personnel have successfully designed and developed prototype receiver cavities.

\section{Business Development Opportunities}

This project successfully leveraged LDRD funds with funds from the United States Air Force ISUS ATD Program. We have enhanced our potential and value as a partner with the USAF and will continue to perform collaborative research work with them. 


\section{Weight in Motion Sensor and Signal Analysis Technology LDRD8516}

\section{J. G. Rodriguez}

The INEL has been working with the Idaho Transportation Department (ITD) in developing a new capability for a weigh-in-motion (WIM) sensor. This WIM sensor is fabricated using an inexpensive four conductor cable. The WIM cable performs as a strain gauge with the output affected by the load applied. Customized electronics were fabricated to provide signal conditioning for the data acquisition system and to measure the microstrain induced by a vehicle traveling over the WIM sensor. The WIM signal posesses two inherent components, strain $C$ associated with the vehicle and the number of axles the weight is distributed over. Measured strain in the WIM sensor provides weight and the number of axles provides vehicle classification. Vehicle classification coupled with WIM information provides a significant discrimination advantage currently not available with existing commercial available WIM systems.

Research has concentrated on theoretical analysis of pavement materials, static and dynamic measurements of the WIM sensor in the laboratory and INEL bus lot with a prototype system. In addition, static laboratory experiments using an instrumented hammer were performed to determine the acoustic response due to loading. The goal of the theoretical analysis is to gain insight and understanding of the experimental measurements done in the laboratory and bus lot. Static experiments with concrete blocks studied the effects of loading on the transmission of acoustic pulses.

\section{Objectives}

- Theoretically analyze pavement to a static load to determine the location of the minimum and maximum stresses.

- Test in the laboratory using acoustic transmission and WIM sensor.
- Develop data acquisition software to capture and analyze INEL bus lot data.

- Develop an electronic design for signal conditioning.

- Develop a system prototype.

Accomplishments

Although a truthful theoretical model is intractable at this early stage of the project, a simplified model can provide helpful insight and qualitative understanding to the problem. The first model we looked at was a stationary vehicle with the road modeled as a threedimensional half-space with two top layers. The contact area between the road and each tire was assumed to be circular and the load pressure was constant. All the consequent displacements, strains and stresses were then calculated by using the finite element method. From the examples that we have studied, this model suggests the following results. First, the spatial locations that seem to suffer the largest strains or stresses are those located either directly under or very closely under the center of a wheel. The points that have the largest displacement field, however, are usually farther from the center of the wheel. These locations seem to depend on the combination of wheel and the relative distance between neighboring wheels. For example, when an axle has a set of dual wheels on one side of the vehicle, the points under the center point between these dual wheels suffer the largest displacement. From the cases we have calculated, the largest strains show up in the second top layer while the largest stresses and displacements show up in the top layer.

We conducted experiments with concrete blocks to study the effects of loading on the transmission of acoustic pulses through concrete. The concrete blocks used for the experiments contain no aggregate, only course sand. They are a standard size, intended for use in the construction industry. Block dimensions are $76 / 8$ in.wide, 35/8- in. high, and 155/8-in. long.

An Instron Model 4505 Testing System was used to load the samples. The samples were placed in a plywood sand box, so that a layer of sand, approximately 1/2-in deep, supported the block. The plywood holding the sand was 5/8-in. thick. The sand box containing the sample was supported in the Instron by a 1/2-in. steel plate. Loads were applied to a cylindrical steel bar that was positioned perpendicular to the long axis of the block, across the 75/8-in. side. A diagram of the experimental setup appears in the figure to the right.

A B\&K Signal Analyzer was used to acquire acoustic pulse transmission data. An acoustic pulse was initiated with the tap of a hammer on the end of the block. A transducer on the other end of the block detected the transmitted pulse. The hammer was instrumented with an accelerometer so that the shape of the hammer impulse would be known. The transfer function of the block was calculated by deconvolving the hammer impulse from the transmitted pulse. Several loads, ranging from 500 pounds to 10500 pounds, were applied in two sets of experiments on two nominally identical blocks. Measurements involved the time series response when a variety of loads were applied to the two samples. The changes in the time series response with increasing load are quite noticeable and consistent between the two samples. The changes in the frequency domain response with increasing load are also quite noticeable. The conclusion from this set of experiments is that load- 


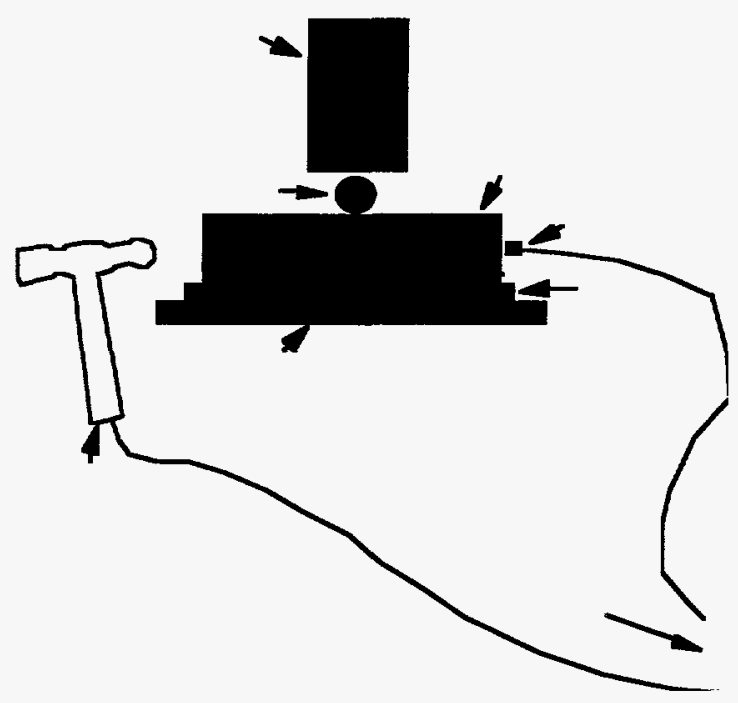

Experimental Setup for Test Conducted to Study Effects of Loading

ing has a measurable effect on the transmission of acoustic pulses through the concrete samples. However, further investigation is needed since this effect may be due to changing boundary conditions that will not be present as load is applied to a concrete roadbed.

The Bus lot experiments used three vehicles; INEL bus, Datsun, and a Nova. These bus lot experiment represent this year completed work. This effort included the software development and signal conditioning required to capture, collect, and analyze, data collected using the sensor.

A portable PC-based data acquisition and analysis system was developed to support field tests of the wire loop sensor.

Software was developed which incorporates a great deal of flexibility to permit data acquisition under a variety of conditions. Analog-to-digital operation commences when an external trigger is received. This trigger comes from an infrared motion detector. The motion detector is usually positioned to detect motion 20 or 30 feet away from the wire loop. Upon initiation, $A / D$ operation continues until the first of three possible events occurs (a) expiration of the user-specified time limit, (b) exhaustion of extended memory, or (c) termination by key input from the user.

Additional software was developed to process the sometimes very large records collected with the data acquisition system. Capabilities include, lowpass filtering, windowed FFTs, subsampling, and plotting. Times series data as long as a million or more samples can be operated upon, depending upon the amount of available extended memory.

Further work will concentrate on integrating the modeling with the experimental/field measurement tasks. The second model and technique is just beginning. In this approach, the road is considered as a two-dimensional half-space with one top layer. The surface load, in the beginning of this approach, is a stationary pressure which is spatially oscillatory and extends from minus infinity to positive infinity on the surface. Consequent fields (displacement, stress and strain) can then be calculated analytically. The overall process of this approach is a combination of analytical and numerical computation. It is hope that a clearer understanding of the pavement dynamics under load will allow predication capabilities. This will provide better discrimination and analysis of the WIM sensor signal for commercial vehicle applications.

\section{New or Increased Technical Capabilities}

During this project, the INEL has gained a better understanding of pavement induced stress/strain related to vehicle weight. Analytical modeling of road surfaces (pavements) has provided a I and unique capability that could be beneficial to the ACETS program. Also, the WIM LDRD activities have provided new partnerships with the State of Idaho's Transportation Department and the University of Idaho. Classification of vehicle type will increase measurement accuracy, system performance, and provide information related to the type of traffic. These new features will give State Highway Departments nationally essential information for highway management. Traffic information related to vehicle type and weight gives Highway department road load usage (capacity), pavement reliability design, vehicle weight enforcement, and maintenance guidance.

\section{Business Development Opportunities}

Current efforts have been to develop partnerships with ITD, the University of Idaho, and Federal Highway Administration. This relationship will assist in developing new business and obtaining commercial partnerships by allowing the INEL to install and test this sensor design on an existing road way. Companies selling commercial WIM systems will also be solicited in the future. The successful implementation of this prototype design will demonstrate the overall robustness of the system as a viable commercial product. 


\section{Communications Spectrum Needs for Short-Range Intelligent Vehicle Highway Systems \\ LDRD 8517}

\section{W. R. Croxford, A. A. Anderson}

Many IVHS applications and advanced transportation applications, such as multimodal, emergency, and automated port-of-entry will use dedicated frequencies for short-range communications. The optimum frequencies for these applications are not well understood. In order to deploy these systems and new technologies more effectively, an analysis of spectrum characteristics is needed. Short-range communications between roadside and vehicles can be a reliability problem, particularly for the transmission of data and information. This is a recommended research problem by IVHS America and FHVVA. The project is in support of transportation initiative (Management Integration of National Energy Strategy) and the State of Idaho Out-of-Service Testing Program. Follow-on field testing is proposed for FY-96.

The major objective is to evaluate and determine spectrum needs for short-range IVHS communications. This research will explore which frequencies are appropriate for shortrange communications for specific IVHS applications, including State of Idaho storm warning and out-of-service operational tests. The results will be a recommended frequency or spectrum for each application and for follow-on tests.

Potential benefits include increased reliability of data transmission for radio frequency $(R F)$ shorthaul applications (between roadside and vehicle), reduced need for multiple antennas on vehicles, support to transportation initiative, and support to the State of Idaho service testing program.

\section{Objectives}

- Recommend suitable frequencies and technologies for RF shorthaul communications between vehicle and roadside.

- Recommend frequencies for follow-on testing.

- Discuss findings with organizations and companies.

- Prepare a final report.

Accomplishments

Communications characteristics were determined for each IVHS application for short-haul communications between vehicle and roadside. The IVHS applications considered were emergency (Mayday), advanced traveler information system (ATIS), navigation, electronic credentials, and out-ofservice information. Characteristics considered were RF distance, message length, data rate, frequency band, bandwidth and communications protocol.

Research was conducted on the characteristics of different technologies and frequencies available for short-haul communications (one mile or less). Current radio technologies and associated frequencies investigated were as follows: Land Mobile Radio (Narrowband), Spread Spectrum Radio (Wideband), Cellular Telephone, Personal Communications Systems (PCS), Broadcast transmitters, HF Shortwave Radio, Satellite (GPS) and microwave radio.

Analysis of frequency bands were conducted from $9 \mathrm{Khz}$ to 400 Ghz for urban, suburban, and rural roadside environments for application to short-haul communication, $\mathrm{s}$ including those frequencies already assigned to IVHS (220 Mhz and 902 to $928 \mathrm{Mhz}$ ). Issues of interference, atmospheric conditions, traff1c obstructions were considered in the analysis. Frequency issues for short-haul RF include traff1c obstructions (moving and stationary), multipath due to and moving objects (Rayleigh Fading), multipath due to reflections from large objects, RF shadowing, noise, RF interference and absorptive losses (oxygen, dust and water vapor).

$R F$ prediction techniques and models were used to study frequency and propagation requirements. Models used include Bullington's model, Epstein and Peterson's model, Picquenard's, Okumara's model, Longley-Rice and Ray Tracing. The results of the study show the most favorable frequency bands for short-haul communications are 400 to $900-\mathrm{Mhz}$ and 2.4 to 4.0-Mhz HF bands. Wideband spread spectrum radio in the 400 to $900 \mathrm{Mhz}$ band is recommended.

New or Increased Technical Capabilities

Frequency and propagation studies have been used to support the State of Idaho Out of Service project. Follow-on frequency tests are needed in FY-96 to verify analysis results.

\section{Business Development Opportunities}

Three patent ideas as a result of studies are being pursued. Two regard on-vehicle antennas, one is a Ray-Trace method for radio propagation studies. Further infield testing needs to be conducted in FY-96 to confirm study results.

We responded to an invitation to make a presentation to the August 1995 IVHS America Telecommunications Committee meeting.

\section{Developing a Nonlinear Electromagnetic Modeling Capability Using the Finite-Difference Time Domain Method LDRD 8518}

D. A. Frickey

The advent of laser technology has brought with it the emerging sci- 
ence of nonlinear optics. When the intensity (electromagnetic energy) is great enough, nonlinear phenomena are induced. These nonlinear phenomena have presented almost unlimited possibilities for the development of new technologies. However, the nonlinear nature of this wave propagation has made analysis almost impossible in all but the simplest cases. For this reason, computer simulation is a potentially invaluable tool for the analysis and synthesis of nonlinear optical processes.

The finite-difference time-domain (FDTD) method is a computer simulation method which has been used in a wide variety of electromagnetic simulation problems. Because of the simplicity of the method, it has shown remarkable flexibility in simulating physical phenomena. This method, however, has mainly been restricted to one or two dimensions. The reason is that the computational intensity limits the size of the physical space that can be simulated.

The first goal of this project was the development of a true, three dimensional simulation of a fiber optic cable. The limitations on size were overcome by the development of a new boundary condition which substantially reduced the problem space. It was determined that a meaningful simulation could only be achieved by initializing the problem with stable, guided modes, thus reducing the length of optical fiber to be simulated. This brings the problem within the capabilities of today's supercomputers. A three-dimensional simulation program would make possible a systematic study of nonlinear switching mechanisms, an enormous advantage over laborious trial and error experiments.

\section{Objectives}

- The development of software to simulate pulse propagation in a fiber optic cable.

- The simulation of nonlinear switching mechanisms in a fiber optic cable.

\section{Accomplishments}

Computer simulation capabilities were developed toward the simulation of nonlinear switching mechanisms in a fiber optic cable. A basic simulation of a threedimensional cable was achieved. This was made possible by the development of a new boundary condition which substantially reduced the amount of computer resources needed. Standard methods to date have needed to use huge numbers of cells over which to calculate, which overwhelms even today's supercomputers.

The size of the core, along with the dielectric constants of the core and the cladding, determines which modes can propagate. It is also the core which contains the nonlinear material, so it is the area of primary interest. The cladding cannot be ignored, however, because a substantial portion of energy, in the form of an evanescent wave, is still a part of the propagating pulse.

There are two things that can be done to substantially reduce the transverse portion of the FDTD space. First of all, it can be assumed that the background medium of the problem space is the dielectric constant of the cladding. The boundary between the cladding with the free space outside plays no role in the simulation because none of the energy reaches this boundary. Second, even though there is an evanescent wave in the cladding, it decays rather rapidly. If the problem space can be truncated in such a way that the evanescent wave is absorbed without impact on the rest of the wave, then the size of the problem space can be substantially reduced.

Fortunately, a recent development in FDTD has made such a truncation possible. The Perfectly Matched Layer (PML) was devel- oped as a means of absorbing any outgoing waves in an FDTD calculation without reflecting spurious components back into the problem space. This PML was modified and adapted to the problem of modeling an optical fiber. Now, as few as 10 cells outside the core should be necessary to model the evanescent portion of the wave. With this, the transverse dimension of the problem space is reduced substantially

\section{New or increased technical capability}

The simulation methods developed during this project form a basis for the field of nonlinear optical simulation.

Business development opportunities.

This project involved no contact with private business. Further development of this simulation capability could support ongoing projects at INEL or lead to new projects which may have future commercialization possibilities.

\section{Electrochemical-based Processing Technologies LDRD 8614}

\section{F. Glenn, J. K. Partin}

The objective of this project is to develop new electrochemical-based processing technologies. During FY-95, two novel electrochemical processes were investigated: electrochemical synthesis of powdered metals at an Ice electrode and laser-assisted electrosynthesis.

The ice electrode is a metal substrate coated with a thin layer of ice. It has been shown that metal powders can be produced by electrodeposition in and on this layer of ice. The benefits of powdered metal production at an ice electrode are the ability to produce unique powder shapes and continuous metal production as a result of the 
distinctive nature of the ice electrode.

Laser-assisted electrosynthesis is a technique in which the working electrode in a synthesis cell is irradiated with laser beam during an electrochemicalIy driven reaction. By increasing the electrochemical reactivity of specific bonds with laser irradiation unwanted side reactions were reduced and product yields increased. The process will, therefore, increase energy efficiency and reduce the cost associated with separating products from byproducts. In addition, the process could provide a technique to synthesize new specialty chemicals and pharmaceuticals. Development offers the possibility of an extraordinary advance in the competitive position of the U.S. chemical and pharmaceutical industries.

\section{Objectives}

\section{Electrochemical synthesis}

- Demonstrate a new electrochemical process to produce metal powders.

- Produce metal powders having uniform shape and size distribution.

- Explore new uses for ice coated electrodes.

Laser-assisted Electrosynthesis

- Investigate the effects of laser irradiation on organic electrosynthesis reactions.

- Demonstrate proof-of-principle for this process.

Accomplishments

In FY-95, the following experiment was used to demonstrate proof-of-concept of Laser-assisted Electrosynthesis. The electrochemical oxidation of p-aminophenol in aqueous acid was carried out on a rotating disk electrode. The oxidation was carried out without laser irradiation and with the electrode irradiated at two different wavelengths $(10.6 \mu$ and at $2.99 \mu)$.

The effect of laser irradiation on the reaction products is shown in the figure below. The product of the reaction at the non-irradiated

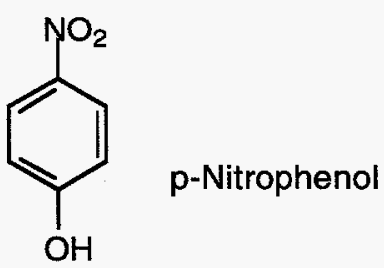<smiles>Nc1ccc(O)cc1</smiles>

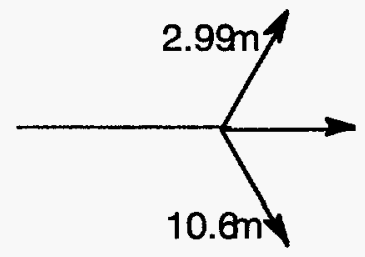<smiles>O=C1C=CC(=O)C=C1</smiles>

\section{Benzoquinone}

p-Aminophenol

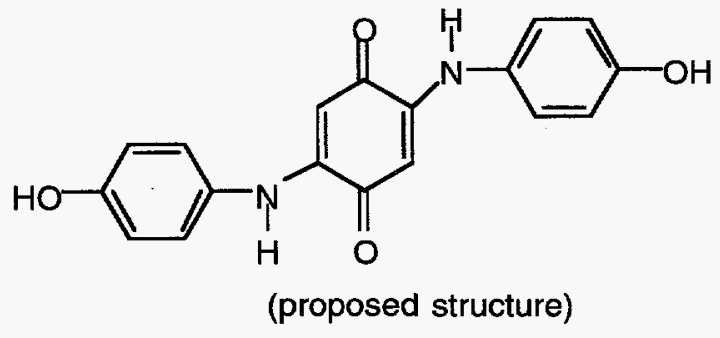

Electrochemical Oxidation of $p$-aminophenol electrode is benzoquinone. With the electrode irradiated at $2.99 \mathrm{~m}$, the reaction product was determined by GC/MS to be p-nitrophenol. Laser irradiation at $10.6 \mathrm{~m}$ during electrolysis produced a purple precipitate believed to be 1,4 bis(4-hydroxyanilino)-p-benzoquinone. While the exact structure of the $10.6 \mathrm{~m}$ product has not yet been confirmed, it is clear that laser irradiation of the electrode during an electrosynthesis reaction can and does influence the direction taken by the electrochemical reaction.

The results of the these proofof-concept experiments demonstrate the tremendous potential of combining laser irradiation with electrochemistry. The results indicate that it may be possible to diala-product simply by changing the laser wavelength. However, these results leave a myriad of unanswered questions concerning exactly how the laser alters the normal mechanism of the reaction and how to best utilize the laser irradiation to obtain a desired product. These questions will be addressed during the FY-96 efforts.

Electrochemical Synthesis of Powdered Metals at an Ice Electrode

To demonstrate the synthesis of powdered metals, a small scale laboratory apparatus was constructed that would allow a thin layer of ice to be formed and maintained on a metal substrate. Initial ice formation resulted in a frostlike ice that consisted of a network of ice dendrites and unfrozen solution. In and on this surface a variety of metals were electrodeposited.

Copper was continuously electrodeposited at the ice-coated electrode from a quiescent $0.1 \mathrm{M}$ CuSO4 solution at a constant potential of $-1.5 \mathrm{~V}$. Deposition was initiated $2 \mathrm{~min}$. after the initial formation of ice on the metal substrate. Immediately upon application of 
the potential, a grainy, copper-colored coating appeared in and on the ice layer. The cooling rate was then adjusted so that the thickness of the ice layer remained constant during deposition.

This experiment demonstrates that powdered metals can indeed be produced at an ice coated electrode. The shape and size of the powdered metal can be controlled by pulsing the applied potential and varying the length of the pulse. Some of the metal particles obtained during these experiments are unique. These metal powders are currently being test to determine their catalytic properties.

\section{New or Increased Technical Capabilities}

For the INEL, the development of new electrochemical processing technologies offers the potential for strengthening the laboratory's intellectual property portfolio and expanding its customer base. For powder metal production, the ice electrode offers the potential of developing a continuous production process with the ability to control the shape and size of the powders produced. For Laser-assisted Electrosynthesis, the FY-95 results clearly demonstrated the enormous potential of Laser-assisted processing.

In the chemical, metals and pharmaceutical industries, a new process for producing a product and new synthesis routes to that product are always patentable. These patents often produce sizeable returns on investment. Patents derived from the Ice Electrode process (U.S. Patent 5,200,054) and Laser-assisted Electrosynthesis (EGG-PI-708) could, therefore, form the basis for several spinoff businesses and provide a royalty stream to the INEL.

\section{Business Development Opportunities}

To date, no external business contacts have been initiated.

\section{Nonaqueous Processing in the Textile Industry}

LDRD 8616

\section{W. A. Propp, M. D. Argyle,}

\section{A. G. Chambers}

The major objective of this project is to develop nonaqueous processes for application in the textile industry, particularly in the sizing process. It is anticipated that these processes will both improve the competitiveness of the U.S. industry and result in significant waste minimization at the same time. This project has been organized using a multidisciplinary team composed of scientists and engineers. An integrated technical approach was employed based on the systems engineering approach successfully used by the collaborators not only for other projects here at the INEL, but also in support work conducted at Rocky Flats for environmental remediation. The final results of this project were anticipated to be nonaqueous processes applicable to the textile industry that would be more efficient, potentially produce higher quality products, and produce significantly less waste than those used currently. Thus, overall, these processes would reduce costs and improve competitiveness of the U.S. industry.

\section{Objectives}

As originally funded, this phase of the project would consist of laboratory studies to evaluate the solubility of selected sizing agents in supercritical fluid (SCF) carbon dioxide and generate the solubility information required for laboratory-scale process engineering studies to be carried out in later phases. After the project was initiated, management redirected it to achieve the following objectives:

- Conduct sufficient laboratory studies to support developing a proposal for submittal to the AMTEX Slashing Initiative.
- Interact with industry, other DOE laboratory personnel, and others involved in AMTEX.

- Develop a conceptual design for a slashing process using SCF carbon dioxide-based solvent systems.

- Conduct proof-of-concept studies of the slashing process to support preparation of a patent application for the technology.

- Prepare and submit a patent application for the technology.

Accomplishments

This project met all of its FY-95 technical objectives. The proposal submitted as part of the objectives was endorsed by the industry partners of AMTEX and was selected for funding as part of the AMTEX Slashing Initiative. However, the initiative was a victim of the current budget cuts, so funding was never received even though authorized.

The proof-of-concept studies to reduce the INEL technology concept to practice were successful.

A patent application covering the proposed technology was subsequently filed.

\section{New or Increased Technical Capabilities}

There have been no long-term benefits to the INEL from this project. The expertise developed from this project has not been considered for use on other similar projects currently underway at the INEL. Internal funding for followon work related to other textile industry processes has not been forthcoming. As a result, project personnel have all returned to their home organizations for reassignment. No further future work in this area is anticipated.

\section{Business Development Opportunities}

As indicated above, industry was very supportive of the INEL 
slashing concept. However, no specifics are available at this time.

\section{Offline Manufacturing} Simulation

LDRD 8708

\section{G. A. Cordes, J. M. Zabriskie, J. W. Rhudy}

This project demonstrated that PC-level microcomputer software can be used for graphical offline programming and simulation. User screens and interface software allow the nonexpert user to retrieve design information from a data file, look at the design in a visualization package, generate code for the EMCO milling machine, mill the parts, pass the design file to the Macintosh platform, generate code for the CRS 7-axis robot, and perform a weld simulation using the robot and the parts. A more expert user can create a MASTERCAM design file within the same environment.

Robots and numerically controlled machine tools for welding and other manufacturing tasks are generally programmed on-line- taught appropriate motions one step at a time, using the actual parts to be handled as models. Offline programming is more efficient, because these time-consuming teaching steps can be compressed, and it can also use part models that exist only electronically, such as CAD representations, to test the manufacturing viability of a design, and to store and modify complex programs for a variety of parts.

An estimated 30,000 small scale manufacturing facilities exist in the U.S. Typically, these facilities are equipped with PC-level (IBM, Apple) computers and employ machinists and lower-level engineering personnel. The project will bring offline programming and simulation into this $C A D /$ CAM environment to provide efficiency in the work cell without requiring a high level of CAD/CAM expertise from the staff or the use of expensive software and hardware.
Objectives

- Determine file transfer requirements.

- Implement input operations.

- Implement robot control.

- Select test object and implement offline cycle.

- Prepare documentation, including the final report and a video.

\section{Accomplishments}

This project successfully met all the objectives listed above and completed a demonstration of offline simulation and manufacturing using PC-level microcomputer software. The demonstration software and hardware remains in place and operational for technical tours. A video of the demonstration is available for off-site presentations.

Major accomplishments were identification of off-the-shelf software most appropriate for the application and the laboratory work cell; identification of the file transfer techniques that are effective in moving design files and machine code among the IBM PC, the EMCO mill, the Macintosh, and the CRS 7-axis robot; development of user interface software for the robot movements; and the successful demonstration of non-trivial milling and assembly of two parts using all elements of the software and hardware involved in the research. A process flow diagram was developed involving file transfer paths among the various computers, software packages, and manufacturing machines.

The 3-D model of the parts to be milled was constructed using CAD software and then transferred to both a computer aided manufacturing system and to the robot control program for use in placement of the parts and assembly on the robot work table. The design models were created using several CAD programs, including AutoCad and MASTERCAM and were exported in a portable DXF format for use in the machining operation. The models were used to generate machine code to operate EMCO F1 mill through postprocessors supplied by CNC Software Corporation. The machine command sequences were transferred from an IBM PC through a communications link to the EMCO milling machine where the parts were constructed. The same DXF model files were also transferred to a Macintosh where they were used as a basis for the offline manipulation of the CRS robot.

Software was developed in the CAD package programming language to allow a user to automatically generate a file of robot instructions for tool and part positioning operations and a file of named locations in space. Both files are then downloaded to the robot for use. The advantage of using named locations allows references to parts and positions regardless of the specific workcell arrangement. The workcell can be rearranged, or even the process shifted to a completely different workcell, and the program will still run perfectly. Although the software developed is unique to the $\mathrm{CAD}$ program and the robot controller with respect to syntax, the methodology and procedure are universal to this type of application. Revision to allow operation with different $C A D$ programs and robot controllers is a straightforward matter.

Additional software was developed to allow an operator to control the robot via an intuitive graphical interface, removing dependence on the restrictive teach pendant or command line terminal methods. The software was built in Hypercard. In the indirect 
mode, the programs can be run independently of the computer interface.

A procedure was also developed for generating computer animation sequences (QuickTime movies) from robot time history information. This is accomplished by periodically recording robot joint encoder positions with time stamps.

The demonstration began with creation of the design files and visualization of the milling process (shown in the figure below). After generation of the milling machine code, two parts fitting into the other were machined. Part B was milled with the recessed design to accept the projected design of Part A. The demonstration continued with transfer of the design files to the 3-D CAD package on the Macintosh and user generation of the assembly commands. The assembly commands were transferred to the robot memory. To complete the demonstration, the CRS robot selected the gripper tool from the

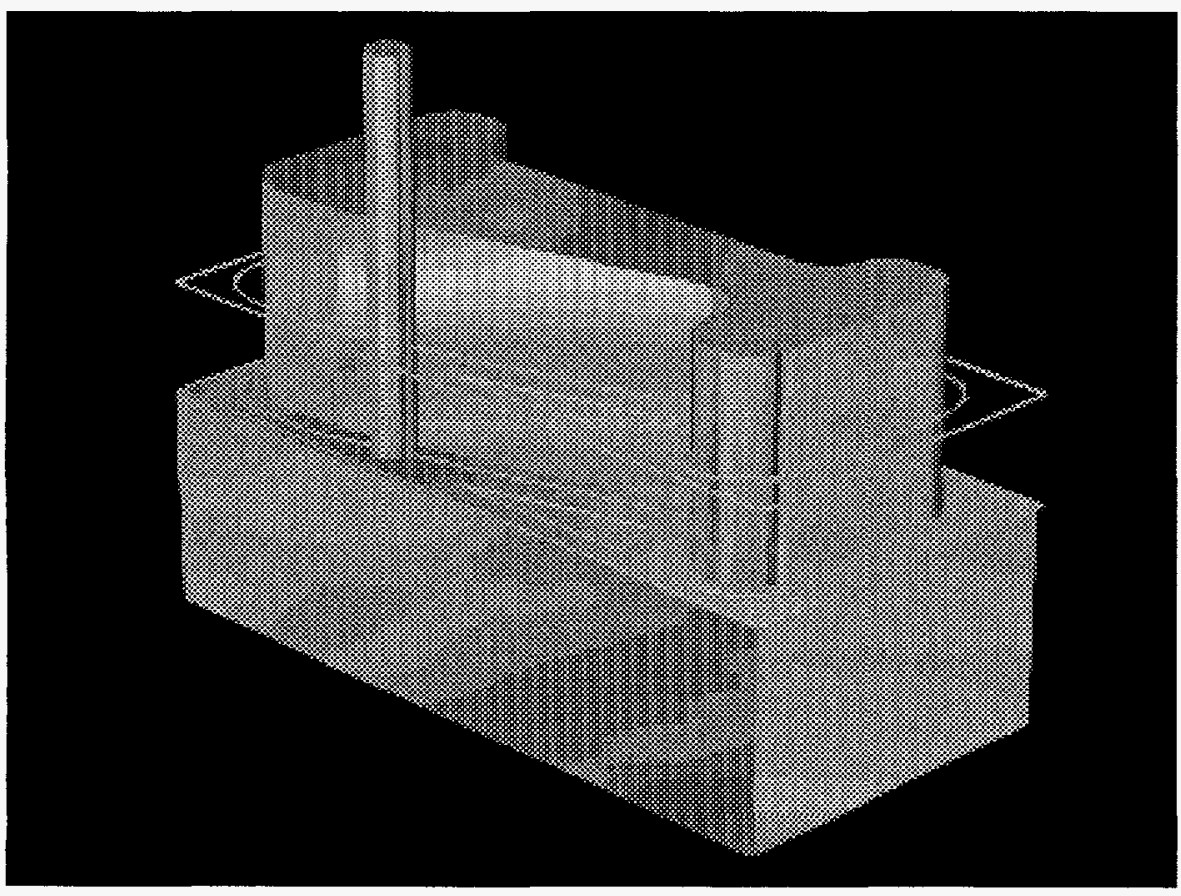

Machined Part A, with Visualization of the End Mill and Tool Paths storage holders, picked up and moved Part $B$ into position, then picked up Part $A$ and assembled it with Part B. The gripper tool was then returned to its storage position. The project video illustrates the demonstration.

\section{New or Increased Technical Capabilities}

A capability was developed to design parts using MASTERCAM on an IBM PC and successfully machine the part on the EMCO F1 mill after first visualizing the finished part. This capability has been in demand by other projects. The INEL now has the ability to use design files from the MASTERCAM package as input to the 3-D CAD package and program the CRS 7-axis robot through a user interface and computer network interface with the opportunity to obtain feedback from the robot on its movements. The capability to freely transfer parts design files in appropriate computer file formats is also new, as are the alternate modes for programming the robot.

\section{Business Development Opportunities}

Two industrial contacts were made during the course of the year, Lockheed Martin Missiles and Space (LMMS) and NewFocus. LMMS, Missiles Systems Division, is researching agile manufacturing under a variety of funding sources including ARPA and internal budgets. We will continue this contact in the coming year because of their interest in linking with small businesses, including electronic transfer of machine and part design data files. New Focus is a small business in Sunnyvale, California that manufactures and markets photonics tools. Under ARPA funding they are researching offline simulation and manufacturing for their robot and milling machine. During FY-96 they will visit INEL and discuss opportunities for future collaboration and joint work. A software disclosure was submitted to the Technology Transfer Office.

\section{Spray Forming Titanium into Billet and Sheet}

\author{
LDRD 8712
}

\section{A. D. Watkins}

The process of reducing titanium, melting sponge, remelting, forging large ingots, and rolling to produce sheet has high fixed costs. Developing technology that can circumvent part of this process will lower fixed costs and allow titanium to enter new markets (e.g., automotive). Spray forming billet and sheet directly from molten titanium is one technique that will lower fixed costs considerably by eliminating ingot casting and subsequent forging operations. Spray forming of titanium alloys is accomplished by atomizing a molten stream of metal and directing semi-solid particles onto a substrate. This project addresses 
refitting improving the gas atomizer for superior titanium spray followed by micro-characterization of the sprayed material.

The development of process equipment to spray titanium initially started by designing a cold wall crucible spray system using existing spray casting equipment and a small cold wall crucible that had a capacity to melt only $10 \mathrm{lb}$ of titanium. Tests using this equipment and a $100 \mathrm{~kW}$ power supply were run to try and melt a solid rectangular charge of titanium weighing approximately $10 \mathrm{lb}$. The titanium charge was partially melted, but the equipment was unable to fully melt the charge in the crucible. Power input limitations to the charge during melting were experienced that necessitated design changes.

\section{A larger diameter chamber was} built that had a larger working area. $A$ $20^{\prime \prime}$ diameter cross was added to the middle of the spray forming chamber. This allowed the addition of a linear slide that incorporated a heated preform. A larger cold wall crucible assembly was also designed and built. $A$ new $150 \mathrm{~kW}$ induction power supply was installed and tested. These initial tests indicated that a SCR and diode failed on startup of the power supply. figure below shows a schematic of the gas atomizing equipment and the cold wall crucible with atomizing die that were used for these tests. Titanium is a very reactive metal especially at high temperatures. The wall of the crucible is internally cooled by water allowing titanium to solidify against the crucible wall, thus creating a buffer layer to reduce contamination between the crucible wall and the molten titanium. As the inductive field is applied around the crucible, molten titanium from the molten center fills the lower nozzle. Another inductive field is applied to the nozzle which maintains the titanium in its molten state and allows a molten stream to flow out of the nozzle into the atomizer die. Gas jets in the atomizer die impinge onto the molten stream of titanium causing the stream to break-up into small droplets and to be deposited onto a solid substrate.
Initial tests only partially melted the titanium charge and revealed several design and operational problems:

- That the magnetic field partially coupled with the chamber wall of the gas atomizer. This raised the temperature of the wall to well over $100^{\circ} \mathrm{C}$ and the experiment was terminated before the inert atmosphere was lost due to O-ring seal degradation.

- That there was an imbalance between the coil design and the load on the power supply. This severely limited the amount of power that could be coupled with the titanium charge and resulted in a partially melted charge of titanium.

Several coil designs were tried that resulted in only marginal gains in the amount of energy that was coupled to the titanium charge. This series was terminated

\section{Objectives}

- Refit the inert gas atomizer with a new die and cold-wall crucible.

- pray titanium onto a drum substrate.

- Analyze microstructure of sprayed titanium.

- Spray LCB alloys on linear substrates.

- Investigate spray forming system.

- Analyze microstructure of LCB spray deposits.

Accomplishments

The gas atomizer at the INEL Research Center was refitted with cold wall crucible technology. The

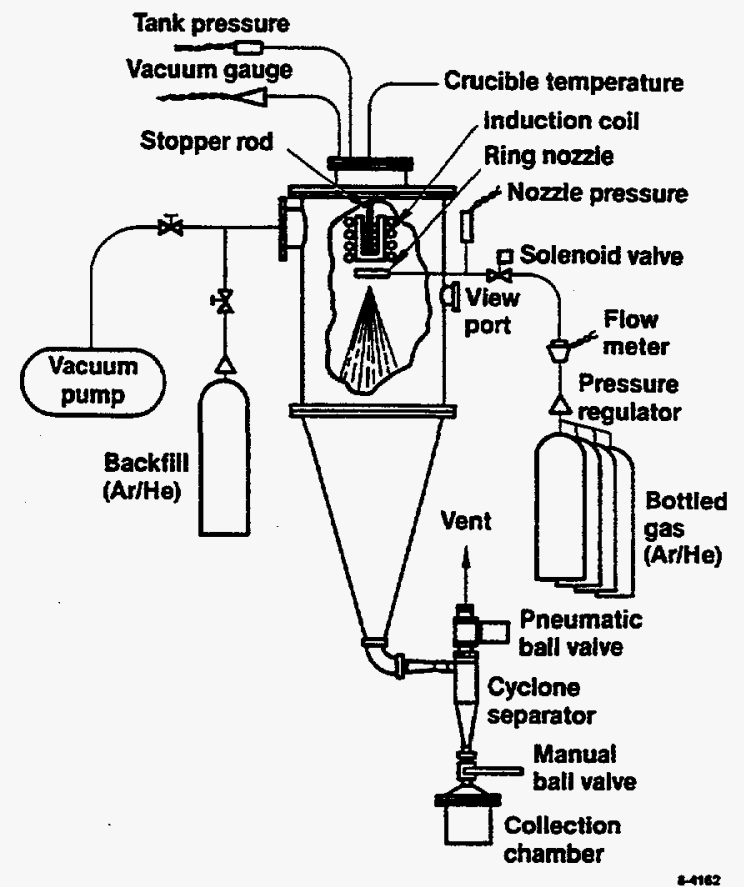

Gas Atomizer Located at the INEL Research Center 
because of the power supply that was being used was moved to the May Street Facility.

Another cold wall crucible, that had different dimensions, was fabricated and built to increase the magnetic coupling with the titanium charge and reduce the coupling with the atomizer wall. A new $150-\mathrm{kW}$ power supply was purchased. Initial test on the power supply indicated that there were problems with an SCR and diode in the main power circuit. A requisition for these parts was submitted, but in order to reduce indirect spending the order was not placed with the power supply manufacturer.

\section{New or Increased Technical Capabilities}

With the installation of the cold-wall crucible, the INEL now has the capability to melt and atomize reactive metals, such as titanium, vanadium, niobium, and silicon. This capability is not only needed for spray forming and atomization of reactive metals such as titanium, but also serves as a test bed for casting and refining these metals.

\section{Business Development Opportunities}

The thrust of the spray forming process is to exploit the need for light weight material in transportation applications. The development of low cost titanium processing technology is of particular interest to the automotive industry, and collaboration with the U.S. Automotive Materials Partnership (US AMP) will be initiated. General Electric Corporate Research \& Development, Crucible Research, and Rohr Inc. have all expressed interest in titanium spray forming.

\section{Industrially Compatible Laser Ultrasonics}

LDRD 8806

\author{
K. L. Telschow, R. T. Allemeier, \\ T. C. Chatters, J. Lee
}

The use of laser sources and detectors for ultrasonic measurements of materials is gaining acceptance for nondestructive evaluation both during and after processing. Work at INEL has shown how this technique can be implemented in industrial circumstances on materials with rough and highly absorbing surfaces. This measurement method offers fully noncontacting capability, high speed and exceptional flexibility, all factors that lend it very desirable for in situ process monitoring and control. However, currently only laboratory setups are available for demonstrating the applicability of the technique to process monitoring. This is not sufficient, as industry would like to see the technology used at their facilities before committing funds for capital and labor training. Both the generation and detection methods can be made portable and compact for application in many processing situations by using laser diode systems. This project has investigated the use of laser diode systems for detection with the goal of developing a system suitable for demonstration in industrial facilities. The work has resulted in a small unit driven by a compact laser diode pumped solid state laser. The performance of this unit has been measured and the sensitivity determined to be better than $10^{-4} \mathrm{~nm} / \mathrm{Hz}^{-1 / 2}$ collecting $100 \mu \mathrm{W}$ of light reflected from the material surface. The unit is usable from $0.5 \mathrm{MHz}$ to above $50 \mathrm{MHz}$.

\section{Objectives}

- Determine laser system suitable for portable operation.
- Redesign laboratory setup into a compact rugged unit.

- Fabricate the interferometer feedback control stabilizer.

- Account for instrument vibrations and alignment requirements.

- Test and calibrate instruments.

\section{Accomplishments}

The approach that has been chosen is based on using a selfbeating interferometer of the confocal Fabry-Perot design. This approach uses all the speckles recovered from the sample surface reflection and can be used on rough surfaces. A long coherence length laser is focused onto a vibrating material surface. The reflected beam is phase modulated by the ultrasonically moving surface resulting in a very small Doppler shift in frequency, proportional to the surface velocity. A rough surface finish produces a diffusely reflected beam containing many speckles that is collected and fed into the interferometer. The Fabry-Perot interferometer essentially causes interference between each speckle and itself, such that the output from all the speckles is modulated in the same way by the ultrasonic motion. The main difficulties of this approach are (a) obtaining a suitable laser, (b) maintaining the interferometer tuned to the unshifted laser light frequency, and (c) collection of the scattered light and channeling it through the interferometer.

The experimental setup for ultrasonic detection using the Fabry-Perot interferometer is shown in the figure on the top of the following page. Several different solid state lasers were evaluated for this project. The first was a 


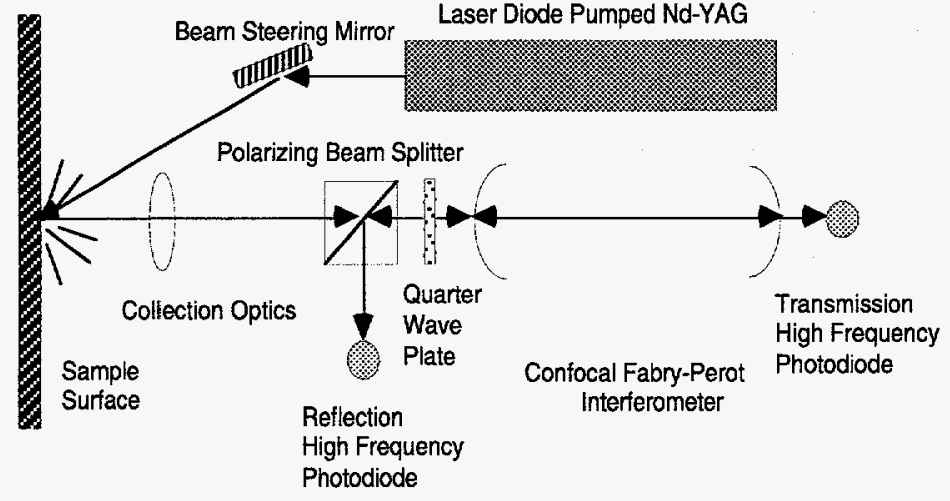

Laser Ultrasonic Detection Setup Using a Fabry-Perot Interferometer

low-power laser diode that did not possess the required coherence, although single mode behavior was observed. The next choice was a laser diode driven optical diode amplifier combination produced by Spectra Diode Inc. This laser had high power $(700 \mathrm{~mW}, 980 \mathrm{~nm})$, single frequency behavior and a moderate coherence length. However, it displayed a large phase noise such that it could only be used at very low frequencies. Operation was demonstrated at acoustic frequencies of less than about $50 \mathrm{KHz}$ by passing the beam through the sound wave radiation from a loudspeaker at distance of $60 \mathrm{~cm}$. Laser noise prevented application at higher ultrasonic frequencies.

A new laser system employing a laser diode to pump a solid state laser (Nd:YAG) has been obtained from Lightwave Inc. These lasers became in 1994 and exhibit very long coherence lengths and low phase noise. These lasers are expensive yet, but offer the characteristics needed for portable ultrasonic measurement as outlined here. A model 126, $1064 \mathrm{~nm}$ single frequency $500 \mathrm{~mW}$ laser was purchased with a linewidth of less than $10 \mathrm{KHz}$. This laser has proven to be well matched for our application. It is small, about $3 \times 3 \times 6 \mathrm{cu}$ inches, solid state, and very stable. Its only drawback is operation in the near infrared (invisible) with a lower sensitivity due to the longer wavelength than the argon laser $(514 \mathrm{~nm}$ ) used conventionally. A contact piezoelectric transducer $(10 \mathrm{MHz})$ generated and detected ultrasonic waves in a plate of Inconel, 6-mm thick, from the back side. The detection of ultrasonic motion at a surface was simulated by phase modulating the laser beam with an electro-optic modulator that could be sinewave driven up to about $60 \mathrm{MHz}$. In this manner the entire system response could be accurately measured as a function of frequency and ultrasonic surface displacement. This phase modulator produces a shift corresponding to a displacement of $91 \mathrm{~nm} /$ volt input. The signal to noise ratio is about 2000 at $10 \mathrm{MHz}$ for the spectrum analyzer bandwidth of $1 \mathrm{KHz}$. This results in an overall ultrasonic displacement sensitivity of about $10-4 \mathrm{~nm} / \sqrt{\mathrm{Hz}}$. For wideband operation, this corresponds to about $3 \mathrm{~nm}$ sensitivity for $10 \mathrm{MHz}$ bandwidth, commonly used in ultrasonic measurements. This is adequate sensitivity for many ultrasonic applications where noncontact portability is required. Personnel contributing to this project included both principal investigators, a postdoctoral appointee, and an electronics technician. Major equipment items, such as the laser power supply, high power laser diode and interferometer optics, were purchased from other programs that will utilize this technology for on-site laser ultrasonic demonstrations and evaluations.

\section{New or Increased Technical Capability}

This project has been successful in reducing the size of the conventional laser ultrasonic detection system to a size of about $1 \times 0.5 \times$ 2-cu feet in a manner that is robust, stable and portable. It does not require an optical table or specialized vibration isolation equipment. It can be operated like other pieces of portable electronic equipment. This is a new capability for INEL. Field uses are now possible for laser ultrasonic detection from materials with rough surfaces using this device.

\section{Business Development Opportunities}

This project has been driven by the needs of many industrial partners for on-site demonstration of laser ultrasonic measurement capability. The device developed on this project will be used at several industrial sites under allied projects., including Molten Metal Depth Sensing, a CRADA consortium including Retech, Viking Metallurgical, General Electric, Teledyne Wah Chang, Teledyne Allvac, and Axel Johnson; NDE of Superconductors, a DOE-BES project including IGC Advanced Superconductor and American Superconductor. Development of this technology for the microelectronics industry is also underway through a CRADA with Simpex Technologies of California. Capabilities of this device are being considered for use in potentially new projects and proposals involving Howmet Corporation for in situ monitoring of the metal casting process and by 
Harvest Technologies Co. and Phillips Petroleum for in situ monitoring of hydrocarbon deposits during the refining process.

\section{Optimization of Automobile Component Shapes}

\section{LDRD 9508}

\section{D. Landon, R. W. Johnson}

Optimization is a mathematical science that can be applied to engineering design to modify existing or initial designs to maximize performance; because the optimization and analysis of the design are computerbased, the potential for shortening the design cycle is great. We call this technology Engineering Design Optimization. Engineering Design Optimization (EDO) is still an emerging technology and is not nearly as widespread as it should be. The overall goal of this project was to apply EDO to internal fluid flow and heat transfer problems of interest to the automobile and other industries to demonstrate the effectiveness and usefuiness of the technology. The specific objectives for the present proposal were to combine existing fluid flow simulation software with existing optimization software to analyze and optimize the shape of internal flow components. The optimization and analysis software are complemented by advanced arbitrary shape deformation software that provides the capability to change the component shape as part of the computerized design and optimization loop. The objectives that were achieved include optimization of the shape of a channel with a 90-degree bend to minimize pressure drop and heat loss and the optimization of the shape of an electronic cooling channel to maximize the heat transfer coefficient from the heated wall to the coolant. The results have been publicized through presentations at three engineering conferences, through publication of an article in an engineering newsletter and through numerous presentations given to engineering groups within the automobile and other industries.

\section{Objectives}

- Link existing optimization and shape deformation software to a commercial CFD code (FIX- aces of the heaters (chips) that are being cooled by the flow of fluid in the cooling channel.

\section{Accomplishments}

In order for the optimization software to be able to control the optimization problem, it had to be able to call the engineering analysis code, in this case, a computational fluid dynamics (CFD) code. The first objective was to write a simple interface to allow communication between the optimizer and the CFD code. The interface was not written as a general interface between the two codes; it was problem specific. A more general, user-friendly interface would require considerably more time and effort.

The first internal flow component to be optimized concerned the steady, laminar, incompressible flow of air in a two-dimensional channel with a 90-degree bend. Air enters the channel at a temperature higher that the channel wall temperature. Heat is lost through the walls of the channel to the atmosphere. Initially, the objective

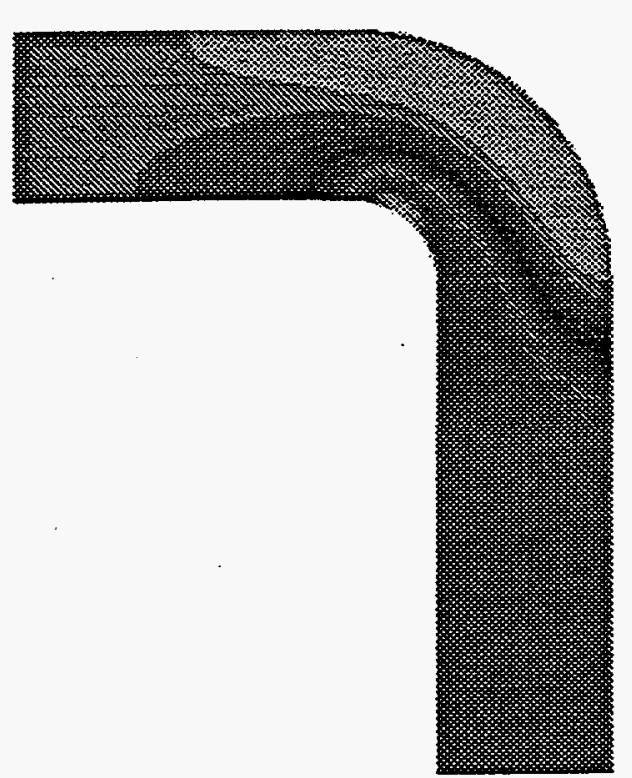

(a) Initial Shape

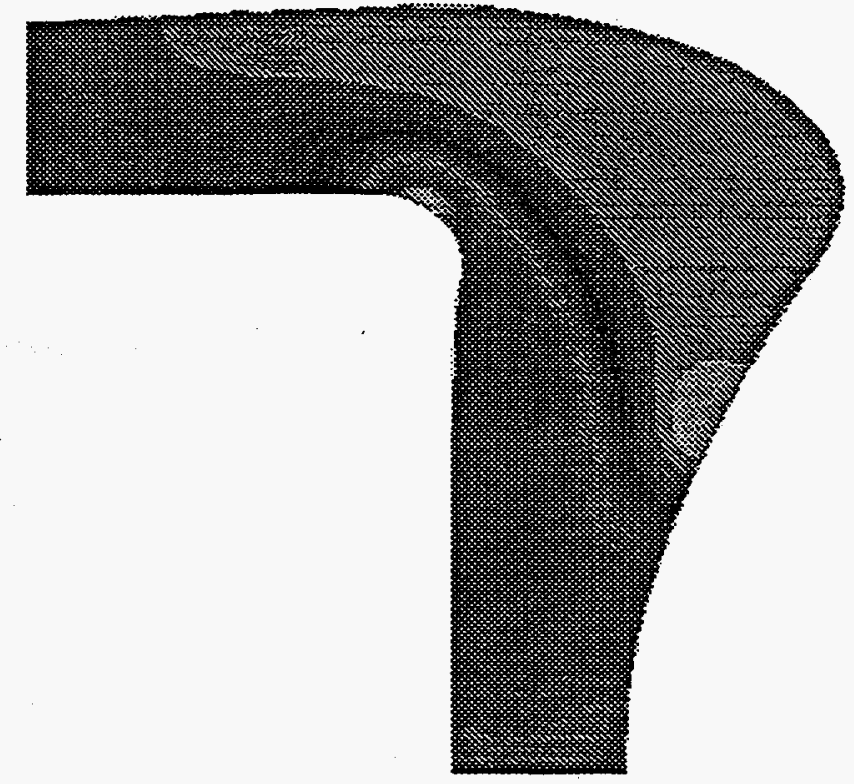

(b) Optimized Shape

Initial and Optimized Shapes and temperature Contours for a Channel with a 90-degree Bend; the Objective Was to Minimize the Heat Loss and Pressure Drop through the Bend 
of the optimization problem was defined to be the minimization of pressure drop through the channel. A commercial CFD code (FIDAP) was used to provide the flow and heat transfer analyses for the channel designs. The optimizer was constrained to maintain the width and locations of the bend inlet and outlet and to avoid violating the space on the inside of the bend (where some other component may be located). Using the arbitrary shape deformation software, the optimizer changed the shape of the bend automatically to meet the objective. As an example, the figure below illustrates the geometry and pressure contours of the initial and optimized channel designs for minimal pressure drop. We see that the outer wall of the channel bend moves outward considerably; the optimal design yields a $42 \%$ reduction in pressure drop.

For the second optimization problem, the objective of the previous optimization problem was modified to simultaneously minimize the heat loss from the air through the channel walls to the atmosphere and the pressure drop through the bend. As it happens, the two objectives are competing since minimization of the pressure drop pushes toward a larger radius bend while minimization of the heat loss leads to a bend with smaller area for heat transfer. Based on the same constraints specified for the previous problem, the optimizer found a new optimal shape where the pressure drop has been reduced by $14 \%$ and the heat loss has been reduced by $5 \%$ from the initial design.

The third component to be optimized was first suggested by an Idaho State University professor, Professor Antonio Campo. The problem was to improve the heat transfer in a two-dimensional cooling channel with several discrete heaters mounted on one wall to represent the cooling of a series of electronic chips. The objective was to minimize heater-face temperatures; the distribution of wall temperatures for the three cases was predicted.

The final component that has been optimized is a more difficult problem: a three-dimensional exhaust duct from a General Motors car. The exhaust duct has an S-curve in it to connect the exhaust manifold to the catalytic converter. The objective function is to minimize the pressure drop in the duct. Maintaining a constant duct cross-section, a pressure drop reduction of $20.4 \%$ was achieved. Further pressure drop reduction is expected if the cross-sectional area is allowed to change.

\section{New or Increased Technical Capabilities}

The capability to apply optimization methodologies to find optimal solutions to real engineering problems could become a unique niche for the INEL. That is, the INEL can become the leader in developing and using optimization tools coupled with engineering analysis tools to solve real engineering problems. The computerbased optimization tools not only find optimal solutions, they do so very efficiently with the very important result of shortening design cycles. Our overall goal is to develop our optimizing tools that allow us to go directly to industry and help them solve their engineering problems; we expect that some of the designs will be patentable.

\section{Business Development Opportunities}

Direct collaborative relationships have been established with automotive engineering departments. These people are interested in having us optimize specific components for which they can then produce a rapid prototype to test to validate our optimal designs. If proven useful, they wish to license our software. The departments with whom we have such collaborations include

- General Motors, Engineering Mechanics Department

- General Motors, Powertrain Group

- Ford Motor Company, Engine Research Department

- Ford Motor Company, Climate Control Division.

\section{Mediator \\ LDRD 9511}

\section{A. Dean}

The primary objective of this project is to demonstrate that mediator technology can be used to effectively and efficiently resolve end-user information access issues and to establish an understanding of mediator technology capabilities at the INEL. A secondary objective is to develop a data access product that will use intelligent intervention to mediate between end user workstation applications and databases to simplify access to data and cut information processing costs.

Mediators provide intelligence for understanding, selecting, accessing, merging, and manipulating data. They provide a level of knowledge that an end user may not know or understand about the data and applications. Because of this intelligence, mediators provide consistent responses to questions regardless of who asks the mediator a question. In addition, mediators integrate information from multiple sources without having to redesign existing databases or change existing operational systems.

The purpose of this project is to allow the Data Access Products (DAP) Directorate to partner with an INEL end-user area (Environmental Operations) and the mediator technology developers (Stanford University and ISX Corporation) to resolve a specific end-user information access issue, 
train DAP personnel in mediator technology capabilities and uses, and to develop a basic set of mediator building block products (i.e., mediator toolkit) for use at the INEL and possible commercialization. This project will focus the mediator technology on solving an accessibility and using problem for Environmental Operations.

\section{Objectives}

- Form an integrated product team with Stanford University and ISX Corporation.

- Conduct knowledge acquisition interviews with Environmental Operations personnel and select a domain scenario.

- Develop a long range visionary demonstration using the selected domain scenario.

- Present initial prototype architecture and visionary demonstration to INEL personnel.

\section{Accomplishments}

A subcontract was issued in June, 1995, with Stanford University and ISX Corporation. This allowed the INEL to leverage and capitalize on mediator research efforts already conducted by academia and private industry by including representatives from Stanford University and the ISX Corporation on the product team. Stanford has extensive experience in research and database domains as well as intimate familiarity with mediator research conducted by the Advanced Research Projects Agency (ARPA). ISX has acted as a mediator technology broker; specifically in using ARPA-developed concepts in engineering and manufacturing for the F-22 project.

During July, 1995, several knowledge acquisition interviews were conducted by the Integrated Product Team comprised of INEL DAP personnel and Stanford University and ISX Corporation representatives. These interviews resulted in a specific Environmental Operations data access problem being identified and selected as the project domain scenario. This selected domain scenario will be the basis for the mediator development work in FY-96.

Stanford University and ISX Corporation representatives then developed a visionary demonstration that visually describes the approach and an initial prototype computer hardware and software architecture that will be implemented using mediator technology to resolve the selected domain scenario. This visionary demonstration was delivered in August, 1995, and presented to the Environmental Operations Branch and DAP personnel.

\section{New or Increased Technical Capabilities}

Work in FY-95 was specifically oriented to identifying an actual end user problem on which to apply the mediator technology. An actual technical solution for this problem will be developed in FY-96. At this time, any new or increased technical capability at the INEL is limited to an improved understanding of mediators and their capabilities and additional insight into what software products will be used in developing mediator applications. The mediator prototype to be developed in FY-96 will require use of software that provides the following functionality:

- User interface builder

- Persistent object store

- C++ compilers

- Module interoperability

- IP/IPX conversion

- User database access.

Business Development Opportunities

A working relationship was formed with Stanford University and ISX Corporation to partner with the INEL in developing mediator applications for a selected Environmental Operations data access scenario. Both Stanford and ISX are interested in participating in the project to gain additional knowledge about the uses of and development of mediator applications in a nondefense oriented user domain. Application of mediator technology to an Environmental Operations scenario broadens the specific domains to which this technology has been successfully applied. This will make it easier to generalize the development of mediator applications and add to a mediator development toolkit. As a result, the probability of commercialization opportunities for this technology will be increased.

\section{Gas-Driven Photovoltaic Generators LDRD 9512}

$$
\text { R. L. Moore }
$$

A gas driven photovoltaic generator is a device used to generate electricity by photovoltaic conversion of photons emitted from a heat source heated by the combustion of a gas. The objective of this project was to develop a simple thermal system model that couples the combustion process with the energy generation process of a gas-driven photovoltaic electrical generator. The simple model was used to help assess the overall thermal efficiency of a proposed generator whose size is on the same order as a battery pack used to power a cellular phone. The efficiency of such a generator relies heavily on recuperation, both optical and thermal, to achieve high system efficiency. The model of the gas driven photovoltaic generator considers both opti$\mathrm{cal}$ and thermal recuperation. 


\section{Objectives}

- Develop a simple thermal system model of a gas driven photovoltaic electrical generator.

- Evaluate generator performance as related to optical and thermal recuperation.

\section{Accomplishments}

It has been proposed that powering a cellular phone with a small photovoltaic generator in place of batteries may be feasible. If this were the case, it could lead to a large profitable market for the developer of such a product. The photovoltaic generator as envisioned would allow the cellular phone to be operated continuously for times far greater than that associated with the life time of batteries.

There are many questions associated with the development of a miniature photovoltaic generator that must be answered before such a device can become a reality. This project was initiated to address the question of what is the maximum overall thermal efficiency such a device can realistically achieve.
Experts in the field believe the overall thermal efficiency could be as high as $20 \%$ if both optical and thermal recuperation are used.

A simple Mathcad model of a small photovoltaic generator was developed. The model consists of a combustion process where butane gas is mixed stoichiometrically with air and ignited. The energy from the combustion of the butane with air is used to heat a filament (emitter), which in turn emits photons collected by a photocell and converted to electricity. The emitter and photocell are coupled by means of a three-band radiation transport model. Ytterbial optical properties were used for the emitter, and silicon oxide properties were applied to the PV cell. The emissivity used for Ytterbial and silicon oxide is shown in the figure below.

The results from the model show that the thermal efficiency of the generator is a very strong function of it ability to recuperate thermal energy from the combustion chamber exhaust gas. The thermal efficiency ranges from $4.25 \%$ with

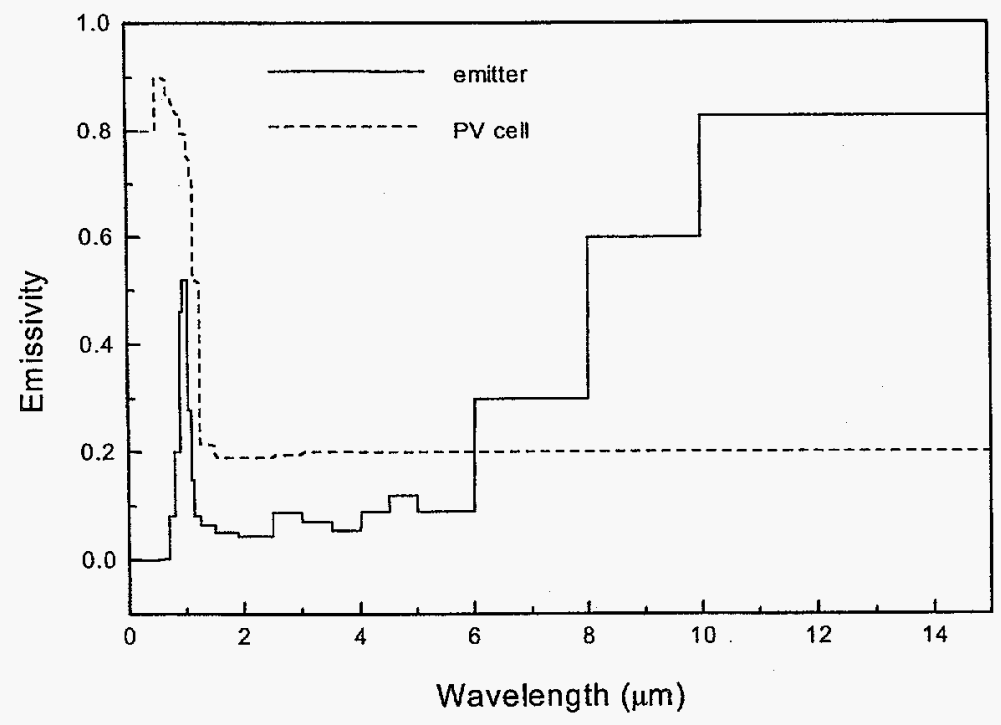

no thermal recuperation (air inlet temperature equal to $300 \mathrm{~K}$ ) to $11.5 \%$ with substantial thermal recuperation (air inlet temperature equal to $1300 \mathrm{~K}$ ).

\section{Measurement of Crude Oil Corrosivity} LDRD 9514

D. F. Glenn

Corrosion is a matter of particular concern in the petroleum industry. Corrosivity measurements are necessary in order to determine if today's boatload of crude is significantly different from the last one received. Current methods for evaluating crude corrosivity involve fractionation of the oil followed by time consuming weight loss testing of metal coupons in each fraction. The development of a rapid method to determine the corrosiveness of crude oil to the metals in the refinery has a clear dollar value.

The objective of this $L D R D$ effort was to determine the applicability of Electrochemical Impedance Spectroscopy in the determination of the corrosivity of crude oil. To this end, six crude oil samples were obtained from Exxon and the impedance spectra of each crude oil on different metals was obtained. It was determined that impedance spectroscopy could indeed detect differences in crude oils and the technique holds promise for the development of a rapid method for the determination of crude oil corrosivity.

\section{Objectives}

The objective of this effort was to obtain electrochemical impedance spectra on several metals immersed in different crude oils and to determine from this whether or not the technique might be developed in a viable method for the determination of crude oil corrosivity. 


\section{Accomplishments}

Electrochemical impedance spectroscopy is an electrochemical technique whereby a series of discrete ac signals (a spectrum) is passed between two electrodes immersed in the solution of interest and the resultant current and phase shift determined. From this data, the impedance of the metal in a given solution can be calculated. This impedance, between the metal and the solution, is related to the charge transfer kinetics which is in turn related to the corrosion rate of metal in a given solution.

To determine the applicability of Electrochemical Impedance Spectroscopy in the determination of the corrosivity of crude oil, six crude oil samples (three crude oils and three crude oil fractions) were acquired from an Exxon refinery. The impedance spectra of different metals immersed in each crude oil sample was obtained. The crude oils were diluted 50/50 with hexane and the impedance spectra obtained on 1020 steel, iron, copper and silver electrodes. Each spectrum contained 41 discrete frequencies ranging from 100,000 to $0.05 \mathrm{~Hz}$ at $700 \mathrm{mV}$ rms.

The experimental consists of an electrochemical potentiostat, a phase-sensitive lock-in amplifier, and a computer with dedicated software. A standard three-electrode arrangement was used for the cell itself.

The figure below shows an example of the raw data obtained on three crude oils using 1020 steel electrodes. These data are shown in the Bode format in which the real component of impedance is plotted versus the frequency of the applied ac signal. Of interest in these data is the straight line formed at the left-hand side of the plot. It is this impedance that is related to the resistance to electron transfer and therefore can be related to the corrosivity of the oil. The simple interpretation of this data is that the smaller is the impedance, the more corrosive is the oil. Therefore, sample $A$ is more corrosive than $B$, which is more corrosive than sample $C$.

The data obtained in these experiments clearly demonstrate that electrochemical impedance spectroscopy can detect differences in various crude oils and crude oil fractions. This is very encouraging

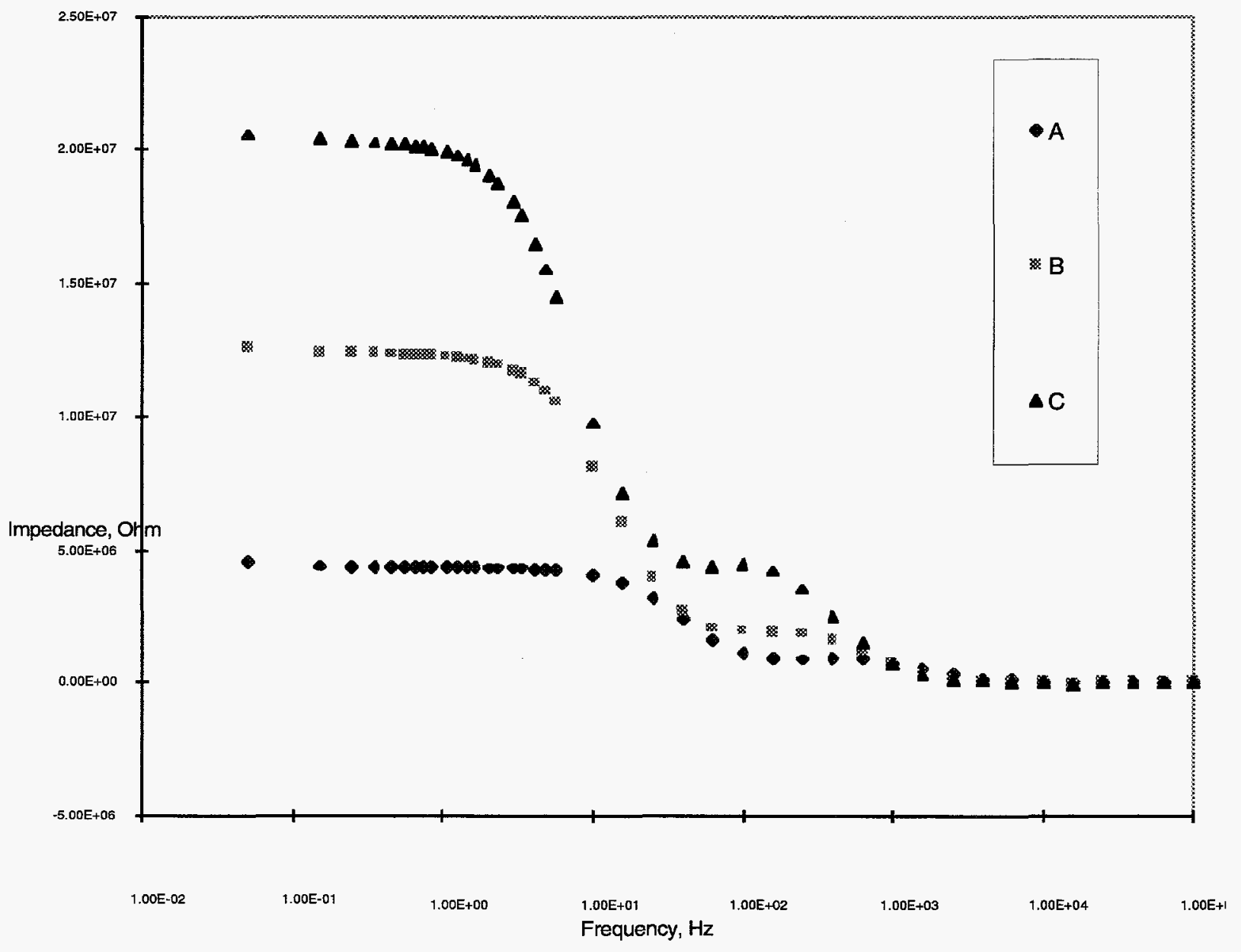

Bode Format Impedance Spectra for Crude Oil Samples A, B, and C 
and presents the real possibility that, with some further development, this technique can be used to determine the corrosivity of crude oil.

\section{New or Increased Technical Capabilities}

Electrochemical impedance spectroscopy is a relatively new technique in electrochemistry. This technique has been little used at the INEL until this project. The development of expertise in this area is of value to the INEL for the measurement and study of various electrochemical phenomena. Electrochemical Impedance Spectroscopy has applications in the study of corrosion, metal coatings, semiconductors, batteries, and fuel cells.

\section{Business Development Opportunities}

This project was initiated in an effort to establish a working relationship with the petroleum industry. The crude oil and crude oil fractions for this project were supplied by the Exxon Corporation. They have expressed a strong interest in using the results of this effort to improve their current methods for the determination of crude oil corrosivity. Further, they have expressed an interest in funding further development of this technique for their specific needs.

\section{Installation of Precession X-Ray Camera for Single Crystal Analysis} LDRD 9515

\section{H. O'Brien, J. R. Kennedy}

The purpose of this small yearend task is to install a precession $x$-ray camera donated by the National Institute and Standards and Technology to add needed single-crystal analytical capability to the INEL.
Objectives

- Install precession camera in existing IRC $x$-ray cage.

This simple project was initiated with only severn weeks left in the fiscal year. The task was not completed at the time of this reporting.

\section{Accomplishments}

Precession x-ray cameras allow the analysis of the crystal structure of new and unknown single crystals. Most materials and solid state chemistry laboratories have this capability. It has long been needed as a general scientific asset at the NEL. It should contribute to a variety of projects in the future.

\section{Business Development Opportunities}

While no direct business development is envisioned, it is likely that this capability will contribute to projects having commercial potential.

\section{Direct Production of Low-Cost, High-Quality Nanocrystalline Metal Powders LDRD 9516 \\ B. A. Detering}

The purpose of FY-95 efforts at the INEL were to support and compliment INEL/URC-funded research at the University of Idaho (UofI) for synthesis of nanomaterials and characterization of these materials to delineate their unique characteristics and potential industrial applications. The plasma fast quench (PFQ) technology has been demonstrated for synthesis of nanomaterials and limited funding was used to design, construct, and test a PFQ device and process for synthesis of nanocrystalline silver powder at the INEL. Work during FY-96 was to focus on synthesis of titanium and titanium alloy nano- materials, including titanium metal and titanium/aluminum alloys for automotive applications. This FY96 work directly complimented and supported work on synthesis of these materials by mechanical alloying methods being performed at the U of I. A silver powder was successfully synthesized during September 1995. Limited time did not allow for physical characterization of this material. The material was synthesized in a straight tube reactor without the quench nozzle. Two industrial contacts were made and expressed interest in this material as a replacement for hazardous solder in fabrication of electronics and in biomedical plastics.

\section{Objectives}

- Design a PFQ system for synthesis of nano-phase silver materials.

- Modify and adapt the current PFQ system for synthesis this material.

- Solicit and develop industrial collaborations with identified industrial partners.

- Make one trip to the U of I to discuss the course of interaction with INEL/URC collaborators, and to assess the university program plan and progress.

- Submit a FY-96 LDRD project proposal to continue development and applications of the PFQ process for nano-phase powder synthesis.

- File an invention idea record with LITCO for the nano-powder synthesis technology.

\section{Accomplishments}

A PFQ system design for synthesis of nanophase silver powder was completed by September 1 , 1995. 
Modifications of the PFQ hardware for synthesis of the materials was completed and tested on September 11, 1995.

Industrial contacts were made with Dr. Nancy Scoville of ThermoTrex, Inc., Waltham, Massachusetts and Dr. William Hendrickson of AVEKA, Inc., Woodbury, Minnesota.

One trip was made to the University of Idaho campus on September 7-8, 1995 to meet with Professor Froes and Professor Prisbrey (the INEL/URC collaborators) to discuss the course of interaction and to assess the university program plan and progress.

Two LDRD proposals were submitted that would fund continuation of the work.

Insufficient data were generated in FY-95 to justify filing an invention disclosure. Depending on available funding, at least one invention disclosure should be filed in FY-96.

New or Increased Technical Capabilities

The limited data obtained here indicate that the rapid quench nozzle is not necessary to produce nanocrystalline silver powder. Silver powder was produced during one test using a reaction chamber of constant diameter $(0.022-\mathrm{m}$ diameter $\times 1.0$-length) operated at low pressure $(0.2 \mathrm{~atm})$. This test indicates that for silver and other nanocrystalline materials, the expensive design and construction of converging/diverging nozzles may be eliminated. This would drastically reduce the capital and operational costs associated with commercial production of these materials.

\section{Business Development Opportunities}

Contacts were made at both AVEKA, Inc., of Woodbury, Minnesota and ThermoTrex, Inc., of Waltham, Massachusetts. AVEKA is a small company specializing in coating fine powders, and ThermoTrex is a producer of medical technology. AVEKA showed interest in silver powders as a way of producing conducting epoxy cements for the electronics industry as a replacement for hazardous soldering of electronic components. ThermoTrex was interested in using nanocrystalline silver powder in bio-plastics to inhibit bacterial infections when these materials are placed in the human body. Some examples of potential applications are catheters, artificial valves, and artificial hearts.

\section{Petroleum Tanks Inspection Delivery System \\ LDRD W091}

\section{W. T. Zollinger}

In the petroleum and chemical industries, there is a relatively new requirement to inspect the bottoms of storage tanks for corrosion and leaks. The current method of inspecting the tank floors is to drain the tank, decontaminate and clean the interior, install ventilation, and allow workers to enter the tank with inspection equipment. This costs between $\$ 50,000$ to $\$ 250,000$ depending on the size of the tank, its age, commodity and hazard classification, and type of inspection, and may take the tank out of service for up to a month. The inspection could be performed using a remote controlled vehicle for $\$ 10,000$ to $\$ 20,000$ in several days while the tank remains in service. The INEL magnetic wheeled wall crawler is being redesigned to fulfill this role.

The economic benefits to the U.S. petroleum industry and local economy will be substantial. The members of the American Petroleum Institute estimate the costs of coming into compliance with the new inspection standard, API 653, to be in the billions of dollars using current methods. Cutting those costs by a factor of 10 will have a great impact on their competi- tiveness worldwide. The local economy will also benefit from the commercialization of this technology. As a part of the licensing contract, the manufacturing facilities for the commercial inspection systems are to be located in the INEL area. This will both create jobs in the local area and require services from local industry, infusing the local economy with an estimated six million dollars in the first three years. Solex Environmental Services is currently negotiating the details of an initial CRADA with Lockheed to continue the commercialization process.

\section{Objectives}

- Design modifications needed for submergence.

- Fabricate a submergible prototype.

- Test and evaluate system performance.

- Initiate technology transfer to private industry.

Accomplishments

The schedule of the project was accelerated in the first quarter of FY-95 to achieve specific design goals in order to asses the system's feasibility. This information was used successfully to petition DOE for additional funding to fully commercialize the system. $\$ 400 \mathrm{~K}$ was obtained mid year for this purpose, with the promise of an additional $\$ 600 \mathrm{~K}$ for FY-96. At that time, the entire project was rescoped and became a program under the DOE Office of Technology Development. The technical objectives for this LDRD were then transferred to that program, and expanded so as to produce a commercial prototype ready for immediate transfer into the private sector.

Design modifications needed for submergence were completed ahead of schedule and fabrication of the vehicle was pursued through the end of the LDRD funding. At this point, additional programmatic funding was procured and the 
goals developed were rescoped to include additional capabilities needed to perform a complete petroleum tank inspection.

The base vehicle was reworked to increase its speed and capability. Larger diameter magnetic wheels were integrated to increase the payload from $20 \mathrm{lbs}$ to $75 \mathrm{lbs}$ and allow the vehicle to maneuver over weld seams more easily. Weight of the vehicle was reduced by fabricating the frame from graphite composite panels. The motors were also resized to gain higher speeds, to allow inspection of the acreage of a storage tank bottom in the target range of two to three days. Fabrication of the new base vehicle is complete.

The control system and ultrasonic (UT) inspection equipment were integrated to reduce commercial hardware costs, and a positioning gyro was added. The original UT system was controlled by a separate Amdata UT control computer and software which added about $\$ 50 \mathrm{~K}$ to the price of the sys- tem. The UT inspection functions have been integrated into the vehicle control computer using portable UT preprocessors, reducing the UT system costs to below $\$ 15 \mathrm{~K}$. To meet explosive certification requirements, onboard temperature and pressure sensors have been added, and an inert gas purge/pressurization system is being designed into the vehicle. In addition, an independent positioning gyro was added to supplement the dead reckoning tracking system and improve data mapping. An onboard controller was added to coordinate data collection from all the onboard sensors. Conceptual design of the control system is complete, with fabrication underway. Programming of the upgraded control software will begin following hardware fabrication.

The current programmatic schedule is being maintained, projecting completion of the basic prototype and a demonstration within the year. Once budgets are decided upon and if the FY-96\$600
$\mathrm{K}$ is approved, development of a radiocontrolled version with added capabilities will be pursued.

New or Increased Technical Capabilities

Following development, which extends into FY-96, the INEL will capable of inspecting fuel storage tanks such as the emergency diesel fuel tanks on-line, without facility shut downs. This will become more important as EPA inspection requirements become more stringent.

\section{Business Development Opportunities}

A CRADA is currently being negotiated with Solex Environmental Systems of Houston, Texas, which includes licensing agreements for commercialization. End users of the inspection technology have been used throughout the project to specify design requirements and market information, including Exxon, Shell, and Mobil Oil. Numerous other companies in the industry were contacted by Thermo Technology Ventures in identifying the best avenue for commercialization. 


\title{
Long-Term Research Initiatives
}

\author{
Strategic Thrust
}

ine experientia nihil sufficienter sciri potest. Without expe-
rience, nothing can be certainly known.

- Roger Bacon,

On Experience, Both Spiritual

and Practical, about 1250 


\section{Molecular Biology LTRI 0010}

\section{T. E. Ward}

The objective of this LTRI is to develop the research capabilities of the INEL in the area of molecular biology. This is being approached through investigation of the genetic characteristics and capabilities of microorganisms that have the potential to be used in bioprocesses developed by the Biotechnologies organization. Biological processes have a number of advantages compared to chemical and physical processes. They generally take place at ambient temperature and pressure, saving energy, and also produce less toxic and hazardous waste. The research conducted under this LTRI will provide the capability of manipulating the genetic characteristics of the microorganisms to be used in the various bioprocesses. These microbes can be provided with either enhanced or entirely new capabilities. This work thus supports and can potentially contribute to all projects in the Biotechnologies organization. Continuing the strategy of evolving the program in response to changing circumstances, projects have been started in a several of new areas where there appear to be opportunities, and completed or terminated in other areas in which good progress was not being made or which did not appear to support the future direction of the Biotechnologies organization. The past year's projects have included construction and analysis of a recA mutant of Acidiphilium, construction and testing of potential transposons for acidophilic cells, studies on arsenic resistance genes in acidophiles, studies on bacterial chromate reduction, cloning of genes for production of a specific organic chemical, and development of an RNAbased assay to analyze the composition of microbial mixtures.

\section{Objectives}

- Construct and analyze $r e c A$ mutants of Acidiphilium.
- Construct and analyze potential transposons for Acidiphilium.

- Characterize a chromate reducing enzyme in E. coli.

- Study activity of arsenic resistance genes in acidophiles.

- Start cloning the genes for production of a specific organic chemical.

- Begin development of an assay for determining the composition of unknown mixtures of microbes.

\section{Accomplishments}

Construction and analysis of a recA- mutant of Acidiphilium

Construction of a rec $A^{-}$ Acidiphilium strain for use as a host in genetic experiments was successfully completed. A recA gene inactivated in vitro by insertion of a tetracycline resistance gene was constructed in a vector that replicates in Acidiphilium, and this plasmid was introduced into Acidiphili$u m$ cells that were grown for several generations to allow a double crossover to occur. These cells were then transformed again using a second plasmid, which is incompatible with the first and contains a different antibiotic resistance gene (chloramphenicol). After transformation with the second plasmid, these cells were inoculated onto plates containing both antibiotics. Since the two plasmids are incompatible, they cannot coexist in the same cell. One way for a cell to become resistant to both antibiotics is for the tetracycline gene in the recA segment in the first plasmid to have integrated into the recA gene in the chromosome by means of a double crossover. Colonies that were resistant to both antibiotics were tested for sensitivity to UV light, a characteristic of recAcells. A colony was identified that exhibited much greater sensitivity to UV light than wild type cells, and was thus a candidate recA- mutant. The DNA of this strain was analyzed, and the results showed that a tetracycline gene had integrated into the recA gene in the chromosome of this strain, exactly as expected. Thus, a recAstrain has been successfully developed.

Investigation of potential transposons for Acidiphilium cells.

Potential transposons for Acidiphilium cells had previously been constructed by introducing a tetracycline resistance gene separately into two different sites in an insertion sequence isolated from Thiobacillus ferrooxidans by Dr. David Holmes. This DNA construct was inserted into a vector that replicates in Acidiphilium, and the resulting plasmid was introduced into Acidiphilium by electroporation. Tetracycline resistant transformants were obtained. They were grown for several generations to allow transposition of the potential mobile element into the chromosome, and then transformed again with a second plasmid that is incompatible with the first and that contains an alternative antibiotic resistance gene, similar to the strategy used in the $r e c A$ work. These cells were then inoculated onto plates containing both antibiotics. This strategy is designed to select for cells in which the first antibiotic resistance gene has integrated into the chromosome. Numerous colonies resistant to both antibiotics were obtained, but analysis of their DNA showed that no integration of the potential transposon into the chromosomes of the cells had occurred. Thus, functional transposons for these acidophilic cells were not produced.

Characterization of a chromate reducing enzyme from $E$. coli. Chromate is a major toxic waste problem for both DOE and U.S. industry. Several bacteria have the ability to reduce chromate to chromium (III), a much less toxic 
and less mobile form of this element. It had previously been shown that whole cells of $E$. coli, a standard laboratory bacterium, have this ability, and that this activity can also be detected in cell free extracts of this bacterium. This year, major progress was made on characterization of this enzyme. The enzyme has a broad activity maximum around $\mathrm{pH} 6.5$. The crude extract was fractionated on a gel filtration column

(Sephadex G100), which separates proteins on the basis of size. The peak of chromate reducing activity trailed the major peak of protein, demonstrating that this enzyme is smaller than the majority of proteins in E. coli. Preparative isoelectric focusing demonstrated that this enzyme has a $\mathrm{pH}$ of approximately 4.7. The enzyme seems to be fairly stable on storage in both the refrigerator and freezer, and is relatively stable to heating at $60^{\circ} \mathrm{C}$ for 30 minutes, indicating much greater stability than the typical $E$. coli protein. The enzyme can be renatured to give apparently good recovery of activity after running on preparative SDS acrylamide gels, staining, eluting, removal of SDS on a Dowex column, and dialysis to renature. This is presumptive evidence for a relatively small protein, and possibly for an active monomer. On SDS gels, bands that have most consistently shown activity migrate at molecular weights of approximately 33-35 and $24-25 \mathrm{kd}$.

Study of arsenic resistance genes in acidophiles. Arsenic resistance genes are being studied as an alternative to antibiotic resistance genes for use in practical situations. We had previously shown that such genes could be introduced into Acidiphilium, significantly increasing their tolerance of arsenic. In order to better understand the mechanism of arsenic resistance, the detailed DNA struc- ture of a set of uncharacterized arsenic resistance genes was determined through construction of a detailed restriction map combined with hybridization studies. The results show that this set of arsenic resistance genes shows several similarities to a previously characterized set of such genes, but with some significant differences, which may help to explain the different results observed when these two sets of genes were separately introduced into the same Acidiphilium strain.

Cloning of the genes for production of a specific organic chemical. Of the various alternative routes for biological synthesis of this particular chemical, one starts with an inexpensive hydrocarbon, involves three intermediates, and requires three enzymes to convert the hydrocarbon to the desired chemical. The enzymes that catalyze this conversion are part of a pathway responsible for metabolizing the hydrocarbon to provide carbon and energy to a particular bacterium. Our strategy is to isolate from that bacterium just the genes for the enzymes required to convert the hydrocarbon to the desired product, to increase their level of expression, and to transfer them into other bacteria to produce cells expressing high levels of these enzymes. Such cells should be very efficient at converting the hydrocarbon substrate to the desired chemical. An industrial partner is working with us to develop a process to produce the same organic chemical using an alternative route. The difficulty has been that, although the genes for the first enzyme in the pathway have been extensively studied, very little is known about the genes coding for the rest of the enzymes in the pathway. Therefore, following the literature, we isolated a 10,000 -base-pair $(10 \mathrm{~kb})$ fragment of DNA that contains at least the genes for the components of the first enzyme in the pathway, and has approximately $4000 \mathrm{bp}$ in addition, which might code for additional enzymes of the pathway. In order to determine which genes are present on this DNA fragment (in addition to those for the first enzyme), bacteria containing this fragment of DNA were exposed to the hydrocarbon substrate for a few days. A portion of the cells and medium was extracted with an organic solvent, and the extract was concentrated and analyzed by HPLC along with an authentic sample of the desired product. A small peak with the retention time of the desired product was present in the extract from cells which contained the $10 \mathrm{~kb}$ DNA fragment, but not in the extract of control cells that did not contain this DNA fragment, indicating that all the genes required for this conversion are probably located on this 10-kb DNA fragment. This encouraging preliminary result must be confirmed and strengthened.

Development of an assay to determine the composition of microbial mixtures. Samples from the environment, bioprocesses, both sterile and nonsterile, food safety analysis, bioremediation operations, etc., often contain mixtures of microorganisms. An assay is needed to determine the composition of such samples simply and rapidly without having to culture the microbes in the samples. Many bacteria from environmental samples are not readily cultured, and any growth that occurs is invariably selective, thus creating the need for an assay that does not involve culturing. The assay we are developing analyzes the small ribosomal RNA molecules, termed $5 S$ rRNA, which are present in all bacteria. $5 \mathrm{~S}$ rRNA molecules from different bacterial species have different sequences, the degree of dif- 
ference reflecting the evolutionary relatedness of the various bacteria. These sequence differences also affect the shapes and stabilities of the three-dimensional structures into which the $5 \mathrm{~S}$ rRNAs spontaneously fold in aqueous solutions. To perform the assay, microbes in the sample are collected and lysed, and total RNA is isolated and analyzed using a special type of gel electrophoresis termed denaturing gradient gel electrophoresis

(DGGE). In this method, a gradient of denaturing chemical is formed perpendicular to the direction of electrophoresis. The sample is loaded in a horizontal band across the top of a slab gel containing this gradient. As the band of $5 S$ rRNA migrates down the gel, different amounts of denaturant in different areas of the gel cause continuously increasing degrees of unfolding of the RNA molecule and a resulting change in migration rate. These effects differ for the different 5S RNAs, which consequently run slightly differently from each other, revealing the number of different bacteria in the sample, and from the intensities of the bands, the relative abundance of the various species in the mixture. As yet, the position and shape of the bands cannot be used to identify individual species, but this capability may be developed if enough different species can be run to provide a computer library against which to compare any new band pattern, especially if marker $5 S$ rRNA molecules are included with each sample. An alternative method to identify individual bands involves transfer of the bands to membranes, followed by hybridization with probes specific for various groups of microorganisms. Three manuscripts are currently in preparation describing these various results.
New or Increased Technical Capabilities

We can currently perform all the standard molecular biologygenetic engineering techniques and procedures (gene isolation, cloning, PCR, DNA synthesis, sequencing, etc.). This would not have been possible without the support of this LTRI. We can effectively support a variety of projects of the Biotechnologies organization, which contributes to the integrated nature of the Biotechnology program at the INEL.

The capabilities and expertise that are due to this LTRI have resulted in several direct funded projects for the Biotechnology organization over the years. Many of these have come from DOE-Fossil Energy and the Bureau of Mines (DOI). The most recent example of this is a new task under the Alternative Feedstocks Program of the DOE-Office of Energy Efficiency, obtained as a direct result of the INEL's molecular biology expertise. Approximately $\$ 225 \mathrm{~K}$ was obtained in FY-95, and additional funding for FY-96 has also been secured. This project involves development of a biological process for production of a specific organic chemical from glucose. A commercial partner is heavily involved in this project, and a CRADA has been established. Work under the LTRI in support of this particular area is being expanded through investigation of an alternative approach to production of the same organic chemical, using an inexpensive hydrocarbon as a starting material instead of glucose. This project will involve genetic manipulations to produce appropriate bacterial strains, followed by bioreactor studies to optimize production of the chemical.

\section{Business Development Opportunities}

INEL currently has a CRADA with General Electric Co. to devel- op a process to produce a specific organic chemical from glucose. The funding for the INEL portion of the work is being provided by the Alternative Feedstocks Program as described above. GE has an internal customer that will use this organic chemical. This customer doesn't really care how this chemical is produced, as long as the price is right. Research under the LTRI is investigating an alternative process for producing this chemical. GE is aware of this alternative route and would have been interested in including this work in the current CRADA, or in establishing another CRADA to cover it, but they currently have no matching funds to put toward this project. If additional funding became available to them, they would probably be interested in a CRADA in this area as well.

\section{Nuclear Physics LTRI 0020}

\author{
R. Aryaeinejad, J.D. Cole, \\ M.W. Drigert, R.C. Greenwood, \\ J.W. Mandler
}

The basic objectives of this project are (a) to provide world class stature and peer recognition to the INEL in selected areas of nuclear physics and (b) to transfer advanced instrumentation developments and basic nuclear physics data (in particular, data pertaining to the nuclear fuel cycle) into programmatic and missionrelated marketing areas at the INEL. Both of these objectives are important components of the effort to make the INEL the leading applied engineering laboratory in the DOE system. In order for the INEL to get this recognition, it must possess the capability to rapidly transfer emerging technologies from the basic research arena to the applied engineering arena (i.e., into useful products for the government and private sector). This capability 
relies heavily on maintaining close ties with the research community and knowing what technological advances are being made there. This, in turn, can only be fully realized by having an active involvement and peer recognition in the technical research community. During FY-95, tasks were successfully pursued in several major areas to meet these objectives. We continued our participation with a number of collaborations, performing work in the areas of heavy-ion interactions and studies of nuclear structure off the line of beta stability. These efforts resulted in increased capabilities for the INEL in advanced data acquisition and processing and in new techniques for isotope detection and assay. We are leading the work on the on-line error monitoring of the data acquisition system for the E877 Experiment. During FY-95, an R\&D 100 Award and one fully executed patent were received for an assay technique developed on the initiative. In addition, the work resulted in 14 papers either published or accepted for publication in refereed journals and 23 technical conference presentations by us or collaborators.

\section{Objectives}

The approach taken by the Nuclear Physics LTRI is to (a) develop and sustain a base capability in nuclear physics research that is making a significant contribution to this discipline and is therefore able to acquire top technical personnel; (b) strengthen the ties between the INEL and regional, national, and international university nuclear physics groups; and (c) provide strong technical support for the development of technology transfer, together with the basis for independent spinoff programs, and scientific and technical support to the programmatic and missionrelated marketing activities of the laboratory. Specific FY-95 technical objectives were as follows:

\section{Studies of Heavy-Ion Interactions}

- Continue involvement of our activities concerning heavy-ion interaction physics. This includes leading the work on the on-line error monitoring of the data acquisition system for the E877 Experiment and continuing our involvement in the planning for PHENIX collaboration at RHIC (relativistic heavy ion collider at Brookhaven National Laboratory). Shorter term goals were to (a) develop a new, more modular data acquisition system during FY-95 and (b) finish and have ready for testing a cross-correlation system for analysis of multi-dimensional coincidence data. This provides an environment for development and use of high-speed, distributed data acquisition systems, and it gives INEL personnel involvement in the development and testing of new electronics and of new types of detectors.

Studies of Nuclear Structure Off the Line of Beta Stability

- Continue participation in experiments with fragment mass analyzer (FMA) and Compton-suppressed Ge detector array at ANL to look at charged particle evaporation channels in the light actinides for the study of stable octupole deformation. This will give us experience in using a new piece of equipment that has a greater sensitivity for recognition of small signals in large backgrounds and pushes the sensitivity of gamma-ray spectrometry.

- Continue analysis for odd mass isotopes to search for rotational bands, including performing an angular correlation experiment to get information on gamma-ray multipolarities and level spins and an experiment at Berkeley to detect fragment mass and gamma rays at the same time from ${ }^{252} \mathrm{Cf}$. These experiments will provide some of the basic nuclear information needed to apply GNAT to the identification of fissionable material for arms control and nonproliferation and for waste management applications. This also provides the project for the pre-freshman program.

- Continue the complementary studies of the structure of neutron-rich nuclei off the line of beta stability using the INEL ${ }^{252} \mathrm{Cf}$-based isotope separator system (ISOL). These nuclear structure data have direct applicability to arms control and non-proliferation. We are exploiting the unique capabilities of the ISOL to explore in a systematic manner selected properties of heretofore inaccessible neutron-rich nuclides in the mass region $107<\mathrm{A}<122$.

\section{Pre-Freshman Program}

- Continue involvement in the pre-freshman program by providing realistic research work for the students. This program exposes the students to how scientific research is actually performed and enhances the teacher's knowledge in the scientific method. This is a high visibility, positive image program for Lockheed Idaho in the southeast Idaho community as well as Washington D.C.

\section{Accomplishments}

Studies of Heavy-Ion Interactions We participated in data collection and on-line analysis for the Sept-Oct, 1994 experimental run of the E877 Experiment at the Alternating Gradient Synchrotron (AGS) at BNL.

We completed development of the initial version of a network-distributed VME-based data acquisition system for increased data 
throughput, which is a spinoff of our work on the E877 Experiment, was completed. With our present computers, a sustained data transfer of about 3 Mbytes/sec has been measured. The speed of the transfer has been limited by the processing power of the CPUs we have.

This is well above the experimental limitations imposed by the maximum speed at which data can be written to an 8-m tape, which for a single drive is $500 \mathrm{kbytes} / \mathrm{sec}$. This improved data acquisition concept has recently been used in arms control applications.

Development of an on-line error monitoring method for the Oct-Dec 1995 run for the E877 experiment was completed. The cross-correlation analysis program was written and awaits testing. A stand-alone program aimed at carrying out a systematic study of the use of the technique on different multi-dimensional data sets with varying signal-to-noise characteristics is almost operational. The next phase of the development will be to make the necessary modifications to allow the program to process large multi-dimensional data sets.

The conversion of the VAX$P A K$ analysis package to run on the Nuclear Physics Silicon Graphics workstations was completed and resulted in a significant improvement in program speed, especially in the display of twodimensional data histograms where an order of magnitude improvement has been realized. The different versions of the analysis and replay programs share a common data format between the VAX VMS, VAX ULRIX, and SGI IRIX versions so that data can be seamlessly shared across network file service (NFS) mounted file systems.

Studies of Nuclear Structure Off the Line of Beta Stability

A follow-up experiment to the ${ }^{194} \mathrm{Po}$ FMA/Experiment last year was performed using the Argonne-Notre Dame BGO to look for delayed gamma-rays in ${ }^{194} \mathrm{Po}$. This is part of a series of experiments investigating the interplay of collective and singleparticle structures in the neutrondeficient Po nuclei.

We continued the research activity associated with the INEL ISOL facility in an active collaboration with the University of Idaho and with the Nuclear Science project. During FY-95, the research effort focused on analysis and interpretation of data, including the following:

Using the recently completed total absorption gamma-ray spectrometer (TAGS) analysis methodology, which employs Monte Carlo generated photon and electron response functions and undertakes a complete accounting for all photon, electron, and internal-conversion electron summed cascades, it has been demonstrated conclusively that the TAGS data are invaluable in assessing the completeness of deduced decay schemes for deformed rare-earth radionuclides and in providing new. information on previously undetected $\beta$-feeding components. Using the TAGS system, precise values were obtained for the ground-state (defined to include all levels below about 100 to $150 \mathrm{keV}$ ) $\beta$-branching intensities of several lanthanide radionuclides.

A decay scheme has been established for the first time for the new isotope ${ }^{155} \mathrm{Nd}$, which includes population of some 36 levels in $155 \mathrm{Pm}$ and establishment of the rotational band structures for the lowest 9 energy levels. Decay schemes have been established for a ${ }^{154} \mathrm{Pm}$ isomeric pair. This work, combined with the pre- viously completed ${ }^{154} \mathrm{Nd}$ decay, provides unambiguous evidence for a $4^{+}$spin-parity assignment to $154 \mathrm{Pm}$ (2.7 $\mathrm{min}$ ).

An experiment was performed at LBL with ${ }^{252} \mathrm{Cf}$ using Gammasphere and 36 detectors to further study three-fold and higher coincidences. Over 30 gigabytes of triples data were collected. Analysis of the data from these experiments was initiated. We are building a matrix of a gamma-gammagamma coincidences cube essential to these data and are looking at one or two fragments at a time. Primary emphasis is being placed on odd mass nuclei (which could be odd proton or odd neutron), which are not well known. From these data, we investigated the nuclear structure of odd-A 109,111 Ru nuclides and identified several new gamma rays in these isotopes.

Analysis of $x$-ray/gamma-ray coincident data taken at the INEL was performed, and appropriate two dimensional matrices ( $x-x$ and $x$-gamma) were built. Besides providing basic data required for developing systems to detect and identify fissile material, these data allow us to study the nuclear structure of some odd-N and odd- $\mathrm{Z}$ nuclei.

Basic studies of the prompt gamma rays produced in the fission process resulted in the identification of sets of high energy gamma rays in $\mathrm{Te}-\mathrm{Zr}$ and Te-Mo fission fragment pairs that are suitable for use in fissile material identification in nuclear weapons nonproliferation and waste management activities.

We installed Fitek and Radware softwares on our Silicon Graphics computer. The former is software for a wide range of peak-fitting applications, and the latter allow us to perform data analysis in three-dimensional space very quickly. 
A new detection system consisting of three DUET Ge detectors and eight neutron detectors was developed. The data acquisition system incorporating fast ADCs and fast digital electronics was implemented, and we are in the process of upgrading to the VMEbased computer system with FERA readout capability instead of using the slow CAMAC bus readout, which had been used.

The application of prompt gamma rays from fission was implemented in assaying radioactive waste drums at the RWMC using our gamma-neutron setup. We were able to identify ${ }^{240} \mathrm{Pu}$ in real 55-gal waste drums in the presence of a high activity concentration of ${ }^{241} \mathrm{Am}$. The coincidence technique used in our measurement reduces the background and increases the signal-to-noise ration significantly, which is essential in assaying very high-level wastes as the background problem causes misidentification of some radionuclides.

\section{Pre-Freshman Program}

The Pre-Freshman Program continued. The group was able to identify several rotational bands in odd mass isotopes and to identify new isotopes. This resulted in the publication of a paper.

\section{Publications}

Recognition, Patents, and

We were the recipient of the prestigious 1995 R\&D 100 Award for development of the Gamma Neutron Assay Technique (GNAT) for identification and assay of nuclear material.

A fully executed patent was received for GNAT for use in nuclear weapons nonproliferation activities and in fissile materials waste management work.

During FY-95, the work on the Nuclear Physics LTRI resulted in 14 papers either published or accepted for publication in refereed journals and 23 papers presented (by us or collaborators) at technical conferences. Data obtained from the ISOL studies are included in the Nuclear Data Sheets.

\section{New or Increased Technical Capability}

By our significant contributions to selected areas of nuclear physics, the technical reputation of LITCO in selected areas of experimental nuclear physics has been maintained and enhanced. This has resulted in our ability to transfer advanced instrumental developments and basic nuclear physics data pertaining to the nuclear fuel cycle into applied engineering programmatic and mission-related marketing areas at the INEL. State-of-the-art instruments and techniques, developed for the research community, have successfully been transferred to the INEL and utilized in a number of thrust areas (e.g., arms control and nonproliferation, waste management, nuclear operations, and technical transfer to industries). The network distributed VME-based data acquisition system has greatly increased our abilities in data throughput. The VAXPAK analysis package, which runs on the Nuclear Physics Silicon Graphics workstations, resulted in a significant improvement in program speed. The cross-correlation analysis program enhances our ability to process large, multi-dimensional data sets. The nuclear data acquired has enhanced our abilities to develop isotope detection and assay systems.

\section{Business Development Opportunities}

The nuclear data and enhanced capabilities provided by the Nuclear Physics LTRI puts us in a position to develop new isotope detection and assay techniques to address the needs in the areas of waste management and arms control and nonproliferation. The enhanced data acquisition and processing capabilities put us in a position to develop data acquisition and control systems for a multitude of applications, including process control for industry.

\section{Condensed Matter LTRI 0030}

\section{H. Sellers, C. D. VanSiclen, R. A. Anderl}

The Condensed Matter LTRI is intended to support basic condensed matter/materials research in areas of current or future interest to the INEL. The research primarily addresses defect phenomena, in recognition that defects generally control material properties. In keeping with the engineering missions of the INEL, the LTRI research considers structural (rather than electronic) defects, in particular their generation, interaction, and evolution, and their effects on material properties and behavior in various environments. As such, the $L T R I$ supports a wide variety of INEL and national interests; over FY-95, for example, LTRI research and personnel benefited INEL programs in advanced materials processing, fusion safety, and waste management as well as CRADAs with the automotive industry. The Condensed Matter LTRI is unique among the LTRIs in conducting both experimental and theoretical research.

This LTRI has historically maintained very close ties with applied research programs at the INEL, in order that the (more basic) LTRI research activities complement and enhance those efforts. The LTRI has additionally sought out collaborations with regional and national universities and with the other national laboratories both to utilize expertise available elsewhere and to increase LITCO/INEL visibility within the technical community. The Condensed Matter LTRI also emphasizes journal publication and symposia participation to disseminate the research. 


\section{Objectives}

- Improve the properties of inert gas atomized hard magnetic powders through a better understanding of the correlations between alloy chemistry, particle microstructure, and the powder size-dependent magnetic properties.

- Understand the nature of binary and ternary intermetallic phase transitions by in situ studies of the temperaturedependent electrical and magnetic properties.

- Understand the anomalous increase in the A.C. electrical resistivity of ferromagnetic materials near their Curie temperature (and other phase transitions).

- Develop new electrical and magnetic instrumentation techniques for the characterization of materials.

- Develop a self-consistent description of solute diffusioninduced grain boundary migration, in conjunction with an experimental program at the INEL.

- Complete a study of inert gas bubble migration in solids.

- Pursue research opportunities in surface/interface physics, in particular those relating to crystal growth and atom diffusion.

\section{Accomplishments}

For several years, LTRI researchers have collaborated with General Motors/MAGNEQUENCH on the use of inert gas atomization as an alternative rapid solidification processing route for the production of rare earth permanent magnets based on $\mathrm{Nd}_{2} \mathrm{Fe}_{14} \mathrm{~B}$. Earlier experiments at the INEL using the alloy composition in commercial production (produced by the melt spinning process) proved it to be unsuitable for atomization due to an inadequate cooling rate. Subsequently, it was discovered that the composition could be modified to produce better properties and that gas atomization was an excellent method for studying the correlation between the material's microstructure and its magnetic properties (because of the range of cooling rates experienced by different powder particle sizes). Experiments were carried out at the National Synchrotron Light Source to determine the crystal and phase structures as a function of alloy composition, particle size, and heat treatment. Results of collaborations with Brookhaven National Laboratory and Utah State University were presented at the 1995 Intermag conference in San Antonio, Texas. When it became apparent that the increase in the rare earth content required to prevent undesirable phase formation also limited the magnetic properties (because of the reduction in the iron content), Dr. Dan Branagan was hired as post doc to explore more revolutionary ways of improving the quenchability of these alloys without sacrificing the magnetic properties. His initial experiments have shown that insightful alloying can improve the magnetic properties by allowing for an increase of the iron content over what was previously thought possible. This research has recently received funding from DOE-BES and will continue (and expand) under their sponsorship. Much of this research is presented in the two papers, Phase Composition and Magnetic Characteristics of Inert Gas Atomized $\mathrm{RE}_{2} \mathrm{Fe}_{14} \mathrm{~B}$ Based Powders and Magnetic Hardening Mechanism of As-Gas Atomized Nd(Dy)-Fe-B Powders Bonded Magnet, to be published in IEEE Transactions in Magnetics.

Collaborative work with Dr. Richard Wright over the last several years has shown the applicabili- ty of using electromagnetic analysis techniques to study the phase transformations in high temperature structural intermetallics (such as $\mathrm{Fe} 3 \mathrm{Al}$ ). In the past, several candidate high-strength (also wear and corrosion resistant) alloys were studied, but a need was seen for more basic studies on carefully prepared series of samples. This would allow for an in-depth understanding of the relationship between the phase transitions and the electromagnetic response. Ternary Fe-A1-Ti alloys were prepared and studied to determine the effect of substitutions. The most recent research focuses on the electrical and magnetic properties of binary $\mathrm{Fe}-\mathrm{Al}$ alloys with a wide range of compositions. The equilibrium binary phase diagram has been determined using these methods, and it is in good agreement with other experimental and theoretical results. The magnetic properties were found to depend only on the nearest neighbor environment and could be modeled accurately. These results are presented in a paper, Electrical and Magnetic Properties of Binary Fe3Al Alloys, to be submitted for journal publication in FY-96.

Work on the ferromagnetic iron aluminides indicated that the ac electrical resistivity showed an anomalous response close to the ferromagnetic/paramagnetic transition. This phenomenon had been occasionally observed, but not explained in the literature. We have developed a model based on the total magnetic energy of the system that indicates that, when close to the Curie temperature, the magnetocrystalline anisotropy (which tends to align the individual moments with certain crystallographic axes) is weak, and the direction of the spins randomizes, scattering conduction electrons excessively. From this, we could predict that there should be certain 
other instances when the total magnetocrystalline anisotropy was small where the resistivity was affected. Pure samples of the transition element ferromagnets ( $\mathrm{Fe}$, $\mathrm{Ni}$, and $\mathrm{Co}$ ) were studied, and the anomaly was indeed found at temperatures well below Tc, because of a change in the easy axis of magnetization. This work is presented in a paper, On the Electrical Properties of Ferromagnetic Materials, to be submitted for journal publication in early FY-96.

With additional LDRD funding, techniques were developed to detect microstructural changes within stainless steel 304 due to mechanical deformation. Dr. Ken Telschow has begun to apply a SQUID sensor as a technique for nondestructive evaluation (NDE), and a quantitative measurement of the weakly ferromagnetic (due to fatigue-induced magnetic precipitates) samples was necessary for calibration of this new technique. Samples were removed from a fatigued specimen so as not to induce additional transformations and the amount of ferromagnetic martensite was measured with the LTRI vibrating sample magnetometer (VSM). The excellent correlation between the saturation magnetization (measured by the VSM) and the remnant magnetization (measured by the SQUID) demonstrated the complementary nature of the two techniques and will allow SQUID results to be more quantitative. Methodologies were then developed to use the SQUID sensor to spatially resolve the martensite content of large fatigue specimens. These techniques are included in the paper, Electromagnetic Measurements for Materials Characterization, to be published in Reviews of Quantitative Non-Destructive Evaluation.

Collaborative research with Oak Ridge National Laboratory, performed over FY-94, has recently been published: Radiation-Induced Electrical Degradation in Crystalline A12O3: A Bulk Effect, Journal of Nuclear Materials 219, 176 (1995). A description of this research is provided in the LTRI FY-94 Project Report.

During processing of rapidly solidified powders, the initial, interlocking dendritic structure of the powder particles is replaced by a granular microstructure that characterizes the sintered solid material. The evolution of the microstructure is believed to be due to grain nucleation at the dendrites followed by grain boundary (GB) migration. In the absence of stresses, the GB motion is driven by the increase in entropy that occurs as the solute distribution in a region becomes more homogeneous. The most favorable diffusion path for solute atoms is within. the GB, so solute atoms swept up by the moving GB are expected to diffuse within the $G B$ in response to the solute concentration gradient there, and then to be deposited in the new grain as the GB moves on. This scenario has been formulated in a model consisting of a set of highly nonlinear differential equations and boundary conditions. Given the initial solute distribution, the GB velocity and final solute distribution can be calculated. This research is coordinated with a DOE-BES experimental program at the INEL that will measure these quantities in situ for aluminum powder particles containing a dilute concentration of silver. Publication of this research is anticipated in FY-96.

Development of a novel, vacancy-diffusion mechanism by which gas bubbles in solids may preferentially migrate towards grain boundaries and free surfaces, begun during FY-94, has been completed and is presented in the paper, Migration of Gas Bubbles in Solids Towards Vacancy Sources,
Philosophical Magazine Letters 72, 41 (1995). It is shown that gas bubbles will migrate up a vacancy concentration gradient (produced, for example, by annealing the material) since vacancy capture is anisotropic at the bubble surface while vacancy emission into the bulk is isotropic. Conflicting experimental data on inert gas bubble distributions taken over several decades are thus reconciled, and a clear experimental signature for this mechanism is provided. This is an important paper because it provides a convincing explanation for the anomalously rapid diffusion of inert gases to grain boundaries observed in fusion reactor materials. (The competing mechanism of thermal resolution of gas atoms from bubbles and their subsequent diffusion to the grain boundaries is simply not physically viable.) This work should influence various aspects of reactor research; more immediately, this mechanism will be incorporated into gas transfer codes used to design new reactors and to monitor aging reactors. This mechanism may additionally account for the gas release behavior found during annealing of irradiated beryllium samples here at the INEL. The application to these experimental results is discussed in the paper, Tritium and Helium Release From Irradiated Beryllium, by R. A. Anderl et al., presented last May at the Fifth Topical Meeting on Tritium Technology in Fission, Fusion, and Isotopic Applications held in Italy.

A catalog of mechanisms for diffusion of large adatom (and vacancy) clusters on metal surfaces, inspired by recently published experiments and drawing on LTRI expertise in crystal growth and diffusion processes, is presented in the paper, Single Jump Mechanisms for Large Cluster Diffusion on Metal Surfaces, Physical Review Letters 75, 1574 (1995). Clear exper- 
imental signatures are provided for the various mechanisms, including circular cluster diffusion by correlated adatom evaporation and subsequent condensation at the cluster periphery, and faceted cluster diffusion by evaporation and condensation of facet ledges. Both these particular mechanisms are novel, and both are verified by experiment. This is an important paper because it resolves a fundamental issue in cluster dynamics at surfaces, and so has implications for studies of thin film and crystal growth, catalysis, and other surface processes.

Research performed during FY-94 has been published: Indium Adatom Diffusion and Clustering on Stepped Copper Surfaces, Physical Review B 51, 7796 (1995).

Aspects of this work were also presented at the American Physical Society March meeting in San Jose, California. A description of this research is provided in the LTRI FY-94 Project Report.

Recent experiments have shown that an initial coverage of a fraction of a monolayer of antimony promotes two-dimensional, layer-by-layer homoepitaxial growth at silver surfaces. This is a very interesting discovery, as such layer-by-layer growth required for microdevice fabrication is otherwise achieved only for particular (and very restrictive) atom deposition conditions; more generally, three-dimensional islands are formed on the surface. Total energy calculations performed under the LTRI show that the proposed mechanisms for this surfactantinduced growth mode (the surfactant atoms nucleate clusters, or lower the energy barrier for interlayer transport) are not viable. Instead, the surfactant atoms (e.g., antimony), which are embedded in the topmost atomic layer of the host (e.g., silver), pin the cluster edges and thereby slow the lateral growth of the clusters. The resulting cluster size is too small to permit nucleation of second-layer clusters, so that the supersaturation of deposited adatoms is accommodated by increased first-layer cluster nucleation on the substrate. Thus, a high density of very small clusters is created, which is well known to lead to layer-by-layer growth. Upon completion of an atomic layer, the buried surfactant atoms diffuse towards the surface and the cluster nucleation and growth cycle begins anew. An invention disclosure record, Method for Promoting AtomicallySmooth Homoepitaxial Growth at Metal Surfaces (LTT-PI-161), which discusses other potential surfactant-host systems as well as applications of this phenomenon, has been submitted to the Technology Transfer Office, and a scientific paper, Step-Pinning Mechanism for Surfactant-Mediated Layer-byLayer Homoepitaxial Growth, has been submitted for journal publication.

\section{New or Increased Technical Capability}

We have continued to refine our electromagnetic measurement techniques; we now make some of the most precise measurements at high temperatures in the world.

With Dr. Ken Telschow, we began the development of a system and methodology for the use of a HTSC SQUID sensor as an NDE technique. Because of its recent

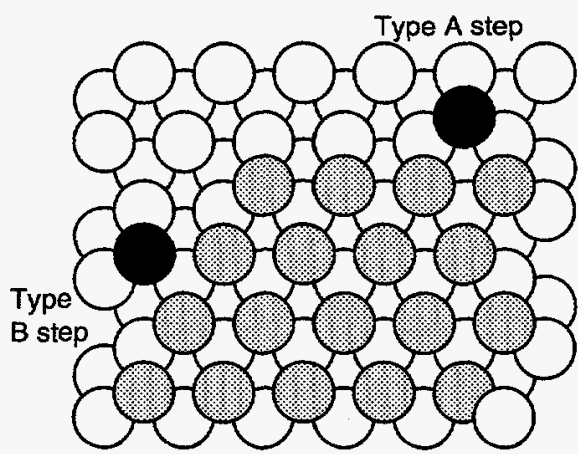

Close-packed, Type A and type B Steps on the $\mathrm{Cu}$ (111) Surface arrival on the market, we are among the first to consider this application.

In response to interest by funding sponsors and industry collaborators, we have initiated construction of an additional gas atomizer to provide more capacity. The new atomizer will have additional features to enhance our current capabilities, and is being designed at minimal cost in order to facilitate eventual transfer of the technology of small, inexpensive atomizers.

Dr. Dan Branagan was hired to bring expertise in alloy design and evaluation, and to initiate collaborations with Ames Laboratory, a traditional rival of the INEL in the area of gas atomization.

LTRI publications, presentations, and collaborations continue to increase national awareness of the technical capabilities residing at the INEL.

\section{Business Development Opportunities}

Visi Trak (NDA) contacted Dr. Sellers to investigate magnetic proximity sensing techniques. Dr. Sellers visited their plant to assess what the INEL could contribute.

Marlow Industries (NDA) contacted Dr. Sellers to seek assistance in developing a production facility for gas atomization of semiconductor thermoelectric elements. Our experience with atomizers and atomization should prove valuable to the design of the new plant.

\footnotetext{
The shaded circles are $\mathrm{Cu}$ atoms at surface lattice sites in the upper terrace; the open circles are $\mathrm{Cu}$ atoms at sites in the lower terrace and below. The two dark circles are In adatoms at trapping sites at the two types of steps. the letters $A$ and $B$ superposed on the upper terrace indicate the two distinct adatom binding sites on a $\mathrm{Cu}(111)$ terrace.
} 
CRADAs with the two GM divisions Delphi and MAGNEQUENCH, with which LTRI personnel are involved, continue

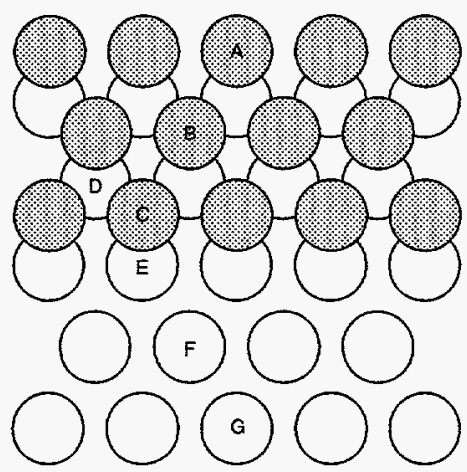

Type A step on the Cu (111) Surface to be fruitful. We anticipate that success in the two research areas will lead to commercialization activities.
The shaded circles are $\mathrm{Cu}$ atoms at surface lattice sites in the upper terrace; the open circles are $\mathrm{Cu}$ atoms at sites in he lower terrace and below. The two dark circles are In atoms at trapping sites at the two types of steps. the letters $A$ and $B$ superposed on the upper terrace indicate the two distinct adatom binding sites on a $\mathrm{Cu}(111)$ terrace.

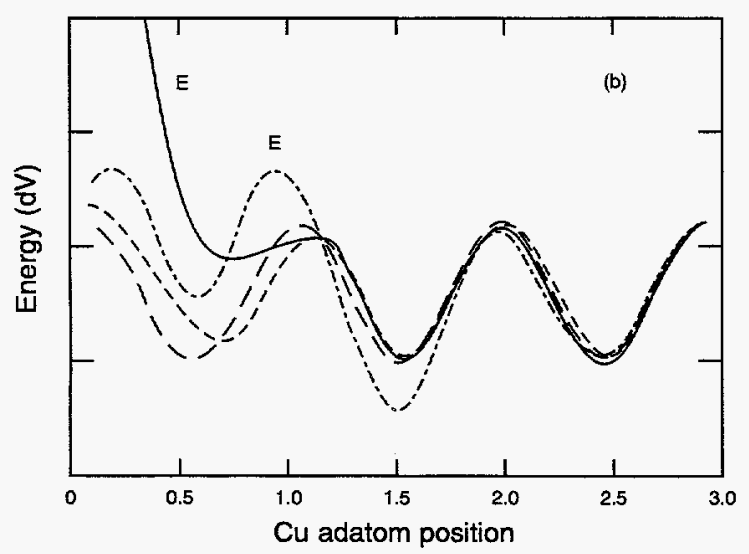

The sinusoidal dashed curve represents the energy variation of the system as a Cu adatom migrates along the base of the step: the alternating minima and maxima correspond to equilibrium sites $(0 \mathrm{eV})$ and saddle points $(0.25 \mathrm{eV})$, respectively. The $\mathrm{Cu}$ adatom position is given in units of the surface lattice spacing (2.5562 $\AA$ ) measured along the step from the surface atom labeled E. Similarly, the dotted curve and the curves labeled $E$ and $F$ represent the energy variation of the system when surface atoms at sites $C, E$, and $f$, respectively, are replaced by an in atom.

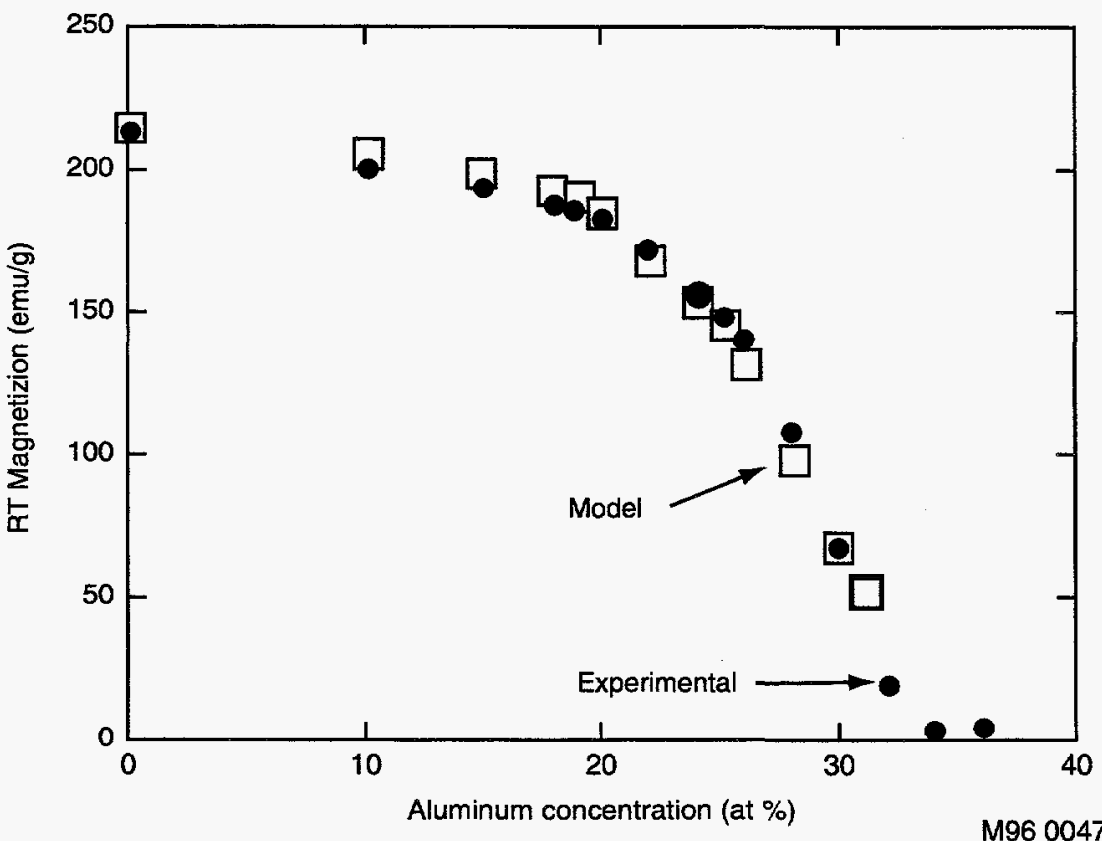

Fe-Al alloys crystallize into several different intermetallic phase structures $\left(\alpha, \mathrm{DO}_{3}\right.$, and $\left.\mathrm{AB} 2\right)$ depending on their composition. An electrical and magnetic study of these materials has illustrated the nature of phase transitions in this system and show that the magnetic properties result from $\mathrm{Fe}$ nearest-neighbor interactions only. The model results illustrated are derived from an examination of the $\mathrm{Fe}$ population surrounding each $\mathrm{Fe}$ atom, in each crystal structure. These materials have many potential industrial applications because of their strength at high temperature, and their wear and corrosion resistance. 


\section{Theoretical Chemistry}

LTRI 0040

\section{R. A. LaViolette}

The Theoretical Chemistry LTRI concentrated on molecular or mesoscopic modeling for four distinct research topics: Condensation of metal particles in a plasma quench reactor; ionic oxide materials, diffusion in the presence of random traps; and a novel energetic material. Research in these areas has produced, for FY-95, eight articles in refereed journals, two articles for refereed proceedings, six invited presentations, and one contributed presentation. Research in each area has contributed to the INEL's modeling capability, by providing new models or techniques (some unique to the INEL) for molecular or mesoscopic modeling of materials or processes employed in industrial or remediation applications.

Research in three of the four areas has resulted in proposals to the INEL's University Consortium, the EPA, and DOE, respectively.

\section{Objectives}

- Finish a model of nucleation of metal particles in the plasma quench reactor.

- Implement new theory of nucleation in a steady-state code.

- Calculate nucleation rates and particle size distributions for a variety of metals in a model argon plasma quench reactor.

- Develop a molecular theory and model of ionic oxide materials.

- Implement lattice dynamics calculations for solids.

- Implement molecular dynamics calculations for liquids.
- Generalize our stochastic model of diffusion in the presence of random traps.

- Identify the most typical events in anomalous diffusion (previous analysis only considered averages).

- Begin investigation of a novel high-energy density material.

- Calculate electronic structure of various solids of spin polarized metastable excited tripletstate (1s2s) helium, including band structure, the density of states, and the zero-temperature equation of state.

- Calculate electronic structure of various antiferromagnetic configurations of triplet helium

\section{Technical Accomplishments}

The INEL has been one of the leaders in developing plasma quench technology. This effort was intended to provide a view of the nucleation process for metals condensing in a plasma quench reactor presently inaccessible via experiment. We first developed a theory of the nucleation process in a steady-state nozzle flow, and then a code to implement the theory. Our code (nozzle) requires only basic information about the flow of the non-condensing carrier gas (e.g., temperature, density, velocity), which is available either from experiment or routine computational fluid dynamics calculations, and basic about the condensing metal information (e.g., melting point, surface tension, vapor pressure), which is available from the literature. For the first time, we are able to observe, via the model, the steady-state supersaturation, nucleation rate, mean particle number, mean particle size, and particlesize distribution, at any point inside the reactor. So far, 11 metals have been examined. Besides our ability to accurately predict primary- particle size distributions, we are able to explain the origins of multiple particle size distributions. The results are summarized in a journal publication and two proceedings from invited presentations., This work was carried out in collaboration with Dr. R.A. Berry here at the INEL, and Dr. R.L. McGraw at Brookhaven National Laboratory.

Most geologic substances (e.g., soils, sand, and rocks) and ceramics consist of ionic oxides, sulfides, or nitrides. We have developed a new theory for the ground-state molecular properties of ionic (nontransition metal) oxide materials. With it, we calculate the molecular interactions among cations and oxygen ions from first principles, i.e., without empirical input. With these molecular interactions in hand, we can calculate the thermodynamic and thermoelastic properties of any of the phases of ionic oxide materials. We have recently begun to extend our work on oxides to sulfides and nitrides. So far, we have calculated thermodynamic and thermoelastic properties for solids of all of the rare-earth oxides, some perovskites (both oxide and sulfide), alumina, aluminum nitride, and the as yet unsynthesized sulfur analogue to ferroelectric barium titanate (the archetypal material for solid state RAM). We have also begun to calculate the properties of liquid $\mathrm{CaO}$ and liquid alumina. Detailed results are contained in five journal articles (three in print, one in press, and one just submitted) and one unpublished invited presentation. This work was carried out in collaboration with Dr. A. Chizmeshya, formerly an AWU/INEL Postdoctoral Fellow, and Prof. G.H. Wolf and his students in the Materials Research Group at Arizona State University.

One of the significant prior accomplishments for Theoretical Chemistry was the discovery of 
anomalous diffusion in the presence of random traps. In that work, we discovered, among other things, that the average properties of the diffusing plume were dominated by rare events, namely, ballistic trajectories rather than Brownian trajectories. We have since extended our work, in which we focused only on average properties, to those corresponding to the most typical events. This analysis is called for because of the unusually wide separation between rare and typical events. One of the remarkable results of this analysis shows that even the typical trajectories still show anomalies. Results of this and previous work were contained in an invited presentation at a national geoscience conference. A full-length article is in preparation, in collaboration with Prof. D.H. Dunlap at the University of New Mexico and Prof. P.E. Parris at the University of Missouri-Rolla (of whom each have visited the INEL under AWU auspices).

Both chemical propellants and explosives probably will see little more than incremental improvements in energy or power release, as has been the case to date since World War II, unless a method is found to incorporate and stabilize excited electronic states in energetic materials. Through a firstprinciples solid-state electronic structure calculation, we have discovered that a hypothetical solid of spin-polarized excited triplet-state helium atoms $\left(\mathrm{He}^{*}\right)$ does not spontaneously decay to the ground state through conversion to singlet states. Our calculation is the first of its kind; the result just quoted and suggests that a solid of spinpolarized $\mathrm{He}^{*}$ might be metastable. Here, metastable would mean that the solid might have a lifetime of between a few seconds and a few hours. An ounce of such a material would release $13.6 \mathrm{MJ}$ of energy and 680 TW of power, or about 100 times the energy and 5,000 times the power of an ounce of TNT (N.B. commercial dynamite is between $20 \%$ and $60 \%$ TNT). Further work is needed to assess other potential destabilizing influences before the feasibility of condensing such a phase can be determined. These first results have been presented at a national physics meeting, at two invited university colloquia, and have been published as a letter in a physics journal. A fulllength follow-on to the letter is expected to be completed at the end of FY-95. This work was carried out in collaboration with Dr.

T.J. Godin, formerly an AWU/INEL postdoctoral fellow, and Dr. A.C. Switendick, a consultant for the INEL whose contract ends this year.

\section{New or Increased Technical Capabilities}

The code for modeling nucleation and condensation of metals in a plasma quench reactor is a new capability for and unique to the INEL. If plasma quench processing becomes important at the INEL, this capability is now ready to assist.

The theory and code for molecular modeling of ionic oxides, sulfides, and nitrides is a new capability for the INEL, and is presently shared only with the Materials Research Group at Arizona State University. As it matures, it is expected to see application to geochemical (including soil remediation) problems, to problems of detection of hazardous materials bound to geologic media, and to modeling of ceramics for industrial applications. Work in this area is expected to continue under another LDRD.

The theory and code for diffusion and infiltration of contaminant plumes in the presence of random traps is expected to become a substantial portion of a new LTRI ded- icated to flow in porous geologic media (C.P. Thomas Ph.D., PI). It is expected to be employed in assessing natural and biological or chemical in situ soil remediation schemes. It also has applications to modeling a new class of reactive polymer membranes for chemical separations that are being proposed for development at the INEL. This modeling effort therefore is expected to continue for membrane applications under another LDRD, in addition to the applications to remediation anticipated under the new LTRI.

If our prediction of the metastability of a new energetic material results in an attempt to synthesize it, it will almost certainly require a cold positron plasma as a coolant. The INEL is a center of expertise in the production of positrons for plasmas and microscopy. Therefore, if condensation of excited triplet helium is feasible, the INEL would be among the most suitable sites to pursue its synthesis.

\section{Business Development Opportunities}

Our modeling expertise for the plasma quench reactor has been incorporated into a proposal to the INEL's University Consortium from the University of Idaho. (Three years, $\$ 665 \mathrm{~K}$ ).

Our modeling expertise for plumes infiltrating porous geologic material in the presence of random traps led to a joint proposal with the University of New Mexico to the Environmental Protection Agency. Although our bid was not successful, we expect to resubmit on the next call. (Three years, $\$ 300 \mathrm{~K})$.

Our elucidation of the electronic structure of condensed spin polarized excited triplet-state helium has led to a solicitation from the Office of Advanced Energy Programs, Basic Energy Sciences (DOE-ER) for a proposal. A joint 
proposal with the University of Nevada, Reno and the University of Missouri, Rolla, is in preparation. (Three years, $\$ 1.1 \mathrm{M}$ )

A list of the relevant papers described in this summary is available form R. A. LaViolette.

\section{Computational Mechanics} LTRI 0050

R. W. Douglass

The Long-Term Research Initiative in Computational Mechanics was established to provide a mechanism through which basic technical competence in computational mechanics could be maintained at the Laboratory and so that the Laboratory might be more nationally competitive in computationally based research and development. To that end, in fiscal year 1995, the project supported two major research emphases: basic computational capability development and specific applications areas. The first emphasis area was directed at providing core computing capabilities for new and/or developing computing ideas and needs. These projects included advanced iterative solution methods, higher-order numerical methods, advanced (parallelivector) computing methods, advanced CFD graphics development, and interfacial dynamics. The second area focused on specialized applications of computation including improved chemistry models for industrial process model development and bioremediation and sub-surface transport models for environmental analysis. The LTRI-CM staff published 11 journal articles and presented nine conference papers (one invited). Contacts with industry have been made, and university collaborations have been either initiated or maintained. The copyrighted software tool Xcuber, an improved graphics product for 2- and 3-D animation and display of vector and scalar data, is moving toward licensing.

\section{Objectives}

\section{Computational Capability Devel-} opment

This category funds new algorithmic areas and their improvement to provide INEL core modeling capabilities in support of multiple programmatic areas.

- Advanced Iterative Solution Methods

- Complete dissertation on development of advanced iteration algorithms.

- Continue doctoral dissertation research on iterative methods for kinetic modeling of collisional plasmas.

- Publish an article on damped artificial compressibility schemes.

- Higher-Order Numerical Methods

- Publish papers on higher-order methods for fluid simulation codes.

- Complete dissertation on a finite element technique with spatially varying time steps.

- Advanced Computing Methods

- Continue research on scalability for parallel computation of compressible flows

- Publish or present research on parallel computation

- Advanced CFD Graphics Development

- Continue enhancement/ improvement of Xcuber, a copyrighted software tool.

- Publish work based on Xcuber

- Interfacial Dynamics

- Publish research on fractal analysis of interfacial mixing of two fluids.

\section{Applications Areas}

The objective of this category is to develop modeling tools and implement advanced computational methods in support of new and existing INEL programmatic areas.

- Industrial Processing

- Develop a capability to simulate multicomponent two-temperature plasmas for chemical synthesis and materials processing.

- Develop ability to model flows with heterogeneous chemistry.

- Environmental Analysis

- Develop capability for simulating in situ biodegradation of organic materials.

- Develop capability to model sub-surface colloid-facilitated transport of contaminants.

Establish relationship with Lockheed Martin Corporate Task Force on Advanced Computing

\section{Accomplishments}

Newton-Krylov Algorithms for Low Mach Number Compressible Fluid Flow and Heat Transfer Problems (Ph.D. Dissertation Research), $P$. R. $\mathrm{McHugh}$

Newton-Krylov algorithms were investigated for solving strongly coupled, nonlinear systems of partial differential equations arising in computational fluid dynamics. An appropriate system of nonlinear algebraic equations are linearized using Newton's method. Preconditioned Krylov subspace iterative algorithms then solve the linear systems on each Newton iteration. Mesh sequencing, adaptive damping, pseudotransient relaxation, and parameter continuation techniques are used to improve solution efficiency and simplified using numerical Jacobian evaluation. The final examination was passed in March 1995. 
P. R. McHugh was invited to the Workshop on Iterative Methods for Large Scale Nonlinear Problems (paper and poster). Publications: three journal articles and three conference presentations.

Kinetic Modeling of Collisional Plasmas (Ph.D. Dissertation

Research), V. A. Mousseau

The objective is to develop numerical methods that will make kinetic modeling of realistic problems viable. The integro-differential nature of the kinetic equations results in large matrices because of their bandwidth and number of unknowns, which has historically prohibited a fully coupled solution. The Matrix-Free Newton-Krylov iteration technique was implemented and has shown at least an order of magnitude speed-up over other methods for computing a steady state solution of a model problem. Publications: one journal article in preparation.

Damped Artificial Compressibility Iteration Scheme for Implicit Calculations of Unsteady Incompressible Flows, J. D. Ramshaw and P. R. $\mathrm{McHugh}$

An artificial compressibility iteration scheme was developed that accelerates convergence of the iteration algorithm. This was done using a new procedure for setting the artificial compressibility parameter and by introducing an artificial bulk viscosity into the momentum equation. This scheme was used to solve the nonlinear equations resulting from a fully implicit time differencing scheme for unsteady incompressible flow. Publications: two journal articles. Higher-Order Numerical Methods, H.D. Nguyen

The objective is to develop a method to accurately solve the equations arising in fluid dynamics and in heat and mass transfer. A spectral method was developed for problems in spherical and cylindrical coordinate systems by combin- ing the accuracy of spectral methods and the generality of finite element methods. This hybrid method has been applied to twophase convective heat transfer problems. Publications: one journal article, one in review, and one conference paper.

A Finite Element Technique with Spatially Variable Time-Steps (Ph.D. Dissertation Research), P. G. Jacobs

Finite element schemes for transient convection-diffusion problems on grids with local refinement in time and space were studied. An efficient preconditioner for the composite problem was presented. Theoretical results include stability analysis, some error results, and convergence results. Numerical results illustrating the known theoretical results and demonstrating expected results were found. The final examination was passed in July 1995.

Scalability of Preconditioners for Parallel Computation of Compressible Fluid Flow (Ph.D. Dissertation Research), G. A. Hansen

A general parallel solution technique for non-linear algebraic systems is the goal of this research. Three capabilities make this research unique and establish the INEL as a leader in this area: (1) the fully coupled robust solution of poorly conditioned, highly non-linear systems without user-tuning of the algorithms, (2) a scalable, parallel solution technique based on preconditioner, Jacobian, and matrix-free algorithm scalability on commodity hardware, and (3) the wide applicability of parallel techniques to diverse engineering problems. A two-dimensional, low Mach number flow over a backward-facing step forms a challenging test problem. An inexact Newton technique is used to linearize the associated algebraic system, and a preconditioned Krylov subspace method is used to solve the resulting linear system. The Jaco- bian formation, Schwarz preconditioner formation and application, and the matrix-free approximation within the Krylov technique are parallelizable. This task has been quite successful in that (1) on commodity symmetric multiprocessing hardware typically employed in engineering tasks, super-linear efficiencies of the solution algorithm are easily obtained ( 2.2 speedup on two processors on the SGI Onyx) and (2) the solution technique scales very well to larger machines (CRAY C-90) where speedups greater than 13 were obtained on 16 processors, after vectorization. Publications: two conference presentations.

Advanced Computing Methods, H.D. Nguyen

The goal of this work was to demonstrate the feasibility of functional parallelism versus domain decomposition for parallel/distributed CFD computations. Such parallelism can be achieved by using the Galerkin method with appropriate linearization. The nonlinear convection terms in both the momentum and energy equations of the model problem were linearized by means of the secondorder Adams-Bashforth scheme. Results indicated a speedup of more than three when executing on a network of four DEC alpha workstations. Publication: one conference presentation. Hansen

Xcuber Development, G. A.

Development of Xcuber and, for distributed/parallel execution Pcuber, is being continued on an as-needed basis.

Cuber and its derivatives are INEL copyrighted software (in FY94). License agreements and other applications are being explored. Publication: one conference presentation.

Interfacial Dynamics: Mixing at Two-Fluid Interfaces, $R$. W. Douglass This project is aimed at simulating the evolution and characteri- 
zation of unstable fluid interfaces using dimensional analysis and fractal theory with the ultimate goal of being able to correlate interfacial growth and transport rates. Publication: one journal article in review.

Diffusion Formulation for Multicomponent Two-Temperature Plasmas $\&$ Heterogeneous Chemistry Model Development, J. D. Ramshaw and C. H. Chang

Two-temperature plasma formulations provide an enhanced capability for plasma modeling applied to chemical synthesis and materials processing. Currently, the ideal magnitohydrodynamics (MHD) case, where electron mass is neglected, was completed. The model will be enhanced to include the resistive (non-ideal) MHD case. The heterogeneous chemistry project was initiated to model many material/waste processing concepts proposed by the INEL, including black-liquor gasification for paper industries and material synthesis using plasma quench reactors such as $\mathrm{TiO} 2$ synthesis, UF6 reduction, and natural gas conversion. The most important enhancement to the currently available model is to include fully coupled chemical kinetics of gas-phase reactions and condensation kinetics. Equilibrium condensation can be determined at this time. More realistic condensation kinetics will be developed along with generalization of particle logic for nucleation.

Biodegradation \& Sub-Surface Transport Phenomena, H.D. Nguyen

A two-dimensional finite element-based computer code is being written for simulating in situ biodegradation of organic materials. Plans are to include additional phenomena to simulate the transport in an aerated compost pile undergoing biodegradation. This modeling tool will enhance the development of bioremediation and composting technologies cur- rently being studied by the Biotechnology LTRI under R. Cherry. The sub-surface transport project aims at enhancing understanding of the fate of contaminants in geological media and the coupling between transport mechanisms. The mechanism being studied is colloid-facilitated transport, considered one of the important pathways for contaminant migration and is speculated to be the cause for the discrepancy between predicted data and field observations. Publications: one journal in press plus one in preparation.

Establish relationship with Lockheed Martin Corporate Task Force on Advanced Computing (R.W. Douglass).

Contact with Dr. P. Raj, Chair of the Task Force, was established in July, 1995 by R.W. Douglass.

\section{New or Increased Capability}

\section{Advanced Iterative Solution}

\section{Methods}

- Established INEL as a world class leader in the research and application of advanced Newton methods for solution of highly nonlinear algebraic systems of equations.

- Improved capability to solve highly nonlinear, coupled algebraic systems.

- Invited to attend Workshop on Iterative Methods for Large Scale Nonlinear Problems held at Utah State University.

\section{Higher-Order Methods}

- Enhanced expertise in applying spectral methods to complex flow problems.

\section{Advanced Computing Methods}

- Attained new capability in applying parallel and vector processors to complex applications.

- Expertise in message-passing methods for distributed processors.
- Expertise in developing and implementing scalable parallel algorithms into applications.

- Expertise in vector processors.

- Expertise in applying shared memory processors to applications

- Expertise in Cray C-90 architectures

Invitation (Dr. Keyes, ICASE) on parallel solution methods for a combustion problem (Sixth SIAM International Conference on Numerical Combustion.)

- Invitation to demonstrate parallel techniques at the 31st National Heat Transfer Conference.

Advanced CFD Graphics Development

- Improved capability to animate/analyze two- and threedimensional scalar/vector data sets.

- Expertise in X11, Motif, and GL libraries.

- Expertise in parallel methods for graphics (Parallel Virtual Machine).

- New capability in applying functional parallelism (vis-avis, domain decomposition) to CFD applications.

\section{Interfacial Dynamics}

- Expertise in the dynamics and scaling of two-fluid mixing.

\section{Industrial Processing}

- World class expertise in modeling plasma dynamics.

- New ability to model combustion, combustion flame spraying, complex chemical reactions.

- Expertise in design/scaleup/optimization of plasma/advanced chemistry applications. 


\section{Environmental Analysis}

- New modeling capability for simulation of in situ biodegradation of organic materials.

- Enhanced capability to model sub-surface transport of contaminants by including colloidfacilitated transport mechanisms.

Business Development Opportunities

Internal Business Units

- Implementing advanced iterative methods, higher-order differencing methods, and parallel solution techniques into RELAP5 (Advanced Nuclear Energy Products) via a FY-96 LDRD proposal.

- Black liquor gasification for paper industries depends on the optimization and control of condensation kinetics. An LDRD proposal for FY-96 including model development was submitted.

- Concepts based on nonequilibrium thermal processes for material/chemical synthesis have been proposed as LDRD projects for FY-1996.

- Joint FY-96 LDRD submitted (with Robotics) on experiments and model development for cryogenic liquid Nitrogen cutting.

- FY-96 LDRD submittal (Advanced Computer Interface Department) on development of a generic object-oriented user interface for input specification and computational steering of software

External Business Units

- Collaboration with Computational Mechanics, Co., Knoxville, TN through a URC proposal for FY-96 and bringing the President of the company, Dr. A. J. Baker to INEL for a seminar and staff discussions. Possible CRADA.

- Collaboration with Dr. Darrell Pepper of the University of Nevada-Las Vegas and president of a small business on parallel and advanced iterative solution methods and Xcuber. $\mathrm{He}$ is awaiting further development of Xcuber for finite element grids and for $\mathrm{PC}$ execution. Possible CRADA.

\section{Nonconventional Bioprocessing}

LTRI 0060

\section{R. S. Cherry}

Composting, biofiltration, and in situ bioremediation all involve bacterial conversion of materials in complex, poorly characterized systems of air, solids, free water, and, possibly, a second nonaqueous liquid phase.

This LTRI work addresses design and operations problems pertinent to all these processes. By understanding the closely coupled physical, chemical, and biological processes that control the behavior of these systems and developing instruments to measure the key variables, this LTRI contributes to producing better bioremediation and waste biotreatment systems. Other applications of the work are in bioleaching of mineral ores and biopulping of wood chips to make paper. The long-term goal is to make biofilters, composting, and other related technologies more useful by making it possible to design them with confidence for given feed materials and to ensure, by advanced monitoring and control techniques, that these processes will perform well under field conditions.

\section{Objectives}

This LTRI supported three main projects during FY-95. All are related to the design and operation of biofilters and compost beds. They support the LTRI's topic area of bioprocessing of solid and gas phase materials.

- Develop rigorous design procedures for passively aerated compost piles.

- Develop on-line instrumentation and processes for monitoring and controlling the water content of biofilters.

- Develop a better understanding of what limits the rates of biological activity in biofilters, and test physical and biological means of increasing those rates.

\section{Accomplishments}

The first major task was to develop a computational model of a passively aerated compost pile for use in rigorous design to achieve specified performance. This involves simulating the biological reactions and the heat and mass transfer within the pile to determine the final results for a given initial configuration. The difficult aspect of this problem is that oxygen is supplied by natural convection driven by the heat generated in the core of the pile, which depends on the amount of oxygen available. The Computational Mechanics LTRI assisted with initial evaluation of existing finite difference models that might be adapted to this problem. The survey showed that none of the models are really appropriate or capable of solving the problem, so we began developing our own models. The first was an analytical model to determine the vertical air flow between the perforated pipes that introduce air into the pile.

Although it is highly simplified by assuming a temperature profile rather than calculating it, this model provides a basis for setting the pipe spacing. The second model, still under development, is far more complex. It is a one- 
dimensional model composed of differential equations for heat and mass balancing that are integrated numerically. This model allows for variable pressure, temperature, biological reaction rates, oxygen, and water content in the bed. Choosing proper boundary conditions is especially difficult, as we have found that both diffusion and convection are significant at the boundaries for heat, water vapor, and oxygen.

In support of this modeling work, we ran several full-scale test piles at the Bonneville County landfill during winter 1995. These generated data have been used to validate the early versions of the numerical model. We also did some laboratory experiments to determine values of physical constants such as the gas permeability and density of compost. In addition, Dr. William Ball at Johns Hopkins University began to measure the rate at which organics equilibrate with compost and high organic content soils; in some cases, this can take hundreds of days and have major implications for the rate of bioremediation of contaminants on such materials.

We also did some work on composting as a way of treating No. 2 fuel oil contamination of surface soils. Laboratory work this year has shown that composting works well for the initial degradation of hydrocarbons, but the residual material degrades much more slowly. However, before this work was fully completed, the responsible group decided to landfill the drummed waste rather than treat it by composting. The tests were suspended at that point.

The second major task in FY-95 was instrumentation and control of biofilters. The instrumentation part of this testing used a modified soil moisture probe on-line in the biofilter. We have already shown that this time-domain reflectometry probe works satisfactorily in off-line trials. In on-line work, we have found that compost is a much lossier medium than soil, so probe designs that work in soil must be modified to decrease the probe spacing to maintain signal strength. We also tested a phasedomain reflectometer that worked well but would be difficult to interface with our data collection system because it is a compact consolidated unit intended for soil use. We also did some work with tensiometers being developed by the INEL geophysics group. While those instruments ultimately proved too slow in response to be useful, this was itself a valuable finding. These data show that the hydraulic conductivity of compost is very low, so that water added to the bed moves through it extremely slowly, with times measured in days. This greatly limits what strategies for water addition ought to work. It has suggested a line of research, just beginning at the end of FY-95, into measuring the basis for how bed amendments such as bark, polystyrene beads, and sand might improve hydraulic conductivity and overall biofilter performance.

A large effort in this area during FY-95 was to complete construction of a large bench-scale biofilter unit in which we can test various strategies for measuring the water content of a biofilter and, when necessary, adding water evenly throughout the bed, a task that is not as simple as it appears. Early results with the water probes have shown that local heat production by bacterial degradative activity causes evaporation and drying of the bed, leading to a local loss of activity that lets organic vapors move further into the bed. Consequently, a distinct front of drying and activity moves through the bed; this result has apparently not been reported before as a possible cause of biofilter operating prob- lems even if water content is controlled by humidifying the inlet air stream. A master's student in chemical engineering has been working on a thesis related to this issue to determine how the amount of water on compost affects the accessibility of hydrocarbon vapors to bacteria at various points on the surface and in the pores of a highly irregular compost particle. The LTRI's principal investigator is supervising this thesis project. Separately, we measured the changes in the residence time distribution (the amount of time flowing gas spends in the vessel) of a biofilter as it dried out from intentionally poor water control. The data show dramatic effects of evaporation on the system as pores formerly filled with water become accessible to the gas phase.

In other instrumentation work, we began work on a fiber optic probe of biological activity in the bed. This project was subcontracted to Dr. Ken Reardon at Colorado State University, who has assembled the necessary equipment and made preliminary measurements on suspensions of fluorescent beads in suspension. The next work will use a specially designed probe head to measure fluorescence in soils and beds of solids.

In the final task, we have almost completed work on improving the biodegradation of light hydrocarbons in biofilters by using watersoluble polymers to increase their bioavailability. A patent application was filed on this idea in December 1994. The polymer itself is not degraded by the bacteria, but by increasing hydrocarbon solubility in water it increases the biodegradation rate of hexane vapors by a factor of about three. Preliminary results in biofilters confirm these results that were obtained in batch tests in serum vials. The concept also works with several related polymers and with 
the gases isobutane and isopentane. These gases were tried in support of a field trial to be done with Envirogen Inc. and their client, Southern California Edison. Although the first trial in December 1994 did not work for nontechnical operational reasons, these new data (requested by the client) offer incentive for a second test.

This work was performed primarily by three postdoctoral researchers (Nancy Lynch, James Kastner, and Peter Gostomski). The first two left during the year for permanent positions elsewhere, while an offer of a permanent position at the INEL for Dr. Gostomski is pending now. To replace the two who left, another chemical engineering postdoctorate has been hired (David Thompson), and a regular chemical engineering employee (Kevin Gering) has agreed to continue with the compost modeling project on a parttime basis. In addition, the LTRI employed an AWU summer student in 1995.

\section{New or Increased Technical Capabilities}

This work has improved our fundamental understanding of the design and operation of biofiltration and composting processes. In particular, our understanding of the behavior of water in these solid-phase biological systems is advancing quite rapidly, both in terms of identifying the phenomena and modeling them mathematically. This will be of great use, as this work is applied to in situ bioremediation problems in the future. We also expect this information to be useful to other DOE and industryfunded programs in the Biotechnology Department.

\section{Business Development Opportunities}

The INEL has begun to receive notice for its work in composting, which has been featured in several newsletters after press releases and conference presentations. These notices have led to several inquiries from Idaho dairy interests with major manure disposal problems. We have made contact with Rust Engineering about their planned commercial composting facility in the Twin Falls area, and we may ultimately be involved with that as a full-scale facility for testing our ideas. We have also made initial contact with the U.S. Department of Agriculture, Forest Products Laboratory, and an industry-supported research program on possible application of the composting modeling work in biopulping, the fungal degradation of lignin before wood chips are made into paper. Newsletter coverage of the polymer work to increase bioconversion rates of organic vapors led to an unsolicited request from Envirogen Inc. for a field trial. During the coming year, we intend to repeat the first trial, which was inconclusive for nontechnical reasons.

A list of presentations, publications, and patents can be obtained from the principal investigator, $R$. S. Cherry.

\section{Radiation Measurements LTRI 0070}
A. J. Caffrey, R. Aryaeinejad, A. E. Egger, R. J. Gehrke, J. K. Hartwell, R. G. Helmer, L. O. Johnson, E. B. Nieschmidt, M. H. Putnam

TheThe objective of the Radiation Measurements Long-Term Research Initiative is the development of advanced radiation measurement instruments and techniques. The accomplishments of the research initiative will be applied, as appropriate, to programmatic activities at INEL and published in peer-reviewed journals.

This LTRI addresses radiation measurement problems within the DOE complex, at civilian nuclear reactors, and in other U.S. industries. In essence, the LTRI is aimed to apply recent advances from the physics laboratory to real-world problems.

\section{Objectives}

Analytical techniques for radiation measurements

The LTRI has explored analytical methods to improve the accuracy and efficiency of radiation measurements, including:

- Interactive gamma-ray spectrum analysis

- Automatic energy calibration of field gamma-ray spectrometers

- Monte Carlo modeling of radiation detection efficiency.

\section{Radiation measurement systems}

The LTRI has conducted laboratory development of the following instruments:

- A list-mode waste-drum assay system

- Position-sensitive radiation detection methods

- Room-temperature semiconductor detectors.

\section{Accomplishments}

The FY-95 Radiation Measurements LTRI research has explored analytical methods for radiation measurements and the development of new radiation detection systems.

Data analysis methods for radiation measurements interactive gammaray spectrum analysis

A new interactive program for precise analysis of gamma-ray spectra is a major activity of this LTRI. Spectrum analysis is of fundamental importance in applied measurements of radioactivity at DOE and commercial reactors, at waste management sites, and for basic research in nuclear physics and radiochemistry. 
This program allows the user to identify peaks and define peak regions, either by hand or via an automatic search algorithm. In the process of fitting complex or closely spaced peak regions, the program automatically adds Gaussian components to the region in order to improve the quality of the fit. The process often results in several analyses for one peak region; these can be reviewed both graphically and numerically. The user can visually determine the best results, archive them, and discard the rest, or edit the fit parameters and experiment further.

The program's interactive user interface is expected to markedly improve spectral analysis by increasing peak detection sensitivity and peak fitting reliability. It should also dramatically reduce the turnaround time for spectral analyses.

\section{Automatic calibration of field} gamma-ray spectroscopy systems

Establishment of the frequency, wavelength, or energy scale and assessment of spectral data quality are fundamental concerns for any spectroscopy system. In field applications of gamma-ray spectroscopy, these problems are usually more severe than in the laboratory, since field instruments may encounter rough handling, wide temperature swings, frequently interrupted electrical power, and indifferent operators.

To improve the accuracy and reliability of field gamma-ray measurements, we have recently developed an automatic gain adjustment and energy calibration method for both field and laboratory gammaray spectrometers; in addition, the associated software monitors the quality of the spectrometer data, and it simplifies system operation.
Monte Carlo modeling of detector efficiency for complex and extended sources

Frequently, the detection efficiency of a radiation measurement system is not well understood except in simple counting geometries, for example point sources. Outside of the laboratory setting, radiation measurements often involve complex, extended geometries, self-shielding corrections, and other matrix effects; hence, large systematic errors in the detection efficiency are possible.

We are improving our computational methods for generating peak and total efficiencies for gamma rays measured by Ge semiconductor detectors for both point and extended sources. Our principal computational method is Monte Carlo.

\section{Radiation instrumentation}

List-mode waste drum assay system

Passive and active neutron interrogation systems are expected to play an important role for the detection of fissionable materials in the waste drums stored at the INEL. The characterization of the drums is a prerequisite before shipment to a permanent repository.

A list-mode system capable of storing data from each individual neutron counter is under development. A list mode approach has probably not been pursued until now because of the very high data rates generated by an active neutron system, one hundred kilobytes to one megabyte per second. Since magnetic media cannot follow data at these rates, we have taken advantage of the falling price of computer random access memory, and store the list-mode data in a 128-megabyte array. The data are transferred to tape after the experiment ends for archiving and replay.
A custom module has been designed and constructed for the list-mode data system. It provides amplification and discrimination for each detector. For each event, it notes the time from a fast clock, encoded as 28 bits, and the detector identification, encoded in 4 bits. The 32-bit data word is written to memory by direct memory access without central processor unit intervention.

A fast PC will control the acquisition module and store the data. This unit will also be used for data sorting and analysis.

Position-sensitive neutron detection As a first step toward developing a neutron tomographic imaging system for waste drums, we have tested the position resolution of a one meter long ${ }^{3} \mathrm{He}$ detector. The detector has a resistive anode, and the axial position of an event within it is determined by charge division. The resolution for neutrons, as expected, is the order of one centimeter.

We have also tested the position resolution of a scintillating fiber bundle and have achieved a gamma-ray source position resolution of about two centimeters. This instrument may have applications in safeguarding special nuclear materials.

Room temperature semiconductor detectors

A new generation of room temperature semiconductor detectors, especially the PIN silicon diode and the cadmium-telluride diode, seems ready for several INEL applications, including lowlevel plutonium monitoring. We are testing several detectors of each type as detectors for $\mathrm{x}$-rays and low-energy gamma rays. We have also developed a novel palm-sized detector for low energy and thermal neutrons using lithium glass scintillators. 
Simple multiparameter data system

A simple multiparameter data acquisition system has been developed further in order to bridge the gap between single-parameter multi-channel analyzers and the complex minicomputer-based systems found in physics laboratories. The new system uses commercial software and runs on an Apple Macintosh PC. It was applied in the testing of several of the instruments listed above.

\section{New or Increased Technical Capabilities}

While the instruments and methods explored under this LTRI are useful for a wide variety of radiation counting applications, the following INEL applications directly benefit from this research.

Waste management and environmental measurements

The list-mode drum monitor, of course, addresses a highly visible INEL waste management issue, the assay of the Rocky Flats waste drums. In addition, the improved analytical methods are directly applicable to gamma-ray spectroscopy of the drums and the analysis of environmental samples, for example low-level $\mathrm{Pu}$ assays of dirt from Pit 9. The spectrum autocalibration method may find application in the characterization of mixed waste before incineration.

The improved spectroscopy methods have already found application in a DOE-funded arms control project. In order to design a new, spectrum-blind version of the PINS Chemical Assay System, the manual calibration of the gammaray spectrum energy scale had to be replaced by an automatic technique, developed under this LTRI.

\section{Nuclear operations}

The improved spectroscopy methods are also applicable to reactor effluent monitoring and dismantlement/decommisioning projects. We have received inquiry on the application of these methods to decommissioning facilities at Oak Ridge and ArgonneNational Laboratories.

\section{Business Development Opportunities}

The following INEL business opportunities have arisen as a direct result of this LTRI.

\section{PINS Arms Control RED}

Our development of automatic energy calibration techniques for gamma-ray spectrometers and the GAUSS IX spectrum analysis code has led directly to increased funding from our Department of Energy and Defense Nuclear Agency customers.

\section{CRADA with EGEG Ortec}

The advanced techniques explored in the LTRI have caused our CRADA partner, EG\&G Ortec, to extend the PINS CRADA agreement for another year. Ortec recently has begun production of the INEL-developed PINS system as a commercial product.

\section{GAUSS IX}

The GAUSS IX gamma-ray spectrum analysis code presently runs on Unix engineering workstations as a stand-alone product. We are currently modifying this code to operate under the Windows 95 system on IBM PCs. We anticipate that the Windows version will be attractive to a commercial partner.

\section{Other}

Los Alamos has expressed interest in applying the positionsensitive counting techniques to characterizing the neutron field in upcoming tests of accelerator tritium breeding at LAMPF. We have conducted tests to study
PGNAA monitoring of liquid sulfur/phosphorus mixture ratios for Monsanto Corp.

\section{Nuclear Fuels and Materials LTRI 0080}

\section{B. H. Rabin, W. J. Carmack, L. Lundberg, D. J. Varacalle}

The projects carried forward in the Nuclear Fuels and Materials Long-Term Research Initiative (NF\&M LTRI) in FY95 included (a) Synthesis and Characterization of Carbides and Carbonitrides, (b) Aerosol Technology, (c) Refractory Material Thermal Spray Coatings and Monoliths Fabrication Studies, (d) Refractory Material Melting Studies, and (e) Actinide Laboratory Development. INEL capabilities and expertise were successfully developed in all of these areas. Several business opportunities were developed as a result of research performed under this LTRI. This LTRI was deemed not relevant to the future interests of the INEL and consequently terminated at the end of FY-95.

\section{Objectives}

The original objective of the NF\&M LTRI, which was started in April of 1993, was to revitalize the capability to conduct research and development on nuclear fuels and materials at the INEL in order to support the design and testing of new reactor concepts. However, as a result of the recognition of a wider and changing spectrum of long term research needs both inside the INEL and nationally and as a result of discussions amongst the LTRI participants, our internal project monitors, INEL project managers, and outside contacts, we expanded our focus to include pro- 
jects relating to behavior of irradiated nuclear fuels and materials, storage of spent nuclear fuel, and storage of nuclear waste. The objectives of each individual project are presented below.

\section{Synthesis and Characterization of} Carbides and Carbonitrides

The FY-95 objectives were to synthesize thin films of selected carbonitride systems using state-ofthe art ion-beam assisted physical vapor deposition (PVD) methods, to conduct detailed materials characterization studies using $x$-ray diffraction, SEM, TEM, Auger Spectroscopy, XPS, microhardness, etc., and to address and establish fundamental relationships regarding the structure, phase equilibria, and mechanical, thermophysical and optical properties of these materials.

\section{Aerosol Technology}

A special need exists for calculating the region of aerosol particle size distributions centered below about $0.3 \mu \mathrm{m}$. A prime objective of FY-95 was to develop and document $\mathrm{C}$-code functions that can be assembled to perform pre- and posttest analysis of aerosol transport and deposition during advanced in situ particle characterization. These functions were intended primarily as wellresearched building blocks. At least one assembled code was to be produced to analyze data being generated in on-going aerosol characterization projects. Papers describing the results of the research were to be written and submitted for publication in a refereed journal.

\section{Refractory Material Thermal} Spray Coatings and Monoliths Fabrication Studies

The objective of this LTRI task was to perform studies on advanced thermal spray methods for producing coatings and monoliths of refractory and reactive materials that are of interest to the nuclear industry. The materials of prime interest for FY-95 were to be tungsten and tungsten-base alloys. A facility for applying coatings under inert conditions was to have been developed to support these efforts.

\section{Refractory Material Melting Studies}

This project was developed to study melting of uranium bearing refractory carbides whose solidus temperatures range to $4250 \mathrm{~K}$. The melting behavior of various carbide and carbonitride compositions that contain two or more of the metals zirconium, niobium or hafnium were to be studied to demonstrate a high power rf-heated melting apparatus developed in FY-94. A two-dimensional infrared detector array was to be purchased and installed in the rf-heated melting apparatus to allow for spectral as well as spatial detection of radiant energy from the heated sample.

\section{Actinide Laboratory Development}

The objective of this task was to obtain and develop laboratory space capable of meeting the requirements set for high temperature testing and fabricating uranium bearing material samples. A state-of-the-art thermal spray system was to be installed into an inert glove box and brought to operational status during this fiscal year.

\section{New or Increased Technical Capabilities}

Synthesis and Characterization of Carbides and Carbonitrides

Construction and testing of the state-of-the-art ion-beam assisted deposition system was completed and this facility is now supporting a wide range of existing and new research projects involving advanced materials and processing of novel thin films and coatings. A series of $1-\mu \mathrm{m}$-thick $\mathrm{Zr}: \mathrm{Nb}$ nitride films was produced and preliminary characterization was carried out using $x$-ray diffraction and surface analysis. Direct nitride formation was confirmed in films produced at high nitrogen partial pressures with elevated substrate temperatures. The films contained high residual stresses.

\section{Aerosol Technology}

Appropriate C-Code functions were written, and optimum parameters for vapor deposition of paraffin wax onto polystyrene particles were investigated. The parameters examined were temperature, particle size, and area of evaporation. To find the ideal temperature for this deposition, tests were executed varying the rate of temperature increase and the temperature range. In addition, the effects of the size of the polystyrene particles on the amount of deposition was explored. Finally, the amount of wax vapor entering the vapor deposition chamber was examined by varying the area through which the wax evaporated.

\section{Refractory Material Thermal} Spray Coatings and Monoliths Fabrication Studies

Accomplishments included development of plasma spray processes for spray forming tungsten- and tungsten-rhenium alloygraphite composite tubes for use in high temperature thermionic devices. In this process, the reactive tungsten deposit was protected only by the inert gas and hydrogen used to produce the plasma and carry the pure metal powder feedstock. The deposition targets were located in ambient air, and the temperature of the targets was uncontrolled. Various substrate materials were investigated besides graphite including steel, stainless steel and aluminum. Bulk density measurements revealed that as- 
deposited monoliths were typically no more than $65 \%$ of theoretical density. Heat treatments using combinations of vacuum and hydrogen at temperatures as high as $2500 \mathrm{~K}$ produce product densities in excess of $90 \%$. Twinwire electric arc (TWEA) methods were also tested for depositing tungsten using inert gases for the carrier gas, as opposed to air, but even though the method looked promising, success was limited because of the fact the TWEA torches used were not designed to handle the very high temperatures associated with the arc-melting of tungsten.

\section{Refractory Material Melting} Studies

During FY-95, this task focused on developing state of-the-art algorithms to be used in conjunction with spectral intensity measurements of very high temperatures using an optical monochromator. This technique allows temperature measurements on a variety of solids, liquids, and plasmas over a temperature range extending from room temperature to plasma temperatures $(>10,000 \mathrm{~K})$.

Actinide Laboratory Development.

A new thermal spray system facilities was acquired with GPCE funds and installed at TAN (WRRTF). Installation of this equipment into an inert glovebox with HEPA filtered exhaust was begun.

\section{New or Increased Technical Capabilities}

Synthesis and Characterization of Carbides and Carbonitrides

The two materials processing techniques and expertise developed under this project have added entirely new capabilities for producing and studying a wide variety of novel materials of interest to the DOE and private industry for use in advanced energyrelated applications.

\section{Aerosol Technology}

This LTRI task focused on developing the INEL's basic aerosol production, measurement, and analysis capabilities. The continued development of capabilities has been coupled with business oriented opportunities. For instance, prior to the development of measurement facilities under this LTRI, the PHEBUS on-line aerosol monitor (OLAM) project was conducted jointly with Sandia National Laboratory because the INEL did not have aerosol measurement capabilities. Using equipment and capabilities established under support of the NF\&M LTRI, the PHEBUS/OLAM calibration work was conducted at the INEL. This work conducted at the INEL would have been conducted at Sandia if the NF\&M LTRI had not developed an aerosol measurement capability.

\section{Refractory Material Thermal} Spray Coatings and Monoliths Fabrication Studies

Components for an inert atmosphere thermal spraying facility have been collected and partially assembled. The thermal spray equipment is installed at TAN (WRRTF) and operational in room ambient conditions.

\section{Actinide Laboratory Development.}

Facilities are in place at TAN (WRRTF) for heating materials to very high temperatures in controlled environments, including high vacuum and hydrogen. Facilities are also in place to handle and process reactive materials in high purity inert environments.

\section{Business Development Opportunities}

Synthesis and Characterization of Carbides and Carbonitrides

The ion-beam assisted deposition system is now being used to support additional ongoing programs at the INEL including research on graded coatings, improved corrosion resistant aluminum alloy coatings, and solid oxide fuel cells for power generation.

\section{Aerosol Technology}

Through this LTRI task a number of technology transfer opportunities were pursued. Contact was made with Idaho Supreme Potatoes in Shelley, Idaho and technical assistance provided in the area of aerosol measurement. In addition contact was made with AMI of Pocatello, Idaho in relation to enhanced cleanroom filtration technology.

Aerosol measurements in nuclear reactor accident experiments and processes have been required for a number of years. Aerosols play a very important roll in accident initiation and progression. The INEL has been involved in the development and research of key aerosol measurement instruments as well as in the post accident analysis of aerosol data.

Aerosol behavior makes up a significant portion of the Severe Accident Analysis Group's business. Currently the Severe Accident Analysis Group is conducting programmatic funded projects in Calcine Offgas Analysis Experiments, Fusion Safety aerosol production, measurement, and behavior, and PHEBUS/OLAM development and calibration. The Severe Accident Analysis group is also involved in an LDRD to characterize the offgas system proposed for a high level waste vitrification facility.

There are numerous business opportunities in non-nuclear aerosol behavior and the capabilities developed under the LTRI are directly applicable to these areas. All industries are coming under increased particulate (aerosol) emission restrictions. The state of Idaho is currently establishing restrictive particulate emission cri- 
teria for all industries. The Severe Accident Analysis Group currently is performing research in the areas of Mllegal Substance Detection and Measurement and in developing advanced particulate control technology for offgas systems. Currently, funding amounts to approximately $\$ 200,000$ in these areas.

\section{Refractory Material Thermal} Spray Coatings and Monoliths Fabrication Studies

Inquiries have been made to the INEL to spray form tungsten components for solar heated space power and propulsion system development.

\section{Actinide Laboratory Development.}

The business opportunities can be developed especially in the areas of high temperature space power and propulsion development.

\section{Experimental Thermal Science}

LTRI 0090

D. M. McEligot, K. G. Condie, G. E. McCreery, M. G. McKellar, K. A. Shinpaugh, C. M. Stoots

The overall goal of this $L T R I$ is to extend internationally recognized capabilities in basic experimental thermal science (ETS) in applications important to present and future missions of the INEL. Thermal sciences, such as heat transfer and fluid mechanics, are involved in almost all programs at the INEL. Studies conducted under this ETS LTRI concentrated in the areas of heat, mass, and momentum transfer in (a) complex turbulent flows, (b) porous media, and (c) two-phase particulate flows. Our efforts also focused on development of a versatile, unique matched-indexof-refraction (MIR) flow facility. We selected generic experimental projects and other related activities that would support applications in all four present
Strategic Thrust Areas, the Centers of Excellence, and/or other present and future areas of importance to INEL. Main accomplishments for FY-95 have been development of the MIR system, and development of a facility for investigating waste remediation techniques. Six technical papers have been published or accepted for publication in archival journals in the field; six papers were presented at national and international technical conferences; collaboration, review, and technical seminars have been provided by about twelve university faculty; and informal collaboration has been conducted with firms in the vehicle and agricultural industries. Also, we joined the Stanford Thermosciences Industrial Affiliates. This LTRI has extended experimental capabilities, both in equipment and staff expertise, for INEL and for local, regional, national and international thermal fluid mechanics communities, and is providing technology to improve U.S. competitiveness in the vehicle industry.

\section{Objectives}

The overall goal of this LTRI is to extend internationally recognized capabilities in basic thermal engineering experimentation in applications important to the missions and future of INEL.

- Enhance technology transfer by participating in ongoing INEL applied research.

- Identify knowledge gaps (and basic research activities to bridge these gaps) that represent barriers to development activities in main INEL mission areas.

- Support INEL analytical and code development efforts via basic laboratory experiments.

- Collaborate with university and national laboratory peer groups.

- Increase publication in leading basic ETS journals.
- Attract and retain highly qualified scholars in experimental heat transfer and fluid mechanics.

- Increase staff involved in basic research related to INEL's future.

\section{Accomplishments}

The ETS LTRI is primarily structured in terms of individual research projects within the three areas of concentration (complex turbulent flows, porous media, and two-phase particulate flows). Recruiting of outstanding new experimental thermal scientists is accomplished via the Postdoctoral Fellowship program of the Associated Western Universities. During FY-95, Dr. Kevin A. Shinpaugh of Virginia Polytechnic Institute joined us as an AWU Fellow.

The main accomplishments for FY-95 are (a) development of the MUR system, and (b) development of a facility for investigating waste remediation techniques. Our approach for selecting and developing research topics for this LTRI is mission-oriented. Heat transfer and fluid mechanic needs are identified in areas of importance to the present or future missions of INEL, as in the Strategic Thrust Areas. Basic experimental questions are then determined and an appropriate generic experiment is designed. Results are generalized to the extent possible, so they will be useful both for the initial application and for applications identified later in other fields found to correspond to the same generic situation.

Results are published in the appropriate journals. Brief descriptions of the FY-95 accomplishments in the experimental projects and facility development follow.

Nonsteady flows with heat transfer, Keith Condie, Kevin Shinpaugh, and J. Robert Mondt (GM Delphi). Pulsating flows occur in vehicle exhaust systems, hypothe- 
sized accident scenarios for nuclear reactor safety studies, and pulmonary flows in the human body. To examine the effects of entry conditions and pulsating flow, we conducted experiments in which we took heat transfer measurements on a contemporary exhaust system on a recent commercial V-6 engine. A typical S-shaped takedown pipe was heated electrically and was instrumented to determine local wall temperatures and related heat transfer parameters. For further insight, outlet air temperatures and cyclical inlet pressure fluctuations were also measured.

Inlet conditions included pulsed and steady air flow through the exhaust manifold. Six cylinder operation of the engine as a compressor provided a pulsating inlet flow with moderate amplitude fluctuations. With steady flow, slight differences in wall temperature profiles were observed for flow from different exhaust ports. A numerical analysis and empirical correlations predicted still higher wall temperatures. Compared to the Dittus-Boelter correlation, the convective heat transfer parameters for flow from the actual manifold showed convective enhancement factors of the order of two. Non-dimensional frequencies in pulsed flow fell in the range 0.045 $<\omega^{+}<0.97$, corresponding to highfrequency flows rather than quasisteady flows; in contrast to most heat transfer experiments, the cyclical pressure fluctuations were not near-sinusoidal. Results showed enhancement factors of about three to seven.

We presented the results of this study at the 27th International Symposium on Automotive Technology and Automation in Aachen, Germany, in October, at the SAE International Congress in Detroit in March, and at the 2nd Vehicle Thermal Management Systems
Conference, sponsored by

I.Mech.E/SAE in London, England, in May.

Rapidly scanning laser

Doppler velocimeter (RSLDV)

measurements of a wing-body

junction, Kevin Shinpaugh, and

Professor R. L. Simpson (VPI). Dr.

Shinpaugh's technical paper on his design and development of the

RSLDV was accepted for publication in a special issue of Measurement Science and Technology (J. Phys. E.).

Turbulence structure in laterally converging flows, D. M. McEligot, Professor Dr. H. Eckelmann (Max Planck Institute), and Professor R. S. Brodkey (Ohio State University). This study applied various analyses to examine data on the modification of turbulence structure by streamwise pressure gradients induced by flows through convergences. The purpose was to provide information about flows in vehicle exhaust manifolds, gas turbines, industrial exhaust stacks, nuclear reactor plena, and many other configurations in practice, and to provide information to support research in computational fluid dynamics. Strong favorable pressure gradients have been found to reduce viscous drag by altering the structure of the turbulent velocity fluctuations. We studied the turbulence structure, in order to provide bases for incorporating the effects of such favorable streamwise pressure gradients into models for turbulent boundary layers and internal turbulent flows.

Earlier, at the Max Planck Institut für Strömungsforchung, McEligot and Eckelmann obtained simultaneous time series measurements with an $\mathrm{x}$-probe and a wall shear stress sensor in an oil channel both for fully-developed and for laterally-converging flows. Current efforts aim at determining how the coherent behavior of the turbulence near the wall is affected by the convergence-induced pressure gradients. Results calculated to date include conditionally-sampled statistics determined by the VITA detection technique, by quadrant-splitting, and by pattern recognition techniques. The simultaneous transient structure of events across the viscous layer has been deduced by multiple detection with an extension of the VITA approach. These results were presented at an invited talk, Coherent Structures in the Viscous Layers of Flows in Converging Ducts, at a special symposium honoring Professor Brodkey at the Annual Meeting of the American Institute of Chemical Engineers last November.

Turbulence structure in heated gas flows, D. M. McEligot, A. M. Shehata (Xerox), M. F. Taylor (deceased, University of Arizona), and Professor J. D. Jackson (University of Manchester, UK). Forced convection of gases and gas mixtures with significant property variation occurs in many applications: superheated steam, gascooled nuclear reactors, vehicle exhaust systems, and solar collectors. This project analyzed measurements taken earlier by D. M. McEligot and his colleagues in order to obtain further basic insight into forced convection of gases with variable properties and to evaluate application of proposed turbulence models to this situation. We have completed the analyses. The results are presented in a technical paper, The Turbulent Prandtl Number in the Near-wall Region for Low-Prandtl-number Gas Mixtures, which is being published by the International Journal of Heat and Mass Transfer. A technical report, Turbulence Structure in the Viscous Layer of Strongly Heated Gas Flows, is nearing completion; it will form the bases for two technical journal papers on our experiments and their numerical predic- 
tion, to be written in collaboration with Professor J. Derek Jackson of the Nuclear Power Program at the University of Manchester in England.

Maximum allowable heat flux for a horizontal tube bundle, D. M. McEligot, K. G. Condie, and Professor V. K. Dhir (UCLA). Heat exchangers with horizontal tube bundles occur in some designs for advanced nuclear power plants, stab-in configurations in the petroleum industry, and other pool heaters. We presented our approximate analysis for the critical heat flux and discussed comparisons to the few related experiments in an invited talk at a special technical symposium honoring Professor John H. Lienhard IV at the National Heat Transfer Conference in Portland in August.

Steam injection for removal of chlorinated solvents in fractured porous media, Glenn McCreery, Mike McKellar, Mel Herd, and Professor Kent S. Udell (University of California). One of our main successes has been our work on heat transfer and phase change in porous media, cited by INEL Environmental Operations as the LTRI project of the highest priority of importance to our mission. Subsequently, the Chief Scientist and Chief Engineer determined that the topic of porous media warrants a separate LTRI starting in FY-96, so our efforts in this area will be transferred.

Of potential environmental concern are chlorinated solvents and organic compounds in the vesicular basalt flows that lie above the Snake River aquifer at the INEL. The steam injection remediation process has been demonstrated by Professor K. S. Udell to be effective for the removal of hydrocarbons and organic compounds from heterogeneous porous media. It has been suggested that this steam extraction technique may be a cost-effective approach to removing contaminants from the basalt above the aquifer at INEL. However, the basic phenomena involved are not understood sufficiently for accurate design predictions to be made for this application.

Fundamental laboratory-scale experiments will be performed to model the steam-enhanced extraction process of removing liquid contaminants in fractured porous media (see the figure below). The process consists of steam injection into a porous medium that is partially or completely saturated with contaminated water. The steam condenses and sweeps contaminants with the condensation front

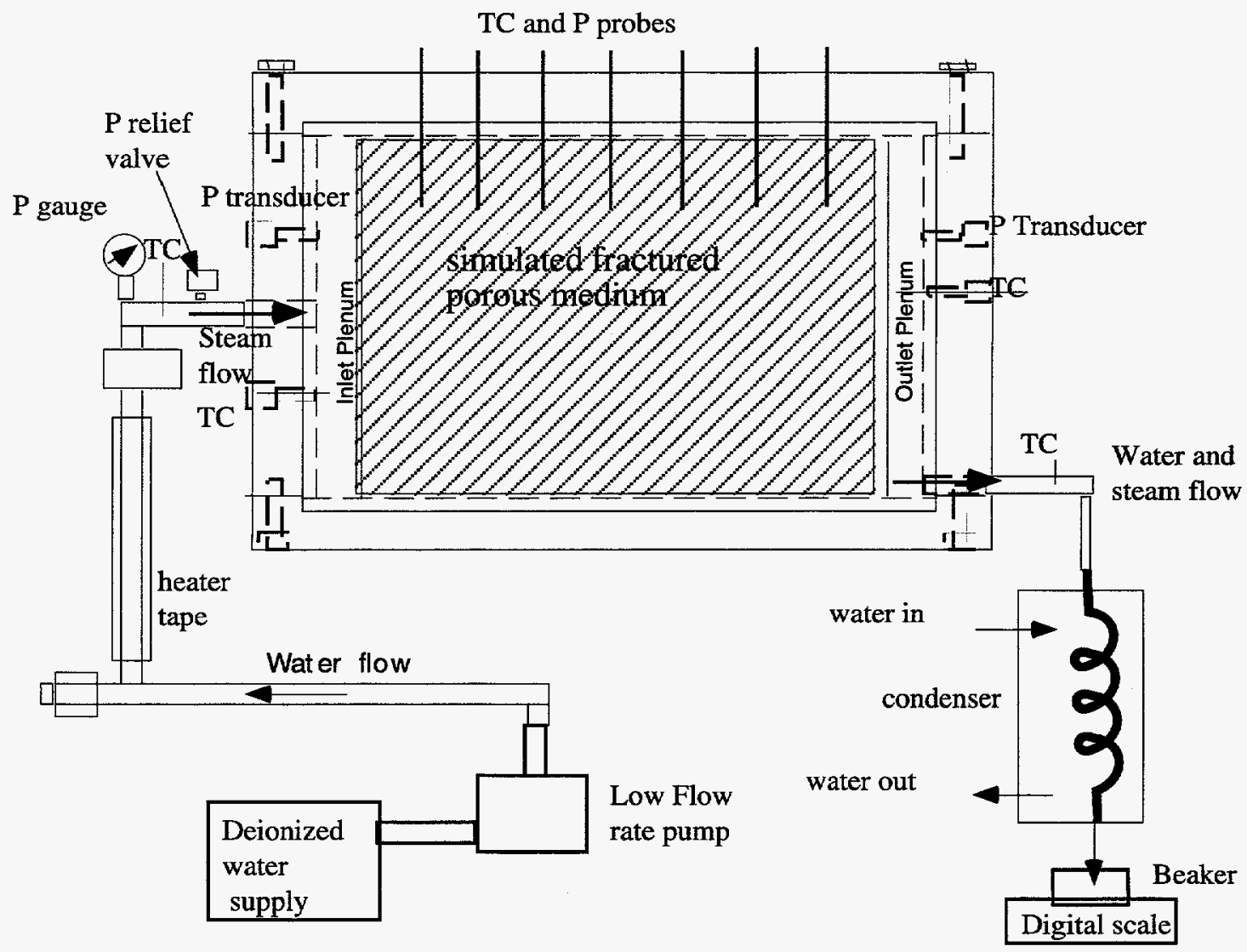

Laboratory apparatus for fundamental studies of steam injection into fractured porous media. Reproduced from technical report by McCreery and McKellar [1995]. 
toward a low pressure region established by a vacuum (and water) pump.

Design features of the test section include

- A modular and removable test section

- Windows for visual, video, and photographic access

- A thermochromic liquid crystal sheet adjacent to the back window for mapping the condensation front

- Temperature and pressure measurements within the inlet and outlet plena and at select locations within the simulated fracture gaps

- A removable top section of the frame for insertion of additional simulated fracture fill material (if it compacts)

- Baffle plates to direct the flow of steam and water.

The test section is well insulat- ed with argon-filled triple-pane windows, stainless-steel walls, and removable Styrofoam blocks.

Although we designed the apparatus for simulation of the steamenhanced extraction process, the apparatus can also be used to simulate other waste extraction processes in fractured porous media, such as vapor vacuum extraction. During FY-95 we completed the test section for steam injection, and we conducted checkout tests. We performed preliminary approximate analyses to deduce the porosity and permeability from calibration experiments. We conducted isothermal steady and transient flow experiments and wrote a draft technical report presenting the results to date.

Droplet formation in liquid jet impingement, Glenn McCreery, Dr. Dennis Kincaid (USDA Agricultural Research Service, Kimberley, Idaho). We conducted an experimental investigation of droplet formation from a jet impinging on a circular plate. This generic type of spray nozzle is commonly used for center pivot agricultural irrigation and for fire protection; a comparable situation exists with some injection systems for emergency core cooling of commercial nuclear reactors. Results included short duration photographs of nozzle jet flow, liquid film flow, and water drop formation. We derived drop diameter distributions using our phase doppler particle analyzer. The study and its results are presented in a technical paper accepted for publication in the International Journal of Multiphase Flow.

Matched-index-of-refraction facility, Carl Stoots, Keith Condie, Blaine Merkely (retired), Professor R. F. Blackwelder (University of Southern California), Professor P. Bradshaw (Fellow, Royal Society, Stanford), and Professor Dr. F. Durst (University Erlangen, Germany). Our key experimental facility will be the MIR system (see the figure below), recommended by our international advisory com-

The temporary plate, with circular fitting for pump- and leak-testing, is to be replaced by $4 \times 2$ foot rectangular test section for concept testing for the ACETS program.

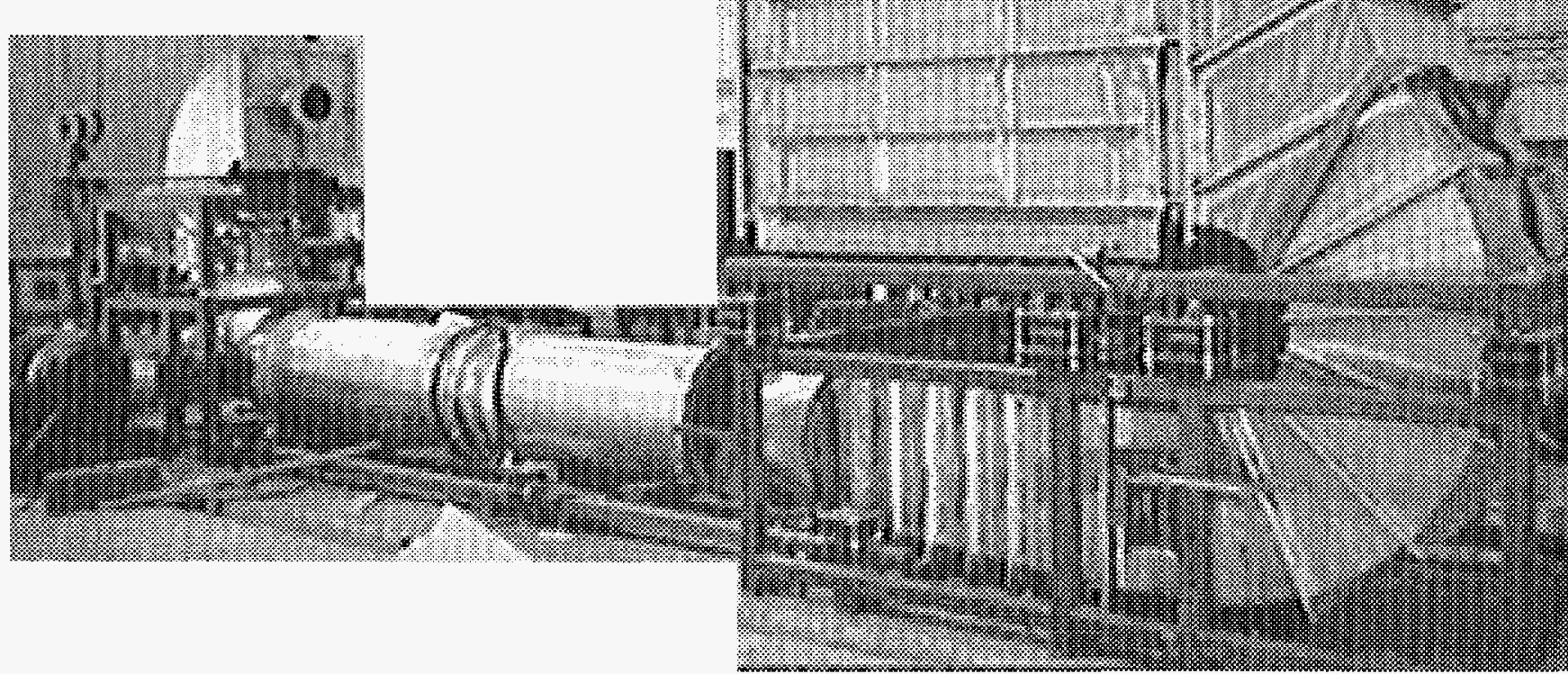

INEL Matched-Index-of-Refraction System during Fabrication at Idaho Steel Products, Idaho Falls, Idaho 
mittee. It will provide unique capabilities to investigate basic fluid dynamics in complex turbulent flows and two-phase particulate flows, as well as flows in porous media and other complex geometries. In each of the areas of concentration, complex geometries hamper pointwise measurements, particularly by non-intrusive optical means. If a transparent solid is immersed in a transparent fluid, and the solid and fluid have the same indices of refraction, the solid optically disappears. This measurement technique allows one to perform experiments and make measurements that otherwise would be impossible. About twenty fundamental experiments have been identified that would benefit from the advantages of such a flow facility. While past researchers have employed the advantages of matching index of refraction, this project is unique in that it intends to index match in a "large" measurement system as well as in moderate-sized test sections.

The system is similar to water tunnels used in hydrodynamics research, except that the proposed fluid is a mineral oil to match the index-of-refraction of quartz test specimens. The maximum design flow rate is about $5400 \mathrm{gpm}$. For application to the ACETS program, the first test section has been redesigned to provide a flow cross section of two by four feet. For this system, a review committee has evolved with authorities from universities internationally and from national laboratories.

During FY-95, we completed the structural analyses, and we finalized the general system design interactively with Idaho Steel Products Co. of Idaho Falls, the fabricator. Oil supply, air suspension, and oil temperature control systems were designed and mostly procured. A laboratory room at the Water Reactor Research Test Facility (WRRTF) was prepared for installation of the
MIR system, the floor was sealed against leaks, and oil storage tanks and electrical power components were installed. System instrumentation was specified, honeycomb and screens for flow conditioning were ordered, and additional instrumentation ports were installed. Contractions were designed for the ACETS test section. As of this writing, the system is scheduled for leak testing (with a "test section simulator" in place) in late September 1995, with delivery to WRRTF to occur shortly afterward.

\section{New or Increased Technical Capabilities}

The major new experimental facilities developed by this LTRI have been the MIR system and the laboratory-scale apparatus for investigating waste remediation techniques, both mentioned and pictured above. In the process, some of our technical staff's expertise was extended to improved capabilities in the thermal, fluid mechanics, and structural design of temperature- and vibration-controlled oil flow experiments. The capability to construct prototype flow nozzles was developed for two-phase particulate flows. Techniques for measuring high temperature, non-steady flows and heat transfer in our V- 6 engine/compressor experiments were improved. Also, some of the staff extended their abilities in data acquisition, reduction, analysis, and presentation.

Additional activities for the benefit of INEL in general and of the LTRI involve collaboration with university faculty and membership in the Stanford Thermosciences Industrial Affiliates. Faculty provided technical seminars covering topics that are important to INEL programs beyond the LTRI alone; these seminars update and expand the fundamental understanding of the INEL staff in the areas involved. During FY-95, seminars were presented on design of thermal fluid mechanic experiments and experimental uncertainty analysis, heat transfer in fluidized beds, fluid mechanics measurements by index-matching techniques, techniques to measure concentrations in turbulent flows, heat transfer from porous media, and effects of freestream turbulence on heat transfer. Also, the LTRI staff, representing INEL, joined the Stanford Thermosciences Industrial Affiliates. Through Stanford, the Affiliate membership gives us better access to resources in this country and a powerful connection to what is being done in the rest of the thermoscience world. Membership provides direct, informal, person-to-person technical assistance from Stanford faculty, and other benefits as well.

\section{Business Development Opportunities}

No participants in this LTRI serve full time under LTRI support. Therefore, all participants contribute directly to INEL business units, either via externally supported programs or LDRD projects supported by the Product Directors. Donald M. McEligot assists the technetium project, the Boron Neutron Capture Therapy project, NRC studies, and proposals to the USCAR/ESST program. Keith Condie provides experimental guidance to the NRC program. Glenn McCreery supports NRC studies and agricultural initiatives. Michael McKellar assists NRC and waste remediation programs. Carl Stoots obtained, via the DOE/USCAR/ESST program, two externally-funded projects that he now leads.

The scope of this LTRI is being expanded with the initial test section for the MIR system being built to permit concept evaluation for wind testing aspects of the 
Advanced Combined Environments Test Station (ACETS) and other initiatives of NITTEC. The MIR system also is a facility where the potential exists to establish collaborative research with foreign countries as part of the offset credits program of the Lockheed Martin Corporation.

Studies of nonsteady flow involve informal collaboration with GM Delphi Division (J. R. Mondt) and Ford Research Laboratory
(D. L. Hartsock) in the area of vehicle exhaust heat transfer. This project has the potential for support and a CRADA from the DOE/USCAR/ESST program, with liaison being handled by S. M. Sorensen of INEL. A white paper has been submitted to the $\mathrm{DOE} / \mathrm{BES}$ heat transfer program for fundamental studies of nonsteady flow. Collaboration, a CRADA, and external support for two-phase particulate flows, con- centrating on droplet formation by spray plate impingement, are under discussion with Valmont Irrigation Company (Dr. J. Chapman) and Nelson Irrigation (B. Nelson) via P. E. Reep of the INEL agricultural product area. D. M. McEligot and G. E. McCreery have submitted a proposal to the NASA Microgravity Fluid Physics program for basic experiments on droplet detachment and formation under reduced gravity conditions. 
Appendix A

Author Index 
A

Akers, D. W. 136

Allemeier, R. T. 225

Anderl, R. A. 103, 242

Anderson, A. A. 218

Anderson, P. A. 70

Antill, R. I. 171

Apel, W. A. 32, 53

Appelhans, A. W. 140

Argyle, M. D. 221

Aryaeinejad, R. 239,254

Atkinson, D. A. 71

Austad, W. E. 185

\section{B}

Baker, J. D. 89,103

Bala, G. A. 46

Barklund, C. R. 162, 163

Barrett, K. B. 197, 198

Bauer, W. F. 13

Bechtold, T. E. 176,177

Becker, G. K. 35

Beelman, R. J. 94

Bennett, R. G. 105

Bergeson, G. C. 186

Berry, R. A. 155

Bishop, C. W. 101

Brasier, J. E. 89

Brown, B. W. 178

Bruneel, F. W. 119

C

Caffrey, A. J. 254

Carlson, N. M. 144, 213

Carmack, W. J. 256

Carver, C. 75

Chambers, A. G. 221

Chang, G. S. 96

Chatters, T. C. 121,225

Cherry, R. S. 128, 198, 201, 252

Church-Riddle, K. A. 171

Clark, D. E. 159

Cole, G. H. 149, 164

Cole, J. D. 239

Colwell, F. S. 54

Condie, K. G. 259

Cordes, G. A. 222

Cowan, R. L. 140

Crawford, T. M. 209

Croxford, W. R. 218

$\mathrm{D}$

Dean, L. A. 228

Dearien, J. A. 152

Deason, V. A. 137, 208, 209

Delmore, J. E. 140

Derr, K. W. 188

Detering, B. A. 232

Dicke, C. A. 48
Douglass, R. W. 249

Downs, W. C. 39

Drigert, M. W. 239

E

Edwards, A. J. 42

Egger, A. E. 189, 191, 254

Epstein, J. S. 212

Erickson, A. W. 64, 175

Erickson, V. A. 167

$\mathrm{F}$

Filby, E. E. 74,

Fincke, J. R. 63, 126, 131, 143, 148

Fink, R. K. 190, 206

Freeman, L. E. 42

Frickey, D. A. 218

G

Garrard, D. M. 212

Garwick, S. 121

Gehrke, R. J. 25, 57, 65, 254

Gering, K. L. 18

Ginosar, D. M. 20

Glenn, D. F. $142,219,230$

Gombert, D. 84, 85, 107

Grandy, J. D. 156

Greenwood, R. C. 239

Groenewold, G. S. 140

$\mathrm{H}$

Heaps, R. J. 212

Hamilton, M. A. 31, 197

Hampton, N. L. 45

Harker, Y. D. 35,91

Harmon, J. F. 105

Hartenstein, S. D. 73

Hartwell, J. K. 254

Haulenbeek, K. K. 119

Hawkes, B. D. 180

Hawkes, G. L. 194

Hawkes, W 180

Helmer, R. G. 254

Hess, J. R. 202

Hong, B. C. 167

Hoskinson, R. L. 134, 194

Hubbell, J. M. 40, 203

Hunt, G. L. 150,165

I

Ingram, J. C. $58,59,140$

$\mathrm{J}$

James, J. L. 72

Janikowski, S. K. 20, 66

Johnson, L. O. 254

Johnson, K. R. 167

Johnson, R. W. 227

Jones, J. L. 105
K

Kaplan, B. L. 182

Keating, E. P. 171

Kennedy, J. R. 12, 232

Kessinger, G. F. 77, 176

Knecht, D. A. 77

Kong, P. C. 156, 161, 173, 175

Korth, G. E. 142

Kotter, D. K. 44, 75

Kunerth, D. C. 119

Kurkowski, D. J. 138, 207

L

Landon, M. D. 164, 227

Larsen, D. G. 192

Lassahn, G. D. 119

LaViolette, R. A. 247

Lee, B. D. $14,32,129$

Lee, J. 225

Lehman, R. M. 67

Lessing, P. A. 173

Lickson, R. S. 23

Liekhus, K. J. 21

Losinski, S. J. 192

Lundberg, L. 256

M

MacDonald, P. E. 136

MacDowall, R. D. 146,171

Mandler, J. W. 192, 239

Marshall, D. W. 80

McAtee, R. E. 27

McCreery, G. E. 259

McEligot, J. 259

McIsaac, C. V. 30

McKay, M. D. 78, 151

McKeller, M. G. 259

Miller, B. G. 214

Miller, C. S. 167

Miller, S. J. 69

Mills, J. I. 33, 152, 182

Mincher, B. J. 21

Montierth, L. M. 98,99

Moore, G. A. 12

Moore, R. L. 93, 229

Morrison, J. L. 183

Motloch, C. G. 204

Mousseau, K. C. 194

N

Nelson, W. R. 180

Nieschmidt, E. B. 254

Nigg, D. W. 17, 90, 97, 98

Noah, K. S. 15

$\mathrm{O}$

O'Brien, C. C. 112

O'Brien, M. H. 64, 212, 232

Olson, A. L. 27 
$\mathrm{P}$

Partin, J. K. 219

Polk, R. E. 41

Polman, J. K. 52

Propp, W. A. 221

Pryfogle, P. A. 51, 130

Putnam, M. H. 254

\section{R}

Rabin, B. H. $124,212,256$

Raymond, R. H. 167

Renner, J. L. 196

Repetti, T. E. 62, 209

Reuter, W. G. 29, 114, 213

Rhudy, J. W. 222

Richins, W. 180

Rinehart, B. N. 36,51

Roberto, F. F. 61

Robertson, E. P. 46

Rodriguez, J. G. 216

Rogers, J. W. 89, 103

Rogers, R. D. 31, 130

Rosentreater, J. J. 18

Ryskamp, J. M. 92

$\mathrm{S}$

Schafer-Perini, A. L. 56

Schnitzler, B. G. 103

Schwieder, P. R. 192
Sebo, D. E. 207

Sellers, C. H. 119, 136, 242

Shah, V. N. 136

Shinpaugh, K. A. 259

Shook, G. M. 49, 196

Shurtliff, R. M. 82

Siahpush, A. S. 133

Siemer, D. D. 81

Smartt, H. B. 168,206

Smith, R. W. 48

Snow, E. 119

Snyder, M. W. 167

Snyder, S. C. 161

Stacey, M. R. 153

Stone, M. L. 122

Stoots, C. M. 259

Swank, W. D. 131, 148

$\mathrm{T}$

Taylor, P. L. 208

Telschow, K. L. 119, 121, 136, 225

Thomas, C. P. 46

Turick, C. E. 22

Turner, T. D. 133,180

$\mathrm{V}$

Van Siclen, C. D. 242

Varacalle, D. J. 26, 256

Venhuizen, J. R. 91
W

Ward, T. E. 199,237

Watkins, A. D. 156, 161, 223

Watts, K. D. 65

Welker, K. D. 167

Wheeler, F. J. 17,98

White, G. J. 38

Wiedert, L. L. 170

Wilding, B. 180

Williamson, R. L. 210, 212

Wolf, J. R. 26

Wolfram, J. H. 9, 111

Woods, G. K. 139

Wright, R. B. 10

Wright, R. N. 125, 126, 143

Y

Yoon, W. Y. 98, 105

Z

Zabriskie, J. M. 222

Zollinger, W. T. 83, 233 


\section{Appendix B \\ Project Number and Title Index}




\section{Project}

Number

Title

\section{National Integrated \\ Environmental Management}

Assessment Methodology for Enhanced Bioremedi-ation of Hydrocarbons in the Petroleum Industry .............

Catalyst-Assisted Sonochemical Treatment of Hazardous Organic Wastes ...

Synthesis, Characterization, and the Modes of Interaction in Volatile Organic Compound Sensor Materials .........

262 Integration of Sample Introduction Techniques, Torch Design, and Optics for Determining Trace Metals in Biological Samples with Inductively Coupled Plasma Atomic Emission Spectrometer

Removal of Chlorinated Solvents from Air Streams Using Anaerobic

Biofiltration ..................

$306 \quad$ Using Sawdust for Microbial

Abatement of Acid Mine Drainage ....

1172 High-Energy Computational Dosimetry for Neutron Radiotherapy ..........

1201 Photocatalytic Treatment of Anionic

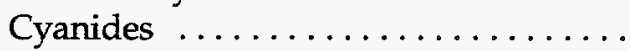

Catalytic Destruction of Halogenated Organics at Supercritical Fluid Conditions .................

1203 Destruction of PCBs in Oil Using Gamma Irradiation and Ozone ......

1233 Bioprocess Development for the Remediation of CR(VI) and U(VI)Contaminated Soils and Groundwater . .

Ultraviolet Volatile Organic Compound Destruction Technology, Liner Lifetime and By-product Testing with Hazardous Waste Disposal Considerations .......

\section{Project}

Number

Title

Page

2104 Portable Photon Analysis Spectrometer for TRU and $\gamma$ Assay . . . . . . . . . . 25

3203 Conceptual Design of a 55-Gallon Waste Container Refurbishment System . .

3710 Photodissociation of Mercuric Chloride Treatability Study ..............

4078 INEL-University of Idaho Laboratory for Liquid Extraction and Ion Exchange Research ..................

4090 Fracture Mechanics-Based Design

Methodologies for Metal Matrix

Composite Material ............

4110 Develop, Build, and Test Assay System to Measure Transuranic and Fission Product Contamination in Subsurface Soil .......................

4171 Biodecontamination of Massive

Concrete Structures . . . . . . . . . . .

4172 Catalyst-Assisted In Situ

Bioremediation ...............

4204 Sustainable Water Resources

Management ................

4275 High-Sensitivity Nondestructive Analysis for Transuranic Waste Examination for Characterization ............ 35

$4374 \quad$ Flexible Fish Conduit Passage System ... 36

4550 Development of a Methodology for Assessment of Power Plant Emissions on Air Quality-Related Values ........

4579 Passive Containment of Volatile

Organic Soil Vapors .............

4581 Development of Vadose Zone

Monitoring Instruments ..........

4863 Intelligent Sensor Data Acquisition

Analysis .................... 


\section{Project}

Number

Title
Project

Number
Title
Page

4864 Modeling Underground Structures ....

4865 Neural Analysis Techniques for Field

Deployable Environment Sensors .....

5203 Grouping and Modeling Ecosystem

Components for Ecological Risk

Assessment

5205 Water Shutoff in Producing Oil Wells

Using Bacteria ................

5301 Estimating Sorption Properties of Clay Materials ...................

5303 Identification of a Surfactant/Alcohol/ Polymer System for Safe Mobilization of TCE ....................

5601 Aquatic Vascular Plant Control .......

5608 Bioprocessing of Indigo Textile Dye

Waste Effluent ...............

5614 Removal of Methanol from Forest

Products Industry Off-gases by

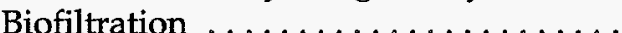

6503 Using Nutrient-Diffusing Substrata to Conduct Rational Bioremediation .....

6701 Intermediate/Field Scale Model for Predicting Microbial Transport .......

7907 Prompt Gamma Neutron Activation Analysis Methods Development for Waste Assay Applications ..........

8104 Real-Time Asbestos Analysis by Raman Spectroscopy ............... 58

8106 Rapid Agrochemical Sensor ..........

8403 In Situ Biodegradation of High-Energy Materials ...................

8409 Rapid Laser Assay of Surface

Contamination During Decontamination and Decommissioning

8414 Measuring Component Ratio in Oil/Water/Gas Mixtures and Determining Flow Regime...$\ldots \ldots \ldots$

8509 Polyethylene Sulfur Cement: Feasibility as a Waste Encapsulation Material for Low-Level and Mixed Wastes .....

8514 In-Field Assay for Parts-per-Million Levels of Chromium in Environmental Media ....................

8615 Low-Temperature Reductive Catalytic Destruction of Halogenated Organics ..

9510 Periphyton Communities for Environmental Monitoring...$\ldots \ldots \ldots \ldots$

9513 Tolo Lake Mammoth Excavation ......

W007 Alternate Material Stainless Steel Lined Concrete ...................

W016 Oxidative Processes for the Remediation of Contaminated Soils ..........

W019 Solid Phase Microextraction ..........

W027 Synthesis and Characterization of $\mathrm{Pt} / \mathrm{Pd}$ Compounds for a Fiber Optic Based Sensor for VOCs ............

W029 ICPP Testbed Framework and Field Tests .....................

W032 Electrocatalytic Vapor Sensor .......

W043 Characterization of Fuel Processing/ Waste Form Chemistry by High-Temperature Mass Spectrometry ........

W089 Telepresence for Mobile Robotics in Nuclear Environments ...........

W097 Sodium-Bearing Waste Treatment Technology for Separating the Nitrate Anion and Alkali Salts Using Ion-Exchange ..

W104 The Effect of Thermal Treatment of INEL Soil Prior to Cementitious Solidification ....................

64

65

66




\section{Project}

Number

Title
Project

Page

Number

Title

Page

7909 Tritium Production by Irradiation

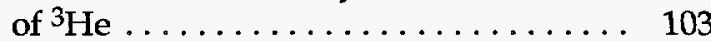

Environmental Samples for Remote

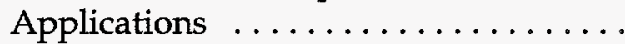

W111 Position Tracking for Remote Subsurface Characterization Systems .......

W122 Evaluation of Delphi Oxidation for Activated Carbon ...............

W123 Evaluation of EET Proprietary

Decontamination ...............

Nuclear Operations and Nuclear Materials

1102 ATR Radioisotope Production and

Purification Study ..............

1171 Neutron Delivery System for

Fast-Neutron Radiotherapy ..........

1173 Real-time Measurement Dosimetry for Boron Neutron Capture Therapy .... 91

2108 Mixed Oxide Fuels Testing in the

Advanced Test Reactor............

2111 General Purpose Time-Dependent

Particle Transport with Isotope

Generation and Depletion ..........

2205 Development of a Simplified Version of SCDAP/RELAP5 for Simulator Applications .............. 94

2207 Nonfertile Fuel Fabrication Development and Irradiation Performance

Assessment ................ 96

2302 Photoneutron Studies for Radiotherapy .. 97

2303 Feasibility Study of Using INEL-

Developed Transport Tools to Calculate Photodynamic Dosimetry ........ 98

2304 Kinetic Modeling of the ITER

Dissipative Divertor ............ 99

5304
9517. Technetium Separation System ...... 105

W03 On-Site F-18 Production . . . . . . . . 105

W036 WERC University Design Competition .. 107

National Infrastructure

Technology Test/Evaluation

5610 Microstructural Influences on

Biocorrosion ............... 111

7603 Advanced Combined Environments

Test Station Scoping Studies . . . . . . . 112

8501 Characterization of Aged Materials with NDE and Internal Friction Measurements .................. 114

\section{National Derived Use Technology Transfer Center}

230 Nondestructive Examination of BottomCrimped Seam on 55-gallon Drums ... 119

$231 \quad$ High Transsition Temperature Squid (Superconducting Quantum Interference Devices) Nondestructive Evaluation ................

232 Robust Nondestructive Evaluatoin Using Photorefractive Nonlinear Optics ....................

248 Polymers-Water Separations, Ozone/Oxygen Separations, Platform, and Laser-Assisted Polymerization .... .

251 Iron Aluminide Filters for Improved Efficiency Fossil Energy Conversion ...

252 Wear Coatings for Aluminum

Automobile Engine Components ..... 125

253 Factors That Determine the Microstructure of Spray-Deposited Materials .... 126 
Project

Number

Title

Page

305 Microbiology of Sugar Plant Flume

Systems ................

1206 Alkylation Catalysts for Operation at

Supercritical Fluid Conditions .......

1241 Mixed Microbial Encapsulated

Fertilizer Amendments ...........

1301 Sensors for the Control of Thermal

Spray Processes ...............

1302 Heat Transfer Through Solid Cryogen . .

1303 Concentration Technologies .........

2278 Site-Specific Technologies for

Agriculture: Pilot Project . . . . . . . . .

2501 Development of Nondestructive

Fatigue Monitor ............

3302 Advanced Barcode (Holotag) Reader . .

3601 Transportation Network Core Software . .

3711 Optical Sensor Development for High-

Voltage Metering and Protection

Applications ................

4061 Development of Chemically Selective Surfaces for Adsorption and Detection of Gas-Phase Contaminants . . . . . . . .

Alloys by Design

4076 Improved Process Control

Thermocouples

4080

National Infrastructure Renewal ......

144

4081

Optical Measurement Technology ....

146

4082 Velocity Sensor for Control of Thermal Spray .................

4103 Hybrid Electric Vehicle Evaluation ....

4105 Demand Side Management Control ... System for Electric Vehicles

\section{Project}

Number

Title

Page

4151 Development of Intelligent Cooperative

Miniature Robot Systems ..........

4157 Natural Gas Locomotives and

Transportable Power ............

4184 Loom Feedback Controls . . . . . . . . .

4203 Highly Dynamic Materials Process

Modeling Using Particle Methods . . . . .

4205 Advanced Plasma Processes for Black

Liquor Gasification ..............

4206 Joining and Testing of Small-Scale

Automotive Components

4208 Process Control Development for Ultracapacitor Electrodes by Liquid

Injection Plasma Deposition .......

4395 Miniature Alternative Power Supply,

Solid Oxide Fuel Cell . . . . . . . . . . .

4396 Miniature Alternative Power Supply

Thermo-photovoltaic ............

4468 Optimization-Based SIMPLEV

164

4469 Hybrid Vehicle Component Hardwarein-the-Loop Feasibility Study . . . . . . . .

Software Metrics Program

4479

Manufacturing Research

168

4480

Building Materials

170

4481 Smart HVAC Systems Design

4482 High-Temperature Porous Cerment

Filters for the Paper and Pulp Industry . .

4483 Silent Discharge Plasma for Treatment of Automobile Exhaust . . . . . . . . . . .

4485 Evaluation of Liquid Metal Technologies Applicable to Radioactively Contaminated Scrap Metal Recycling ............ 


\section{Project}

Number
Title

\section{Project}

Page

Number
Title

Page
4486

INEL Metal Recycle: Induction Melting . .

177

4487 Process Industries-Industrial

Improvement Using Integrated Process

Evaluation ...............

4488 Depleted Uranium Flywheels for

Stationary Applications ..........

4758 Simulation-based Prototyping for

Manufacturing-based Engineering ....

4759

Readiness ................

4860

Butterfat Management

4862 Advanced Process Diagnostics System . .

4866 Miniature Tag and Covert

Communicator

4952

INEL Multimedia Object Database ....

4953 General User Interface Development

Environment ...............

4954 Intelligent Data Access and Retrieval

System ..................

4955

$C++$ SAGE $\ldots \ldots \ldots \ldots \ldots \ldots$

4956 Fractional Frame Augmentation .....

5201 Development of Prompt Gamma Assay Method for Mining of Phosphate Ore . .

5204 Develop Intelligent Farm Management System Database Prototype ..........

5306 Measurement of Potato Water Content . .

5308 Numerical Investigation of Dynamic Pressure Seals in Geologically Active Basins ..................

5604 Evaluation of Potential Antimicrobial Properties of Malted Barley and Its Agricultural Applications
5606 Biological System for Organic Acid Removal with Minimal Effluent ..... 198

5609 Isolation of Gossypol Degrading Bacteria ...................

5615 System for Growing Conifer Embryos . . 201

6111 Improved Transformation and

Regeneration Techniques for Cotton ...

6808 Compressed Gas Safety Valve ... . . . .

7105 Internal-Reforming Solid Oxide Fuel Cell Modeling ................

7203 Advanced Methods for Adaptive Control for Automated Traffic Management ................

7204 System Dynamics Model for Intermodalism . . . . . . . . . . . . .

8415 Alternate Joining Techniques for Textiles ...................

8416 Real-Time, On-Loom Knit and Hand Characterization Using Diffractive Optical Methods ...............

8506 Micromechanical Analysis Capability for Composite Materials ............

8507 Metal Matrix Composites from Novel Nitride Preforms .............

8510 Cooperative Industrial Research with General Electric Lighting Products ....

8511 Lifetime Extension and Increased Reliability for Refinery Structures .....

8515 Solar Bimodal Technologies

Demonstration ...............

8516 Weight in Motion Sensor and Signal Analysis Technology ............

8517 Communications Spectrum Needs for Short-Range Intelligent Vehicle Highway Systems Communications ...... 
Project

Number

Page

8518 Developing a Nonlinear Electromagnetic Modeling Capability Using the Finite-

Difference Time Domain Method .....

8614 Electrochemical-based Processing

Technologies

8616 Nonaqueous Processing in the Textile Industry $\ldots \ldots \ldots \ldots \ldots \ldots \ldots$

8708 Off-Line Manufacturing Simulation ...

8712 Spray Forming Titanium Alloys into Billet and Sheet

8806 Industrially Compatible Laser

Ultrasonics ..................

9508 Optimization of Automobile Component Shapes ............. 227

9511 Mediator

9512 Gas-Driven Photovoltaic Generators . . . 229

9514 Measurement of Crude Oil Corrosivity .. 230

9515 Installation of Precession X-Ray

Camera for Single Crystal Analysis ...

\section{Project}

Number

Title

Page

9516 Direct Production of Low-Cost High-

Quality Titanium Powder by

Mechanical Alloying

W091 Petroleum Tanks Inspection Delivery

System ....................

\section{Long-Term Research Initiatives}

$10 \quad$ Molecular Biology . . . . . . . . . . . . 237

$20 \quad$ Nuclear Physics . . . . . . . . . . . . . 239

$30 \quad$ Condensed Matter $\ldots \ldots \ldots \ldots \ldots, 242$

40 Theoretical Chemistry $\ldots \ldots \ldots \ldots, 247$

$50 \quad$ Computational Mechanics ......... 249

60 Nonconventional Bioprocessing ..... 252

$70 \quad$ Radiation Measurements ......... 254

$80 \quad$ Nuclear Fuels and Materials $\ldots \ldots \ldots .256$

$90 \quad$ Experimental Thermal Science ....... 259 


\section{Appendix C \\ Project Performance Measures}




\begin{tabular}{|c|c|c|c|c|c|c|c|c|c|c|c|c|c|}
\hline & & \multicolumn{12}{|c|}{ Numbers } \\
\hline Project & Title & 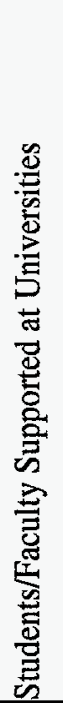 & 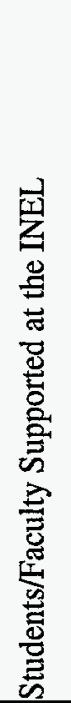 & 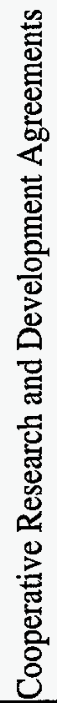 & 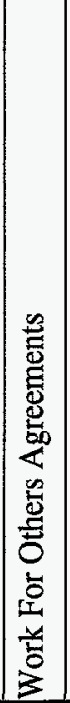 & 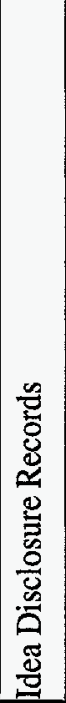 & 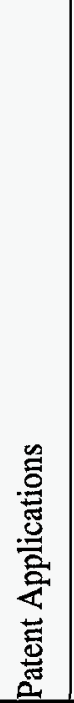 & 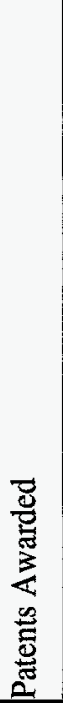 & 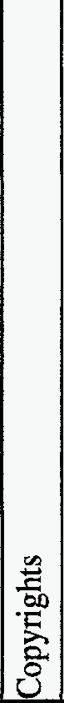 & 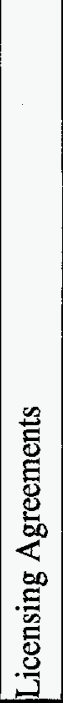 & 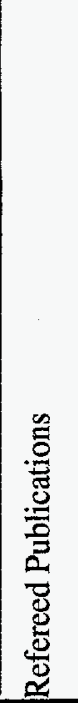 & 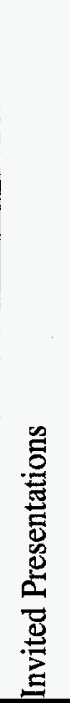 & 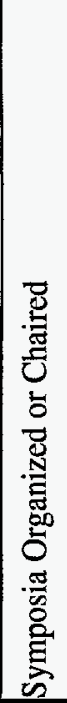 \\
\hline 0010 & Molecular Genetics & $\frac{1}{1}$ & & & & & & & & & 5 & & \\
\hline 0020 & Nuclear Physics & & 3 & & & & & & & & 36 & 4 & \\
\hline 0030 & Condensed Matter & 1 & 1 & 2 & & & 1 & & & & 6 & 3 & \\
\hline 0040 & Theoretical Chemistry & 1 & & & & & & & & & 9 & 5 & 1 \\
\hline 0050 & Computational Mechanics & 1 & 1 & & & & & & & & 11 & 10 & 3 \\
\hline 0060 & Nonconventional Bioprocessing & 3 & 5 & & & & 1 & & & & 6 & 6 & \\
\hline 0070 & Radiation Measurements & 2 & 3 & 1 & & 1 & & & 1 & & 1 & & \\
\hline 0080 & Nuclear Fuels and Materials & 2 & 3 & & & & & & & & & & \\
\hline 0090 & Experimental Thermal Science & 5 & 1 & & & & & & & & 6 & 12 & 2 \\
\hline 0102 & $\begin{array}{l}\text { Assessment Methodology for Enhanced } \\
\text { Bioremediation of Hydrocarbons in the Petroleum } \\
\text { Industry }\end{array}$ & 2 & 1 & & & & & & & & 3 & & \\
\hline 0230 & $\begin{array}{l}\text { Nondestructive Examination of Bottom Crimped } \\
\text { Seam on } 55 \text { Gallon Drums }\end{array}$ & & & & & & & & & & & & \\
\hline 0231 & High Tc Squid-Sensor NDE & & 1 & & & & & & & & & & \\
\hline 0232 & Robust NDE using photorefractive nonlinear optics & & 2 & & & 1 & & & & & & & \\
\hline 0242 & $\begin{array}{l}\text { Catalyst Assisted Sonochemical Treatment of } \\
\text { Hazardous Organic Wastes }\end{array}$ & & & & & & & & & & & & \\
\hline 0247 & $\begin{array}{l}\text { Synthesis, Characterization, and the Modes of } \\
\text { Interaction in VOC Sensor Materials }\end{array}$ & & & & & & & & & & & & \\
\hline 0248 & $\begin{array}{l}\text { Polymers - Water Separations, Ozone/Oxygen } \\
\text { Separations, Platform, and Laser Assisted } \\
\text { Polymerization }\end{array}$ & & 0.5 & 1 & & 1 & 1 & & & & & & \\
\hline 0251 & $\begin{array}{l}\text { Iron Aluminide Filters for Improved Efficiency } \\
\text { Fossil Energy Conversion }\end{array}$ & & & & & & 1 & 1 & & & & & \\
\hline 0252 & $\begin{array}{l}\text { Wear Coatings for Aluminum Automobile Engine } \\
\text { Components }\end{array}$ & & & & & & & & & & & & \\
\hline 0253 & $\begin{array}{l}\text { Factors Which Determine the Microstructure of } \\
\text { Spray Deposited Materials }\end{array}$ & 1 & 1 & & & & & & & & & & \\
\hline 0262 & $\begin{array}{l}\text { Integration of sample introduction techniques, torch } \\
\text { design and optics for the determination of trace } \\
\text { metals in biological samples with ICP-AES }\end{array}$ & & & & & & & & & & & & \\
\hline 0303 & $\begin{array}{l}\text { Removal of Chlorinated Solvents from Air Streams } \\
\text { using Anaerobic Biofiltration }\end{array}$ & 1 & & & & & & & & & & & \\
\hline 0305 & Microbiology of Sugar Plant Flume Systems & & & 1 & & & & & & & & & \\
\hline 0306 & $\begin{array}{l}\text { Utilization of Sawdust for Microbial Abatement of } \\
\text { Acid Mine Drainage }\end{array}$ & & 1 & & & & & & & & & & \\
\hline 1102 & ATR Radioisotope Production and Purification Study & & & & & & & & & & & & \\
\hline 1171 & Neutron Delivery System Study & & & & & & & & & & & & \\
\hline 1172 & High Energy Computational Dosimetry & & & & & & & & & & & & \\
\hline
\end{tabular}




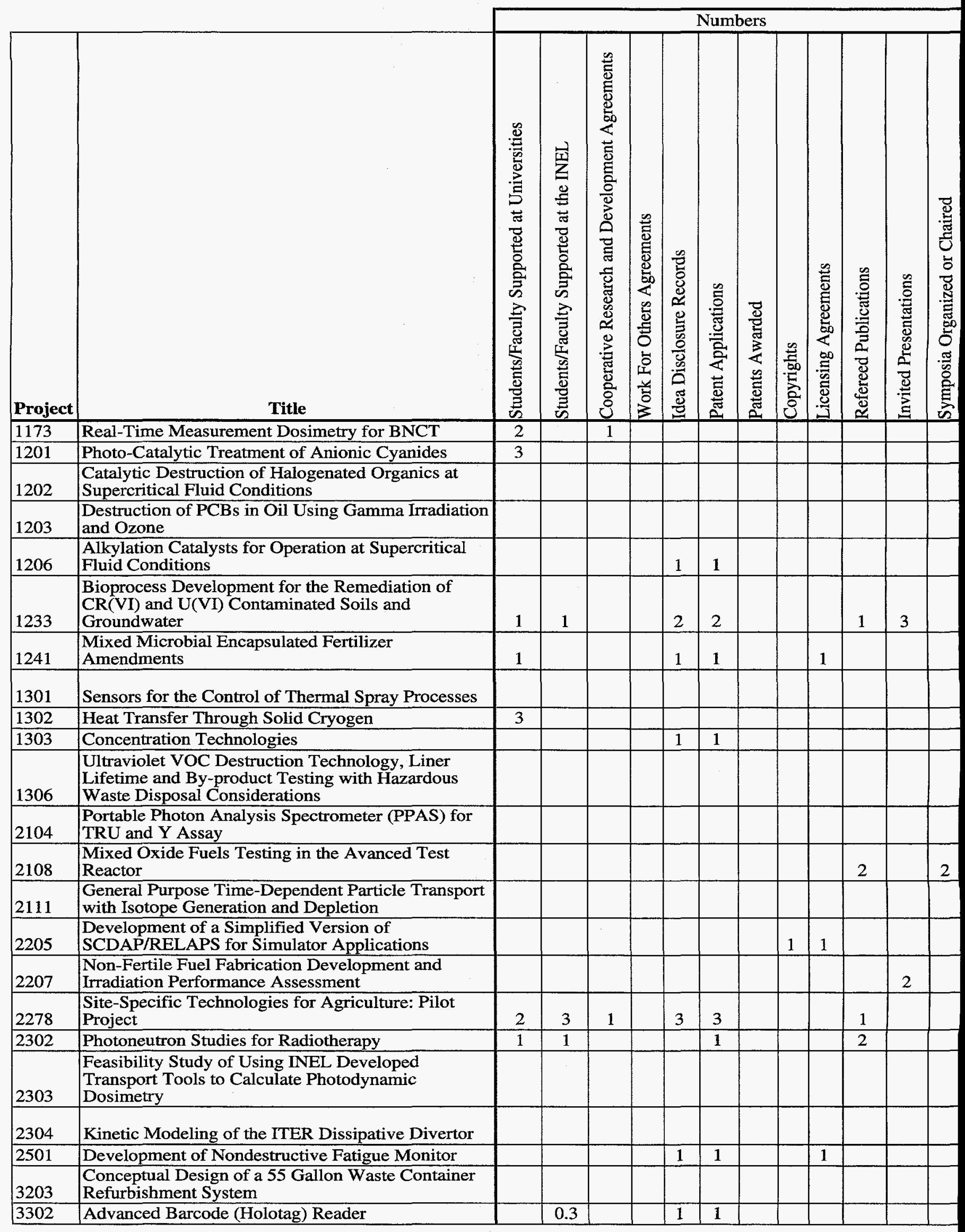




\begin{tabular}{|c|c|c|c|c|c|c|c|c|c|c|c|c|c|}
\hline & & & & & & & Tumb & & & & & & \\
\hline Project & \begin{tabular}{|c|} 
Title \\
\end{tabular} & 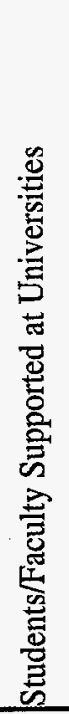 & 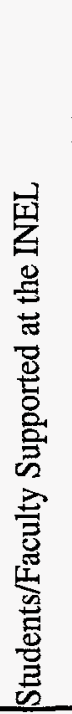 & 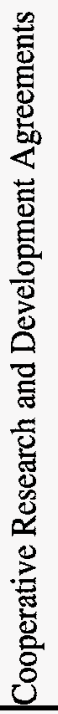 & 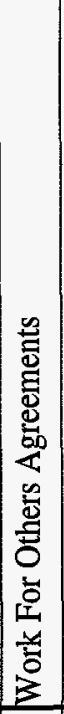 & 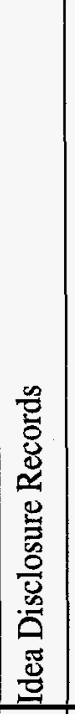 & 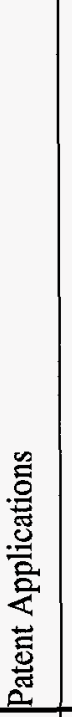 & 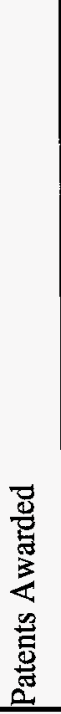 & 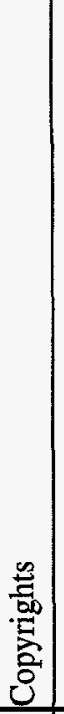 & 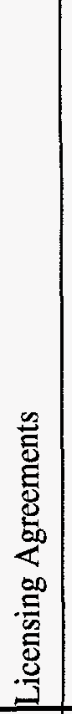 & 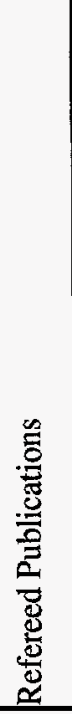 & 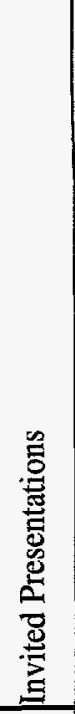 & 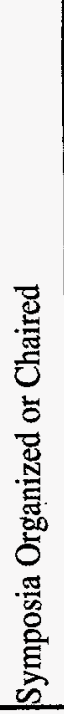 \\
\hline \begin{tabular}{l|l}
3601 & 7 \\
\end{tabular} & Transportation Network Core Software & & & & & & & & & & & & \\
\hline 3710 & $\begin{array}{l}\text { Photodissociation of Mercuric Chloride Treatability } \\
\text { Study }\end{array}$ & 2 & 2 & & & & & & & & & & \\
\hline 3711 & $\begin{array}{l}\text { Optical Sensor Development for High Voltage } \\
\text { Metering and Protection Applications }\end{array}$ & 1 & 1 & & & 1 & 1 & & & 1 & & & \\
\hline 4061 & $\begin{array}{l}\text { Development of Chemically Selective Surfaces for } \\
\text { Adsorption and Detection of Gas-Phase } \\
\text { Contaminants }\end{array}$ & & & & & & & & & & 3 & 2 & \\
\hline 4073 & \begin{tabular}{|l|} 
Alloys by Design \\
\end{tabular} & & & & & & & & & & & & \\
\hline 4076 & Improved Process Control Thermocouples & & & & & & & & & & & & \\
\hline 4078 & $\begin{array}{l}\text { INEL-U of I Laboratory for Liquid Extraction and } \\
\text { Ion Exchange Research }\end{array}$ & 3 & & & & & & & & & 1 & & \\
\hline 4080 & National Infrastructure Renewal & 4 & & 1 & & & & & & & & & \\
\hline 4081 & Optical Measurement Technology & & 0.3 & & & 1 & 1 & & & & & & \\
\hline 4082 & Velocity Sensor for Control of Thermal Spray & & & & & 1 & 1 & & & & 2 & & \\
\hline 4090 & $\begin{array}{l}\text { Fracture Mechanics-Based Design Methodologies for } \\
\text { Metal Matrix Composite Material }\end{array}$ & & & 2 & & & & & & & & & \\
\hline 4103 & \begin{tabular}{|l|} 
Hybrid Electric Vehicle Evaluation \\
\end{tabular} & & & & & & & & & & & & \\
\hline 4105 & $\begin{array}{l}\text { Demand Side Management Control System for } \\
\text { Electric Vehicles }\end{array}$ & & & & & & & & & & & & \\
\hline 4110 & $\begin{array}{l}\text { Develop, Build, and Test Assay System to Measure } \\
\text { TRU and Fission Product Contamination in } \\
\text { Subsurface Soil }\end{array}$ & 1 & & & & 2 & 2 & & & 1 & 2 & & \\
\hline 4151 & $\begin{array}{l}\text { Development of Intelligent Cooperative Miniature } \\
\text { Robot Systems }\end{array}$ & 5 & & & & & & & & & & & \\
\hline 4157 & Natural Gas Locomotives and Transportable Power & & & & & & & & & & & & \\
\hline 4171 & Biodecontamination of Massive Concrete Structures & & & & & & & & & & & & \\
\hline 4172 & Catalyst Assited in-Situ Bioremediation & 1 & & 1 & & & & & & & & & \\
\hline 4184 & Loom Feedback Controls & & & & & 1 & 1 & & & & & & \\
\hline 4203 & $\begin{array}{l}\text { Highly Dynamic Materials Process Modeling Using } \\
\text { Particle Methods }\end{array}$ & 1 & & & & & & & & & & & \\
\hline 4204 & Sustainable Water Resources Management & & & & & & & & & & & & \\
\hline 4205 & $\begin{array}{l}\text { Advanced Plasma Processes for Black Liquor } \\
\text { Gasification }\end{array}$ & & & & & 1 & 1 & & & & 1 & & 1 \\
\hline 4206 & $\begin{array}{l}\text { Joining and Testing of Small Scale Automotive } \\
\text { Components }\end{array}$ & & & 1 & & 1 & 1 & & & & & & \\
\hline 4208 & $\begin{array}{l}\text { Process Control Development for Ultracapacitor } \\
\text { electrodes by Liquid Injection Plasma Deposition } \\
\text { (LIPD) }\end{array}$ & & 1 & & & & & & & & & & \\
\hline
\end{tabular}




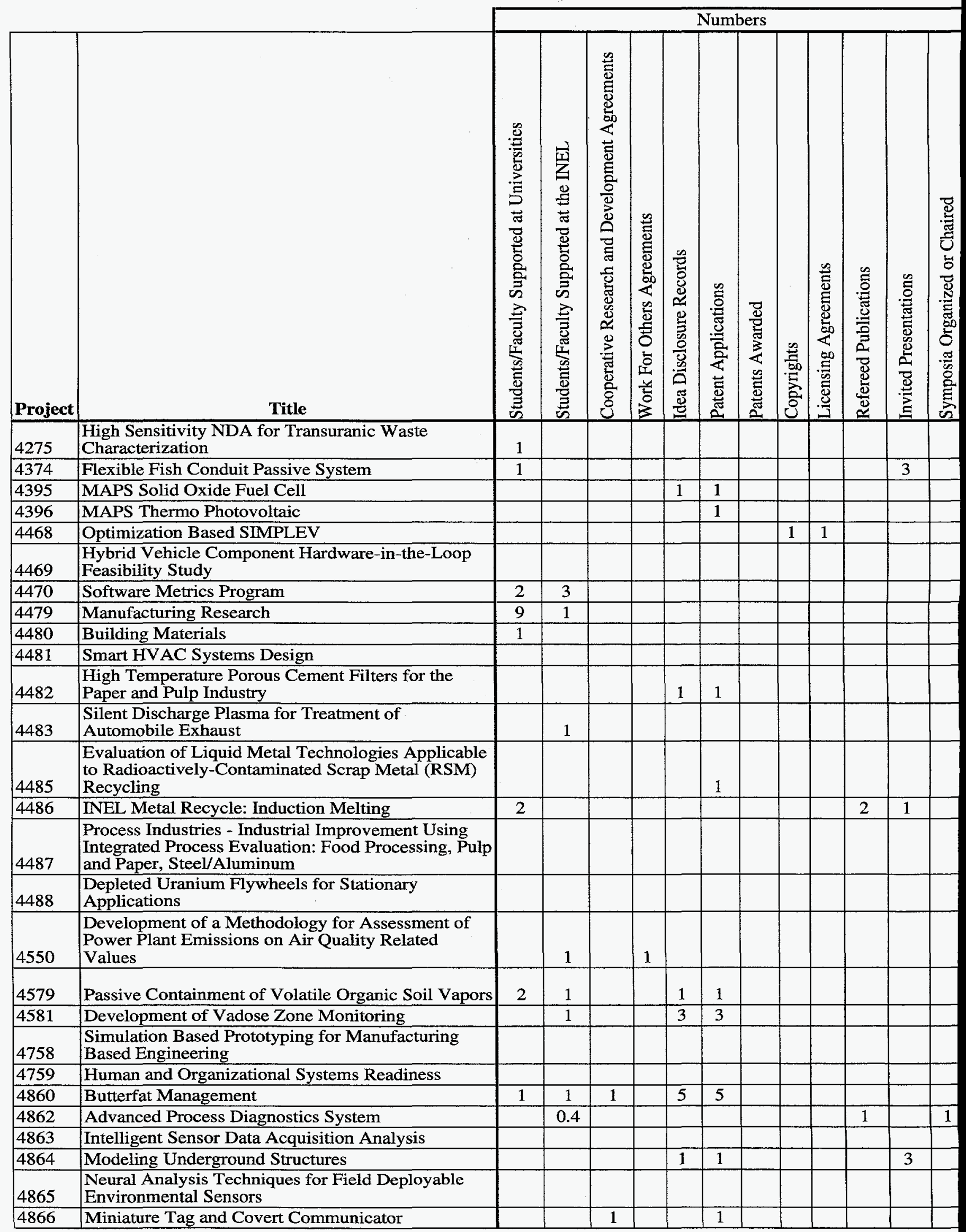




\begin{tabular}{|c|c|c|c|c|c|c|c|c|c|c|c|c|c|}
\hline & & \multicolumn{12}{|c|}{ Numbers } \\
\hline Project & Title & 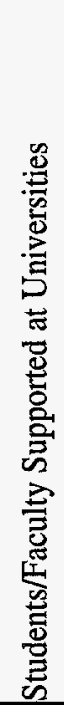 & 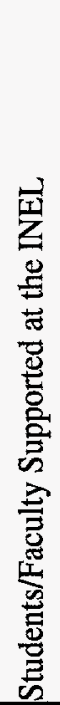 & 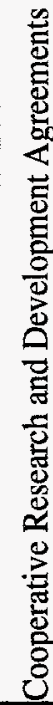 & 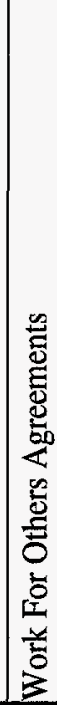 & 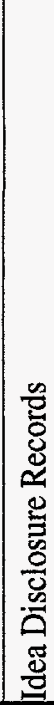 & 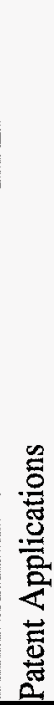 & 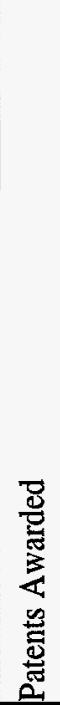 & 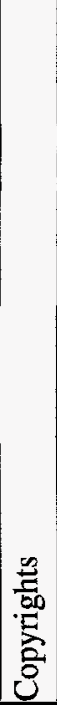 & 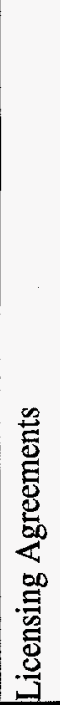 & 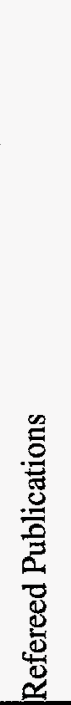 & 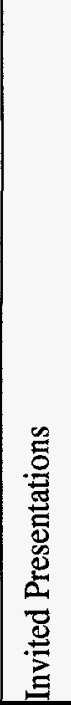 & 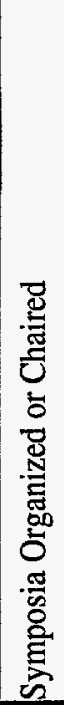 \\
\hline 4952 & INEL Multimedia Object Database & & & & & & & & & & & & \\
\hline 4953 & General User Interface Development Environment & & 2 & & & & & & & & & & \\
\hline 4954 & Intelligent Data Access and Retrieval System & & & & & & 1 & & & & & & \\
\hline 4955 & $\mathrm{C}++\mathrm{SAGE}$ & & 1 & & & & & & & & & & \\
\hline 4956 & Fractional Frame Augmentation & & & & & & & & & & & & \\
\hline 5201 & $\begin{array}{l}\text { Development of Prompt Gamma Assay Method for } \\
\text { Mining of Phosphate Ore }\end{array}$ & & & & & 2 & 2 & & & 1 & & & \\
\hline 5203 & $\begin{array}{l}\text { Grouping and Modeling Ecosystem Components for } \\
\text { Ecological Risk Assessment }\end{array}$ & & & & & & & & & & & 2 & \\
\hline 5204 & $\begin{array}{l}\text { Develop Intelligent Farm Management System } \\
\text { Database Prototype }\end{array}$ & & & & & 1 & 1 & & & & & & \\
\hline 5205 & $\begin{array}{l}\text { Water Shutoff in Producing Oil Wells Utilizing } \\
\text { Bacteria }\end{array}$ & & & & & 1 & 1 & & & & & & \\
\hline 5301 & Estimating Sorption Properties of Clay Materials & & & & & & & & & & & & \\
\hline 5303 & $\begin{array}{l}\text { Identification of a Surfactant/Alcohol/Polymer } \\
\text { System for Safe Mobilization of TCE }\end{array}$ & 2 & & & & & & & & & & & \\
\hline 5304 & Automated Neutron Probe Monitoring & & & & & 1 & 1 & & & & & & \\
\hline 5306 & Measurement of Potato Water Content & & & & & 1 & 1 & & & & & & \\
\hline 5308 & $\begin{array}{l}\text { Numerical Investigation of Dynamic Pressure Seals } \\
\text { in Geologically Active Basins }\end{array}$ & & & & & & & & & & & & \\
\hline 5601 & Aquatic Vascular Plant Control & & & 1 & & & & & & 1 & & & \\
\hline 5604 & $\begin{array}{l}\text { Evaluation of Potential Antimicrobial Properties of } \\
\text { Malted Barley and its Agricultural Applications }\end{array}$ & 2 & & & & & & & & & & & \\
\hline 5606 & $\begin{array}{l}\text { Biological System for Organic Acid Removal with } \\
\text { Minimal Effluent }\end{array}$ & & & & & 1 & 1 & & & & & & \\
\hline 5608 & Bioprocessing of Indigo Textile Dye Waste Effluent & & & & & 1 & 1 & & & & & & \\
\hline 5609 & Isolation of Gossypol Degrading Bacteria & & & & & & & & & & & & \\
\hline 5610 & Microstructural Influences on Biocorrosion & & & & & & & & & & & & \\
\hline 5614 & $\begin{array}{l}\text { Removal of Methanol from Forest Products Industry } \\
\text { Off-gases by Biofiltration }\end{array}$ & & & & 1 & & & & & & & & \\
\hline 5615 & System for Growing Conifer Embryos & & & & & 7 & 7 & & & & & & \\
\hline 6111 & $\begin{array}{l}\text { Improved Transformation and Regeneration } \\
\text { Techniques for Cotton }\end{array}$ & 3 & 2 & & & & & & & & & & \\
\hline 6503 & $\begin{array}{l}\text { Nutrient Diffusing Substrata to Conduct Rational } \\
\text { Bioremediation }\end{array}$ & 1 & & & & 1 & 1 & & & & & 4 & \\
\hline 6701 & $\begin{array}{l}\text { An Intermediate to Field Scale Model for Predicting } \\
\text { Porous Media Microbial Transport }\end{array}$ & & & & & & & & & & & & \\
\hline 6808 & Compressed Gas Safety Valve & & & & & & & & & & & & \\
\hline 7105 & Internal-Reforming SOFC Modeling & 1 & & & & & & & 1 & & 1 & 2 & \\
\hline
\end{tabular}




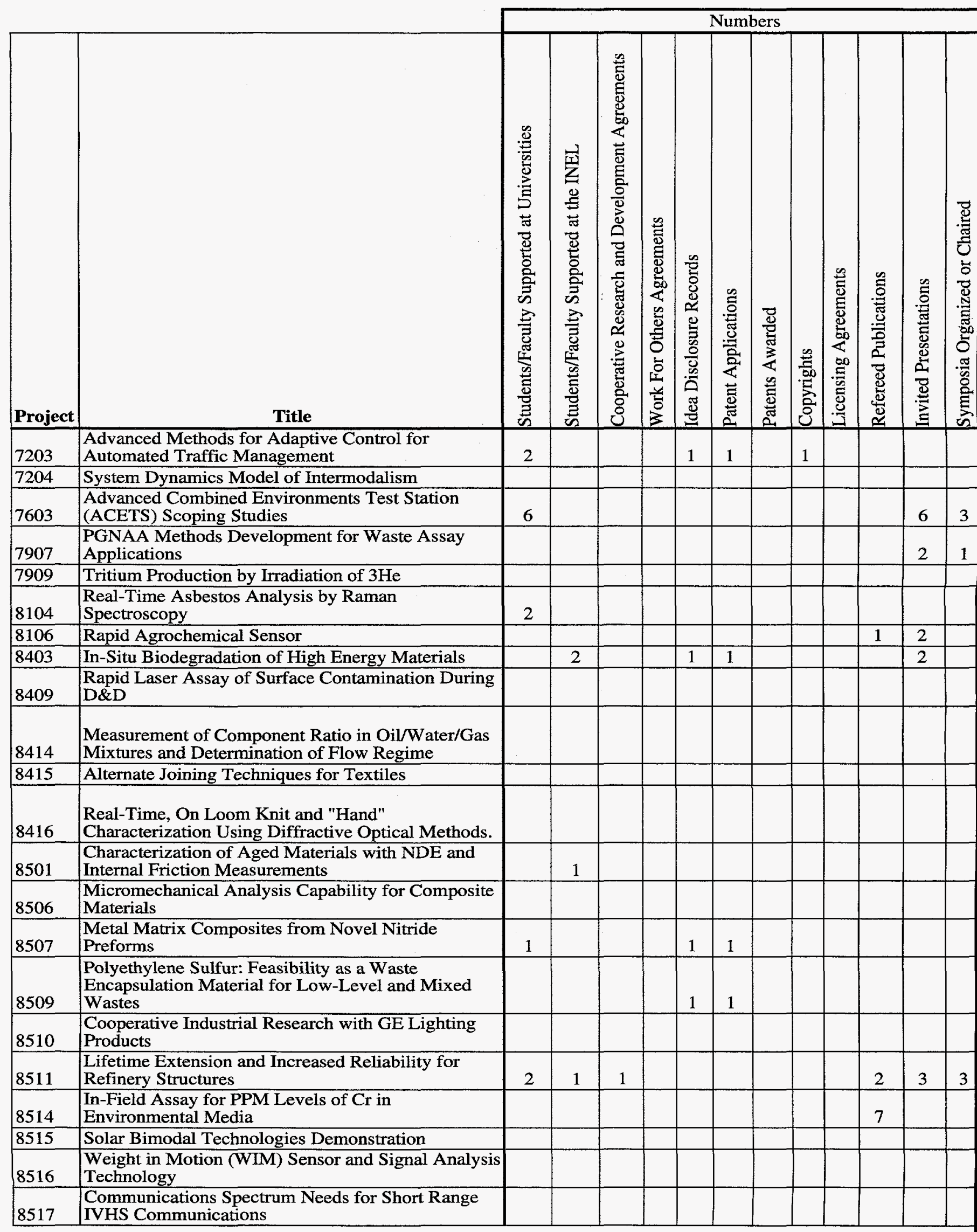




\begin{tabular}{|c|c|c|c|c|c|c|c|c|c|c|c|c|c|}
\hline \multirow[b]{2}{*}{ Project } & & \multicolumn{12}{|c|}{ Numbers } \\
\hline & Title & 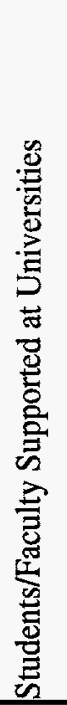 & 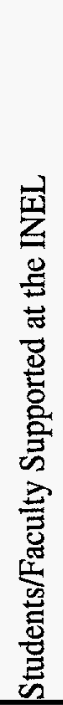 & 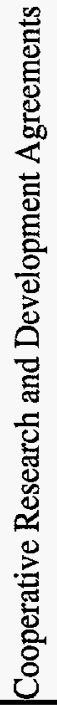 & 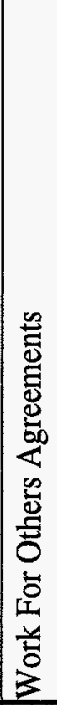 & 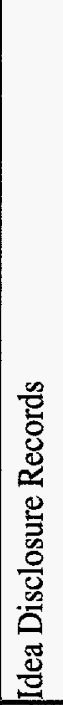 & 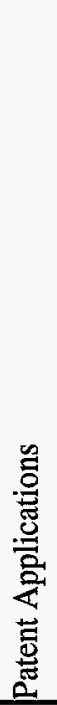 & 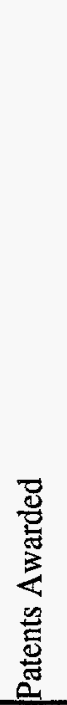 & $\begin{array}{l}0 \\
\text { 总 } \\
\text { 帘 } \\
0\end{array}$ & 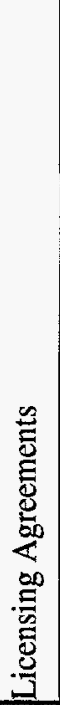 & 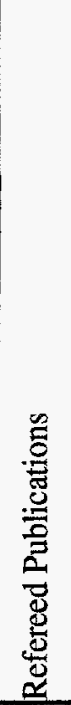 & 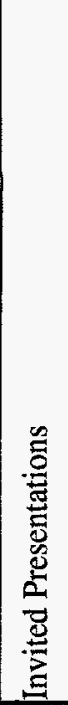 & 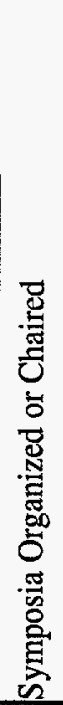 \\
\hline 8518 & $\begin{array}{l}\text { Developing a Nonlinear Electromagnetic Modeling } \\
\text { Capability using the Finite-Difference Time Domain } \\
\text { Method }\end{array}$ & 1 & & & & & & & & & 1 & & \\
\hline 8614 & Electro Chemical-Based Processing Technologies & & & & & 2 & 2 & 1 & & & & 1 & \\
\hline 8615 & $\begin{array}{l}\text { Low temperature Reductive Catalytic Destruction of } \\
\text { Halogenated Organics }\end{array}$ & & & & & 1 & 1 & & & & & & \\
\hline 8616 & Nonaqueous Processing in the Textile Industry & & & & & & & & & & & & \\
\hline 8708 & Off-Line Manufacturing Simulation & & & & & & & & & & 2 & & \\
\hline 8712 & Spray Forming Titanium Alloys into Billet and Sheet & & & & & & & & & & & & \\
\hline 8806 & Industrially Compatible Laser Ultrasonics & & & 1 & & 1 & 1 & & & & & & \\
\hline 9508 & Optimization of Automobile Component Shapes & & & & & & & & & & & 4 & 7 \\
\hline 9510 & $\begin{array}{l}\text { Periphyton Communities for Environmental } \\
\text { Monitoring }\end{array}$ & 1 & 1 & & & & & & & & & & \\
\hline 9511 & Mediator & 1 & & & & & & & & & & & \\
\hline 9512 & Gas Driven Photovoltaic Generators & & & & & & & & & & & & \\
\hline 9513 & Tolo Lake Mammoth Excavation & 4 & & & & & & & & & & & \\
\hline 9514 & Measurement of Crude Oil Corrosivity & & & & & & & & & & & & \\
\hline 9515 & $\begin{array}{l}\text { Installation of Precession X-Ray Camera for Single } \\
\text { Crystal Analysis }\end{array}$ & 1 & & & & & & & & & & & \\
\hline 9516 & $\begin{array}{l}\text { Direct Production of Low Cost High Quality } \\
\text { Titanium Powder by Mechanical Alloying }\end{array}$ & & & & & & & & & & & & \\
\hline 9517 & Technetium & 3 & & & & & & & & & & & \\
\hline W007 & Alternate Material for Stainless Steel Lined Concrete & 1 & 1 & & & & & & & & & & \\
\hline W016 & $\begin{array}{l}\text { Oxidative Processes for the Remediation of } \\
\text { Contaminated Soils }\end{array}$ & 2 & & & & & & & & & & & \\
\hline W019 & Solid Phase Microextraction & & & & & & & & & & & & \\
\hline W027 & $\begin{array}{l}\text { Synthesis and Characterization of Pt/Pd Compounds } \\
\text { for a Fiber Optic Based Sensor for VOCs }\end{array}$ & 1 & & 1 & & 1 & 1 & & & & 2 & & \\
\hline W029 & ICPP Testbed Framework and Field Tests & & & & & & & & & & & & \\
\hline W030 & On-Site F-18 Production & 1 & & & & 1 & 1 & & & & & & \\
\hline W032 & Electrocatalytic Vapor Sensor & & & & & & & & & & & & \\
\hline W036 & WERC University Design Competition & 10 & & & & & & & & & & & \\
\hline W043 & $\begin{array}{l}\text { Characterization of Fuel Processing/Waste Form } \\
\text { Chemistry by High Temperature Mass Spectrometry }\end{array}$ & & & & & & & & & & & & \\
\hline W089 & $\begin{array}{l}\text { Telepresence for Mobile Robotics in Nuclear } \\
\text { Environments }\end{array}$ & & 1 & & & 1 & 1 & & 1 & & & & \\
\hline W091 & Petroleum Tanks Inspection Delivery System & & & & & 1 & 1 & & & 1 & & & \\
\hline
\end{tabular}




\begin{tabular}{|c|c|c|c|c|c|c|c|c|c|c|c|c|c|}
\hline & & \multicolumn{12}{|c|}{ Numbers } \\
\hline Project & $\begin{array}{c}\text { Title } \\
\end{array}$ & 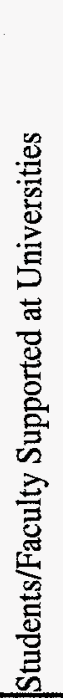 & 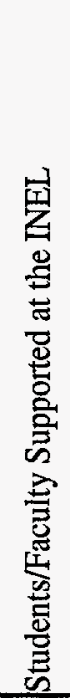 & 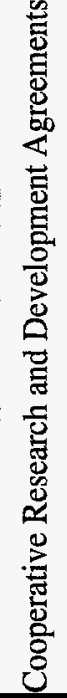 & 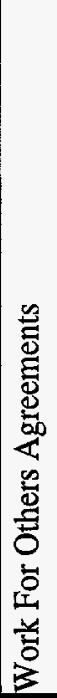 & 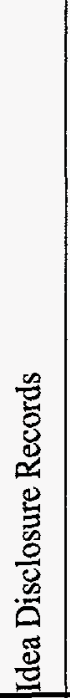 & 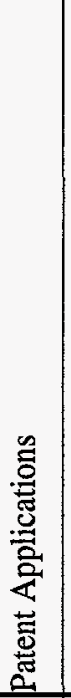 & 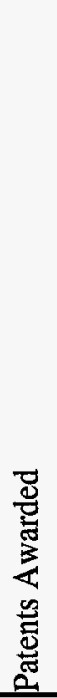 & 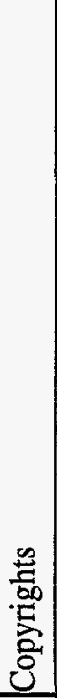 & 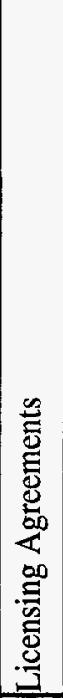 & 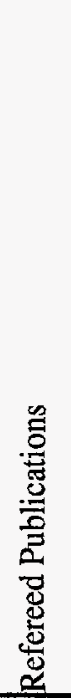 & 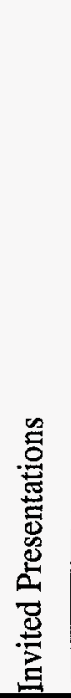 & 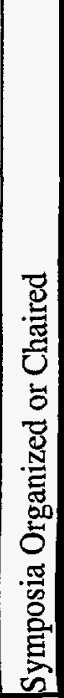 \\
\hline W097 & $\begin{array}{l}\text { Sodium-Bearing Waste Treatment Technology for } \\
\text { Separating the Nitrate Anion and Alkali Salts Using } \\
\text { Ion-Exchange }\end{array}$ & 2 & & & & & & & & & & & \\
\hline W104 & $\begin{array}{l}\text { The Effect of Thermal Treatment of INEL Soil Prior } \\
\text { to Cementitious Solidification }\end{array}$ & 1 & & & & & & & & & & & \\
\hline W109 & $\begin{array}{l}\text { Automatic Pressurized Filtering of Environmental } \\
\text { Samples for Remote Applications }\end{array}$ & & & & & & & & & & & & \\
\hline W111 & $\begin{array}{l}\text { Position Tracking for Remote Subsurface } \\
\text { Characterization Systems }\end{array}$ & & & & & & & & & & & & \\
\hline W122 & Evaluation of Delphi Oxidation for Activated Carbon & & & & & & & & & & & & \\
\hline W123 & Evaluation of EET Proprietary Decontamination & & & & & & & & & & & & \\
\hline & $\begin{array}{ll}\text { TOTALS } & \text { TOTA }\end{array}$ & & 54.5 & 18 & 2 & 61 & 67 & 2 & 6 & 9 & 117 & 82 & \\
\hline
\end{tabular}




\section{Appendix D \\ Relevance to Major National Programs}




\begin{tabular}{|c|c|c|c|c|c|c|c|c|c|c|c|c|c|c|}
\hline Project & Project Name & 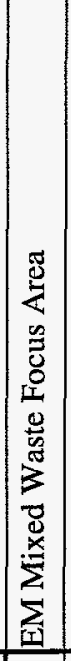 & 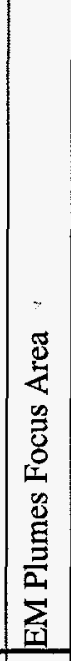 & 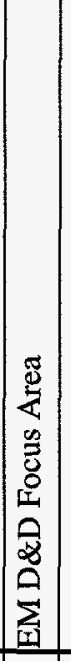 & 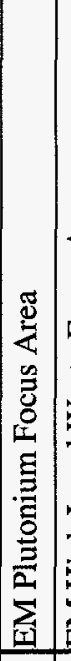 & 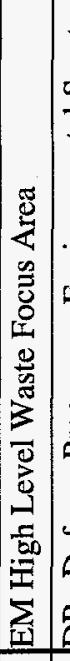 & 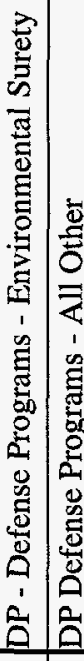 & 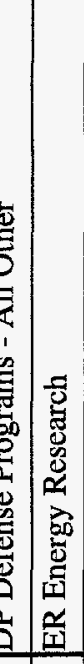 & 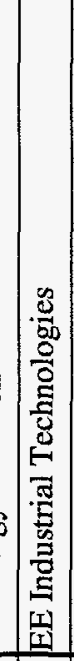 & 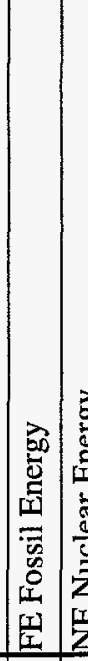 & 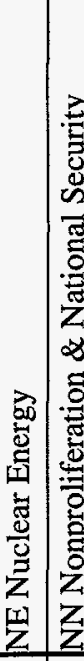 & 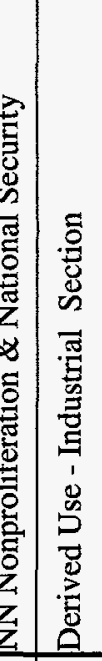 & 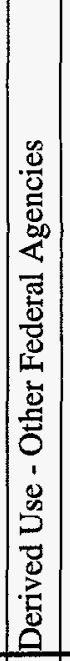 & 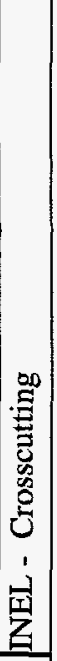 \\
\hline 0102 & $\begin{array}{l}\text { Assessment Methodology for Enhanced Bioremediation } \\
\text { of Hydrocarbons in the Petroleum Industry }\end{array}$ & & $\mathrm{x}$ & & & & & & & $X$ & & & & \\
\hline 0230 & $\begin{array}{l}\text { Nondestructive Examination of Bottom Crimped Seam } \\
\text { on } 55 \text { Gallon Drums }\end{array}$ & $\mathrm{X}$ & & & & & & & $\mathrm{X}$ & & & $\mathrm{X}$ & & \\
\hline 0231 & High Tc Squid-Sensor NDE & $\mathrm{x}$ & & & & & & $\mathrm{X}$ & $\mathrm{X}$ & & & $\mathrm{X}$ & & \\
\hline 0232 & Robust NDE using photorefractive nonlinear optics & $\mathrm{x}$ & & & & & & $\mathrm{x}$ & $\mathrm{x}$ & & & $\mathrm{X}$ & & \\
\hline 0242 & $\begin{array}{l}\text { Catalyst Assisted Sonochemical Treatment of Hazardous } \\
\text { Organic Wastes }\end{array}$ & $\mathrm{x}$ & & & & & & $\mathrm{X}$ & $\mathrm{X}$ & & & $\mathrm{x}$ & & \\
\hline 0247 & $\begin{array}{l}\text { Synthesis, Characterization, and the Modes of } \\
\text { Interaction in VOC Sensor Materials }\end{array}$ & & $\mathrm{X}$ & & & & & & & & & & & \\
\hline 0248 & $\begin{array}{l}\text { Polymers - Water Separations, Ozone/Oxygen } \\
\text { Separations, Platform, and Laser Assisted } \\
\text { Polymerization }\end{array}$ & & & & & & & & $\mathrm{X}$ & & & $\mathrm{X}$ & & \\
\hline 0251 & $\begin{array}{l}\text { Iron Aluminide Filters for Improved Efficiency Fossil } \\
\text { Energy Conversion }\end{array}$ & & & & & & & $\mathrm{X}$ & $\mathbf{x}$ & $\mathrm{X}$ & & $\mathrm{X}$ & & \\
\hline 0252 & $\begin{array}{l}\text { Wear Coatings for Aluminum Automobile Engine } \\
\text { Components }\end{array}$ & & & & & & & & $\mathrm{X}$ & & & $\mathbf{X}$ & & \\
\hline 0253 & $\begin{array}{l}\text { Factors Which Determine the Microstructure of Spray } \\
\text { Deposited Materials }\end{array}$ & & & & & & & $\mathrm{X}$ & $\mathrm{x}$ & & & $\mathrm{X}$ & & \\
\hline 0262 & $\begin{array}{l}\text { Integration of sample introduction techniques, torch } \\
\text { design and optics for the determination of trace metals in } \\
\text { biological samples with ICP-AES }\end{array}$ & $\mathrm{x}$ & & & & & & $\mathrm{X}$ & & & & & & \\
\hline 0303 & \begin{tabular}{|l|} 
Removal of Chlorinated Solvents from Air Streams \\
using Anaerobic Biofiltration
\end{tabular} & & $\mathrm{X}$ & & & & & & & & & & & \\
\hline 0305 & Microbiology of Sugar Plant Flume Systems & & & & & & & & & & & $\mathrm{X}$ & & \\
\hline 0306 & $\begin{array}{l}\text { Utilization of Sawdust for Microbial Abatement of Acid } \\
\text { Mine Drainage }\end{array}$ & & & & & & & & & & & $\mathrm{X}$ & & \\
\hline 1102 & ATR Radioisotope Production and Purification Study & & & & & & & & & & $\mathbf{X}$ & $\mathrm{X}$ & & \\
\hline 1171 & Neutron Delivery System Study & & & & & & & & & & $\mathbf{X}$ & $X$ & & \\
\hline 1172 & High Energy Computational Dosimetry & & & & & & & & & & $\bar{X}$ & $\mathrm{X}$ & & \\
\hline 1173 & Real-Time Measurement Dosimetry for BNCT & & & & & & & & & & $\mathrm{X}$ & $\mathrm{x}$ & & \\
\hline 1201 & Photo-Catalytic Treatment of Anionic Cyanides & & & & & & & $\mathrm{X}$ & & & & $\mathrm{X}$ & & \\
\hline 1202 & $\begin{array}{l}\text { Catalytic Destruction of Halogenated Organics at } \\
\text { Supercritical Fluid Conditions }\end{array}$ & $\mathrm{X}$ & & & & & & $\mathrm{X}$ & $\mathrm{X}$ & & & $\mathrm{X}$ & & \\
\hline
\end{tabular}




\begin{tabular}{|c|c|c|c|c|c|c|c|c|c|c|c|c|c|c|c|c|}
\hline Project & \begin{tabular}{|l} 
Project Name \\
\end{tabular} & 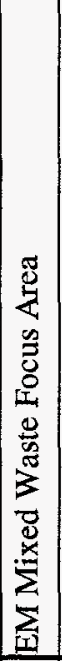 & 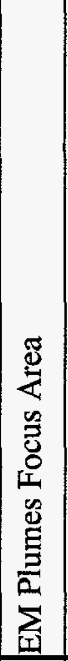 & 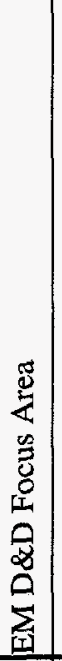 & 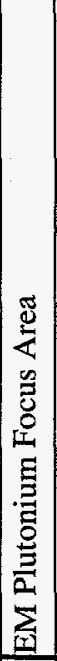 & 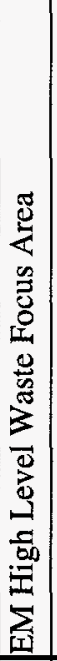 & 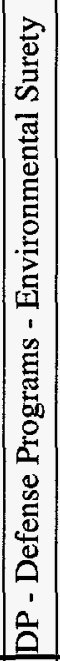 & 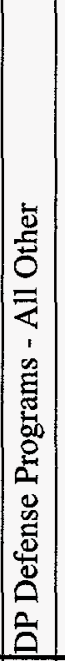 & 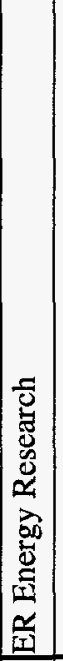 & 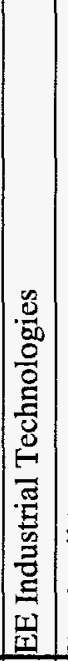 & 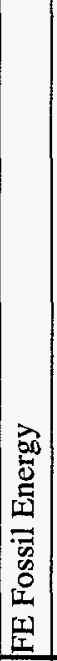 & 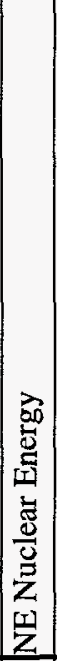 & 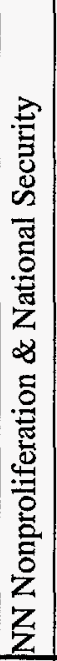 & 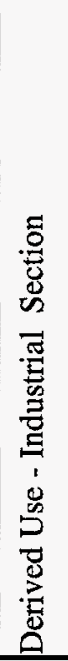 & 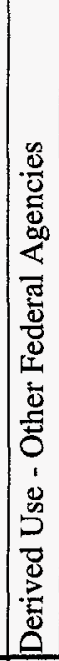 & 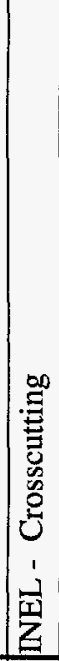 \\
\hline 1203 & $\begin{array}{l}\text { Destruction of PCBs in Oil Using Gamma Irradiation } \\
\text { and Ozone }\end{array}$ & $\mathrm{X}$ & & & & & & & & & $\mathrm{X}$ & & & $\mathrm{X}$ & & \\
\hline 1206 & $\begin{array}{l}\text { Alkylation Catalysts for Operation at Supercritical Fluid } \\
\text { Conditions }\end{array}$ & & & & & & & & & $\mathrm{X}$ & $\mathbf{X}$ & & & $\mathrm{X}$ & & \\
\hline 1233 & $\begin{array}{l}\text { Bioprocess Development for the Remediation of CR(VI) } \\
\text { and U(VI) Contaminated Soils and Groundwater }\end{array}$ & & $\mathbf{X}$ & & & & & & & & & & & $\mathbf{X}$ & & \\
\hline 1241 & Mixed Microbial Encapsulated Fertilizer Amendments & & & & & & & & & & & & & $X$ & & \\
\hline 1301 & Sensors for the Control of Thermal Spray Processes & & & & & & & & $\mathrm{X}$ & $\mathrm{X}$ & $\mathrm{X}$ & & & $\mathrm{X}$ & & \\
\hline 1302 & Heat Transfer Through Solid Cryogen & & & & & & & & & $\mathrm{X}$ & $\mathrm{X}$ & & & $\mathrm{X}$ & $\mathrm{X}$ & \\
\hline 1303 & Concentration Technologies & & & & & & & & & & & & & $\mathrm{X}$ & & \\
\hline 1306 & $\begin{array}{l}\text { Ultraviolet VOC Destruction Technology, Liner } \\
\text { Lifetime and By-product Testing with Hazardous Waste } \\
\text { Disposal Considerations }\end{array}$ & & $\mathbf{X}$ & & & & & & & & & & & $\mathrm{X}$ & & \\
\hline 2104 & $\begin{array}{l}\text { Portable Photon Analysis Spectrometer (PPAS) for TRU } \\
\text { and Y Assay }\end{array}$ & $\mathrm{X}$ & & & & & & & & & & $\mathrm{X}$ & & & & \\
\hline 2108 & Mixed Oxide Fuels Testing in the Avanced Test Reactor & & & & & & & & & & & $\mathrm{X}$ & & & & \\
\hline 2111 & $\begin{array}{l}\text { General Purpose Time-Dependent Particle Transport } \\
\text { with Isotope Generation and Depletion }\end{array}$ & & & & & & & $\mathrm{X}$ & & & & $\mathrm{X}$ & $\mathrm{X}$ & & & \\
\hline 2205 & $\begin{array}{l}\text { Development of a Simplified Version of } \\
\text { SCDAP/RELAPS for Simulator Applications }\end{array}$ & & & & & & & & & & & $\mathrm{X}$ & & $\mathrm{X}$ & & \\
\hline 2207 & $\begin{array}{l}\text { Non-Fertile Fuel Fabrication Development and } \\
\text { Irradiation Performance Assessment }\end{array}$ & & & & & & & $\mathrm{X}$ & & & & $\mathbf{X}$ & & & & \\
\hline 2278 & Site-Specific Technologies for Agriculture: Pilot Project & & & & & & & & & & & & & $\mathrm{X}$ & & \\
\hline 2302 & Photoneutron Studies for Radiotherapy & & & & & & & & & & & $\mathrm{X}$ & & $\mathrm{X}$ & & \\
\hline 2303 & $\begin{array}{l}\text { Feasibility Study of Using INEL Developed Transport } \\
\text { Tools to Calculate Photodynamic Dosimetry }\end{array}$ & & & & & & & & & & & $\mathrm{X}$ & & $\mathrm{X}$ & & \\
\hline 2304 & Kinetic Modeling of the ITER Dissipative Divertor & & & & & & & & $\mathrm{X}$ & & & & & & & \\
\hline 2501 & Development of Nondestructive Fatigue Monitor & & & & & & & & & $\mathrm{X}$ & $\mathrm{X}$ & & & $\mathrm{X}$ & & \\
\hline 3203 & $\begin{array}{l}\text { Conceptual Design of a } 55 \text { Gallon Waste Container } \\
\text { Refurbishment System }\end{array}$ & & $\mathrm{X}$ & & & & & & & & & & & $\mathrm{X}$ & & \\
\hline 3302 & Advanced Barcode (Holotag) Reader & $\mathrm{X}$ & & & & & & & & & & & & $\mathrm{X}$ & & \\
\hline 3601 & Transportation Network Core Software & $\mathrm{X}$ & & & & & & & & $\mathrm{X}$ & & & & $\mathrm{X}$ & & \\
\hline
\end{tabular}




\begin{tabular}{|c|c|c|c|c|c|c|c|c|c|c|c|c|c|c|c|}
\hline Project & Project Name & 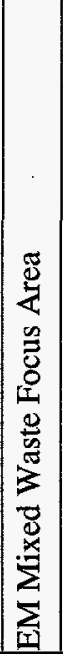 & 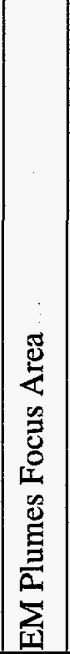 & 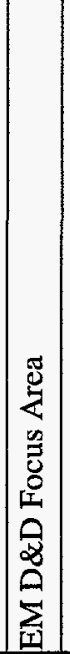 & 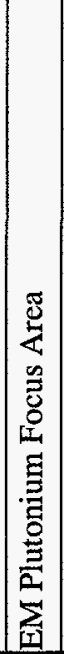 & 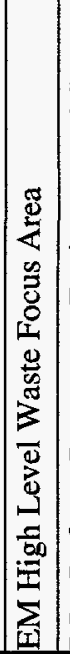 & 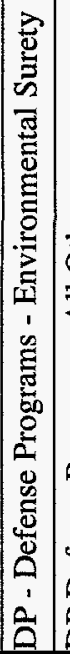 & 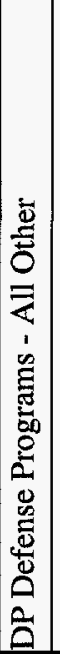 & 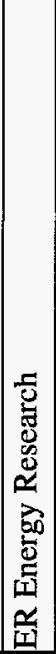 & 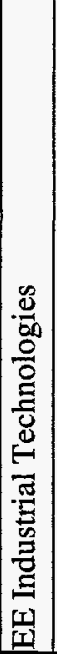 & 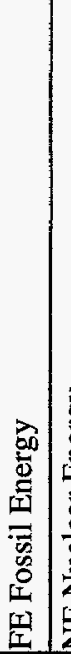 & $\begin{array}{cc} & \\
& \\
0 \\
0\end{array}$ & 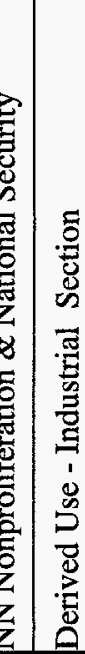 & 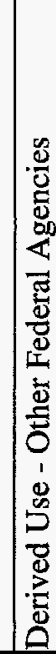 & 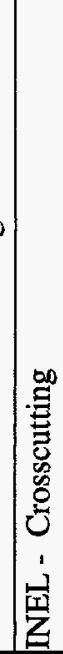 \\
\hline 3710 & $\begin{array}{l}\text { Photodissociation of Mercuric Chloride Treatability } \\
\text { Study }\end{array}$ & $\mathrm{X}$ & & & & & & & $\mathrm{X}$ & & & & $\mathrm{X}$ & & \\
\hline 3711 & $\begin{array}{l}\text { Optical Sensor Development for High Voltage Metering } \\
\text { and Protection Applications }\end{array}$ & & & & & & & & & & & & $\mathrm{X}$ & & \\
\hline 4061 & $\begin{array}{l}\text { Development of Chemically Selective Surfaces for } \\
\text { Adsorption and Detection of Gas-Phase Contaminants }\end{array}$ & $\mathrm{X}$ & & & & & & & & & & & & & \\
\hline 4073 & Alloys by Design & & & & & & & & $\mathrm{X}$ & $\mathrm{X}$ & & & $\mathrm{X}$ & & \\
\hline 4076 & Improved Process Control Thermocouples & $\mathrm{X}$ & & & & & & & & $\mathrm{X}$ & & & $\mathrm{X}$ & & \\
\hline 4078 & $\begin{array}{l}\text { INEL-U of I Laboratory for Liquid Extraction and Ion } \\
\text { Exchange Research }\end{array}$ & & $\mathrm{X}$ & & & & & & & $\mathrm{X}$ & & & $\mathrm{x}$ & & \\
\hline 4080 & National Infrastructure Renewal & & & $\mathrm{X}$ & & & & & & $\mathrm{x}$ & $\mathrm{X}$ & & $\mathbf{X}$ & $\mathrm{X}$ & \\
\hline 4081 & Optical Measurement Technology & & & $\mathrm{X}$ & & & & $\mathrm{X}$ & $\mathbf{X}$ & & & & $\mathrm{X}$ & $\mathrm{X}$ & \\
\hline 4082 & Velocity Sensor for Control of Thermal Spray & & $\mathrm{X}$ & & & & & & $\mathrm{X}$ & $X$ & & & $X$ & & \\
\hline 4090 & $\begin{array}{l}\text { Fracture Mechanics-Based Design Methodologies for } \\
\text { Metal Matrix Composite Material }\end{array}$ & & & & & & & & $\mathbf{X}$ & $\mathbf{X}$ & & & $\mathbf{X}$ & & \\
\hline 4103 & Hybrid Electric Vehicle Evaluation & & & & & & & & & & & & $\mathrm{X}$ & & \\
\hline 4105 & $\begin{array}{l}\text { Demand Side Management Control System for Electric } \\
\text { Vehicles }\end{array}$ & & & & & & & & & $\mathrm{X}$ & & & $\mathrm{X}$ & & \\
\hline 4110 & $\begin{array}{l}\text { Develop, Build, and Test Assay System to Measure } \\
\text { TRU and Fission Product Contamination in Subsurface } \\
\text { Soil }\end{array}$ & & & & & & & & & $\mathrm{X}$ & & & $\mathrm{X}$ & & \\
\hline 4151 & $\begin{array}{l}\text { Development of Intelligent Cooperative Miniature } \\
\text { Robot Systems }\end{array}$ & $\mathrm{X}$ & & & & & & & & $\mathrm{X}$ & & & $\mathrm{X}$ & & \\
\hline 4157 & Natural Gas Locomotives and Transportable Power & & & & & & & & & $\mathrm{X}$ & & & $\mathrm{X}$ & & \\
\hline 4171 & Biodecontamination of Massive Concrete Structures & & & $\mathrm{X}$ & & & & & & & & & $\mathrm{X}$ & & \\
\hline 4172 & Catalyst Assited in-Situ Bioremediation & & $\mathbf{X}$ & & & & & & & & & & $\mathrm{X}$ & & \\
\hline 4184 & Loom Feedback Controls & & & & & & & & & $\mathrm{X}$ & & & $\mathrm{X}$ & & \\
\hline 4203 & $\begin{array}{l}\text { Highly Dynamic Materials Process Modeling Using } \\
\text { Particle Methods }\end{array}$ & & & & & & & & & $\mathrm{X}$ & & & $\mathbf{X}$ & & \\
\hline 4204 & Sustainable Water Resources Management & & $\mathrm{X}$ & & & & & & & & & & & & \\
\hline 4205 & $\begin{array}{l}\text { Advanced Plasma Processes for Black Liquor } \\
\text { Gasification }\end{array}$ & & & & & & & & & $\mathrm{X}$ & & & $\mathrm{X}$ & & \\
\hline 4206 & $\begin{array}{l}\text { Joining and Testing of Small Scale Automotive } \\
\text { Components }\end{array}$ & & & & & & & & & $\mathrm{X}$ & & & $\mathrm{X}$ & & \\
\hline 4208 & $\begin{array}{l}\text { Process Control Development for Ultracapacitor } \\
\text { electrodes by Liquid Injection Plasma Deposition } \\
\text { (LIPD) }\end{array}$ & & & & & & & & & $\mathrm{X}$ & & & $\mathrm{X}$ & & \\
\hline
\end{tabular}




\begin{tabular}{|c|c|c|c|c|c|c|c|c|c|c|c|c|c|c|c|c|}
\hline Project & . & 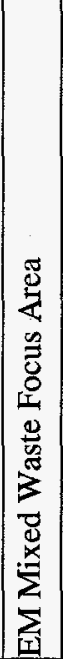 & 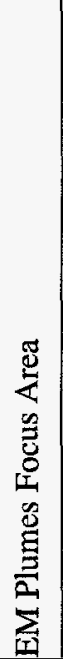 & 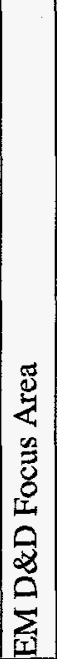 & 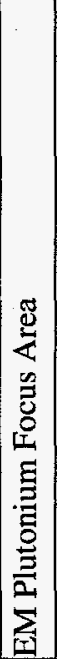 & 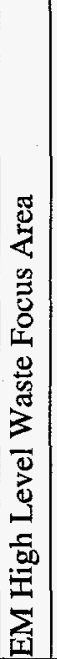 & 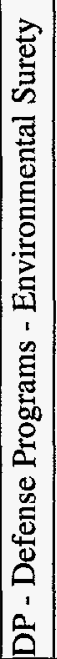 & 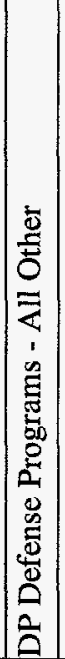 & 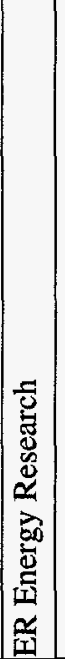 & 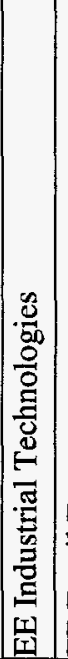 & 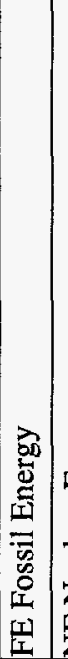 & 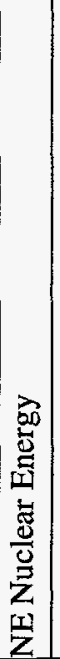 & 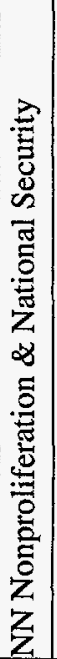 & 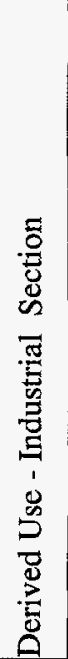 & 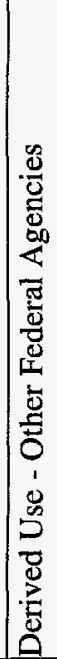 & 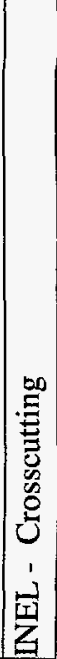 \\
\hline 4275 & $\begin{array}{l}\text { High Sensitivity NDA for Transuranic Waste } \\
\text { Characterization }\end{array}$ & $\mathbf{X}$ & & & & & & & & & & $X$ & & & & \\
\hline 4374 & Flexible Fish Conduit Passive System & & & & & & & & & & & & & $\mathbf{X}$ & & \\
\hline 4395 & MAPS Solid Oxide Fuel Cell & & & & & & & & $\mathrm{X}$ & $\mathrm{X}$ & & & & $\mathrm{X}$ & & \\
\hline 4396 & MAPS Thermo Photovoltaic & & & & & & & & $\mathrm{X}$ & $\mathrm{X}$ & & & & $\mathbf{X}$ & & \\
\hline 4468 & Optimization Based SIMPLEV & & & & & & & & & $\mathrm{X}$ & & & & $\bar{X}$ & & \\
\hline 4469 & $\begin{array}{l}\text { Hybrid Vehicle Component Hardware-in-the-Loop } \\
\text { Feasibility Study }\end{array}$ & & & & & & & & & $\mathrm{X}$ & & & & $\mathbf{X}$ & & \\
\hline $4 \overline{470}$ & Software Metrics Program & $\mathrm{X}$ & & & & & & & & & & & & & & $\mathrm{X}$ \\
\hline 4479 & Manufacturing Research & $\mathrm{X}$ & & & & & & & & $\mathrm{X}$ & & & & $\mathrm{X}$ & & \\
\hline 4480 & Building Materials & & & $\mathrm{X}$ & & & & & & $\mathrm{X}$ & & & & $\mathbf{X}$ & & \\
\hline 4481 & Smart HVAC Systems Design & $\mathrm{X}$ & & & & & & & & $\mathrm{X}$ & & & & $\bar{X}$ & & \\
\hline 4482 & $\begin{array}{l}\text { High Temperature Porous Cermet Filters for the Paper } \\
\text { and Pulp Industry }\end{array}$ & & & & & & & & & $\mathrm{X}$ & & & & $\mathbf{X}$ & & \\
\hline 4483 & $\begin{array}{l}\text { Silent Discharge Plasma for Treatment of Automobile } \\
\text { Exhaust }\end{array}$ & $\mathrm{X}$ & & & & & & & & $\mathbf{X}$ & & & & $\mathbf{X}$ & $\mathbf{X}$ & \\
\hline 4485 & $\begin{array}{l}\text { Evaluation of Liquid Metal Technologies Applicable to } \\
\text { Radioactively-Contaminated Scrap Metal (RSM) } \\
\text { Recycling }\end{array}$ & & & $\mathbf{X}$ & & & & & & & & & & $X$ & & \\
\hline 4486 & INEL Metal Recycle: Induction Melting & $\mathrm{X}$ & & & & & & & & $\mathrm{X}$ & & & & $\mathrm{X}$ & & \\
\hline 4487 & $\begin{array}{l}\text { Process Industries - Industrial Improvement Using } \\
\text { Integrated Process Evaluation: Food Processing, Pulp } \\
\text { and Paper, Steel/Aluminum }\end{array}$ & & & & & & & & & $\mathrm{X}$ & & & & $\mathrm{X}$ & & \\
\hline 4488 & $\begin{array}{l}\text { Depleted Uranium Flywheels for Stationary } \\
\text { Applications }\end{array}$ & & & & & & & & & $\mathbf{X}$ & & & & $\mathrm{X}$ & & \\
\hline 4550 & $\begin{array}{l}\text { Development of a Methodology for Assessment of } \\
\text { Power Plant Emissions on Air Quality Related Values }\end{array}$ & $\mathrm{X}$ & & & & & & & & $\mathrm{X}$ & & & & $\mathrm{X}$ & & \\
\hline 4579 & Passive Containment of Volatile Organic Soil Vapors & & $\mathrm{X}$ & & & & & & & & & & & & & \\
\hline 4581 & Development of Vadose Zone Monitoring & & $\mathrm{X}$ & & & & & & & & & & & & & \\
\hline 4758 & $\begin{array}{l}\text { Simulation Based Prototyping for Manufacturing Based } \\
\text { Engineering }\end{array}$ & & & & & & & & & $\mathbf{X}$ & & & & $\mathrm{X}$ & & \\
\hline 4759 & Human and Organizational Systems Readiness & $\mathrm{X}$ & & & & & & & & & & & & & & $\mathrm{X}$ \\
\hline 4860 & Butterfat Management & & & & & & & & & $\mathrm{X}$ & & & & $\mathrm{X}$ & & \\
\hline 4862 & Advanced Process Diagnostics System & $\mathbf{X}$ & & & & & & & & $\mathrm{X}$ & & & & $\mathrm{X}$ & & \\
\hline 4863 & Intelligent Sensor Data Acquisition Analysis & $\mathrm{X}$ & & & & & & & & $\mathrm{X}$ & $\mathrm{X}$ & & & $\mathrm{X}$ & & \\
\hline 4864 & Modeling Underground Structures & & & $\mathrm{X}$ & & & & & & & & & & & & \\
\hline 4865 & $\begin{array}{l}\text { Neural Analysis Techniques for Field Deployable } \\
\text { Environmental Sensors }\end{array}$ & & $\mathrm{X}$ & & & & & & $\mathrm{X}$ & $x$ & & & & & & \\
\hline 4866 & Miniature Tag and Covert Communicator & $\mathrm{X}$ & & & & & & $\mathrm{X}$ & & & & & $\mathrm{X}$ & & & \\
\hline
\end{tabular}




\begin{tabular}{|c|c|c|c|c|c|c|c|c|c|c|c|c|c|c|c|c|}
\hline Project & $\begin{array}{r}\text { Project Name } \\
\end{array}$ & 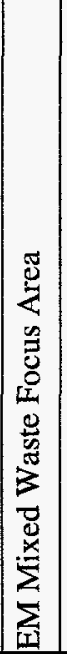 & 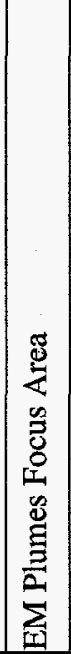 & 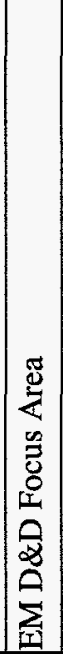 & 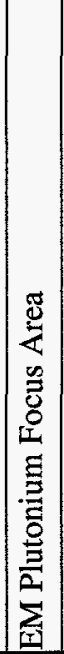 & 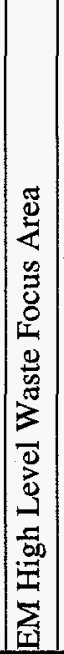 & 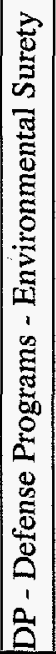 & 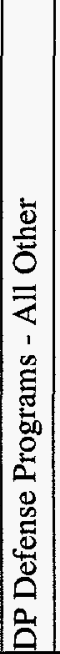 & 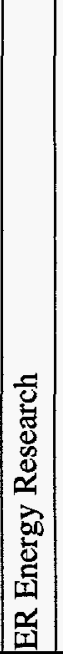 & 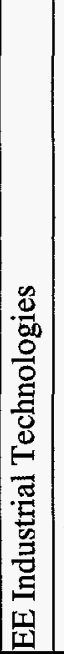 & 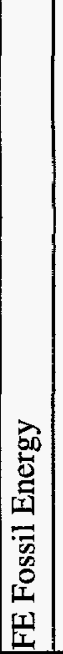 & 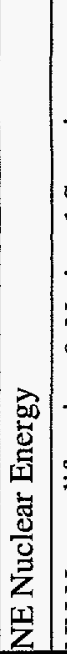 & 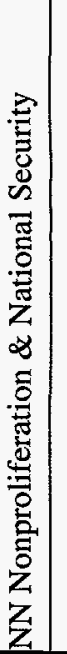 & 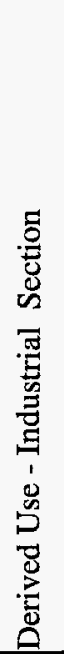 & 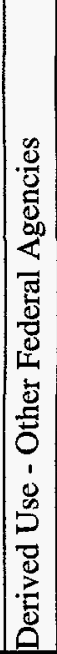 & 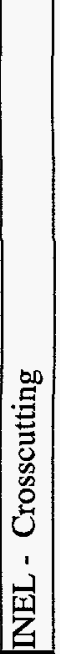 \\
\hline 4952 & INEL Multimedia Object Database & & $\mathrm{X}$ & & & & & & & & & & & & & $\mathrm{X}$ \\
\hline 4953 & General User Interface Development Environment & & $\mathrm{X}$ & & & & & & & & & & & & & $\mathrm{X}$ \\
\hline 4954 & Intelligent Data Access and Retrieval System & $\mathrm{x}$ & & & & & & & & & & & & & & $\mathrm{X}$ \\
\hline 4955 & $\mathrm{C}++$ SAGE & & & & & & & & & & & & & & & $\mathbf{X}$ \\
\hline 4956 & Fractional Frame Augmentation & & & & & & & & & & & & & & & $\mathbf{X}$ \\
\hline 5201 & $\begin{array}{l}\text { Development of Prompt Gamma Assay Method for } \\
\text { Mining of Phosphate Ore }\end{array}$ & & & & & & & & & $\mathrm{X}$ & & & & $\mathbf{X}$ & & \\
\hline 5203 & $\begin{array}{l}\text { Grouping and Modeling Ecosystem Components for } \\
\text { Ecological Risk Assessment }\end{array}$ & & $\mathrm{X}$ & & & & & & & $\mathrm{X}$ & & & & $\mathbf{X}$ & & \\
\hline 5204 & $\begin{array}{l}\text { Develop Intelligent Farm Management System Database } \\
\text { Prototype }\end{array}$ & & & & & & & & & & & & & $\mathbf{X}$ & & \\
\hline 5205 & Water Shutoff in Producing Oil Wells Utilizing Bacteria & & & & & & & & & & $\mathrm{x}$ & & & $\mathrm{X}$ & & \\
\hline 5301 & Estimating Sorption Properties of Clay Materials & & & & & & & & & & $\mathrm{X}$ & & & $\mathbf{X}$ & & \\
\hline 5303 & $\begin{array}{l}\text { Identification of a Surfactant/Alcohol/Polymer System } \\
\text { for Safe Mobilization of TCE }\end{array}$ & & $\mathrm{X}$ & & & & & & & & & & & $\mathrm{X}$ & & \\
\hline 5304 & Automated Neutron Probe Monitoring & & $\mathrm{X}$ & & & & & $\mathbf{X}$ & & & & & & & & \\
\hline 5306 & Measurement of Potato Water Content & & & & & & & & & & & & & $\bar{X}$ & & \\
\hline 5308 & $\begin{array}{l}\text { Numerical Investigation of Dynamic Pressure Seals in } \\
\text { Geologically Active Basins }\end{array}$ & & & & & & & & & & $\mathrm{X}$ & & & $\mathrm{X}$ & & \\
\hline 5601 & Aquatic Vascular Plant Control & & & & & & & & & & & & & & & \\
\hline 5604 & $\begin{array}{l}\text { Evaluation of Potential Antimicrobial Properties of } \\
\text { Malted Barley and its Agricultural Applications }\end{array}$ & & & & & & & & & & & & & $\mathrm{X}$ & & \\
\hline 5606 & $\begin{array}{l}\text { Biological System for Organic Acid Removal with } \\
\text { Minimal Effluent }\end{array}$ & & & & & & & & & & $\mathrm{x}$ & & & $\mathrm{X}$ & & \\
\hline 5608 & Bioprocessing of Indigo Textile Dye Waste Effluent & & & & & & & & & & & & & $\mathrm{X}$ & & \\
\hline 5609 & Isolation of Gossypol Degrading Bacteria & & & & & & & & & & & & & $\mathrm{X}$ & & \\
\hline 5610 & Microstructural Influences on Biocorrosion & & & & & & & & $\mathbf{X}$ & $\mathrm{X}$ & $\mathrm{X}$ & & & $\mathrm{X}$ & & \\
\hline 5614 & $\begin{array}{l}\text { Removal of Methanol from Forest Products Industry Off } \\
\text { gases by Biofiltration }\end{array}$ & & & & & & & & & $\mathbf{X}$ & & & & $\mathrm{X}$ & & \\
\hline 5615 & System for Growing Conifer Embryos & & & & & & & & & $\mathrm{X}$ & & & & $\mathbf{X}$ & & \\
\hline 6111 & $\begin{array}{l}\text { Improved Transformation and Regeneration Techniques } \\
\text { for Cotton }\end{array}$ & & & & & & & & & & & & & $\mathrm{X}$ & & \\
\hline 6503 & $\begin{array}{l}\text { Nutrient Diffusing Substrata to Conduct Rational } \\
\text { Bioremediation }\end{array}$ & & $\mathrm{X}$ & & & & & & & & & & & & & \\
\hline
\end{tabular}




\begin{tabular}{|c|c|c|c|c|c|c|c|c|c|c|c|c|c|c|c|}
\hline Project & Project Name & 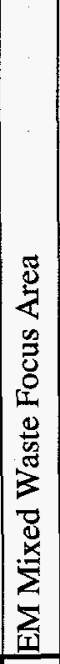 & 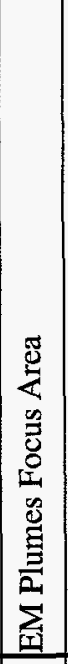 & 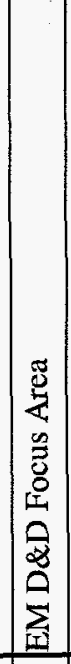 & 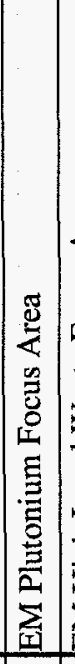 & 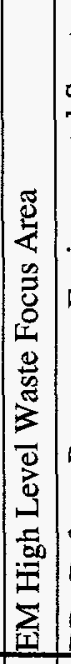 & 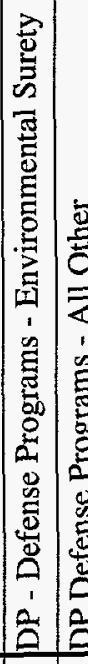 & 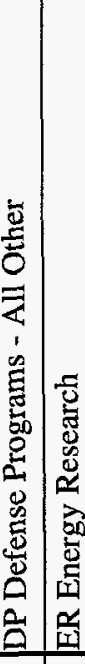 & 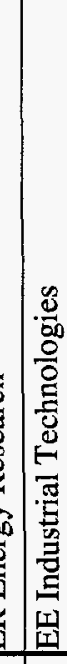 & 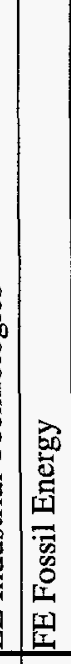 & 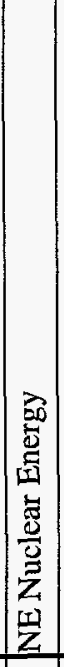 & 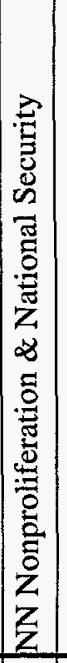 & 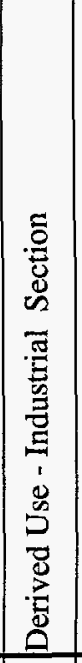 & 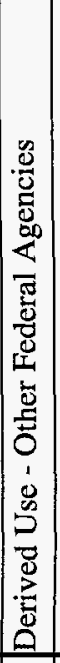 & 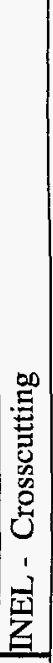 \\
\hline 6701 & $\begin{array}{l}\text { An Intermediate to Field Scale Model for Predicting } \\
\text { Porous Media Microbial Transport }\end{array}$ & & $\mathrm{X}$ & & & & & & & & & & & & \\
\hline 6808 & Compressed Gas Safety Valve & & & & & & & & & & & & $\mathrm{X}$ & & \\
\hline 7105 & Internal-Reforming SOFC Modeling & & & & & & & & $\mathrm{X}$ & & & & $\mathrm{X}$ & & \\
\hline 7203 & $\begin{array}{l}\text { Advanced Methods for Adaptive Control for Automated } \\
\text { Traffic Management }\end{array}$ & & & & & & & & $\mathrm{X}$ & & & & $\mathrm{X}$ & & \\
\hline 7204 & System Dynamics Model of Intermodalism & & & & & & & & $X$ & & & & & & $\bar{x}$ \\
\hline 7603 & $\begin{array}{l}\text { Advanced Combined Environments Test Station } \\
\text { (ACETS) Scoping Studies }\end{array}$ & $\mathrm{x}$ & & & & & & $\mathrm{X}$ & & $\mathrm{x}$ & & & $\mathrm{X}$ & $\mathrm{X}$ & \\
\hline 7907 & $\begin{array}{l}\text { PGNAA Methods Development for Waste Assay } \\
\text { Applications }\end{array}$ & & $x$ & & & & & & & & & & $\mathrm{X}$ & & \\
\hline 7909 & Tritium Production by Irradiation of $3 \mathrm{He}$ & & & & & & & $\mathrm{X}$ & & & $\mathrm{X}$ & & & & \\
\hline 8104 & Real-Time Asbestos Analysis by Raman Spectroscopy & & & $\mathrm{x}$ & & & & $\mathrm{X}$ & & & & & $\mathrm{X}$ & & \\
\hline 8106 & Rapid Agrochemical Sensor & & & & & & & & & & & & $\mathrm{X}$ & & \\
\hline 8403 & In-Situ Biodegradation of High Energy Materials & & $\mathrm{X}$ & & & & & $\mathrm{X}$ & & & $\mathrm{x}$ & $\mathrm{x}$ & & & \\
\hline 8409 & $\begin{array}{l}\text { Rapid Laser Assay of Surface Contamination During } \\
\text { D\&D }\end{array}$ & & & $\mathrm{X}$ & & & & & & & & & $\mathrm{X}$ & & \\
\hline 8414 & $\begin{array}{l}\text { Measurement of Component Ratio in Oil/Water/Gas } \\
\text { Mixtures and Determination of Flow Regime }\end{array}$ & & & & & & & & & $\mathrm{x}$ & & & $x$ & & \\
\hline 8415 & Alternate Joining Techniques for Textiles & & & & & & & & & & & & $\mathrm{X}$ & & \\
\hline 8416 & $\begin{array}{l}\text { Real-Time, On Loom Knit and "Hand" Characterization } \\
\text { Using Diffractive Optical Methods. }\end{array}$ & & & & & & & & & & & & $\mathrm{X}$ & & \\
\hline 8501 & $\begin{array}{l}\text { Characterization of Aged Materials with NDE and } \\
\text { Internal Friction Measurements }\end{array}$ & & & & & & & $\mathrm{X}$ & $\mathrm{X}$ & $x$ & & & & & \\
\hline 8506 & $\begin{array}{l}\text { Micromechanical Analysis Capability for Composite } \\
\text { Materials }\end{array}$ & & & & & & & $\mathrm{X}$ & $\mathrm{X}$ & $\mathrm{X}$ & & & $\mathrm{x}$ & & \\
\hline 8507 & Metal Matrix Composites from Novel Nitride Preforms & & & & & & & $\mathrm{X}$ & $\mathrm{x}$ & $x$ & & & $\mathrm{x}$ & & \\
\hline 8509 & $\begin{array}{l}\text { Polyethylene Sulfur: Feasibility as a Waste } \\
\text { Encapsulation Material for Low-Level and Mixed } \\
\text { Wastes }\end{array}$ & $\mathrm{x}$ & & & & & & & $\mathrm{X}$ & & & & $\mathrm{X}$ & & \\
\hline 8510 & $\begin{array}{l}\text { Cooperative Industrial Research with GE Lighting } \\
\text { Products }\end{array}$ & & & & & & & $\mathrm{X}$ & $\mathrm{X}$ & & & & $\mathrm{x}$ & & \\
\hline 8511 & $\begin{array}{l}\text { Lifetime Extension and Increased Reliability for } \\
\text { Refinery Structures }\end{array}$ & & & & & & & & $\mathrm{X}$ & $\mathrm{x}$ & & & $\mathrm{X}$ & & \\
\hline 8514 & $\begin{array}{l}\text { In-Field Assay for PPM Levels of Cr in Environmental } \\
\text { Media }\end{array}$ & & $\mathrm{x}$ & & & & & & & & & & $\mathrm{x}$ & & \\
\hline 8515 & Solar Bimodal Technologies Demonstration & & & & & & & & & & & & & $\mathrm{X}$ & \\
\hline
\end{tabular}




\begin{tabular}{|c|c|c|c|c|c|c|c|c|c|c|c|c|c|c|c|c|}
\hline Project & $\begin{array}{l}\text { Project Name } \\
\end{array}$ & 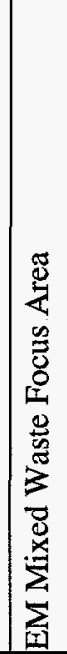 & 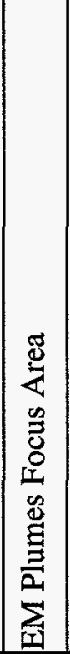 & 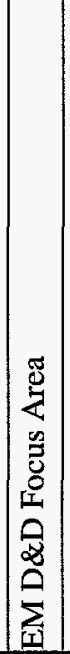 & 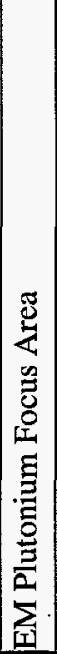 & 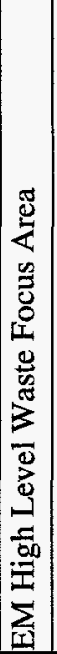 & 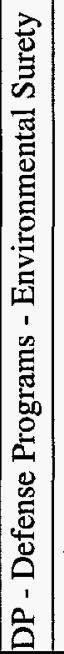 & 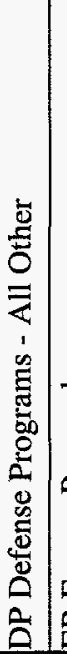 & 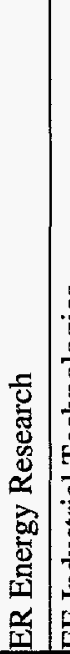 & 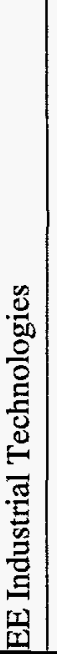 & 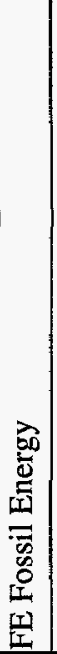 & 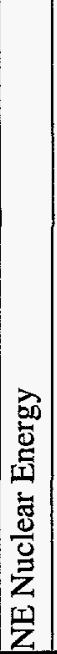 & 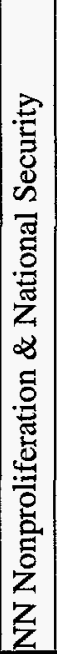 & 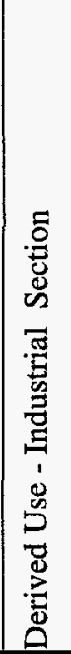 & 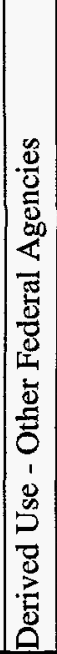 & 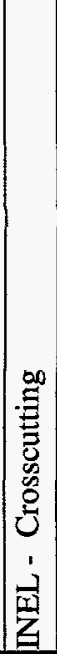 \\
\hline 8516 & $\begin{array}{l}\text { Weight in Motion (WIM) Sensor and Signal Analysis } \\
\text { Technology }\end{array}$ & & $\mathrm{X}$ & & & & & & & $\mathrm{X}$ & & & & $\mathrm{x}$ & $\mathrm{X}$ & \\
\hline 8517 & $\begin{array}{l}\text { Communications Spectrum Needs for Short Range } \\
\text { IVHS Communications }\end{array}$ & & & & & & & & & $\mathrm{X}$ & & & & $\mathrm{X}$ & $\mathrm{x}$ & \\
\hline 8518 & $\begin{array}{l}\text { Developing a Nonlinear Electromagnetic Modeling } \\
\text { Capability using the Finite-Difference Time Domain } \\
\text { Method }\end{array}$ & & & & & & & & $\mathrm{X} X$ & $\mathrm{X}$ & & & & $\mathrm{X}$ & & \\
\hline 8614 & Electro Chemical-Based Processing Technologies & & $\mathrm{X}$ & & & & & & & $\mathrm{X}$ & & & & $\mathrm{x}$ & & \\
\hline 8615 & $\begin{array}{l}\text { Low temperature Reductive Catalytic Destruction of } \\
\text { Halogenated Organics }\end{array}$ & & $\mathrm{X}$ & & & & & & $\mathrm{X}$ & $\mathrm{X}$ & & & & $\mathrm{X}$ & & \\
\hline 8616 & Nonaqueous Processing in the Textile Industry & & & & & & & & & & & & & $\mathrm{x}$ & & \\
\hline 8708 & Off-Line Manufacturing Simulation & $\mathbf{X}$ & & & & & & & & $\mathrm{X}$ & & & & $\mathrm{X}$ & & \\
\hline 8712 & Spray Forming Titanium Alloys into Billet and Sheet & $\mathrm{X}$ & & & & & & & & $\mathrm{X}$ & & & & $\mathrm{x}$ & & \\
\hline 8806 & Industrially Compatible Laser Ultrasonics & $\bar{x}$ & & & & & & & $\mathrm{X}$ & $\bar{X}$ & & & & $\bar{x}$ & & \\
\hline 9508 & Optimization of Automobile Component Shapes & & & & & & & & & $\mathrm{X}$ & & & & $\mathrm{X}$ & & \\
\hline 9510 & Periphyton Communities for Environmental Monitoring & & & & & & & & & & & & & & & \\
\hline 9511 & Mediator & & & & & & & & & & & & & & & $\mathrm{X}$ \\
\hline 9512 & Gas Driven Photovoltaic Generators & & & & & & & & \begin{tabular}{|l|l|l}
$X$ & $X$ \\
\end{tabular} & $\mathrm{X}$ & & & & $\mathrm{X}$ & & \\
\hline 9513 & Tolo Lake Mammoth Excavation & & & & & & & & & & & & & & $\bar{X}$ & \\
\hline 9514 & Measurement of Crude Oil Corrosivity & & & & & & & & & $\bar{X}$ & $\bar{X}$ & & & $\mathrm{X}$ & & \\
\hline 9515 & $\begin{array}{l}\text { Installation of Precession X-Ray Camera for Single } \\
\text { Crystal Analysis }\end{array}$ & & & & & & & & $\mathrm{X}$ & & & & & $\mathrm{x}$ & & \\
\hline 9516 & $\begin{array}{l}\text { Direct Production of Low Cost High Quality Titanium } \\
\text { Powder by Mechanical Alloying }\end{array}$ & & & & & & & & $\mathrm{X}$ & $\mathrm{X}$ & & & & $\mathrm{X}$ & & \\
\hline 9517 & Technetium & & & & & & & & & & & $\mathrm{X}$ & & $\mathrm{X}$ & & \\
\hline W007 & Alternate Material for Stainless Steel Lined Concrete & $\mathrm{x}$ & & & & & & & & $\mathrm{X}$ & & & & $x$ & & \\
\hline W016 & $\begin{array}{l}\text { Oxidative Processes for the Remediation of } \\
\text { Contaminated Soils }\end{array}$ & & $\mathrm{X}$ & & & & & & & & & & & $\mathrm{x}$ & & \\
\hline \begin{tabular}{|l|l|} 
W019 \\
\end{tabular} & Solid Phase Microextraction & $\mathrm{X}$ & & & & & & & $\mathbf{X}$ & $\mathrm{X}$ & & & & $\mathrm{X}$ & & \\
\hline W027 & $\begin{array}{l}\text { Synthesis and Characterization of Pt/Pd Compounds for } \\
\text { a Fiber Optic Based Sensor for VOCs }\end{array}$ & & & & & & & & $\mathrm{X}$ & & & & & $\mathrm{X}$ & & \\
\hline W029 & ICPP Testbed Framework and Field Tests & $\mathrm{X}$ & & & & & & & & & & $\mathrm{X}$ & & & & \\
\hline W030 & On-Site F-18 Production & & & & & $\mathbf{X}$ & & & & & & $\mathrm{X}$ & & $\mathrm{X}$ & & \\
\hline W032 & Electrocatalytic Vapor Sensor & $\mathrm{X}$ & & & & & & & & & & & $\mathrm{X}$ & $\mathrm{X}$ & & \\
\hline W036 & WERC University Design Competition & & & & & & & & & & & & & & & $\mathbf{X}$ \\
\hline
\end{tabular}




\begin{tabular}{|c|c|c|c|c|c|c|c|c|c|c|c|c|c|c|c|c|}
\hline Project & Project Name & 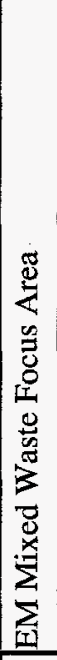 & 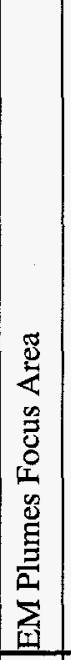 & 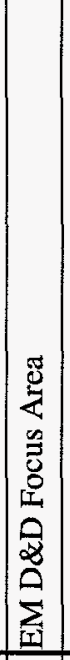 & 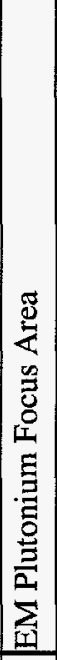 & 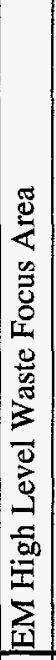 & 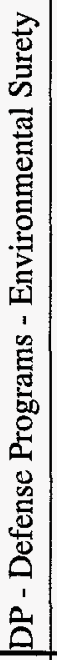 & 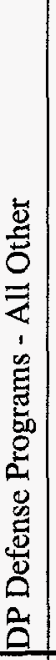 & 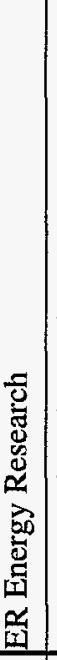 & 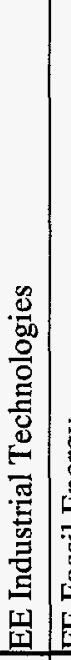 & 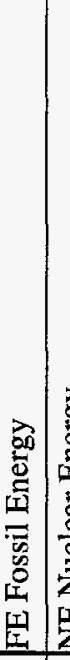 & 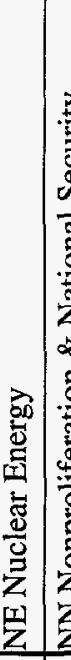 & 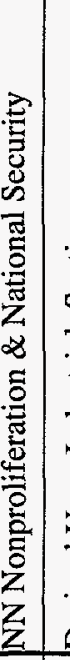 & 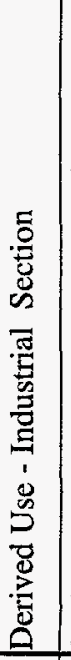 & 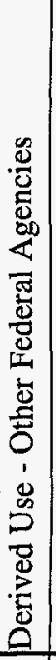 & 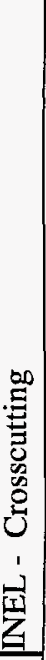 \\
\hline W043 & $\begin{array}{l}\text { Characterization of Fuel Processing/Waste Form } \\
\text { Chemistry by High Temperature Mass Spectrometry }\end{array}$ & & & & & $\mathrm{X}$ & & & & $\mathrm{X}$ & & & & $\mathrm{X}$ & & \\
\hline W089 & $\begin{array}{l}\text { Telepresence for Mobile Robotics in Nuclear } \\
\text { Environments }\end{array}$ & & & $\mathrm{x}$ & & & & & & & & $\mathrm{X}$ & & $\underline{x}$ & & \\
\hline W091 & Petroleum Tanks Inspection Delivery System & & & & & & & & & & $\mathbf{X}$ & & & $x$ & & \\
\hline W097 & $\begin{array}{l}\text { Sodium-Bearing Waste Treatment Technology for } \\
\text { Separating the Nitrate Anion and Alkali Salts Using Ion- } \\
\text { Exchange }\end{array}$ & & & & & & & & & & & & & & & \\
\hline W104 & $\begin{array}{l}\text { The Effect of Thermal Treatment of INEL Soil Prior to } \\
\text { Cementitious Solidification }\end{array}$ & & $\mathrm{x}$ & & & & & & & & & & & & & \\
\hline W109 & $\begin{array}{l}\text { Automatic Pressurized Filtering of Environmental } \\
\text { Samples for Remote Applications }\end{array}$ & & $\mathrm{x}$ & & & & & & & & & & & $\mathrm{X}$ & & \\
\hline W111 & $\begin{array}{l}\begin{array}{l}\text { Position Tracking for Remote Subsurface } \\
\text { Characterization Systems }\end{array} \\
\end{array}$ & & $\mathrm{X}$ & & & & & & & & & & & $X$ & & \\
\hline W122 & Evaluation of Delphi Oxidation for Activated Carbon & & $\mathrm{X}$ & & & & & & & & & & & $\mathrm{X}$ & & \\
\hline W123 & Evaluation of EET Proprietary Decontamination & & $\mathrm{X}$ & & & & & & & & & & & $\mathrm{x}$ & & \\
\hline 0010 & Molecular Genetics & & $\mathrm{X}$ & & & & & & & $\mathrm{X}$ & & & & $\bar{X}$ & & $\bar{X}$ \\
\hline 0020 & Nuclear Physics & & $\mathrm{X}$ & & & & & $\mathrm{X}$ & & $\mathrm{X}$ & & & & $\overline{\mathbf{X}}$ & & $\overline{\mathrm{X}}$ \\
\hline 0030 & Condensed Matter & $\mathrm{X}$ & & & & & & & & \begin{tabular}{|l|l|l}
$\mathrm{X}$ & $\mathrm{X}$ & $\mathrm{x}$ \\
\end{tabular} & $\bar{X}$ & $\bar{X}$ & & $\bar{X}$ & & $\bar{X}$ \\
\hline 0040 & Theoretical Chemistry & & $\mathrm{X}$ & & & & & & & $\overline{\mathrm{X}}$ & & & & $\bar{X}$ & & $\bar{X}$ \\
\hline 0050 & Computational Mechanics & & & & & & & & & $\bar{X}$ & & & & $\bar{X}$ & & $\bar{X}$ \\
\hline 0060 & Nonconventional Bioprocessing & & $\mathrm{X}$ & & & & & & & $\mathrm{X}$ & & & & $\bar{X}$ & & $\mathrm{X}$ \\
\hline 0070 & Radiation Measurements & & & $\mathbf{X}$ & & & & & & & & $\bar{X}$ & & $\bar{X}$ & & $\bar{X}$ \\
\hline 0080 & Nuclear Fuels and Materials & & $\mathrm{X}$ & & & & & & & & & $\bar{X}$ & & $\overline{\mathbf{x}}$ & & $\overline{\mathbf{X}}$ \\
\hline 0090 & Experimental Thermal Science & $\bar{X}$ & & & & & & & $\mathrm{X}$ & $\mathrm{X}$ & 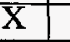 & & & $\mathrm{X}$ & & $\mathbf{X}$ \\
\hline
\end{tabular}




\section{Appendix E Publications}




\section{LTRI 0010}

Bruhn, D.F. and F.F. Roberto, 1995, "Introduction of Arsenic Resistance Genes into Acidiphilium," in preparation.

Bruhn, D.F., J. Li, F.F. Roberto, S. Silver, and B. Rosen, 1995, "Comparison of the Restriction Maps of the Arsenic Resistance Regions of Plasmids R773 and R46," in preparation.

Stoner, D.L., C.A. Browning, D.K. Bulmer, T.E. Ward, and M.T. MacDonell, 1995, "Direct 5S rRNA Assay for Microbial Community Characterization," in preparation.

Stoner, D.L., C.A. Browning, D.K. Bulmer, T.E. Ward, and M.T. MacDonell, 1995, "Direct 5 S rRNA Assay for Monitoring Mixed-Culture Bioprocesses," submitted.

Ward, T.E. and D. Bulmer, 1995, "Cloning of the Acidiphilium recA Gene and Construction of recAcidiphilium Mutants," in preparation.

\section{LTRI 0020}

Barrette, Jean, for the E877 Collaboration (BNL, GSI, INEL, McGill Univ., Univ. of Pittsburgh, Univ. Univ. of de Sao Paulo, SUNY Stony Brook, Wayne State Univ.), J. Barrette, R. Bellwied, S. Bennett, P. Braun-Munzinger, W.E. Cleland, M. Clemen, J.D. Cole, T.M. Cormier, G. David, J. Dee, Y. Kwon, R. Lacasse, A. Lukaszew, Q. Li, T.W. Ludlam, S.K. Mark. S. McCorkle, R. Matheus, D.

Miskowiec, J.T. Murgatroyd, E. O'Brien, S. Panitkin, T. Piazza, C. Pruneau, M.N. Rao, M. Rosati, N.C. da Silva, S. Sedykh, U. Sonnadara, J. Stachel, N. Starinsky, E.M. Takagui, S. Voloshin, G. Wang, J.P. Wessels, C.L. Woody,
N. Xu, Y. Zhang, and C. Zou, 1995, "Directed Flow and Particle Production in $\mathrm{Au}+\mathrm{Au}$ Collisions from Experiment 877," submitted to QM95.

Barrette, J., R. Bellwied, S. Bennett, P. Braun-Munzinger, W.E. Cleland, M. Clemen, J.D. Cole, T.M. Cormier, G. David, J. Dee, O. Dietzsch, M.W. Drigert, J.R. Hall, T.K. Hemmick, N. Herrmann, B. Hong, Y. Kwon, R. Lacasse, A. Lukaszew, Q. Li, T.W. Ludlam, S.K. Mark, S. McCorkle, R. Matheus, E. O'Brien, S. Panitkin, T. Piazza, C. Pruneau, M.N. Rao, M. Rosati, N.C. daSilva, S. Sedykh, U. Sonnadara, J. Stachel, N. Starinsky, E.M. Takagui, S. Voloshin, G. Wang, J.P. Wessels, C.L. Woody, N. Xu, Y. Zhang, and C. Zou, 1995, “Charged Particle Pseudorapidity Distributions in $\mathrm{Au}+\mathrm{Al}, \mathrm{Cu}, \mathrm{Au}$ and $\mathrm{U}$ Collisions at 10.8 A.GeV/c," Phys. Rev. C51, 3309 .

Barrette, J., R. Bellwied, S. Bennett, P. Braun-Munzinger, W.E. Cleland, M. Clemen, J. Cole, T.M. Cormier, G. David, J. Dee, O. Dietzsch, M. Drigert, S. Gilbert, J.R. Hall, T.K. Hemmick, N. Herrmann, B. Hong, C.L. Jiang, Y. Kwon, R. Lacasse, A. Lukaszew, Q. Li, T.W. Ludlam, S. McCorkle, S.K. Mark, R. Matheus, E. O'Brien, S. Panitkin, T. Piazza, C. Pruneau, M.N. Rao, M. Rosati, N.C. daSilva, S. Sedykh, U. Sonnadara, J. Stachel, H. Takai, E.M. Takagui, S. Voloshin, G. Wang, J.P. Wessels, C.L. Woody, N. Xu, Y. Zhang, Z. Zhang, and C. Zou (E877 Collaboration ), 1994, "Observation of Anisotropic Event Shapes and Transverse Flow in Ultrarelativistic $\mathrm{Au}+\mathrm{Au}$ Collisions," Phys. Rev. Lett. 73, 2532.

Bersch, R., for the E877 Collaboration (BNL, GSI, INEL, McGill Uni- versity, Univ. of Pittsburgh, SUNY-Stony Brook, Univ. of Sao Paulo, Wayne State University, Yale University), 1995, "A Geometric Charge Division Pad Chamber for $\mathrm{Au}+\mathrm{Au}$ Collisions at the AGS," Bull. Am. Phys. Soc. 40,920 .

Butler-Moore, K., R. Aryaeinejad, J.D. Cole, Y. Dardenne, R.C.

Greenwood, J.H. Hamilton, A.V. Ramayya, W.C. Ma, B.R.S. Babu, J.O. Rasmuessen, M.A. Stoyer, S.Y. Chu, K.E. Gregorich, S. Asztalus, S.G. Prussin, K. Moddy, and R. Lougheed, "Evidence of Unique Parity Band Structure in NeutronRich Odd-A Ru Isotopes," Phys. Rev. C. (in press).

Butler-Moore, K., R. Aryaeinejad, J.D. Cole, Y. Dardenne, R.C. Greenwood, and H.M. Winston, 1995, "Application of X-rays in Prompt Spectroscopy of ${ }^{252} \mathrm{Cf}$ Fission Fragment," Nucl. Instr. and Methods A361, 245.

Drigert, M.W., J.D. Cole, E.L. Reber, and J.M. Young, "Report on a Field-Portable VME-based Distributed Data Acquisition System," presented at Ninth Conference on Real-Time Computer Applications in Nuclear, Particle, and Plasma Physics, May 23-26, East Lansing, Michigan.

Fornal, B., R.H. Mayer, I.G. Bearden, Ph. Benet, R. Broda, P.J. Daly, Z.W. Grabowski, I. Ahmad, M.P. Carpenter, P.B. Fernandez, R.V.F. Janssens, T.L. Khoo, T. Lauritsen, E.F. Moore, and M.W. Drigert, 1994, "Gamma-Ray Studies of Neutron-Rich sdf Shell Nuclei Produced in Heavy Ion Collisions," Phys. Rev. C49, 2413.

Hall, J.R., for the E877 Collaboration (BNL, GSI, INEL, McGill University, U. of Pittsburgh, U. of Sao 
Paulo, SUNY-Stony Brook, Wayne State University), 1994, "Charged Particle Multiplicity in $\mathrm{Au}+\mathrm{Au}$ Collisions at AGS Energy," Bull. Am. Phys. Soc. 39, 1418.

Hamilton, J.H., A.V. Ramayya, J. Kormicki, W.-C. Ma, Q. Lu, D. Shi, J.K. Deng, S.J. Zhu, A. Snadelescu, W. Greiner, G.M. Ter-Akopian, Yu Ts, Oganessian, G.S. Popeko, A.V. Daniel, J. Kliman, V. Polhorsky, M. Morhac, J.D. Cole, R. Aryaeinejad, I.Y. Lee, N.R. Johnson, and F.K. McGowan, 1994, "Zero Neutron Emission in Spontaneous Fission of ${ }^{252} \mathrm{Cf}$ : a Form of Cluster Radioactivity," J. Phys. G20, L85.

Hamilton, J.H., G.M. Ter-Akopian, Yu. Ts. Oganessian, A.V. Daniel, J. Kormicki, G.S. Popeko, A.V.

Ramayya, Q. Lu, K. Butler-Moore, W.-C. Ma, S. Cwiok, W.

Nazarewicz, W. Greiner, A. Sandulescu, J.K. Deng, D. Shi, J. Kliman, M. Morhac, J.D. Cole, R. Aryaeinejad, S.J. Zhu, R. Babu, N.R. Johnson, I.Y. Lee, F.K. McGowan, and J.X. Saladin, 1995, "A New Spontaneous Fission Mode for ${ }^{252}$ Cf: Hyperdeformation, Cluster Radioactivity, New Levels," Presented at European Physical Society, XV Nuclear Physics Divisional Conference, Low Energy Nuclear Dynamics, April 1822, 1995, St. Peterburg, Russia.

Hamilton, J.H., Q.-H. Lu, S.J. Zhu, K. Butler-Moore, A.V. Ramayya, W.-C. Ma, B.R.S. Babu, T.N. Ginter, J. Kormicki, J.K. Deng, D. Shi, L.K. Peker, J.O. Rasmussen, M.A. Stoyer, S.Y. Chu, K.E. Gregorich, M.F. Mohar, S. Prussin, J.D. Cole, R. Aryaeinejad, N.R. Johnson, I.Y. Lee, F.K. McGowan, G.M. TerAkopian, and Yu. Ts. Oganessian, 1995, "Extended Identical Bands in $98,100 \mathrm{Sr}$ and $108,110 \mathrm{Ru}$ and New High Spin States in ${ }^{98-102} \mathrm{Zr}$ and 112-116Pd," International Conference on Exotic Nuclei and Atomic Masses, June 19-23, 1995, Arles France. Hamilton, J.H., G.M. Ter-Akopian, Yu. Ts. Oganessian, J. Kormicki, S.J. Zhu, M.G. Wang, Q.-H. Lu, K. Butler-Moore, A.V. Ramayya, W.C. Ma, B.R.S. Babu, D. Shi, J.K. Deng, G.S. Popeko, A.V. Daniel, W. Greiner, A. Sandulescu, J.D. Cole, R. Aryaeinejad, J. Kliman, V. Polhorsky, M. Morhac, N.R. Johnson, I.Y. Lee, F.K. McGowan, and L.K. Peker, 1995, "Structure of Neutron-Rich Nuclei and Neutron Multiplicities in Spontaneous Fission," Phys. Rep. 101-100.

Hamilton, J.H., A.V. Ramayya, S.J. Zhu, G.M. Ter-Akopian, Ts.Yu. Oganessian, J.D. Cole, J.O. Rasmussen, and M. Stoyer, 1995, "New Insights from Studies of Spontaneous Fission with Large Detector Arrays," Prog. Part. Nucl. Phys. 35, 635-704.

Kliman, J., V. Polhorsky, M. Morhac, G.M. Ter-Akopian, Yu Ts, Oganessian, G.S. Popeko, A.V. Daniel, J.H. Hamilton, K. ButlerMoore, A.V. Ramayya, W.-C. Ma, Q. Lu, J.K. Deng, J. Kormicki, J.D. Cole, R. Aryaeinejad, R.C. Greenwood, S.S. Harrill, N.D.

Lohstreter, I.Y. Lee, N.R. Johnson, and F.K. McGowan, 1994, "Correlated Yields and the Emissions of Neutrons of Mo/Ba Fragments from ${ }^{252} \mathrm{Cf}$," Phys. Rev. Lett. 73 , 1477.

Kwon, Y., for E877 Collaboration (BNL, GSI, INEL, McGill University, Univ. of Pittsburgh, SUNYStony Brook, Univ. of Sao Paulo, Wayne State University, Yale University), 1995, "Realistic Simulation of E877 Spectrometer Through GEANT," Bull. Am. Phys. Soc. 40,920 .

Lu, Q., K. Butler-Moore, S. Zhu, J.H. Hamilton, B.R.S. Babu, A.V.
Ramayya, W.C. Ma, V.E. Oberacker, J. Kormicki, D. Shi, J.K. Deng, D.B. Wang, L.K. Peker, J.D. Cole, R. Aryaeinejad, Y.X. Dardenne, M.Drigert, M.R. Johnson, I.Y. Lee, F.K. McGowan, G.M. Ter-Akopian, Yu Ts, Oganessian, J.O. Rasmussen, M.A. Stoyer, S.Y. Chu, K.E. Gergorich, M. Mohar, J.M. Nitschke, K. Moody, R. Lougheed, S.G. Prussin, and S. Asztalos, 1994, "New Level Structures in Neutron Rich Nuclei from Spontaneous Fission," Conference on Physics from Large Gamma-ray Detector Arrays, LBL, Aug. 2-6, 1994.

Lu, Q., K. Butler-Moore, J.H. Hamilton, B.R.S. Babu, A.V. Ramayya, W.C. Ma, V.E. Oberacker, J. Lormicki, D. Shi, J.K. Deng, S. Zhu, D.B. Wang, L.K. Peker, J.D. Cole, R. Aryaeinejad, Y.X. Dardenne, M. Drigert, N.R. Johnson, I.Y. Lee, F.K. McGowan, G.M. TerAkopian, Yu. Oganessian, J.O. Rasmussen, M.A. Stoyer, S.Y. Chu, K.E. Gregorich, M. Mohar, J.M. Nitschke, K. Moody, R. Lougheed, S.G. Prussin, and S. Asztalos, 1994, "Identical Yrast and GammaVibrational Bands in $108,110 \mathrm{Ru}, "$ Bull. Am. Phys. Soc. 39, 1393.

Lu, Q.-H., K. Butler-Moore, S.J. Zhu, J.H. Hamilton, A.V. Ramayya, V.E. Oberacker, W.-C. Ma, B.R.S. Babu, J.K. Deng, J. Kormicki, J.D. Cole, R. Aryaeinejad, Y.X. Dardenne, M. Drigert, L.K. Peker, J.O. Rasmussen, M.A. Stoyer, S.Y. Chu, K.E. Gregorich, I.Y. Lee, M.F. Mohar, J.M.

Nitschke, N.R. Johnson, F.K. McGowan, G.M. Ter-Akopian, Yu. Ts. Oganessian, and J.B. Gupta, "Structure of 108, 110, 112Ru: Identical Bands in 108, ${ }^{110} \mathrm{Ru}$," Phy. Rev. C.

Miskowiec, D., for the E877 Collaboration (BNL, GSI, INEL, McGill Univ., Univ. of Pittsburgh, SUNY Stony Brook, Univ. of Sao 
Paulo, Wayne State Univ.), 1995,

"Pion-Pion Correlations in $\mathrm{Au}+\mathrm{Au}$ Collisions at AGS Energy," submitted to QM95.

Moore, E.F., W. Henning, R.V.F. Janssens, T.L. Khoo, I. Ahmad, D. Blumenthal, M.P. Carpenter, B. Crowell, D. Gassman, R.G. Henry, R. Lauritsen, C.J. Lister, S.J. Sanders, M.W. Drigert, and D. Nisius, 1994, "Search for the Twophonon Octupole Vibration State in ${ }^{208} \mathrm{~Pb}$," Conference on Physics from Large Gamma-ray Detector Arrays, LBL, Aug. 2-6, 1994.

Moore, E.F., M.P. Carpenter, Y. Liang, R.V.F. Janssens, I. Ahmad, I.G. Bearden, P.J. Daly, M.W. Drigert, B. Formal, U. Garg, Z.W. Grabowski, H.L. Harrington, R.G. Henry, T.L. Khoo, T. Lauritsen, R.H. Mayer, D. Nisius, W.

Nazarewicz, W. Reviol, M. Sferrazza, and T. Werner, 1995, "Collectivity of Dipole Bands in ${ }^{196 \mathrm{~Pb} \text {," }}$ Phys. Rev. C51, 115.

Murgatroyd, J.T., for the E877 Collaboration (BNL, GSI, INEL, McGill University, U. of Pittsburgh, U. of Sao Paulo, SUNYStony Brook, Wayne State University), 1994, "Transverse Energy Production in $\mathrm{Au}+\mathrm{U}$ Collision at AGS Energy," Bull. Am. Phys. Soc. $39,1418$.

Panitkin, S., for E877 Collaboration (BNL, GSI, INEL, McGill University, Univ. of Pittsburgh, SUNY-Stony Brook, Univ. of Sao Paulo, Wayne State University, Yale University), 1995, "Two-Photon Correlations in $10.8 \mathrm{ANGeV} / \mathrm{C}$ $\mathrm{Au}+\mathrm{Au}$ Central Collisions," Bull. Am. Phys. Soc. 40, 935.

Soramel, F., T.L. Khoo, Ph. Benet, K.B. Beard, R.V.F. Janssens, I. Ahmad, I. Bearden, M.P. Carpenter, P.J. Daly, M.W. Drigert, B. For- nal, U. Garg, Z. Grabowski, T. Lauritsen, Y.Liang, R. Mayer, E.F. Moore, W. Reviol, and D. Ye, 1995, "Search for Entrance-Channel Dependence in the Population of SD Bands of 191Hg," Phys. Lett. B350, 173.

Stoyer, M.A., J.O. Rasmussen, F. $\mathrm{Chu}$, K.E. Gregorich, I.Y. Lee, M.F. Mohar, J.M. Nitschke, K. Moody, R. Lougheed, S.G. Prussin, S. Asztalos, R. Aryaeinejad, J.D. Cole, Y.X. Dardenne, M.W. Drigert, J.H. Hamilton, Q.H. Lu, W.C. Ma, and A.V. Ramayya, 1994, “Gammasphere Study of ${ }^{242} \mathrm{Pu}$ Spontaneous Fission Gamma Rays," Conference on Physics from Large Gamma-ray Detector Arrays, LBL, Aug. 2-6, 1994.

Ter-Akopian, G.M., J.H. Hamilton, Yu Ts, Oganessian, J. Kormicki, G.S. Popeko, A.V. Daniel, A.V. Ramayya, Q. Lu, K. Butler-Moore, W.-C. Ma, J.K. Deng, J. Kliman, V. Polhorsky, M. Morhac, W. Greiner, A. Snadelescu, J.D. Cole, R. Aryaeinejad, N.R. Johnson, I.Y. Lee, and F.K. McGowan, 1994, "Neutron Multiplicities and Yields of Correlated $\mathrm{Zr}$-Ce and $\mathrm{Mo}-\mathrm{Ba}$ Fragment Pairs in Spontaneous Fission of ${ }^{252} \mathrm{Cf}$," Phys. Rev. Lett. $73,1477$.

Ter-Akopian, G.M., J.H. Hamilton, Yu Oganessian, J. Kormicki, G.S. Popeko, A.W. Daniel, K. ButlerMoore, A.V. Ramayya, W.C. Ma, Q. Lu, J.K. Deng, J. Kliman, V. Polhorsky, M. Morhac, J.D. Cole, R. Aryaeinejad, N.R. Johnson, I.Y. Lee, and F.K. McGowan, 1994, "Yields and Neutron Multiplicities of Correlated Pairs in Spontaneous Fission of ${ }^{252} \mathrm{Cf}$," Conference on Physics from Large Gamma-ray Detector Arrays, LBL, Aug. 2-6, 1994.

Voloshin, Sergei A., for E877 Collaborations (BNL, GSI, INEL,
McGill University, University of Pittsburgh, SUNY Stony Brook, University of Sau Paulo, Wayne State University), 1995, "dNch/dh Distributions in $\mathrm{Au}+\mathrm{Al}, \mathrm{Cu}, \mathrm{Au}$, and U Collisions," submitted to QM95.

Wessels, J., for E877 Collaboration (BNL, GSI, INEL, McGill University, Univ. of Pittsburgh, SUNYStony Brook, Univ. of Sao Paulo, Wayne State University, Yale University), 1995, “Quantitative Analysis of Flow Observed in $\mathrm{Au}+\mathrm{Au}$ Collisions at the AGS," Bull. Am. Phys. Soc. 40, 936.

Zhang, Yingchao and Johannes P. Wessels for the E877 Collaboration (BNL, GSI, INEL, McGill Univ., Univ. of Pittsburgh, SUNY Stony Brook, Univ. of Sao Paulo, Wayne State Univ.), 1995, “Energy Flow and Particle Spectra with Respect to the Reaction Plane for Au on Au Collisions at AGS Energies," submitted to QM95.

Zhu, S.J., J.H. Hamilton, A.V. Ramayya, B.R.S. Babu, Q. Lu, W.C. Ma, T.N. Ginter, M.G. Wang, J. Kormicki, J.K. Deng, D. Shi, J.D. Cole, R. Aryaeinejad, J.O. Rasmussen, M.A. Stoyer, S.Y. Chu, K.E. Gregorich, M.F. Mohar, S.G. Prussin, Ter-Akopian, and Y.T. Oganessian, N.R. Johnson, I.Y. Lee, and F.K. McGowan, 1995, "Identification of Levels in ${ }^{160} \mathrm{Sm}$ and High Spin States in $156,158 \mathrm{Sm}$ Nuclei," J. Phys. G: Nucl. Part. Phys. 21 L57.

Zhu, S.J., M.G. Wang, Q. Lu, J.H. Hamilton, A.V. Ramayya, W.-C. Ma, J. Kormicki, B.R.S. Babu, D. Shi, J.K. Deng, I.K. Peker, J.O. Rasmussen, M.A. Stoyer, S.Y. Chu, K.E. Gregorich, M.F. Mohar, S. Asztalos, S.G. Prussin, J.D. Cole, R. Aryaeinejad, Y.X. Dardenne, M. Drigert, K.J. Moody, R.W. 
Lougheed, J.F. Wild, N.R. Johnson, I.Y. Lee, F.K. McGowan, G. TerAkopian, and Y.T. Oganessian, "Octupole Deformation in 142,143 Ba and New Band Structure in Neutron-Rich Ba-Isotopes," Phys. Lett. B (accepted for publication).

Zhu, S.J., J.H. Hamilton, Q.H. Lu, A.V. Ramayya, M.G. Wang, B.R.S. Babu, T.N. Ginter, W.-C. Ma, J.K. Deng, D. Shi, J. Kormicki, J.D. Cole, R. Aryaeinejad, N.R. Johnson, I.Y. Lee, F.K. McGowan, TerAkopian, Y.T. Oganessian, J.O. Rasmussen, M.A. Stoyer, S.Y. Chu, K.E. Gregorich, M.F. Mohar, and S.G. Prussin, "Identification of ${ }^{152} \mathrm{Ce}$ and Unexpected Variations in Moments of Inertia With Neutron Number and Spin in 142${ }^{148} \mathrm{Ba},{ }^{144-152} \mathrm{Ce}$, and ${ }^{146-156} \mathrm{Nd}^{\prime \prime}, J$. Phys. G: Nucl. Part. Phys. (accepted for publication).

Zhu, S.J., M.G. Wang, Q. Lu, J.H. Hamilton, A.V. Ramayya, W.-C. Ma, J. Kormicki, B.R.S. Babu, T.N. Ginter, D. Shi, J.K. Deng, I.K. Peker, J.O. Rasmussen, M.A. Stoyer, S.Y. Chu, K.E Gregorich, M.F. Mohar, S.G. Prussin, J.D. Cole, R. Aryaeinejad, N.R. Johnson, I.Y. Lee, F.K. McGowan, G. Ter-Akopian, and Ts. Yu. Oganessian, 1995, "Octupole Deformation in Neutron-Rich ${ }^{142,143} \mathrm{Ba}$ and ${ }^{144} \mathrm{Cs}$ and Identical Ground and Octupole Bands in $144,146 \mathrm{Ba}$," International Conference on Exotic Nuclei and Atomic Masses, June 19-23, 1995, Arles France.

Zhu, S.J., J.H. Hamilton, A.V. Ramayya, Q. Lu, W.-C. Ma, B.R.S. Babu, J. Kormicki, T.N. Ginter, D. Shi, J.K. Deng, I.K. Peker, J.O. Rasmussen, M.A. Stoyer, S.Y. Chu, K.E Gregorich, M.F. Mohar, S.G. Prussin, J.D. Cole, R. Aryaeinejad, N.R. Johnson, I.Y. Lee, F.K. McGowan, G. Ter-Akopian, and
Ts. Yu. Oganessian, 1995, "Identification of Levels in ${ }^{152} \mathrm{Cs},{ }^{160} \mathrm{Sm}$ and Higher Spin States and Identical Bands in Neutron-Rich $\mathrm{Ce}, \mathrm{Nd}$, Sm Isotopes," International Conference on Exotic Nuclei and Atomic Masses, June 19-23, 1995, Arles France.

Zou, C., for E877 collaboration (BNL, GSI, INEL, McGill University, Univ. of Pittsburgh, SUNYStony Brook, Univ. of Sao Paulo, Wayne State University, Yale University ), 1995, "A Measurement of Inclusive Photon Production in $10.8 \mathrm{ANGeV} / \mathrm{c}$ Au-Au Central Collisions," Bull. Am. Phys. Soc. 40, 935.

\section{LTRI 0030}

Hu, J., C. Pan, Y. Wang, W. Lai, R. Zao, and C.H. Sellers, 1995, "Magnetic Hardening Mechanism of As Gas Atomized Nd(Dy)-Fe-B Powders Bonded Magnet," IEEE Transactions on Magnetics (in press).

Lewis, L.H., C.H. Sellers, and V. Panchanathan, 1995, "Phase Composition and Magnetic Characteristics of Inert Gas Atomized RE ${ }_{2} \mathrm{Fe}_{14} \mathrm{~B}$ Based Powders," IEEE Transactions on Magnetics (in press).

Sellers, C.H., and T.A. Hyde, 1995, "Electromagnetic Measurements for Materials Characterization," Reviews of Quantitative Non Destructive Evaluation (in press).

Van Siclen, C. DeW., 1995, "Single Jump Mechanisms for Large Cluster Diffusion on Metal Surfaces," Physical Review Letters 75, 1574.

Van Siclen, C. DeW., 1995, "Indium Adatom Diffusion and Clustering on Stepped Copper Surfaces," Physical Review B 51, 7796. Van Siclen, C. DeW., 1995, “Migra- tion of Gas Bubbles in Solids Towards Vacancy Sources," Philosophical Magazine Letters 72, 41.

Zong, X., C. Chen, S. Liu, Z. Wu, Y. Chen, R. Zhang, J. Zhu, Y. Chen, B. Evans, R. Gonzalez, and C. H. Sellers, 1995, "RadiationInduced Electrical Degradation in Crystalline $\mathrm{Al}^{203}$ : A Bulk Effect," Journal of Nuclear Materials 219, 176.

\section{LTRI 0040}

Chizmeshya, A., R.A. LaViolette, and G.H. Wolf, 1995, "Thermoelastic Properties and Equation of State of AlN and BeO," J. Mat. Res. (submitted).

Chizmeshya, A., F.M. Zimmerman, R.A. LaViolette, and G.H. Wolf, 1994, "Variational Charge Relaxation in Ionic Crystals: An Efficient Treatment of Statics and Dynamics," Phys. Rev. B 50, 15559.

Ekbundit, S., A. Chizmeshya, R.A. LaViolette, and G.H. Wolf, 1995, "Experimental and Theoretical Study of the High Pressure Behavior of CaS and MgS," Phys. Rev. B (accepted for publication and in press).

Hemati, M., A. Chizmeshya, G.H. Wolf, P.H. Poole, J. Shao, and C.A. Angell, 1995, "Crystalline-Amorphous Transition in Silicate Perovskites," Phys. Rev. B 51, 14841.

LaViolette, R.A., R.A. Berry, and R. McGraw, 1995, "Homogeneous Nucleation of Metals in a Plasma Quench Reactor," Plasma Chem. Plasma Proc. (to appear).

LaViolette, R.A., T.J. Godin, and A.C. Switendick, 1995, "First-Principles Calculation of the Electronic Structure of Condensed SpinPolarized Excited Triplet State Helium," Phys. Rev. B 52, R5487. 
LaViolette, R.A., R.A. Berry, and R. McGraw, 1994, "Nucleation of Metals in a Steady-State Gas Flow" (extended abstract), Proc. INEL Computing Symposium, Idaho Falls, Oct. 4-7, edited by G.L. Mesina.

McGraw, R. and R.A. LaViolette, 1995, "Fluctuations, Temperature, and Detailed Balance in Classical Nucleation Theory," J. Chem. Phys. 102, 8983.

Smith, K.H., E. Shero, A. Chizmeshya, and G.H. Wolf, 1995, "The Equation of State of Polyamorphic Germania Glass: A Two-Domain Description of the Viscoelastic Response," J. Chem. Phys. 102, 6851.

\section{LTRI 0050}

McHugh, P.R. and D.A. Knoll, 1994, "Comparison of Standard and Matrix-Free Implementations of Several Newton-Krylov

Solvers," AIAA J. 32, 2394-2400.

McHugh, P.R. and J.D. Ramshaw, 1995, "Damped Artificial Compressibility Iteration Scheme for Implicit Calculations of Incompressible Flow," Int'l. J. Numer. Meth. Fluids 21, 141-153.

Murray, P.E., "Thermal Strain and Viscous Flow of Glass Films," J. Applied Physics 76, 723-727.

Nguyen, H.D., S. Paik, and R. W. Douglass, 1995, “Unsteady Mixed Convection About a Rotating Circular Cylinder with Small Fluctuations in the Free-Stream Velocity," Int'l J. Heat Mass Transfer (in press). Nguyen, H.D. and S. Paik, 1995, "Thermal Convection in a Spherical Enclosure Containing a fluid Core and a Porous Shell, Int'l J. Heat Mass Transfer, (in press).
Nguyen, H.D., S. Paik, and R.W. Douglass, 1994, "Study of DoubleDiffusive Convection in Layered Anisotropic Porous Media," Numerical Heat Transfer, B 26, 489-505.

Nguyen, H.D., S. Paik, and R.W. Douglass, 1995, "Convective Transport about a Rotating Cylinder with a Surface Reaction of Arbitrary Order," AIChE J. (in press).

Nguyen, H.D. and S. Paik, 1994, "Unsteady Mixed Convection from a Sphere in Water-Saturated Porous Media with Variable Surface Temperature/Heat Flux," Int'l J. Heat Mass Transfer 37, 17831793.

Nguyen, H.D., S. Paik, R.W. Douglass, and I. Pop, 1995, "Unsteady Non-Darcy Reaction-Driven Flow from an Anisotropic Cylinder in Porous Media," Chemical Engineering Science, (in press).

Nguyen, H.D., S. Paik, and A. Rood, 1994, "Effects of Thermally Generated Convection on the Migration of Radionuclides in Saturated Geologic Formation," Int'l J. Eng'rg Sci. 32, 1605-1614.

Nguyen, H.D. and S. Paik, 1995, "Solution Domain Decomposition with Finite Difference Methods for Partial Differential Equations," Numerical Methods P.D.E's 11, 453466.

\section{LTRI 0060}

Cherry, R.S., "Residence Time Distribution in a Compost Biofilter as a Function of Water Content," (in draft form for Biotechnology Techniques (to be submitted December 1995).

Gostomski, P.A., J.B. Sisson, and R.S. Cherry, "Water Content Effect on Biofilter Performance for the Biodegradation of Aromatic Hydrocarbons," Journal of the Air and Water Management Association (internal review in progress, to be submitted October 1995).

Kastner, J.R. and R.S. Cherry, 1994, "Enhanced Biodegradation of Sparingly Soluble Gases Using a Water Soluble Polymer," Proceedings of Seventh International IGT Symposium on Gas, Oil, and Environmental Technology, Colorado Springs, CO, December.

Lynch, N.J., H.D. Nguyen, and R.S. Cherry, 1995, “Design of Passively Aerated Compost Piles: Air Flow in a Passively Aerated Pile," Proceedings of the Science of Composting Conference, Bologna, Italy, June.

Lynch, N.J. and R.S. Cherry, 1995, "Winter Composting Using the Passively Aerated Windrow Compost System," Compost Science and Utilization, (internal review in progress, to be submitted October 1995).

Lynch, N.J. and R.S. Cherry, "A Mathematical Model for the Passively Aerated Windrow Compost System," Biotechnology and Bioengineering (internal review in progress, to be submitted October 1995).

\section{LTRI 0090}

McCreery, G.E., and C.M. Stoots, 1995, "Water Drop Formation in Spray Plate Nozzles," Int. J. Multiphase Flow, (accepted pending revisions).

McCreery, G.E., 1995, “Liquid Flow and Vapor Formation in a Flat Heat Pipe," Heat Transfer Engineering 15, 33-41. 
McEligot, J., and D.M. McEligot, 1994, "Some Research Needs in Convective Heat Transfer for Industry," J. Fluids Engr. 116, 398404.

McEligot, D.M. and M.F. Taylor, 1995, "The Turbulent Prandtl Number in the Near-Wall Region for Low-Prandtl-Number Gas Mixtures," Int. J. Heat Mass Transfer (in press).

Shinpaugh, K.A. and R.L. Simpson, 1995, "A Rapidly Scanning Two-Velocity Component Laser Doppler Velocimeter," Measurement Science and Technology (J. Phys. E), (accepted).

Stoots, C.M. and R.V. Calabrese, 1995, "The Mean Velocity Field Relative to a Rushton Turbine Blade," AIChE Journal 41, 1-11.

\section{LDRD 0102}

Peccia, J., 1995, "The Bioavailability of Selected Aromatic Hydrocarbons: An Extension from Saturated to Unsaturated Soils," Masters Thesis, Montana State University.

Wolfram et al., 1995, "Scale-up Implications of Respirometrically Determined Microbial Kinetic Parameters," Applied Biotechnology for Site Remediation, pp. 300-304.

Wolfram et al., "Engineering Scale-up of In-Situ Bioremediation Processes: A Guideline for Biotreatability for the INEL," Manual.

\section{LDRD 1233}

Turick, C.E., W.A. Apel, and N. S. Carmiol, 1995, "Isolation of Hexavalent Chromium Reducing Anaerobes from Hexavalent
Chromium Contaminated and Noncontaminated Environments," Applied Microbiology and Biotechnology (in press).

\section{LDRD 2108}

Sterbentz, J.W., J.M. Ryskamp, S.C. Mason, and G.S. Chang, 1994, "Mixed Oxide Fuels Testing in the Advanced Test Reactor," Trans. Am. Nucl. Soc., 71, 301.

Terry, W.K., J.M. Ryskamp, J.W. Sterbentz, G.S. Chang, R.G. Ambrosek, and S.L. Hayes, 1995, "Opportunities for Mixed Oxide Fuel Testing in the Advanced Test Reactor to Support Plutonium Disposition," INEL-95/0384, Idaho National Engineering Laboratory.

\section{LDRD 2278}

Hoskinson, R.L., 1995, “Using GIS in the National Site-Specific Technologies for Agriculture (National SST4Ag) Project," presented at the June 1995 Annual International ASAE Meeting in Chicago, IL, paper no. 953239.

\section{LDRD 2302}

Nigg, D.W., H.E. Mitchell, Y.D. Harker, W.Y. Yoon, and J.L. Jones, 1994, "An Accelerator-Based Epithermal Photoneutron Source for BNCT," Proceedings of the Sixth International Symposium on Neutron Capture Therapy for Cancer, Kobe, Japan, October (in press).

Nigg, D.W., H.E. Mitchell, Y.D. Harker, W.Y. Yoon, J.L. Jones, and J.F. Harmon, "Epithermal Photoneutron Source Studies for BNCT," CONF-940976, March.

\section{LDRD 4061}

Delmore, J.E., G.S. Groenewold, E.M. Harrison, and J.C. Ingram, 1995, "Secondary Ion Mass Spectrometric Study of Vapor Phase Adsorption of Organic Acids on Basic Substrates," Int. J. Mass Spectrom. Ion Proc. (to be submitted).

Groenewold, G.S., R.L. Cowan, J.E. Delmore, J.C. Ingram, and A.D. Appelhans, 1995, "Characterization of Bis-(phenoxy)phosphazene Polymers Using Static Secondary Ion Mass Spectrometry," Polymer (to be submitted).

Groenewold, G.S., J.C. Ingram, R.L. Cowan, J.E. Delmore, and A.D. Appelhans, "Detection of Adsorbed Contaminants on Phosphazene Polymers Using Static SIMS" (in preparation).

\section{LDRD 4078}

Carleson, T.E., N.A. Chipman, and C.M. Wai (Editors), 1995, Separation Processes in Nuclear Waste Management, CRC Press, Boca Raton, FL.

\section{LDRD 4082}

Fincke, J.R., W.D. Swank, and D.C. Haggard, 1995, "Feedback Control of the Subsonic Plasma Spray Process: System Model," Thermal Spray Science \& Technology, C.C. Berndt and S. Sampath (editors), ASM International, August.

Fincke, J.R., W.D. Swank, and D.C. Haggard, 1995, "Feedback Control of the Subsonic Plasma Spray Process: Controller Performance," Thermal Spray Science \& Technology, C.C. Berndt and S. Sampath (editors), ASM International, August. 


\section{LDRD 4110}

Brey, R., T. Islam, and L. Johnson, "Use of Triple Proportional Counter Beta Detector for Analysis of $90 \mathrm{Sr}$ Surface Contamination," Health Physics (to be published).

Brey, R., T. Islam, and L. Johnson, "Evaluation of Vendor-Supplied Decontamination Agents for the Decontamination of Lead Bricks, Shot, and Chips," Health Physics (to be published).

\section{LDRD 4205}

Grandy, J.D. and P.C. Kong, 1995, "Energy Considerations for Steam Plasma Gasification of Black Liquor and Chemical Recovery," Proceedings of the 12th International Symposium on Plasma Chemistry, Vol. II, p. 1089.

\section{LDRD 4486}

Worcester, S.A., L.G. Twidwell, D.J. Paolini, T.A.Weldon, and R.E. Mizia, 1994, "Decontamination of Metals by Melt Refining/Slagging," Proceedings of the 1994 International Symposium on Liquid Metal Processing and Casting, Vacuum Metallurgy Division of the American Vacuum Society, Santa Fe, New Mexico, September.

"Decontamination and Decarburization of Radioactively Contaminated Stainless Steel by the Vacuum Induction Melting Process," to be published.

\section{LDRD 4862}

Fryer, Michael O., 1995, "System Health Monitoring," Proceedings of the 41st International Instrumenta- tion Symposium, Aurora, CO, May, sponsored by the Aerospace Industries and Test Measurement Divisions of the Instrument Society of America, pp. 509-519.

\section{LDRD 7105}

Motloch, C.G. and R.G. Bennett, 1995, "Thermo-Chemical Modeling of Internal-Reforming SOFCs at the Idaho National Engineering Laboratory," Proceedings of the Fourth International Symposium on Solid Oxide Fuel Cells (SOFC-IV) Yokohama, Japan, June 18-23.

\section{LDRD 8106}

Ingram, J.C., G.S. Groenewold, A.D. Appelhans, and J.E. Delmore, "Direct Surface Analysis of Pesticides on Soil, Leaves, Grass, and Stainless Steel by Static Secondary Ion Mass Spectrometry, "Environmental Science \& Technology (in preparation).

\section{LDRD 8511}

Carlson, N.M. and R.E. Rice, 1995, "NDE for Lifetime Extension and Increased Reliability for Refinery Structures," Inspectioneering Journal, (submitted, refereed, to go to press in October or November).

Reuter, W.G., A.M. Porter, N.M. Carlson, 1995, "Plant Life Extension and Reliability Issues," Nondestructive Evaluation of Aging Utilities, SPIE Vol. 2454, pp. 47-55.

\section{LDRD 8514}

Currie, L.A., 1968, "Limits for Qualitative Detection and Quantitative Determination," Anal Chem 40, 586-593.
Gehrke, R.J., K.D. Watts, E.W. Killian, M.H. Putnam, and R.G. Helmer, 1994, "PASS, an Extended-Range Ge Spectrometer for Radionuclide Analysis via L x-, gRay Spectrometry," Nuclear Instruments and Methods in Physics Research A353, 109.

Gehrke, R.J. and R.G. Helmer, 1975, "Nickel-59 Excitation Source for X-Ray Fluorescence Analysis of Carbon and Low Alloy Steels," X-Ray Spectrometry 4, 77-84.

Hartwell, J.K. and S.G. Goodwin, 1989, IEEE Trans. Nucl. Sci. NS-36 615.

Johnson, L.O., E.W. Killian, R.G. Helmer, and R. Coates, 1981, IEEE Trans. Nucl. Sci. NS-28, 638.

Reeves, J. H. et al., 1995, "A TubeExcited X-Ray Fluorescence Spectrometer for Use In Small-Diameter Boreholes, Journal of Radioanalytical and Nuclear Chemistry 193, 1, 93-98

Watts, K.D. and R.J. Gehrke, 1995, “EDXRF Measurement of PPM Concentration Levels of Chromium in Soil Using 59Ni," (Submitted for publication in September).

\section{LDRD 8518}

"A Simplified PML for Use with the FDTD Method," (submitted for publication in: IEEE Microwave and guided letters).

\section{LDRD 8614}

Glenn, D. F. and J.C. Ingram, 1994, "Electrodeposition at an Ice-Coated Electrode," J. Electrochem. Soc. 141, 9, p. L113. 


\section{LDRD 8708}

Cordes, Gail A., John M.

Zabriskie, and J. Wess Rhudy, 1995, "Avoiding the Teach Pendant", invited paper at the 1995 ASME Robotics and Automation Symposium, May, 1995, Idaho Falls, Idaho.

\section{LDRD W027}

Exstrom, C.L., J.R. Sowa, Jr., C.A. Daws, D. Janzen, K.R. Mann, G.A. Moore, F.F. Stewart, 1995, "Inclusion of Organic Vapors by Crystalline, Solvatochromic [Pt(ary] isonitrile)4][Pd(CN)4] Compounds-Vapochromic Environmental Sensors," Chemical Materials 1995, 15-17.
Hartenstein, S.D., G.A. Moore, D.W. Daniels, B.H. Clark, 1994, Field Demonstration of Vapochromic Compound-Based Volatile Organic Compound Sensor, INEL-94-0206, November. 


\section{Appendix F \\ Acronyms}


A/O ratios ACETS

ACIP

ACM

AEC

AFB

AGS

AIM

AIPM

ALC

AMD

ANL

APC

APT

AQRVs

ARPA

ARPF

ATR

ATRC

AWU

BASS

BIRL

BNCT

BNFL

BPA

BPLU

BWR

CAA

CCL4

CFD

$\mathrm{cfm}$

CGCC

CGSV

CHA

CLB

CLP

CLPP

$\mathrm{CN}^{-}$

$\mathrm{CNO}^{-}$

Corps

$\mathrm{Cr}$

CRADA

CSTRs

CTAPS

CVD

CVISN

D.I.

$D \& D$

DAP

DEC aqueous to organic phase ratios

Advanced Combined Environments Test Station

Advanced Computer Interface Products asbestos-containing

Atomic Engineering Corporation

Air Force Base

Alternating Gradient Synchrotron

Air Induction Melting

Authenticated In-Plant Monitoring

Air-Line Coax

acid mine drainage

Argonne National Laboratory

air pollution control

accelerator-based tritium production

Air Quality Related Values

Advanced Research Projects Agency

actinide-reduced plutonium fuel

Advanced Test Reactor

ATR critical reactor

Associated Western Universities

Benthic Algal Substrate Sampler

Basic Industry Research Laboratory

Boron Neutron Capture Therapy

British Nuclear Fuel plt

Bonneville Power Administration

Border Profiled Lower-Upper

Boiling Water Reactor

Clean Air Act

carbon tetrachloride

computational fluid dynamics

cubic feet per minute

coal gasification combined cycle

compressed gas safety valve

Cyclohexyl-amine

community-level bioassay

Contract Laboratory Procedures

community-level physiological profiles

cyanide

cyanate

U.S. Army Corps of Engineers

Chromium

Cooperative Research and Development

Agreement

continuous stirred tank reactors

Contingency Theater Air Control System

Automated Planning System

chemical vapor deposition

Commercial Vehicle Information Systems

Network

de-ionized

decontamination and decommissioning

Data Access Products

Digital Equipment Corporation
DEG

DEM

DGGE

DLLS

DOE

DOE-ER

e-beam

EBSL

EDI

EDO

EDOF

EDXRF

EDXS

EFGM

EOPM

EPA

ERG

ETS

FADOF

FAQs

FEM

FET

FMA

FMPS

FTIR

GB

GC

GCMS

GFCR

GNAT

GPB

GPS

GRIS

GRNN

GUIDE

GUI

HDPE

HE

$\mathrm{He}^{*}$

HTML

HVAC

IAQ

ICP-AES

ICPP

IDC

IET

IGC-AS

IMPA

IMTRP diethylene glycol monomethyl ether

diffuse element methods

denaturing gradient gel electrophoresis

Dynamic Link Libraries

Department of Energy

Office of Advanced Energy Programs, Basic

Energy Sciences

electron beam

ecologically-based screening level

electronic data interchange

Engineering Design Optimization

Electronically Dithered Optical Filter

energy dispersive $x$-ray fluorescence

energy dispersive $x$-ray spectroscopy

element-free Galerkin method

electro-optical phase modulator

Environmental Protection Agency

Materials and Aerospace Corporation

Experimental Thermal Science

Faraday Anomalous Dispersion Optical Filter

Frequently Asked Questions

finite element numerical

field effect transistor

Fragment Mass Analyzer

field matric potential sensor

Fourier Transform Infrared

grain boundary

gas chromatography

gas chromatography with mass spectrometry

gas filter correlation radiometry

Gamma Neutron Assay Technique

gas phase bioreactors

Global Positioning System

gamma-ray imaging system

neural network algorithms

Graphical User Interface Development

Environment

Graphical User Interface

high density polyethlene

high explosives

helium atoms

Hyper Text Markup Language

heating, ventilation, and air conditioning

indoor air quality

Inductively Coupled Plasma - Atomic

Emission Spectroscopy

Idaho Chemical Processing Plant

intelligent distributed control

Initial Engine Test

Intermagnetics General Corporation

Advanced Superconductor

isopropylmethylphosphonic acid

Inorganic Membrane Technology Research

Program 


\begin{tabular}{|c|c|c|c|}
\hline INEL & Idaho National Engineering Laboratory & ODBMS & object database \\
\hline IPP & In-flight Particle Pyrometer & ODBMS & object-oriented database \\
\hline IPST & Institute of Paper Science and Technology & OPC & ordinary Portland concrete \\
\hline IPT & Integrated Product Team & ORNL & Oak Ridge National Laboratory \\
\hline IS-Antibodies & Individual-Specific Antibodies & OT & object technology \\
\hline ISOL & isotope separator system & PAF & particle and force \\
\hline ISU & Idaho State University & PAWS & Programmable Automated Welding Systems \\
\hline ITD & Idaho Transportation Department & PCA & principle components analysis \\
\hline \multirow[t]{2}{*}{ ITER } & International Thermonuclear Experimental & PCB & polychlorobiphenyl \\
\hline & Reactor & PCC & Portland cement concrete \\
\hline ITS & Intelligent Transportation Systems & PCE & perchloroethylene \\
\hline KAP & Kuck and Associates pre-processor & PC & personal computer \\
\hline LANL & Los Alamos National Laboratory & PDA & Personal Data Assistant \\
\hline LBL & Lawrence Berkeley Laboratory & PDT & Photodynamic therapy \\
\hline LBLOCA & large break loss-of-coolant accident & PFBC & pressurized fluidized bed combustors \\
\hline LDPE & low density polyethylene & PGNAA & prompt gamma neutron activation analysis \\
\hline LDRD & laboratory directed research and development & PGNAA & prompt gamma-ray neutron activation analysis \\
\hline LHGR & Linear Heat Generation Rate & PID & proportional-integral-differential \\
\hline LIBS & Laser Induced Breakdown Spectroscopy & PIGUI & Platform Independent GUI \\
\hline LIPD & Liquid Injection Plasma Deposition & PLFA & phospholipid fatty acid \\
\hline \multirow[t]{2}{*}{ LMAS } & Lockheed Martin Aeronautical Systems & PMAA & polymethacrylic acid \\
\hline & Company & PML & Perfectly Matched Layer \\
\hline LMIT & Lockheed Martin Idaho Technologies & PMT & photomultiplier tube \\
\hline LMMS & Lockheed Martin Missiles and Space & PNGV & Partnership for a New Generation of Vehicles \\
\hline \multirow[t]{2}{*}{ LMRDD } & Lockheed Martin Research and Development & $\mathrm{PP}$ & polypropylene \\
\hline & Division & PPAS & Portable Photon Analysis Spectrometer \\
\hline LNG & Liquefied Natural Gas & PRC & Photorefractive crystals \\
\hline LTRI & long term research initiative & PRI & Positron Resources Inc. \\
\hline \multirow[t]{2}{*}{ LTRI-CM } & Long Term Research Initiative in Computa- & PS & Polystyrene \\
\hline & tional Mechanics & PSD & Prevention of Significant Deterioration \\
\hline LWR & light water reactor & PTI & Process Technologies, Inc. \\
\hline \multirow[t]{2}{*}{ MCCS } & Multi-spectral Chemical Characterization & PTO & Patents and Trademark Office \\
\hline & System & PTU & pan and tilt unit \\
\hline MDFE & multi-directional finite difference & PUFs & \\
\hline MDI & diphenylmethane diisocynate & $\mathrm{PuO}_{2}$ & plutonia \\
\hline MG & mixed gas & PVC & polyvinyl chloride \\
\hline MHD & magnitohydrodynamics & PWR & Pressurized Water Reactor \\
\hline MIMS & Modular Integrated Monitoring System & QCM & quartz crystal microbalance \\
\hline MIR & Matched-Index-of-Refraction & $\mathrm{QP}$ & quasi-particle \\
\hline MLI & Multi-Layer Insulation & RAL & Remote Analytical Laboratory \\
\hline $\mathrm{MMC}$ & Metal matrix composites & RCRA & Resource Conservation and Recovery Act \\
\hline MOX & mixed oxide & RDD & Research and Development Center \\
\hline MSC & Manufacturing Sciences Corp. & RF & Radio Frequency \\
\hline MWLS & moving weighted least squares & RKH & reproducing kernel Hilbert \\
\hline NABOR & 'neighbor' node method & RKPM & reproducing kemel particle methods \\
\hline NCT & neutron capture therapy & ROD & Record of Decision \\
\hline \multirow[t]{2}{*}{ NCTPC } & National Center for Tooling and Precision & RSCS & remote subsurface characterization system \\
\hline & Components & RSLDV & Rapidly-Scanning Laser Doppler Velocimeter \\
\hline \multirow[t]{2}{*}{ Nd:YAG } & neodymium-doped yttrium aluminum gar- & RSM & radioactively-contaminated scrap metal \\
\hline & net & RSM & Radioactive Scrap Metal \\
\hline NDE & nondestructive evaluation & RSP & rapidly solidified processing \\
\hline NDS & & RWMC & Radioactive Waste Management Complex \\
\hline NFS & Network File Service & $\mathrm{SC}$ & sulfur concrete \\
\hline NIJ & National Institute of Justice & SCADA & supervisory control and data acquisition \\
\hline $\mathrm{NO}$ & nitric oxide & SCF & supercritical fluid \\
\hline
\end{tabular}


SEG

SEM

SETAC

$\mathrm{SF}_{6}$

SHS

SIMS

SLERAs

SLM

SNL

SOFC

SPC

SPH

SPME

SQUID

SRB

SST4Ag

SVE

SVOCs

TAGS

TAN-SMC

TBP

TCE

TCL

TCLP

$\mathrm{TiO}_{2}$

TNT
Scientific Ecology Group

Secondary Electron Microscope

Society of Environmental Toxicology and

Chemistry

sulfur hexafluoride

self propagating combustion synthesis

secondary ion mass spectrometry

screening-level ecological risk assessments

Spatial Light Modulator

Sandia National Laboratory

solid oxide fuel cells

sulfur polymer cement

smoothed particle hydrodynamics

Solid phase microextraction

superconducting quantum interference device

sulfate-reducing bacteria

Site-Specific Technologies for Agriculture soil vapor extraction

semivolatile organic compounds

total absorption gamma-ray spectrometer

Test Area North-Specific Manufacturing

Capability

Tri- $n$-butyl phosphate

trichloroethene

Target Compound List

Toxicity Characteristic Leaching Procedure titanium dioxide

trinitrotoluene
TRU

TRVs

UM

$\mathrm{UO}_{2}$

UofU

URC

US AMP

USU

UT

UV

VIM

VOC

VSC

VSM

VVE

W

WERC

WEST

WGPu

WIM

WMD

WRRTF

WSU

WWW

XRD transuranic

toxicity reference values

University of Minnesota

urania

University of Utah

University Research Consortium

U.S. Automotive Materials Partnership

Utah State University

ultrasonic

ultraviolet

Vacuum Induction Melting

volatile organic compound

vibrating sample magnetometer

vibrating sample magnetometer

vapor vacuum extraction

width

Waste Management Education and Research Western Energy Supply and Transmission weapons-grade plutonium

Weigh-In-Motion

weapons of mass destruction

Water Reactor Research Test Facility

Washington State University

World-Wide Web

$\mathrm{X}$-ray Diffraction 
F-6

Appendix F-Acronyms

Idaho National Engineering Laboratory LDRD FY 1995 Annual Report 